\title{
ENERGY HARVESTING FROM ELLIPTICAL MACHINES: \\ DC-DC CONVERTER DESIGN \\ USING SEPIC TOPOLOGY
}

\begin{abstract}
A Thesis
presented to

the Faculty of California Polytechnic State University, San Luis Obispo
\end{abstract}

\author{
In Partial Fulfillment \\ of the Requirements for the Degree \\ Master of Science in Electrical Engineering
}

By

Martin Kou

June 2012 
(C) 2012

Martin Kou

ALL RIGHTS RESERVED 


\section{COMMITTEE MEMBERSHIP}

TITLE:

ENERGY HARVESTING FROM ELLIPTICAL MACHINES: DC-DC CONVERTER DESIGN USING SEPIC TOPOLOGY
AUTHOR:
Martin Kou
DATE SUBMITTED: June 2012
COMMITTEE CHAIR: $\quad$ Dr. David B. Braun, Professor, Electrical Engineering
COMMITTEE MEMBER: Dr. Ahmad Nafisi, Professor, Electrical Engineering
COMMITTEE MEMBER: $\quad$ Dr. Taufik, Professor, Electrical Engineering 


\begin{abstract}
Energy Harvesting from Elliptical Machines: DC-DC Converter Design Using SEPIC
\end{abstract}

Topology

Martin Kou

Cal Poly's ongoing Energy Harvesting from Exercise Machines (EHFEM) project is a very convenient and cost-effective way for generating DC power from physical exercise and sending it back to the electrical grid as $\mathrm{AC}$ power, providing a renewable energy source for the future. The EHFEM project consists of numerous subprojects involving converting different types of exercise machines for power generation. This project is a continuation of one of the previous subprojects, specifically involving an elliptical machine, and focuses on improving system functionality at different machine settings without altering the elliptical user's experience by selecting a new DC-DC converter design, while keeping the other system components intact. The new proposed DC-DC converter design is based on a non-isolated, PWM-switching single-ended primary inductor converter (SEPIC) topology, as opposed to the resonant zero-current switching/zero-voltage switching (ZCS/ZVS) topology-based off-the-shelf DC-DC converter that the previous project utilized, which had poor system functionality at high physical input levels (greater than $30 \mathrm{~V}$ input) from the elliptical trainer. This project proves that a PWM-switching SEPIC topology provides a functional DC-DC converter design for DC power generation and inverter interfacing from a dynamic input voltage generator because of its wide input voltage range, high power driving capability and inherent voltage step-up and step-down functions. The proposed DC-DC converter supplies up to 288 watts of power and outputs 36 volts, and simultaneously takes 5-65 volts from its input depending on the elliptical user's physical input level. This project details the new DC-DC converter's design and construction processes, compares its topology to other existing DC-DC converter topologies and analyzes unfeasible designs as well as the overall system's performance when converting the generated DC power to $\mathrm{AC}$ power, and documents any potential problems when used for this specific application.

Keywords: DC-DC converter, elliptical machine, energy harvesting, SEPIC, sustainability 


\section{ACKNOWLEDGEMENTS}

First, I would like to thank Dr. David Braun, Dr. Dale Dolan, Dr. Ahmad Nafisi and

Dr. Taufik for their help and guidance for my thesis project as well as for their guidance through my university career. I would also like to thank the following people who contributed to this project:

- Paul Grierson, who provided the Precor elliptical machine to Cal Poly's EE department.

- Sharon Kirk and Curtis Kahnberg from Enphase, who donated two Enphase micro-inverters.

- Dr. John S. Dunning, Cal Poly Mechanical Engineering Department Research Scholar, who provided access to the BK Precision XLN3640 power supply and BK Precision $85181.2 \mathrm{~kW}$ electronic load used for low input voltage tests.

Furthermore, I would like to extend my thanks to my fellow EHFEM team members Alvin Hilario, Greg Hollister, Nicholas Lovgren, Michelle Lum and Jonathan Yuen for their help with my project. Additionally I'd like to thank Ben Braun, Jason Delgadillo and Juan Chavez for helping gather and provide data for my project. Finally, I would like to thank my family and friends for supporting me throughout my university career; I do not know where I would be today without them. 


\section{TABLE OF CONTENTS}

LIST OF TABLES $\quad$ Page

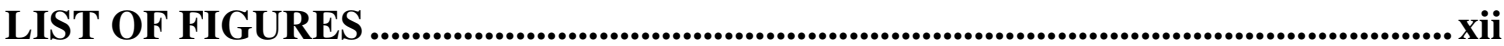

CHAPTER 1: INTRODUCTION....................................................................................... 1

1.1 Design Requirements ........................................................................................................................................

1.2 Project Specifications..................................................................................................................................

1.3 Primary Constraints ................................................................................................................................4

CHAPTER 2: PROJECT PLANNING AND ABET SENIOR PROJECT

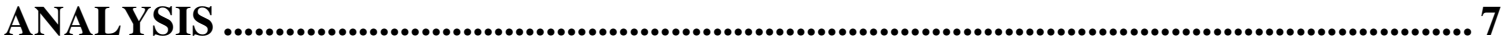

2.1 Project Timeline .................................................................................................................................................

2.2 Estimate of Parts Cost and Labor ............................................................................................................9

2.3 ABET Senior Project Analysis.............................................................................................................10

2.3.1 Economic …………………………………………………………………………10

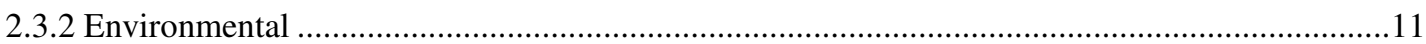

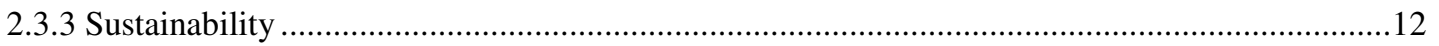

2.3.4 Manufacturability ……………………………………………………………………12

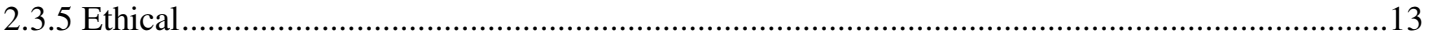

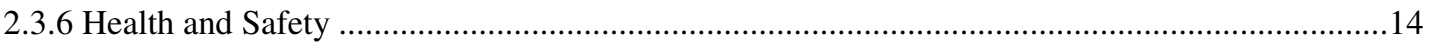

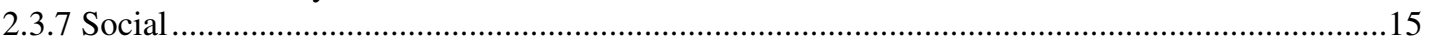

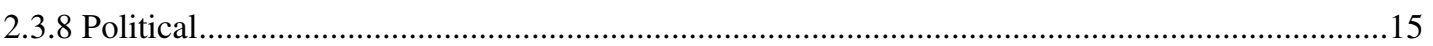

CHAPTER 3: SYSTEM COMPONENT CHARACTERIZATION AND

DC-DC CONVERTER TOPOLOGY SELECTION........................................................ 17

3.1 Precor EFX 546i Elliptical Trainer Characterization …………………………………...................17

3.2 Enphase M175-24-240 Micro-Inverter Specifications and Characterization .................................25

3.3 Converter Topology Selection ........................................................................................................26

3.3.1 Comparison of Hard-Switching, Non-Isolated Topologies .....................................................29

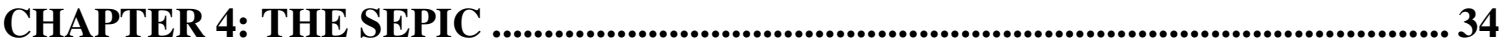

4.1 SEPIC Introduction ………………………..................................................................................................34

4.2 Basic SEPIC Operation ......................................................................................................................35

4.3 SEPIC Operation in Continuous Conduction Mode (CCM) ...........................................................36

4.3.1 SEPIC Operation in CCM with Switch Closed …………………………………………….....38

4.3.2 SEPIC Operation in CCM with Switch Open.........................................................................39

CHAPTER 5: FIRST DESIGN PHASE AND SIMULATION RESULTS ................42

5.1 Basic SEPIC Design .........................................................................................................................42

5.2 PWM Controller Component Selection ............................................................................................50

5.2.1 Programming Thresholds with the LTC1871's RUN Pin.............................................................52

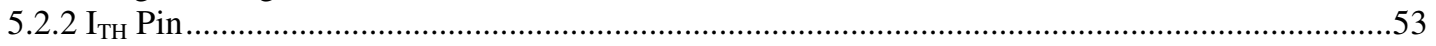

5.2.3 Output Voltage Programming with the FB Pin ..........................................................................56

5.2.4 Switching Frequency Programming with the FREQ Pin ..........................................................56

5.2.5 MODE/SYNC Pin Operation ..................................................................................................5

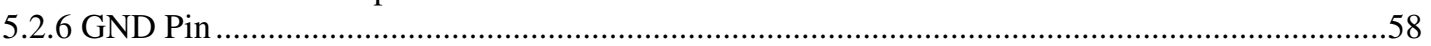

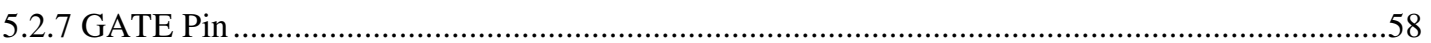

5.2.8 $\mathrm{INTV}_{\mathrm{CC}}$ Regulator Bypassing and Operation ( $\left.\operatorname{INTV}_{\mathrm{CC}} \mathrm{Pin}\right)$..............................................58

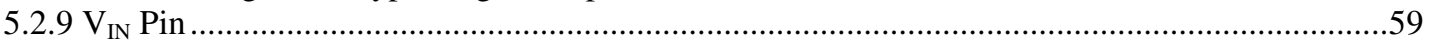

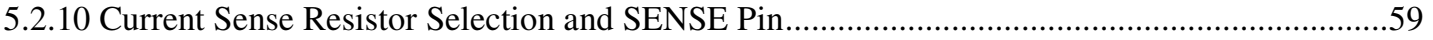

5.3 Switching MOSFET and Diode Selection .............................................................................................60

5.4 Base SEPIC Simulation Implementation and Output Voltage Results .........................................61

5.4.1 Base SEPIC Simulation Switching Waveform Analysis at 65V Input..........................................62

5.4.2 Base SEPIC Efficiency Analysis ...................................................................................................68 
CHAPTER 6: SEPIC SNUBBER CIRCUIT ....................................................... 73

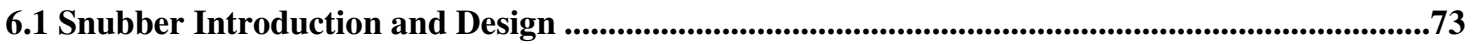

6.2 Snubber Operation Analysis .................................................................................................................78

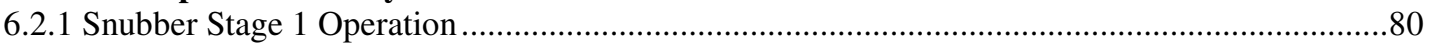

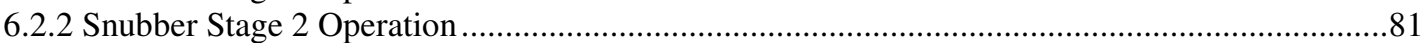

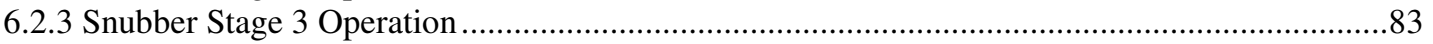

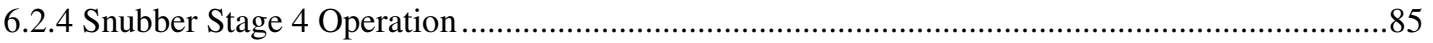

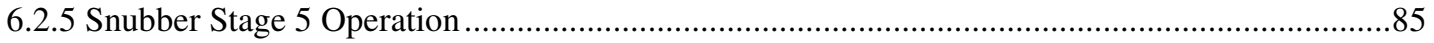

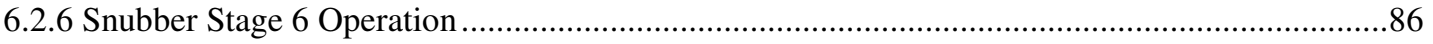

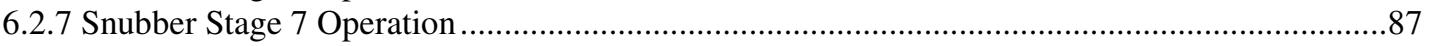

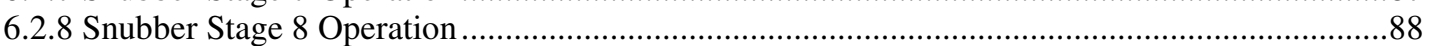

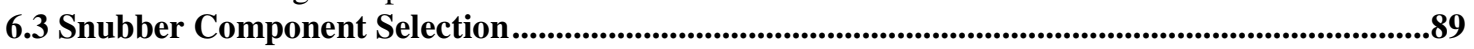

6.4 SEPIC Simulation Results with Snubber ..................................................................................92

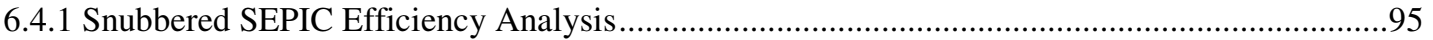

CHAPTER 7: PRECOR EFX 546i OUTPUT VOLTAGE RIPPLE AND

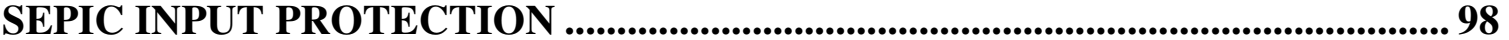

7.1 Precor EFX 546i Output Voltage Ripple .........................................................................................................98

7.2 Input Overvoltage and Overcurrent Protection Circuit..............................................................................99

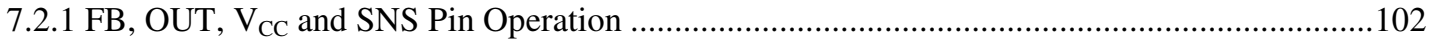

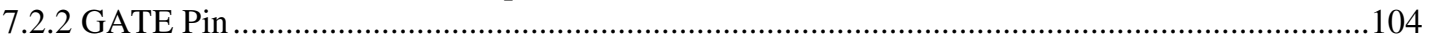

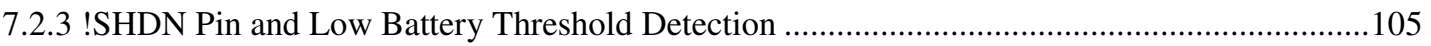

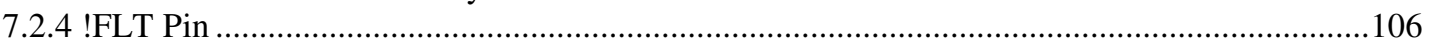

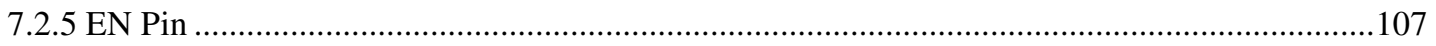

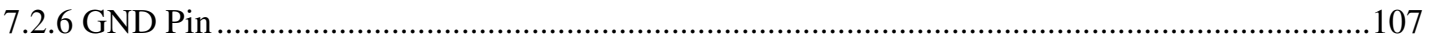

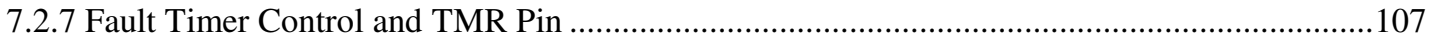

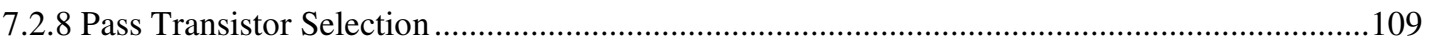

7.3 Protected SEPIC Simulation Results...........................................................................111

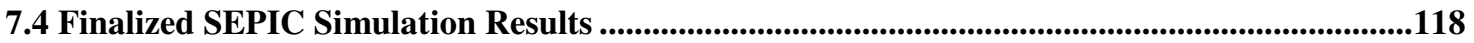

CHAPTER 8: THERMAL CONSIDERATIONS ................................................ 129

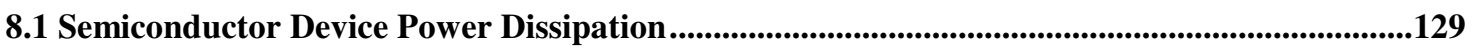

8.2 Heatsink Thermal Resistance Ratings...........................................................................131

8.3 Heatsink Selection and Component Temperature Recalculation ........................................................133

CHAPTER 9: PCB LAYOUT ........................................................................... 140

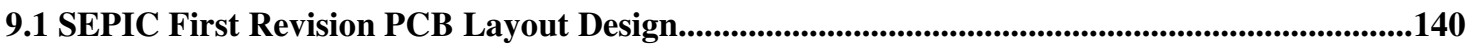

9.2 SEPIC Second Revision PCB Layout Design.......................................................................................147

CHAPTER 10: HARDWARE TESTING...................................................................... 158

10.1 Component Soldering Process ............................................................................................................................158

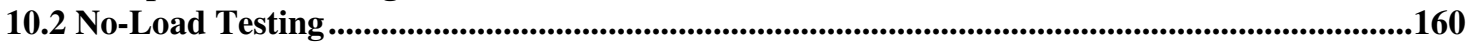

10.3 Load Testing ...........................................................................................................................................164

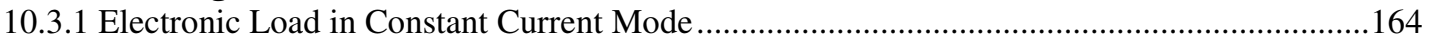

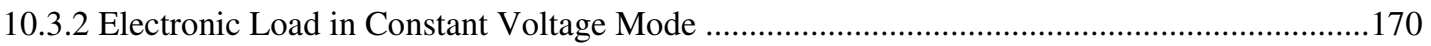

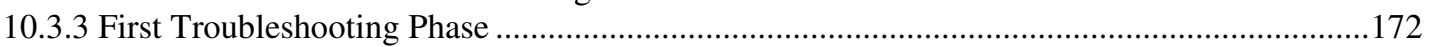

10.3.3 Constant Voltage Load Tests Using Non-Current Limited Input Supply .................................185

10.3.4 Constant Voltage Load Tests Using Non-Voltage and Current Limited Input Supply.............203

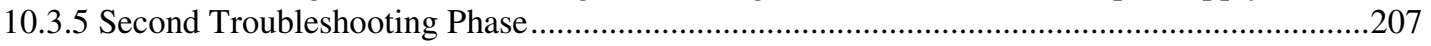

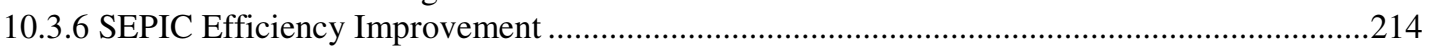

10.4 Precor Elliptical Trainer Full System Hardware Testing ......................................................234

CHAPTER 11: CONCLUSION, FUTURE IMPROVEMENTS AND

FUTURE EHFEM PROJECTS............................................................................... 245

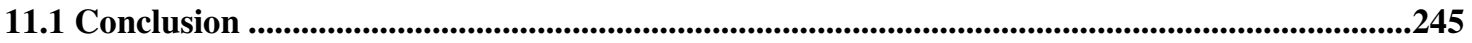

11.2 Future SEPIC Improvements ..........................................................................................................................246

vii 


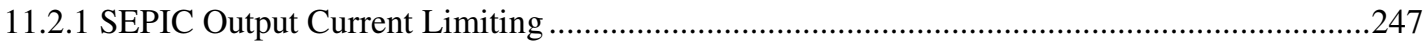

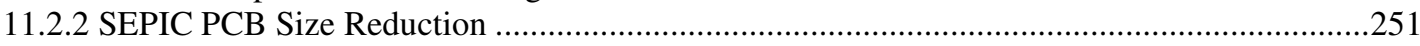

11.2.3 Elliptical Trainer Battery Conservation ............................................................................251

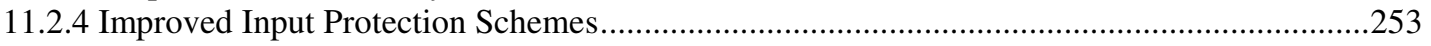

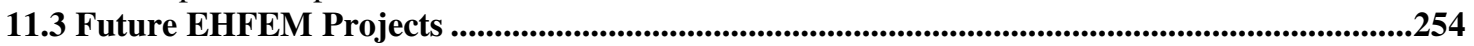

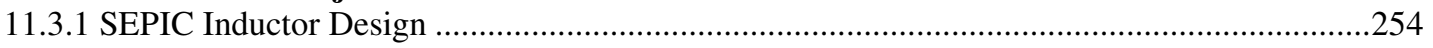

11.3.2 Inverter Control Mechanism......................................................................................258

11.3.3 Inverter Design ............................................................................................................259

APPENDIX A: ĆUK CONVERTER DESIGN ......................................................... 261

A1.1 PWM Controller Decision Matrix ............................................................................................262

A1.2 Non-Inverting Ćuk Converter Design using National LM5022 PWM Controller ..................268

APPENDIX B: INTERLEAVED BUCK-BOOST CONVERTER DESIGN ........ 274

B1.1 Interleaved, Multi-Stage Buck-Boost Converter Design Using LT3845 and

LTC3862 PWM Controllers .................................................................................................2274

B1.2 Single-Phase Input and Output Stage Implementation........................................................276

B1.3 Two-Phase Input Stage and Single-Phase Output Stage Implementation................................286

B1.4 Four-Phase Input Stage and Single-Phase Output Stage Implementation................................290

B1.5 Two-Phase Input and Output Stage Implementation ...........................................................295

B1.6 Single-Phase Input and Output Stage Implementation at $500 \mathrm{kHz}$ Switching

Frequency ...........................................................................................................................299

B1.7 Single-Phase Input Stage and Two-Phase Output Stage Implementation at 500 kHz

Switching Frequency .......................................................................................................................302

B1.8 Two-Phase Input and Output Stage Implementation Analysis at $500 \mathrm{kHz}$ and 350

kHz Switching Frequencies .......................................................................................................305

B1.9 Four-Phase Input Stage and Two-Phase Output Stage Implementation...............................336

B1.10 Component Downsizing and Optimization Process.............................................................333

B1.11 Linear LTC6908-1 External Oscillator Configuration ............................................................381

B1.12 Test Circuit for LTC6908-1 External Oscillator .............................................................384

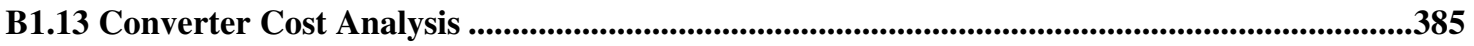

B1.14 Converter Downsize Proposal .................................................................................................389

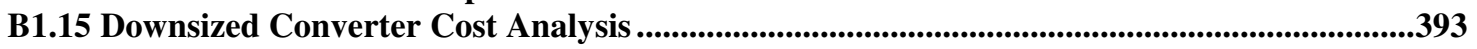

APPENDIX C: FINAL SEPIC NETLIST ............................................................ 399

REFERENCES............................................................................................................................. 401 


\section{LIST OF TABLES}

Table

Page

Table A-1: Physical Exercise Input and Electric Output Results for Participant \#1 ...................................19

Table A-2: Physical Exercise Input and Electric Output Results for Participant \#2 ..................................20

Table A-3: Physical Exercise Input and Electric Output Results for Participant \#3 ..................................21

Table A-4: Physical Exercise Input and Electric Output Results for Participant \#4 ................................22

Table B-1: Finalized SEPIC Component BOM …...............................................................122

Table C-1: SEPIC Semiconductor Component Average Steady-state Power Dissipation with

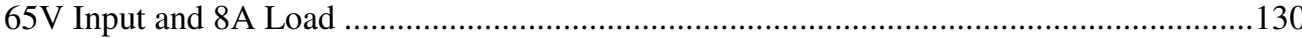

Table C-2: SEPIC Semiconductor Component Average Steady-state Power Dissipation with 27V Input and 8A Load

Table D-1: Finalized SEPIC BOM with PCB and Heatsink Costs

Table E-1: SEPIC Initial Load Tests with 0.2A Constant Current Electronic Load, TPS-4000 and GW Instek Power Supplies

Table E-2: SEPIC Initial Load Tests with 0.25A Constant Current Electronic Load, TPS-4000 and GW Instek Power Supplies.

Table E-3: SEPIC Initial Load Tests with 0.3A Constant Current Electronic Load, TPS-4000 and GW Instek Power Supplies

Table E-4: SEPIC Initial Load Tests with 0.35A Constant Current Electronic Load, TPS-4000 and GW Instek Power Supplies.

Table E-5: SEPIC Initial Load Tests with 0.4A Constant Current Electronic Load, TPS-4000 and GW Instek Power Supplies

Table E-6: SEPIC Initial Load Tests with 0.45A Constant Current Electronic Load, TPS-4000 and GW Instek Power Supplies.

Table E-7: SEPIC Initial Load Tests with 0.5A Constant Current Electronic Load, TPS-4000 and GW Instek Power Supplies

Table E-8: SEPIC Initial Load Tests with 0.6A Constant Current Electronic Load, TPS-4000 and GW Instek Power Supplies

Table E-9: SEPIC Initial Load Tests with 0.7A Constant Current Electronic Load, TPS-4000 and GW Instek Power Supplies

Table E-10: SEPIC Initial Load Tests with 0.8A Constant Current Electronic Load, TPS-4000 and GW Instek Power Supplies.

Table E-11: SEPIC Initial Load Tests with 0.9A Constant Current Electronic Load, TPS-4000 and GW Instek Power Supplies.

Table E-12: SEPIC Initial Load Tests with 1A Constant Current Electronic Load, TPS-4000 and GW Instek Power Supplies

Table E-13: SEPIC Initial Load Tests with 1.5A Constant Current Electronic Load, TPS-4000 and GW Instek Power Supplies.............................................................................. 168

Table E-14: SEPIC Initial Load Tests with 2A Constant Current Electronic Load, TPS-4000 and GW Instek Power Supplies

Table E-15: SEPIC Initial Load Tests with 2.2A Constant Current Electronic Load, TPS-4000 and GW Instek Power Supplies.

Table E-16: SEPIC Initial Load Tests with 2.3A Constant Current Electronic Load, TPS-4000 and GW Instek Power Supplies.

Table E-17: SEPIC Initial Load Tests with Constant Voltage Electronic Load, TPS-4000 and GW Instek Power Supplies 
Table E-18: SEPIC Initial Load Tests with 160 Strides/Min. Theoretical Input Voltages from [1], Constant Voltage Electronic Load, TPS-4000 and GW Instek Power Supplies

Table E-19: Modified Feedback and Gate Nodes SEPIC Load Tests with Constant Voltage Electronic Load, TPS-4000 and GW Instek Power Supplies ...

Table E-20: Modified Feedback and Gate Nodes SEPIC Load Tests with 160 Strides/Min. Theoretical Input Voltages from [1], Constant Voltage Electronic Load, TPS-4000 and GW Instek Power Supplies.

Table E-21: Modified Feedback and Gate Nodes SEPIC Load Tests with Constant Voltage Electronic Load, Agilent E3630A and BK XLN3640 Power Supplies

Table E-22: Modified Feedback and Gate Nodes SEPIC Load Tests with 160 Strides/Min. Theoretical Input Voltages from [1], Constant Voltage Electronic Load, Agilent E3630A and BK XLN3640 Power Supplies ....

Table E-23: SEPIC Load Tests with $0.02 \mu$ F Gate Capacitor, Constant Voltage Electronic Load, Agilent E3630A and BK XLN3640 Power Supplies

Table E-24: SEPIC Load Test with 0.02 $\mu$ F Gate Capacitor Using 160 Strides/Min. Theoretical Input Voltages from [1], Constant Voltage Electronic Load, Agilent E3630A and BK XLN3640 Power Supplies

Table E-25: SEPIC Load Tests with 0.02 $\mu$ F Gate Capacitor, Constant Voltage Electronic Load, Agilent E3630A and BK XLN3640 Power Supplies

Table E-26: SEPIC Load Test with $0.02 \mu$ F Gate Capacitor Using 160 Strides/Min. Theoretical Input Voltages from [1], Constant Voltage Electronic Load, Agilent E3630A and BK XLN3640 Power Supplies....

Table E-27: SEPIC Load Tests with $0.02 \mu$ F Gate Capacitor, Additional $470 \mu \mathrm{F}$ Output Capacitor, Constant Voltage Electronic Load, Agilent E3630A and BK XLN3640 Power Supplies

Table E-28: SEPIC Load Test with $0.02 \mu \mathrm{F}$ Gate Capacitor and Additional $470 \mu \mathrm{F}$ Output Capacitor Using 160 Strides/Min. Theoretical Input Voltages from [1], Constant Voltage Electronic Load, Agilent E3630A and BK XLN3640 Power Supplies

Table E-29: SEPIC Load Tests with $0.02 \mu \mathrm{F}$ Gate Capacitor, Additional $470 \mu \mathrm{F}$ Output Capacitor, Constant Voltage Electronic Load and BK 9153 Power Supplies.....

Table E-30: SEPIC Load Test with $0.02 \mu \mathrm{F}$ Gate Capacitor and Additional $470 \mu \mathrm{F}$ Output Capacitor, Using 160 Strides/Min. Theoretical Input Voltages from [1], Constant Voltage Electronic Load and BK 9153 Power Supplies .... 206

Table E-31: SEPIC Load Tests with LTC1871 Current Sense Pin Filter and BK 9153 Power Supplies.

Table E-32: SEPIC Load Test with LTC1871 Current Sense Pin Filter, Using 160 Strides/Min.

Theoretical Input Voltages from [1] and BK 9153 Power Supplies.

Table E-33: SEPIC Load Tests with LTC1871 Current Sense Pin Filter and GATE Pin Damping Filter and BK 9153 Power Supplies

Table E-34: SEPIC Load Test with LTC1871 Current Sense Pin Filter and GATE Pin Damping Filter, Using 160 Strides/Min. Theoretical Input Voltages from [1] and BK 9153 Power Supplies...

Table E-35: Final SEPIC BOM

Table E-36: SEPIC Test Results with Precor Elliptical Trainer Input and Constant $10 \Omega$ Load.

Table A.A-1: PWM Controller Decision Matrix - Characteristics of Each Controller IC. 265

Table A.A-2: PWM Controller Decision Matrix - Preference Ratings for Each Controller IC 266

Table A.A-3: PWM Controller Decision Matrix - Weighted Final Preference Ratings for Each Controller IC .267

Table B.B-1: Controller IC Components Cost for Converter 385

Table B.B-2: Transistor Components Cost for Converter..... 386 


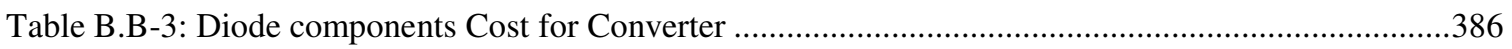

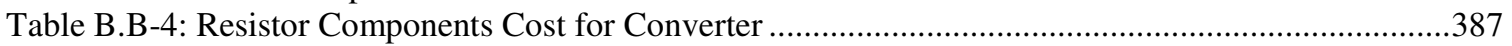

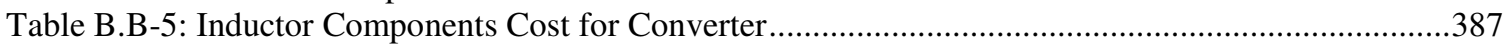

Table B.B-6: Capacitor Components Cost for Converter......................................................................388

Table B.B-7: Controller IC Components Cost for Downsized Converter .............................................394

Table B.B-8: Transistor Components Cost for Downsized Converter ................................................394

Table B.B-9: Diode Components Cost for Downsized Converter......................................................394

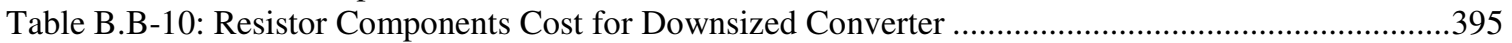

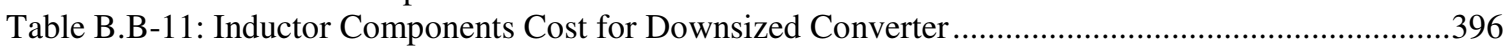

Table B.B-12: Capacitor Components Cost for Downsized Converter ................................................396 


\section{LIST OF FIGURES}

Figure Page

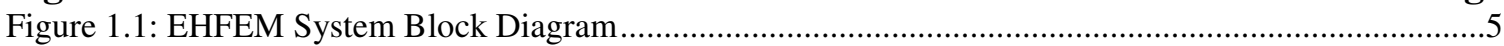

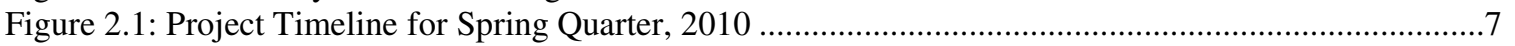

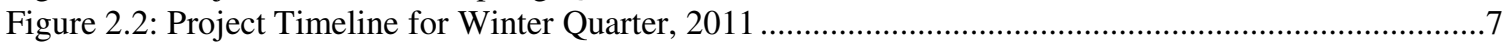

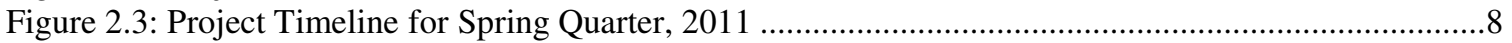

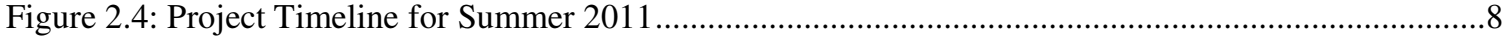

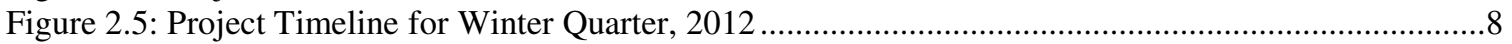

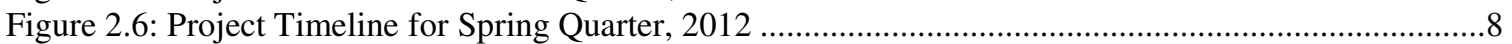

Figure 3.1: Elliptical DC Output Power for Participants \#3 and 4 vs. Resistance Level..............................22

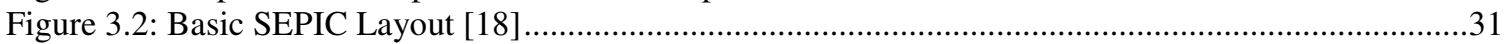

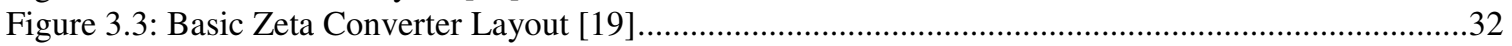

Figure 4.1: Crucial SEPIC Component Waveforms [26] ........................................................................

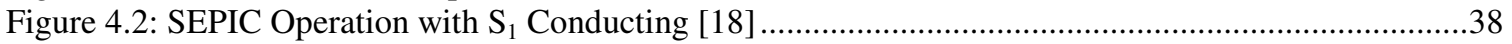

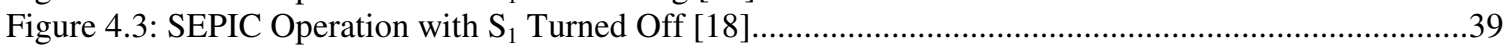

Figure 4.4: SEPIC Component Voltage Waveforms in CCM Operation [27]..............................................41

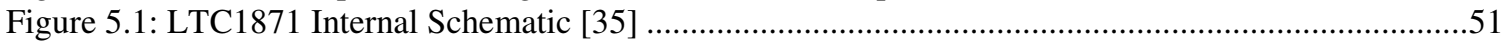

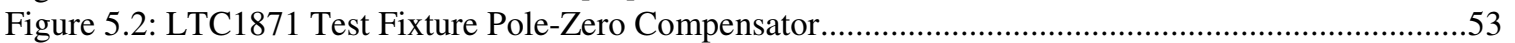

Figure 5.3: Error Amplifier Equivalent Circuit ....................................................................................53

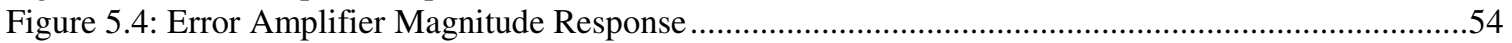

Figure 5.5: Linear Technology's Recommended LTC1871 Base SEPIC [35] ..........................................55

Figure 5.6: LTC1871 Controller Frequency Resistor Values and Corresponding Frequencies [35].............57

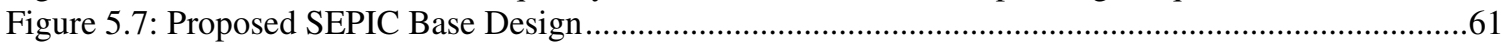

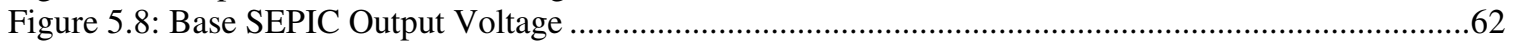

Figure 5.9: Base SEPIC Crucial Component Waveforms Maximum Input Voltage, Full Load and Steady-state ............................................................................................................6

Figure 5.10: Base SEPIC Switch Drain-Source Voltage and Inductor Voltages Maximum Input Voltage, Full load and Steady-state ....................................................................................66

Figure 5.11: Base SEPIC Inductor Currents at Maximum Input Voltage, 25\% Load and Steadystate ......

Figure 5.12: Base SEPIC Input and Output Power at Maximum Input Voltage, Full load and Steady-state

Figure 5.13: Base SEPIC Output Voltage and Switch Power Dissipation Waveforms...

Figure 5.14: Base SEPIC Switching Diode Reverse Voltage and Forward Current Stress

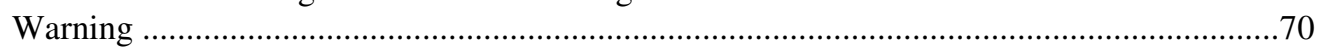

Figure 5.15: Base SEPIC Switching Diode Operation in Converter Steady-State Mode ...............................71

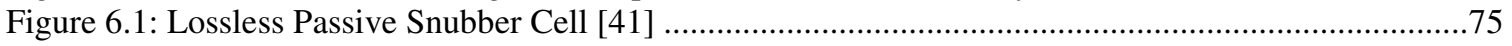

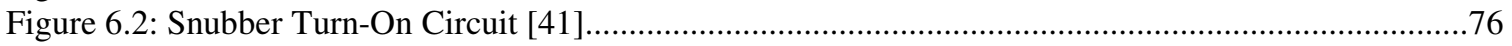

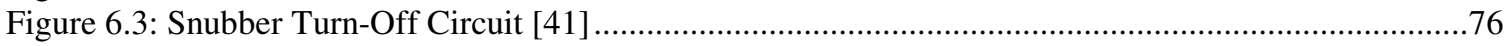

Figure 6.4: Basic SEPIC with Lossless Passive Snubber Cell [41] ........................................................77

Figure 6.5: Key Waveforms of Boost Converter with Lossless Passive Snubber [41] ....................................79

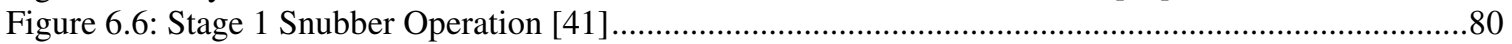

Figure 6.7: Stage 2 Snubber Operation [41]

Figure 6.8: Stage 3 Snubber Operation [41]

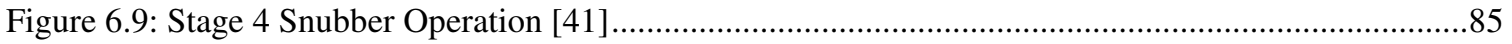

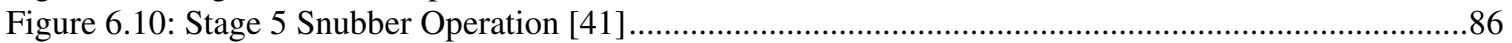

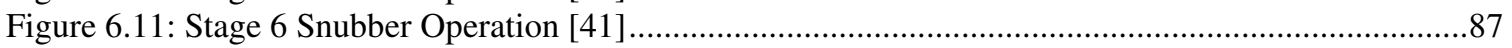

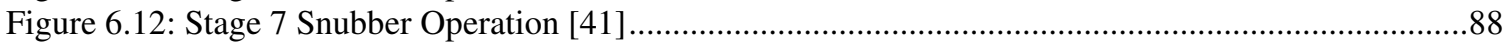

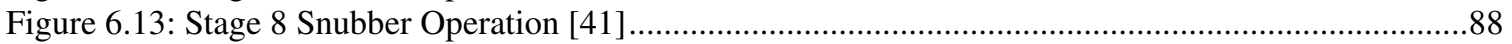

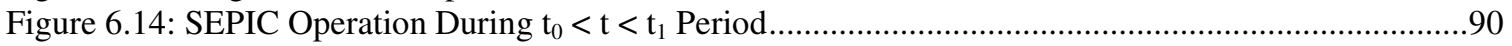

Figure 6.15: SEPIC Simulation Circuit with Passive Lossless Snubber .....................................................92 
Figure 6.16: SEPIC Output Voltage and $\mathrm{Q}_{1}$ Power Dissipation with Snubber at Full Load and Steady-state Operation

Figure 6.17: SEPIC Switching Diode Power Dissipation with Snubber at Full Load and Steadystate Operation

Figure 6.18: Snubber Diode $\mathrm{D}_{4}$ Reverse Voltage and Forward Current Stress Warning .............................95

Figure 6.19: SEPIC with Snubber Input and Output Power at Full Load and Steady-state

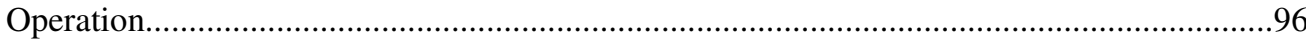

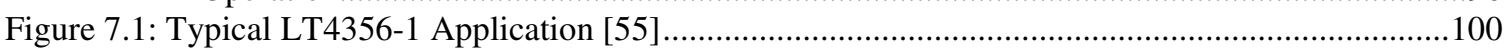

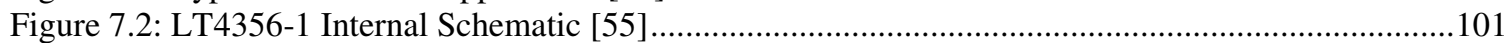

Figure 7.3: Linear Technology's LT4356-1 Sample Test Fixture Circuit..................................................105

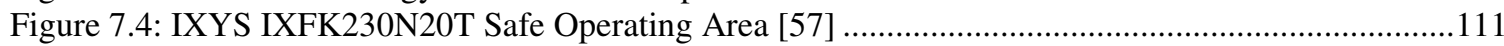

Figure 7.5: Complete SEPIC Schematic............................................................................................112

Figure 7.6: Protected SEPIC Output Voltage and $\mathrm{Q}_{1}$ Power Dissipation at Full Load and Steady-

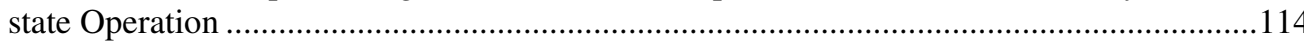

Figure 7.7: Protected SEPIC Input Voltage, Clamped Input Voltage and Q_OVP Power

Dissipation at Full Load and Steady-state Operation...

Figure 7.8: Protected SEPIC Input Voltage, Clamped Input Voltage and Q_OVP Power Dissipation at Full Load and Steady-state Operation with 1000 $\mu$ F Input Capacitor..............117

Figure 7.9: Finalized SEPIC Schematic Before PCB Layout. 119

Figure 7.10: Finalized SEPIC Output Voltage and Q_MAIN Power Dissipation with Parasitic Component Values .126

Figure 7.11: Finalized SEPIC Converter Input and Output Power with Parasitic Component Values 127

Figure 8.1: Ohmite MV-102-55E TO-247 and TO-264 Heatsink [63] ..................................................134

Figure 8.2: Aavid Thermalloy 531202B02500G TO-202 and TO-220 Heatsink [64] ...............................135

Figure 8.3: Ohmite WA-T247-101E TO-247 Heatsink [65] .......................................................................136

Figure 8.4: Aavid Thermalloy 574502B00000G TO-220 Heatsink [66] ...................................................137

Figure 9.1: Linear Technology's Suggested LTC1871 Boost Converter Layout [35] ................................140

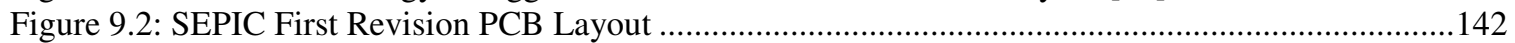

Figure 9.3: SEPIC First Revision PCB Layout Top Copper Layer ..........................................................143

Figure 9.4: SEPIC First Revision PCB Layout Bottom Copper Layer......................................................144

Figure 9.5: SEPIC First Revision PCB Layout Silkscreen Layer............................................................145

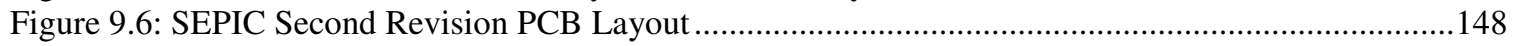

Figure 9.7: SEPIC Second Revision PCB Layout Top Copper Layer.....................................................150

Figure 9.8: SEPIC Second Revision PCB Layout Bottom Copper Layer ................................................151

Figure 9.9: SEPIC Second Revision PCB Layout Silkscreen Layer ......................................................152

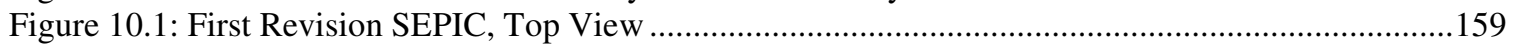

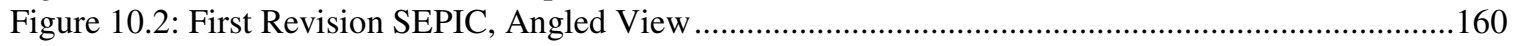

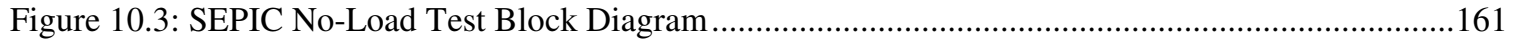

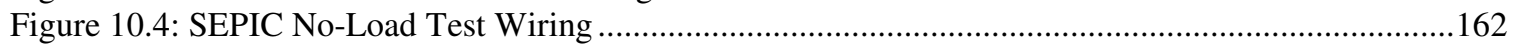

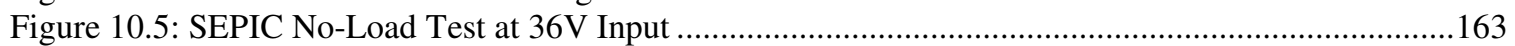

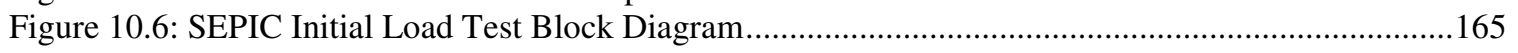

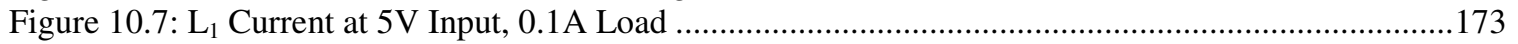

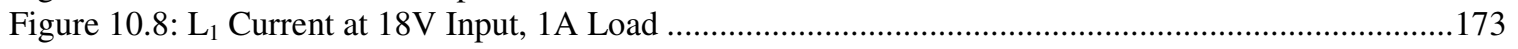

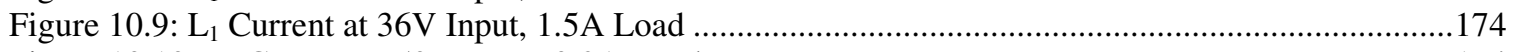

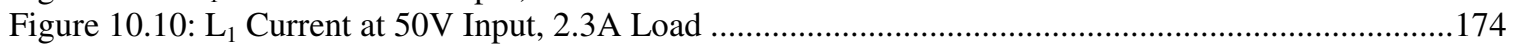

Figure 10.11: Q_MAIN Switching Trajectory at 5V Input and 0.1A Load .............................................176

Figure 10.12: Q_MAIN Switching Trajectory at 36V Input and 1.46A Load...........................................176

Figure 10.13: Q_MAIN Switching Trajectory at 50V Input and 2.3A Load ............................................176

Figure 10.14: Finalized SEPIC Output Voltage, Q_MAIN Switching Trajectory, L $_{1}$ Current and Feedback and Sense Node Voltages from LTSpice Simulation... 
Figure 10.15: Finalized SEPIC with $0.01 \mu$ F Feedback Capacitor Output Voltage, Q_MAIN Switching Trajectory, $\mathrm{L}_{1}$ Current and Feedback and Sense Node Voltages from LTSpice Simulation ....

Figure 10.16: Finalized SEPIC with $0.01 \mu \mathrm{F}$ Feedback Capacitor and $0.01 \mu \mathrm{F}$ Q_MAIN Gate Capacitor Output Voltage, Q_MAIN Switching Trajectory, $\mathrm{L}_{1}$ Current and Feedback and Sense Node Voltages from LTSpice Simulation.

Figure 10.17: Finalized SEPIC with $0.01 \mu$ F Feedback Capacitor and $0.01 \mu \mathrm{F}$ Q_MAIN Gate Capacitor Test Setup Block Diagram

Figure 10.18: Finalized SEPIC with $0.01 \mu$ F Feedback Capacitor and $0.02 \mu \mathrm{F}$ Q_MAIN Gate Capacitor Output Voltage, Q_MAIN Switching Trajectory, L $_{1}$ Current and Feedback and Sense Node Voltages from LTSpice Simulation

Figure 10.19: Q_MAIN Switching Trajectory with $0.02 \mu \mathrm{F}$ Gate Capacitor at $5 \mathrm{~V}$ Input and 0.03 A Load

Figure 10.20: Q_MAIN Switching Trajectory with $0.02 \mu \mathrm{F}$ at $36 \mathrm{~V}$ Input and 2.484A Load

Figure 10.21: SEPIC Output Voltage Ripple at 36V Input and 2.164A Load.

Figure 10.22: SEPIC Output Voltage Ripple at 36V Input and 2.231 A Load With Additional $470 \mu \mathrm{F}$ Output Capacitor 200

Figure 10.23: Q_MAIN Switching Trajectory with $0.02 \mu \mathrm{F}$ Discrete Gate Capacitor and Additional $470 \mu \mathrm{F}$ Capacitor at $5 \mathrm{~V}$ Input and $0.085 \mathrm{~A}$ Load.

Figure 10.24: Q_MAIN Switching Trajectory with $0.02 \mu \mathrm{F}$ Discrete Gate Capacitor and Additional $470 \mu \mathrm{F}$ Capacitor at $36 \mathrm{~V}$ Input and 2.231 A Load....

Figure 10.25: Finalized SEPIC with $0.01 \mu \mathrm{F}$ Feedback Capacitor, Additional $470 \mu \mathrm{F}$ Output

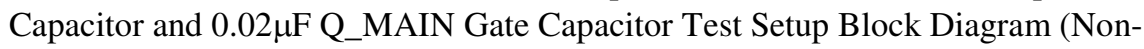
Voltage and Current Limited)

Figure 10.26: LTC1871 Current Sense Pin Switching Waveform at 36V Input and 2.28A Load ...............207

Figure 10.27: LTC1871 Current Sense Pin RC Low Pass Filter ............................................................208

Figure 10.28: LTC1871 Current Sense Pin Waveform With Low Pass RC Filter In Steady-State Operation. .209

Figure 10.29: SEPIC Maximum Load Current Driving Ability vs. Input Voltage with LTC1871

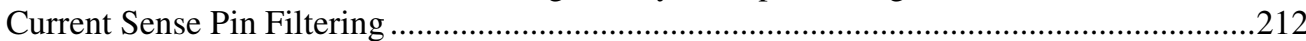

Figure 10.30: SEPIC Efficiency vs. Input Voltage with LTC1871 Current Sense Pin Filtering ..................213

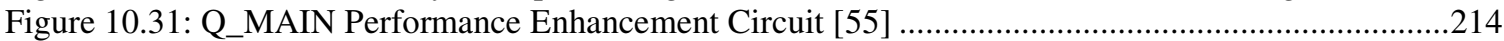

Figure 10.32: LTC1871 GATE Pin Damping Filter..... 215

Figure 10.33: SEPIC Maximum Load Current Driving Ability vs. Input Voltage with LTC1871 Current Sense Pin Filtering and GATE Pin Damping Filter .219

Figure 10.34: SEPIC Efficiency vs. Input Voltage with LTC1871 Current Sense Pin Filtering and GATE Pin Damping Filter 220

Figure 10.35: LTC1871 Current Sense Pin Switching Waveform with All SEPIC Performance Enhancement Modifications at 36V Input and 2.748A Load. .222

Figure 10.36: Q_MAIN Gate Switching Trajectory with All SEPIC Performance Enhancement Modifications at 36V Input and 2.748A Load 223

Figure 10.37: SEPIC Output Voltage Ripple at 30V Input and 1.838A Load (Boost Mode) ......................224

Figure 10.38: SEPIC Output Voltage Ripple at 36V Input and 2.748A Load (Buck-Boost Mode)............225

Figure 10.39: SEPIC Output Voltage Ripple at 60V Input and 7.498A Load (Buck Mode) .......................226

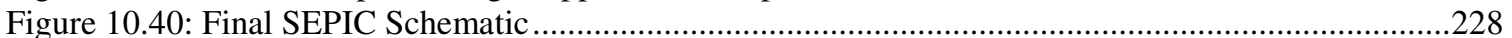

Figure 10.41: SEPIC Testing with Precor Elliptical Trainer, Angled View.............................................235

Figure 10.42: SEPIC Testing with Precor Elliptical Trainer, Instrument and Grid Panel View ..................236

Figure 10.43: SEPIC Load Current Driving Ability vs. Elliptical Training Resistance Level with Precor Elliptical Trainer Input 240

Figure 10.44: SEPIC Efficiency vs. Elliptical Training Resistance Level with Precor Elliptical Trainer Input 240

Figure 11.1: Active Overvoltage Crowbar [72]...... 248 


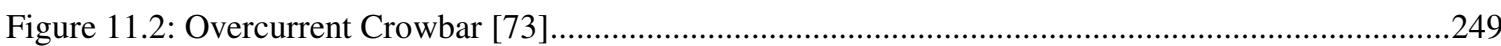

Figure 11.3: DC-DC Converter Output Current Limiter Using Multiplier [53] .........................................250

Figure 11.4: Elliptical Trainer Battery Minimum Voltage Threshold Detection Circuit [10].....................252

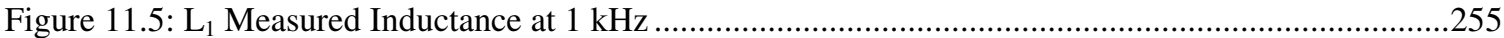

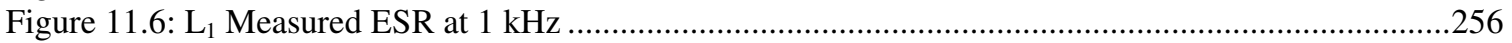

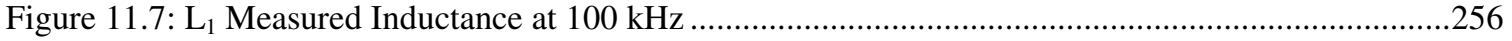

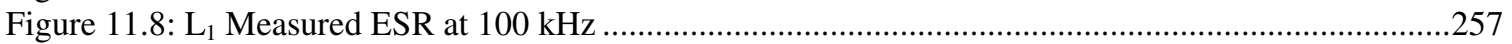

Figure 11.9: Possible Control Scheme for Enphase M175-24-240 Micro-Inverter [10] ...........................259

Figure A-1: Non-Inverting Cuk Converter Using National LM5022 PWM Controller ...............................269

Figure A-2: Non-Inverting Cuk Converter Performance Characteristics at 60V Input, No Load, Using National LM5022 PWM Controller.......................................................................220

Figure A-3: Non-Inverting Cuk Converter Performance Characteristics at 60V Input, Full Load, Using National LM5022 PWM Controller........................................................................22

Figure B-1: Single-Phase, Multi-Stage Buck-Boost Converter Design....................................................277

Figure B-2: Single-Phase Buck-Boost Converter Individual Stage Output Voltages at 60V Input,

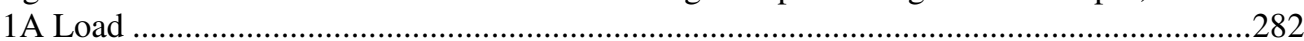

Figure B-3: Single-Phase Buck-Boost Converter Individual Stage Output Voltages at 60V Input, 2A Load .283

Figure B-4: Single-Phase Buck-Boost Converter Individual Stage Output Voltages at 60V Input,

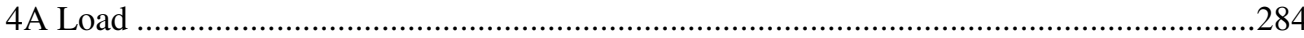

Figure B-5: Single-Phase Buck-Boost Converter Individual Stage Output Voltages at 60V Input,

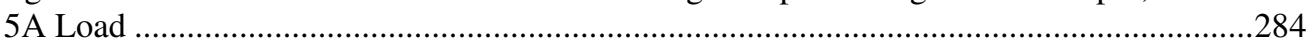

Figure B-6: Single-Phase Buck-Boost Converter Individual Stage Output Voltages at 60V Input,

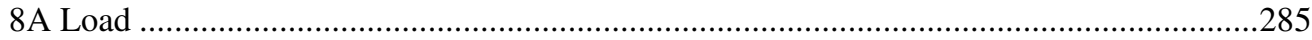

Figure B-7: Two-Phase Buck, Single-Phase Boost, Multi-Stage Buck-Boost Converter Design ...............287

Figure B-8: Two-Phase Input, Single-Phase Output Buck-Boost Converter Individual Stage Output Voltages at 60V Input, 8A Load .............................................................................28

Figure B-9: Two-Phase Input, Single-Phase Output Buck-Boost Converter Individual Stage

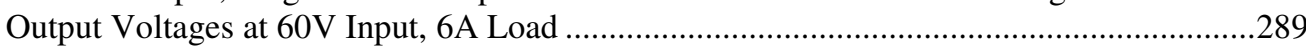

Figure B-10: Two-Phase Input, Single-Phase Output Buck-Boost Converter Individual Stage Output Voltages at 60V Input, 4.75A Load

Figure B-11: Four-Phase Buck, Single-Phase Boost, Multi-Stage Buck-Boost Converter Design ..............291

Figure B-12: Four-Phase input, Single-Phase Output Buck-Boost converter Individual Stage Output Voltages at 60V Input, 8A Load ........................................................................292

Figure B-13: Four-Phase Buck, Two-Phase Boost, Multi-stage Buck-Boost Converter Design .................293

Figure B-14: Four-Phase Input, Two-Phase Output Buck-Boost Converter Individual Stage Output Voltages at 60V Input, 8A Load

Figure B-15: Two-Phase Buck, Two-Phase Boost, Multi-Stage Buck-Boost Converter Design .................296

Figure B-16: Two-Phase Input, Two-Phase Output Buck-Boost Converter Individual Stage Output Voltages at 60V Input, 8A Load

Figure B-17: Two-Phase Input, Two-Phase Output Buck-Boost Converter Individual Stage Output Voltages at 60V Input, 8A Load, Using $500 \mathrm{kHz}$ Switching Frequencies for Each Stage

Figure B-18: Single-Phase, Multi-Stage Buck-Boost Converter Design with 500 kHz Switching Frequency for Each Stage

Figure B-19: Single-Phase, Multi-Stage Buck-Boost Converter Individual Stage Output Voltages at $60 \mathrm{~V}$ Input, 8A Load, Using $500 \mathrm{kHz}$ Switching Frequencies for Each Stage......

Figure B-20: Single-Phase Buck, Two-Phase Boost, Multi-Stage Buck-Boost Converter Design .......... 301

Figure B-21: Single-Phase Buck, Two-Phase Boost, Multi-Stage Buck-Boost Converter Output Voltage with 60V Input and 8A Load. 
Figure B-22: Inductor Current of $\mathrm{L}_{1}$ (Input Stage) of Dual-Phase Buck-Boost Converter Under Maximum Input and Load..

Figure B-23: Inductor Current of $\mathrm{L}_{4}$ (Input Stage) of Dual-Phase Buck-Boost Converter Under Maximum Input and Load......

Figure B-24: Inductor Current of $\mathrm{L}_{2}$ (Output Stage) of Dual-Phase Buck-Boost Converter Under Maximum Input and Load.

Figure B-25: Inductor Current of $\mathrm{L}_{3}$ (Output Stage) of Dual-Phase Buck-Boost Converter Under

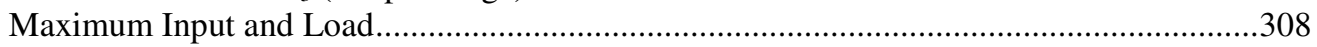

Figure B-26: Inductor Current of $\mathrm{L}_{3}$ (Output Stage) of Dual-Phase Buck-Boost Converter Under Maximum Input and Load.....

Figure B-27: Inductor Current of $\mathrm{L}_{8}$ (Output Stage) of Dual-Phase Buck-Boost Converter Under Maximum Input and Load.

Figure B-28: $\mathrm{Q}_{1}$ Drain Current (Input Stage) of Dual-Phase Buck-Boost Converter Under Maximum Input and Load......

Figure B-29: $\mathrm{Q}_{2}$ Drain Current (Input Stage) of Dual-Phase Buck-Boost Converter Under Maximum Input and Load.....

Figure B-30: $\mathrm{Q}_{4}$ Drain Current (Output Stage) of Dual-Phase Buck-Boost Converter Under Maximum Input and Load

Figure B-31: $\mathrm{Q}_{5}$ Drain Current (Output Stage) of Dual-Phase Buck-Boost Converter Under Maximum Input and Load.

Figure B-32: $\mathrm{Q}_{1}$ and $\mathrm{Q}_{2}$ Power Dissipation of Dual-Phase Buck-Boost Converter Under Maximum Input and Load.

Figure B-33: Q 4 Power Dissipation of Dual-Phase Buck-Boost Converter Under Maximum Input and Load.

Figure B-34: Q5 Power Dissipation of Dual-Phase Buck-Boost Converter Under Maximum Input and Load.

Figure B-35: Two-Phase Buck, Two-Phase Boost, Multi-Stage Buck-Boost converter Design With Improved Diodes and MOSFETs

Figure B-36: Output Voltage and $\mathrm{Q}_{1}$ Power Dissipation of Dual-Phase Buck-Boost Converter Under Maximum Input and Load, Using Improved Diodes and MOSFETs

Figure B-37: Zoomed-in View of $\mathrm{Q}_{1}$ Power Dissipation Transients of Dual-Phase Buck-Boost Converter Under Maximum Input and Load.....

Figure B-38: Width of Largest $Q_{1}$ Power Dissipation Transient Under Maximum Input and Load ...........323

Figure B-39: $Q_{1}$ Drain-Source Voltage and Drain Current Under Maximum Input and Load....................324

Figure B-40: $Q_{1}$ Drain-Source Voltage and Drain Current at Turn-On Transition Under Maximum Input and Load....

Figure B-41: $Q_{1}$ Drain-Source Voltage, Gate Voltage, Gate-Source Voltage and Drain Current at Turn-On Transition Under Maximum Input and Load. 326

Figure B-42: Q 2 Power Dissipation of Dual-Phase Buck-Boost Converter Under Maximum Input and Load, Using Improved Diodes and MOSFETs

Figure B-43: Q 4 Power Dissipation of Dual-Phase Buck-Boost Converter Under Maximum Input and Load, Using Improved Diodes and MOSFETs

Figure B-44: Q5 Power Dissipation of Dual-Phase Buck-Boost Converter Under Maximum Input and Load, Using Improved Diodes and MOSFETs

Figure B-45: $\mathrm{Q}_{1}$ Gate Voltage of Dual-Phase Buck-Boost Converter Under Maximum Input and Load

Figure B-46: $\mathrm{Q}_{4}$ Gate Voltage of Dual-Phase Buck-Boost Converter Under Maximum Input and Load

Figure B-47: Two-Phase Buck, Two-Phase Boost, Multi-Stage Buck-Boost Converter Design with $350 \mathrm{kHz}$ Switching Frequency and IRF510 and HUFA76413 MOSFETs

Figure B-48: Output Voltage, Buck Stage Output Voltage and $\mathrm{Q}_{1}$ Power Dissipation of TwoPhase Buck, Two-Phase Boost, Multi-Stage Buck-Boost Converter Design with $350 \mathrm{kHz}$ Switching Frequency and IRF510 and HUFA76413 MOSFETs..... 
Figure B-49: Four-Phase Buck, Two-Phase Boost, Multi-Stage Buck-Boost Converter Design with $300 \mathrm{kHz}$ Switching Frequency and IRF510 and HUFA76413 MOSFETs .

Figure B-50: Output Voltage, Buck Stage Output Voltage and $\mathrm{Q}_{1}$ Power Dissipation of FourPhase Buck, Two-Phase Boost, Multi-Stage Buck-Boost Converter Design with $300 \mathrm{kHz}$ Switching Frequency and IRF510 and HUFA76413 MOSFETs.

Figure B-51: Output Voltage, Buck Stage Output Voltage and $Q_{1}$ Power Dissipation of FourPhase Buck, Two-Phase Boost, Multi-Stage Buck-Boost Converter Design in Steady-State Operation with $300 \mathrm{kHz}$ Switching Frequency and IRF510 and HUFA76413 MOSFETs

Figure B-52: Output Voltage, Buck Stage Output Voltage and $\mathrm{Q}_{1}$ Power Dissipation of FourPhase Buck, Two-Phase Boost, Multi-Stage Buck-Boost Converter Design in Steady-State Operation with $100 \mathrm{kHz}$ Switching Frequency and IRF510 and HUFA76413 MOSFETs

Figure B-53: $\mathrm{Q}_{1}$ Drain-Source Voltage, Gate Voltage, Power Dissipation and Drain Current at Turn-On Transition Under Maximum Input and Load

Figure B-54: Basic Turn-Off Snubber Circuit.

Figure B-55: Basic Turn-On Snubber Circuit 344

Figure B-56: Two-Phase Buck, Two-Phase Boost, Multi-Stage Buck-Boost Converter Design with $100 \mathrm{kHz}$ Switching Frequency and Turn-On and Turn-Off Snubbers

Figure B-57: Output Voltage, Buck Stage Output Voltage and $\mathrm{Q}_{1}$ Power Dissipation of TwoPhase Buck, Two-Phase Boost, Multi-Stage Buck-Boost Converter Design with $100 \mathrm{kHz}$ switching frequency and Turn-On and Turn-Off Snubbers .

Figure B-58: Output Voltage, Buck Stage Output Voltage and $\mathrm{Q}_{1}$ Power Dissipation of TwoPhase Buck, Two-Phase Boost, Multi-Stage Buck-Boost Converter Design in Steady-State Operation with $100 \mathrm{kHz}$ Switching Frequency and Turn-On and Turn-Off Snubbers

Figure B-59: Output Voltage, Input Current and $\mathrm{Q}_{1}$ Power Dissipation of Two-Phase Buck, Two-Phase Boost, Multi-Stage Buck-Boost Converter Design in Steady-State Operation with $100 \mathrm{kHz}$ Switching Frequency and Turn-On and Turn-Off Snubbers.

Figure B-60: $\mathrm{Q}_{1}$ Power Dissipation, Drain-Source Voltage and Drain Current of Two-Phase Buck, Two-Phase Boost, Multi-Stage Buck-Boost Converter Design in SteadyState Operation with $100 \mathrm{kHz}$ Switching Frequency and Turn-On and Turn-Off Snubbers.

Figure B-61: Simplified Two-Phase Output Boost Converter Stage

Figure B-62: Four-Phase Buck, Two-Phase Boost, Multi-Stage Buck-Boost Converter Design with $100 \mathrm{kHz}$ Switching Frequency and Turn-On and Turn-Off Snubbers

Figure B-63: Output Voltage, Buck Stage Output Voltage and $Q_{1}$ Power Dissipation of FourPhase Buck, Two-Phase Boost, Multi-Stage Buck-Boost Converter Design with $100 \mathrm{kHz}$ Switching Frequency and Turn-On and Turn-Off Snubbers

Figure B-64: Output Voltage, Buck Stage Output Voltage and $Q_{1}$ Power Dissipation of FourPhase Buck, Two-Phase Boost, Multi-Stage Buck-Boost Converter Design in Steady-State Operation with $100 \mathrm{kHz}$ Switching Frequency and Turn-On and Turn-Off Snubbers

Figure B-65: $\mathrm{Q}_{1}$ Power Dissipation, Drain-Source Voltage and Drain Current of Four-Phase Buck, Two-Phase Boost, Multi-Stage Buck-Boost Converter Design in SteadyState Operation with $100 \mathrm{kHz}$ Switching Frequency and Turn-On and Turn-Off Snubbers......

Figure B-66: $\mathrm{Q}_{1}$ Switching Trajectory at Maximum Power Dissipation of Four-Phase Buck, Two-Phase Boost, Multi-Stage Buck-Boost Converter Design in Steady-State Operation with $100 \mathrm{kHz}$ Switching Frequency and Turn-On and Turn-On Snubbers... 
Figure B-67: Output Voltage, Buck Stage Output Voltage and $\mathrm{Q}_{1}$ Power Dissipation of FourPhase Buck, Two-Phase Boost, Multi-Stage Buck-Boost Converter Design with $100 \mathrm{kHz}$ Switching Frequency and Turn-On and Turn-Off Snubbers

Figure B-68: Turn-On and Turn-Off Snubber Resistor Power Dissipation of Four-Phase Buck, Two-Phase Boost, Multi-Stage Buck-Boost Converter Design .360

Figure B-69: Input Current of Four-Phase Buck, Two-Phase Boost, Multi-Stage Buck-Boost Converter Design

Figure B-70: Output Voltage, Boost Stage Output Voltage and $\mathrm{M}_{1}$ Power Dissipation of FourPhase Buck, Two-Phase Boost, Multi-Stage Buck-Boost Converter Design with $100 \mathrm{kHz}$ Switching Frequency and Turn-On and Turn-Off Snubbers

Figure B-71: Output Voltage, Boost Stage Output Voltage and $\mathrm{M}_{2}$ Power Dissipation of FourPhase Buck, Two-Phase Boost, Multi-Stage Buck-Boost Converter Design with $100 \mathrm{kHz}$ Switching Frequency and Turn-On and Turn-Off Snubbers

Figure B-72: Output Voltage, Boost Stage Output Voltage, Inductor Currents, Diode Currents and $\mathrm{M}_{1}$ and $\mathrm{M}_{2}$ Drain Currents Four-Phase Buck, Two-Phase Boost, Multi-Stage Buck-Boost Converter Design with $100 \mathrm{kHz}$ Switching Frequency and Turn-On and Turn-Off Snubbers

Figure B-73: Output Voltage, Boost Stage Output Voltage and $\mathrm{M}_{1}$ and $\mathrm{M}_{2}$ Drain-Source Voltages for Four-Phase Buck, Two-Phase Boost, Multi-Stage Buck-Boost Converter Design with $100 \mathrm{kHz}$ Switching Frequency and Turn-On and Turn-Off Snubbers.

Figure B-74: Output Stage for Four-Phase Buck, Two-Phase Boost, Multi-Stage Buck-Boost Converter Design with $100 \mathrm{kHz}$ Switching Frequency and Turn-On and Turn-Off Snubbers.

Figure B-75: Output Voltage, Buck Stage Output Voltage and $\mathrm{M}_{1}$ Power Dissipation of Modified 100 kHz Two-Phase Boost, Multi-Stage Buck-Boost Converter Design

Figure B-76: Steady-State Output Voltage, Buck Stage Output Voltage and $\mathrm{M}_{1}$ power Dissipation of Modified $100 \mathrm{kHz}$ Two-Phase Boost, Multi-Stage Buck-Boost Converter Design ....

Figure B-77: Steady-State Input Current, Output Voltage, Buck Stage Output Voltage and $\mathrm{M}_{1}$ Power Dissipation of Modified $100 \mathrm{kHz}$ Two-Phase Boost, Multi-Stage BuckBoost Converter Design ....

Figure B-78: Output Voltage, Buck Stage Output Voltage, $\mathrm{M}_{1}$ Drain Current and $\mathrm{M}_{1}$ Power Dissipation of Modified $100 \mathrm{kHz}$ Two-Phase Boost, Multi-Stage Buck-Boost Converter Design

Figure B-79: Optimized 100 kHz Two-Phase Boost, Multi-Stage Buck-Boost Converter Design ..............375

Figure B-80: Output Voltage, Buck Stage Output Voltage and $Q_{1}$ Power Dissipation of Optimized 100 kHz Two-Phase Boost, Multi-Stage Buck-Boost Converter Design

Figure B-81: Output Voltage, Buck Stage Output Voltage, and $\mathrm{M}_{1}$ Power Dissipation of Optimized $100 \mathrm{kHz}$ Two-Phase Boost, Multi-Stage Buck-Boost Converter Design

Figure B-82: Optimized 100 kHz Two-Phase Boost, Multi-Stage Buck-Boost Converter Design with ES1D Snubber Diodes

Figure B-83: Steady-State Output Voltage, Buck Stage Output Voltage and $\mathrm{Q}_{1}$ Power Dissipation of Optimized $100 \mathrm{kHz}$ Two-Phase boost, Multi-Stage Buck-Boost Converter Design with ES1D Snubber Diodes

Figure B-84: Steady-State Output Voltage, Buck Stage Output Voltage, and $\mathrm{M}_{1}$ Power Dissipation of Optimized $100 \mathrm{kHz}$ Two-Phase Boost, Multi-Stage Buck-Boost Converter Design with ES1D Snubber Diodes

Figure B-85: LTC6908-1 Configuration for use with the LT3845A Controllers .382

Figure B-86: Complete 100 kHz Two-Phase Boost, Multi-Stage Buck-Boost Converter Design with LTC6908-1 External Oscillators 383

Figure B-87: Test Circuit for LTC6908-1 External Oscillator .384 
Figure B-88: Downsized 100 kHz Two-Phase Boost, Multi-Stage Buck-Boost Converter Design with LTC6908-1 External Oscillators...

Figure B-89: Output Voltage, Buck Stage Output Voltage and $\mathrm{Q}_{1}$ Power Dissipation of Downsized 100 kHz Two-Phase Boost, Multi-stage Buck-Boost Converter Design.............391

Figure B-90: Output Voltage, Buck Stage Output Voltage and $\mathrm{Q}_{1}$ Power Dissipation of Downsized $100 \mathrm{kHz}$ Two-Phase Boost, Multi-Stage Buck-Boost Converter Design.....

Figure B-91: Output Voltage, Buck Stage Output Voltage and $\mathrm{Q}_{1}$ and $\mathrm{Q}_{3}$ Power Dissipation of Downsized 100 kHz Two-Phase Boost, Multi-Stage Buck-Boost Converter Design ............393 


\section{NOTE TO THE READER:}

Thank you for your interest in the Energy Harvesting from Elliptical Machines project. When reading this report, please note that this project continues the research and work completed in the first phases of the project, Energy Harvesting from Exercise Machines Self-Generating Elliptical Machines [1] and Energy Harvesting from Elliptical Machines: DC Converter Troubleshooting [1]. 


\section{CHAPTER 1: INTRODUCTION}

This project provides the existing Precor elliptical trainer machine from Dr. Braun's ongoing energy harvesting from exercise machines (EHFEM) project with a functional, self-designed DC-DC converter necessary for the energy harvesting system to provide AC power for the electrical grid. Harvesting energy from physical exercise provides a sustainable energy source for the future and reduces dependence on alreadyscarce natural resources. In addition, it also provides monetary benefits because selfgenerated electricity from physical exercise reduces net energy consumption and results in utility cost savings. However, energy harvesting from physical exercise also requires additional modifications as well electronic devices for the harvesting device (in this project, an elliptical trainer). Those required additional components include a DC-DC converter as well as a grid-tie inverter. This project focuses on the DC-DC converter portion of the EHFEM energy harvesting system. DC-DC converters are widely used today in electronic applications for providing a stable DC voltage from another (usually rectified) DC voltage level. This stable DC voltage is in turn used for powering the main circuit in an electronic device, such as a computer, MP3 player or cellular phone. The main power source for the EHFEM power generation system is Precor's EFX 546i elliptical trainer, which generates a rectified DC voltage from its onboard 6-phase generator [2]. This generated DC voltage varies in magnitude in proportion to the user's

physical exercise level (which includes adjustable physical resistance and incline levels), thus the generated DC voltage magnitude is unstable if the user does not maintain a constant physical exercise level. This project also selects the commercially-available 
Enphase M175-24-240 micro-inverter for the EHFEM system's inverter component. The Enphase M175-24-240 micro-inverter used for taking user-generated DC voltages from the elliptical trainer and sending $\mathrm{AC}$ power back to the power grid requires a stable DC input voltage range for proper operation [3]. Therefore, this project designs a DC-DC converter for providing a stable DC voltage (from a user-generated DC voltage from physical exercise on the elliptical machine) as the input to a power inverter that converts its DC input voltage into a stable $240 \mathrm{~V}_{\mathrm{RMS}} \mathrm{AC}$ voltage to feed back to the electrical grid. There are many different types of DC-DC converter circuit topologies available, such as buck (step-down), boost (step-up) and buck-boost (step-up or down. Furthermore, these topologies are broken down into other sub-categories, such as non-isolated and isolated (the latter makes use of a transformer), as well as PWM-switching converters (otherwise known as hard-switching converters) and soft-switching converters. The non-isolated, PWM-switching single-ended primary inductor converter (SEPIC) topology used in this project outputs a stable DC voltage either greater than or less than its input voltage, which is what the elliptical energy harvesting system requires for generating power from its users' variable stride rate and inputted physical training resistance level. The previous off-the-shelf resonant zero-current switching/zero-voltage switching (ZCS/ZVS) based soft-switching Vicor Maxi 28V DC-DC converter used in one of the previous elliptical projects did not function with the energy harvesting system at all at input voltage levels above $30 \mathrm{~V}$, because of its limited input voltage range (9-36V) [4]. This project proves that a self-designed PWM-switching SEPIC topology provides a functional DC-DC converter design for DC power generation and inverter interfacing from a dynamic 
voltage generation system. Based on user exercise data in this report as well as [1], this thesis project defines in sections 1.1 and 1.2 the electrical and mechanical design constraints for its designed DC-DC converter, based on Precor elliptical user exercise data from [1].

\subsection{Design Requirements}

This section outlines this project's DC-DC converter's design requirements listed below:

- Conformity to UL 1741 specifications [5]

- Conformity to IEEE 1547 specifications [6]

- Conformity to PG\&E safety requirements [7]

- Conformity to the National Electric Code (NEC) [8]

- No long-term costs to ASI or Cal Poly

- No change in the Precor elliptical trainer's user experience with the new system

- The system's long-term operational benefits must be greater than its total implementation costs.

- Must be functionally compatible with existing elliptical power generation system (sans current Vicor Maxi 28V DC-DC converter) at all input resistance levels

- Must meet all specifications outlined in the next section

These requirements are safety considerations as well as economic considerations for maintaining a safe exercise experience for the elliptical trainer's user as well as providing minimal operation costs to Cal Poly should it implement this power generation system. The next section describes quantitative specifications for this project's converter.

\subsection{Project Specifications}

- Output voltage step-down (buck) and step-up (boost) functions

- Non-inverting, PWM-switching SEPIC circuit topology

- 8-60V nominal DC input from the elliptical trainer

- $5 \mathrm{~V}$ minimum DC input from the elliptical trainer

- $65 \mathrm{~V}$ absolute maximum DC input from the elliptical trainer

- $6.5 \mathrm{~A}$ absolute maximum input current (DC) at $65 \mathrm{~V}$ input

- $7.5 \mathrm{~A}$ nominal maximum output current (DC) at $60 \mathrm{~V}$ input

- $8 \mathrm{~A}$ absolute maximum output current (DC) at $65 \mathrm{~V}$ input

- $36 \mathrm{~V}$ DC nominal output (feeds into the Enphase micro-inverter) 
- 288W maximum power output

- Efficiency at $60 \mathrm{~V}$ input must be $\geq 75 \%$ (individual DC-DC converter)

- Line regulation when input changes from $8-60 \mathrm{~V}$ must be $\leq 5 \%$

- Peak-to-peak output voltage ripple must be $\leq 10 \%$

- Both DC-DC converter inductors must run in continuous conduction mode (CCM) down to $25 \%$ maximum output load current (2A, which occurs around $31 \mathrm{~V}$ input according to table E-34)

- Overall input impedance to the converter must be $10 \Omega$, in order to keep the elliptical trainer's eddy current braking system intact

- Must physically fit within the Precor EFX 546i elliptical trainer's mechanical confines

In addition to the converter's requirements and specifications outlined in this section and section 1.1, section 1.3 outlines further electrical design constraints that this project's converter must satisfy.

\subsection{Primary Constraints}

The following constraints control and influence the converter design approach for this project:

- The DC-DC converter needs to be able to tolerate the wide input voltage range that users generate from the elliptical trainer.

- The DC-DC converter's input impedance needs to match that of the elliptical machine's output resistor coils $(10 \Omega)$, in order to keep the elliptical trainer's eddy current braking system intact so that it does not alter the end-user's physical exercise experience. In Spring Quarter 2010, this project's author measured the EFX 546i elliptical trainer's resistor coils having $9.7 \Omega$ series and parallel nominal resistance, no capacitance, $0.0151 \mathrm{mH}$ series inductance at $1 \mathrm{kHz}$ with no parallel inductance, and $0.017 \mathrm{mH}$ series inductance at $120 \mathrm{~Hz}$ with no parallel inductance. Thus it is still possible for this project's DC-DC converter to have some additional input inductance and not affect the elliptical machine's braking system.

- The DC-DC converter must be compatible with all other existing system components, including the Enphase micro-inverter.

- The system and DC-DC converter must overall ensure safe electrical operation for the end-user, components and electrical grid, per UL, IEEE, NEC and PG\&E safety requirements. 
- Voltage, current and power levels in the system should never exceed individual components' absolute maximum ratings (including individual components within the DC-DC converter).

Figure 1.1 shows the EHFEM power generation system block diagram.

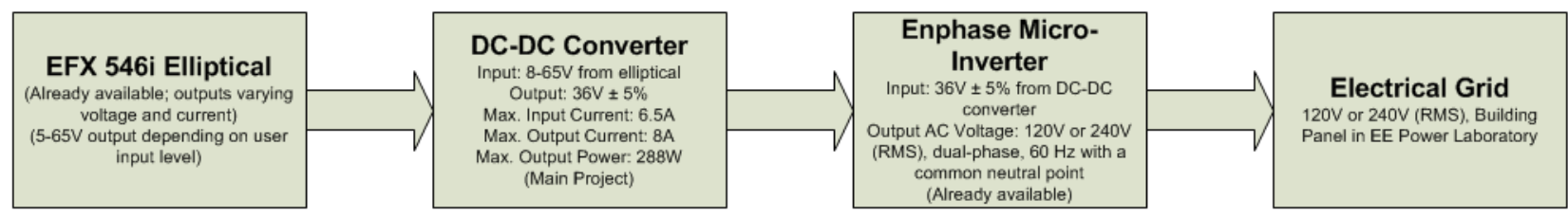

Figure 1.1: EHFEM System Block Diagram

From figure 1.1, at the EHFEM system's input stage, an elliptical trainer user exercises on the Precor EFX 546i and generates an unstable rectified DC voltage. That rectified DC voltage is unstable because of the user's volatile and unpredictable exercise habits and levels. That unstable DC voltage feeds into a DC-DC converter. The DC-DC converter's purpose is converting that unstable user-generated DC-voltage to a stable DC voltage level that the Enphase Micro-Inverter requires for properly converting to the 240V RMS AC voltage (at the system's output stage) that the electrical grid uses. Sections 3.1 and 3.2 describe each stage's quantitative requirements in greater detail. Like the previous elliptical energy harvesting project in [1], all system components except for the DC-DC converter are readily available. This project focuses on the second part of the block diagram in figure 1.1, however, unlike what occurred in previous projects [1], this DC-DC converter uses discrete components and is more functional with the elliptical energy harvesting system at input voltages above $30 \mathrm{~V}$, while still following required specifications and physical space constraints. Designing this DC-DC converter and meeting all project requirements, specifications and constraints requires project 
planning, topology selection, EHFEM system characterization, design, simulation, hardware testing and troubleshooting phases.

The remainder of this report documents from this project's planning, converter topology selection, EHFEM system characterization, design, design simulation and hardware testing and troubleshooting phases, from project inception to completion. These project phases span several academic quarters. This report also provides detailed appendices documenting designs that this project deemed unfeasible based on its requirements, constraints and specifications. Those unfeasible designs contributed to this project's long timeframe and report's length, but helped narrow down a feasible DC-DC converter design for this project. The next chapter describes this project's planning phase, analyzes this project's lifecycle costs and explains how this project fits in with ABET's senior project guidelines. 


\section{CHAPTER 2: PROJECT PLANNING AND ABET SENIOR PROJECT}

\section{ANALYSIS}

\subsection{Project Timeline}

Figures 2.1 to 2.6 show this project's design timeline during each quarter that the author contributed work towards the project.

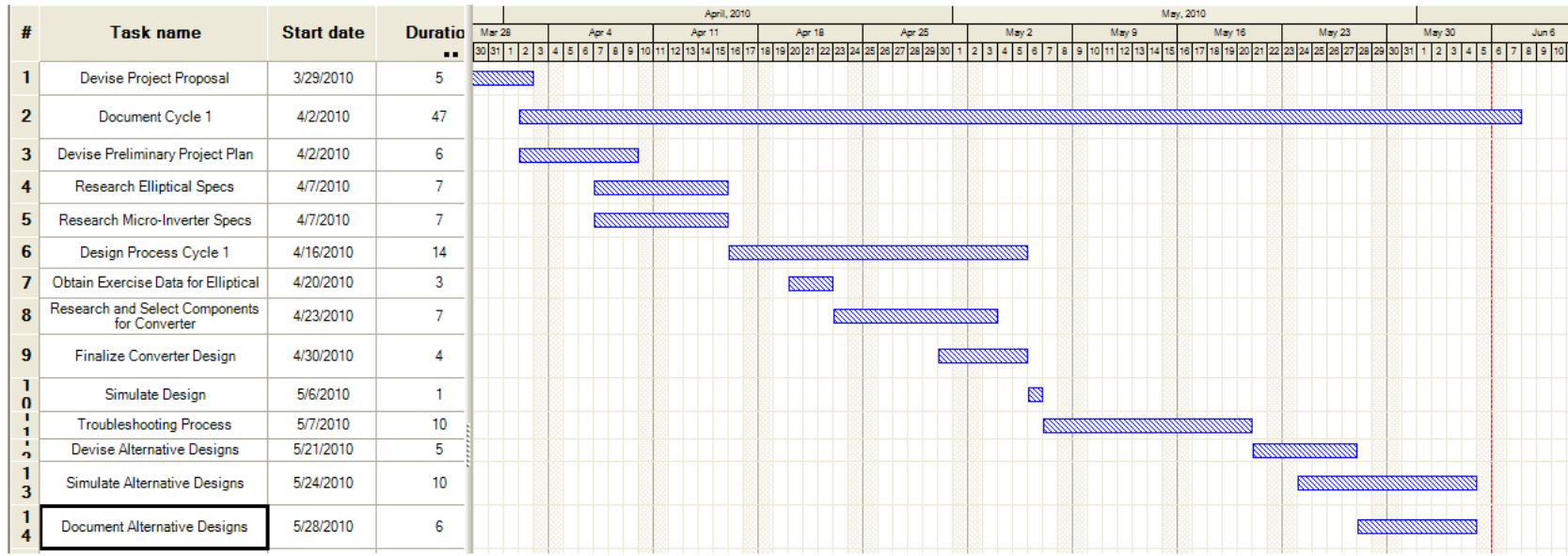

Figure 2.1: Project Timeline for Spring Quarter, 2010

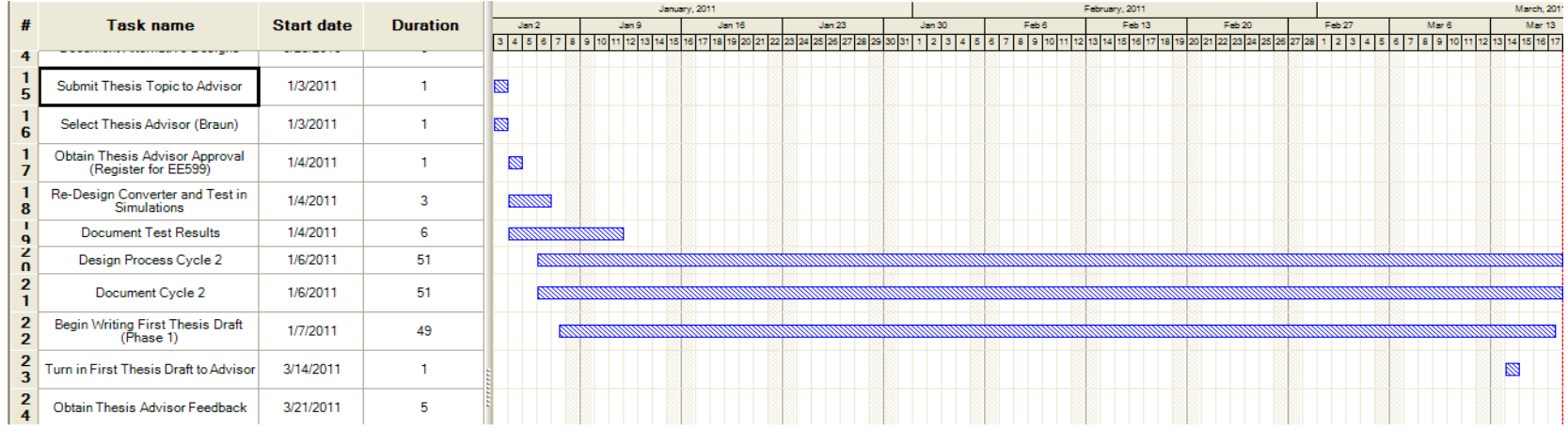

Figure 2.2: Project Timeline for Winter Quarter, 2011 


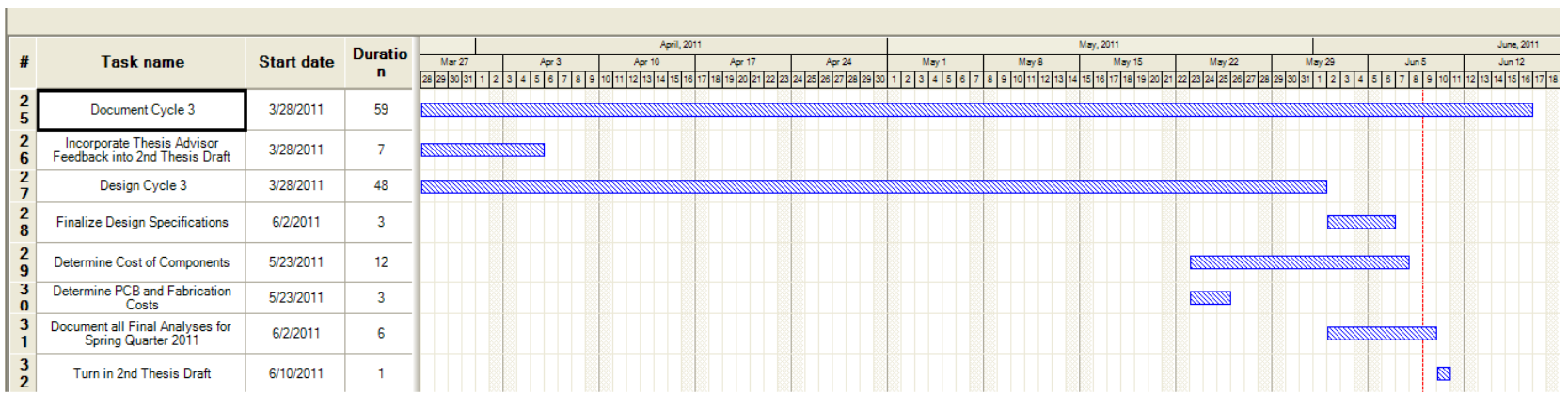

Figure 2.3: Project Timeline for Spring Quarter, 2011

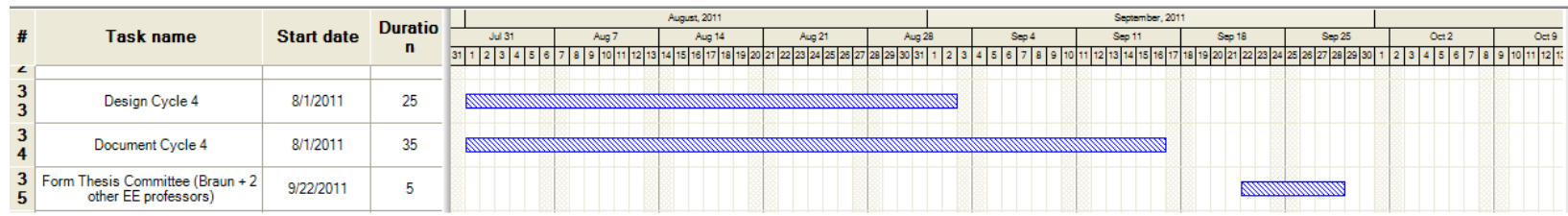

Figure 2.4: Project Timeline for Summer 2011

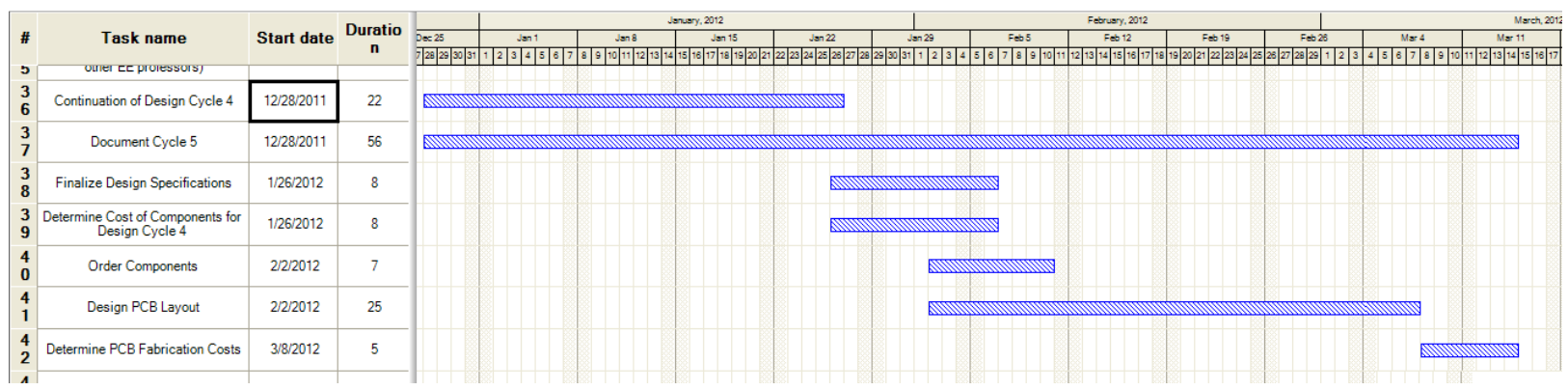

Figure 2.5: Project Timeline for Winter Quarter, 2012

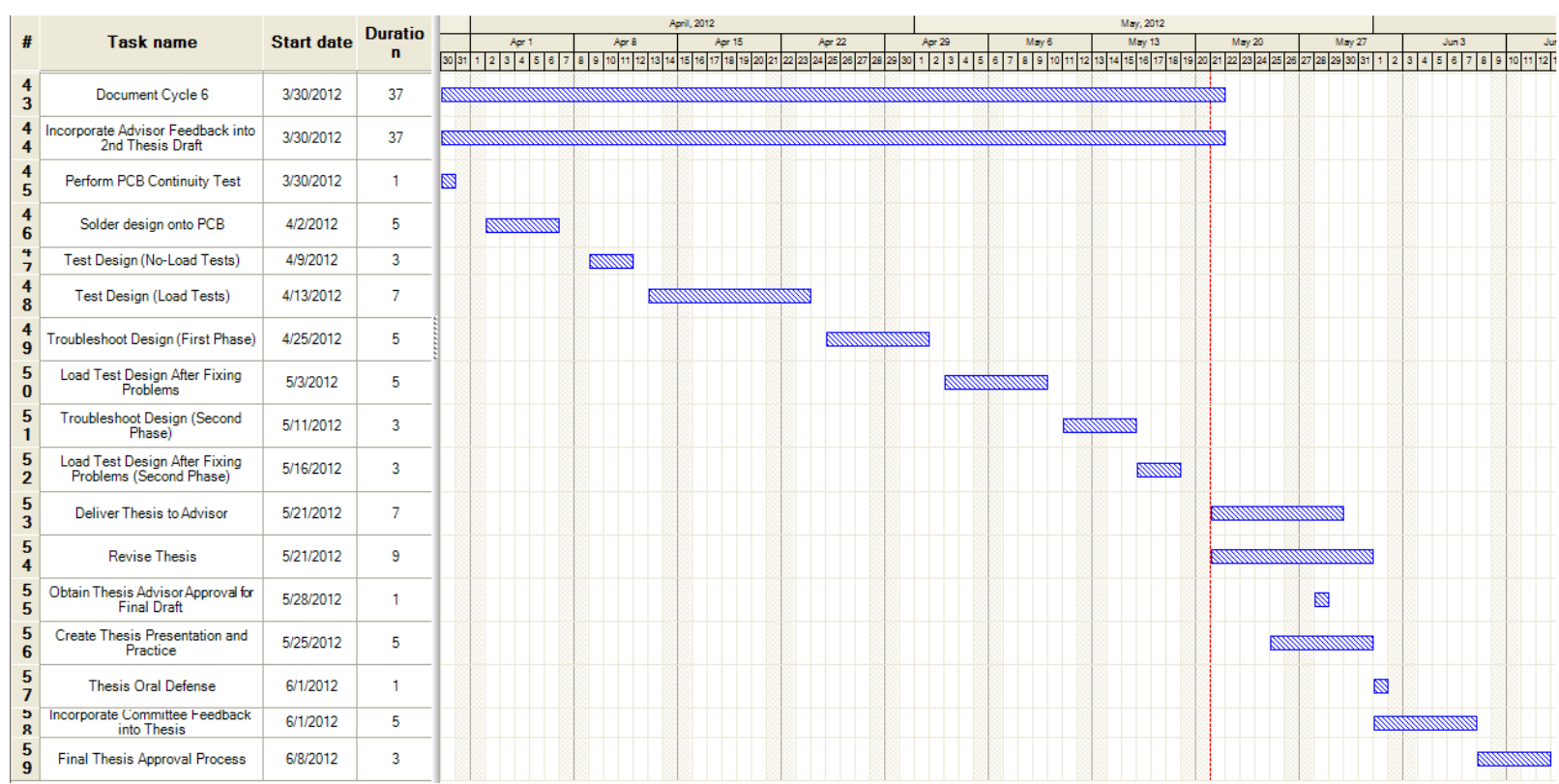

Figure 2.6: Project Timeline for Spring Quarter, 2012 
The Gantt charts in figures 2.1 to 2.6 measure duration in days. This project required 6 quarters for completion because of the author's priority for focusing on completing nonthesis coursework. Furthermore, the project also underwent a few converter design stages that resulted in unfeasible designs for the EHFEM power generation system, adding delay to this project's completion. Refer to appendices A and B for more details on these unfeasible designs. During this project's final two quarters the author completed the printed circuit board (PCB) layout as well as soldered all required components onto the PCB for this project's DC-DC converter. Section 2.2 details this project's estimated component costs for its implementation into the EHFEM power generation system.

\subsection{Estimate of Parts Cost and Labor}

Monetary costs (which include lifecycle costs) as well as payback period affect this project's DC-DC converter's implementation feasibility into the EHFEM power generation system. This section provides an initial estimate of component costs for the converter.

- PWM controller for controlling transistor switching in converter - \$8-10 (for final design; samples for prototype design are free)

- DC-DC converter circuit components (Includes resistors, capacitors, Schottky power diodes and power MOSFETs) - \$60-90

- PCB (for final converter design): \$65-300, depending on number of layers, board size and trace sizes.

- Labor: $~ 9$ hours per week (minimum); has ranged between 10 to 12.5 hours per week throughout this project's timeline. Furthermore, this labor figure also includes time lost (roughly 4-5 hours per week) due to projects and assignments for other courses outside of thesis.

In the worst case scenario, such a converter would cost $\$ 400$ (excluding the chassis and any other shielding material), which is almost twice that of Vicor Maxi 28V DC-DC 
converter previously used in an earlier project [1]. Therefore, if this project's converter does end up costing that much, then its lifecycle cost must remain low and its payback period must be short. Section 10.3.6 describes the final converter component cost in greater detail. Section 2.3.1 discusses the converter's lifecycle costs as well as the EHFEM system's payback period.

\subsection{ABET Senior Project Analysis}

\subsubsection{Economic}

Economic feasibility of this project's converter requires the EHFEM system paying itself off over its lifespan and reducing the Cal Poly Recreation Center's energy costs, as using electricity costs money. Thus in the long run the project's implementation and maintenance costs must equal that of its payback value. Eventually, the project will also pay back to the school, thus the school generates profit off this project, and the total amount of money saved over such a period of time will be greater than the project's implementation and maintenance costs. Implementing such a system in a frequently-used gym such as Cal Poly's Recreation Center can also raise electricity conservation awareness and reduce overall energy consumption in the city of San Luis Obispo. Furthermore, it can also draw attention from potential donors and sponsors that may frequent Cal Poly's Recreation Center, leading to reduced overall system lifecycle costs. According to Dr. David Braun's EHFEM project proposal in [9], for an entire power generation system with $80 \%$ DC-DC converter efficiency and $90 \%$ inverter efficiency, the added cost for modifying a single exercise machine for energy harvesting such as the Precor elliptical trainer must lie under $\$ 360$, in order to achieve a zero system 
lifecycle cost after ten years operation. A zero lifecycle cost after ten years means that the EHFEM system completely offsets its initial system costs (which include implementation) after ten years. This $\$ 360$ maximum added cost value derives from a typical $100 \mathrm{~W}$ generation figure from exercise, along with $0.5 \%$ system efficiency degradation, 12 hours per day machine usage for 41 weeks out of a year and $\$ 0.12$ per $\mathrm{kWh}$ electricity cost for the first year while increasing 3\% annually [9]. This $\$ 360$ figure also assumes that the DC-DC converter and inverter do not require replacement over time. This project's DC-DC converter and the Enphase micro-inverter contribute to the added cost for the EHFEM system. Using a DC-DC converter with $75 \%$ efficiency further reduces this added cost allowance. According to [1], an individual Enphase M175-24-240 micro-inverter costs \$209. Therefore, this project should keep its total DCDC converter costs at $\$ 120$ or below for meeting the $\$ 360$ maximum added system cost for achieving zero EHFEM system lifecycle cost after ten years operation. A DC-DC converter that costs $\$ 400$ in the worst case as mentioned in section 2.2 would not suffice for achieving this maximum added system cost value. However, if this project does not offset the initial system costs from usage in Cal Poly's Recreational Center after ten years, reduced campus electricity usage from electricity conservation awareness by students and faculty as well as monetary donations to Cal Poly's Recreational Center contribute as implicit benefits to Cal Poly as a result of implementing this project.

\subsubsection{Environmental}

Making this converter environmentally friendly requires this project designing it using the fewest materials possible (while still maintaining safety and functionality 
standards) so that it does not leave a large footprint on the environment when system retirement is necessary. Additionally, the components used in this project require safe disposal when necessary. Furthermore, this project benefits the environment in the long run by using a renewable energy source (in this case, physical exercise) that does not release any harmful by-products and because that energy source is renewable, humans do not need to further tap for already scarce resources in the environment for meeting future electricity needs.

\subsubsection{Sustainability}

Sustainability is the main purpose of this project - this project allows for sustainability by creating electricity from physical exercise, which would otherwise be wasted. Physical exercise is always renewable and sustainable from those who use Cal Poly's Recreation Center. Powering the Recreation Center using physical exercise, allows for a reusable energy source in the future without depleting or harming any other resources.

\subsubsection{Manufacturability}

This project needs must be affordable to the end user, and its manufacturing cost as well as materials cost must be as low as possible, while maintaining functionality and performance requirements listed in sections 1.1-1.3. Furthermore, the converter design must be simple, easy to fabricate and be easily modified in order to allow for future modifications that may enhance efficiency or performance of the converter. 


\subsubsection{Ethical}

As with any engineering project, this project requires its converter's design, manufacture, implementation and disposal processes conducted in an ethical manner. From the author's standpoint, this project is ethical only if everyone benefits from it in the long run. Ethical issues that arise include constructing this project's DC-DC converter using RoHS components alongside leaded components for cost reduction, as well as monetary benefits for the EHFEM system's end user and provider.

Using cheaper, leaded components in this project's DC-DC converter for reducing added system costs provides increased monetary benefits for individuals and organizations (such as gyms and fitness centers) but at the same time harms the environment when an EHFEM system component or the system itself requires retirement. Using purely RoHS components for this project's DC-DC converter reduces the available component choice and may drive up the converter's cost, increasing the system's overall payback period. Therefore, this project attempts using as many environmentally-friendly components as possible for its DC-DC converter while simultaneously maximizing operation reliability such that the converter does not require frequent replacement and disposal.

The other ethical issue that arises is that people who use the EHFEM system in organizations such as gyms and fitness centers would receive no monetary reimbursement from generating electricity from their own exercise. However, organizations that provide the exercise equipment already pay for the equipment's implementation and operation costs receive monetary reimbursement from user exercise 
for covering those costs, in addition to fees such as gym membership. The users enjoy added health benefits. Thus, in that situation both the provider and user benefit from system implementation. Applying this case to Cal Poly's Recreation Center yields a similar scenario. Because this project eventually will lower the Recreation Center's operating costs, students will enjoy lower tuition costs. Also because this project helps save the school power, areas and communities with great power needs can use that saved power. Finally, the school gains environmentally-friendly power generation equipment while at the same time supporting this project for future senior project students pursuing a sustainable senior project. Therefore, everyone who this project affects benefits in the long run.

\subsubsection{Health and Safety}

Health and safety are crucial aspects of this project, as the various components of this project transfer and output electrical energy at lethal voltage, current and power levels. This project designs its converter in a manner such that it does not shock, burn or physically injure in any other way its end-user. Also, because this project's initial DC-DC converter prototype has components hand-soldered onto a $\mathrm{PCB}$, this project must take all safety precautions into account during the soldering process. Food and liquids are also commonplace on or near gym equipment in standard gym settings, thus for future extensions of this project, the chassis for this converter must properly shield the electronic components from such dangerous disturbances.

Implementing an energy harvesting system using exercise machines such as the Precor elliptical trainer also provides health benefits for its users. Raising awareness 
about generating electricity from exercise encourages people to exercise more frequently because of potential monetary benefits such as cutting electricity costs. The EHFEM system helps its users burn calories from bodily exercise. In turn, exercising more frequently leads to healthy weight maintenance and potentially reduces the risk of harmful diseases such as heart attacks, obesity and strokes.

\subsubsection{Social}

Gaining wide acceptance of this project's converter in the business world requires using visually pleasing and user-friendly converter components. In other words, this converter must not get in the way of the elliptical machine's user such that it alters their exercise experience. Thus this project designs its converter in such a manner. However the EHFEM system's most crucial aspect is its energy-saving ability while simultaneously providing a renewable energy source - currently "green" machines such as hybrid vehicles are among the largest social trends, particularly among the younger generation of the population. By having more institutions and gyms adopt this type of project, it attracts more people to exercise because of its "green" aspect and in turn help save more power in the long run, emphasizing the sustainability aspect of this project.

\subsubsection{Political}

Finding ways for the United States of America to use less energy and conserve it has been a large part of various political debates for a long time in the past, and such debates are still ongoing. Because this project uses purely human energy and not any natural resources (except for those used in producing the components for this project), this project helps institutions and businesses save money while at the same time conserve 
more electrical energy, which is already a costly and scarce resource. Eventually this project would reach outside of Cal Poly's Recreation Center and expand to other institutions and businesses worldwide, helping cut down the world's dependence for scarce natural resources for energy production. 


\section{CHAPTER 3: SYSTEM COMPONENT CHARACTERIZATION AND DC-DC CONVERTER TOPOLOGY SELECTION}

\subsection{Precor EFX 546i Elliptical Trainer Characterization}

For this project's DC-DC converter, the Precor elliptical machine's power characteristics and physical specifications, the Enphase micro-inverter's specifications, and exercise tests based on the elliptical machine's user input by typical Cal Poly students govern the converter's design requirements and specifications. This project's DC-DC converter also must not modify the end user's experience at all, with the only difference from a standard elliptical trainer machine being that it harvests and sends electrical energy back to the electrical grid. After the user-generated power flows through the DC-DC converter, the Enphase micro-inverter converts the user-generated DC voltage from the DC-DC converter and outputs a $240 \mathrm{~V}_{\mathrm{RMS}} \mathrm{AC}$ voltage, with a maximum power of 175 watts [3], which suffices for residential or commercial applications if many of these modified machines simultaneously work in parallel.

Designing the DC-DC converter using specifications outlined in sections 1.1-1.3 first requires gathering data on how hard typical Cal Poly students exercise on an elliptical trainer. The Precor EFX 546i elliptical trainer has 20 preset physical resistance settings (corresponding to resistance levels 1-20 on the machine), as well as an incline modifier. The elliptical trainer also dumps its output power generated from user exercise into an onboard $10 \Omega$ resistor load. That power becomes heat when dumped. Any resistance change to the elliptical trainer's $10 \Omega$ load results in an altered physical resistance that the user feels when exercising, hence causing an altered user experience 
[10]. Therefore this project's DC-DC converter must maintain a $10 \Omega$ input impedance during user exercise for maintaining the elliptical trainer user's exercise experience. The elliptical trainer also has a digital readout that measures the user's exercise speed in strides per minute. For the preliminary exercise tests, this project keeps the machine's incline constant while modifying the test subject's resistance levels in steps of one level. Tables A-1 to A-4 and figure 3.1 show the results of these exercise tests. For the first two exercise participants, the author could not perform current (and thus power) measurements, until he devised a method in which he could perform such measurements for the last two participants. 
Table A-3-1: Physical Exercise Input and Electric Output Results for Participant \#1

\begin{tabular}{|c|c|c|}
\hline \multicolumn{3}{|c|}{ Test Participant \#1 } \\
\hline Resistance Level & Exercise Rate (Strides/min.) & Output Voltage (V) \\
\hline 1 & 154 & 0.04 \\
\hline 2 & 150 & 8.85 \\
\hline 3 & 154 & 11.84 \\
\hline 4 & 148 & 13.51 \\
\hline 5 & 148 & 16.23 \\
\hline 6 & 154 & 19.01 \\
\hline 7 & 150 & 21.59 \\
\hline 8 & 152 & 23.79 \\
\hline 9 & 148 & 27.33 \\
\hline 10 & 150 & 29.56 \\
\hline 11 & 138 & 31.6 \\
\hline 12 & 134 & 34.07 \\
\hline 13 & 112 & 33.81 \\
\hline 14 & 112 & 34.13 \\
\hline 15 & 104 & 34.69 \\
\hline 16 & 86 & 30.37 \\
\hline 17 & 82 & 28.7 \\
\hline 18 & 72 & 27.86 \\
\hline 19 & 68 & 28.82 \\
\hline 20 & 72 & 30.72 \\
\hline
\end{tabular}


Table A-3-2: Physical Exercise Input and Electric Output Results for Participant \#2

\begin{tabular}{|c|c|c|}
\hline \multicolumn{3}{|c|}{ Test Participant \#2 } \\
\hline Resistance Level & Exercise Rate (Strides/min.) & Output Voltage (V) \\
\hline 1 & 188 & 0.04 \\
\hline 2 & 166 & 9.87 \\
\hline 3 & 158 & 12.76 \\
\hline 4 & 170 & 15.29 \\
\hline 5 & 148 & 16.29 \\
\hline 6 & 162 & 20.45 \\
\hline 7 & 150 & 22.54 \\
\hline 8 & 174 & 26.5 \\
\hline 9 & 164 & 29.26 \\
\hline 10 & 158 & 30.75 \\
\hline 11 & 172 & 35.63 \\
\hline 12 & 162 & 37.3 \\
\hline 13 & 160 & 40.01 \\
\hline 14 & 115 & 37.48 \\
\hline 15 & 104 & 37.16 \\
\hline 16 & 98 & 33.98 \\
\hline 17 & 100 & 36.9 \\
\hline 18 & 80 & 29.22 \\
\hline 19 & 94 & 34.9 \\
\hline 20 & 100 & 42.84 \\
\hline
\end{tabular}


Table A-3-3: Physical Exercise Input and Electric Output Results for Participant \#3

\begin{tabular}{|c|c|c|c|c|}
\hline \multicolumn{5}{|c|}{ Test Participant \#3 } \\
\hline $\begin{array}{l}\text { Resistance } \\
\text { Level }\end{array}$ & $\begin{array}{l}\text { Exercise Rate } \\
\text { (Strides/min.) }\end{array}$ & $\begin{array}{l}\text { Output Voltage } \\
\text { (V) }\end{array}$ & $\begin{array}{l}\text { Output Current } \\
\text { (A) }\end{array}$ & $\begin{array}{l}\text { Power } \\
\text { (W) }\end{array}$ \\
\hline 1 & 124 & 0.03 & 0 & 0 \\
\hline 2 & 130 & 8.08 & 0.78 & 6.3024 \\
\hline 3 & 130 & 10.56 & 1.02 & 10.7712 \\
\hline 4 & 126 & 12.97 & 1.32 & 17.1204 \\
\hline 5 & 130 & 15.66 & 1.53 & 23.9598 \\
\hline 6 & 130 & 16.75 & 1.7 & 28.475 \\
\hline 7 & 129 & 20.23 & 1.97 & 39.8531 \\
\hline 8 & 128 & 21.86 & 2.15 & 46.999 \\
\hline 9 & 129 & 25.72 & 2.45 & 63.014 \\
\hline 10 & 130 & 27.15 & 2.77 & 75.2055 \\
\hline 11 & 128 & 31.11 & 2.9 & 90.219 \\
\hline 12 & 124 & 31.33 & 3.17 & 99.3161 \\
\hline 13 & 132 & 34.81 & 3.56 & 123.9236 \\
\hline 14 & 126 & 37.45 & 3.66 & 137.067 \\
\hline 15 & 128 & 38.77 & 3.84 & 148.8768 \\
\hline 16 & 125 & 40.37 & 4.09 & 165.1133 \\
\hline 17 & 115 & 34.32 & 3.85 & 132.132 \\
\hline 18 & 98 & 37.2 & 3.52 & 130.944 \\
\hline 19 & 90 & 39.52 & 3.53 & 139.5056 \\
\hline 20 & 80 & 29.81 & 2.81 & 83.7661 \\
\hline
\end{tabular}


Table A-3-4: Physical Exercise Input and Electric Output Results for Participant \#4

\begin{tabular}{|c|c|c|c|c|}
\hline \multicolumn{2}{|c|}{ Test Participant \#4 } \\
$\begin{array}{c}\text { Resistance } \\
\text { Level }\end{array}$ & $\begin{array}{c}\text { Exercise Rate } \\
\text { (Strides/min.) }\end{array}$ & $\begin{array}{c}\text { Output Voltage } \\
(\mathbf{V})\end{array}$ & $\begin{array}{c}\text { Output Current } \\
(\mathbf{A})\end{array}$ & $\begin{array}{c}\text { Power } \\
(\mathbf{W})\end{array}$ \\
\hline 1 & 130 & 0.03 & 0 & 0 \\
\hline 2 & 130 & 9.27 & 0.75 & 6.9525 \\
\hline 3 & 130 & 11.42 & 1.08 & 12.3336 \\
\hline 4 & 130 & 13.01 & 1.33 & 17.3033 \\
\hline 5 & 130 & 15.43 & 1.56 & 24.0708 \\
\hline 6 & 130 & 17.41 & 1.77 & 30.8157 \\
\hline 7 & 130 & 20.08 & 2.01 & 40.3608 \\
\hline 8 & 130 & 22.39 & 2.23 & 49.9297 \\
\hline 9 & 130 & 25.09 & 2.42 & 60.7178 \\
\hline 10 & 130 & 29.16 & 2.66 & 77.5656 \\
\hline 11 & 130 & 30.15 & 2.91 & 87.7365 \\
\hline 12 & 130 & 33.16 & 3.2 & 106.112 \\
\hline 13 & 130 & 33.24 & 3.05 & 101.382 \\
\hline 14 & 130 & 37.07 & 3.55 & 131.5985 \\
\hline 15 & 130 & 37 & 3.17 & 117.29 \\
\hline 16 & 120 & 33.89 & 2.49 & 84.3861 \\
\hline 17 & 90 & 27 & 1.85 & 49.95 \\
\hline 18 & 90 & 32 & 3.49 & 111.68 \\
\hline 19 & 90 & 29 & 2.11 & 61.19 \\
\hline 20 & 80 & 20 & 2.63 & 52.6 \\
\hline
\end{tabular}

\section{Elliptical Output DC Power vs. Training Resistance Level}

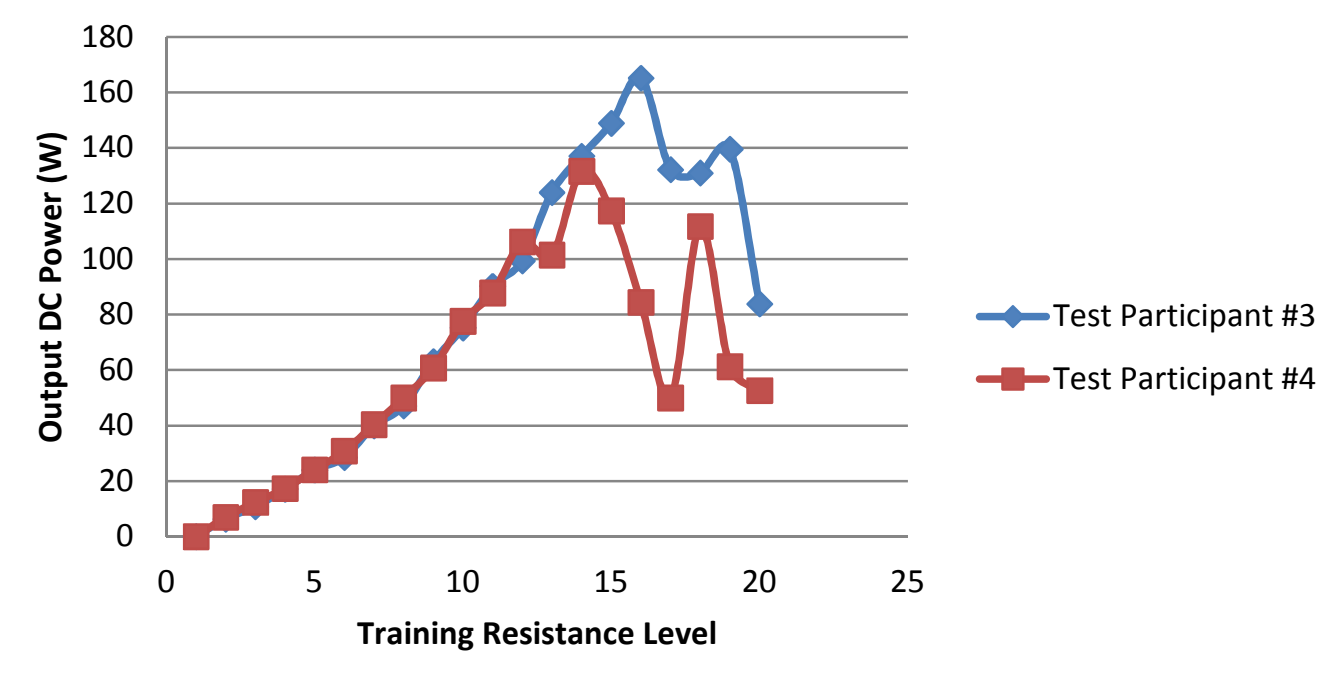

Figure 3.1: Elliptical DC Output Power for Participants \#3 and 4 vs. Resistance Level 
As expected, the user's physical exercise rate decreases as the elliptical machine's physical resistance level increases. Output power falloffs occur at higher training resistance levels because of the participants' inability to maintain a 120 strides per minute exercise pace. This physical resistance relies on an eddy current braking system within the elliptical machine for making a workout more difficult for the elliptical trainer's user at higher physical resistance settings, hence the lower exercise rates at higher resistance settings. However, increasing the physical resistance level helps generate more output power as long as the user exercises at a constant rate relative to lower resistance levels. In the case of participants \#3 and 4, the author attempted having both participants maintain to the best of their abilities a 130 strides per minute exercise rate. Out of the four participants, the maximum output voltage obtained from the elliptical trainer was $42.84 \mathrm{~V}$ and the maximum output current obtained was 4.09A. This corresponds to a maximum output (input to the DC-DC converter) power of $175.2 \mathrm{~W}$, which is well under the $288 \mathrm{~W}$ converter output load power specification that this project set. The reason this project set a higher specification was for headroom, after analyzing exercise data from [1]'s project report (in that report, the group members reported a 350W output from the elliptical trainer on a participant performing 160 strides per minute at resistance level 20). Furthermore, that same group obtained a $60 \mathrm{~V}, 6 \mathrm{~A}$ output from the elliptical trainer when their participant exercised at 160 strides per minute at resistance level 20. Therefore, this project selects $65 \mathrm{~V}$ as its peak input voltage - with the additional $5 \mathrm{~V}$ as headroom. The project also selects $6.5 \mathrm{~A}$ as its nominal maximum output current level at $60 \mathrm{~V}$ input from the Precor elliptical trainer. The next section selects an absolute maximum output current 
level. Even with these set maximum specifications, the converter requires input and output protection from voltage and current levels that exceed those specifications. For basic protection from such conditions this converter requires input and output fuses rated for their set maximum current levels. The results in tables A- 1 to A- 4 also show that the elliptical trainer does not begin outputting current until it reaches at least $8 \mathrm{~V}$ output. Therefore, this project selects $5 \mathrm{~V}$ as its minimum required input voltage for converter output regulation - the $3 \mathrm{~V}$ from the minimum $8 \mathrm{~V}$ input is for headroom, likewise with the maximum input voltage selection process. This project selects $8-60 \mathrm{~V}$ as the nominal input voltage range based on exercise data from tables A-1 to A-4 as well as [1]. Also, according to a survey that same group performed, however, most Cal Poly students using the ASI Recreation Center typically exercise between resistance levels 5 to 10 at speeds between 140 to 180 strides per minute - translating to a $60-100 \mathrm{~W}$ power generation range [1]. This range is far below the physical exercise level and consequently, the maximum electrical levels that the elliptical machine could theoretically output. Later in the DC-DC converter testing process, this project also discovered that the Precor elliptical trainer does not output a ripple-free DC voltage [10]. Section 7.1 describes this problem in greater detail and it results in minor changes to this project's DC-DC converter later on. Section 3.2 explains the reasoning for the $288 \mathrm{~W}$ maximum converter output load power level, as well as specifications from the Enphase micro-inverter that govern this project's DC-DC converter's design. 


\subsection{Enphase M175-24-240 Micro-Inverter Specifications and Characterization}

The current Precor EFX 546i-based EHFEM system uses an Enphase M175-24240 micro-inverter for power inversion and sending generated power to the electrical grid. This inverter complies with FCC Part 15 Class B, UL 1741 and IEEE 1547 standards, making it safe for this project's EHFEM system [3]. This inverter also features maximum power tracking. The inverter activates its maximum power tracking feature if its input voltage is between 25 and $40 \mathrm{~V}$. The inverter can also tolerate up to $54 \mathrm{~V}$ maximum input voltage, along with a recommended 8A maximum input current (though it can tolerate up to 10A maximum input short circuit current) [3]. This project uses Enphase's 8A maximum input current specification as the output current for its DCDC converter. Therefore, this project selects $8 \mathrm{~A}$ as its DC-DC converter's maximum output (load) current. Enphase also recommends 210W as the inverter's input power, though its datasheet does not specify a maximum input power level [3]. On its output side, the inverter also outputs $175 \mathrm{~W}$ of power (maximum), 750mA nominal RMS current and 240V nominal RMS AC voltage (varying between $211-264 V_{\text {RMS }}$ ) at $60 \mathrm{~Hz}$ nominal frequency (varying between $59.3-60.5 \mathrm{~Hz}$ ) with at least 0.95 power factor [3]. Enphase also states that the M175-24-240's peak efficiency is 95\% [3]. EHFEM project member Alvin Hilario also concluded that the inverter's optimal input voltage is $36 \mathrm{~V}$ for obtaining peak efficiency from it [10]. Therefore, this project selects $36 \mathrm{~V}$ as its required DC-DC converter output voltage. However, the DC-DC converter still requires a maximum average input current specification. Obtaining that value requires estimating the DC-DC converter's efficiency. This project uses a conservative, $75 \%$ efficiency 
estimate at full load (65V input, $8 \mathrm{~A}$ output load current with $36 \mathrm{~V}$ output) for its DC-DC converter. Equations (3.1) and (3.2) obtain the converter's maximum average input current.

$$
\begin{gathered}
\eta=\frac{P_{\text {OUT }}}{P_{I N}}=\frac{V_{\text {OUT }} I_{\text {OUT }}}{V_{I N} I_{I N}} \\
I_{I N}=\frac{V_{\text {OUT }} I_{\text {OUT }}}{\eta V_{I N}}=\frac{(36 \mathrm{~V})(8 \mathrm{~A})}{(0.75)(65 \mathrm{~V})}=5.91 \mathrm{~A}
\end{gathered}
$$

$\mathrm{V}_{\text {OUT }}$ denotes the converter's average output voltage, IOUT denotes its average output current, $\mathrm{V}_{\mathrm{IN}}$ denotes its average input voltage, and $\mathrm{I}_{\mathrm{IN}}$ denotes its average input current. (3.2) determines this project's DC-DC converter's required maximum input current as 5.91A, but this project selects $6.5 \mathrm{~A}$ for headroom. The $6.5 \mathrm{~A}$ limit also helps the converter maintain a $10 \Omega$ input resistance at $65 \mathrm{~V}$ input, helping maintain the elliptical user's exercise experience. After selecting the converter's electrical specifications, this project selects a topology for its DC-DC converter. Sections 3.3 and 3.3.1 documents this project's entire DC-DC converter topology selection process.

\subsection{Converter Topology Selection}

Numerous topologies exist for wide-input DC-DC conversion. However, the topology selected for this project's DC-DC converter must tolerate the requirements and specifications outlined in sections 1.1-1.3. Furthermore, the selected topology must minimize circuit complexity and component count while maintaining functionality as well as safety to both the EHFEM system and the elliptical trainer user. The previous offthe-shelf converter used by the EHFEM group in [1] shut down at input voltages above $30 \mathrm{~V}$ and in turn caused physical resistance loss to the elliptical trainer's user, creating a 
safety hazard for physical injuries. Furthermore, that same EHFEM group also reported that "at higher resistance levels, if the user decreases pace, he or she feels a large spike of physical resistance against their feet" [1]. This sudden "spike of physical resistance" not only alters the elliptical user's exercise experience, but like the physical resistance loss phenomenon mentioned earlier, it also creates a safety hazard for physical injuries to the elliptical trainer's user. Therefore it is crucial that this project's DC-DC converter maintains a steady $10 \Omega$ input impedance, which prevents such abrupt physical resistance changes during user exercise. Furthermore, this project's DC-DC converter must also function and output voltage and current throughout the specified 5-65V input range, which helps prevent sudden physical resistance loss to the elliptical trainer's user. Also, the previous-off-the-shelf converter used by the EHFEM group in [1] peaked at $80 \%$ efficiency, therefore this project's DC-DC converter's efficiency must be as high as possible for minimizing its parts cost, which in term minimizes its payback period for achieving zero life-cycle cost, as outlined in section 2.3.1 [1].

Selecting an appropriate DC-DC converter topology also requires consideration for typical operation from typical user exercise levels on the elliptical trainer. As mentioned in section 3.1, typical elliptical users in the Cal Poly Recreational Center exercise between training resistance levels 5 and 10, which translates to between $15-30 \mathrm{~V}$ typical input for the DC-DC converter. With the DC-DC converter outputting 36V, this means that the converter will typically operate in voltage step-up (boost) mode. Although this EHFEM system requires a topology with both boost and voltage step-down (buck) modes because of its $5-65 \mathrm{~V}$ input range specification, this project puts more emphasis on 
a converter topology with very efficient boost mode operation, because high efficiency in typical operation settings reduce the EHFEM system's payback period.

Firstly, DC-DC converters with both boost and buck functions are available in non-isolated and isolated topologies. Isolated topologies typically use a transformer for providing isolation between the converter's input and output stages. Such topologies include flyback, forward, push-pull, half-bridge and full-bridge DC-DC converters. Selecting an appropriate, commercially-available transformer for these topologies that fits this project's converter's electrical requirements can be difficult because of turns-ratio considerations as well as current-carrying capacity of each winding. Not having an appropriate, commercially-available transformer also resorts the project to using a custom transformer, which can significantly increase the converter's cost and control complexity. A push-pull converter, for instance, requires two switches and a transformer with four windings [11]. Half-bridge converters require a 3-winding transformer and two switches, and full bridge converters require four switches in addition to a 3-winding transformer, resulting in very complex converter circuitry and increased component count. Flyback and forward converters are the simplest of the isolated converter topologies, but have very low power density, making it unsuitable for high current output loads that this project requires [11]. Therefore, such converter topologies are unfeasible for this project. Non-isolated topology-based converters typically have a lower parts count and their operation isn't as complex as isolated converters. UL standards also dictate that operating voltages above $42.5 \mathrm{~V}$ require isolation between input and output for preventing user access [10]. However, the Precor elliptical trainer outputs voltages above 42.5V and uses 
a hefty enclosure, preventing access to internal components for typical end-users.

Furthermore, the Enphase micro-inverter already contains isolation transformers between its DC input and AC output branches. Therefore, a non-isolated based converter is appropriate for this project's EHFEM system.

The second consideration in topology selection for this project's DC-DC converter is whether to use a hard-switching or resonant soft-switching topology. The Vicor Maxi 28V DC-DC converter that the previous EHFEM group in [1] used is a resonant ZCS/ZVS (soft-switching) architecture [4]. However, this converter did not function with the EHFEM system at higher input voltages and also altered the elliptical user's experience, thus making its topology unsuitable for this project's EHFEM system. Resonant soft-switching converters typically yield high efficiency [12], but typically have more complex circuitry than hard-switching converters, driving up converter cost [13]. Furthermore, a resonant soft-switching converter is not necessary for achieving high efficiencies with the typical 15-30V exercise output range [10]. Therefore, a hardswitching, non-isolated topology is the best choice for this project's EHFEM system. Section 3.3.1 compares different hard-switching, non-isolated topologies and selects the appropriate topology for this project.

\subsubsection{Comparison of Hard-Switching, Non-Isolated Topologies}

Perhaps the most well-known hard-switching, non-isolated DC-DC converter topologies with boost and buck functions are the buck-boost and Ćuk topologies. Their basic implementations, however, are unsuitable for this project because they output a voltage with an inverted polarity with respect to their inputs [14]. Therefore, this project 
requires variations of such topologies if implemented into the EHFEM system. Obtaining a non-inverted output voltage polarity from either a buck-boost or Ćuk converter requires additional components such as switches or transformers, increasing circuit complexity and component count $[15,16]$. Although Enphase's M175-24-240 datasheet does not explicitly state that the M175-24-240 micro-inverter requires a positive polarity DC voltage input, this project assumes that the datasheet's specified input voltage notation implies that the micro-inverter requires positive input DC voltage polarity [3]. Furthermore, adding switches to a DC-DC converter requires additional control circuitry, usually additional or more complex control ICs. This problem can significantly increase converter cost as well as decrease operation reliability.

This project at one point considered a four-switch buck-boost topology, as it yields a non-inverted output voltage and high efficiency [17], but its overall complexity and potential cost deemed it unsuitable for this project. Also, early on in the selection process, this project selected a PWM-switching, non-inverting Ćuk DC-DC converter topology, but simulation results uncovered severe load regulation problems during a full load condition (60-65V input, 8A load), thus making it unsuitable for this project. Furthermore, this project was also unable to find a suitable control IC that could properly drive the two switches that the non-inverting Ćuk converter required while still tolerating the 5-65V input specification. Later on, this project's author discovered that the Precor Elliptical trainer's onboard $12 \mathrm{~V}$ battery [1] could power and bias a control IC, bypassing the $5-65 \mathrm{~V}$ input range requirement for the control IC. However, by that time, the author proposed the DC-DC converter design that this project uses and analyzes. This project 
also experimented with a non-inverting, multi-phase PWM switching cascaded buckboost converter in simulations. Such a converter consists of a buck converter input stage and boost converter output stage connected in cascade. However, this project scrapped that design because of its circuit complexity, high component count, high cost and low efficiency (87.1\% maximum) relative to its monetary cost. Refer to appendices A and B for more information on the failed non-inverting Ćuk and cascaded buck-boost designs as well as their simulation results.

After recognizing the non-inverting Ćuk and cascaded buck-boost design's unfeasibility, this project examined two underutilized hard-switching, non-isolated topologies: the single-ended primary inductor converter (SEPIC) and Zeta topologies. SEPICs and Zeta converters are essentially modified Ćuk converters, but their basic implementations output a non-inverted polarity voltage with respect to their input voltages. Figures 3.2 and 3.3 show the SEPIC and Zeta converters in their basic implementations $[18,19]$.

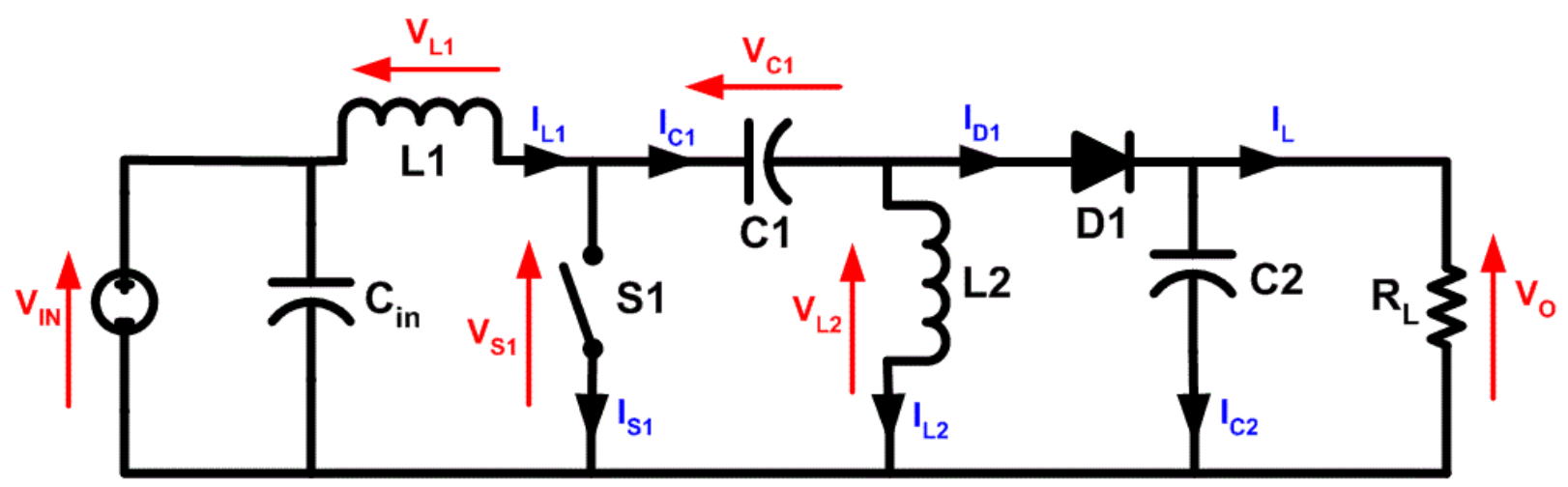

Figure 3.2: Basic SEPIC Layout [18] 


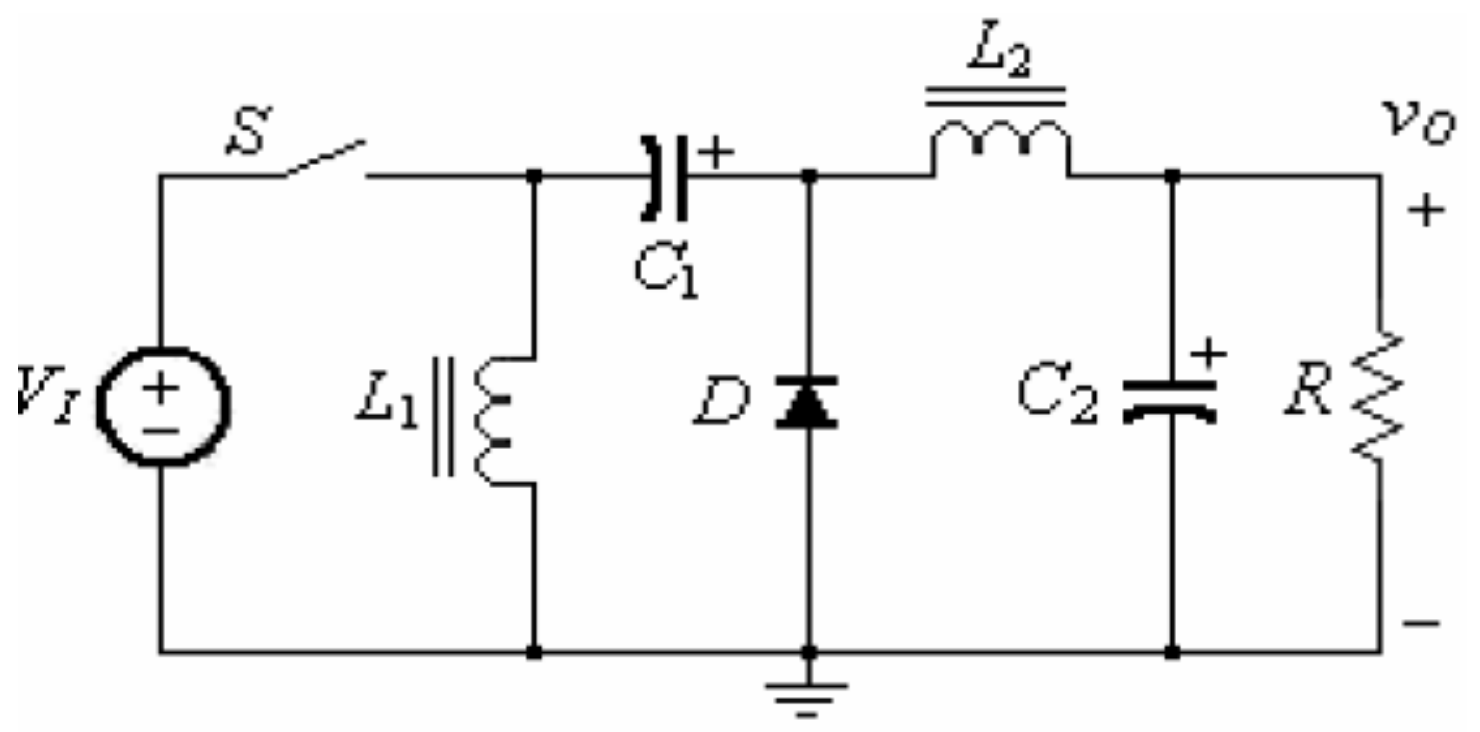

Figure 3.3: Basic Zeta Converter Layout [19]

The basic SEPIC and Zeta converter implementations both use two inductors for energy exchange and outputting a non-inverted voltage, while also performing both boost and buck functions. Most implementations of both converters magnetically couple the two inductors onto a single core, saving physical PCB space as well as cost. However, section 5.1 indicates that using a magnetically coupled inductor for such converter implementations is unfeasible for this project, because of high current requirements resulting from the $8 \mathrm{~A}$ maximum output load current requirement described in section 1.2. Using two discrete inductors on a SEPIC or Zeta converter, however, while adding cost and requiring additional physical PCB space, is easier than using an isolated topology because high-current inductors are widely available in the commercial market and have a less complex selection process than transformers. Furthermore, both converters' basic implementations maintain a low component count when compared to isolated converters or buck-boost or Ćuk converter variations. From figures 3.2 and 3.3, SEPICs and Zeta converters have identical layouts with respect to each other, except that the inductor, 
switch and diode positions are different. The SEPIC's layout is more akin to that of a boost converter [20,21], while the Zeta converter's layout more resembles that of a buck converter [22]. Section 3.3 indicated that based on elliptical user exercise data, this project's DC-DC converter will typically operate in boost mode. Therefore, this project prefers the SEPIC over the Zeta converter. Both converter topologies yield roughly the same theoretical efficiencies ( 95\%), with the Zeta converter having slightly higher efficiency [23, 24], but because the SEPIC functions more similarly to a boost converter and because this project's DC-DC converter will typically operate in boost mode, the SEPIC is this project's choice. Neither converter is also as efficient as a standard Cuk converter, but the SEPIC and Zeta converters' main advantage is that they output a noninverted voltage while still maintaining a low component count [23]. Furthermore, both converters provide electrical isolation between their inputs and outputs via a coupling capacitor, but they are not typically considered "isolated" topologies because their typical applications do not require transformers. This electrical pseudo-isolation makes it suitable for high power applications such as this project. This project selects the SEPIC as its DC-DC converter topology because of its low component count and its functionality similar to that of a boost converter. Furthermore, it only requires one switch for proper operation (and hence one control IC), thus reducing this project's DC-DC converter's overall circuit complexity. The next chapter provides a brief introduction to the SEPIC topology and its functionality. 


\section{CHAPTER 4: THE SEPIC}

\subsection{SEPIC Introduction}

As mentioned in section 3.3.1, a SEPIC is a type of non-isolated DC-DC converter that can convert a DC voltage to another higher or lower stable DC voltage level at its output. "Single-ended" means that only one switch in the converter controls energy exchange between its components [25].

The main advantage that the SEPIC has over boost and buck-based converter topologies is that it outputs a voltage ( $V_{O}$ in figure 3.2) that is either higher or lower than that of its input voltage $\left(\mathrm{V}_{\mathrm{IN}}\right.$ in figure 3.2) [26], whereas a boost converter can only output a voltage higher than that of its input voltage, and a buck converter can only output a voltage lower than that of its input voltage. The overall layout is similar to that of a boost converter [20,21], with overall good input current characteristics because inductor $\mathrm{L}_{1}$ in figure 3.2 helps keep the input current waveform close to that of a DC waveform, but bad output current characteristics because diode $\mathrm{D}_{1}$ always sends a pulsating current to the converter's output. Essentially, a SEPIC is a cascaded boost/buck-boost converter, with its input stage similar to that of a basic boost converter, and its output stage is similar to that of a basic buck-boost converter. Overall, a SEPIC functions akin to a buck-boost converter, but has the additional advantages of having its output voltage polarity non-inverted with respect to its input voltage [26], having a true shutdown mode - i.e. when switch $S_{1}$ turns off, the converter's output voltage reduces to $0 \mathrm{~V}$, and having isolation between the input and output (from coupling capacitor $\mathrm{C}_{1}$ ) [25]. The capacitive isolation prevents unwanted current from flowing from the input to output 
(and potentially cause premature switch failure) [27]. $\mathrm{S}_{1}$ 's duty cycle controls the SEPIC's output voltage. Typically $S_{1}$ is an electrically controlled switch, such as a power metal oxide semiconductor field effect transistor (MOSFET), power bipolar junction transistor (BJT) or insulated-gate bipolar transistor (IGBT). Its switching actions are controlled by a pulse-width modulation (PWM) or pulse-frequency modulation (PFM) controller. A PWM controller varies $\mathrm{S}_{1}$ 's duty cycle while keeping its switching frequency constant, while a PFM controller varies $S_{1}$ 's switching frequency while keeping its duty cycle constant [28]. The next section describes the SEPIC's rudimentary operation.

\subsection{Basic SEPIC Operation}

The basic SEPIC performs DC-DC voltage conversion through energy exchange between its coupling capacitor and switching inductors $\left(\mathrm{C}_{1}, \mathrm{~L}_{1}\right.$ and $\left.\mathrm{L}_{2}\right)$. $\mathrm{S}_{1}$ controls the energy exchange amount between the capacitor and inductors.

Maximizing energy exchange efficiency (and overall converter efficiency) requires this project's SEPIC design operating in continuous conduction mode (CCM) [29]. In CCM, the currents through $\mathrm{L}_{1}$ and $\mathrm{L}_{2}$ never reach $0 \mathrm{~A}$ for any significant time period relative to $S_{1}$ 's switching period. Discontinuous conduction mode (DCM) occurs if the currents through $\mathrm{L}_{1}$ and $\mathrm{L}_{2}$ remain at $0 \mathrm{~A}$ for any significant time period relative to $\mathrm{S}_{1}$ 's switching period [25]. Operating a SEPIC in DCM yields higher efficiency at lighter current loads [30, 31], but this project's SEPIC requires operating at high current loads (> 1A). A survey performed by the previous EHFEM group in [1] in May 2009 shows that most elliptical trainer users in the Cal Poly ASI Recreational Center exercise at levels that 
do not drive very light loads [1]. Therefore, operating in CCM should yield better overall converter efficiency for this project's SEPIC. A third operation mode known as boundary conduction mode $(\mathrm{BCM})$, occurs when the currents through $\mathrm{L}_{1}$ and $\mathrm{L}_{2}$ drop to $0 \mathrm{~A}$ but immediately rise back to their maximum values. $\mathrm{BCM}$ is the transition point between the CCM and DCM operation modes [29].

\subsection{SEPIC Operation in Continuous Conduction Mode (CCM)}

Operating the SEPIC in CCM means never letting the currents through $\mathrm{L}_{1}$ and $\mathrm{L}_{2}$ reduce to $0 \mathrm{~A}$ - i.e. never letting $\mathrm{L}_{1}$ and $\mathrm{L}_{2}$ completely discharge. When the SEPIC reaches steady-state operation, the average voltage across $C_{1}$ will be equal to that of $V_{\text {IN }}$. Additionally, the average current through $\mathrm{C}_{1}\left(\mathrm{I}_{\mathrm{C} 1}\right)$ is $0 \mathrm{~A}$ in steady-state, because capacitors block DC [32]. When this steady-state phenomenon occurs, $\mathrm{L}_{2}$ is the only source of current to the output load. Thus, $\mathrm{L}_{2}$ 's average current equals that of the output load's, and is independent of $\mathrm{V}_{\mathrm{IN}}$.

In CCM, the sum of the average voltages across the SEPIC's energy storage elements (excluding input and output filter capacitors $C_{\mathbb{I N}}$ and $C_{2}$ ) equal that of the SEPIC's input voltage, as described in (4.1) [18]:

$$
V_{I N}=V_{L 1}+V_{C 1}+V_{L 2}
$$

Since the average voltage across $\mathrm{C}_{1}$ equals that of $\mathrm{V}_{\mathrm{IN}}, \mathrm{V}_{\mathrm{C} 1}$ equals $\mathrm{V}_{\mathrm{IN}}$, leading to (4.2):

$$
V_{L 1}=-V_{L 2}
$$

Under CCM in steady-state, the SEPIC's operation further splits into two operation modes: when $S_{1}$ conducts (turned on or closed) and when it does not conduct (turned off 
or open). Figure 4.1 shows in greater detail the SEPIC's crucial CCM voltage and current waveforms during $\mathrm{S}_{1}$ 's conduction and non-conduction periods [26].

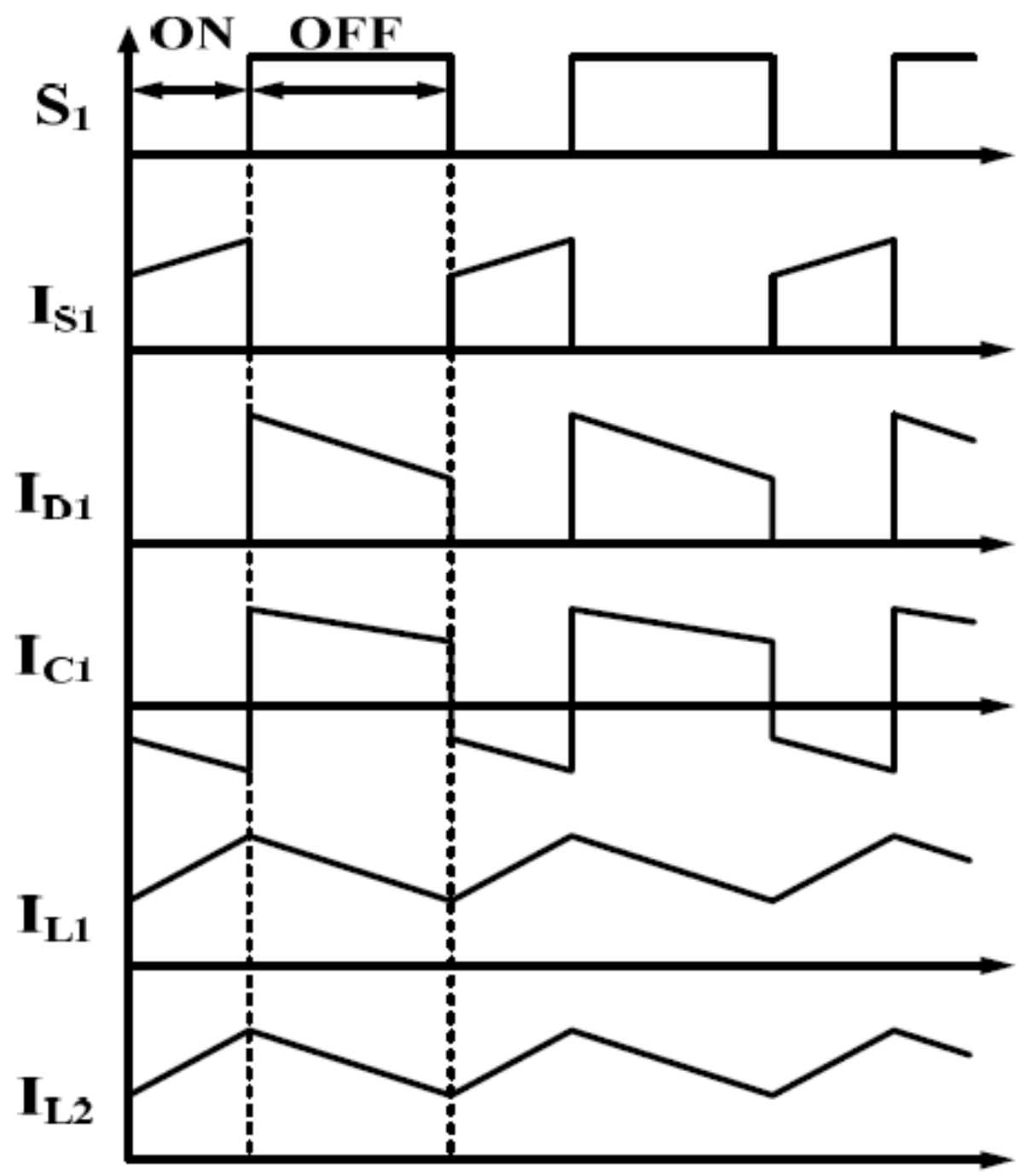

Figure 4.1: Crucial SEPIC Component Waveforms [26]

$S_{1}$ in figure 4.1 refers to the SEPIC's switch $S_{1}$ 's gate voltage, $I_{S 1}$ refers to $S_{1}$ 's drain current, $\mathrm{I}_{\mathrm{D} 1}$ refers to $\mathrm{D}_{1}$ 's forward current, $\mathrm{I}_{\mathrm{C} 1}$ refers to the current flowing through $\mathrm{C}_{1}, \mathrm{I}_{\mathrm{L} 1}$ refers to the current flowing through $\mathrm{L}_{1}$ and $\mathrm{I}_{\mathrm{L} 2}$ refers to the current flowing through $\mathrm{L}_{2}$. Analyzing the SEPIC's entire operation in CCM requires analyzing it in $\mathrm{S}_{1}$ 's conduction and non-conduction modes, which the next section covers in greater detail. 


\subsubsection{SEPIC Operation in CCM with Switch Closed}

Figure 4.2 shows the SEPIC's operation when $\mathrm{S}_{1}$ conducts. Figure 4.1 shows the SEPIC's component waveforms in $\mathrm{S}_{1}$ 's "ON" state [18]. The green arrow denotes $\mathrm{L}_{1}$ and switch $S_{1}$ current flow while the red arrow denotes coupling capacitor $C_{1}$ and $L_{2}$ current flow. Filter capacitors $C_{\mathrm{IN}}$ and $\mathrm{C}_{2}$ are assumed to be in steady-state, thus no current flows through these two components until they discharge. Furthermore, $\mathrm{C}_{\mathrm{IN}}$ and $\mathrm{C}_{2}$ are also assumed to be large enough in capacitance such that the SEPIC's input and output ripple voltages are nearly $0 \mathrm{~V}$.

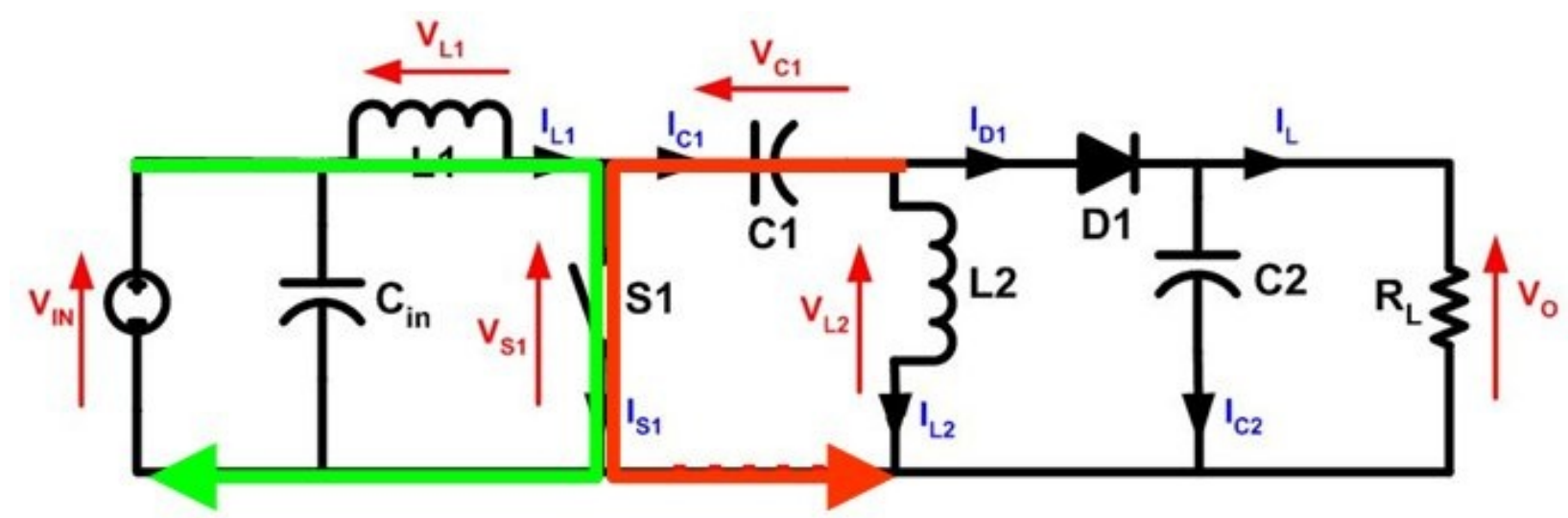

Figure 4.2: SEPIC Operation with $S_{1}$ Conducting [18]

When $S_{1}$ conducts during the first half-switching cycle, the current through $L_{1}\left(I_{L 1}\right)$ increases in the positive direction while the current through $\mathrm{L}_{2}\left(\mathrm{I}_{\mathrm{L} 2}\right)$ increases in the negative direction (or decreases in the mathematical sense). Hence $\mathrm{L}_{1}$ charges via $\mathrm{V}_{\mathrm{IN}}$, while $\mathrm{L}_{2}$ discharges (acting as a source) through $\mathrm{C}_{1}$. $\mathrm{S}_{1}$ remains closed for a short time period (assuming a fast switching frequency $-50 \mathrm{kHz}$ or greater) and during this time period the instantaneous voltage across $\mathrm{C}_{1}\left(\mathrm{~V}_{\mathrm{C} 1}\right)$ equals $\mathrm{V}_{\mathrm{IN}}$. Thus, $\mathrm{V}_{\mathrm{L} 1}$ and $\mathrm{V}_{\mathrm{L} 2}$ both equal approximately $\mathrm{V}_{\mathrm{IN}}$ in magnitude. The only difference between the two voltages is that $\mathrm{V}_{\mathrm{L} 2}$ 's polarity is reversed (i.e. negative) because $\mathrm{L}_{2}$ is discharging. $\mathrm{C}_{1}$ in turn 
discharges and supplies current to $\mathrm{L}_{2}$ in order to store energy in it, so that $\mathrm{L}_{2}$ can supply current to the output load during the next half-switching cycle when $\mathrm{S}_{1}$ no longer conducts. During this entire half-switching cycle, diode $\mathrm{D}_{1}$ does not conduct - in other words, it is reverse-biased, as in figure 4.1. Thus $\mathrm{C}_{2}$ discharges and hence is the only component that helps maintain the output load current when $S_{1}$ is conducting. During the second half-switching cycle, $\mathrm{S}_{1}$ turns off.

\subsubsection{SEPIC Operation in CCM with Switch Open}

Figure 4.3 shows the SEPIC's operation when $\mathrm{S}_{1}$ does not conduct [18]. The red arrow denotes $\mathrm{L}_{2}$ current flow while the green arrow denotes current flow through all other components (except $\mathrm{C}_{\mathrm{IN}}$ and $\mathrm{C}_{2}$ ).

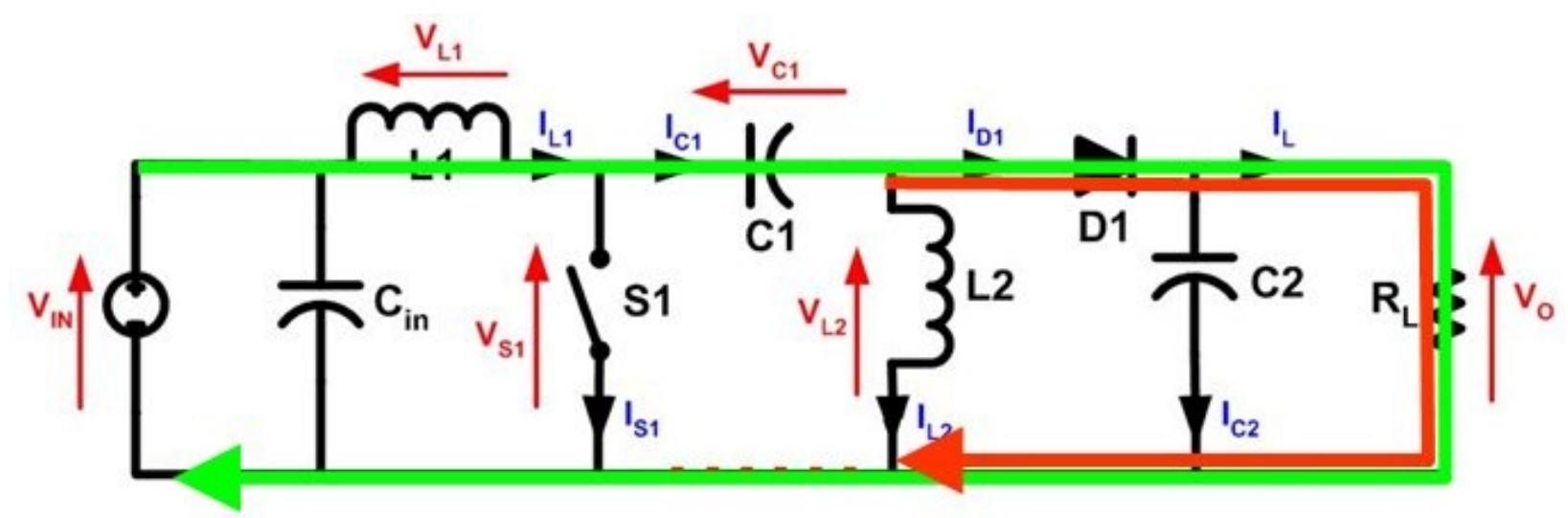

Figure 4.3: SEPIC Operation with $S_{1}$ Turned Off [18]

At the end of one half-switching cycle, $S_{1}$ turns off. The new path for the input current is through $\mathrm{L}_{1}$ and $\mathrm{C}_{1}$. Because current cannot change instantaneously through an inductor [33], $\mathrm{I}_{\mathrm{L} 1}$ and $\mathrm{I}_{\mathrm{L} 2}$ do not immediately change. Thus, $\mathrm{I}_{\mathrm{C} 1}$ equals $\mathrm{I}_{\mathrm{L} 1} \cdot \mathrm{L}_{2}$ continues to discharge, but during this half-switching cycle it discharges into $C_{2}$, thus turning on $D_{1}$ and supplying current $\left(\mathrm{I}_{\mathrm{L} 2}\right)$ to the output load. However, the direction of $\mathrm{I}_{\mathrm{L} 2}$ causes it to 
add to the input current $\left(\mathrm{I}_{\mathrm{L} 1}=\mathrm{I}_{\mathrm{C} 1}\right)$ that already flows to the output load. By Kirchhoff's Current Law, the current through $\mathrm{D}_{1}\left(\mathrm{I}_{\mathrm{D} 1}\right)$ equals:

$$
I_{D 1}=I_{C 1}-I_{L 2}
$$

However, because $\mathrm{I}_{\mathrm{L} 1}=\mathrm{I}_{\mathrm{C} 1}$ :

$$
I_{D 1}=I_{L 1}-I_{L 2}
$$

Thus, when $\mathrm{S}_{1}$ does not conduct, both $\mathrm{L}_{1}$ and $\mathrm{L}_{2}$ supply current to the output load. $\mathrm{V}_{\mathrm{IN}}$ and $\mathrm{L}_{1}$ charge $\mathrm{C}_{1}$ (which discharged during the half-switching cycle when $\mathrm{S}_{1}$ conducted), and $\mathrm{L}_{2}$ continues discharging to the output load until $\mathrm{S}_{1}$ conducts again at the beginning of the next half-switching cycle (when $\mathrm{C}_{1}$ supplies current to charge $\mathrm{L}_{2}$ ). By Kirchhoff's Voltage Law, the voltages across $\mathrm{L}_{1}$ and $\mathrm{L}_{2}\left(\mathrm{~V}_{\mathrm{L} 1}\right.$ and $\left.\mathrm{V}_{\mathrm{L} 2}\right)$ during this half-switching cycle equal:

$$
V_{L 1}=-V_{L 2}=-V_{O}
$$

Hence, $L_{1}$ and $L_{2}$ both discharge and supply current to the output load when $S_{1}$ does not conduct. In both $\mathrm{S}_{1}$ conduction cases, the voltages across $\mathrm{L}_{1}$ and $\mathrm{L}_{2}$ are always both equal in magnitude. Their phases are both equal if the SEPIC's input voltage is less than its output voltage. If the SEPIC's input voltage is greater than its output voltage, then $\mathrm{V}_{\mathrm{L} 1}$ and $\mathrm{V}_{\mathrm{L} 2}$ are $180^{\circ}$ out of phase with respect to one another [27]. Figure 4.4 shows this phenomenon [27]. 


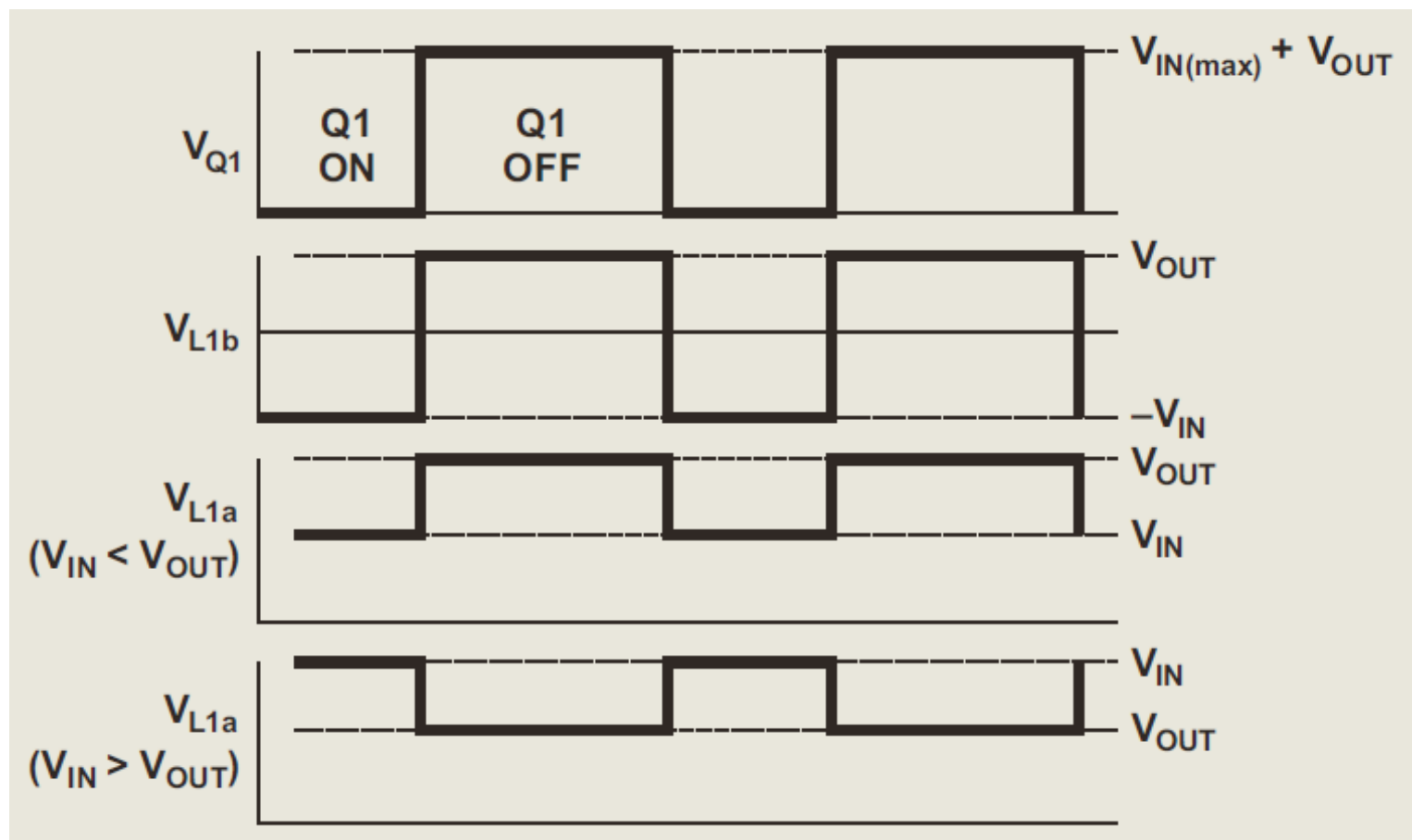

Figure 4.4: SEPIC Component Voltage Waveforms in CCM Operation [27]

$\mathrm{V}_{\mathrm{Q} 1}$ in figure 4.4 refers to $\mathrm{S}_{1}$ 's gate voltage, $\mathrm{V}_{\mathrm{L} 1 \mathrm{a}}$ refers to the voltage across $\mathrm{L}_{1}$ and $\mathrm{V}_{\mathrm{L} 1 \mathrm{~b}}$ refers to the voltage across $\mathrm{L}_{2}$. Understanding the SEPIC's operation is crucial for designing a converter that properly operates under any required specifications. The next section describes this project's SEPIC's design and component selection process. 


\section{CHAPTER 5: FIRST DESIGN PHASE AND SIMULATION RESULTS}

\subsection{Basic SEPIC Design}

Designing the SEPIC for proper operation under the proposed specifications requires selecting the proper component values. The crucial components in the SEPIC are inductors $\mathrm{L}_{1}$ and $\mathrm{L}_{2}$, switching transistor $\mathrm{S}_{1}$, diode $\mathrm{D}_{1}$ and coupling capacitor $\mathrm{C}_{1}$.

The input and output filter capacitors, $\mathrm{C}_{\mathrm{IN}}$ and $\mathrm{C}_{2}$, also need to be large enough in capacitance to minimize input and output voltage ripple. One major disadvantage with the SEPIC topology is that its output voltage ripple is inherently large because a pulsating diode $\left(\mathrm{D}_{1}\right)$ connects to its output. As mentioned in sections 4.3.1 and 4.3.2, $\mathrm{D}_{1}$ conducts when $S_{1}$ turns off and does not conduct when $S_{1}$ turns on, resulting in a pulsating current fed to the converter's output. Hence $\mathrm{C}_{2}$ needs to be large in capacitance to effectively quell any output voltage ripple that results from $\mathrm{D}_{1}$ 's pulsating current.

For $\mathrm{S}_{1}$, a MOSFET is preferable over a BJT because MOSFETs offer higher input impedance and lower voltage drop across its main current path compared to BJTs [34]. Furthermore, a BJT needs to be biased with additional resistors as current differences control BJT switching, unlike MOSFETs, in which voltage differences control switching [34].

From equation (5.20), $\mathrm{L}_{1}$ and $\mathrm{L}_{2}$ can be wound on the same core, resulting in a single magnetically-coupled inductor. Thus, it is reasonable to use a single coupled inductor to house $\mathrm{L}_{1}$ and $\mathrm{L}_{2}$, in order to allow for any potential PCB real estate savings. However, using a single coupled inductor later proves unfeasible, from the required 
calculated specifications in (5.13)-(5.19) as well as physical availability of such a component meeting the required calculated specifications.

The first step in this project's design process was selecting an appropriate switching controller for $\mathrm{S}_{1}$. The SEPIC in this project uses PWM for switching control rather than PFM, for keeping the switching frequency constant. Varying the switching frequency in PFM leads to higher power dissipation (losses) across crucial components if the switching frequency is too high, which typically occurs if the input voltage is very low. Low input voltages (below 36V) and high switching frequencies require the elliptical trainer to supply more input current to the SEPIC and require $\mathrm{S}_{1}$ to conduct more frequently than at high input voltages (above 36V) and low switching frequencies. This results in greater power dissipation stress across crucial components. Therefore PWM was selected as the switching control method for the SEPIC in this project. Linear Technology's LTC1871 PWM controller was selected as the switching controller for the SEPIC in this project, because of its high duty cycle capability (up to 92\%) and programmable switching frequency (via a frequency set resistor) [35]. Furthermore, as with previous designs using Linear Technology's ICs (refer to Appendices A and B), LTSpice contains an LTC1871 simulation model. However, the LTC1871's main caveat is that its maximum input voltage is $36 \mathrm{~V}$ [35]. Using the Precor elliptical trainer's onboard $12 \mathrm{~V}$ battery for supplying the bias input voltage for the controller (which only controls $\mathrm{S}_{1}$ ) while using the elliptical trainer's user-generated exercise as the input source for the SEPIC in general remedies that problem. During Winter Quarter 2012, the author measured the Precor elliptical trainer's onboard battery voltage as $12.16 \mathrm{~V}$. 
Next, this project requires determining LTC1871's operating switching frequency and duty cycle range, as designated by (5.1) to (5.3), taken from Texas Instruments' SEPIC design datasheet: [27] (http://www.ti.com/lit/an/slyt309/slyt309.pdf)

$$
\begin{gathered}
T=\text { One switching cycle period }=\frac{1}{100 \mathrm{kHz}}=10 \mu \mathrm{s} \\
\alpha=\text { Duty cycle for which switch is closed } \\
1-\alpha=\text { Duty cycle for which switch is open }
\end{gathered}
$$

This project selects $100 \mathrm{kHz}$ as the LTC1871's switching frequency as it provides a reasonable tradeoff between component sizes and component power dissipation. Higher switching frequencies result in greater component power dissipation but reduce the inductances required for $\mathrm{L}_{1}$ and $\mathrm{L}_{2}$, while lower switching frequencies result in lower component power dissipation, but increase the inductances required for $\mathrm{L}_{1}$ and $\mathrm{L}_{2}$. Because this project's SEPIC's design requires high power output (288W), component power dissipation is the more crucial issue. Hence, the low $100 \mathrm{kHz}$ switching frequency was selected. The LTC1871 is capable of a $50 \mathrm{kHz}$ minimum switching frequency, but at that level the SEPIC requires larger inductors, resulting in higher overall converter cost.

Equations (5.4) to (5.9) govern the SEPIC's operating duty cycle. $V_{D}$ is $D_{1}$ 's forward voltage drop when it conducts. This project's SEPIC's design calculations assume $0.7 \mathrm{~V}$ for $\mathrm{V}_{\mathrm{D}}$. $\mathrm{D}_{\mathrm{MAX}}$ corresponds to the SEPIC's absolute maximum duty cycle and $\mathrm{D}_{\mathrm{MIN}}$ corresponds to its absolute minimum duty cycle.

$$
\begin{gathered}
D_{M A X}=\frac{V_{O U T}+V_{D}}{V_{I N(\min .)}+V_{O U T}+V_{D}}=\frac{36+0.7}{5+36+0.7}=88 \% \\
\alpha_{M A X}=D_{M A X}=0.88 \\
1-\alpha_{M A X}=1-0.88=0.12 \\
D_{M I N}=\frac{V_{O U T}+V_{D}}{V_{I N \text { (max. })}+V_{O U T}+V_{D}}=\frac{36+0.7}{65+36+0.7}=36 \%
\end{gathered}
$$




$$
\begin{aligned}
\alpha_{M I N} & =D_{M I N}=0.36 \\
1-\alpha_{M I N} & =1-0.88=0.64
\end{aligned}
$$

Therefore, the duty cycle range for the LTC1871 PWM controller for this project's SEPIC is $36 \%$ to $88 \%$, which is well within the LTC1871's operating specifications.

Next, this project's SEPIC's amplification factor range (the ratio between its output voltage to its input voltage) needed to be determined. The amplification factor helps simplify calculating the values rest of the converter's crucial components. Equations (5.10) and (5.11) determine the SEPIC's amplification factor range, assuming $100 \%$ converter efficiency.

$$
\begin{aligned}
& A_{i(\text { min. })}=\frac{V_{\text {OUT }}+V_{D}}{V_{I N}(\max .)}=\frac{\alpha_{M I N}}{1-\alpha_{M I N}}=\frac{0.36}{0.64}=0.5625 \\
& A_{i(\max .)}=\frac{V_{O U T}+V_{D}}{V_{I N}(\min .)}=\frac{\alpha_{M A X}}{1-\alpha_{M A X}}=\frac{0.88}{0.12}=7.33 \overline{3}
\end{aligned}
$$

Thus, this converter's amplification factor ranges from 0.5625 to 7.333 .

The first crucial SEPIC component values that require consideration are switching inductors $\mathrm{L}_{1}$ and $\mathrm{L}_{2}$. The inductances of both inductors should be large enough to keep the SEPIC operating in CCM, but not so large to cause resonance effects because of their adjacency to $\mathrm{C}_{1}, \mathrm{C}_{\mathrm{IN}}$ and $\mathrm{C}_{2}$. Equations (5.12) to (5.19) determine the SEPIC inductors' requirements. In order to determine the proper inductance values for $\mathrm{L}_{1}$ and $\mathrm{L}_{2},(5.12)$ first assumes $100 \%$ converter efficiency and that peak to peak ripple currents through $\mathrm{L}_{1}$ and $\mathrm{L}_{2}$ are approximately $30 \%$ of the maximum input current at the minimum input voltage. Furthermore, (5.12) also assumes that the SEPIC drives a 0.5A maximum load at its minimum input voltage $(5 \mathrm{~V}) . \Delta \mathrm{I}_{\mathrm{L}}$ corresponds to the inductors' peak to peak ripple current and $\mathrm{L}_{1}$ and $\mathrm{L}_{2}$ correspond to their inductances. $\mathrm{I}_{\mathrm{L} 1}$ corresponds to $\mathrm{L}_{1}$ 's peak 
current and $\mathrm{I}_{\mathrm{L} 2}$ corresponds to $\mathrm{L}_{2}$ 's peak current. $\mathrm{I}_{\mathrm{L} 1 \text { (5V input) }}$ corresponds to $\mathrm{L}_{1}$ 's average current at $5 \mathrm{~V}$ SEPIC input, and $\mathrm{I}_{\mathrm{L} 1 \text { (65V input) }}$ corresponds to its average current at $65 \mathrm{~V}$ SEPIC input. $\mathrm{I}_{\mathrm{L} 2 \text { (5V input) }}$ corresponds to $\mathrm{L}_{2}$ 's average current at 5V SEPIC input, and $\mathrm{I}_{\mathrm{L} 2}$ (65V input) corresponds to its average current at $65 \mathrm{~V}$ SEPIC input.

$$
\begin{aligned}
& \Delta I_{L}=I_{I N} \times 30 \%=I_{\text {OUT }} \times \frac{V_{\text {OUT }}}{V_{I N(\text { min. })}} \times 30 \%=0.5 \mathrm{~A} \times \frac{36 \mathrm{~V}}{5 \mathrm{~V}} \times 30 \%=1.08 \mathrm{~A} \\
& L_{1}=L_{2}=L=\frac{V_{I N(\min .)} D_{M A X}}{\Delta I_{L} f_{S W}}=\frac{5 \times 88 \%}{(1.08 \mathrm{~A})(100 \mathrm{kHz})}=40.74 \mu \mathrm{H} \\
& I_{L 1 \text { (peak) }}=I_{I N(\text { max. })}+\frac{\Delta I_{L}}{2}=I_{I N(\text { max. })} \times\left(1+\frac{30 \%}{2}\right)=25 \mathrm{~A} \times\left(1+\frac{30 \%}{2}\right)=28.75 \mathrm{~A} \\
& I_{L 2(\text { peak })}=I_{\text {OUT }}+\frac{\Delta I_{L}}{2}=I_{O U T} \times\left(1+\frac{30 \%}{2}\right)=8 \mathrm{~A} \times\left(1+\frac{30 \%}{2}\right)=9.2 \mathrm{~A} \\
& \overline{I_{L 1(5 V \text { lnput })}}=A_{i(\max .)} I_{\text {OUT }(\min \text { load })}=(7.33 \overline{3})(0.5)=3.667 \mathrm{~A} \\
& \overline{I_{L 1(65 V \text { ınput })}}=A_{i(\text { min. })} I_{\text {OUT }(\max l o a d)}=(0.5625)(8 \mathrm{~A})=4.5 \mathrm{~A} \\
& \overline{I_{L 2(5 \mathrm{~V} \text { input })}}=\overline{I_{\text {OUT }(\mathrm{min} \text { load })}}=0.5 \mathrm{~A} \\
& \overline{I_{L 2(65 \mathrm{~V} \text { input })}}=\overline{I_{\text {OUT }(\mathrm{max} \text { load })}}=8 \mathrm{~A}
\end{aligned}
$$

Winding both SEPIC inductors on the same core (magnetically coupling) halves the required critical inductance:

$$
L_{1}^{\prime}=L_{2}^{\prime}=\frac{L}{2}=\frac{V_{I N(\min .)} D_{M A X}}{2 \Delta I_{L} f_{S w}}=\frac{5(0.88)}{2(1.08 \mathrm{~A})(100 \mathrm{kHz})}=20.37 \mu \mathrm{H}
$$

Because no non-custom commercial coupled inductor physically exists for the inductance and saturation current level that this project's SEPIC requires, this converter uses two discrete inductors. This project's SEPIC uses $60 \mu \mathrm{H}$ for both $\mathrm{L}_{1}$ and $\mathrm{L}_{2}$. This inductance value is significantly higher than but not too different from the calculated critical inductance value in (5.13). This project's SEPIC uses Vishay's IHV28BZ60 power inductor for both $\mathrm{L}_{1}$ and $\mathrm{L}_{2}$. The Vishay IHV28BZ60 has a $60 \mu \mathrm{H}$ nominal inductance with $10 \%$ tolerance, $85 \mathrm{~m} \Omega \mathrm{ESR}$ and a $28 \mathrm{~A}$ saturation current limit. While this is slightly below the $28.75 \mathrm{~A}$ saturation requirement calculated in (5.14), no commercially available, non-custom inductor exists that meets this specification, along with a similar inductance 
value $( \pm 10 \mu \mathrm{H})$. Furthermore, preventative measures later implemented in this project's SEPIC limit its peak input current to 25A. Future variations of this SEPIC design may implement inductors with higher nominal inductance and saturation current ratings. According to Vishay, the IHV28BZ60 does not lose more than $10 \%$ of its rated nominal inductance value if its core saturates [36]. This means that if the IHV28BZ60 saturates, it only loses up to $6 \mu \mathrm{H}$ from its $60 \mu \mathrm{H}$ nominal inductance value, decreasing its inductance to $54 \mu \mathrm{H}$ in the worst case scenario. This value is still well above the calculated critical inductance value from (5.13). Thus, Vishay's IHV28BZ60 is a reasonable choice for $\mathrm{L}_{1}$ and $\mathrm{L}_{2}$.

The SEPIC's next crucial component is its switching $\operatorname{MOSFET}\left(\mathrm{S}_{1}\right)$. Equations (5.21) to (5.23) determine the SEPIC's switching MOSFET's requirements. $\mathrm{V}_{\mathrm{DS}}$ (peak) corresponds to the switching MOSFET's peak drain-source voltage, $\mathrm{I}_{\mathrm{S} 1}$ corresponds to its peak drain current, and $\mathrm{I}_{\mathrm{S} 1(\mathrm{RMS})}$ corresponds to its RMS drain current.

$$
\begin{gathered}
V_{D S(\text { peak })}>1.15\left(V_{I N(\text { max. })}+V_{\text {OUT }}+V_{D}\right)=1.15(65 \mathrm{~V}+36 \mathrm{~V}+0.7 \mathrm{~V})=>116.955 \mathrm{~V} \\
I_{S 1}=I_{L 1(\text { peak })}+I_{L 2(\text { peak })}=28.75 \mathrm{~A}+9.2 \mathrm{~A}=37.95 \mathrm{~A} \\
I_{S 1(R M S)}=I_{\text {OUT }} \sqrt{\frac{\left(V_{I N(\text { min. })}+V_{\text {OUT }}+V_{D}\right) \times\left(V_{O U T}+V_{D}\right)}{V_{I N(\min .)^{2}}}}=0.5 \mathrm{~A} \sqrt{\frac{(5+36+0.7) \times(36+0.7)}{5^{2}}}=3.912 \mathrm{~A}
\end{gathered}
$$

Equations (5.24) to (5.26) determine the SEPIC's switching diode's requirements. $\mathrm{I}_{\mathrm{D} \text { (peak) }}$ corresponds to the switching diode's peak forward current, $\mathrm{I}_{\mathrm{D} \text { (max.) }}$ corresponds to its average forward current and $V_{R}$ corresponds to its required peak reverse voltage rating.

$$
\begin{gathered}
I_{D(\text { peak })}=I_{S 1(\text { peak })}=37.95 \mathrm{~A} \\
\overline{\left.I_{D(\text { max } .)}\right)}=\overline{I_{\text {OUT }(\max .)}}=8 \mathrm{~A} \\
V_{R}>1.15\left(V_{I N(\text { max. })}+V_{\text {OUT }}\right)=1.15(65 \mathrm{~V}+36 \mathrm{~V}) \rightarrow 116.15 \mathrm{~V}
\end{gathered}
$$


Equations (5.27) to (5.30) determine the SEPIC's coupling capacitor's requirements. $\mathrm{C}_{1}$ corresponds to the coupling capacitor's minimum required capacitance value, $\mathrm{I}_{\mathrm{C} 1 \text { (RMS) }}$ corresponds to its RMS current rating, $\mathrm{V}_{\mathrm{C} 1}$ corresponds to its average voltage and $\Delta \mathrm{V}_{\mathrm{C} 1}$ corresponds to its peak to peak voltage ripple. Equation (5.27) assumes that the coupling capacitor's peak to peak voltage ripple is $7.5 \%$ of the SEPIC's maximum input voltage $(65 \mathrm{~V})$.

$$
\begin{aligned}
& C_{1} \geq \frac{I_{\text {OUT }} \times D_{\text {MAX }}}{\Delta V_{C 1}(\text { peak }- \text { peak }) \times f_{\text {sw }}} \geq \frac{I_{O U T} \times D_{\text {MAX }}}{7.5 \% \times V_{I N(\text { max. } .)} \times f_{\text {sw }}} \geq \frac{8 A \times 0.88}{0.075 \times 65 V \times 100 \mathrm{kHz}} \geq 14.44 \mu \mathrm{F} \\
& I_{C 1(R M S)}=I_{\text {OUT }} \times \sqrt{\frac{V_{\text {OUT }+V_{D}}}{V_{I N(\text { min. })}}}=I_{\text {OUT }} \times \sqrt{\frac{D_{M A X}}{1-D_{M A X}}}=8 \mathrm{~A} \times \sqrt{\frac{0.88}{1-0.88}}=21.664 \mathrm{~A} \\
& \overline{V_{C 1}}=V_{I N}=5 \mathrm{~V} \text { to } 65 \mathrm{~V} \\
& \Delta V_{C 1}=\frac{I_{\text {OUT }} D_{M A X}}{C_{1} f_{\text {sw }}}=\frac{8 A(0.88)}{(15 \mu F)(100 \mathrm{kHz})}=4.693 \mathrm{~V}
\end{aligned}
$$

$15 \mu \mathrm{F}$ is the next higher available standard capacitor value, thus $\mathrm{C}_{1}$ is $15 \mu \mathrm{F}$. This project's SEPIC uses a non-polarized capacitor because the voltage across $C_{1}$ reverses polarity during each half-switching cycle. $\mathrm{C}_{1}$ 's RMS current requirement is in reality much lower than in (5.28), since at $5 \mathrm{~V}$ input the SEPIC will not support an 8A output load.

Equations (5.31) to (5.33) determine the SEPIC's output filter capacitor's requirements. $\mathrm{I}_{\text {Cout (RMS) }}$ corresponds to the output filter capacitor's RMS current rating, ESR refers to its equivalent series resistance rating and $\mathrm{C}_{\text {OUT }}$ refers to its minimum required capacitance value. Equations (5.31) and (5.32) also assume a 2\% maximum output filter capacitor voltage ripple.

$$
\begin{gathered}
I_{\text {Cout }(R M S)}=I_{\text {OUT }} \times \sqrt{\frac{V_{\text {OUT }}+V_{D}}{V_{I N(\min .)}}}=I_{\text {OUT }} \times \sqrt{\frac{D_{M A X}}{1-D_{M A X}}}=8 A \times \sqrt{\frac{0.88}{1-0.88}}=21.664 \mathrm{~A} \\
E S R \leq \frac{\Delta V_{\text {OUT }} \times 0.5}{\left.I_{\text {L1 (peak })}+I_{\text {L2 }} \text { peak }\right)}=\frac{36 \times 0.02 \times 0.5}{28.75 A+9.2 A}=0.00949 \Omega=9.49 \mathrm{~m} \Omega
\end{gathered}
$$




$$
C_{\text {OUT }} \geq \frac{I_{\text {OUT }} D_{M A X}}{f_{S W} \Delta V_{\text {OUT }}}=\frac{(8 A)(0.88)}{100 \mathrm{kHz} \times 36 \times 0.02}=97.77 \mu \mathrm{F}
$$

Again, likewise with $\mathrm{C}_{1}, \mathrm{C}_{\text {OUT }}$ 's RMS current requirement is in reality much lower than in (5.31), since at 5V input the SEPIC will not support an $8 \mathrm{~A}$ output load. In order to account for high ESR levels in a real electrolytic capacitor, $\mathrm{C}_{\mathrm{OUT}}$ is $500 \mu \mathrm{F}$, which is over five times the minimum required capacitance value. Furthermore, adding two additional low-ESR ceramic capacitors in parallel at the SEPIC's output $(10 \mu \mathrm{F}$ each $)$ helps offset ESR from the large electrolytic capacitor. Therefore the SEPIC's total output capacitance is $520 \mu \mathrm{F}$.

Recalculating the output voltage ripple percentage with the selected $\mathrm{C}_{\text {OUT }}$ value yields:

$$
\% V_{\text {OUT }} \text { ripple }=\frac{I_{\text {OUT }} D_{\text {MAX }}}{V_{\text {OUT }} C_{\text {OUT }} f_{\text {sw }}} \times 100 \%=\frac{(8 A)(0.88)}{36 V \times 520 \mu F \times 100 \mathrm{kHz}} \times 100 \%=0.376 \%
$$

Thus increasing the $\mathrm{C}_{\text {OUT }}$ value to $520 \mu \mathrm{F}$ reduces the SEPIC's output voltage ripple to $0.376 \%$.

Equations (5.35) and (5.36) determine the SEPIC's input filter capacitor's requirements. $\mathrm{I}_{\mathrm{Cin}(\mathrm{RMS})}$ corresponds to the input filter capacitor's RMS current rating and $\mathrm{C}_{\mathrm{IN}}$ corresponds to its minimum required capacitance value.

$$
\begin{gathered}
I_{C i n(R M S)}=\frac{\Delta I_{L}}{\sqrt{12}}=\frac{1.08 \mathrm{~A}}{\sqrt{12}}=0.3118 \mathrm{~A} \\
C_{I N}=\frac{C_{O U T}}{10}=\frac{97.77 \mu \mathrm{F}}{10}=9.777 \mu \mathrm{F}
\end{gathered}
$$

Likewise with the SEPIC's output filter capacitor, $\mathrm{C}_{\mathrm{IN}}$ is $33 \mu \mathrm{F}$ (a standard capacitor value higher than the minimum calculated $\mathrm{C}_{\mathrm{IN}}$ ) in order to account for high ESR levels in a real electrolytic capacitor. $\mathrm{C}_{\mathrm{OUT}}$ is much larger than $\mathrm{C}_{\mathrm{IN}}$ because the SEPIC's output current 
characteristics are much worse than that of its input's. Now that this project has selected its basic SEPIC components, it requires components for biasing its PWM controller IC.

\subsection{PWM Controller Component Selection}

Selecting proper values for the SEPIC's components ensures proper operation at the specified 5-65V input range. However, its PWM controller also requires proper external components in order to properly bias its internal analog and digital circuitry such that it correctly controls the SEPIC's switch. This section concentrates on selecting the proper components for the LTC1871 PWM controller used for the SEPIC's switch.

Figure 5.1 shows the LTC1871 controller's internal schematic [35]. 


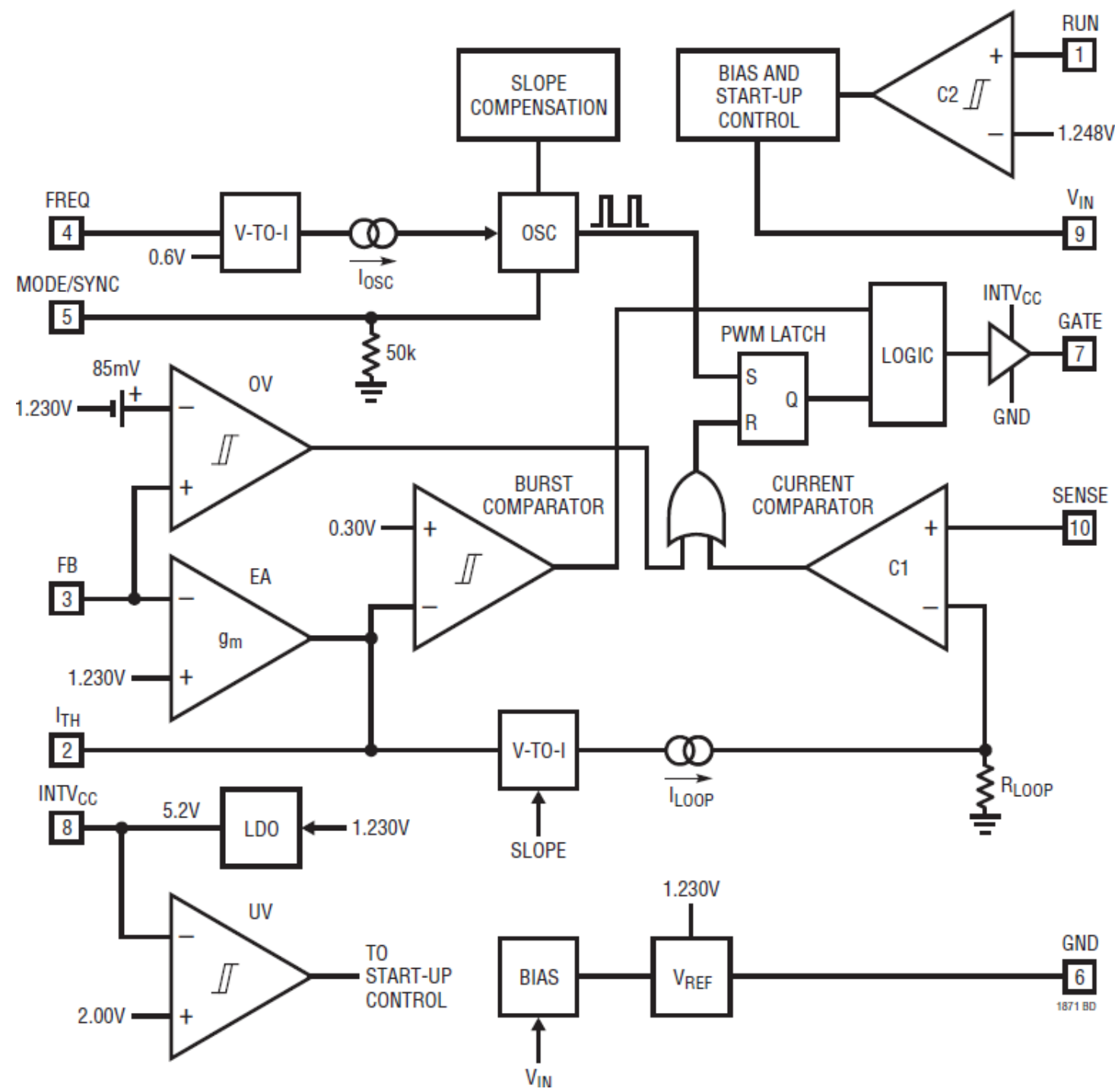

Figure 5.1: LTC1871 Internal Schematic [35]

The MSOP-10 package LTC1871 PWM controller consists of 10 pins that require external biasing components. In numerical order, those pins are RUN, $\mathrm{I}_{\mathrm{TH}}, \mathrm{FB}, \mathrm{FREQ}$, MODE/SYNC, GND (signal ground), GATE, INTV $\mathrm{CC}_{\mathrm{IN}}, \mathrm{V}_{\mathrm{IN}}$ and SENSE. The first pin requiring component selection is the RUN pin. 


\subsubsection{Programming Thresholds with the LTC1871's RUN Pin}

A resistor divider network consisting of $\mathrm{R}_{\mathrm{RUN} 1 \_ \text {MAIN }}$ and $\mathrm{R}_{\mathrm{RUN} 2 \_\mathrm{MAIN}}$ (from figure 5.7) supplies a reference voltage (1.248V according to figure 5.1) to the LTC1871's RUN pin, establishing a turn-on threshold voltage for the controller IC. Along with the internal comparator, this circuit sets the input voltage thresholds at which the LTC1871 controller turns on and off. $\mathrm{V}_{\mathrm{IN}(\mathrm{OFF})}$ is the LTC1871's turn-off threshold and $\mathrm{V}_{\mathrm{IN}(\mathrm{ON})}$ is its turn-on threshold. The RUN pin requires a 1.248V reference voltage to turn on the LTC1871 controller, along with $100 \mathrm{mV}$ hysteresis on the reference voltage for noise immunity [35].

The voltage divider resistor values don't actually matter since the Precor elliptical trainer's on-board battery, a constant, 12V DC voltage source, biases the PWM controller. Therefore, the resistor values were left as the same values from Linear Technology's LTC1871 LTSpice macromodel test fixture circuit $\left(274 \mathrm{k} \Omega\right.$ for $\mathrm{R}_{\mathrm{RUN1} \text { _MAIN }}$ and $133 \mathrm{k} \Omega$ for R $\mathrm{RUN}_{\text {RUMAIN }}$ ). Equations (5.37) and (5.38) determine the LTC1871's turnoff and turn-on input threshold voltages using Linear's test fixture values.

$$
\begin{aligned}
& V_{I N(O F F)}=1.248 \mathrm{~V}\left(1+\frac{R_{R U N 1 . M A I N}}{R_{R U N 2 \_M A I N}}\right)=1.248\left(1+\frac{274 k \Omega}{133 k \Omega}\right)=3.819 \mathrm{~V} \\
& V_{I N(O N)}=1.348 V\left(1+\frac{R_{R U N 1 \text { MAIN }}}{R_{R U N 2 \_ \text {MAIN }}}\right)=1.348\left(1+\frac{274 k \Omega}{133 k \Omega}\right)=4.125 \mathrm{~V}
\end{aligned}
$$

This suffices for a 12V (DC) battery input to the LTC1871's $\mathrm{V}_{\text {IN }}$ pin. The next LTC1871 controller pin that requires component selection is its $\mathrm{I}_{\mathrm{TH}} \mathrm{pin}$. 


\subsection{2 $\mathbf{I}_{\mathrm{TH}}$ Pin}

The LTC1871's $\mathrm{I}_{\mathrm{TH}}$ pin functions as a compensation pin for the internal error amplifier. The LTC1871's internal error amplifier at this pin requires an external compensation circuit. Keeping the error amplifier uncompensated results in higher phase shifts (than a compensated amplifier) from various high frequency poles and zeros [37], which in turn results in unwanted harmonic oscillations. Those oscillations result in undesirable error amplifier operation, which in turn result in undesirable SEPIC MOSFET switching. Linear Technology's LTC1871 LTSpice macromodel test fixture circuit implements an external pole-zero compensation circuit [37], providing the error amplifier with frequency compensation. Figure 5.2 shows the pole-zero compensation circuit from Linear's LTC1871 LTSpice test fixture circuit.

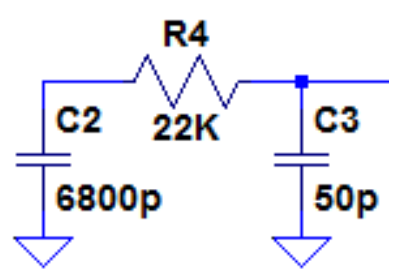

Figure 5.2: LTC1871 Test Fixture Pole-Zero Compensator

$\mathrm{C}_{3}$ and $\mathrm{R}_{4}$ from figure 5.2 connect directly to the LTC1871's $\mathrm{I}_{\mathrm{TH}}$ pin. Figure 5.3 shows an equivalent circuit model of the LTC1871's error amplifier using a Norton-equivalent output circuit [37].

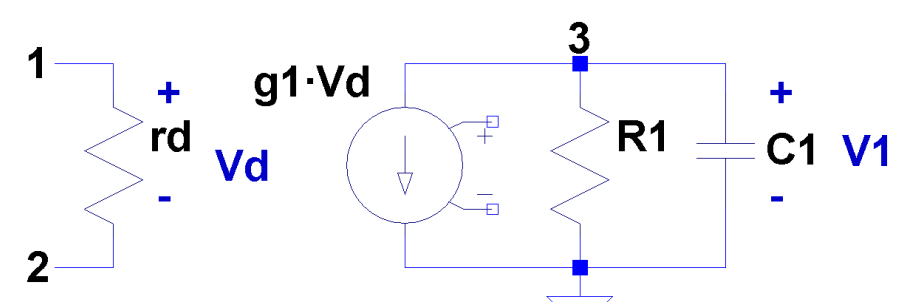

Figure 5.3: Error Amplifier Equivalent Circuit 
$R_{1}$ is the error amplifier's Norton equivalent output resistance and because the amplifier's output voltage does not vary with output current, $\mathrm{R}_{1}$ is very large (i.e. infinite). A Thévenin-equivalent circuit connects $\mathrm{R}_{1}$ is in series with a controlled voltage source (rather than a controlled current source) and sets $\mathrm{R}_{1}$ to $0 \Omega$.

Figure 5.4 shows the error amplifier's magnitude response at each of its pole frequencies $\left(\mathrm{f}_{1}, \mathrm{f}_{2}\right.$ and $\left.\mathrm{f}_{3}\right)$ [37].

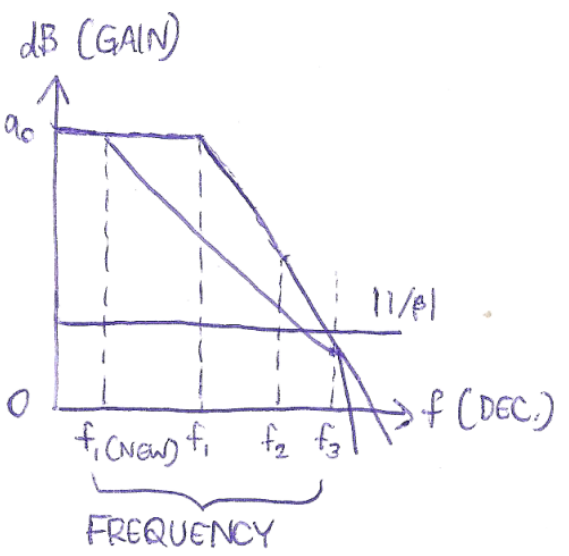

Figure 5.4: Error Amplifier Magnitude Response

$\mathrm{f}_{1 \text { (new) }}$ in figure 5.4 corresponds to the amplifier's first pole frequency after applying compensation.

The pole-zero compensator uses a large capacitor $\left(C_{2}\right.$ in figure 5.3) in series with a resistor $\left(R_{4}\right.$ in figure 5.3), creating a zero frequency $f_{z}$ (much larger than that of $f_{1 \text { (new) }}$ ) that cancels out the error amplifier's second pole frequency $f_{2}$ [37]. Therefore, $f_{z}=f_{2}$. This series combination also creates an additional pole frequency $\left(\mathrm{f}_{4}\right)$ much larger than that of the created $f_{z}$ [37]. The resistor value is much lower than that of the error amplifier's input resistance. $\mathrm{C}_{3}$ 's capacitance is also much smaller than that of $\mathrm{C}_{2}$ 's, lowering the amplifier's first pole frequency from $f_{1}$ to $f_{1(\text { new) }}$ [37]. The lowered first pole 
frequency $\mathrm{f}_{1 \text { (new) }}$ then dominates the amplifier's magnitude response until the new, compensated cut-off frequency $\mathrm{f}_{3}[37]$.

This compensator circuit greatly attenuates any high frequency harmonics that cause undesirable error amplifier operation and hence Linear Technology requires it for proper LTC1871 operation. The compensator's component values were left as nearly the same values from Linear Technology's LTC1871 recommended base SEPIC application circuit shown in figure 5.5 [35].

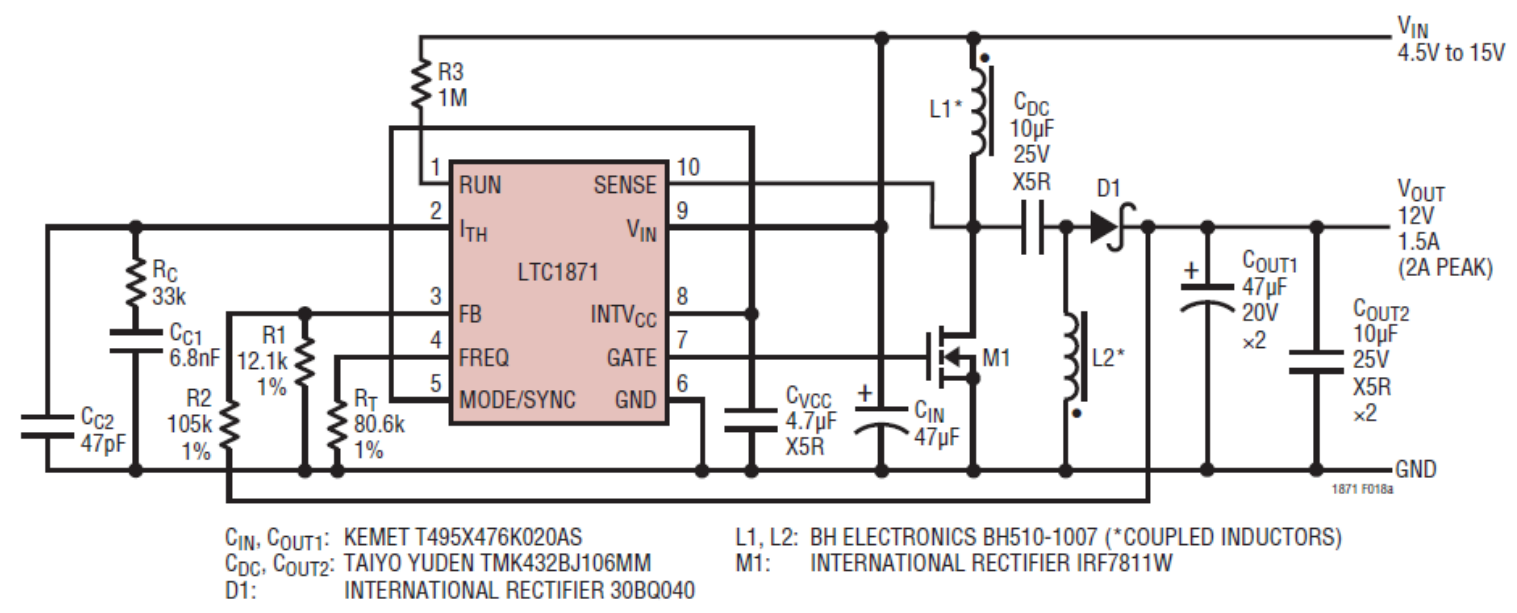

Figure 5.5: Linear Technology's Recommended LTC1871 Base SEPIC [35]

Equations (5.39) to (5.41) determine the compensator's pole frequencies using the selected components from figure 5.5 [37]. $\mathrm{R}_{1}$ denotes the error amplifier's Nortonequivalent output resistance (which is very large).

$$
\begin{gathered}
f_{1(\text { new })} \cong \frac{1}{2 \pi R_{1} C_{c 1}}=\frac{1}{2 \pi\left(R_{1} \text { large }\right)(6800 p F)} \cong 0 \mathrm{~Hz} \\
f_{2}=f_{z}=\frac{1}{2 \pi R_{c} C_{c 1}}=\frac{1}{2 \pi(33 \mathrm{k})(6800 \mathrm{pF})}=704.974 \mathrm{~Hz} \\
f_{4} \cong \frac{1}{2 \pi R_{c} C_{c 2}}=\frac{1}{2 \pi(33 \mathrm{k})(47 \mathrm{pF})} \cong 102 \mathrm{kHz}
\end{gathered}
$$

The selected compensator values suffice at blocking out higher frequency harmonics because attenuation begins at close to DC. Therefore these components suffice for 
biasing the LTC1871's I $\mathrm{T}_{\mathrm{TH}}$ pin for this project's SEPIC. The next pin, FB, determines the SEPIC's output voltage and also requires external components.

\subsubsection{Output Voltage Programming with the FB Pin}

Regulating $\mathrm{S}_{1}$ 's duty cycle with the SEPIC's input voltage requires the LTC1871 controller knowing the SEPIC's set output voltage. A feedback voltage divider resistor network connected to the SEPIC's output sets the SEPIC's output voltage. The pull-down resistor $\left(\mathrm{R}_{\mathrm{FB} 2}\right.$ in figure 5.7) provides the $1.23 \mathrm{~V}$ that the LTC1871's FB pin requires for properly regulating the SEPIC's output at the desired voltage. Equation (5.42) determines the SEPIC's output voltage.

$$
V_{\text {OUT }}=1.23 \mathrm{~V}\left(1+\frac{R_{F B 1}}{R_{F B 2}}\right)
$$

$\mathrm{R}_{\mathrm{FB} 2}$ is $4 \mathrm{k} \Omega$, thus a $36 \mathrm{~V}$ SEPIC output requires the following resistance value for $\mathrm{R}_{\mathrm{FB} 1}$ :

$$
R_{F B 1}=R_{F B 2}\left(\frac{V_{\text {OUT }}}{1.23}-1\right)=4 k \Omega\left(\frac{36}{1.23}-1\right)=113.07 k \Omega
$$

$\mathrm{R}_{\mathrm{FB} 1}$ is $115 \mathrm{k} \Omega$, as it is the next highest commercially available standard resistor value.

After selecting components that determine the SEPIC's output voltage, the LTC1871

controller needs components to set the SEPIC's switching frequency.

\subsubsection{Switching Frequency Programming with the FREQ Pin}

Figure 5.6 below, taken from the LTC1871's datasheet, shows the relationship between the LTC1871's switching frequency for $\mathrm{S}_{1}$ and the chosen frequency set resistor $\left(\mathrm{R}_{\mathrm{T}}\right)[35]:$ 


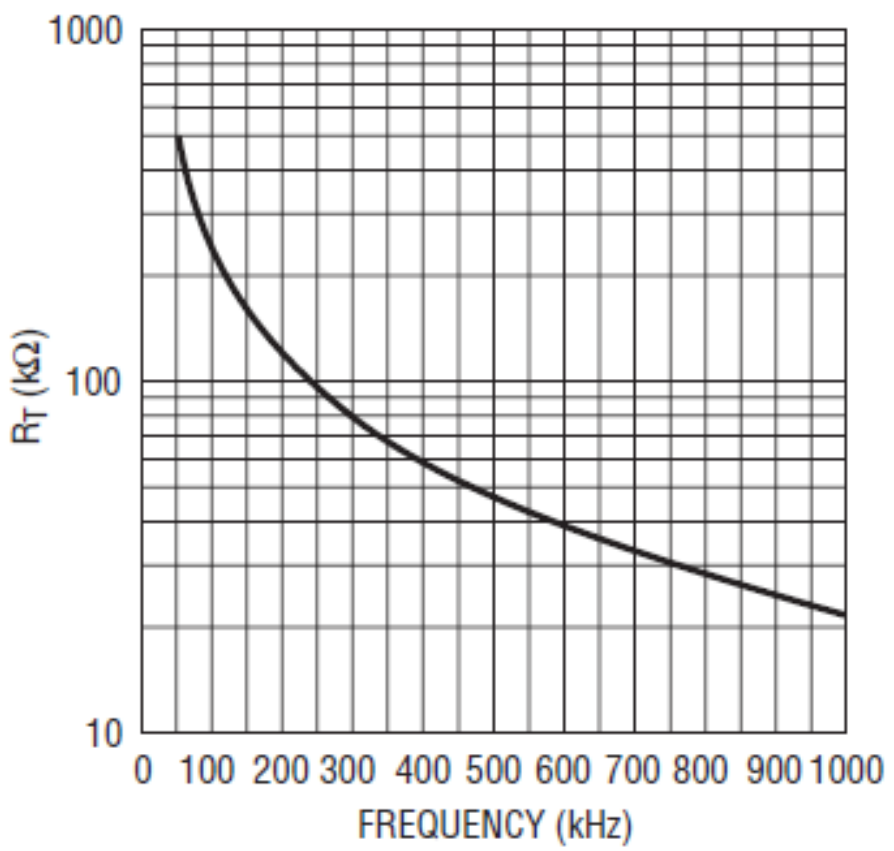

Figure 5.6: LTC1871 Controller Frequency Resistor Values and Corresponding Frequencies [35]

$\mathrm{R}_{\mathrm{T}}$ is the same as $\mathrm{R}_{\mathrm{FREQ}}$ in this project's SEPIC schematic in figure 5.7. For a $100 \mathrm{kHz}$ switching frequency, a $220 \mathrm{k} \Omega$ resistor suffices for $\mathrm{R}_{\mathrm{FREQ}}$, as it is a commonly available value. According to figure 5.6 it also yields a switching frequency very close to that of the desired $100 \mathrm{kHz}$. The next step in biasing the LTC1871 controller after selecting its frequency set resistor is controlling its MODE/SYNC pin.

\subsubsection{MODE/SYNC Pin Operation}

Leaving the MODE/SYNC pin open (unconnected) or connected to ground pulls its voltage low and enables burst-mode operation. The internal $50 \mathrm{k} \Omega$ internal resistor connected to the MODE/SYNC pin (in figure 5.5 above) pulls it low. Tying the MODE/SYNC pin to a voltage source above $2 \mathrm{~V}$ or an external clock disables LTC1871 burst-mode operation and enables continuous mode operation. This project's SEPIC desires continuous PWM operation, as burst mode is desirable only if the SEPIC drives a 
very light load (i.e. low output current) for most of its operation. Thus, this project's SEPIC directly connects the LTC1871's MODE/SYNC pin to the INTV ${ }_{C C}$ pin to provide a $5.2 \mathrm{~V}$ source $(>2 \mathrm{~V})$ for the LTC1871 to operate in continuous mode. Burst mode reduces PWM frequency at light loads and maximizes efficiency at light loads [LTC1871 datasheet]. Operating the SEPIC in burst mode is similar to operating it in DCM, and as mentioned in section 4.2 DCM is only desirable for light loads. Therefore, continuous LTC1871 controller operation yields better overall SEPIC efficiency over burst mode operation. The next section discusses proper LTC1871 controller grounding.

\subsubsection{GND Pin}

The LTC1871's GND pin connects directly to the SEPIC's ground node. Proper, low-noise operation however, requires it connected to a signal ground plane separate from that of a power ground plane. Chapter 9 (PCB Layout) discusses this requirement in greater detail.

\subsubsection{GATE Pin}

The LTC1871's GATE pin feeds the switching signal into $S_{1}$ 's gate and hence connects directly to $\mathrm{S}_{1}$ 's gate. The next section discusses proper INTV $\mathrm{CC}_{\mathrm{CC}}$ pin biasing.

\subsubsection{INTV CC Regulator Bypassing and Operation (INTV $_{\text {CC Pin) }}$}

The LTC1871's INTV $\mathrm{CC}_{\text {r }}$ regulator is a $5.2 \mathrm{~V}$ voltage supply that powers the LTC1871's gate driver and logic circuitry shown in figure 5.1. This regulator supplies up to $50 \mathrm{~mA}$ of current. At the very minimum, the $\mathrm{INTV}_{\mathrm{CC}}$ regulator requires a physically adjacent $4.7 \mu \mathrm{F}$ capacitor connected to $\mathrm{LTC} 1871$ 's INTV $\mathrm{CC}_{\mathrm{CC}}$ pin. This pin requires this capacitor for bypassing the $\mathrm{INTV}_{\mathrm{CC}}$ regulator to ground from the high transient currents 
that the LTC1871's gate driver requires. The SEPIC schematic in figure 5.7 denotes this bypass capacitor as $\mathrm{C}_{\mathrm{VCC}}$. After selecting components for biasing the $\mathrm{INTV}_{\mathrm{CC}}$ pin, biasing the LTC1871's $V_{\text {IN }}$ pin is the next crucial step in the LTC1871 component selection process.

\subsection{9 $\mathrm{V}_{\text {IN }}$ Pin}

The LTC1871's V Vin pin connects to the Precor elliptical trainer's onboard 12V battery's positive terminal. By default its desired terminal is the SEPIC's input (which is directly from the elliptical trainer's output), however because the LTC1871's $\mathrm{V}_{\text {IN }}$ pin can only tolerate up to a $36 \mathrm{~V}$ maximum input voltage, it is instead connected to the elliptical trainer's $12 \mathrm{~V}$ battery. After connecting the LTC1871's $\mathrm{V}_{\mathrm{IN}}$ pin, the last pin that requires component selection is the SENSE pin.

\subsubsection{Current Sense Resistor Selection and SENSE Pin}

The current sense resistor (denoted as $\mathrm{R}_{\mathrm{SNS}}$ in the SEPIC's schematic in figure 5.7) limits the peak current flowing through $S_{1}$. It is not necessary for low power applications (in which $\mathrm{V}_{\mathrm{IN}}$ is less than $36 \mathrm{~V}$, according to Linear Technology's LTC1871 datasheet [35]) because the LTC1871 controller determines $S_{1}$ 's drain current limit using $\mathrm{S}_{1}$ 's drain-source on resistance $\left(\mathrm{R}_{\mathrm{DS} \text { ON }}\right)$. However, adding a discrete sense resistor further reduces $S_{1}$ 's drain current stress by adding resistance. $S_{1}$ 's peak calculated current is 37.95A from (5.22), but requires limiting to $30 \mathrm{~A}$ in order to keep $L_{1}$ from saturating. The LTC1871's SENSE pin operates on a 150mV threshold. Equation (5.44) determines the current sense resistor value required for limiting $S_{1}$ 's peak drain current to $30 \mathrm{~A}$. $\mathrm{V}_{\mathrm{SNS}}$ denotes the LTC1871's SENSE pin voltage. 


$$
R_{S N S}=\frac{V_{S N S}}{I_{D(S 1, M A X .)}}=\frac{150 \mathrm{mV}}{30 \mathrm{~A}}=0.005 \Omega=5 \mathrm{~m} \Omega
$$

Selecting the $\mathrm{R}_{\mathrm{SNS}}$ value completes the LTC1871 biasing component selection process. Before beginning the simulation process however, this project's SEPIC's design requires simulation models for $S_{1}$ and $D_{1}$. The next section describes the selection process for $S_{1}$ and $\mathrm{D}_{1}$.

\subsection{Switching MOSFET and Diode Selection}

Maximizing converter efficiency requires selecting a power MOSFET with low gate charge and a low drain-source on resistance $\left(R_{D S ~ O N}\right)$. A low $R_{D S}$ ON is desirable because it helps minimize $\mathrm{I}^{2} \mathrm{R}$ losses across the MOSFET when high current flows through its drain. Low gate charge is also desirable because less gate charge results in less energy (and hence power) lost across the switching MOSFET during turn-on and turn-off when its total gate capacitance charges and discharges [38]. Furthermore, this project's SEPIC's switching MOSFET also needs to be able to withstand the peak drainsource voltage and drain current requirements outlined in (5.21) to (5.23). Infineon's IPP110N20N3 N-channel power MOSFET satisfies all the above requirements and furthermore, LTSpice has a simulation model available for it. The IPP110N20N3 power MOSFET has a $200 \mathrm{~V}$ maximum $\mathrm{V}_{\mathrm{DS}}$ rating, $11 \mathrm{~m} \Omega$ maximum $\mathrm{R}_{\mathrm{DS}}$ on rating and $65 \mathrm{nC}$ total gate charge [39]. It can also withstand up to 88A continuous drain current and 352A pulsed drain current at $25^{\circ} \mathrm{C}$ ambient operating temperature and $63 \mathrm{~A}$ continuous current at $100^{\circ} \mathrm{C}$ ambient operating temperature [39]. Because it satisfies the requirements outlined in (5.21) to (5.23) and also has a low gate charge and low $\mathrm{R}_{\mathrm{DS} \text { ON }}$, Infineon's IPP110N20N3 is a suitable switching MOSFET for this project's SEPIC. 
For this project's SEPIC's switching diode, the MBR20100CT Schottky diode seems suitable. The MBR20100CT has the highest combined peak reverse voltage and average forward rectified current ratings out of all the pre-packaged diode models available in LTSpice, hence its selection. It also has a 100V peak reverse voltage rating and a 10A (for a single diode) average forward rectified current rating. However, as calculated in (5.25) and (5.26), while the MBR20100CT's average forward rectified current rating is sufficient for the switching diode's required 8A average forward current, its peak reverse voltage rating is not adequate. Using two MBR20100CTs connected in series is thus more feasible, as doing so decreases its peak reverse voltage. However, for initial simulation purposes, only one is used. After selecting its switching MOSFET and diode, the base SEPIC design is ready for simulation.

\subsection{Base SEPIC Simulation Implementation and Output Voltage Results}

Figure 5.7 shows the base SEPIC design schematic after selecting the proper components based on the criteria from sections 5.1 to 5.2.10.

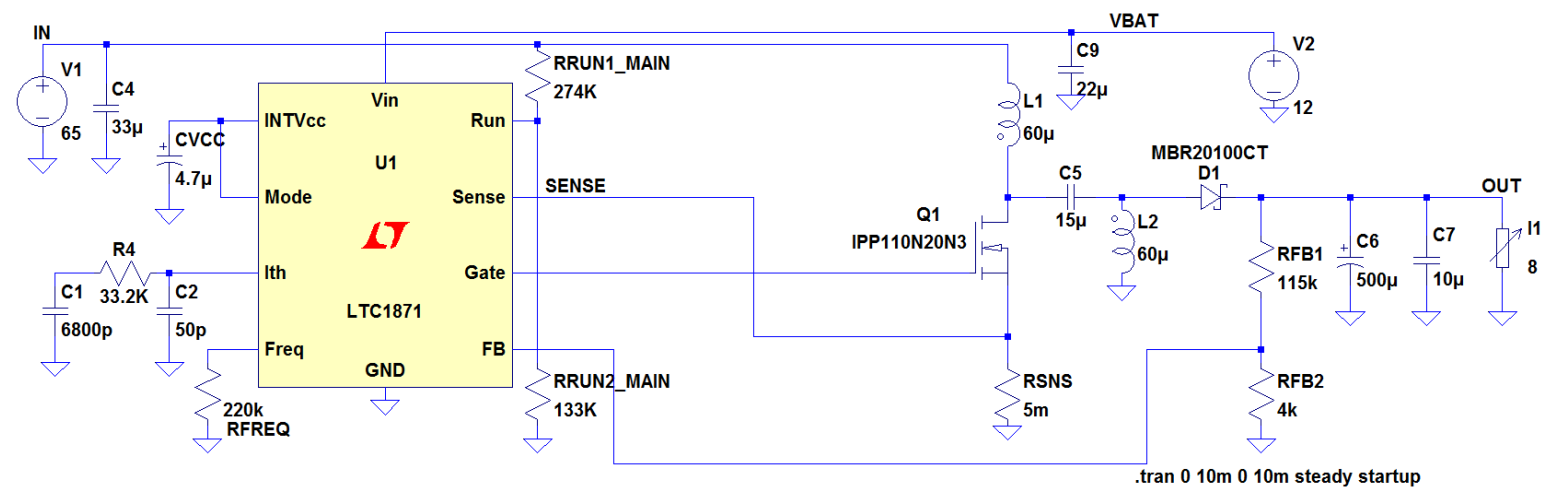

Figure 5.7: Proposed SEPIC Base Design

The initial base SEPIC design is ready for simulation. This SEPIC's first characteristic requiring investigation is its output voltage. Figure 5.8 shows the base SEPIC's output 
voltage in green. This first simulation runs the SEPIC under a full load condition. This project specifies full load condition as $65 \mathrm{~V}$ input while driving an $8 \mathrm{~A}$ load.

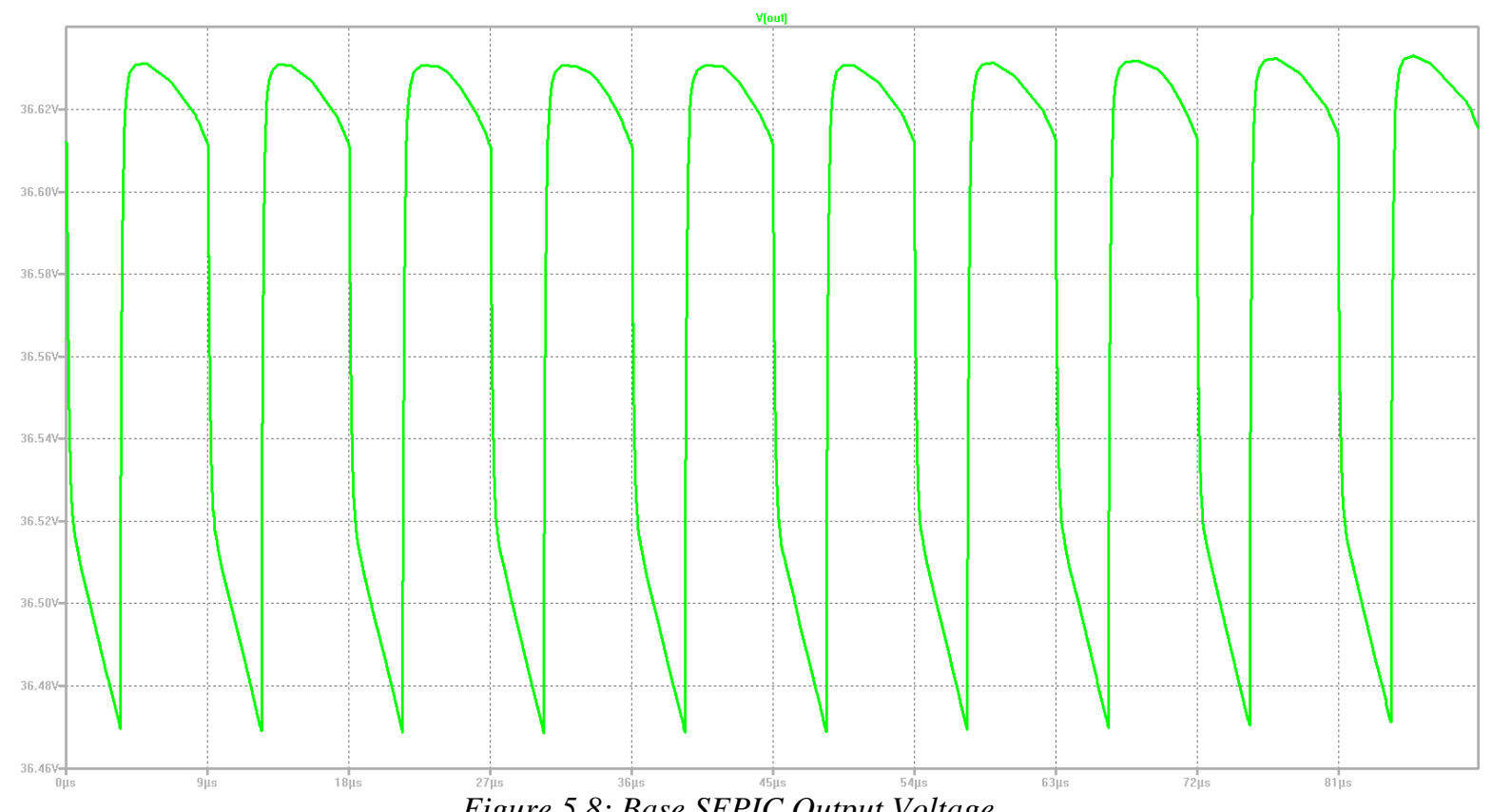

Figure 5.8: Base SEPIC Output Voltage

Average output voltage is $36.577 \mathrm{~V}$ and peak-to-peak ripple voltage is $0.162 \mathrm{~V}$, corresponding to a $0.44 \%$ peak-to-peak output voltage ripple. This initial base SEPIC design outputs the required $36 \mathrm{~V}$ average voltage specified and meets the output voltage ripple specifications outlined in section 1.2. This report's next subsection analyzes other crucial node waveforms in this initial SEPIC design for ensuring that they closely match those in figure 4.1 and $[26,27]$. Having matching simulation waveforms as from figure 4.1 and $[26,27]$ ensures that this initial SEPIC design functions correctly.

\subsubsection{Base SEPIC Simulation Switching Waveform Analysis at 65V Input}

Other crucial SEPIC operation waveforms besides output voltage also require inspection for ensuring proper converter operation. These waveforms include $\mathrm{Q}_{1}$ 's 
switching trajectory and its drain current $\left(\mathrm{I}_{\mathrm{d}}\left(\mathrm{Q}_{1}\right)\right)$, current through switching inductors $\mathrm{L}_{1}$ and $\mathrm{L}_{2}\left(\mathrm{I}_{\mathrm{L} 1}\right.$ and $\left.\mathrm{I}_{\mathrm{L} 2}\right)$, voltage across and current through coupling capacitor $\mathrm{C}_{5}(\mathrm{~V}(\mathrm{~N} 003)-$ $\mathrm{V}(\mathrm{N} 004)$ and $\left.\mathrm{I}_{\mathrm{C} 5}\right)$ and switching diode current $\left(\mathrm{I}_{\mathrm{D} 1}\right)$. Figure 5.9 shows these waveforms with the base SEPIC running in full load and steady-state operation. The light green waveform denotes SEPIC output voltage, blue denotes $\mathrm{Q}_{1}$ gate voltage (switching trajectory), dark green denotes $\mathrm{Q}_{1}$ drain current, red denotes $\mathrm{I}_{\mathrm{L} 1}$, teal denotes $\mathrm{I}_{\mathrm{L} 2}$, V(N003)-V(N004) denotes coupling capacitor voltage, magenta denotes coupling capacitor current, and gray denotes switching diode current.

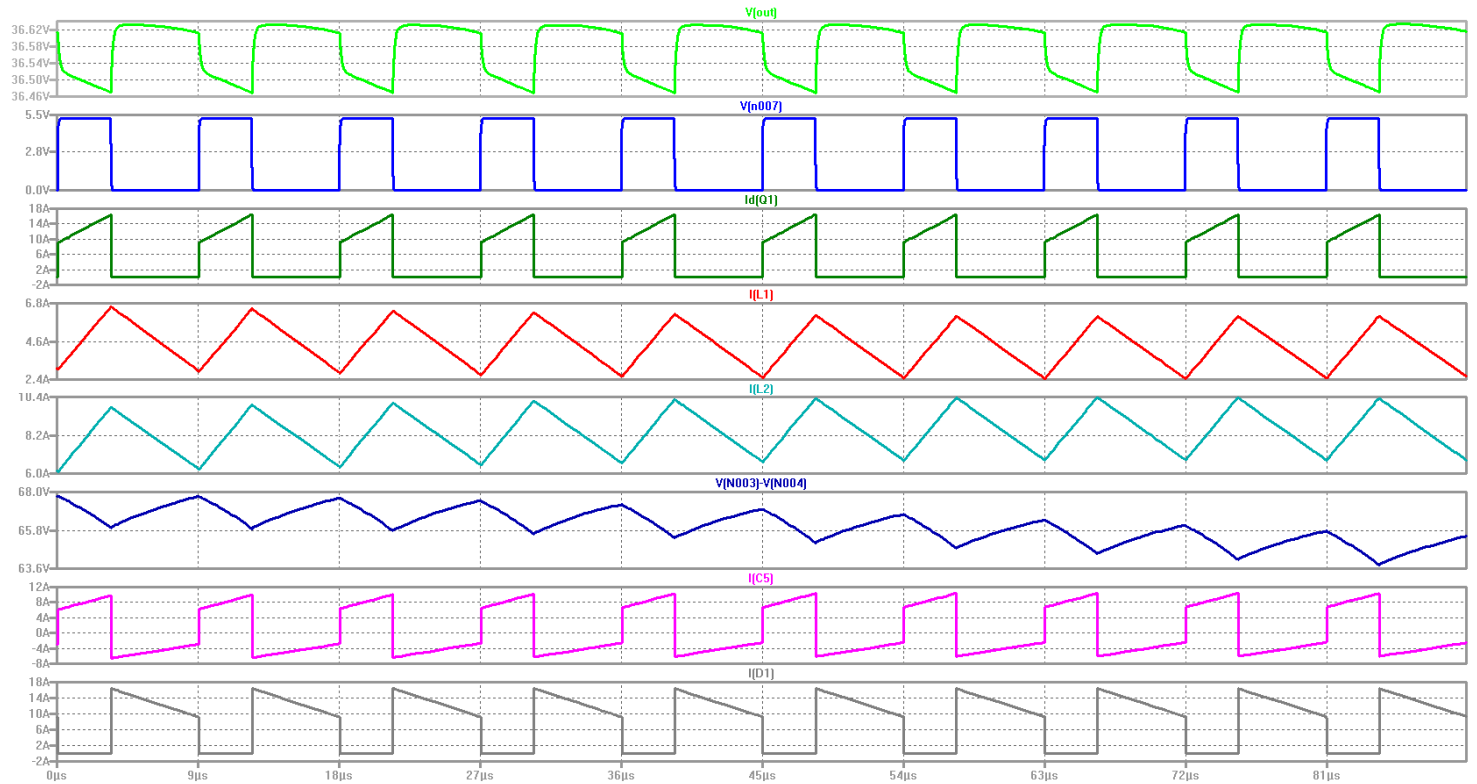

Figure 5.9: Base SEPIC Crucial Component Waveforms Maximum Input Voltage, Full Load and Steadystate

Each waveform is the proper shape according to figure 4.1 and $[26,27]$. The SEPIC's switching period determined from the $\mathrm{Q}_{1}$ switching trajectory waveform in figure 5.9 is $9 \mu$ s, which corresponds to a $111 \mathrm{kHz}$ switching frequency. This switching frequency suffices, as it is close enough to the $100 \mathrm{kHz}$ desired SEPIC switching frequency when using a commonly available $220 \mathrm{k} \Omega$ resistor as the LTC1871 controller's frequency set 
resistor. At the SEPIC's specified 65V maximum input voltage and $8 \mathrm{~A}$ maximum load while in steady-state operation, peak drain current through $\mathrm{Q}_{1}$ is $16.39 \mathrm{~A}$, peak current through $\mathrm{L}_{1}$ is $6.648 \mathrm{~A}$, peak current through $\mathrm{L}_{2}$ is $10.3 \mathrm{~A}$, peak voltage across the coupling capacitor is $67.8 \mathrm{~V}$, peak current through the coupling capacitor is $9.93 \mathrm{~A}$, and peak switching diode current is $16.22 \mathrm{~A}$. Average switching diode current is $8.011 \mathrm{~A}$, which nearly matches that of the SEPIC's average load current. The coupling capacitor's ripple voltage is $2 \mathrm{~V}$ peak-to-peak and its RMS current is 6.292A. Finally, average drain current through $\mathrm{Q}_{1}$ is $4.8 \mathrm{~A}$, average current through $\mathrm{L}_{1}$ is $4.42 \mathrm{~A}$ and average current through $\mathrm{L}_{2}$ is $8.4 \mathrm{~A}$. Furthermore, neither the current through $\mathrm{L}_{1}$ nor the current through $\mathrm{L}_{2}$ decrease to 0A during the entire SEPIC steady-state operation period, thus this SEPIC operates in CCM at full load. The $\mathrm{L}_{1}$ and $\mathrm{L}_{2}$ current waveforms are both also perfect triangular waves, indicating that neither inductor saturates in this SEPIC's steady-state operation.

Overall, these voltage and current values do not place any major electrical stress on the crucial SEPIC components, provided that the physical components can tolerate these electrical stress levels. Of course, all passive components in this base SEPIC simulation are ideal (with no ESR), except for the $85 \mathrm{~m} \Omega$ ESR set in $\mathrm{L}_{1}$ and $\mathrm{L}_{2}$ 's parameters. Furthermore, at $65 \mathrm{~V}$ input, the SEPIC only operates at $36 \%$ duty cycle with a 0.5625 voltage gain from input to output. Its input current (from the elliptical trainer) is lower than that of the output load current. Assuming that the output load current remains constant at 8A, lower SEPIC input voltages require higher input current and thus place higher electrical stress on components connected to the SEPIC's input node - specifically 
$\mathrm{Q}_{1}$ and $\mathrm{L}_{1}$. Thus, these voltage and current values increase at lower SEPIC input voltages and hence are not the highest overall values facing the SEPIC components.

Other waveforms that require analysis are the SEPIC's switch drain-source voltage and inductor voltages. Figure 5.10 shows these waveforms with the base SEPIC running in steady-state operation. The light green waveform denotes SEPIC output voltage, blue denotes $\mathrm{Q}_{1}$ gate voltage (switching trajectory, the magenta waveform and $\mathrm{V}(\mathrm{N} 003)-\mathrm{V}(\mathrm{SENSE})$ denote $\mathrm{Q}_{1}$ drain-source voltage, the red waveform and $\mathrm{V}(\mathrm{IN})$ $\mathrm{V}(\mathrm{N} 003)$ denote $\mathrm{L}_{1}$ voltage $\mathrm{V}_{\mathrm{L} 1}$ and the teal waveform and $\mathrm{V}(\mathrm{N} 004)$ denote $\mathrm{L}_{2}$ voltage $\mathrm{V}_{\mathrm{L} 2}$. 


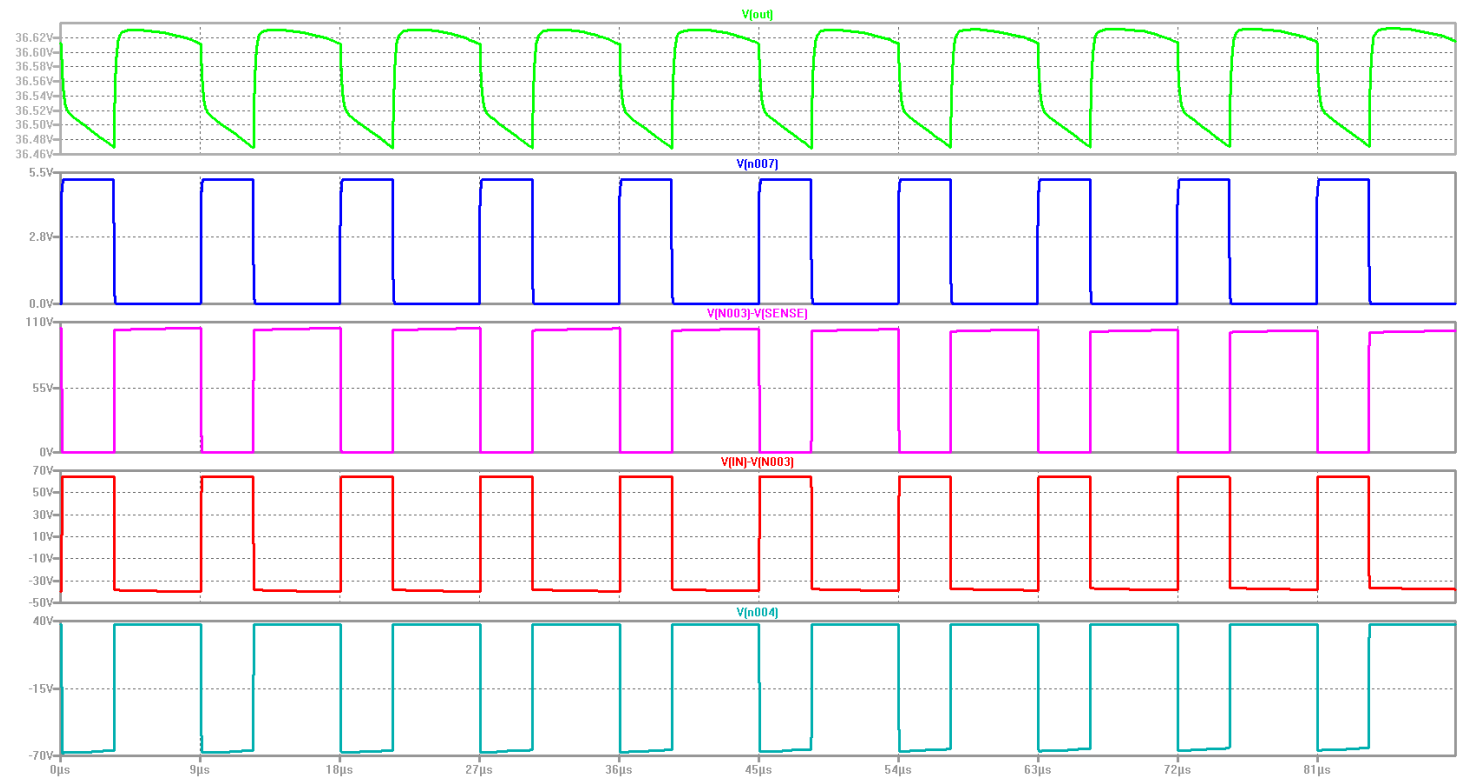

Figure 5.10: Base SEPIC Switch Drain-Source Voltage and Inductor Voltages Maximum Input Voltage, Full load and Steady-state

With 65V input, the SEPIC's input voltage is greater than its output voltage, resulting in $\mathrm{V}_{\mathrm{L} 1}$ and $\mathrm{V}_{\mathrm{L} 2} 180^{\circ}$ out of phase with each other, as specified in [27]. If the SEPIC's input voltage is less than its output voltage, then $\mathrm{V}_{\mathrm{L} 1}$ and $\mathrm{V}_{\mathrm{L} 2}$ would both be in phase with each other (i.e. $0^{\circ}$ phase relative to each other). Peak switch drain-source voltage is $105.63 \mathrm{~V}$, peak $\mathrm{L}_{1}$ voltage is $64.47 \mathrm{~V}$ and peak $\mathrm{L}_{2}$ voltage is $37.16 \mathrm{~V} . \mathrm{L}_{1}$ and $\mathrm{L}_{2}$ 's minimum voltages are the antipodes of each other's peak voltages - minimum $L_{1}$ voltage is $-39.74 \mathrm{~V}$ and minimum $\mathrm{L}_{2}$ voltage is $-66.45 \mathrm{~V}$. Average switch drain-source voltage is $64.91 \mathrm{~V}$ and average $\mathrm{L}_{1}$ voltage is near $0 \mathrm{~V}$. Average $\mathrm{L}_{2}$ voltage is $-1.125 \mathrm{~V} . \mathrm{L}_{2}$ 's average voltage is not quite close to $0 \mathrm{~V}$ because as mentioned in sections 4.3.1 and 4.3.2, $\mathrm{L}_{2}$ alternatively charges (via coupling capacitor $\mathrm{C}_{5}$ ) and discharges when the SEPIC's switch conducts and constantly discharges to the load when the SEPIC's switch does not conduct.

As specified in section 1.2, this project's SEPIC must maintain CCM operation down to $25 \%$ of full load at its output, i.e. a $2 \mathrm{~A}$ load at its output. Thus, the remaining 
waveforms that require analysis are the $\mathrm{L}_{1}$ and $\mathrm{L}_{2}$ current waveforms when the SEPIC operates with a $2 \mathrm{~A}$ load at its output. Figure 5.11 shows these waveforms with the base SEPIC running in steady-state operation with $65 \mathrm{~V}$ input. The green waveform denotes SEPIC output voltage, blue denotes $\mathrm{L}_{1}$ current $\mathrm{I}_{\mathrm{L} 1}$ and red denotes $\mathrm{L}_{2}$ current $\mathrm{I}_{\mathrm{L} 2}$.

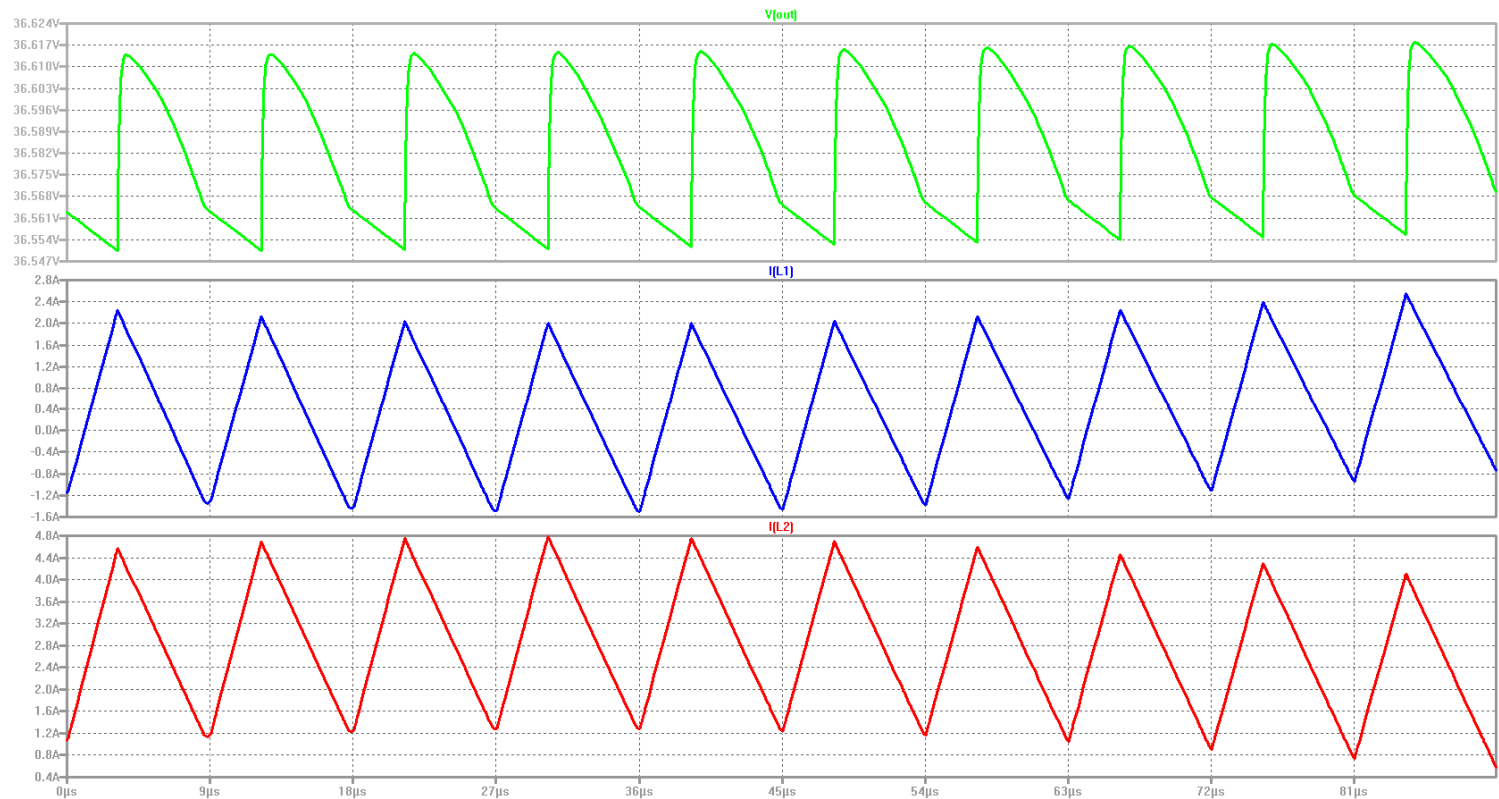

Figure 5.11: Base SEPIC Inductor Currents at Maximum Input Voltage, 25\% Load and Steady-state

Minimum $\mathrm{L}_{1}$ current is $-1.5 \mathrm{~A}$ and minimum $\mathrm{L}_{2}$ current is $0.775 \mathrm{~A}$. The current through both SEPIC inductors never reaches $0 \mathrm{~A}$ for any significant time period relative to the SEPIC's $9 \mu$ s switching period, thus this base SEPIC meets the specification noted in section 1.2 requiring CCM converter operation down to $25 \%$ of output full-load current. After confirming that the base SEPIC outputs the correct voltage and that all crucial waveforms are the proper shape, the next characteristic requiring investigation is its overall efficiency. 


\subsubsection{Base SEPIC Efficiency Analysis}

The ratio between the SEPIC's output power to its input power determines its overall efficiency. Higher efficiency often translates to lower power losses across components and less stress on those components. Lower efficiency means that the SEPIC needs to draw more current from its voltage source (the Precor Elliptical trainer) in order to deliver the same amount of power to the load than if the efficiency was higher. Higher efficiency is more desirable than lower efficiency because it results in lower EHFEM system lifetime costs, as outlined in section 2.3.1. Therefore, this project also focuses on maximizing efficiency on the SEPIC design as much as possible. Figure 5.12 shows the base SEPIC's steady-state input and output power waveforms at $65 \mathrm{~V}$ input and with an 8A load. The green waveform denotes output power and the blue waveform denotes input power.
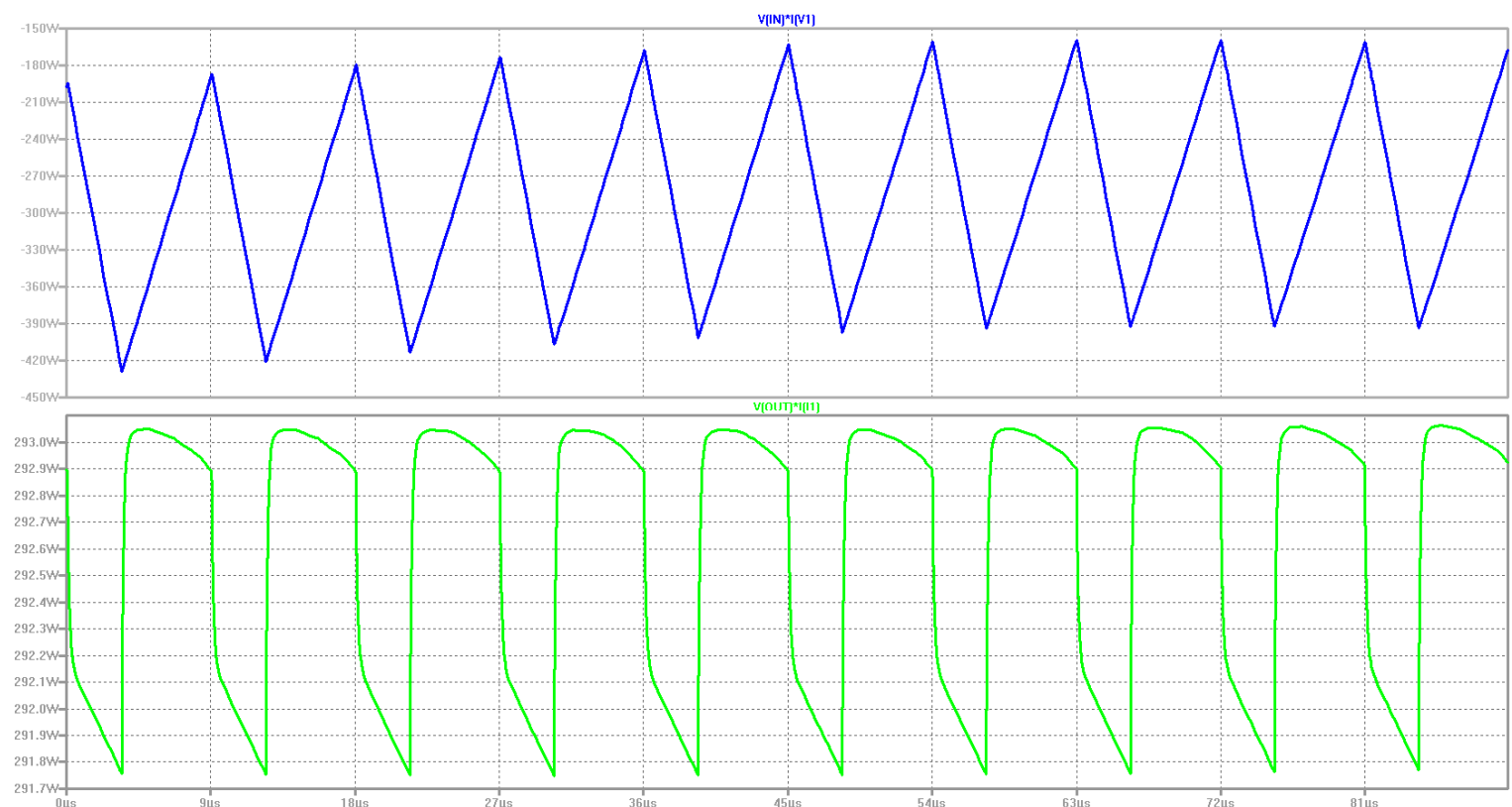

Figure 5.12: Base SEPIC Input and Output Power at Maximum Input Voltage, Full load and Steady-state 
The base SEPIC's average output power at $65 \mathrm{~V}$ input and with an $8 \mathrm{~A}$ load is $292.62 \mathrm{~W}$ and average input power is $328.11 \mathrm{~W}$. Thus, the overall converter efficiency at full load and steady-state is $89.2 \%$. While this overall converter efficiency falls within the SEPIC specification described in section 1.2, operating at near $100 \%$ converter efficiency is more desirable. The components in a real SEPIC are far less ideal than those used in this simulation model. This translates to lower overall converter efficiency on a real SEPIC. Therefore, an investigation on power dissipation (loss) in this SEPIC is necessary. One major component in which such losses occur is in the SEPIC's switching MOSFET, denoted as $\mathrm{Q}_{1}$ in the schematic in figure 5.7.

Figure 5.13 shows the base SEPIC's steady-state output voltage and power dissipation across $\mathrm{Q}_{1}$ at $65 \mathrm{~V}$ input and with an $8 \mathrm{~A}$ load. The green waveform denotes the base SEPIC's output voltage and the blue waveform denotes $\mathrm{Q}_{1}$ 's power dissipation.

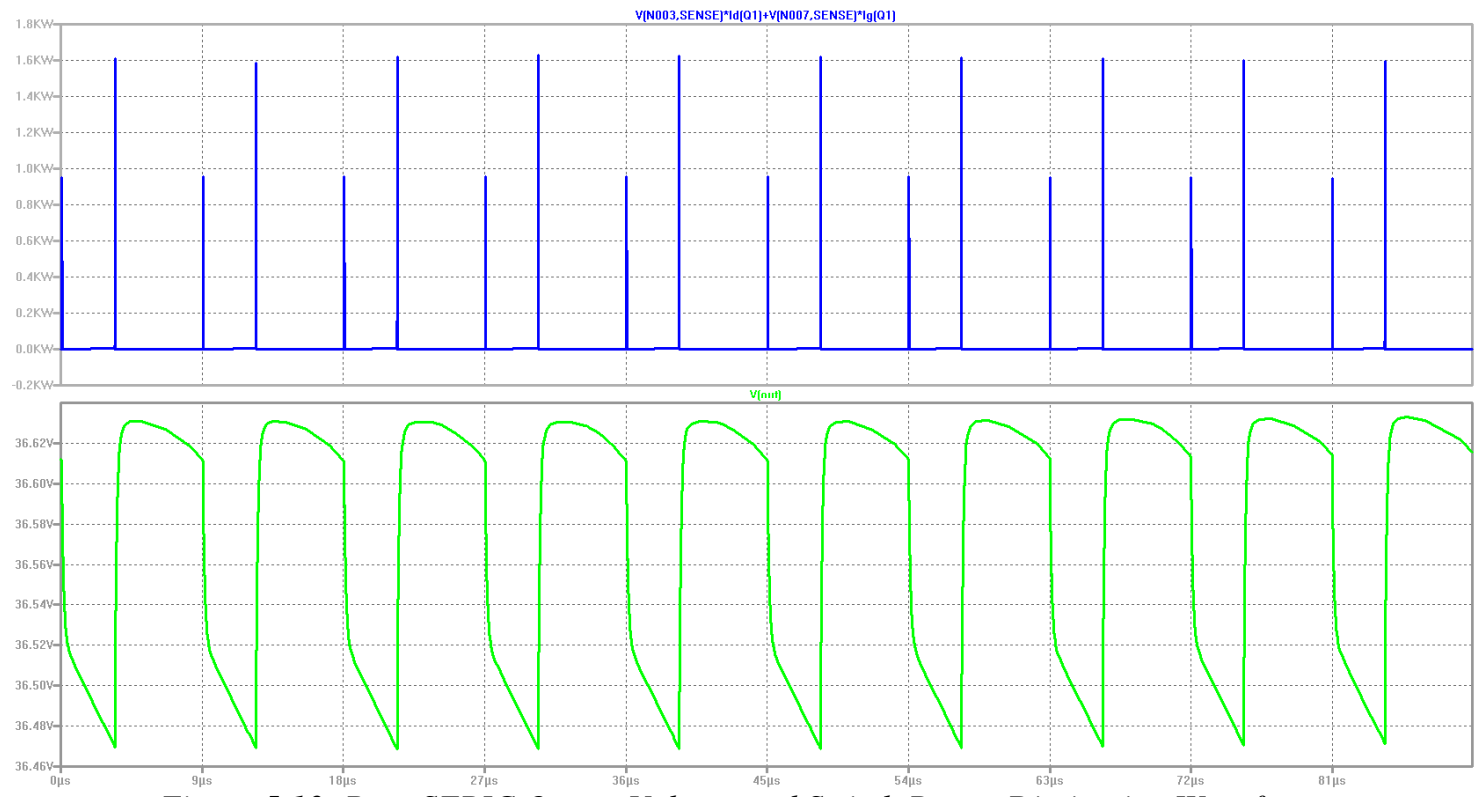

Figure 5.13: Base SEPIC Output Voltage and Switch Power Dissipation Waveforms 
Average power loss across $\mathrm{Q}_{1}$ at full load and steady-state is $2.862 \mathrm{~W}$, which is less than $1 \%$ of the SEPIC's $288 \mathrm{~W}$ output. However, the $1.6 \mathrm{~kW}$ power transients are undesirable for $\mathrm{Q}_{1}$ for prolonged SEPIC operation, as these high power transients reduce $\mathrm{Q}_{1}$ 's operating life and that of the overall converter as well. Thus this base SEPIC requires additional circuitry to reduce the high power transients and power dissipation losses across $\mathrm{Q}_{1}$.

After running the simulation, the LTSpice simulation program also returned a warning message shown in figure 5.14 informing that $\mathrm{D}_{1}$ 's reverse voltage and average forward current reached near their limits during SEPIC steady-state operation.

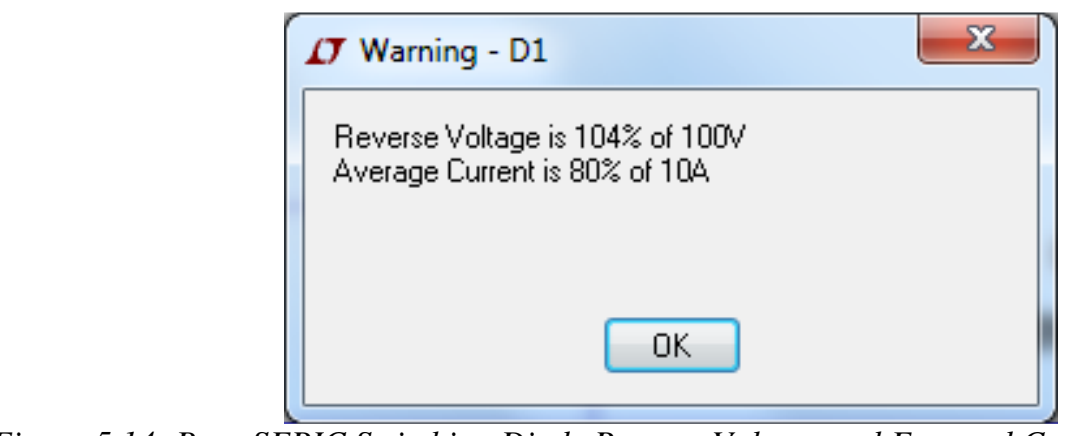

Figure 5.14: Base SEPIC Switching Diode Reverse Voltage and Forward Current Stress Warning

Editing $\mathrm{D}_{1}$ allows for viewing the diode's operating details in the SEPIC's steady-state operation mode. Figure 5.15 shows these details. 


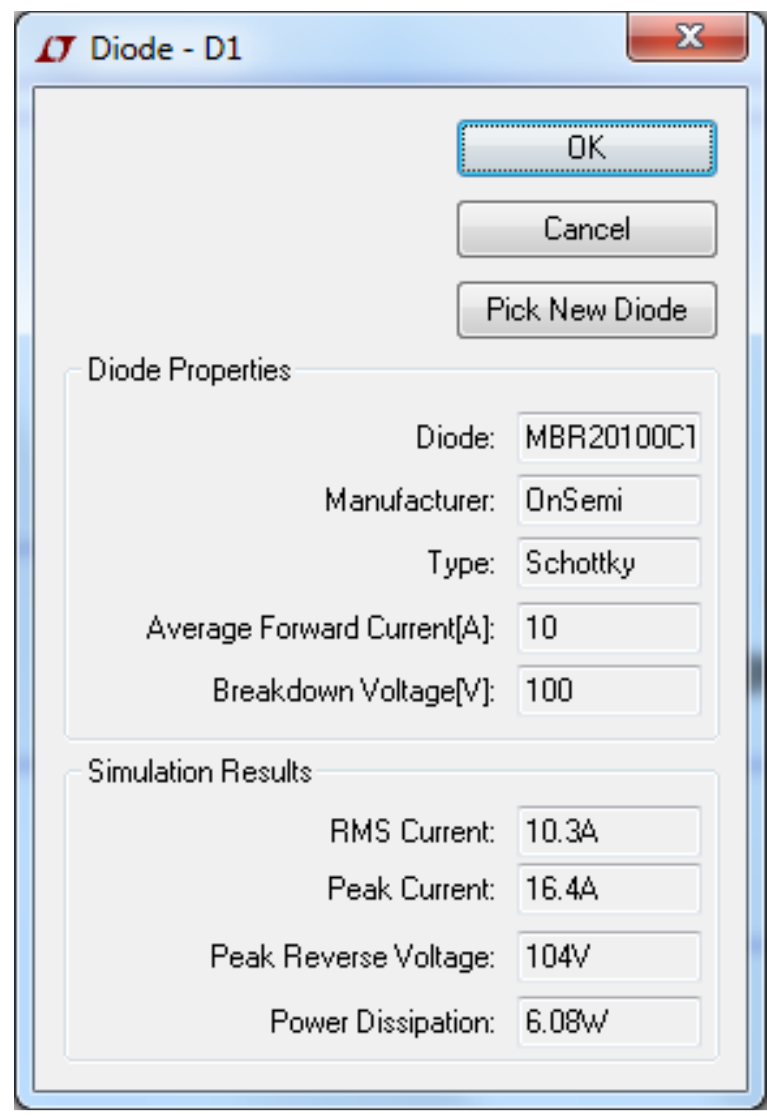

Figure 5.15: Base SEPIC Switching Diode Operation in Converter Steady-State Mode

$\mathrm{D}_{1}$ 's peak reverse voltage reaches $104 \mathrm{~V}$, which is above its $100 \mathrm{~V}$ maximum rating [40].

Furthermore, $\mathrm{D}_{1}$ also dissipates $6.08 \mathrm{~W}$, requiring very effective (and potentially expensive) cooling on the diode. Therefore using one MBR20100CT switching diode in this SEPIC is not sufficient for prolonged SEPIC operation.

Overall, without any further design modifications this initial base SEPIC design is not feasible for implementation into a high-power energy conversion system such as the EHFEM project. The power transients across $\mathrm{Q}_{1}$ reduce its operating life, requiring frequent converter servicing and component replacement throughout the SEPIC's lifetime if implemented into the EHFEM system. Furthermore, using one MBR20100CT for this project's SEPIC's switching diode is also unfeasible since its reverse voltage rises above 
its maximum rated value. Therefore, this SEPIC requires additional circuitry that reduces the power transients (and hence overall dissipation losses) across $\mathrm{Q}_{1}$ as well as additional series-connected MBR20100CT diodes, as prolonged operation is preferable to reduce overall EHFEM system lifetime costs. A snubber cell is one type of circuit that helps reduce switching power transients and overall dissipation losses in a DC-DC converter [41]. 


\section{CHAPTER 6: SEPIC SNUBBER CIRCUIT}

\subsection{Snubber Introduction and Design}

As determined in section 5.4.2, this project's SEPIC's switch theoretically dissipates $2.862 \mathrm{~W}$ with $1.6 \mathrm{~kW}$ transients at $65 \mathrm{~V}$ input while driving an $8 \mathrm{~A}$ load, which is an unreasonable power dissipation stress on the switch even with a heatsink attached to it. Even with a proper cooling mechanism those high power transients may destroy the switch after prolonged SEPIC operation. Furthermore, if this SEPIC drives an 8A load at lower input voltages, the SEPIC's switch's drain current increases because the load requires more power from the input source (the Precor elliptical trainer) in order to maintain a steady $36 \mathrm{~V}$ SEPIC output and $8 \mathrm{~A}$ load current. This drain current increase results in even greater power dissipation and higher power transients across the SEPIC's switch than at maximum input voltage and maximum load current. Thus, this project's SEPIC requires a mechanism for reducing such power dissipation stress and losses. Such losses usually result from the switch's drain-source voltage not decreasing to $0 \mathrm{~V}$ fast enough when the switch turns on and current begins to flow through its drain (resulting in nonzero $\mathrm{V} \times \mathrm{I}$ losses) as well as its drain current not decaying quickly enough to $0 \mathrm{~A}$ when the switch turns off and its drain-source voltage begins increasing. Thus, this project's SEPIC requires an additional circuit that slows down switch drain current rise during switch turn-on and drain-source voltage rise during turn off, such that the switch turns on when its drain-source voltage reaches $0 \mathrm{~V}$ and turns off when its drain current reaches $0 \mathrm{~A}$. Numerous circuits can perform this task, such as a snubber cell or an active clamp [41]. The purpose of adding either of these circuits to the SEPIC is reducing the power 
dissipation stress on its switch as well as electromagnetic interference (EMI) noise from it. This in turn reduces overall power dissipation losses in the converter and improves overall converter efficiency.

A snubber cell, or simply known as a snubber, is more feasible for this project's SEPIC over an active clamp circuit because unlike an active clamp circuit, a snubber can be created from purely passive components and does not require any additional switches (such as MOSFETs), which in turn require additional control circuitry [42-45].

There are many available snubber topologies - some use purely passive components while others employ both passive and active components, known as passive snubbers and active snubbers. Like active clamp circuits, active snubbers require additional switches and those switches require additional control circuitry, adding to the SEPIC's complexity and overall cost. Furthermore, switch control synchronization problems are a likely occurrence if more than one switch is used in an active snubber [41]. Passive snubbers, on the other hand, have simple structures and are thus easy for implementing into a DC-DC converter, while also having a low cost [46]. Passive snubbers are further broken into two sub-categories - those that employ resistors and those that do not. The most well-known type of snubber that employs resistors is a resistor-capacitor-diode (RCD) snubber [46]. However, RCD snubbers and resistive snubbers in general have poor switch power dissipation reduction performance because they incur additional power dissipation losses in their resistors ( $I^{2} \mathrm{R}$ losses) [46].

An alternative passive snubber topology instead replaces resistors with an inductor, thus employing only "theoretically lossless" components [47-51]. The main 
advantages of this snubber topology are its low cost, high reliability and its effective performance in reducing switch power dissipation stress and losses. Thus, the snubber used in this project's SEPIC employs this lossless passive component topology, reducing component costs and snubber power dissipation losses. This snubber circuit only uses inductors, capacitors and diodes. Figure 6.1 shows this lossless passive snubber used in this project's SEPIC [41].

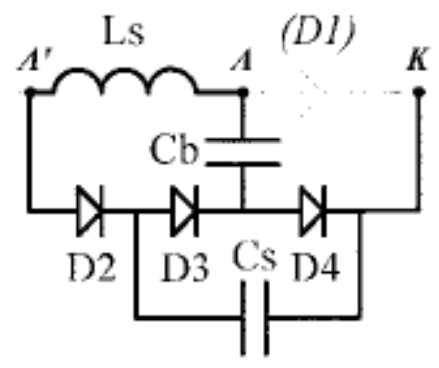

Figure 6.1: Lossless Passive Snubber Cell [41]

Node A denotes the wire connection to the SEPIC's main switching diode $\left(\mathrm{D}_{1}\right)$ 's anode, while $\mathrm{K}$ denotes $\mathrm{D}_{1}$ 's cathode location. Node $\mathrm{A}^{\prime}$ corresponds to $\mathrm{D}_{2}$ 's anode. This snubber is actually a combination of two sub-circuits - one that reduces power dissipation across the SEPIC's switch during its turn-on stage and another that performs the same task during the SEPIC's switch's turn-off stage. These sub-circuits are the snubber's turn-on and turn-off circuits. Figures 6.2 and 6.3 show the snubber's turn-on and turn-off circuits [41]. 


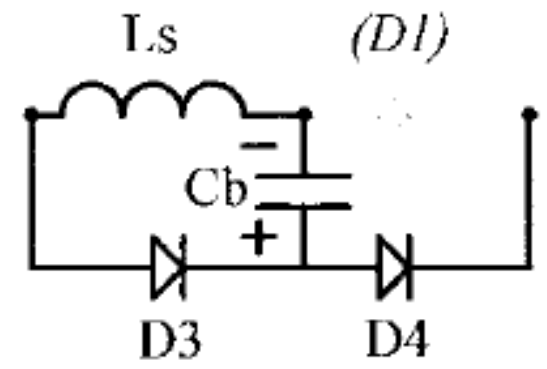

Figure 6.2: Snubber Turn-On Circuit [41]

(DI)

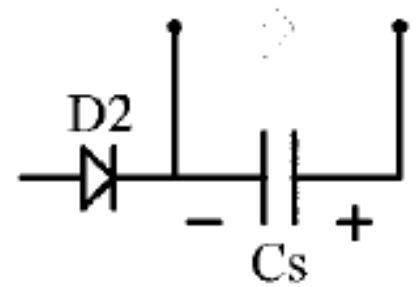

Figure 6.3: Snubber Turn-Off Circuit [41]

Combining the circuits in figures 6.2 and 6.3 results in the complete snubber in figure 6.1. The entire snubber consists of an inductor $\left(\mathrm{L}_{\mathrm{s}}\right)$, two capacitors $\left(\mathrm{C}_{\mathrm{b}}\right.$ and $\left.\mathrm{C}_{\mathrm{s}}\right)$ and three diodes $\left(D_{2}, D_{3}\right.$ and $\left.D_{4}\right)$. Figure 6.4 shows the snubber implemented into a basic SEPIC [41]. 


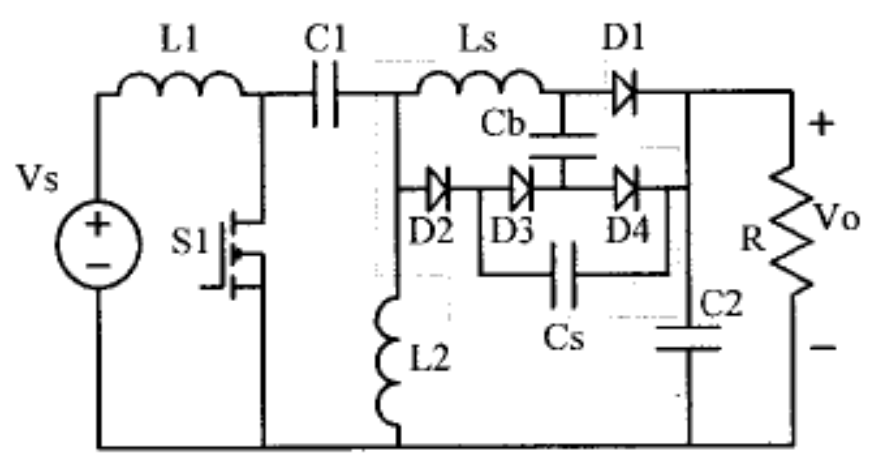

Figure 6.4: Basic SEPIC with Lossless Passive Snubber Cell [41]

The snubber turn-on circuit in figure 6.2 employs an inductor connected in series with $\mathrm{D}_{1}$, which limits $\mathrm{D}_{1}$ 's reverse recovery current increase when $\mathrm{S}_{1}$ turns on. $\mathrm{L}_{1}$ already helps limit $\mathrm{S}_{1}$ 's drain current rise during $\mathrm{S}_{1}$ 's turn-on stage. Diodes $\mathrm{D}_{3}$ and $\mathrm{D}_{4}$ and buffer capacitor $\mathrm{C}_{\mathrm{b}}$ absorb the reverse recovery energy absorbed in both $\mathrm{L}_{\mathrm{s}}$ and $\mathrm{C}_{\mathrm{s}} \cdot \mathrm{C}_{\mathrm{b}}$ then transfers its absorbed energy to the SEPIC's output, completing the energy recovery process. This component combination helps $S_{1}$ achieve zero-current switching (ZCS) turn-on.

The snubber turn-off circuit in figure 6.3 employs a capacitor $\left(\mathrm{C}_{\mathrm{s}}\right)$ connected in parallel with $\mathrm{D}_{1}$, which limits $\mathrm{S}_{1}$ 's drain-source voltage $\left(\mathrm{V}_{\mathrm{DS}}\right)$ rise during its turn-off stage. An additional diode, $D_{2}$, provides isolation between $L_{2}$ and $C_{s}$, preventing $S_{1}, C_{1}$ and $\mathrm{L}_{2}$ from accidentally charging $\mathrm{C}_{\mathrm{s}}$ and resulting in switch turn-on losses from the charge when there is a low-current load or no load at the SEPIC's output. This component combination helps $S_{1}$ achieve zero-voltage switching (ZVS) turn-off (by restricting $\mathrm{S}_{1}$ 's $\mathrm{V}_{\mathrm{DS}}$ rise during turn-off) and also helps $\mathrm{D}_{1}$ achieve both ZVS turn-on and turn-off. 
Compared to DC-DC converters using resonant soft-switching topologies, this snubber only handles small switching transient energy instead of all the energy from the converter's main power path. Thus, the energy circulated in the snubber is much smaller than the energy circulated through the soft-switching circuits in a conventional resonant ZCS/ZVS DC-DC converter [41]. Thus, this project's SEPIC as a whole is expected to have greater efficiency than the previously selected off-the-shelf resonant ZCS/ZVS DCDC converter used by the EHFEM group in [1] in this project while operating with a 5$65 \mathrm{~V}$ input range, when implemented with a passive lossless snubber. The next section describes the snubber's operation during $\mathrm{S}_{1}$ turn-off, turn-on and intermediate stages.

\subsection{Snubber Operation Analysis}

The snubber's principal operation consists of eight separate stages. Figure 6.5 shows key waveforms in a boost converter for each snubber operation stage. Because a SEPIC operates in a similar manner to that of a boost converter [20,21], these waveform shapes are similar for a SEPIC. The only differences lie in waveform magnitudes - in a SEPIC, a secondary inductor $\left(\mathrm{L}_{2}\right)$ also supplies energy to the snubber and the load (with the energy provided to it from coupling capacitor $\mathrm{C}_{1}$ ), thus while the current waveforms through snubber components are the same shape as that of a boost converter, their magnitudes are higher. Therefore these general waveform shapes are also valid for a SEPIC and are thus valid for analyzing each snubber operation stage for this project's SEPIC [41]. 


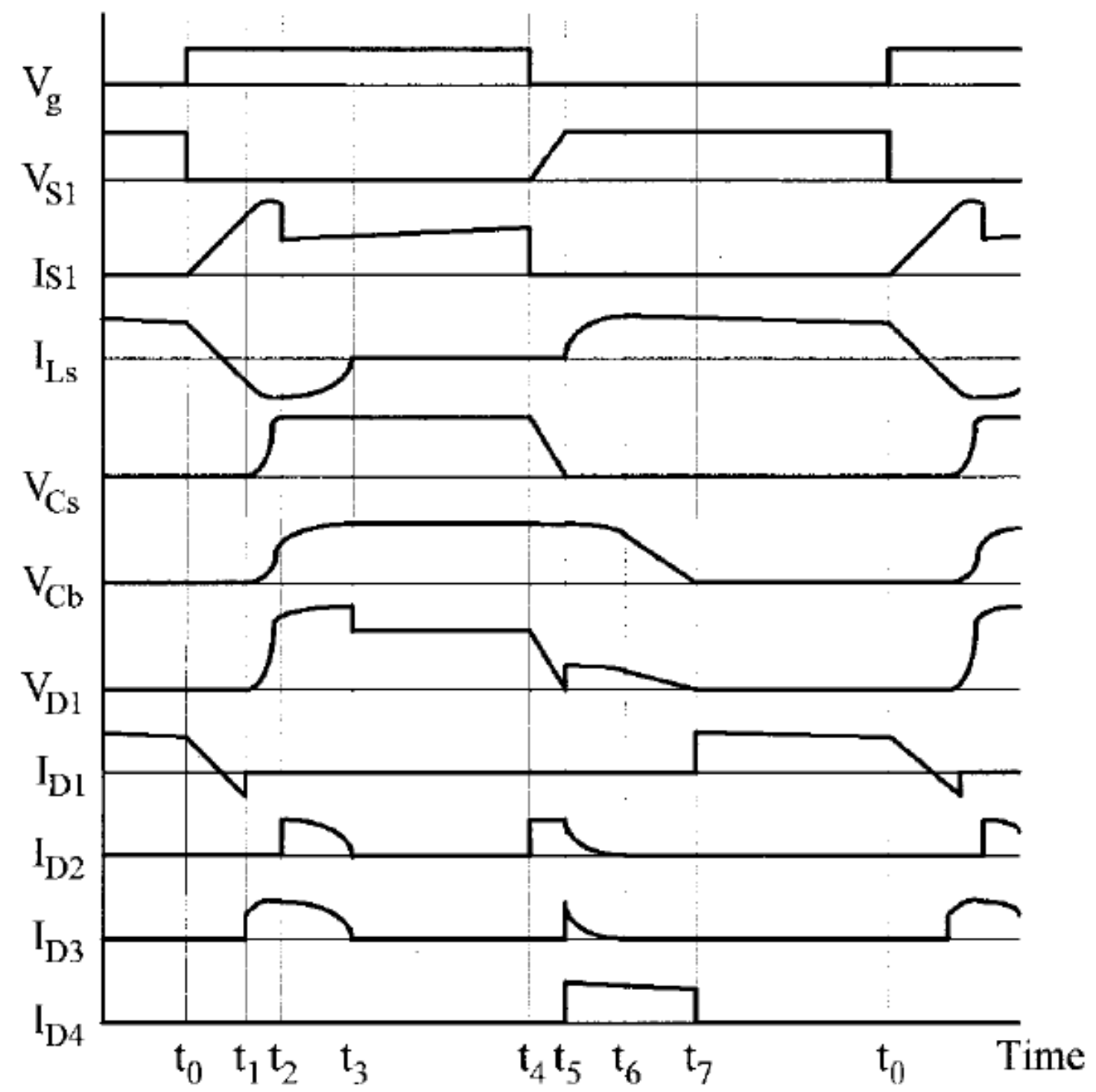

Figure 6.5: Key Waveforms of Boost Converter with Lossless Passive Snubber [41]

In figure $6.5, \mathrm{~V}_{\mathrm{g}}$ denotes $\mathrm{S}_{1}$ 's gate voltage (and its switching trajectory), $\mathrm{V}_{\mathrm{S} 1}$ denotes $\mathrm{S}_{1}$ 's drain-source voltage and $\mathrm{I}_{\mathrm{S}_{1}}$ denotes $\mathrm{S}_{1}$ 's drain current. All other waveforms correspond to voltages across and currents through each respective snubber component. The snubber's first stage (stage 1) operation consists of the time period from $t_{0}$ to $t_{1}$, when $S_{1}$ turns on. Stage 2 consists of $t_{1}$ to $t_{2}$, stage 3 consists of $t_{2}$ to $t_{3}$, stage 4 consists of $t_{3}$ to $t_{4}$, stage 5 consists of $t_{4}$ to $t_{5}$ (when $S_{1}$ turns off), stage 6 occurs during $t_{5}$ to $t_{6}$ and stage 7 
occurs during $t_{6}$ to $t_{7}$. The snubber's final operation stage, stage 8 , occurs during $t_{7}$ to when $\mathrm{S}_{1}$ turns on again at $\mathrm{t}_{0}$. Time $\mathrm{t}_{0}$ designates the beginning of a switching period.

For the purpose of this operation analysis, the SEPIC's output filter capacitance $\mathrm{C}_{2}$ is large enough such that the SEPIC's output voltage $\mathrm{V}_{\mathrm{O}}$ is constant and ripple-free. $\mathrm{C}_{2}$ supplies energy to the load for maintaining load current when $S_{1}$ conducts and $D_{1}$ does not conduct. As long as $\mathrm{C}_{2}$ 's capacitance is large enough, $\mathrm{C}_{2}$ does not affect this snubber cell's operation and this analysis treats it as an open circuit (hence neglecting it). The next section describes the snubber's first operation stage.

\subsubsection{Snubber Stage 1 Operation}

Figure 6.6 shows the SEPIC implemented with the snubber, in the snubber's first operation stage [41].

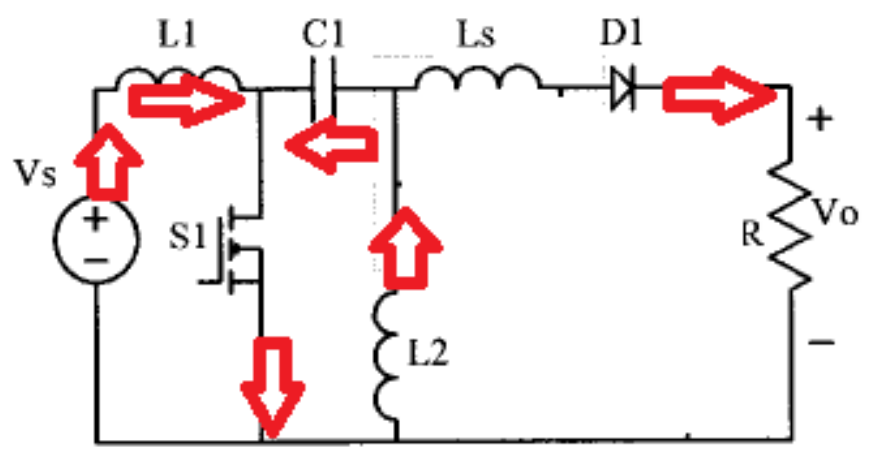

Figure 6.6: Stage 1 Snubber Operation [41]

The red arrows indicate the current flow direction through each branch. The snubber's first operation stage begins with $S_{1}$ turning on at time $t_{0}$. While $S_{1}$ turns on, $D_{1}$ still conducts because it is not ideal; reverse recovery current still flows through it. Snubber inductor $\mathrm{L}_{\mathrm{s}}$ restricts the rate of rise of current through $\mathrm{S}_{1}$, achieving zero-current switching (ZCS) MOSFET turn-on. During $\mathrm{S}_{1}$ turn on, $\mathrm{I}_{\mathrm{L} 1}$, the current through $\mathrm{L}_{1}$, 
increases in the positive direction (from the main voltage source $V_{\mathrm{S}}$ to $\mathrm{S}_{1}$ ) while $\mathrm{I}_{\mathrm{L} 2}$, the current through $\mathrm{L}_{2}$, increases in the negative direction. $\mathrm{V}_{\mathrm{S}}$ is responsible for the $\mathrm{I}_{\mathrm{L} 1}$ increase. As $S_{1}$ turns on, its drain to source voltage decreases to $0 \mathrm{~V}$, allowing the voltage across the converter's coupling capacitor, $\mathrm{V}_{\mathrm{C} 1}$, to equal $\mathrm{V}_{\mathrm{s}}$. Once $\mathrm{S}_{1}$ 's drain-source voltage reaches $0 \mathrm{~V}$, the voltage across $\mathrm{L}_{2}, \mathrm{~V}_{\mathrm{L} 2}$ equals $-\mathrm{V}_{\mathrm{S}} . \mathrm{C}_{1}$ supplies the energy to increase $\mathrm{I}_{\mathrm{L} 2}$ in the negative direction, increasing the stored energy in $\mathrm{L}_{2}$. Equations (6.1) to (6.4) describe the current through $\mathrm{L}_{\mathrm{s}}\left(\mathrm{I}_{\mathrm{Ls}}\right), \mathrm{C}_{1}\left(\mathrm{I}_{\mathrm{C} 1}\right)$ and $\mathrm{L}_{2}\left(\mathrm{I}_{\mathrm{L} 2}\right)$ during this operation stage. Equations (6.1) and (6.2) first show the fundamental time-domain capacitor current and inductor voltage equations necessary for understanding the $\mathrm{I}_{\mathrm{Ls}}$ equations in (6.3) to $(6.4)[33,52]$.

$$
\begin{gathered}
I_{C}=C \frac{d V_{C}}{d t} \\
V_{L}=L \frac{d I_{L}}{d t} \\
I_{L S}(t)=-I_{C 1}\left(t_{0}\right)+I_{L 2}\left(t_{o}\right)-\frac{V_{O}}{L_{S}}\left(t-t_{0}\right) \\
I_{L S}(t)=-C_{1}\left(\frac{\left[V_{C S}(t)-V_{C S}\left(t_{0}\right)\right]}{t-t_{0}}\right)+I_{L 2}\left(t_{o}\right)-\frac{V_{O}}{L_{S}}\left(t-t_{0}\right)
\end{gathered}
$$

Once the SEPIC reaches time $\mathrm{t}_{1}$, the snubber's second operation stage begins.

\subsubsection{Snubber Stage 2 Operation}

At time $t_{1}, D_{1}$ 's reverse recovery phenomenon finishes. Once $D_{1}$ turns off, $D_{3}$ turns on since $\mathrm{V}_{\mathrm{Cs}}$ and $\mathrm{V}_{\mathrm{Cb}}$ both equal $0 \mathrm{~V}$. All energy storage components in the snubber $\left(\mathrm{L}_{\mathrm{s}}, \mathrm{C}_{\mathrm{b}}, \mathrm{C}_{\mathrm{s}}\right.$ ) charge through the first resonant path $\mathrm{V}_{\mathrm{o}}-\mathrm{C}_{\mathrm{s}}-\mathrm{D}_{3}-\mathrm{C}_{\mathrm{b}}-\mathrm{L}_{\mathrm{s}}-\mathrm{C}_{1}-\mathrm{S}_{1}$. The reverse voltage across $D_{1}$ increases to $V_{C s}+V_{C b}$, and charging $C_{s}$ and $C_{b}$ limits the reverse voltage's rise rate. Figure 6.7 shows the SEPIC implemented with the snubber, in the snubber's second operation stage [41]. 


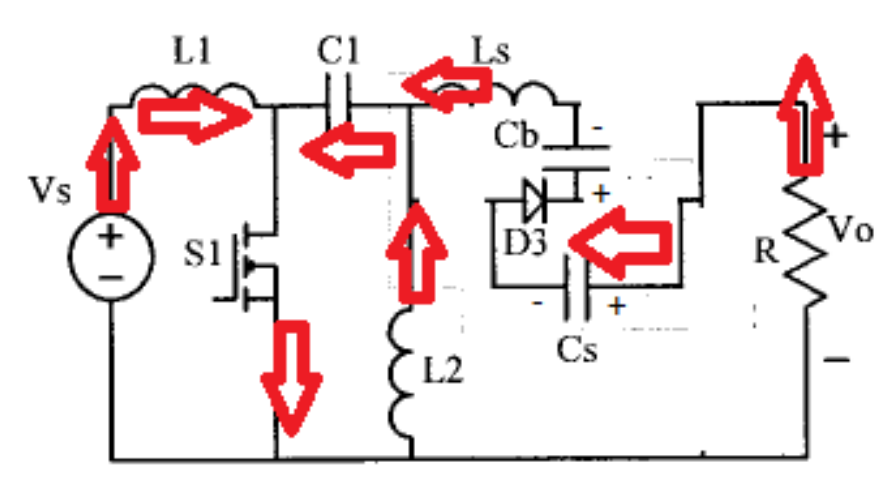

Figure 6.7: Stage 2 Snubber Operation [41]

While $S_{1}$ conducts and $D_{1}$ does not conduct, $C_{2}$ supplies energy to the load for maintaining the load current. Because $\mathrm{C}_{2}$ 's capacitance is large enough for ensuring a constant, ripple-free output voltage, $\mathrm{C}_{2}$ does not affect the snubber's operation.

Equations (6.5) to (6.8) give the snubber inductor current ( $\mathrm{I}_{\mathrm{LS}}$ ), snubber capacitor voltage $\left(\mathrm{V}_{\mathrm{CS}}\right)$, and buffer capacitor voltage $\left(\mathrm{V}_{\mathrm{Cb}}\right)$ during this operation stage.

$$
\begin{gathered}
I_{L S}(t)=-\frac{V_{O}}{Z_{1}} \sin \left(\omega_{1}\left(t-t_{1}\right)\right)-I_{r r} \cos \left(\omega_{1}\left(t-t_{1}\right)\right) \\
V(t)=I_{r r} Z_{1} \sin \left(\omega_{1}\left(t-t_{1}\right)\right)-V_{O} \cos \left(\omega_{1}\left(t-t_{1}\right)\right)+V_{O} \\
V_{C S}(t)=\frac{C_{b}}{C_{s}+C_{b}} V(t) \\
V_{C b}(t)=\frac{C_{s}}{C_{s}+C_{b}} V(t)
\end{gathered}
$$

Where:

$$
\begin{gathered}
I_{r r}=\frac{V_{O}}{L_{S}}\left(t_{1}-t_{0}\right)+I_{L 2}\left(t_{0}\right)+I_{C 1}\left(t_{0}\right)-I_{L 1}\left(t_{0}\right) \\
Z_{1}=\sqrt{\frac{L_{s}\left(C_{S}+C_{b}\right)}{C_{s} C_{b}}} \\
\omega_{1}=\sqrt{\frac{C_{s}+C_{b}}{L_{s} C_{S}+C_{b}}}
\end{gathered}
$$

The snubber slightly increases $S_{1}$ 's peak drain current requirement (which was 37.95A from (5.24) above). By adding the snubber cell, $S_{1}$ 's peak drain current requirement becomes its original determined peak current summed with $\mathrm{I}_{\mathrm{Ls}, \mathrm{p}}$, the snubber inductor's peak current. $\mathrm{I}_{\mathrm{Ls}, \mathrm{p}}$ occurs when $\mathrm{V}_{\mathrm{Cs}}+\mathrm{V}_{\mathrm{Cb}}=\mathrm{V}_{\mathrm{O}}$. 


$$
I_{L s, p}=\frac{\sqrt{V_{O}{ }^{2}+\left(I_{r r} Z_{1}\right)^{2}}}{Z_{1}}
$$

The first resonance stage stops at time $t_{2}$ when $V_{C s}\left(t_{2}\right)=V_{O}$, when diode $D_{2}$ turns on. The snubber inductor current at time $\mathrm{t}_{2}$ is:

$$
I_{L S}\left(t_{2}\right)=\frac{\sqrt{\left(I_{r r} Z_{1}\right)^{2}+V_{O}^{2}-V_{O}\left(\frac{C_{S}}{C_{b}}\right)^{2}}}{Z_{1}}
$$

From (6.13), the energy stored in $\mathrm{L}_{\mathrm{s}}$ and $\mathrm{C}_{\mathrm{s}}$ is:

$$
E_{L s}\left(t_{2}\right)+E_{C b}\left(t_{2}\right)=\frac{1}{2} L_{s} I_{L s}{ }^{2}\left(t_{2}\right)+\frac{1}{2} C_{b} V_{C b}{ }^{2}\left(t_{2}\right)=\frac{1}{2} L_{s} I_{r r}{ }^{2}+\frac{1}{2} C_{s} V_{O}{ }^{2}
$$

Once the SEPIC reaches time $t_{2}$, the snubber's third operation stage begins.

\subsubsection{Snubber Stage 3 Operation}

Once $V_{C s}$ charges to $V_{O}$ at time $t_{2}, D_{2}$ turns on and $C_{s}$ becomes open, beginning a second resonance stage. $\mathrm{I}_{\mathrm{Ls}}$ charges $\mathrm{C}_{\mathrm{b}}$ through the $\mathrm{L}_{\mathrm{s}}-\mathrm{L}_{2}-\mathrm{D}_{2}-\mathrm{D}_{3}-\mathrm{C}_{\mathrm{b}}$ resonant path. $\mathrm{L}_{\mathrm{s}}$ and $\mathrm{C}_{\mathrm{b}}$ perform one-way resonance because of $\mathrm{D}_{2}$ and $\mathrm{D}_{3}$ 's orientations; current cannot flow through $C_{b}$ to $D_{2}$ and $D_{3}$. Figure 6.8 shows the SEPIC implemented with the snubber, in the snubber's third operation stage [41].

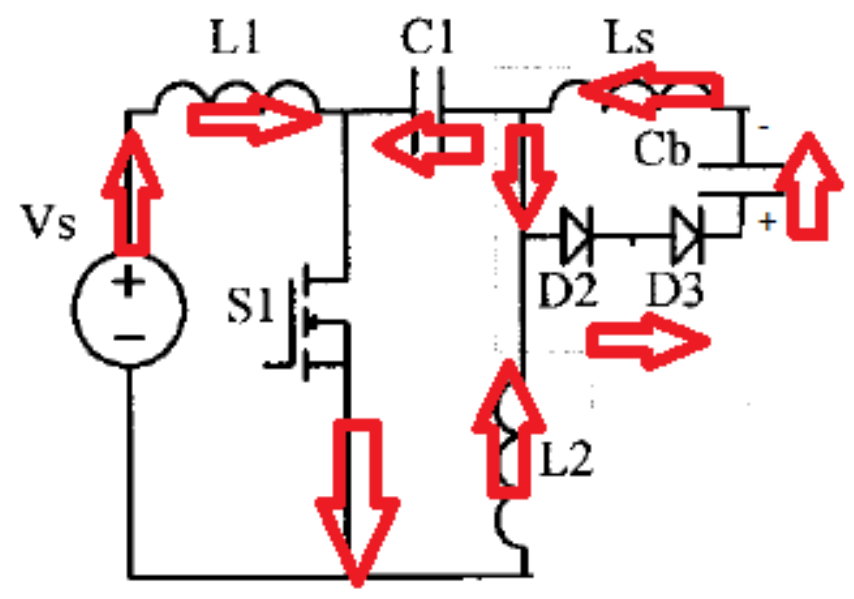

Figure 6.8: Stage 3 Snubber Operation [41] 
$\mathrm{I}_{\mathrm{Ls}}$ and $\mathrm{V}_{\mathrm{Cb}}$ are:

$$
\begin{gathered}
I_{L S}(t)=\frac{C_{s}}{C_{b}} \frac{V_{O}}{Z_{2}} \sin \left(\omega_{2}\left(t-t_{2}\right)\right)-I_{S 2} \cos \left(\omega_{2}\left(t-t_{2}\right)\right) \\
V_{C b}(t)=I_{S 2} Z_{2} \sin \left(\omega_{2}\left(t-t_{2}\right)\right)+\frac{C_{s}}{C_{b}} V_{O} \cos \left(\omega_{2}\left(t-t_{2}\right)\right)
\end{gathered}
$$

Where:

$$
\begin{gathered}
I_{S 2}=\frac{V_{O}}{Z_{1}} \sin \left(\omega_{1}\left(t_{2}-t_{1}\right)\right)+I_{r r} \cos \left(\omega_{1}\left(t_{2}-t_{1}\right)\right) \\
Z_{2}=\sqrt{\frac{L_{s}}{C_{b}}} \\
\omega_{2}=\sqrt{\frac{1}{L_{s} C_{b}}}
\end{gathered}
$$

The second resonance stage stops at time $t_{3}$, when $\mathrm{I}_{\mathrm{Ls}}\left(\mathrm{t}_{3}\right)$ reduces to $0 \mathrm{~A}$. At this stage the energy stored in $L_{s}$ is transfers to $C_{b}$. Thus, the energy stored in $C_{b}$ is:

$$
\frac{1}{2} C_{b}\left(V_{C b}\left(t_{3}\right)\right)^{2}=E_{C b}\left(t_{3}\right)=E_{L S}\left(t_{2}\right)+E_{C b}\left(t_{2}\right)=\frac{1}{2} L_{s} I_{r r}^{2}+\frac{1}{2} C_{s} V_{O}^{2}
$$

Where $\mathrm{E}_{\mathrm{Cb}}\left(\mathrm{t}_{3}\right)$ is the stored energy in $\mathrm{C}_{\mathrm{b}}$ at time $\mathrm{t}_{3}, \mathrm{E}_{\mathrm{Cb}}\left(\mathrm{t}_{2}\right)$ is the stored energy in $\mathrm{C}_{\mathrm{b}}$ at time $t_{2}$, and $E_{L s}\left(t_{2}\right)$ is the stored energy in $L_{s}$ at time $t_{2}$. Buffer capacitor voltage at $t_{3}$ $\mathrm{V}_{\mathrm{Cb}}\left(\mathrm{t}_{3}\right)$ is:

$$
V_{C b}\left(t_{3}\right)=\sqrt{\frac{L_{S} I_{r r}^{2}+C_{S} V_{O}^{2}}{C_{b}}}
$$

The buffer capacitor voltage reaches its peak value at time $t_{3}$. Thus, peak buffer capacitor voltage, $\mathrm{V}_{\mathrm{Cb}, \mathrm{p}}$, is:

$$
V_{C b, p}=V_{C b}\left(t_{3}\right)
$$

$\mathrm{D}_{1}$ freewheels during stages 1-3. The maximum reverse voltage imposed across it is:

$$
V_{R(D 1, \text { max. })}=V_{C b}\left(t_{3}\right)+V_{O}
$$

At time $t_{3}$, the snubber's fourth operation stage begins. 


\subsubsection{Snubber Stage 4 Operation}

Once $\mathrm{I}_{\mathrm{Ls}}$ reduces to $0 \mathrm{~A}$ at time $\mathrm{t}_{3}, \mathrm{D}_{2}$ and $\mathrm{D}_{3}$ simultaneously turn off. Because no current flows through $\mathrm{C}_{\mathrm{b}}$, it maintains a constant voltage. The total energy transferred to $C_{b}$ is the sum of the energy absorbed in $L_{s}$ and $C_{s}$, from (6.20). Figure 6.9 shows the SEPIC implemented with the snubber, in the snubber's third operation stage [41].

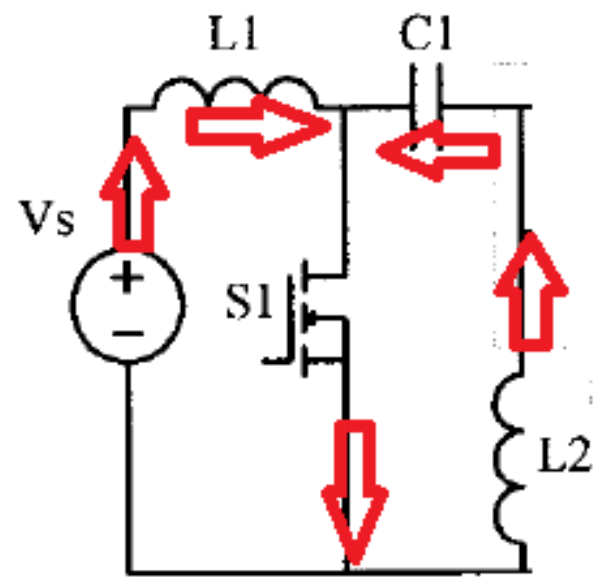

Figure 6.9: Stage 4 Snubber Operation [41]

$S_{1}$ remains turned on and $D_{2}$ remains turned off until time $t_{4}$, when the snubber's fifth operation stage begins.

\subsubsection{Snubber Stage 5 Operation}

At time $t_{4}$, the SEPIC's switch $\mathrm{S}_{1}$ turns off. During this stage, the SEPIC's primary inductor current $\mathrm{I}_{\mathrm{L} 1}\left(\mathrm{t}_{4}\right)$ flows through $\mathrm{D}_{2}$ to discharge $\mathrm{C}_{\mathrm{s}}$ to the output. $\mathrm{L}_{2}$ also discharges through the same path. The voltage polarity across $C_{s}$ causes $D_{3}$ and $D_{4}$ to remain reverse-biased during this stage. Figure 6.10 shows the SEPIC implemented with the snubber, in the snubber's fifth operation stage. 


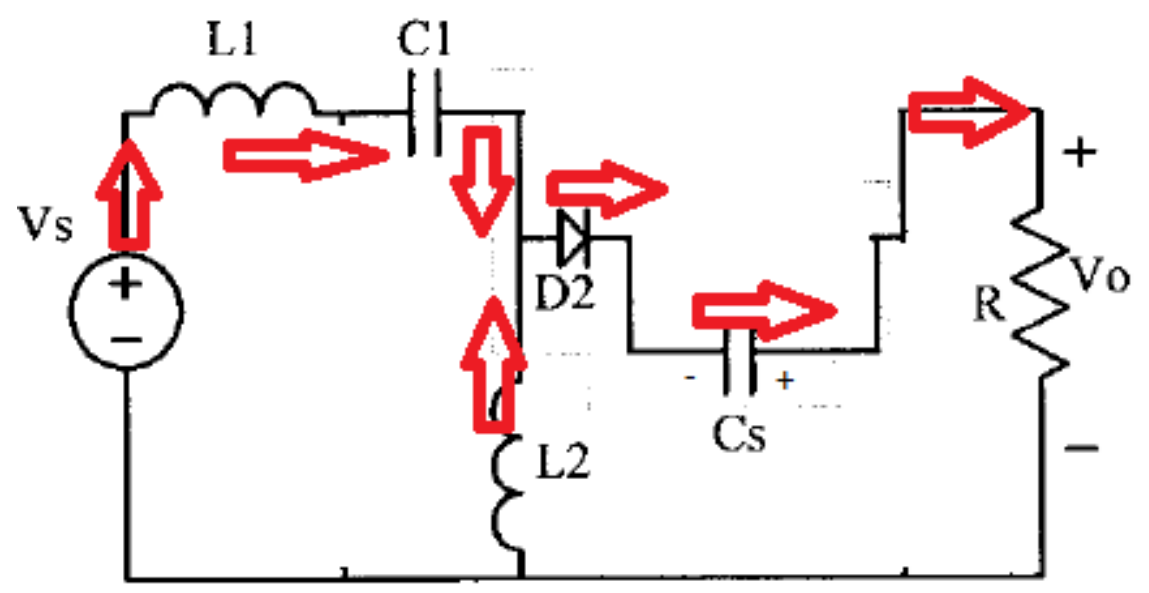

Figure 6.10: Stage 5 Snubber Operation [41]

During this stage the drain-source voltage across $S_{1}$ is:

$$
V_{D S(S 1)}=V_{O}-V_{C S}-V_{C 1}
$$

$\mathrm{V}_{\mathrm{Cs}}$ discharges from $\mathrm{V}_{\mathrm{O}}$ to $0 \mathrm{~V}$, and this in turn helps slow down $\mathrm{S}_{1}$ 's drain-source voltage rise. During stage $4, \mathrm{~V}_{\mathrm{Cs}}$ is:

$$
V_{C s}(t)=V_{O}-\frac{I_{L 1}\left(t_{4}\right)+I_{L 2}\left(t_{4}\right)}{C_{s}}\left(t-t_{4}\right)
$$

Equation (6.25) assumes that $\mathrm{I}_{\mathrm{L} 1}$ and $\mathrm{I}_{\mathrm{L} 2}$ are both constant during this stage. $\mathrm{D}_{3}$ and $\mathrm{D}_{4}$ remain turned off until time $t_{5}$, when the snubber's sixth operation stage begins.

\subsubsection{Snubber Stage 6 Operation}

At time $t_{5}, V_{C s}$ finishes discharging to $0 \mathrm{~V}$ and in turn causes $\mathrm{D}_{3}$ and $\mathrm{D}_{4}$ to turn on. $\mathrm{L}_{\mathrm{s}}$ voltage $\mathrm{V}_{\mathrm{Ls}}$ equals $\mathrm{V}_{\mathrm{Cb}}$ and thus $\mathrm{I}_{\mathrm{L} 1}$ and $\mathrm{I}_{\mathrm{L} 2}$ both increase, discharging $\mathrm{C}_{\mathrm{b}}$ to the output. Figure 6.11 shows the SEPIC implemented with the snubber, in the snubber's sixth operation stage [41]. 


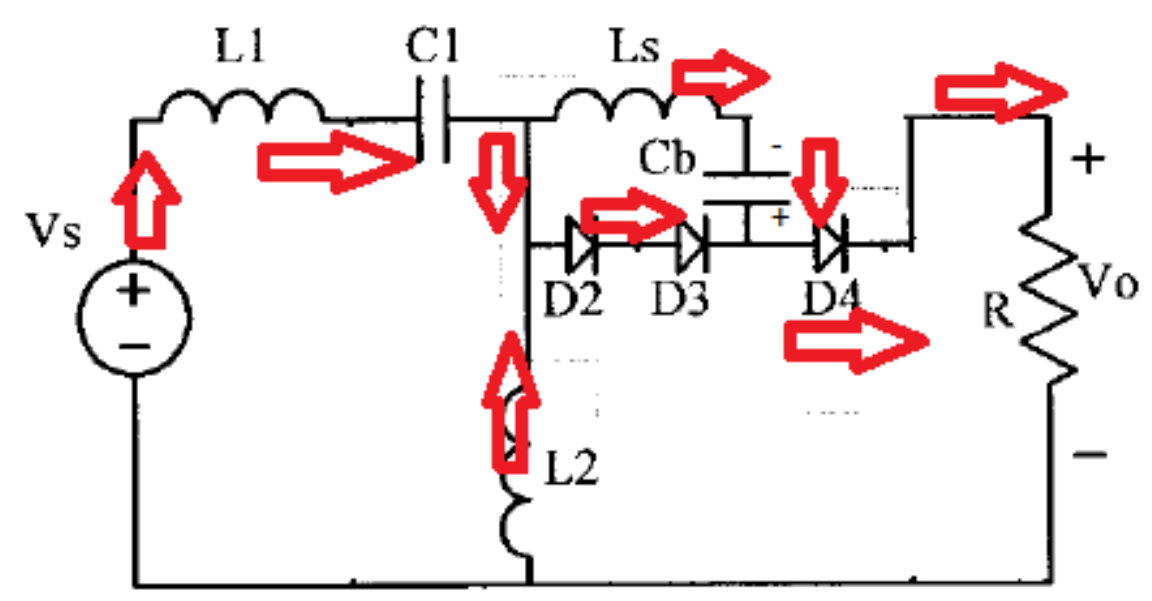

Figure 6.11: Stage 6 Snubber Operation [41]

This phenomenon is similar to the resonance cycle in stage 2 . $\mathrm{I}_{\mathrm{Ls}}$ and $\mathrm{V}_{\mathrm{Cb}}$ are:

$$
\begin{gathered}
I_{L s}(t)=\frac{V_{C b}\left(t_{2}\right)}{Z_{2}} \sin \left(\omega_{2}\left(t-t_{5}\right)\right) \\
V_{C b}(t)=V_{C b}\left(t_{2}\right) \cos \left(\omega_{2}\left(t-t_{5}\right)\right)
\end{gathered}
$$

$D_{2}, D_{3}$ and $D_{4}$ conduct until time $t_{6}$, when the snubber's second to last operation stage begins.

\subsubsection{Snubber Stage 7 Operation}

At time $\mathrm{t}_{6}, \mathrm{I}_{\mathrm{L} 1}$ and $\mathrm{I}_{\mathrm{L} 2}$ both increase to their respective $\mathrm{I}_{\mathrm{L} 1}\left(\mathrm{t}_{6}\right)$ and $\mathrm{I}_{\mathrm{L} 2}\left(\mathrm{t}_{6}\right)$ values. $\mathrm{D}_{2}$ and $\mathrm{D}_{3}$ turn off because of $\mathrm{C}_{\mathrm{b}}$ 's voltage polarity, and $\mathrm{L}_{1}$ and $\mathrm{L}_{2}$ now discharge to the output through the $\mathrm{L}_{\mathrm{s}}-\mathrm{C}_{\mathrm{b}}-\mathrm{D}_{4}$ path. During this stage $\mathrm{C}_{\mathrm{b}}$ also discharges to the output. Figure 6.12 shows the SEPIC implemented with the snubber, in the snubber's seventh operation stage [41]. 


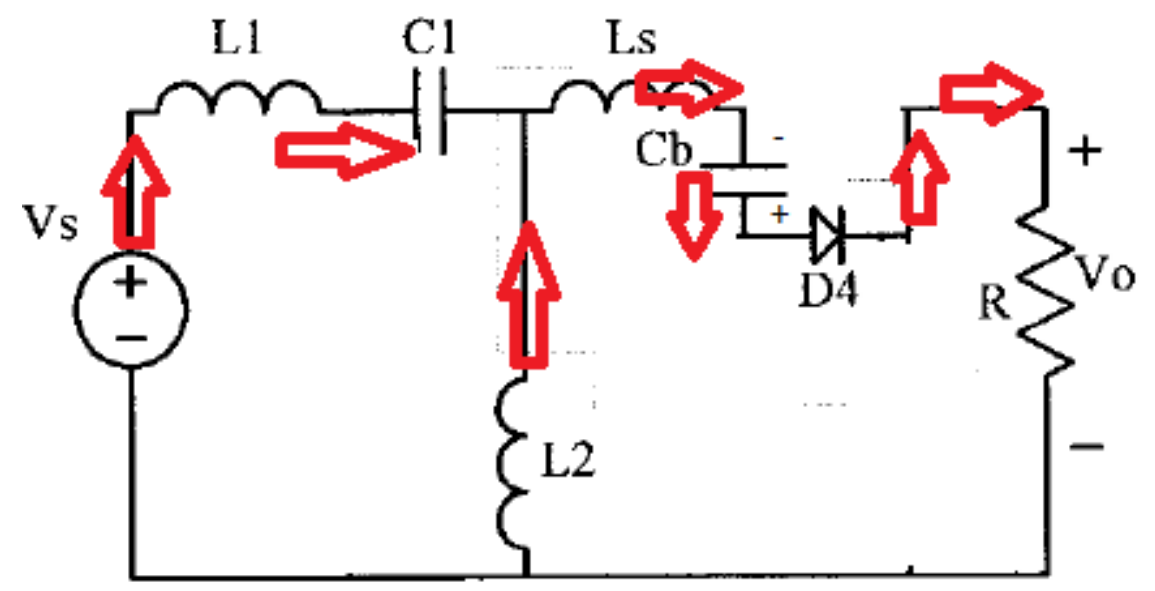

Figure 6.12: Stage 7 Snubber Operation [41]

$\mathrm{V}_{\mathrm{Cb}}$ during this stage is:

$$
V_{C b}(t)=V_{C b}\left(t_{6}\right)-\left[\frac{I_{L 1}\left(t_{6}\right)+I_{L 2}\left(t_{6}\right)}{C_{b}}\right]\left(t-t_{6}\right)
$$

$\mathrm{D}_{1}$ turns on during the next stage, and $\mathrm{C}_{\mathrm{b}}$ 's discharging helps slow $\mathrm{D}_{1}$ 's turn-on process, reducing its power dissipation at turn-on. $\mathrm{D}_{4}$ conducts until the final snubber operation stage.

\subsubsection{Snubber Stage 8 Operation}

Figure 6.13 shows the SEPIC's snubber in its final operation stage [41].

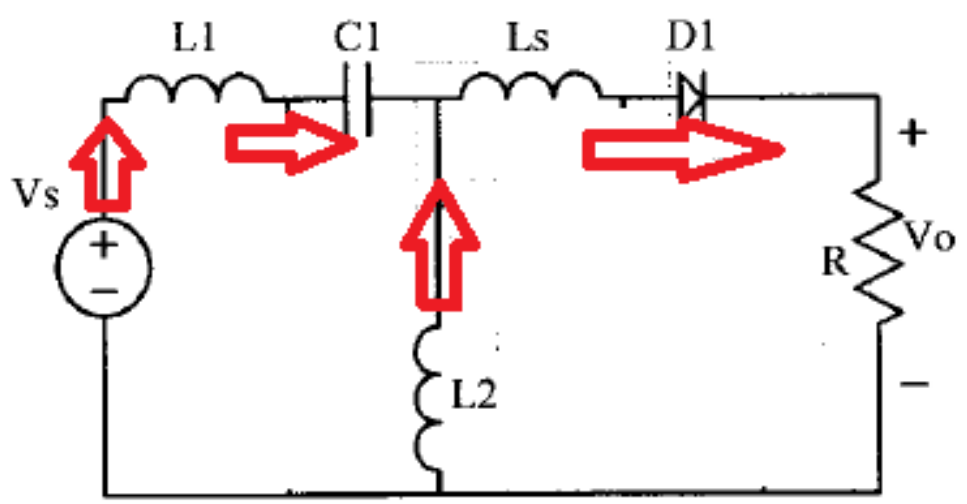

Figure 6.13: Stage 8 Snubber Operation [41] 
During the snubber's final operation stage, $C_{b}$ discharges to $0 \mathrm{~V}$ at time $t_{7}$. Once $C_{b}$ completely discharges to the output, the snubber's energy recovery process finishes. $\mathrm{C}_{\mathrm{b}}$ 's discharge turns off $\mathrm{D}_{4}$ and turns on $\mathrm{D}_{1}$. Turning off $\mathrm{D}_{4}$ helps prevent reversely charging $\mathrm{C}_{\mathrm{s}}$. $\mathrm{L}_{1}$ and $\mathrm{L}_{2}$ continue discharging through the $\mathrm{L}_{\mathrm{s}}-\mathrm{D}_{1}$ path (as in a normal SEPIC without this snubber cell) until $S_{1}$ turns on again at the end of this stage. Once $S_{1}$ turns on again, the snubber's operation cycle restarts at stage 1 and continues through all eight stages as long as the SEPIC is operating with at least $5 \mathrm{~V}$ input voltage. After completing its operation analysis, the next step in designing the snubber is selecting the proper component values for it.

\subsection{Snubber Component Selection}

During stage $6, D_{2}$ and $D_{3}$ should naturally turn off before $C_{b}$ discharges to $0 \mathrm{~V}$, otherwise the residual current from those two diodes turns on $\mathrm{D}_{2}, \mathrm{D}_{3}$ and $\mathrm{D}_{4}$ during the entire $S_{1}$ switching period. Turning on $D_{2}, D_{3}$ and $D_{4}$ during the entire $S_{1}$ switching period is undesirable and results in the snubber operating incorrectly. Thus, the snubber component values must obey the following inequality:

$$
\frac{1}{2} L_{S} I_{F}^{2}<\frac{1}{2} L_{S} I_{r r}^{2}+\frac{1}{2} C_{S} V_{O}^{2}
$$

From (6.29), $I_{F}$ is the main switching diode's average forward current, which is the same as this project's SEPIC's maximum load current (8A). Either $\mathrm{I}_{\mathrm{rr}}$ or $\mathrm{C}_{\mathrm{s}}$ needs to be large in order to satisfy this inequality. However, $\mathrm{C}_{\mathrm{s}}$ needs to be small for minimizing main switching MOSFET current stress as well as main switching diode reverse voltage stress. $10 \mathrm{nF}$ is a reasonable value and selected for $\mathrm{C}_{\mathrm{s}}$. 
Equation (6.9) determines $\mathrm{I}_{\mathrm{rr}}$. Obtaining $\mathrm{I}_{\mathrm{rr}}$ requires determining $\mathrm{t}_{0}$ and $\mathrm{t}_{1}$ from the SEPIC's simulation results. Time $t_{0}$ occurs when $S_{1}$ 's $\left(Q_{1}\right.$ in the SEPIC's schematic in figure 5.7) gate begins conducting, and time $t_{1}$ occurs when $D_{1}$ turns off (when its reverse voltage magnitude becomes large). Figure 6.14 shows the converter's diode reverse voltage trajectory in gray, $\mathrm{I}_{\mathrm{L} 2}$ in teal, $\mathrm{I}_{\mathrm{C} 1}$ in magenta, $\mathrm{I}_{\mathrm{L} 1}$ in red and $\mathrm{Q}_{1}$ 's gate switching trajectory in blue.

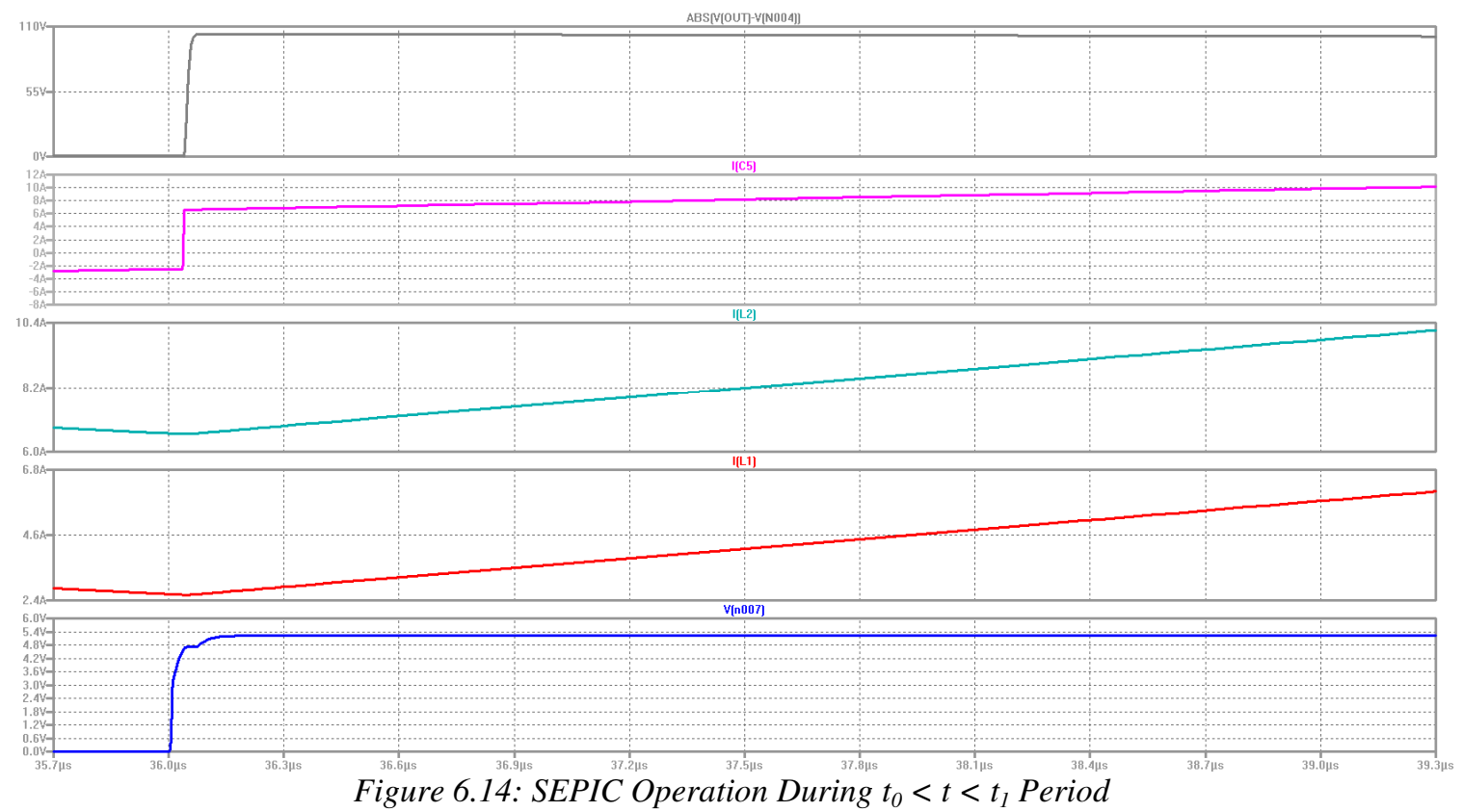

Time $\mathrm{t}_{0}$ occurs when $\mathrm{Q}_{1}$ 's gate begins switching on (VN007 in figure 6.14). Time $\mathrm{t}_{1}$ occurs when $\mathrm{D}_{1}$ turns off and its reverse voltage begins rising (ABS(V(OUT)-V(N004) in figure 6.14). From there, the obtained $\mathrm{I}_{\mathrm{L} 2}, \mathrm{I}_{\mathrm{C} 1}$ and $\mathrm{I}_{\mathrm{L} 1}$ values substitute into (6.30):

$$
I_{r r}=\frac{36 V}{L_{S}}(36.04 \mu s-36.001 \mu s)+6.608 A+(-2.59 A)-2.615 A=\frac{36 V}{L_{S}}(0.039 \mu s)+1.403 A
$$

Plugging in $10 \mathrm{nF}$ for $\mathrm{C}_{\mathrm{s}}$ into (6.29) and solving for $\mathrm{L}_{\mathrm{s}}$ yields the following:

$$
\frac{1}{2} L_{S}(8 A)^{2}<\frac{1}{2} L_{S}\left[\frac{36 V}{L_{S}}(0.039 \mu s)+1.403 A\right]^{2}+\frac{1}{2}(10 n F)(36 V)^{2} \rightarrow 0<L_{S}<360.6 n H
$$


Ideally $\mathrm{L}_{\mathrm{s}}$ should be as close to the maximum value as possible in order for minimizing diode reverse-recovery losses. Thus, this project selects $360 \mathrm{nH}$ for $\mathrm{L}_{\mathrm{s}}$, which is the next lower rated commercially available inductor. This result determines the $\mathrm{C}_{\mathrm{b}}$ capacitance. Keeping $\mathrm{V}_{\mathrm{Cb}}$ at $25 \%$ of $\mathrm{V}_{\mathrm{O}}$ or below $\left(\mathrm{V}_{\mathrm{Cb}} \leq 7.2 \mathrm{~V}\right)$ when the converter's output is $36 \mathrm{~V}$ is preferable. The selected maximum $\mathrm{V}_{\mathrm{Cb}}$ value $(7.2 \mathrm{~V})$ as well as the obtained $\mathrm{L}_{\mathrm{s}}$ value plug into (6.21) for obtaining $\mathrm{C}_{\mathrm{b}}$.

$$
7.2 V=\sqrt{\frac{(360 n H)\left[\frac{36 V}{360 n H}(0.039 \mu s)+1.403 A\right]^{2}+(10 n F)(36 V)^{2}}{C_{b}}} \rightarrow C_{b}=445 n F
$$

The next higher commercially available capacitor value is $470 \mathrm{nF}$. However, when accounting for reverse recovery energy as well as capacitance tolerances, $\mathrm{C}_{\mathrm{b}}$ should be higher than $470 \mathrm{nF}$. Thus, this project selects $\mathrm{C}_{\mathrm{b}}$ as $560 \mathrm{nF}$, which is the next higher commercially available capacitor value after $470 \mathrm{nF}$.

Finally, proper snubber cell operation requires that its resonant frequency determined from (6.19) be much greater than the SEPIC's switching frequency (100 $\mathrm{kHz}$ ). Equations (6.33) and (6.34) determine the snubber's resonant frequency.

$$
\begin{gathered}
\omega_{2}=\sqrt{\frac{1}{(360 n H)(560 n F)}}=2.227 \times 10^{6} \mathrm{rad} / \mathrm{s} \\
f_{2}=\frac{2.227 \times 10^{6}}{2 \pi}=354.5 \mathrm{kHz}
\end{gathered}
$$

The snubber's $354.5 \mathrm{kHz}$ resonant frequency is much greater than the SEPIC converter's $100 \mathrm{kHz}$ switching frequency as well as the $111 \mathrm{kHz}$ switching frequency measured in simulation in section 5.4, thus it should work properly with this project's converter. After selecting proper snubber components, this project implements this passive lossless snubber into the simulation SEPIC circuit. 


\subsection{SEPIC Simulation Results with Snubber}

Figure 6.15 shows the SEPIC with the lossless passive snubber:

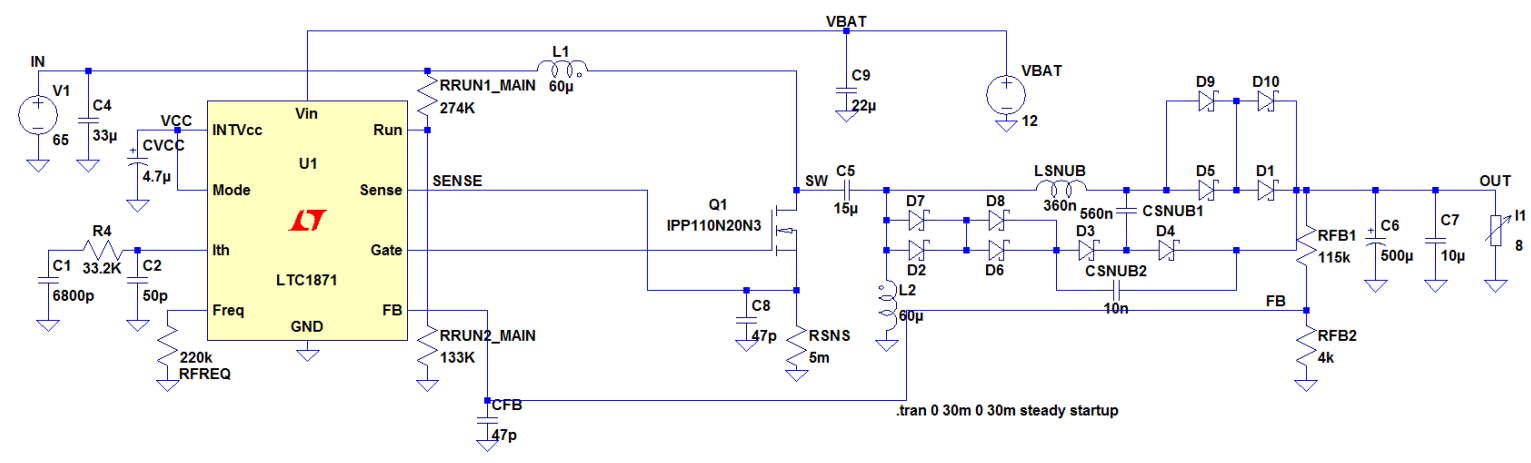

Figure 6.15: SEPIC Simulation Circuit with Passive Lossless Snubber

This implementation uses MBR20100CTs as snubber diodes because of the MBR20100CT's fast reverse recovery time. Also for the SEPIC's main switching diode $\left(D_{1}\right)$, this implementation uses four MBR20100CTs (two in series and two in parallel denoted as $D_{1}, D_{5}, D_{9}$ and $D_{10}$ in figure 6.15) for preventing $D_{1}$ from exceeding its maximum average forward current and reverse voltage ratings. As mentioned in section 5.4, using only one MBR20100CT for the SEPIC's main switching diode resulted in the diode exceeding its electrical limits. This implementation also adds feedback and sense node capacitors $\left(\mathrm{C}_{\mathrm{FB}}\right.$ and $\left.\mathrm{C}_{\mathrm{SNS}}\right)$ for ensuring clean signals for the LTC1871 controller's crucial feedback and sense pins. Simulation results for this SEPIC implementation use a $65 \mathrm{~V}$ input voltage and $8 \mathrm{~A}$ output load (full load), and this project analyzes them in steady-state operation. Figure 6.16 shows the SEPIC's output voltage in green and power dissipation transients across $\mathrm{Q}_{1}$ in blue. 


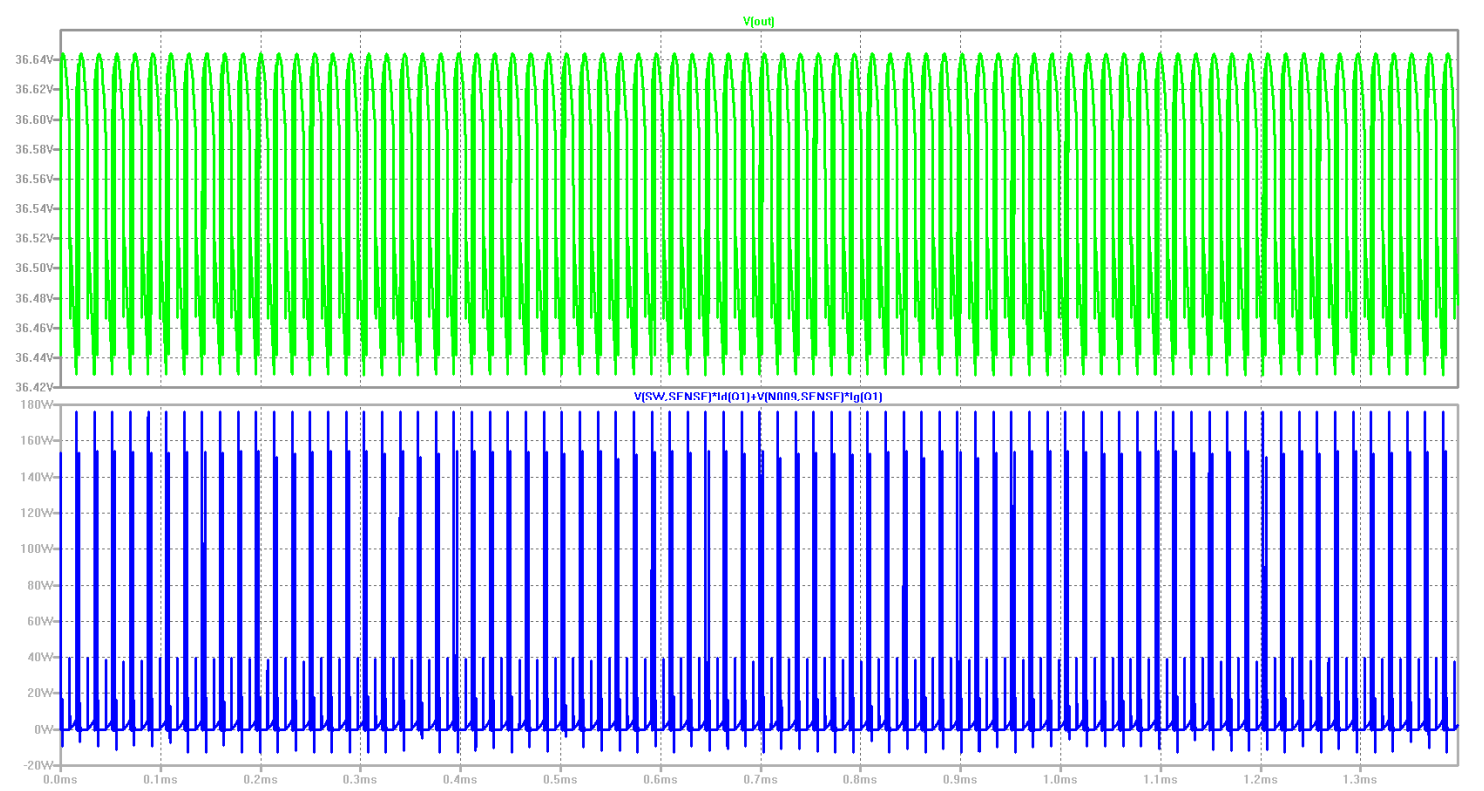

Figure 6.16: SEPIC Output Voltage and $Q_{1}$ Power Dissipation with Snubber at Full Load and Steady-state Operation

Average SEPIC output voltage is $36.572 \mathrm{~V}$, which suffices for the converter's $36 \mathrm{~V}$ output requirement. The snubber reduces $\mathrm{Q}_{1}$ 's peak power dissipation transients to $180 \mathrm{~W}$. These transients were nearly $1.6 \mathrm{~kW}$ (from figure 5.13 ) before implementing the snubber. A $1 \mu \mathrm{s}$ $180 \mathrm{~W}$ transient for is much safer for the IPP110N20N3 power MOSFET $\left(\mathrm{Q}_{1}\right)$ than a $1.6 \mathrm{~kW}$ transient lasting the same time span. Overall average $\mathrm{Q}_{1}$ power dissipation also reduces to $1.8 \mathrm{~W}$ (from $2.862 \mathrm{~W}$ without the snubber), which is a dramatic improvement from the previous implementation from section 5.4. Figure 6.17 shows power dissipation across the SEPIC's main switching diode $\left(\mathrm{D}_{1}\right)$. 


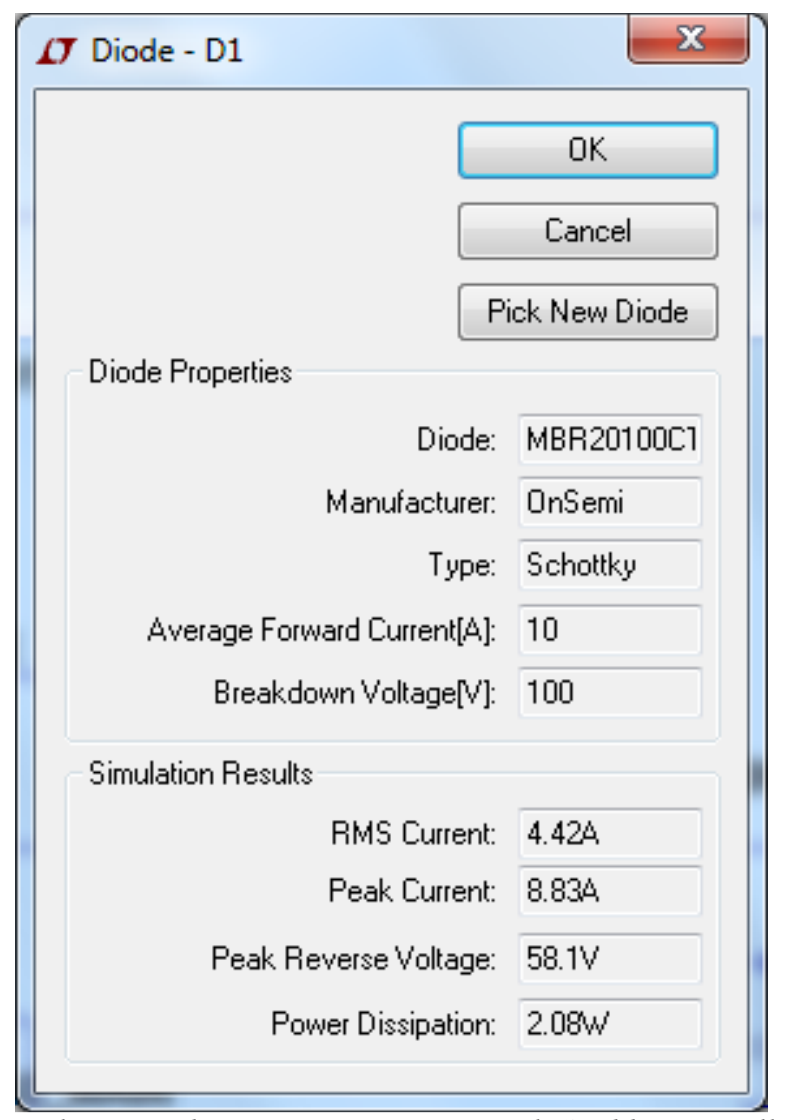

Figure 6.17: SEPIC Switching Diode Power Dissipation with Snubber at Full Load and Steady-state Operation

Using multiple MBR20100CTs as well as a passive lossless snubber greatly reduces power dissipation across $\mathrm{D}_{1}$. Its power dissipation reduces to $2.08 \mathrm{~W}$ (from $6.08 \mathrm{~W}$ in section 5.4, before this project implemented the snubber). Furthermore, it does not reach close to its average forward current limit or exceed its peak reverse voltage limit, unlike the SEPIC implementation from section 5.4. However, one snubber diode requires further attention. That diode is $\mathrm{D}_{4}$ from figure 6.15 and is one of the snubber's diodes. The LTSpice simulation produced the warning message shown in figure 6.18: 


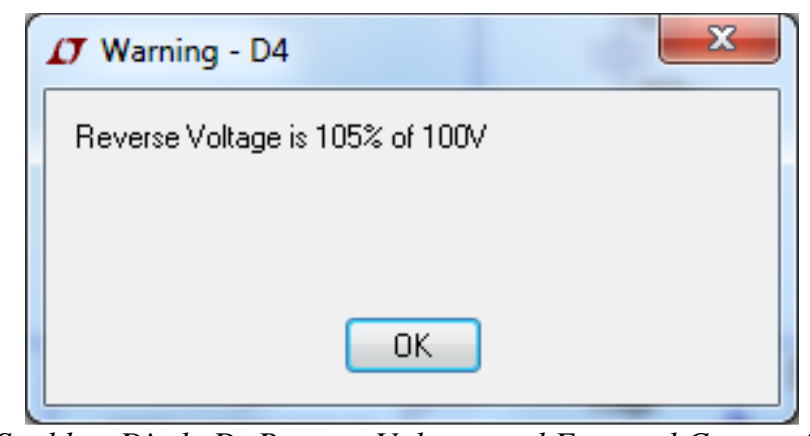

Figure 6.18: Snubber Diode $D_{4}$ Reverse Voltage and Forward Current Stress Warning

$\mathrm{D}_{4}$ 's reverse voltage reaches 105V, which exceeds the MBR20100CT's 100V peak reverse voltage rating. Therefore a single MBR20100CT is not sufficient for tolerating the snubber's output diode stress requirements. Thus, this SEPIC's final implementation uses two MBR20100CTs for its snubber output diode. Now, this project requires an efficiency analysis of its SEPIC when implemented with the designed snubber.

\subsubsection{Snubbered SEPIC Efficiency Analysis}

Likewise with section 5.4.2, this project performs efficiency analysis of the SEPIC with snubber circuit at full-load conditions and steady-state operation. Figure 6.19 shows the snubbered SEPIC's output voltage in green and its input voltage in blue. 


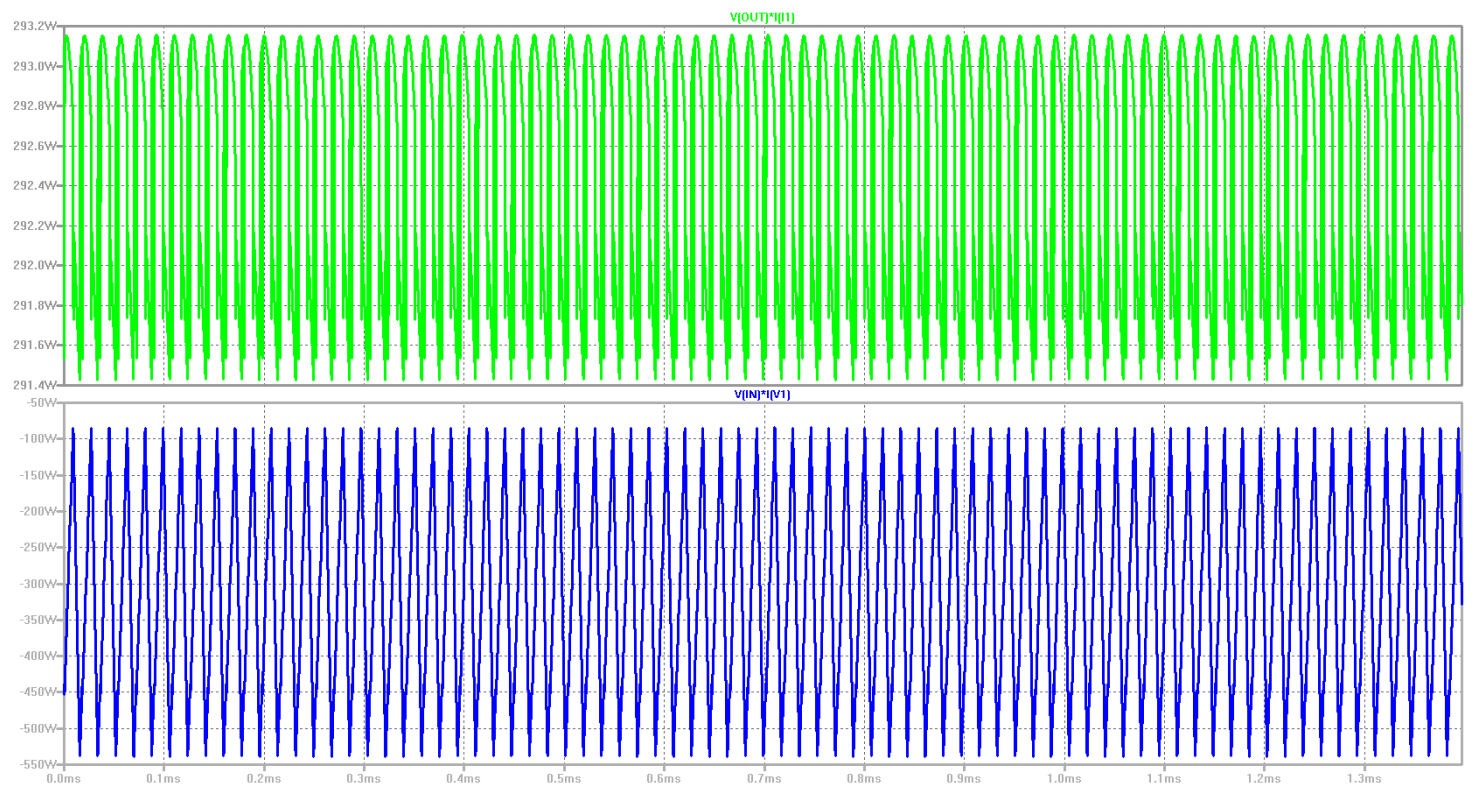

Figure 6.19: SEPIC with Snubber Input and Output Power at Full Load and Steady-state Operation

Average converter output power is $292.58 \mathrm{~W}$ and average input power is $316.69 \mathrm{~W}$. This translates to a $92.4 \%$ overall converter efficiency. This converter efficiency is a slight improvement from the $89.2 \%$ efficiency (described in section 5.4.2) without a snubber. This efficiency gain is still crucial for reducing the EHFEM system's lifecycle costs and this snubber can be fabricated from very low-cost components because of its simplicity. Therefore this project implements the passive lossless snubber circuit designed in this chapter into its SEPIC.

Overall, while adding a snubber increases the SEPIC's cost and overall PCB real estate, the benefits that it offers outweigh its disadvantages.

Implementing a snubber in this project's SEPIC reduces the magnitude of lethal power dissipation transients imposed across its switching MOSFET and diodes, which are the SEPIC's crucial switching components. However, this snubber circuit only protects components connected to the SEPIC's switching nodes. The SEPIC's input node 
requires protection as well from large input ripple voltages that exceed the maximum $65 \mathrm{~V}$ input specification described in section 1.2. As mentioned earlier in section 3.2, the Precor EFX 546i does not output a flat DC voltage; however, this project earlier did not account for this problem in great depth. Therefore during exercise this project's SEPIC receives a rippled DC voltage at its input. The next chapter and section describes those large ripple input voltages in greater detail. 


\section{CHAPTER 7: PRECOR EFX 546i OUTPUT VOLTAGE RIPPLE AND SEPIC INPUT PROTECTION}

\subsection{Precor EFX 546i Output Voltage Ripple}

In Spring Quarter 2011, EHFEM team members Greg Hollister, Alvin Hilario and Nicholas Lovgren described the Precor EFX 546i elliptical trainer outputting a large ripple voltage in addition to its DC voltage [10,53-54]. That ripple voltage was a sawtooth shape and reached a maximum $32 \mathrm{~V}$ peak-to-peak amplitude when the elliptical user exercised at 180 strides per minute and level 17 training resistance level setting [10]. The ripple voltage also reached a $40.7 \mathrm{kHz}$ peak frequency at 100 strides per minute and level 10 training resistance (its minimum frequency was $31.3 \mathrm{kHz}$ at 150 strides per minute and level 15 training resistance) [10]. The overall ripple voltage frequency decreases with the elliptical training resistance level, and its amplitude increases with training resistance level. Therefore, if the elliptical trainer outputs a $65 \mathrm{~V}$ average voltage into this project's SEPIC, its peak will be above the maximum $65 \mathrm{~V}$ input specification. Inputting voltages above $65 \mathrm{~V}$ into the SEPIC may damage its components. Additionally, input currents above $25 \mathrm{~A}$ caused by the input ripple voltage can also damage SEPIC components. Therefore this project's SEPIC requires additional protection at its input node for either clamping input voltages greater than $65 \mathrm{~V}$ and currents greater than $25 \mathrm{~A}$, or completely shutting off operation. An overvoltage and overcurrent protection circuit implemented into the SEPIC's input suffices for those requirements. Section 7.2 describes this project's SEPIC's input overvoltage and overcurrent protection circuit in greater detail. 


\subsection{Input Overvoltage and Overcurrent Protection Circuit}

Dedicated controller ICs for overvoltage and overcurrent protection exist on the consumer market and are readily available from major electronic component suppliers. Therefore implementing an overvoltage and overcurrent protection circuit in this project's SEPIC is much easier with a dedicated controller IC than with discrete components. This project selects Linear Technology's LT4356-1 surge stopper IC as the core of its SEPIC input overvoltage and overcurrent protection circuit. It tolerates up to $100 \mathrm{~V}$ input voltage and clamps short overvoltage and overcurrent transients to user-defined values set by external biasing components [55]. If those overvoltage and overcurrent transients persist for an extended time period, then the LT4356-1 shuts down voltage and current flow to the rest of the SEPIC circuit via a pass transistor (a power MOSFET). Likewise with the selected LTC1871 PWM controller, the LT4356-1 has an MSOP-10 package available, thereby allowing for a small PCB footprint. Furthermore, like the LTC1871, the LT43561 also has an LTSpice model available for simulation. Figure 7.1 shows a typical LT4356-1 application [55]. 


\section{A, 12V Overvoltage Output Regulator}

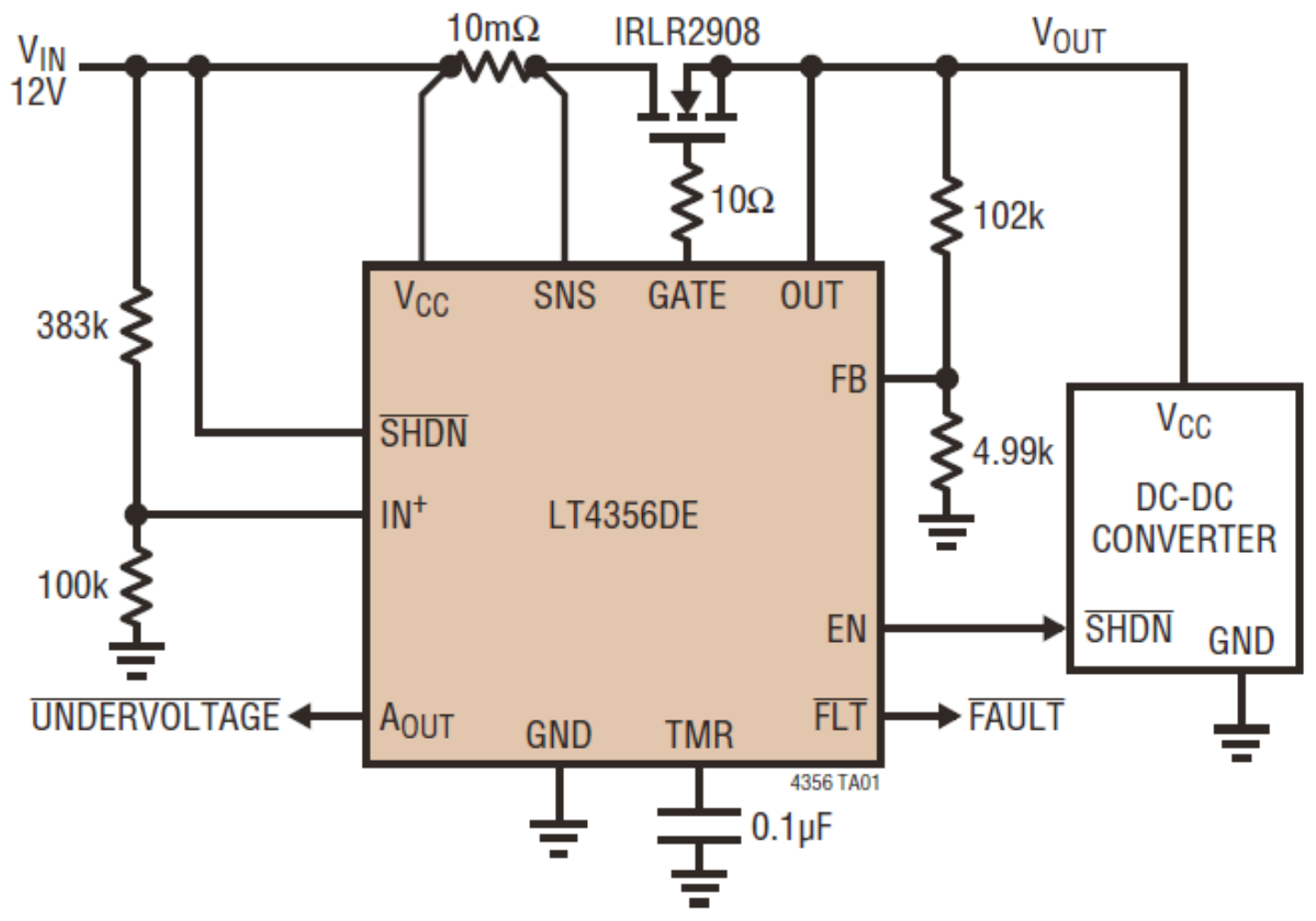

Figure 7.1: Typical LT4356-1 Application [55]

This application from Linear's LT4356-1 datasheet protects a DC-DC converter from input overvoltage and overcurrent transients. A resistor divider network connected to the LT4356-1's FB pin determines the maximum input clamping voltage, and a current sense resistor $(10 \mathrm{~m} \Omega)$ determines the maximum converter input current. The IRLR2908 pass transistor shuts down voltage and current flow to the DC-DC converter if it detects an overvoltage or overcurrent condition for a prolonged time period. The $0.1 \mu \mathrm{F}$ capacitor connected to the LT4356-1's TMR pin determines that time period. This overall LT43561 configuration is similar to the one that this project will implement for its SEPIC. Figure 7.2 shows the LT4356-1's internal schematic [55]. 


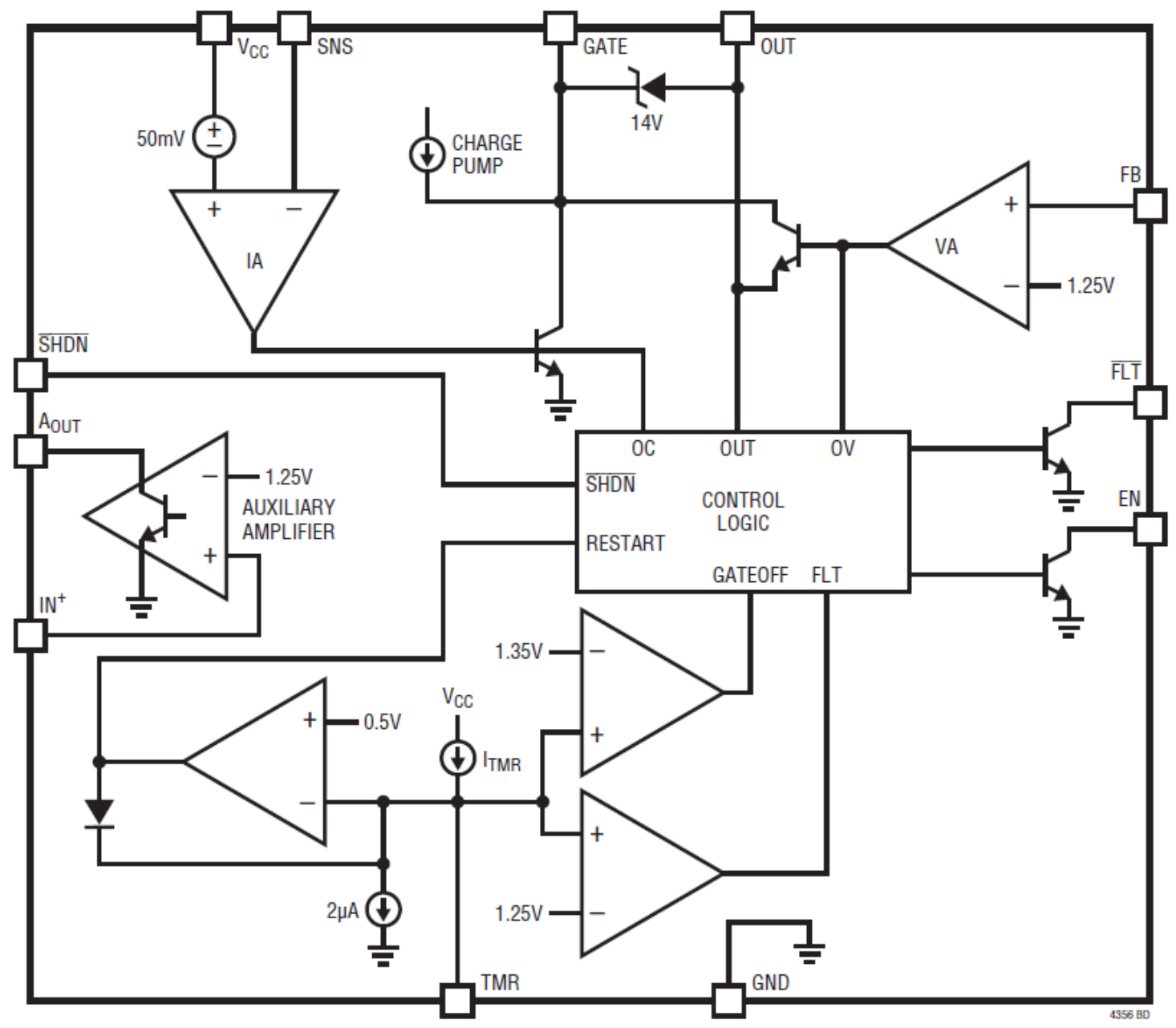

Figure 7.2: LT4356-1 Internal Schematic [55]

The $\mathrm{A}_{\mathrm{OUT}}$ and $\mathrm{IN}^{+}$pins control an internal auxiliary amplifier. The LT4356-1 surge stopper IC is available in DFN-12, MSOP-10 and SOIC-16 packages. The LT4356-1 used in this project's SEPIC is in the MSOP-10 package. The LT4356-1's MSOP-10 package does not contain the internal auxiliary amplifier, hence the $\mathrm{A}_{\mathrm{OUT}}$ and $\mathrm{IN}^{+}$pins are also absent from the IC. These two pins are present in LTSpice's LT4356-1 simulation model. However, this project does not use these pins and hence grounds these pins in simulation models. Likewise with the LTC1871 PWM controller, the MSOP-10 package LT4356-1 surge stopper IC consists of 10 pins that require external biasing components. 
In numerical order, those pins are FB, OUT, GATE, SNS, $V_{\mathrm{CC}}$, !SHDN, !FLT, EN, GND and TMR. The first pins requiring component selection are the IC's feedback (FB), output (OUT), $\mathrm{V}_{\mathrm{CC}}$ and current sense (SNS) pins. The LT4356-1's FB pin controls the protection circuit's output voltage limit (its OUT pin), which is the SEPIC's input voltage limit $(65 \mathrm{~V})$. Its $\mathrm{V}_{\mathrm{CC}}$ pin connects to an external voltage source (in this project's case, the Precor elliptical trainer's output) for supplying the input voltage that it requires. The LT4356-1's SNS pin connects to a current sense resistor for controlling the protection circuit's input current limit for the SEPIC.

\subsubsection{FB, OUT, $\mathrm{V}_{\mathrm{CC}}$ and SNS Pin Operation}

As mentioned in section 7.2, the LT4356-1's FB pin determines the maximum SEPIC input clamping voltage using a resistor divider network. The SEPIC's maximum specified input voltage is $65 \mathrm{~V}$ from the Precor elliptical trainer, therefore this project designs its LT4356-1 protection circuit such that any SEPIC input voltage transients greater than $65 \mathrm{~V}$ clamp to this value. This is the same voltage at the LT4356-1's OUT pin, which for this project connects directly to the SEPIC's clamped input voltage node. Equation (7.1) determines the required voltage divider resistors for setting the clamping voltage.

$$
V_{R E G}=\frac{1.25 V\left(R_{R U N 1 \_O V P}+R_{R U N 2 \_O V P}\right)}{R_{R U N 2 \_O V P}}=65 \mathrm{~V}
$$

$\mathrm{V}_{\mathrm{REG}}$ denotes the converter's maximum desired input voltage (determined as $65 \mathrm{~V}$ from section 1.2) and $R_{\text {RUN1_OVP }}$ and $R_{\text {RUN2_OVP }}$ denote the resistors used for the resistor divider network. $R_{\text {RUN1_OvP }}$ is the resistor connected to the SEPIC's input and $R_{\text {RUN2_OvP }}$ is the resistor connected to ground. They are the $102 \mathrm{k} \Omega$ and $4.99 \mathrm{k} \Omega$ resistors in figure 7.1. 
Linear Technology recommends limiting the current through $\mathrm{R}_{\mathrm{RUN} 1 \_O v P}$ and $\mathrm{R}_{\mathrm{RUN} 2 \_O v P}$ to $250 \mu \mathrm{A}$ during an overvoltage or overcurrent fault condition [55]. Equation (7.2) uses this $\mathrm{R}_{\mathrm{RUN} 2 \_\mathrm{OVP}}$ current value for determining $\mathrm{R}_{\mathrm{RUN} 2 \_\mathrm{OVP}}$.

$$
R_{R U N 2 \_O V P}=\frac{1.25 \mathrm{~V}}{250 \mu \mathrm{A}}=5 \mathrm{k} \Omega
$$

$4.99 \mathrm{k} \Omega$ is the nearest commercially available standard resistor value, thus $R_{\text {RUN2_OvP }}=$ $4.99 \mathrm{k} \Omega$. Therefore, $\mathrm{R}_{\mathrm{RUN} 1 \_ \text {OVP }}$ is the following value:

$$
R_{R U N 1 \_O V P}=\frac{\left(V_{R E G}-1.25 \mathrm{~V}\right) R_{R U N 2 \_} \mathrm{OVP}}{1.25 \mathrm{~V}}=\frac{(65 \mathrm{~V}-1.25 \mathrm{~V}) 5 \mathrm{k} \Omega}{1.25 \mathrm{~V}}=254.49 \mathrm{k} \Omega
$$

$255 \mathrm{k} \Omega$ is the nearest commercially available standard resistor value, thus $\mathrm{R}_{\mathrm{RUN} 1 \_\mathrm{OVP}}=$ $255 \mathrm{k} \Omega$. After selecting the proper resistors for input voltage clamping, the LT4356-1 requires selecting components for SEPIC overcurrent protection. As mentioned in section 7.2, the LT4356-1 determines the SEPIC's maximum input current via a current sense resistor connected to between its $\mathrm{V}_{\mathrm{CC}}$ and $\mathrm{SNS}$ pins. The $\mathrm{V}_{\mathrm{CC}}$ pin directly connects to the Precor elliptical trainer's output for turning on the LT4356-1 IC and can tolerate up to $100 \mathrm{~V}$ (as well as up to 60V reverse voltage input) [55]. That tolerance suffices for this project's SEPIC's 5-65V input range. The SNS pin senses the voltage across the current sense resistor and if that voltage exceeds $50 \mathrm{mV}$ for a prolonged time period, the LT43561 shuts off voltage and current flow to the SEPIC [55]. Figure 7.5 denotes this current sense resistor as $\mathrm{R}_{\mathrm{SNS} \_ \text {Ovp. Equation (7.4) determines the current sense resistor value for }}$ limiting the SEPIC's input current to this project's desired value. This project desires limiting the SEPIC's input current to 25A.

$$
R_{S N S_{-} O V P}=\frac{50 \mathrm{mV}}{I_{I N(\max .)}}=\frac{50 \mathrm{mV}}{25 \mathrm{~A}}=2 \mathrm{~m} \Omega
$$


Earlier in the design process, this project limited SEPIC main switching transistor $\mathrm{Q}_{1}$ 's drain current to 30A through a current sense resistor. However, limiting the SEPIC's input current to $25 \mathrm{~A}$ prevents $\mathrm{L}_{1}$ from saturating (because $\mathrm{L}_{1}$ connects directly to the SEPIC's input node) without significantly compromising the SEPIC's 36V output regulation ability. According to its datasheet, the Vishay IHV28BZ60 inductor saturates when 28A flows through its terminals [36]. In addition to the LT4356-1's output resistor divider and current sense resistor, another crucial component that controls SEPIC shutdown and turn-on during and after a fault condition is the input pass transistor. The next section describes in greater detail biasing the pass transistor.

\subsubsection{GATE Pin}

The LT4356-1's GATE pin controls the input pass transistor (the IRLR2908 Nchannel MOSFET in figure 7.1) that shuts down voltage and current flow to the SEPIC during an overvoltage or overcurrent condition. The GATE pin doesn't require any additional compensation components, but transient input voltage steps greater than $5 \mathrm{~V} / \mu \mathrm{s}$ require connecting a capacitor from the GATE pin to ground. This capacitor helps prevent the pass transistor from self-enhancement [55]. Figure 7.3 shows Linear's LT4356-1 sample test fixture circuit and it includes this additional gate capacitor $\left(\mathrm{C}_{3}\right)$. 


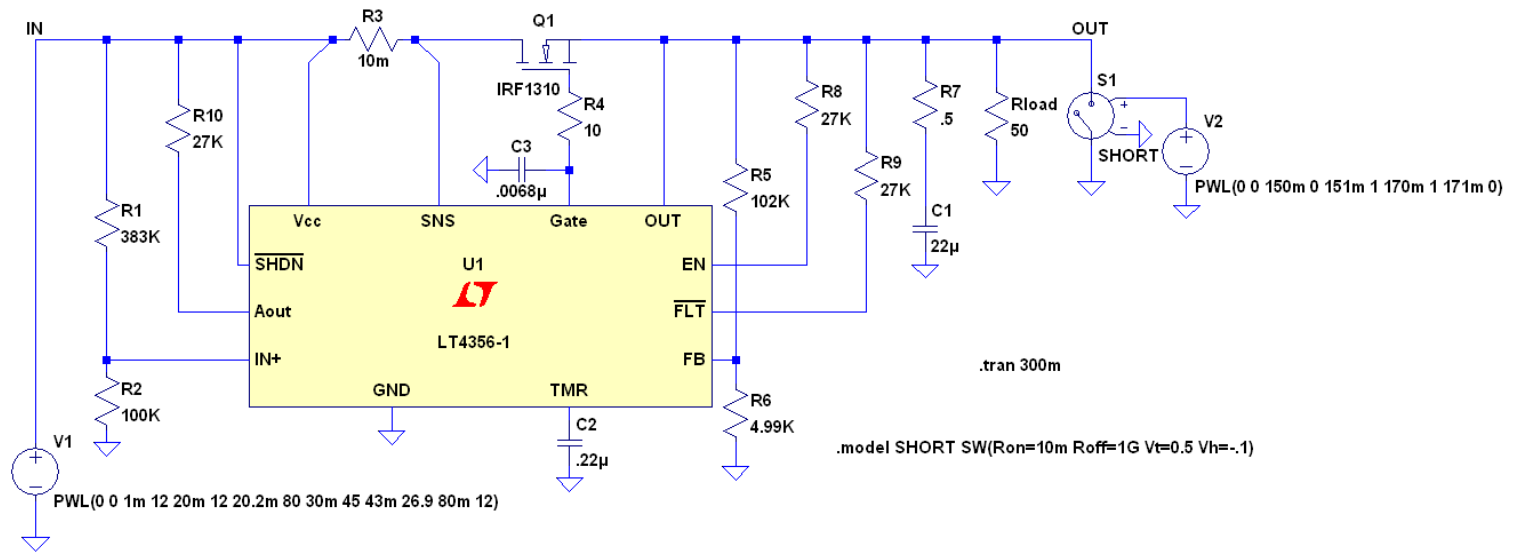

Figure 7.3: Linear Technology's LT4356-1 Sample Test Fixture Circuit

Figure 7.5 denotes this capacitor as CGATE_OVP. Connecting a series resistor with the pass transistor's gate also helps further dampen any oscillations that may occur, ensuring stable overvoltage and overcurrent regulation. Linear's sample test fixture circuit selects $10 \Omega$ for this resistor and $0.0068 \mu \mathrm{F}(6800 \mathrm{pF})$ for its gate capacitor and these values suffice for this project's protection circuit because they are small values that would not cause any signal underdamping. The next section describes the LT4356-1's input undervoltage shutdown control.

\subsection{3 !SHDN Pin and Low Battery Threshold Detection}

The LT4356-1 surge stopper should operate over the full specified 5V-65V range for this project, and operating at input voltages lower than $5 \mathrm{~V}$ does not harm any SEPIC components. Thus this project's SEPIC does not require low battery threshold detection and this project connects the LT4356-1's !SHDN pin directly to the protection circuit's input node. The LT4356-1's !SHDN pin has the same voltage tolerances as the $\mathrm{V}_{\mathrm{CC}}$ pin, thus tying it to the protection circuit's input suffices. The LT4356-1's other shutdown condition is during a prolonged overvoltage or overcurrent fault condition. Physically 
indicating (such as through an LED) when such a fault condition occurs helps simplify troubleshooting the protection circuit if needed. The next section describes implementation of fault condition indicators for this project's SEPIC's protection circuit.

\subsection{4 !FLT Pin}

The LT4356-1's active-low !FLT pin pulls to a logic low during an overvoltage or overcurrent fault condition. This pin connects to any type of fault indicator, such as an LED, and can drive that indicator by sinking up to $3 \mathrm{~mA}$ from an external voltage source. This project indicates an input overvoltage or overcurrent fault by connecting an LED to the !FLT pin, using the Precor elliptical trainer's onboard $12 \mathrm{~V}$ battery as its voltage source. This project uses Lumex's SML-LX1206SRC-TR 1206 package red LED as its fault indicator. Simulations, however, use Nichia's NSCW100 LED as its model because of its availability in LTSpice. Figure 7.5 denotes this LED as FLT_LED. Additionally, this project also requires a series resistor connected to the LED for limiting its current to $3 \mathrm{~mA}$, as its typical forward voltage drop is $1.7 \mathrm{~V}$ [56]. Figure 7.5 denotes this series resistor as $\mathrm{R}_{\mathrm{FLT}}$. Equations (7.5) and (7.6) determine the fault LED's maximum current:

$$
\begin{gathered}
I_{F L T_{-} L E D}=\frac{V_{B A T}-V_{F L T \_L E D}}{R_{F L T}} \\
R_{F L T}=\frac{V_{B A T}-V_{F L T_{-} L E D}}{I_{F L T_{-} L E D}}=\frac{12 V-1.7 V}{3 m A}=3.43 \mathrm{k} \Omega
\end{gathered}
$$

$\mathrm{V}_{\mathrm{BAT}}$ denotes the Precor elliptical trainer's battery voltage, $\mathrm{V}_{\mathrm{FLT} \_ \text {LED }}$ denotes to the LED's forward voltage, $\mathrm{I}_{\mathrm{FLT} \_L E D}$ is its current and $\mathrm{R}_{\mathrm{FLT}}$ is the required resistance value for the LED's current-limiting resistor. $3.6 \mathrm{k} \Omega$ is the next higher available standard resistor value, thus this project selects $3.6 \mathrm{k} \Omega$ for $\mathrm{R}_{\mathrm{FLT}}$. Equation (7.7) recalculates the current flowing through the fault LED using this selected resistor value: 


$$
I_{F L T_{-} L E D}=\frac{V_{B A T}-V_{F L T \_L E D}}{R_{F L T}}=\frac{12 \mathrm{~V}-1.7 \mathrm{~V}}{3.6 \mathrm{k} \Omega}=2.861 \mathrm{~mA}
$$

This current value suffices for the fault LED, as it is below 3mA. After selecting components for the LT4356-1's !FLT pin for driving a fault indicator, the next pin requiring programming is the enable $(\mathrm{EN})$ pin.

\subsubsection{EN Pin}

The LT4356-1's EN pin is its open collector enable output [55]. Linear Technology's LT4356-1 datasheet recommends connecting this pin directly to the DCDC converter's main PWM controller EN, RUN or !SHDN pin. Thus, this project ties this pin to the LTC1871 controller's RUN pin. The next section describes proper grounding for the LT4356-1 surge stopper.

\subsubsection{GND Pin}

Likewise with the LTC1871 PWM controller, the LT4356-1's GND pin connects directly to the SEPIC's ground node. Proper, low-noise operation however, requires it connected to a signal ground plane separate from that of a power ground plane. Chapter 9 (PCB Layout) discusses this requirement in greater detail. After properly connecting the LT4356-1's GND pin, the final pin requiring biasing components is its fault timer (TMR) pin.

\subsubsection{Fault Timer Control and TMR Pin}

The LT4356-1's TMR pin sets the protection circuit's fault condition early warning, fault and cool-down time periods [55]. An external biasing capacitor determines these time periods. Figure 7.5 denotes this capacitor as $\mathrm{C}_{\mathrm{TMR}}$. The current charging up this timing capacitor connected to this pin during fault conditions depends on the voltage 
difference between the LT4356-1's $\mathrm{V}_{\mathrm{CC}}$ and OUT pins. When the voltage across the TMR pin charges to $1.25 \mathrm{~V}$, the !FLT pin pulls low, indicating that the LT4356-1 detects an input overvoltage or overcurrent fault condition. If the condition persists longer, the protection circuit's pass transistor shuts off once the timer capacitor further charges to 1.35V. During this charging period, the LT4356-1 clamps the SEPIC input voltage to $65 \mathrm{~V}$ or the input current to $25 \mathrm{~A}$, depending on what occurs first. The LT4356-1 shuts down voltage and current flow to the SEPIC's input once the timer capacitor charges to $1.35 \mathrm{~V}$. Once the fault condition disappears, no current flows through the TMR pin (thus preventing the timer capacitor from charging) and a $2 \mu \mathrm{A}$ current pulls the TMR pin down, discharging the timer capacitor. Once the timer capacitor discharges to the TMR pin's $0.5 \mathrm{~V}$ retry threshold, the GATE pin pulls high, turning back on the protection circuit's pass transistor and allowing voltage and current to flow into the SEPIC as long as no further prolonged fault condition persists [55]. Linear Technology recommends $1 \mu \mathrm{s}$ early warning time for fault detection, thus this project uses $1 \mu$ s as its early warning time [55]. This value is 10 times lower than this project's SEPIC's $10 \mu$ s switching period, thus this value suffices for proper protection circuit operation. Equation (7.8) determines the $\mathrm{C}_{\mathrm{TMR}}$ value required for $1 \mu$ s early warning time for fault detection:

$$
C_{T M R}=\frac{1 \mu s \cdot 5 \mu \mathrm{A}}{100 \mathrm{mV}}=50 p \mathrm{~F}
$$

$47 \mathrm{pF}$ is the nearest commercially available standard capacitor value, thus this project selects $\mathrm{C}_{\mathrm{TMR}}$ as $47 \mathrm{pF}$. Equation (7.9) determines the total fault time allowed for SEPIC input voltage and current clamping. Equation (7.9) denotes this fault time as toC. 


$$
t_{O C}=\frac{47 p F \cdot 0.85 \mathrm{~V}}{45.5 \mu \mathrm{A}}=0.878 \mu \mathrm{S}
$$

This value suffices for $31.3 \mathrm{kHz}$ input ripple from the Precor elliptical. Therefore, for this project's SEPIC, the LT4356-1 clamps input overvoltage and overcurrent transients with less than $0.878 \mu$ s duration and shuts off voltage and current flow if those transients persist for a longer period. During the clamping time period, the protection circuit's pass transistor must withstand a large power dissipation because a significant voltage difference exists between the pass transistor's drain and source. This difference is between the input voltage (output voltage from the Precor elliptical trainer) and the clamped voltage, and results in $\mathrm{V} \times \mathrm{I}$ dissipation. Figure 7.5 denotes the protection circuit's pass transistor as Q_OVP.

\subsubsection{Pass Transistor Selection}

As described in section 7.2.1, 65V input to the LT4356-1 is the minimum set threshold for the IC's input overvoltage condition. Input overcurrent conditions may occur at lower input voltages. However, at those lower input voltages Q_OVP dissipates less power because its drain-source voltage $\left(\mathrm{V}_{\mathrm{DS}}\right)$ is lower. Thus, this project sets $65 \mathrm{~V}$ as the baseline for determining the required Q_OVP power dissipation during a fault condition. $\mathrm{V}_{\mathrm{CC}(\max .)}$ equals the maximum SEPIC input voltage $\mathrm{V}_{\mathrm{IN}(\max .)}$ because the LT4356-1's $\mathrm{V}_{\mathrm{CC}}$ pin connects to the SEPIC input. Equation (7.10) determines the maximum power dissipation level that Q_OVP must withstand during voltage or current clamping. Equation (7.10) denotes this power dissipation level as $\mathrm{P}_{\mathrm{Q} \_ \text {OVP. }}$

$$
P_{Q_{-} O V P}=\frac{V_{C C(\max .)} \cdot 50 \mathrm{mV}}{R_{S N S_{-} O V P}}=\frac{65 \mathrm{~V} \cdot 50 \mathrm{mV}}{2 m \Omega}=1.625 \mathrm{~kW}
$$


Q_OVP must withstand this power dissipation level for the duration equal to $t_{\mathrm{OC}}$ (determined as $0.878 \mu$ s in (7.9) above). This project selects IXYS's IXFK230N20T for Q_OVP, which has a $1.67 \mathrm{~kW}$ absolute maximum power dissipation rating [57].

Preventing Q_OVP's destruction requires the IXFK230N20T's safe operating area falling within the boundaries set by both $\mathrm{t}_{\mathrm{OC}}$ and $\mathrm{P}_{\mathrm{Q}_{-} \mathrm{OV} P}$. However, the operating voltage and current require extraction from $\mathrm{P}_{\mathrm{Q} \_ \text {ovP }}$ in because the IXFK230N20T's safe operating area plot consists of only operating voltage plotted against operating current [57]. The SEPIC's maximum operating input voltage $\left(\mathrm{V}_{\mathrm{CC} \max }\right)$ is $65 \mathrm{~V}$; now only the current ( $\left.\mathrm{I}_{\mathrm{Q} \_O V P}\right)$ requires extraction from $\mathrm{P}_{\mathrm{Q} \_ \text {OVP: }}$

$$
\begin{gathered}
P_{Q_{-} \text {OVP }}=V_{C C(\max .)} \cdot I_{Q_{-} O V P} \\
I_{Q_{-} O V P}=\frac{P_{Q_{-} O V P}}{V_{C C(\max .)}}=\frac{1.625 \mathrm{~kW}}{65 \mathrm{~V}}=25 \mathrm{~A}
\end{gathered}
$$

Figure 7.4 shows the IXFK230N20T's safe operating area, with the obtained maximum operating voltage and current values from (7.1) and (7.12) marked in blue [57]. 


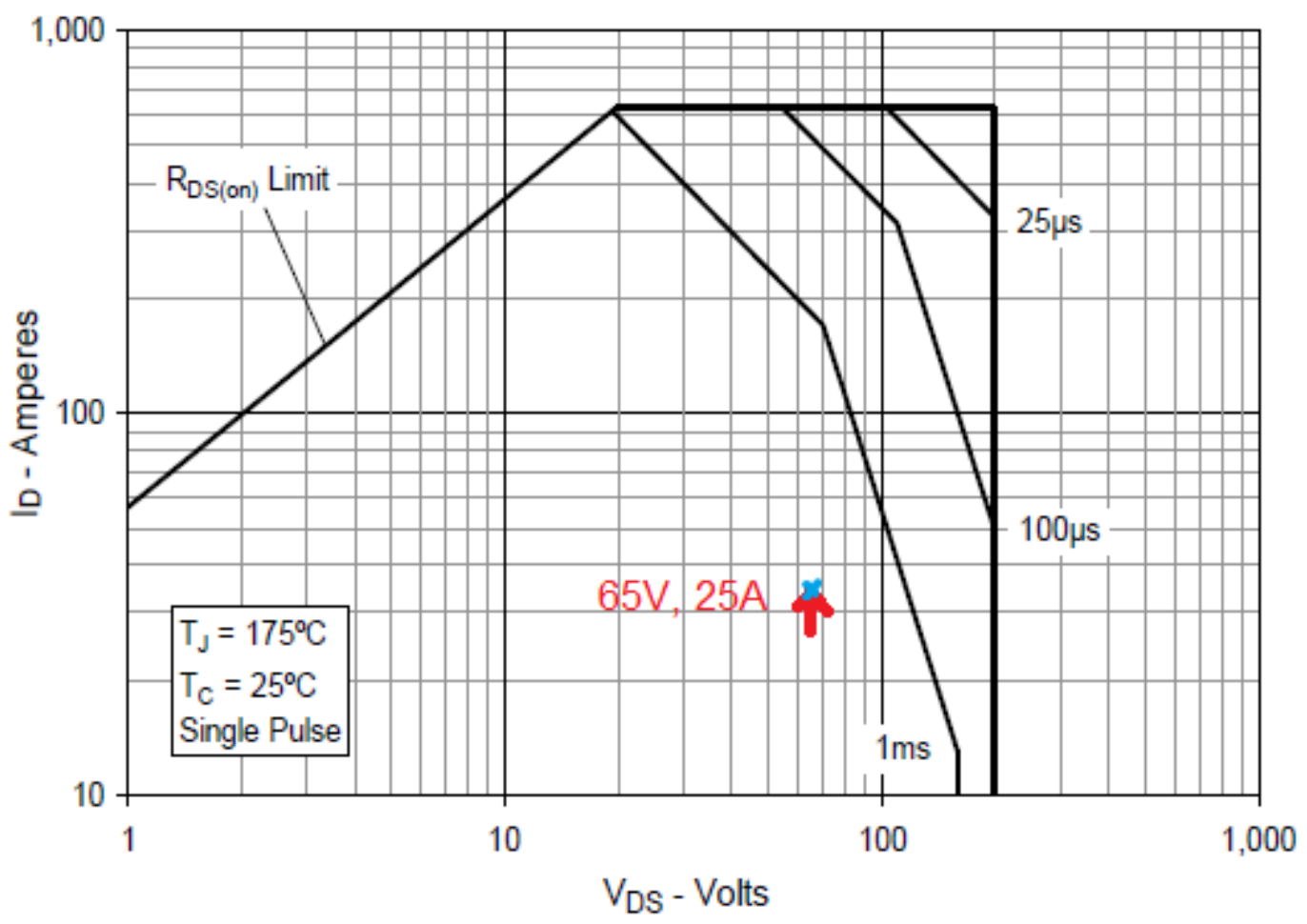

Figure 7.4: IXYS IXFK230N20T Safe Operating Area [57]

From figure 7.4, the IXYS IXFK230N20T withstands 65V operating voltage and 25A operating current for longer than $1 \mathrm{~ms}$ when operating at its $175^{\circ} \mathrm{C}$ maximum junction temperature, which is well above the required $0.878 \mu$ s duration. Thus the IXYS IXFK230N20T qualifies as a suitable transistor for Q_OVP. After selecting all the proper biasing components for the LT4356-1 surge stopper, this protection circuit is ready for implementation into the simulation SEPIC circuit.

\subsection{Protected SEPIC Simulation Results}

Figure 7.5 shows the complete SEPIC circuit implemented with the snubber from chapter 6 and the input overvoltage and overcurrent protection from section 7.2. 


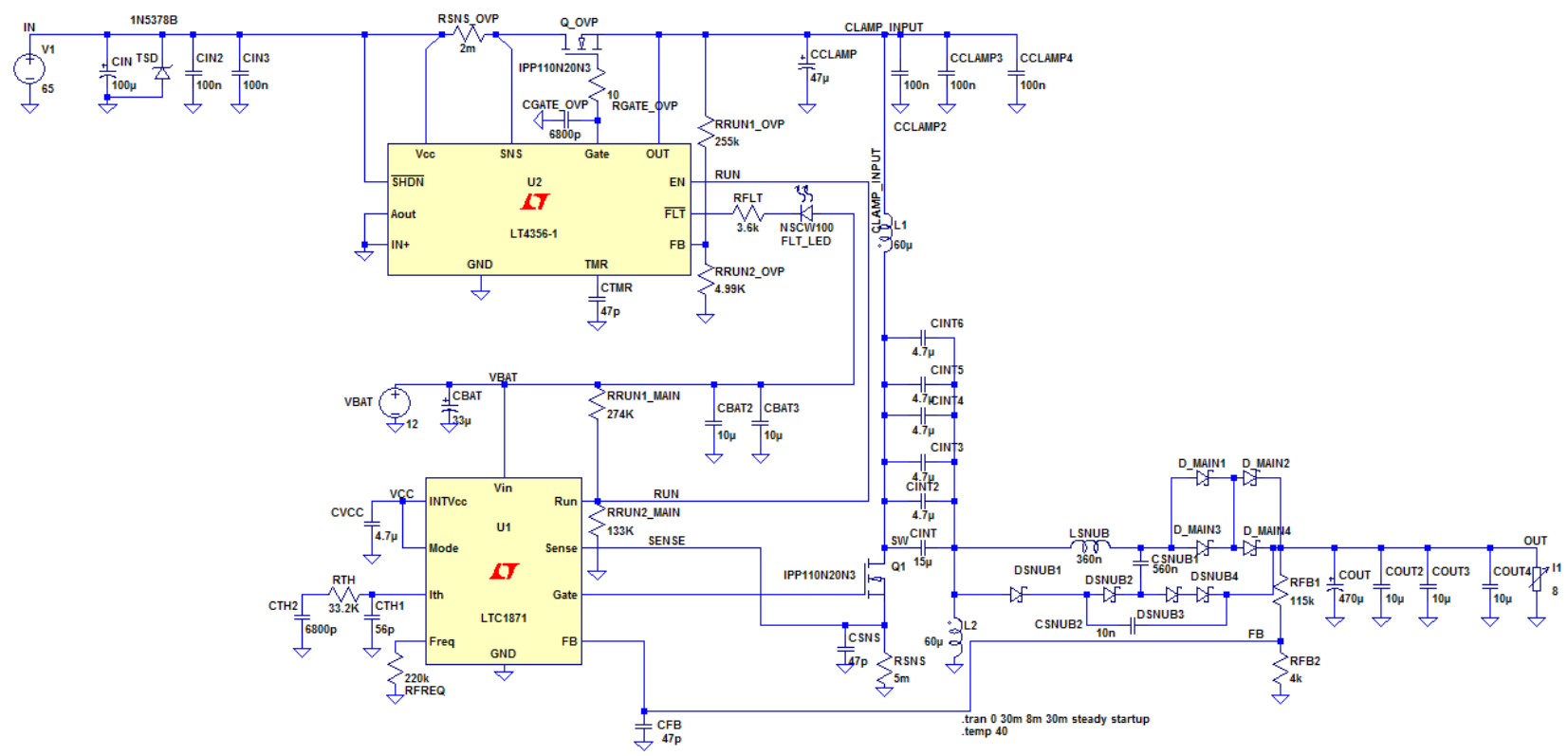

Figure 7.5: Complete SEPIC Schematic

This finalized SEPIC implementation adds ceramic capacitors in parallel with the large electrolytic capacitors at the elliptical input and output nodes, for reducing overall ESR that the electrolytic capacitors add. Furthermore, it also adds additional capacitors $(4.7 \mu \mathrm{F}$ each) in parallel with the coupling capacitor for reducing its ripple voltage and RMS current requirement. Ideally the coupling capacitor's ripple voltage should be low, but not so low that it results in unstable converter operation [27]. This implementation also uses two output snubber diodes (denoted as DSNUB3 and DSNUB4 in figure 7.5) for preventing their reverse voltages from exceeding $100 \mathrm{~V}$. It also increases input filter capacitor $\mathrm{C}_{\mathrm{IN}}$ to $100 \mu \mathrm{F}$ (from $33 \mu \mathrm{F}$ in previous implementations) after taking the elliptical trainer's output voltage ripple into consideration. The LT4356-1 protection circuit's output also contains an additional filter capacitor (denoted as CCLAMP in figure 7.5) for suppressing any ripple voltages that may occur at the protection circuit's output. 
This implementation also adds an input transient suppression diode (a 1N5378B 100V zener diode, denoted as TSD in figure 7.5) for protecting the LT4356-1 IC from potential input voltage ripples that exceed 100V. As described in section 7.1, EHFEM team members measured a $32 \mathrm{~V}$ peak-to-peak ripple from the elliptical trainer's output at higher output voltages, which corresponds to an $81 \mathrm{~V}$ peak input voltage for a $65 \mathrm{~V}$ average output, and although the EHFEM team members did not measure the elliptical trainer's output ripple voltage at $65 \mathrm{~V}$, voltage ripples greater than $100 \mathrm{~V}$ may occur. Also, this implementation also renames all component reference designators to those more understandable to the end user. Unlike previous implementations, this project also simulates this SEPIC at $40^{\circ} \mathrm{C}$ ambient operating temperature, as this temperature will very likely be the same as when a future project encloses this SEPIC inside a confined chassis. Figure 7.6 shows the protected SEPIC's output voltage in green and power dissipation transients across $\mathrm{Q}_{1}$ in blue, while operating at full-load and steady-state conditions. 


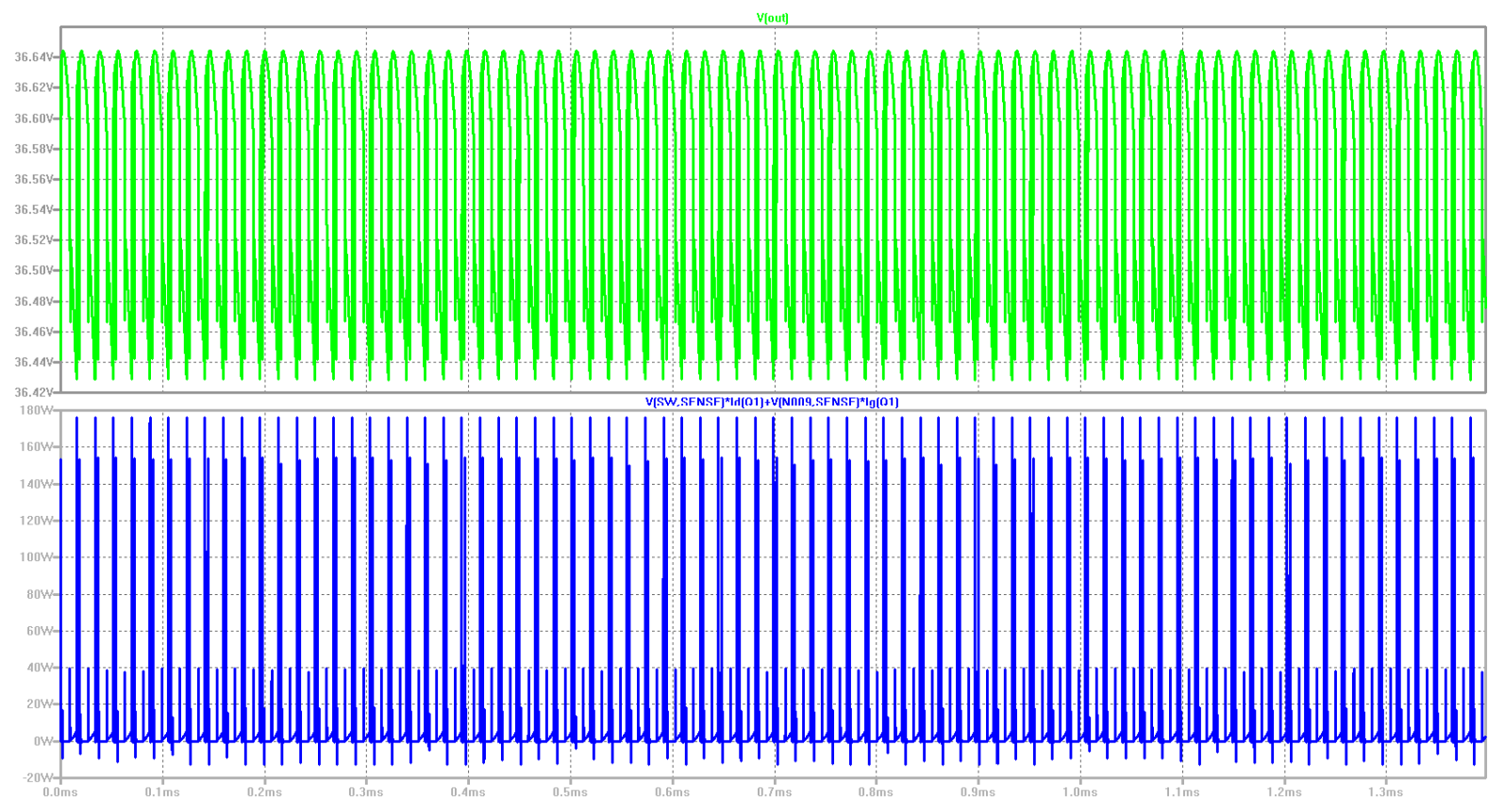

Figure 7.6: Protected SEPIC Output Voltage and $Q_{1}$ Power Dissipation at Full Load and Steady-state Operation

The protected SEPIC's characteristics in steady-state remain unchanged from the unprotected SEPIC's in section 6.4. Its steady-state efficiency also remains unchanged at 92.4\%. The LT4356-1 protection circuit, however, only shows its true advantages at the SEPIC's input during an overvoltage or overcurrent fault condition. Figure 7.7 shows such an example. This next simulation in figure 7.7 changes the SEPIC's main source voltage to a $32 \mathrm{~V}$ peak-to-peak, $31.3 \mathrm{kHz}$ sawtooth wave with $65 \mathrm{~V}$ DC offset $(81 \mathrm{~V}$ peak voltage and 49V minimum voltage). This input waveform uses the SEPIC's 65V maximum DC voltage input specified in section 1.2 as well as the lowest recorded ripple frequency recorded from [10] for testing the LT4356-1 protection circuit's voltage clamping abilities using the longest possible overvoltage transient. Figure 7.7 shows the SEPIC's input voltage in green, LT4356-1 output voltage in blue, as well as Q_OVP's power dissipation in red. 

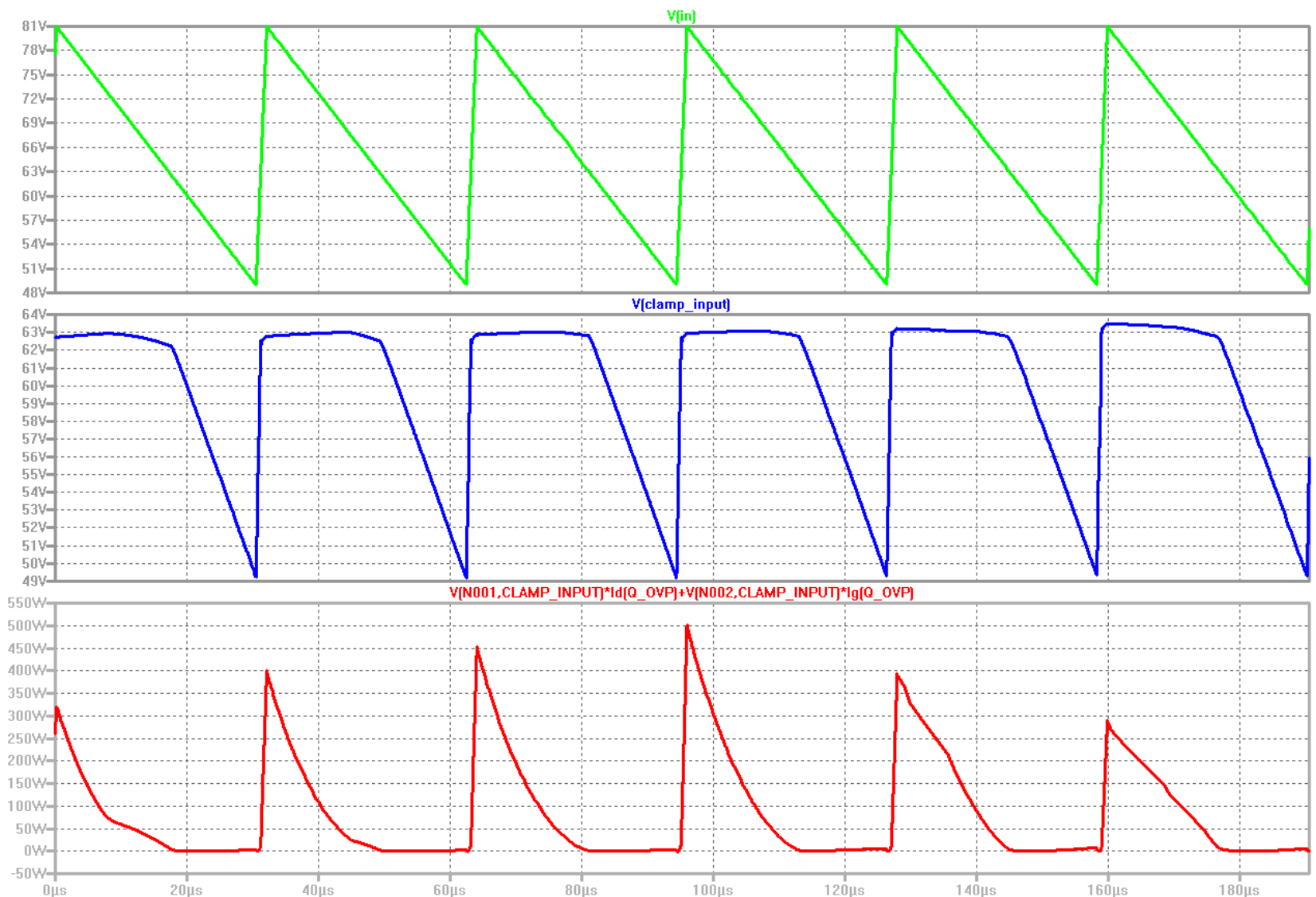

Figure 7.7: Protected SEPIC Input Voltage, Clamped Input Voltage and Q_OVP Power Dissipation at Full Load and Steady-state Operation

Using $100 \mu \mathrm{F}$ for $\mathrm{C}_{\mathrm{IN}}$ effectively quells the input voltage ripple, as the LT4356-1 input protection circuit clamps input voltage transients, as shown in the LT4356-1's output voltage waveform in figure 7.7. However, Q_OVP dissipates $86.223 \mathrm{~W}$ average in steadystate, which requires a very expensive heatsink for cooling. Therefore, this project's SEPIC requires design modifications to its input protection circuit. The only design modification that the input protection circuit requires is changing $\mathrm{C}_{\mathrm{IN}}$ 's capacitance value. Equation (6.1) from section 6.2 is crucial for understanding how capacitance value affects voltage ripple. Voltage ripple is inversely proportional to capacitance value, and increasing $\mathrm{C}_{\mathrm{IN}}$ to $1000 \mu \mathrm{F}$ should reduce input ripple voltage by a factor of 10 . Equations 
(7.13) and (7.14) modify (6.1) and calculate the SEPIC's input peak to peak ripple voltage using the previously defined $100 \mu \mathrm{F}$ as well as new $1000 \mu \mathrm{F} \mathrm{C}_{\mathrm{IN}}$ value as well as the SEPIC's specified 6.5A maximum input current and the $31.3 \mathrm{kHz}$ minimum input voltage ripple frequency determined from section 7.1.

$$
\begin{gathered}
\Delta V_{C}=\frac{I_{C} \Delta t}{C}=\frac{I_{C}}{C \times f}=\frac{6.5 \mathrm{~A}}{(100 \mu \mathrm{F})(31.3 \mathrm{kHz})}=2.08 \mathrm{~V} \\
\Delta V_{C}=\frac{I_{C} \Delta t}{C}=\frac{I_{C}}{C \times f}=\frac{6.5 \mathrm{~A}}{(1000 \mu \mathrm{F})(31.3 \mathrm{kHz})}=0.208 \mathrm{~V}
\end{gathered}
$$

Therefore, increasing $\mathrm{C}_{\mathrm{IN}}$ to $1000 \mu \mathrm{F}$ decreases the peak to peak input voltage ripple by tenfold and a $0.208 \mathrm{~V}$ peak to peak input voltage ripple suffices as a stable input voltage. This peak to peak input voltage ripple reduction should help decrease Q_OVP's power dissipation stress. Future SEPIC revisions will increase $\mathrm{C}_{\mathrm{IN}}$ 's capacitance value again if necessary. This project now re-simulates the waveforms from figure 7.7 using the new $1000 \mu \mathrm{F}$ input capacitor. Figure 7.8 shows these results, with the SEPIC's input voltage in green, LT4356-1 output voltage in blue, as well as Q_OVP's power dissipation in red. 


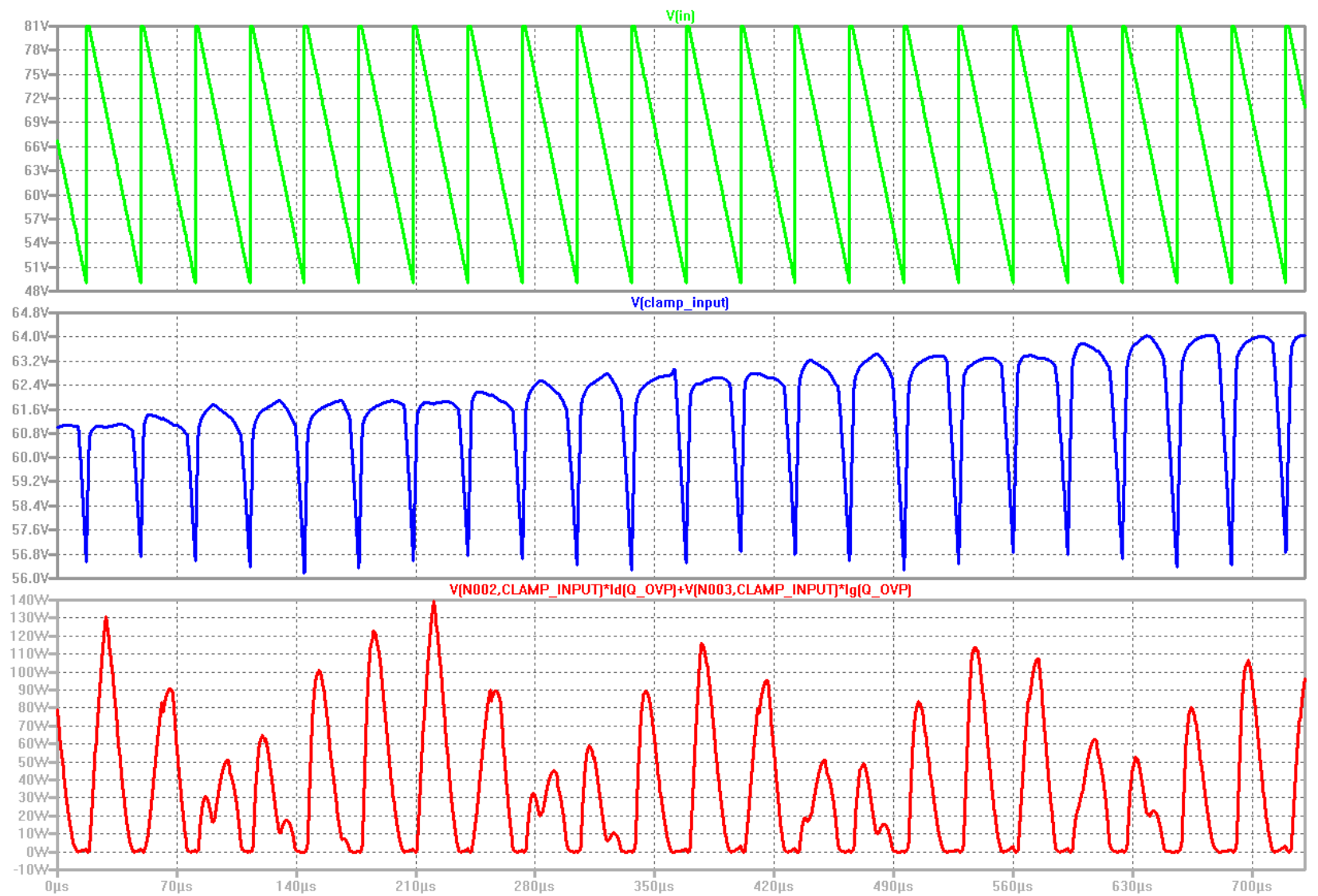

Figure 7.8: Protected SEPIC Input Voltage, Clamped Input Voltage and Q_OVP Power Dissipation at Full Load and Steady-state Operation with $1000 \mu$ F Input Capacitor

With a $1000 \mu \mathrm{F}$ input capacitor Q_OVP's average input voltage clamping power dissipation drops to nearly $12 \mathrm{~W}$ in steady-state, which is an acceptable level for this converter's application, however, such power dissipation still requires a heatsink with low thermal resistance. The LT4356-1 input protection circuit only clamps the input to 64V because of non-ideal $V_{\text {DS }}$ drops across Q_OVP. With a high time-varying input voltage to the SEPIC, C $\mathrm{IN}_{\mathrm{IN}}$ 's RMS current also exceeds 50A according to the same simulation from figure 7.8. One way to remedy this problem is connecting $\mathrm{C}_{\mathbb{I N}}$ in series with the Precor elliptical trainer's onboard $10 \Omega$ resistor load (with the load grounded at one end). This project's SEPIC's PCB layout in Chapter 9 includes an additional 
connector for $\mathrm{C}_{\mathrm{IN}}$, allowing for a connection either to the Precor elliptical trainer's $10 \Omega$ load or ground. Doing so reduces $\mathrm{C}_{\mathrm{IN}}$ 's RMS current to a more acceptable 1A while still maintaining the same LT4356-1 performance as the simulation from figure 7.8. For the remaining simulations as well as synthetic converter hardware tests described in chapter 10 (i.e. using a constant DC power supply as an input source instead of the Precor

elliptical), $\mathrm{C}_{\mathrm{IN}}$ connects to ground for simplicity. The next section details the SEPIC's finalized design before implementing it in PCB layout.

\subsection{Finalized SEPIC Simulation Results}

Figure 7.9 shows the final SEPIC schematic before this project lays it out on a PCB in chapter 9. 


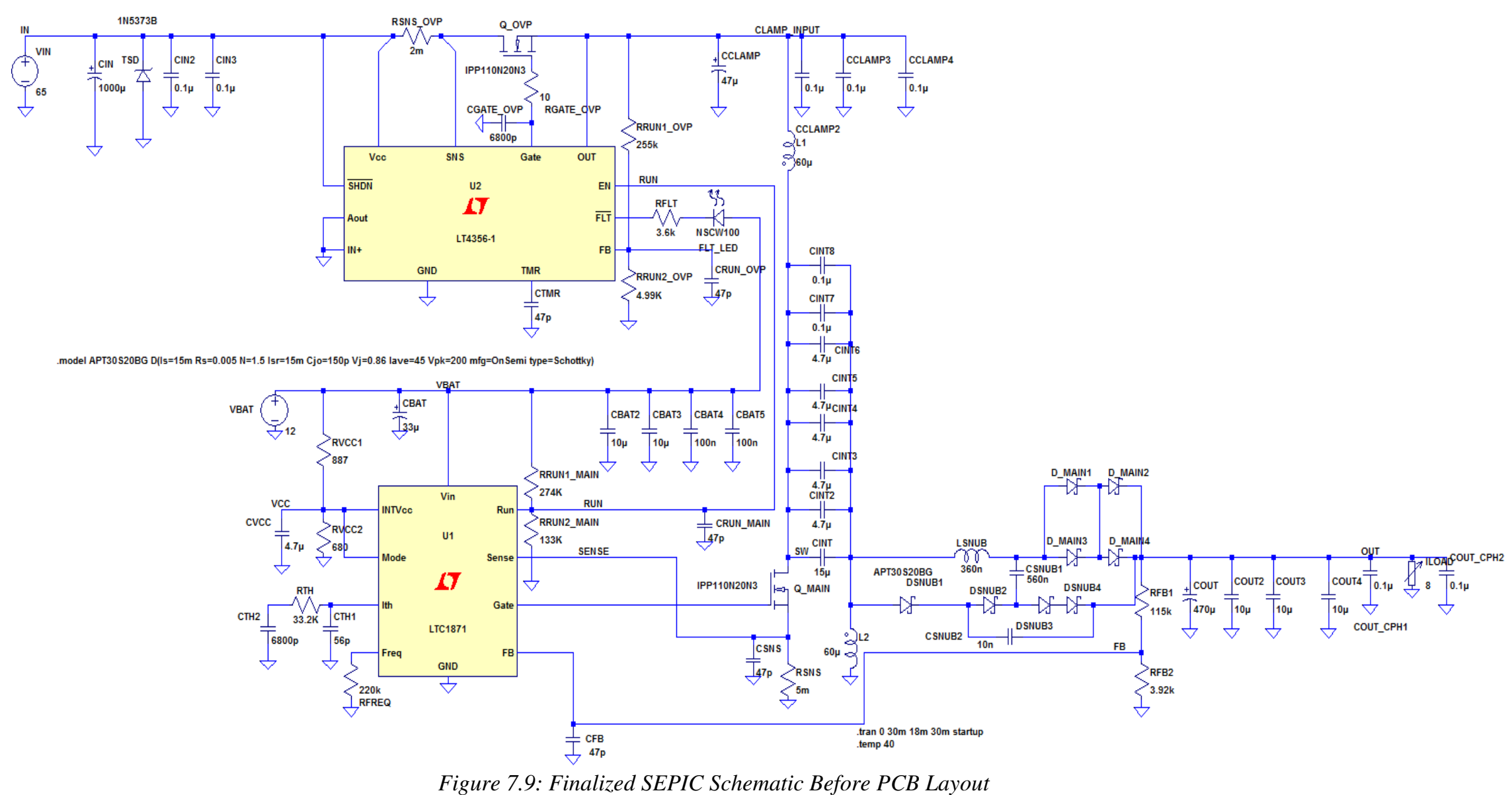


This finalized implementation adds $0.1 \mu \mathrm{F}$ ceramic bypass capacitors to the SEPIC's elliptical input, $12 \mathrm{~V}$ battery input, switching and output nodes. These bypass capacitors prevent resonance effects from occurring at the SEPIC's 100-111 kHz switching frequency, and also prevent high frequency harmonics from causing interference with circuit operation. An example of such resonance effects is that the large input and output capacitors no longer function as capacitors - instead they behave like inductors (with increasing impedance at higher frequencies rather than the opposite) and lose their high frequency signal passing functions [58]. This implementation also renames the SEPIC's main switching transistor $\mathrm{Q}_{1}$ to Q_MAIN. Furthermore, this implementation also adds a resistor divider network consisting of $\mathrm{R}_{\mathrm{VCC} 1}$ and $\mathrm{R}_{\mathrm{VCC} 2}$ at the LTC1871 controller's INTV $_{\mathrm{CC}}$ pin, preventing large inrush current spikes at start-up that may damage the LTC1871 controller. This project selects $\mathrm{R}_{\mathrm{VCC} 1}$ and $\mathrm{R}_{\mathrm{VCC} 2}$ such that the LTC1871's $\mathrm{INTV}_{\mathrm{CC}}$ pin still maintains its required $5.2 \mathrm{~V}$ operation voltage during normal controller operation while simultaneously drawing low current from the Precor elliptical trainer's onboard 12V battery. This implementation also considers using IXYS's IXYS's IXFK230N20T as an alternate transistor for Q_MAIN because of its high power tolerance. Further report sections refer to the IPP110N20N3 as Q_MAIN and the IXFK230N20T as Q_MAIN_ALT (Q_MAIN alternate). Also not shown in figure 7.9 are fuses protecting the converter's main input, battery input and output nodes. UL, IEEE 1547, NEC and PG\&E safety guidelines require these fuses and they are included as offPCB devices during hardware testing. Table B-1 shows this project's SEPIC component 
bill of materials (BOM) with all component part numbers for this finalized converter, sans PCB costs. 
Table B-1: Finalized SEPIC Component BOM

\begin{tabular}{|c|c|c|c|c|c|c|c|}
\hline Controller I.C.s: & Quantity: & $\begin{array}{l}\text { Unit Price } \\
\text { (\$): }\end{array}$ & Total Price: & Components: & Type: & \begin{tabular}{|c|}
$65 V, 8 A$ Required \\
Rating
\end{tabular} & $5 \mathrm{~V}, 0.5 \mathrm{~A}$ \\
\hline LTC1871HMS (10-MSOP) & 1 & 4.83 & 4.83 & U1 & 10-MSOP & $\mathrm{N} / \mathrm{A}$ & $\mathrm{N} / \mathrm{A}$ \\
\hline LT4356-1HMS (10-MSOP) & 2 & 3.70 & 7.40 & U2 & 10-MSOP & $\mathrm{N} / \mathrm{A}$ & $\mathrm{N} / \mathrm{A}$ \\
\hline \multicolumn{3}{|c|}{ Total Cost (\$) } & 12.23 & & & & \\
\hline Transistors: & Quantity: & $\begin{array}{l}\text { Unit Price } \\
\text { (\$): }\end{array}$ & Total Price: & Components: & Type: & $\begin{array}{c}65 \mathrm{~V}, \mathrm{8A} \text { Required } \\
\text { Rating }\end{array}$ & $5 \mathrm{~V}, 0.5 \mathrm{~A}$ \\
\hline Infineon IPP110N20N3 & 1 & 8.06 & 8.06 & Q_MAIN & TO-220-3 & $2.45 \mathrm{~W}$ & $4.01 \mathrm{~W}$ \\
\hline IXYS IXFK230N20T & 1 & 9.50 & 9.50 & Q_OVP & TO-264 & $1.625 \mathrm{~kW}$ & $1.625 \mathrm{~kW}$ \\
\hline \multicolumn{3}{|c|}{ Total Cost (\$) } & 17.56 & & & & \\
\hline Diodes: & Quantity: & $\begin{array}{l}\text { Unit Price } \\
\text { (\$): }\end{array}$ & Total Price: & Components: & Type: & $\begin{array}{c}\text { 65V, 8A Required } \\
\text { Rating }\end{array}$ & $5 \mathrm{~V}, 0.5 \mathrm{~A}$ \\
\hline MBR20100CT (Schottky) & 7 & 0.88 & 6.16 & $\begin{array}{c}\text { D_MAIN1, D_MAIN2, D_MAIN3, } \\
\text { D_MAIN4, DSNUB2, DSNUB3, } \\
\text { DSNUB4 }\end{array}$ & TO-220-3 & $31.11 \mathrm{~A}, 110 \mathrm{~V}$ & $27.72 \mathrm{~A}, 56.46 \mathrm{~V}$ \\
\hline Microsemi APT30S20BG (Schottky) & 1 & 3.67 & 3.67 & DSNUB1 & TO-247 & $39.43 \mathrm{~A}, 15.95 \mathrm{~V}$ & $27.89 \mathrm{~A}, 9.58 \mathrm{~V}$ \\
\hline 1N5378B (Zener) & 1 & 0.57 & 0.57 & TSD & Through-Hole & $100 \mathrm{~V}$ & $100 \mathrm{~V}$ \\
\hline SML-LX1206SRC-TR (LED) & 1 & 0.43 & 0.43 & FLT_LED & 1206 & $\begin{array}{c}1.2 \mathrm{~V} \text { min. forward } \\
\text { drop }\end{array}$ & $\begin{array}{l}1.2 \mathrm{~V} \text { min. } \\
\text { forward drop }\end{array}$ \\
\hline \multicolumn{3}{|c|}{ Total Cost (\$) } & 10.83 & & & & \\
\hline Fuses: & Quantity: & $\begin{array}{l}\text { Unit Price } \\
\text { (\$): }\end{array}$ & Total Price: & Components: & Type: & $\begin{array}{c}\text { 65V, 8A Required } \\
\text { Rating }\end{array}$ & $5 \mathrm{~V}, 0.5 \mathrm{~A}$ \\
\hline $\begin{array}{c}\text { Radio Shack 270-270-1234 30A Blade } \\
\text { Fuse Holder }\end{array}$ & 2 & 2.99 & 5.98 & $\begin{array}{l}\text { Fuse Holder for Input and Output } \\
\text { Fuse }\end{array}$ & $\mathrm{N} / \mathrm{A}$ & $65 \mathrm{~V}, 25 \mathrm{~A}$ & $5 \mathrm{~V}, 25 \mathrm{~A}$ \\
\hline $\begin{array}{c}\text { Cooper Bussmann 270-1085 30A } \\
\text { Blade Fuse }\end{array}$ & 1 & 0.73 & 0.73 & Input Fuse & Blade Fuse & $65 \mathrm{~V}, 25 \mathrm{~A}$ & $5 \mathrm{~V}, 25 \mathrm{~A}$ \\
\hline $\begin{array}{c}\text { Cooper Bussmann 270-1081 10A } \\
\text { Blade Fuse }\end{array}$ & 1 & 0.73 & 0.73 & Output Fuse & Blade Fuse & $36 \mathrm{~V}, 8 \mathrm{~A}$ & $5 \mathrm{~V}, 0.5 \mathrm{~A}$ \\
\hline Bourns SF-0402F200-2 2A Fuse & 1 & 0.68 & 0.68 & Elliptical Battery Fuse & 0402 & $12 \mathrm{~V}, 2 \mathrm{~A}$ & $12 \mathrm{~V}, 2 \mathrm{~A}$ \\
\hline \multicolumn{3}{|c|}{ Total Cost (\$) } & 8.12 & & & & \\
\hline
\end{tabular}


Table B-1 (Continued)

\begin{tabular}{|c|c|c|c|c|c|c|c|}
\hline Resistors: & Quantity: & $\begin{array}{l}\text { Unit Price } \\
\text { (\$): }\end{array}$ & Total Price: & Components: & Type: & $\begin{array}{c}\text { 65V, 8A } \\
\text { Required } \\
\text { Rating }\end{array}$ & $5 \mathrm{~V}, 0.5 \mathrm{~A}$ \\
\hline 274k, 1/8W, 1\%, ERJ-6ENF2743V & 1 & 0.07 & 0.07 & RRUN1_MAIN & 0805 & $0.521 \mathrm{~mW}$ & $0.522 \mathrm{~mW}$ \\
\hline 255k, 1/8W, 1\%, ERJ-6ENF2553V & 1 & 0.07 & 0.07 & RRUN1_OVP & 0805 & $16.289 \mathrm{~mW}$ & $0.094 \mathrm{~mW}$ \\
\hline $220 \mathrm{k}, 1 / 8 \mathrm{~W}, 1 \%$, ERJ-6ENF2203V & 1 & 0.07 & 0.07 & RFREQ & 0805 & $0.002 \mathrm{~mW}$ & $0.002 \mathrm{~mW}$ \\
\hline 133k, 1/8W, 1\%, ERJ-6ENF1333V & 1 & 0.07 & 0.07 & RRUN2_MAIN & 0805 & $0.115 \mathrm{~mW}$ & $0.122 \mathrm{~mW}$ \\
\hline 115k, 1/8W, 1\%, ERJ-6ENF1153V & 1 & 0.07 & 0.07 & RFB1 & 0805 & $11.12 \mathrm{~mW}$ & $12.28 \mathrm{~mW}$ \\
\hline 4.99k, 1/8W, 1\%, ERJ-6ENF4991V & 1 & 0.07 & 0.07 & RRUN2_OVP & 0805 & $0.319 \mathrm{~mW}$ & $0.002 \mathrm{~mW}$ \\
\hline 3.92k, 1/8W, 1\%, RMCF0805FT3K92 & 1 & 0.04 & 0.04 & RFB2 & 0805 & $0.387 \mathrm{~mW}$ & $0.427 \mathrm{~mW}$ \\
\hline 3.6k, 1/8W, 1\%, ERJ-6ENF3601V & 1 & 0.07 & 0.07 & RFLT & 0805 & $32.4 \mathrm{~mW}$ & $32.4 \mathrm{~mW}$ \\
\hline $887,1 / 8 \mathrm{~W}, 1 \%$, ERJ-6ENF8870V & 1 & 0.07 & 0.07 & RVCC1 & 0805 & $<125 \mathrm{~mW}$ & $<125 \mathrm{~mW}$ \\
\hline $680,1 / 8 \mathrm{~W}, 5 \%$, ERJ-6GEYJ681V & 1 & 0.04 & 0.04 & RVCC2 & 0805 & $<125 \mathrm{~mW}$ & $<125 \mathrm{~mW}$ \\
\hline $10,1 / 8 \mathrm{~W}, 1 \%$, ERJ-6ENF10R0V & 1 & 0.07 & 0.07 & RGATE_OVP & 0805 & $13.05 \mathrm{~mW}$ & OW \\
\hline $0.005,5 \mathrm{~W}, 1 \%$, OARSXPR005FLF & 1 & 1.42 & 1.42 & RSNS & SMT (Custom) & $410 \mathrm{~mW}$ & $1.132 \mathrm{~W}$ \\
\hline \multicolumn{3}{|c|}{ Total Cost (\$) } & 5.27 & & & & \\
\hline Inductors: & Quantity: & $\begin{array}{l}\text { Unit Price } \\
\text { (\$): }\end{array}$ & Total Price: & Components: & Type: & $\begin{array}{c}\text { 65V, 8A } \\
\text { Required } \\
\text { Rating }\end{array}$ & $5 \mathrm{~V}, 0.5 \mathrm{~A}$ \\
\hline Vishay IHV28BZ60 $\left(60 \mu \mathrm{H}, \mathrm{I}_{\mathrm{SAT}}=28 \mathrm{~A}\right)$ & 2 & 20.30 & 40.60 & L1, L2 & Through-Hole & $\begin{array}{c}\text { 30.68A (max.), } \\
65 \mathrm{~V}\end{array}$ & $\begin{array}{c}22.37 \mathrm{~A}(\max .), \\
52.22 \mathrm{~V}\end{array}$ \\
\hline $\begin{array}{c}\text { Vishay IHLP4040DZERR36M01 } \\
\left(360 \mathrm{nH}, \mathrm{I}_{\mathrm{SAT}}=60 \mathrm{~A}\right)\end{array}$ & 1 & 2.52 & 2.52 & LSNUB & SMT (Custom) & $\begin{array}{c}\text { 29.3A (max.) } \\
106.25 \mathrm{~V}\end{array}$ & $\begin{array}{c}17.85 \mathrm{~A} \text { (max.), } \\
55.35 \mathrm{~V}\end{array}$ \\
\hline \multicolumn{3}{|c|}{ Total Cost (\$) } & 43.12 & & & & \\
\hline
\end{tabular}


Table B-1 (Continued)

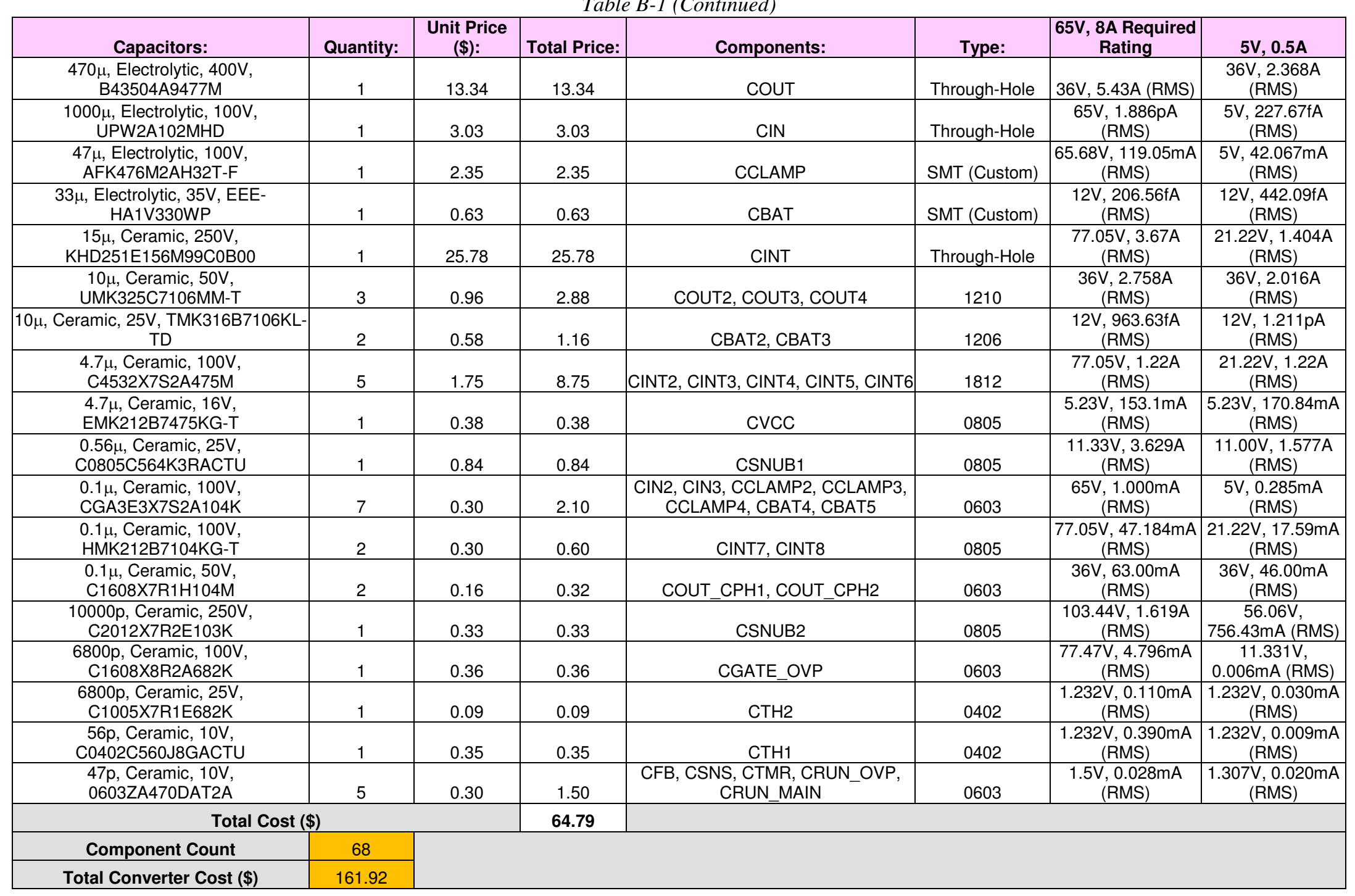


The BOM in table B-1 determines components based on required absolute maximum electrical ratings based on simulation results using the schematic from figure 7.9. Using those maximum electrical ratings, this project determined the most appropriate parts and selected them from component suppliers such as Digi-Key and Mouser Electronics. The most expensive components for this SEPIC are its switching inductors $\left(\mathrm{L}_{1}\right.$ and $\left.\mathrm{L}_{2}\right)$ as well as its coupling capacitor $\left(\mathrm{C}_{\mathrm{INT}}\right)$. This project's SEPIC's switching inductors and coupling capacitors require low ESR as well as high current tolerance (RMS current tolerance on the capacitor). The capacitor selected for $\mathrm{C}_{\mathrm{INT}}$, a Nippon Chemi-Con KHD251E156M99C0B00, is a ceramic capacitor with 250V and 4A RMS current tolerance [59], which suffices for this project's SEPIC. Because it is a ceramic capacitor, its ESR is also inherently low. Using an electrolytic capacitor with similar voltage and RMS current ratings for $\mathrm{C}_{\mathrm{INT}}$ reduces this SEPIC's cost, but requires more parallel ceramic capacitors for offsetting the electrolytic capacitor's high ESR. Selecting all components also helps determine each component's parasitic electrical values such as ESR or parallel capacitance. This project then implemented those parasitic values into the SEPIC's components in the schematic in figure 7.9, for determining how much those parasitic values affect overall converter performance. Because ceramic capacitors typically do not indicate ESR values in their datasheets, this project uses $0.02 \Omega$ as the ESR value for all of its SEPIC's ceramic capacitors. Typical ceramic capacitor ESR values maximize at $0.015 \Omega$, and this project uses a conservative $0.02 \Omega$ value for all of its SEPIC's ceramic capacitors [60]. Figures 7.10 and 7.11 show these results. Figure 7.10 shows the finalized SEPIC's output voltage waveform in green and Q_MAIN's power 
dissipation at full load (65V input, $8 \mathrm{~A}$ load) and in steady-state, with the parasitic component values implemented. Figure 7.11 shows the SEPIC's output power in green and input power in blue. Likewise with the SEPIC implementation in section 7.3, this project also runs these simulations at $40^{\circ} \mathrm{C}$ ambient operating temperature.

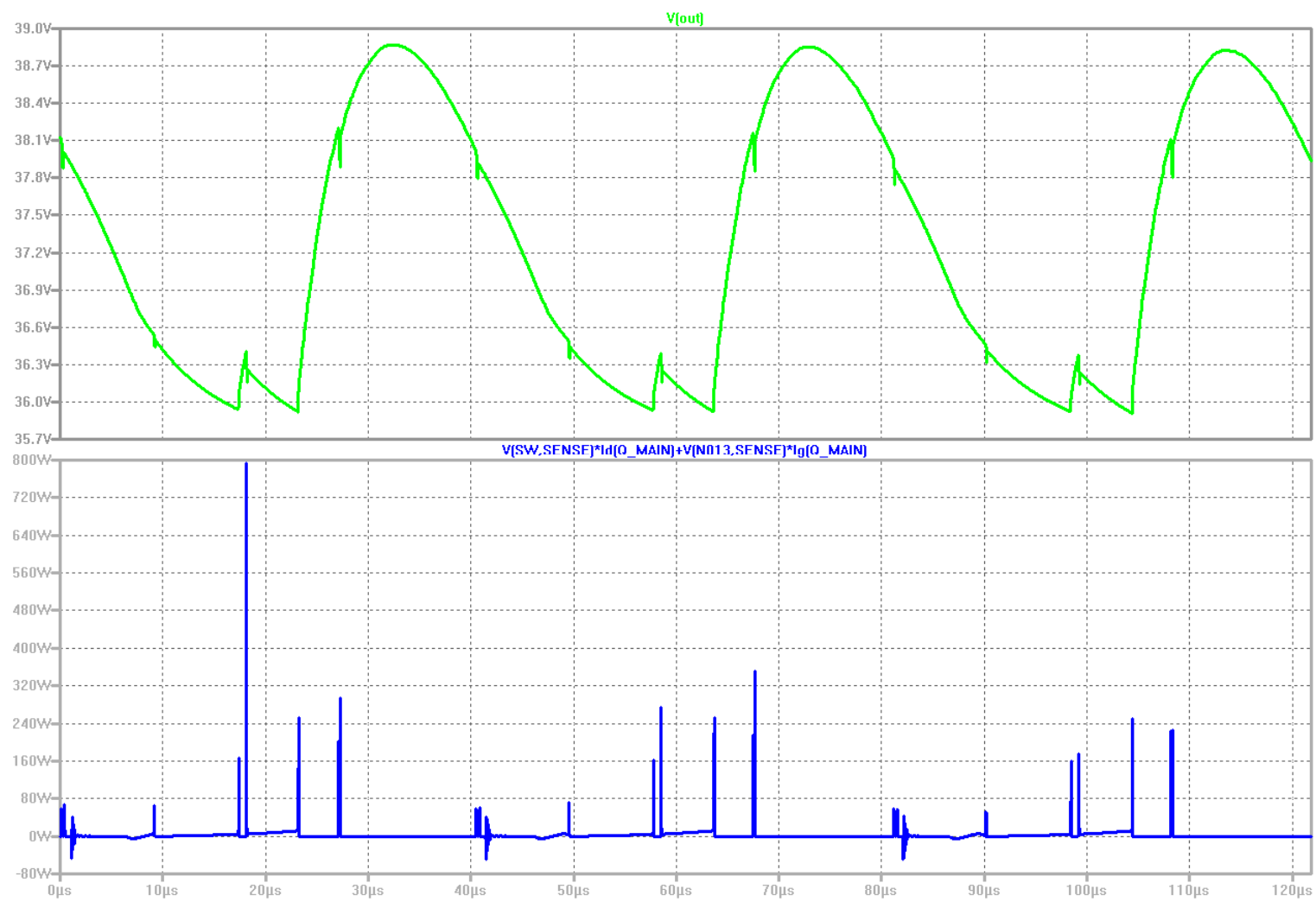

Figure 7.10: Finalized SEPIC Output Voltage and Q_MAIN Power Dissipation with Parasitic Component Values 


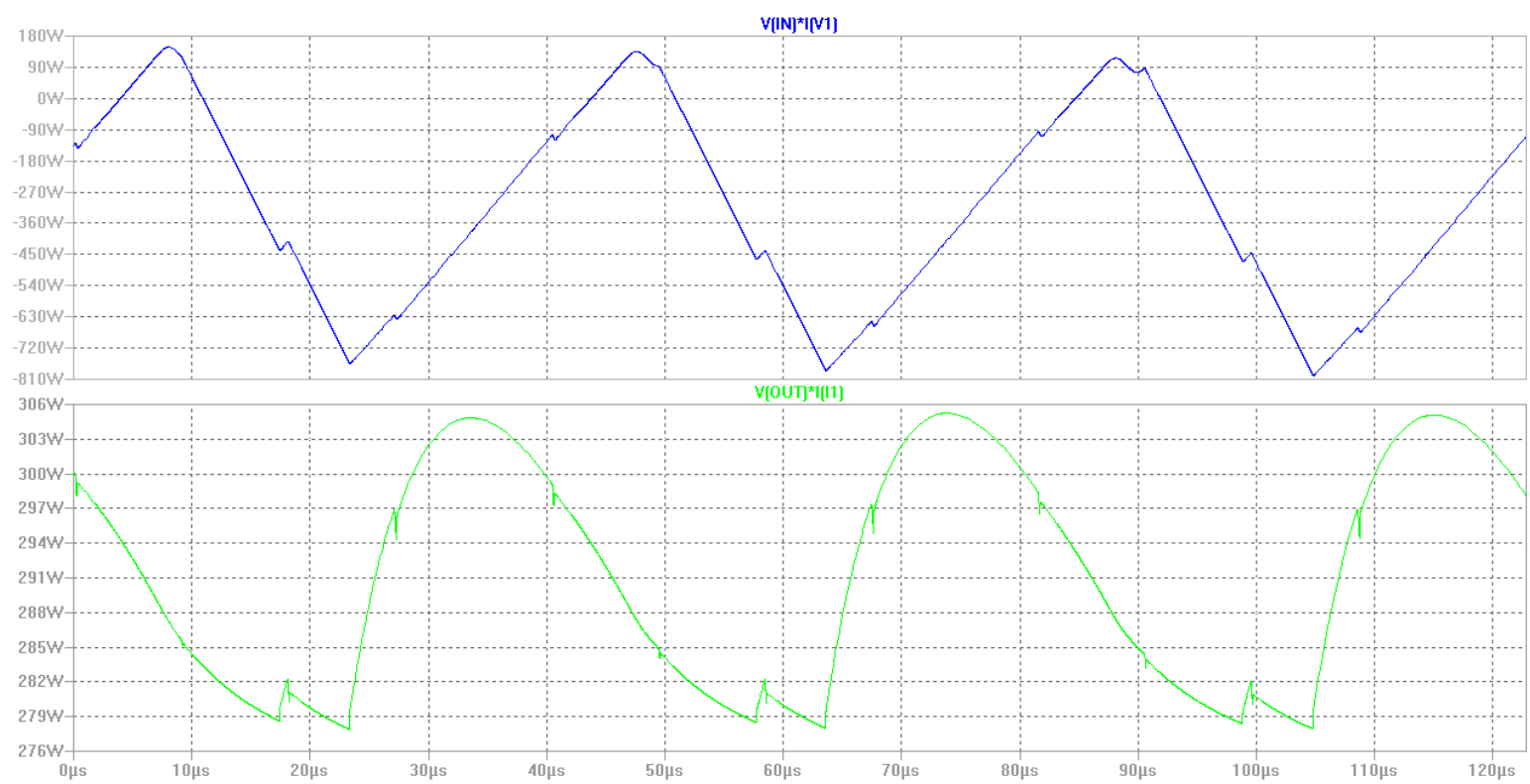

Figure 7.11: Finalized SEPIC Converter Input and Output Power with Parasitic Component Values

From figure 7.10, average SEPIC output voltage is $37.29 \mathrm{~V}$, which is slightly higher than the required $36 \mathrm{~V}$, but nonetheless suffices. The increased output voltage results from changing one of the SEPIC's output feedback resistors, $\mathrm{R}_{\mathrm{FB} 2}$, to $3.92 \mathrm{k} \Omega$ from $4 \mathrm{k} \Omega$. This project changed $\mathrm{R}_{\mathrm{FB} 2}$ 's value for cost reduction and component availability purposes, because $08053.92 \mathrm{k} \Omega$ resistors were available at a much lower price than $4 \mathrm{k} \Omega$ resistors. Peak-to-peak output voltage ripple is $2.959 \mathrm{~V}$, which corresponds to a $7.94 \%$ output voltage ripple. This value is below the $10 \%$ output voltage ripple requirement specified in section 1.2, therefore this value suffices. Average Q_MAIN power dissipation in steadystate is $2.29 \mathrm{~W}$, increased from $1.8 \mathrm{~W}$ from the design in section 6.4 . The parasitic component values caused this power dissipation increase across Q_MAIN, however 2.29W suffices with adequate cooling on Q_MAIN. From figure 7.11, average SEPIC output power is $291.31 \mathrm{~W}$ and average input power is $312.17 \mathrm{~W}$. This translates to a $93.3 \%$ converter efficiency at full load and steady-state, with all parasitic values implemented 
into the SEPIC's components. This is still above the $75 \%$ efficiency specification outlined in section 1.2, therefore this simulated efficiency value suffices. Therefore, even after adding parasitic component values to this project's SEPIC, it still performs better in simulation than the specifications outlined in section 1.2. This project next implements takes the SEPIC from figure 7.9 and determines cooling requirements for components that dissipate more than $1 \mathrm{~W}$ power, as such components experience shortened lifespan and decrease overall converter lifespan if not adequately cooled. 


\section{CHAPTER 8: THERMAL CONSIDERATIONS}

\subsection{Semiconductor Device Power Dissipation}

Because the transistors and diodes in this project's SEPIC are far from ideal, they still dissipate power in the form of heat, as witnessed in previous chapters. Operating such components without heat removal devices result in shortened component lifespan as well as shortened overall SEPIC lifespan. Shortened overall SEPIC lifespan results in frequent component replacement and consequently, increased the EHFEM system's lifecycle costs. Heatsinks are the most common and least expensive type of heat removal devices for semiconductor devices. They also save physical PCB space without using any PCB copper pads (in the case of thermal vias). Therefore, this project employs heatsinks for removing heat from its SEPIC's transistors and diodes for ensuring prolonged converter operation. Determining the maximum power dissipation in Q_MAIN, the main switching diodes (D_MAINx) and snubber diodes (D_SNUBx) requires knowing the lowest possible input voltage at which the SEPIC maintains $36 \mathrm{~V}$ output and drive an $8 \mathrm{~A}$ load (288W output). From simulation, $27 \mathrm{~V}$ was the minimum input voltage at which the SEPIC maintained a 36V output with an 8A load. Realistically, the SEPIC will not drive an $8 \mathrm{~A}$ load at $27 \mathrm{~V}$ input because it must maintain a $10 \Omega$ input resistance, but this conservative estimate ensures that selected heatsinks more than adequately cool components requiring heatsinking and that the SEPIC does not suffer premature failure. Increasing $\mathrm{C}_{\mathrm{IN}}$ to $1000 \mu \mathrm{F}$ in section 7.4 dramatically reduced the average power dissipation across Q_OVP during an overvoltage input condition to $10.56 \mathrm{~W}$, from $60.02 \mathrm{~W}$ with $\mathrm{C}_{\mathrm{IN}}$ at $100 \mu \mathrm{F}$. 
Tables C-1 and C-2 determine from simulation the average steady-state power dissipation across the SEPIC's main semiconductor components. Table C-1 determines average power dissipation when the SEPIC operates with $65 \mathrm{~V}$ input and $8 \mathrm{~A}$ load, and table C-2 determines peak power dissipation when the SEPIC operates with 27V input and 8A load.

Table C-1: SEPIC Semiconductor Component Average Steady-state Power Dissipation with 65V Input and 8 A Load

\begin{tabular}{|c|c|}
\hline SEPIC Component & Average Power Dissipation (W) \\
\hline D_MAIN1 to 4 & 3.436 \\
\hline D_SNUB1 & 1.871 \\
\hline D_SNUB2 & 3.48 \\
\hline D_SNUB3 and 4 & 3.498 \\
\hline Q_MAIN & 2.2 \\
\hline Q_OVP & 0.25 \\
\hline
\end{tabular}

Table C-2: SEPIC Semiconductor Component Average Steady-state Power Dissipation with 27V Input and 8 A Load

\begin{tabular}{|c|c|}
\hline SEPIC Component & Average Power Dissipation (W) \\
\hline D_MAIN1 to 4 & 1.584 \\
\hline D_SNUB1 & 1.932 \\
\hline D_SNUB2 & 3.939 \\
\hline D_SNUB3 and 4 & 4.084 \\
\hline Q_MAIN & 6.0 \\
\hline Q_OVP & 0.53 \\
\hline
\end{tabular}

This project's heatsink selection process considers the higher power dissipation values of the two operation cases above. Also this project rounds up all power dissipation values in thermal calculations, for further conservative thermal resistance requirement estimates. The next section determines each component's heatsink's required thermal resistance ratings. 


\subsection{Heatsink Thermal Resistance Ratings}

Equations (8.1) and (8.2) determine the required thermal resistance rating for each SEPIC semiconductor component's heatsink. Both equations also assume $40^{\circ} \mathrm{C}$ ambient converter operation temperature.

Where:

$$
P_{M A X}=\frac{T_{J M A X}-T_{A}}{R_{\theta J C}+R_{\theta C S}+R_{\theta H A}}
$$

$$
\begin{gathered}
P_{M A X}=\text { Maximum component power dissipation }[\mathrm{W}] \\
T_{J M A X}=\text { Maximum junction operating temperature }\left[{ }^{\circ} \mathrm{C}\right] \\
T_{A}=\text { Ambient air temperature }\left[{ }^{\circ} \mathrm{C}\right] \\
R_{\theta J C}=\text { Junction to case thermal resistance }\left[{ }^{\circ} \mathrm{C} / W\right] \\
R_{\theta C S}=\text { Case to heatsink thermal resistance }\left[{ }^{\circ} \mathrm{C} / W\right] \\
R_{\theta H A}=\text { Heatsink to ambient }(\text { air }) \text { thermal resistance }\left[{ }^{\circ} \mathrm{C} / W\right] \\
R_{\theta H A}=\frac{T_{J M A X}-T_{A}}{P_{M A X}}-R_{\theta J C}-R_{\theta C S}
\end{gathered}
$$

Equation (8.3) calculates Q_OVP's required heatsink thermal resistance, using IXYS's IXFK230N20T datasheet thermal values [57]. Although from tables C-1 and C-2 Q_OVP only dissipates $0.53 \mathrm{~W}$ average power in the worst case scenario, it must withstand $12 \mathrm{~W}$ average should the SEPIC encounter an input overvoltage and overcurrent transient and require Q_OVP clamping that transient.

$$
R_{\theta H A}=\frac{175^{\circ} \mathrm{C}-40^{\circ} \mathrm{C}}{12 W}-0.09^{\circ} \mathrm{C} / W-0.15^{\circ} \mathrm{C} / W=11.01^{\circ} \mathrm{C} / W
$$

Equation (8.4) calculates Q_MAIN_ALT's required heatsink thermal resistance if the project ever necessitated its use. Likewise with (8.3), (8.4) calculates the required heatsink thermal resistance using IXYS's IXFK230N20T datasheet thermal values [57].

$$
R_{\theta H A}=\frac{175^{\circ} \mathrm{C}-40^{\circ} \mathrm{C}}{6 W}-0.09^{\circ} \mathrm{C} / W-0.15^{\circ} \mathrm{C} / W=22.26^{\circ} \mathrm{C} / W
$$


Equation (8.5) calculates Q_MAIN's required heatsink thermal resistance, using Infineon's IPP110N20N3 datasheet thermal values [39].

$$
R_{\theta H A}=\frac{175^{\circ} \mathrm{C}-40^{\circ} \mathrm{C}}{6 W}-0.5^{\circ} \mathrm{C} / W=22.5^{\circ} \mathrm{C} / W
$$

Infineon's IPP110N20N3 datasheet does not specify a case-to-heatsink thermal resistance, thus this project omits $\mathrm{R}_{\theta \mathrm{CS}}$ from Q_MAIN's heatsink thermal resistance calculation. Like IXYS's IXFK230N20T TO-264 power MOSFET, this project expects the IPP110N20N3's case-to-heatsink thermal resistance to be fairly small and thus be neglects it.

Equation (8.6) calculates DSNUB1's required heatsink thermal resistance, using Microsemi's APT30S20BG datasheet thermal values [61].

$$
R_{\theta H A}=\frac{150^{\circ} \mathrm{C}-40^{\circ} \mathrm{C}}{2 W}-0.58^{\circ} \mathrm{C}=54.42^{\circ} \mathrm{C} / \mathrm{W}
$$

Microsemi's APT30S20BG datasheet does not specify a case-to-heatsink thermal resistance, thus this project omits $\mathrm{R}_{\theta \mathrm{CS}}$ from DSNUB1's heatsink thermal resistance calculation. Like IXYS's IXFK230N20T TO-264 power MOSFET, this project expects the APT30S20BG's case-to-heatsink thermal resistance to be fairly small and thus neglects it.

For all diodes using Micro Commerical's MBR20100CT Schottky diode (D_MAIN1 to 4 and DSNUB2 to 4), Micro Commerical's datasheet does not specify either a junction-to-case thermal resistance or a case-to-heatsink thermal resistance [40]. This project omits $\mathrm{R}_{\theta \mathrm{CS}}$ from the heatsink thermal resistance calculation for such diodes, however this project still uses the typical TO-220-3 package transistor $3^{\circ} \mathrm{C} / \mathrm{W}$ thermal resistance value for $\mathrm{R}_{\theta J \mathrm{C}}[62]$. 
Equation (8.7) calculates D_MAIN1, D_MAIN2, D_MAIN3 and D_MAIN4's required heatsink thermal resistance.

$$
R_{\theta H A}=\frac{150^{\circ} \mathrm{C}-40^{\circ} \mathrm{C}}{3.5 W}-3^{\circ} \mathrm{C} / W=28.43^{\circ} \mathrm{C} / W
$$

Equation (8.8) calculates DSNUB2's required heatsink thermal resistance.

$$
R_{\theta H A}=\frac{150^{\circ} \mathrm{C}-40^{\circ} \mathrm{C}}{4 W}-3^{\circ} \mathrm{C} / W=24.5^{\circ} \mathrm{C} / W
$$

Equation (8.9) calculates DSNUB3 and DSNUB4's required heatsink thermal resistance.

$$
R_{\theta H A}=\frac{150^{\circ} \mathrm{C}-40^{\circ} \mathrm{C}}{4.1 W}-3^{\circ} \mathrm{C} / W=23.83^{\circ} \mathrm{C} / W
$$

After calculating component heatsink thermal resistance ratings, this project selects commercially available heatsinks that properly suit the SEPIC's semiconductor components as well as have lower than calculated thermal resistance ratings.

\subsection{Heatsink Selection and Component Temperature Recalculation}

For Q_OVP and Q_MAIN_ALT, this project selects Ohmite's MV-102-55E TO247/TO-264 heatsink. Figure 8.1 shows the heatsink's physical appearance [63]. 


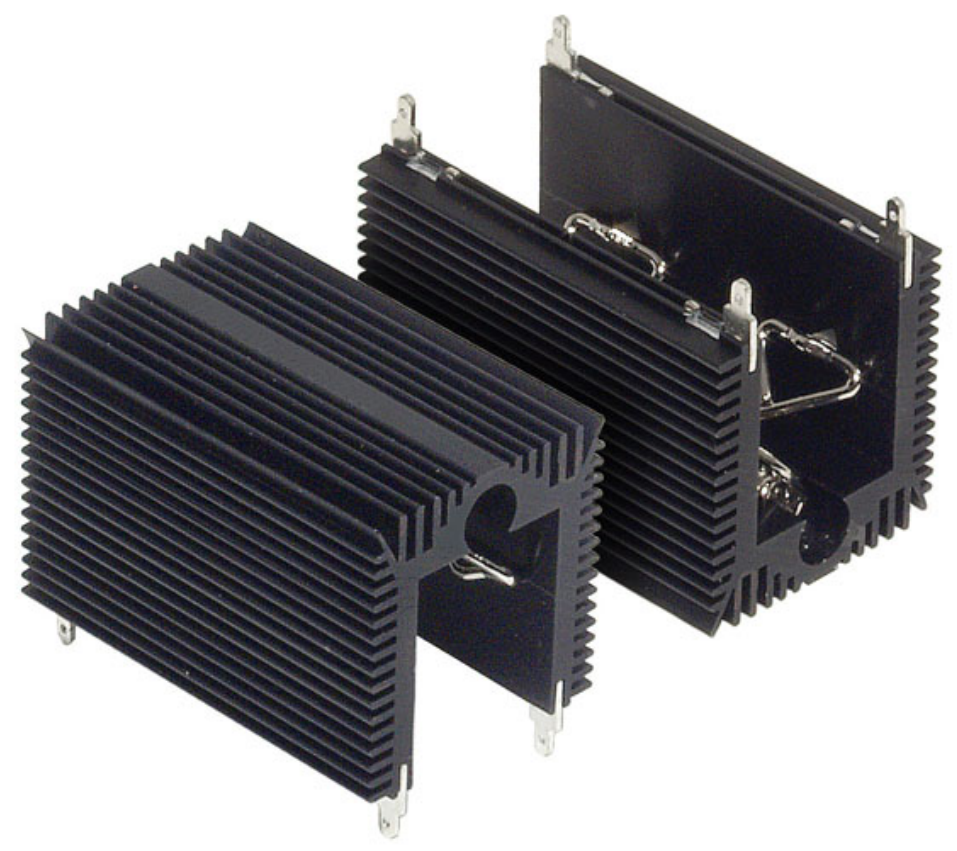

Figure 8.1: Ohmite MV-102-55E TO-247 and TO-264 Heatsink [63]

Ohmite's MV-102-55E heatsink dissipates heat from up to four simultaneous TO-247 or TO-264 components, and has a $6^{\circ} \mathrm{C} / \mathrm{W}$ natural thermal resistance [63]. When attached to a $400 \mathrm{LFM}$ active cooling device, its thermal resistance decreases to $2^{\circ} \mathrm{C} / \mathrm{W}$ [63]. Even without an attached active cooling device, the MV-102-55E's thermal resistance specification is much lower than the required calculated thermal resistance for Q_OVP and Q_MAIN_ALT under the highest stress operating conditions, thus it is reasonable for those components. The smaller version of this heatsink that holds only one component, the MV-xxx-27E series, has a similar natural thermal resistance rating and is more ideal for this project's SEPIC. However, the MV-xxx-27E was unavailable on Digi-Key when 
this project created its SEPIC's bill of materials, thus this project uses the larger $-55 \mathrm{E}$ heatsink instead.

For Q_MAIN, this project selects Aavid Thermalloy’s 531202B02500G TO-2203 heatsink. Figure 8.2 shows the heatsink's physical appearance [64].

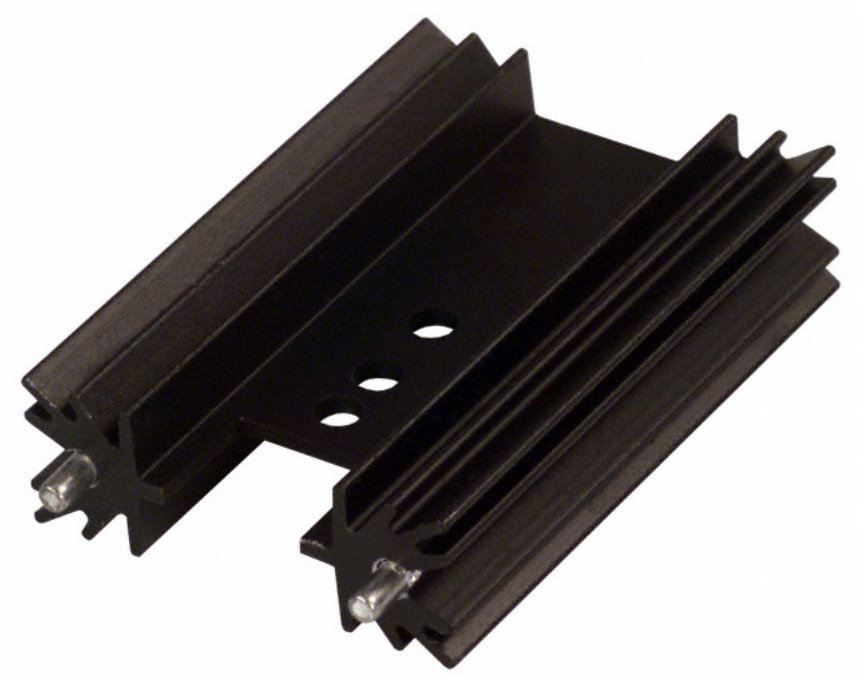

Figure 8.2: Aavid Thermalloy 531202B02500G TO-202 and TO-220 Heatsink [64]

Aavid Thermalloy's 531202B02500G heatsink supports TO-202 and TO-220

components and has a $7.5^{\circ} \mathrm{C} / \mathrm{W}$ natural thermal resistance [64]. When attached to a 600

LFM active cooling device, its thermal resistance decreases to $4^{\circ} \mathrm{C} / \mathrm{W}$ [64]. Likewise with the MV-102-55E used for Q_OVP and Q_MAIN_ALT, the 531202B02500G's thermal resistance specification without any active cooling is much lower than the required calculated thermal resistance for Q_MAIN under the highest stress operating conditions, thus it is reasonable for Q_MAIN. 
This project selects Ohmite's WA-T247-101E TO-247 heatsink for DSNUB1.

Figure 8.3 shows the heatsink's physical appearance [65].

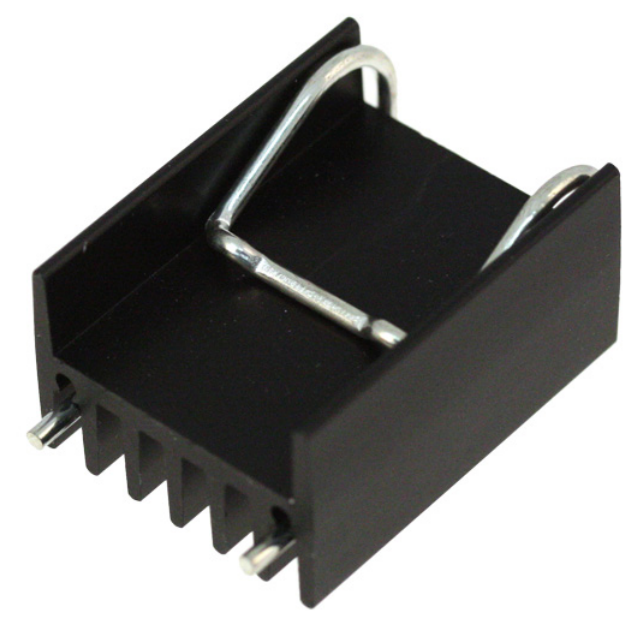

Figure 8.3: Ohmite WA-T247-101E TO-247 Heatsink [65]

Ohmite WA-T247-101E heatsink supports TO-247 components and has a $7^{\circ} \mathrm{C} / \mathrm{W}$ natural thermal resistance [65]. When attached to a 500 LFM active cooling device, its thermal resistance oddly increases to $8^{\circ} \mathrm{C} / \mathrm{W}$ [65]. Thus, active cooling is not required for the WA-T247-101E and in many cases adding active cooling worsens thermal performance. Its natural thermal resistance is much lower than the required calculated thermal resistance for DSNUB1 under the highest stress operating conditions, thus it is reasonable for DSNUB1.

For the remaining D_MAIN and DSNUB diodes, this project selects Aavid Thermalloy's 574502B00000G TO-220-3 heatsink. Figure 8.4 shows the heatsink's physical appearance [66]. 


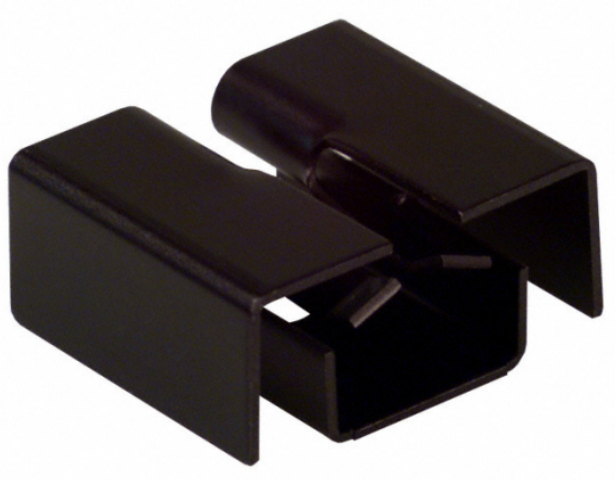

Figure 8.4: Aavid Thermalloy 574502B00000G TO-220 Heatsink [66]

Aavid Thermalloy's 574502B00000G heatsink cools TO-220 components and has a 21. $2^{\circ} \mathrm{C} / \mathrm{W}$ natural thermal resistance. When attached to a $400 \mathrm{LFM}$ active cooling device, its thermal resistance oddly decreases to $8^{\circ} \mathrm{C} / \mathrm{W}$ [66]. Although this heatsink has the worst thermal performance out of all the selected ones, its installation process is simple and it does not require any additional clips, bolts or screws. Its natural thermal resistance is still lower than the required calculated thermal resistance for the DMAIN1-4 diodes and DSNUB2-4 diodes under the highest stress operating conditions, thus it is reasonable for those components.

This project now determines each component's operating junction temperature by using (8.10) for recalculating maximum junction temperatures. (8.10) uses thermal resistance ratings gathered from the selected heatsinks.

$$
T_{J M A X}=P_{M A X}\left(R_{\theta J C}+R_{\theta C S}+R_{\theta H A}\right)+T_{A}
$$

Equation (8.11) calculates Q_OVP's maximum junction temperature with the Ohmite MV-102-55E heatsink.

$$
T_{J M A X}=12 W(0.09+0.15+6)+40^{\circ} \mathrm{C}=114.88^{\circ} \mathrm{C}
$$


Equation (8.12) calculates Q_MAIN_ALT's maximum junction temperature with the Ohmite MV-102-55E heatsink.

$$
T_{J M A X}=6 W(0.09+0.15+6)+40^{\circ} \mathrm{C}=77.44^{\circ} \mathrm{C}
$$

Equation (8.13) calculates Q_MAIN's maximum junction temperature with Aavid Thermalloy's 531202B02500G heatsink.

$$
T_{J M A X}=6 W(0.5+7.5)+40^{\circ} \mathrm{C}=88^{\circ} \mathrm{C}
$$

Equation (8.14) calculates DSNUB1's maximum junction temperature with the Ohmite WA-T247-101E heatsink.

$$
T_{J M A X}=2 W(0.58+7)+40^{\circ} \mathrm{C}=55.16^{\circ} \mathrm{C}
$$

Equation (8.15) calculates D_MAIN1, D_MAIN2, D_MAIN3 and D_MAIN4's maximum junction temperature with Aavid Thermalloy's 574502B00000G heatsink.

$$
T_{J M A X}=3.5 W(3+21.2)+40^{\circ} \mathrm{C}=124.7^{\circ} \mathrm{C}
$$

Equation (8.16) calculates DSNUB2's maximum junction temperature with Aavid Thermalloy's 574502B00000G heatsink.

$$
T_{J M A X}=4 W(3+21.2)+40^{\circ} \mathrm{C}=136.8^{\circ} \mathrm{C}
$$

Equation (8.17) calculates DSNUB3 and DSNUB4's maximum junction temperature with Aavid Thermalloy's 574502B00000G heatsink.

$$
T_{\text {JMAX }}=4.1 W(3+21.2)+40^{\circ} \mathrm{C}=139.22^{\circ} \mathrm{C}
$$

Snubber diodes DSNUB2, DSNUB3 and DSNUB4 operate close to their maximum specified junction temperatures $\left(150^{\circ} \mathrm{C}\right)$ under full load conditions, however, this project selected their heatsinks for saving physical space. Saving physical space reduces 
converter costs by preventing the SEPIC's PCB from becoming too physically large.

Each selected heatsink suffices for properly cooling the SEPIC's semiconductor components and help allow for prolonged SEPIC use. After selecting all the heatsinks for its SEPIC, this project can now design its SEPIC's PCB layout. 


\section{CHAPTER 9: PCB LAYOUT}

\subsection{SEPIC First Revision PCB Layout Design}

Poor PCB layouts jeopardize the functionality of well-designed circuits. The PCB layout for this project's SEPIC followed design guidelines outlined in [67-70] for suppressing any undesirable side-effects such as capacitive crosstalk or resonance. This project's SEPIC's PCB also keeps high power and high frequency switching components such as diodes, MOSFETs and inductors physically as far away as possible from the lowpower control components (e.g. the LTC1871 controller). Figure 9.1 shows a boost converter layout example featured in Linear Technology's LTC1871 datasheet [35].

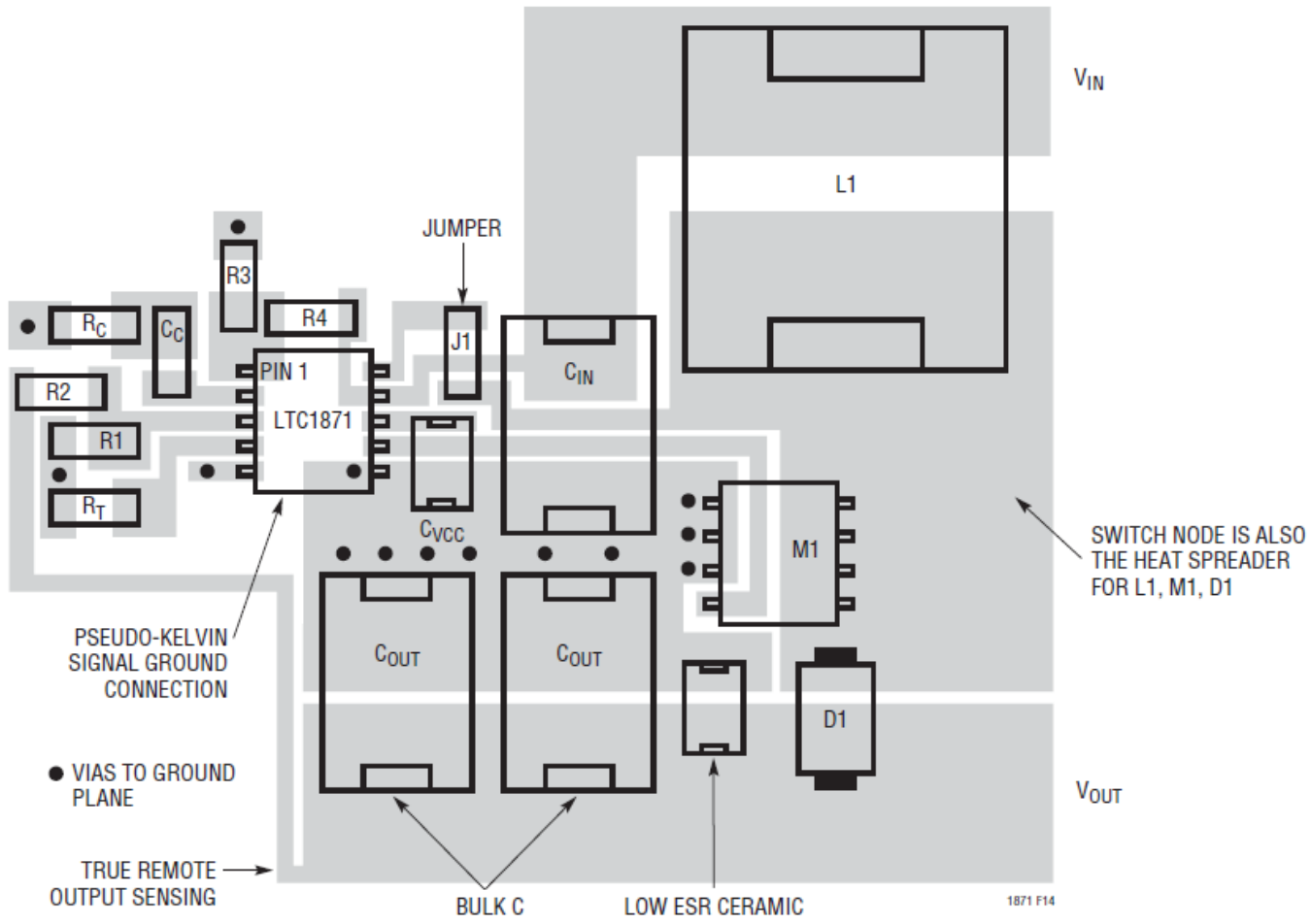

Figure 9.1: Linear Technology's Suggested LTC1871 Boost Converter Layout [35]

Because a SEPIC's overall layout is very similar to that of a boost converter [26, 27], this project uses figure 9.1 as a guideline for determining this project's SEPIC's component 
and trace placement. The SEPIC's first revision PCB layout is similar to that of Linear Technology's suggested LTC1871 boost converter layout in figure 9.1. However, this project reverses the SEPIC's physical input voltage $\left(\mathrm{V}_{\mathrm{IN}}\right)$ location (it is on the left side instead of the right side of the PCB as in figure 9.1) for accommodating the LT4356-1 input protection circuit. This project also minimizes PCB manufacturing costs by manufacturing its SEPIC layout on a two-layer PCB without any silkscreen or solder masking. Silkscreen and solder masking are necessary for a production level or retail SEPIC board for easing troubleshooting as well as for providing additional safety to the end user (from the lack of exposed pads). However, this project does not require silkscreen and solder masking for a prototyping board, which is the PCB type this project uses and tests with the rest of the SEPIC components. Figure 9.2 shows this project's SEPIC first revision PCB layout: 


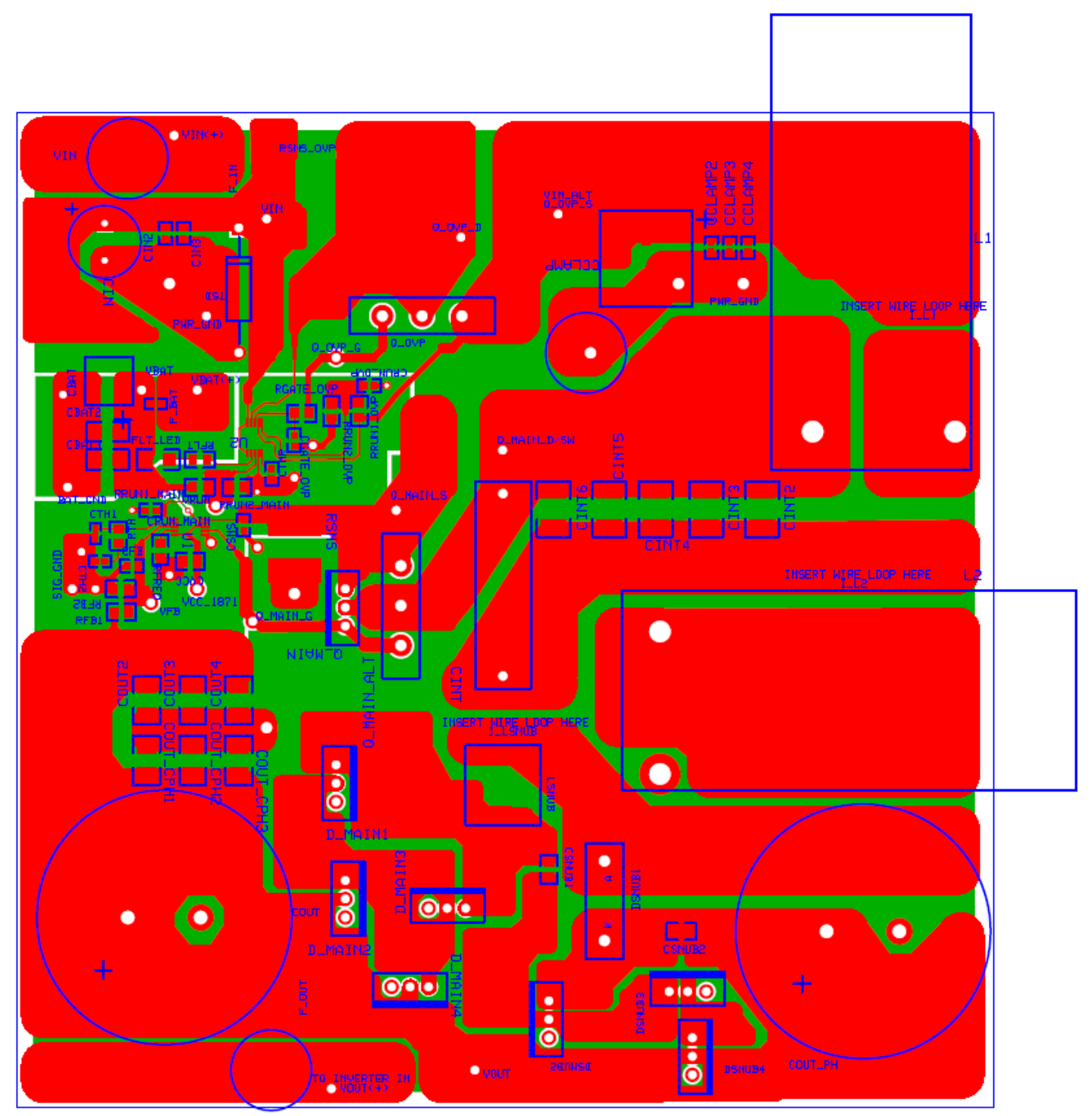

Figure 9.2: SEPIC First Revision PCB Layout

Red designates the PCB's top copper layer while green designates its bottom copper layer. Blue designates its silkscreen layer, but does not show up on the manufactured PCB. Because of this, $\mathrm{L}_{1}$ and $\mathrm{L}_{2}$ can freely extend past the PCB's physical borders, as the Vishay IHV28BZ60 physically stands upward. Figures 9.3, 9.4 and 9.5 show the SEPIC's individual top and bottom copper and silkscreen layers. 


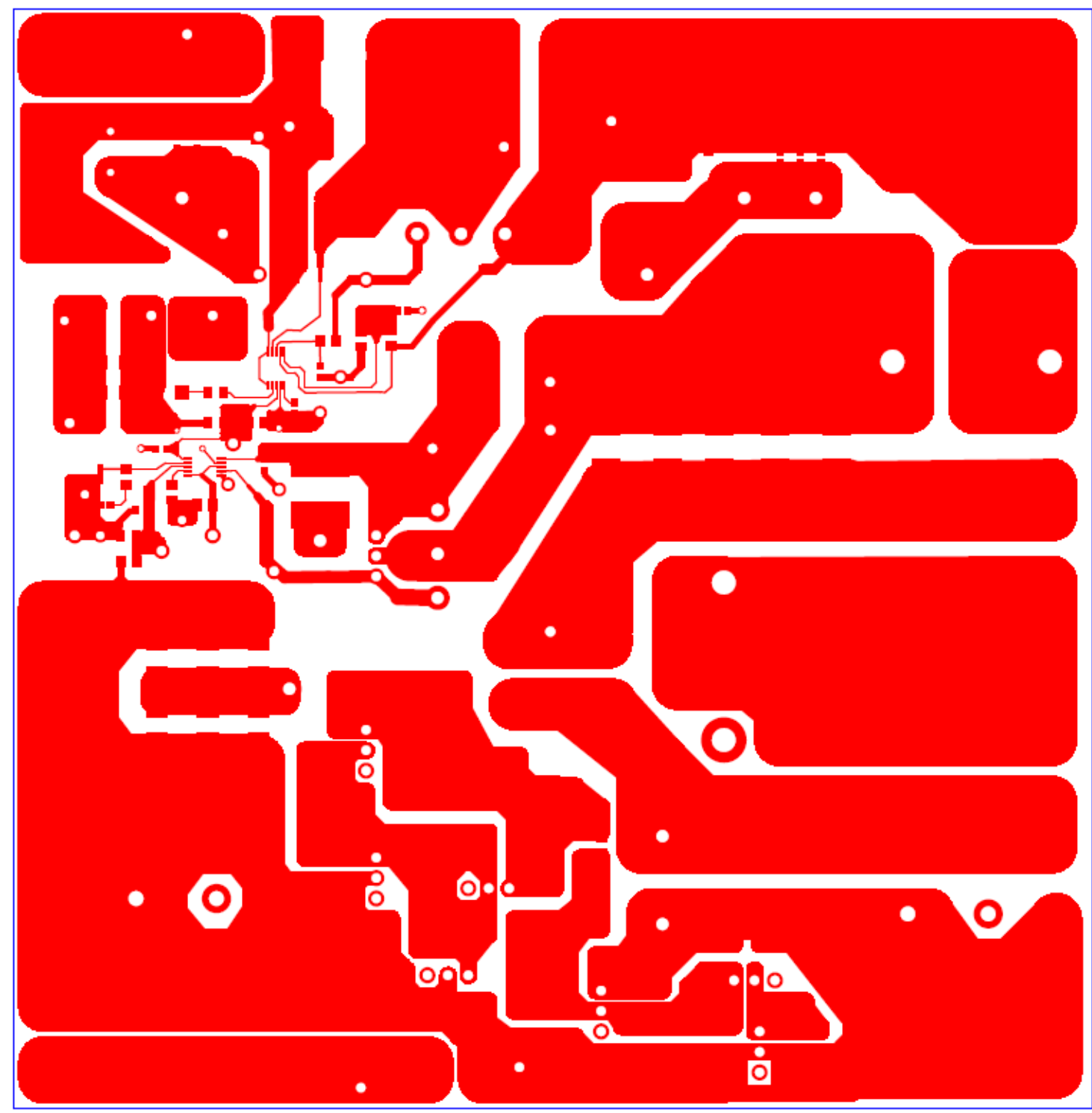

Figure 9.3: SEPIC First Revision PCB Layout Top Copper Layer 


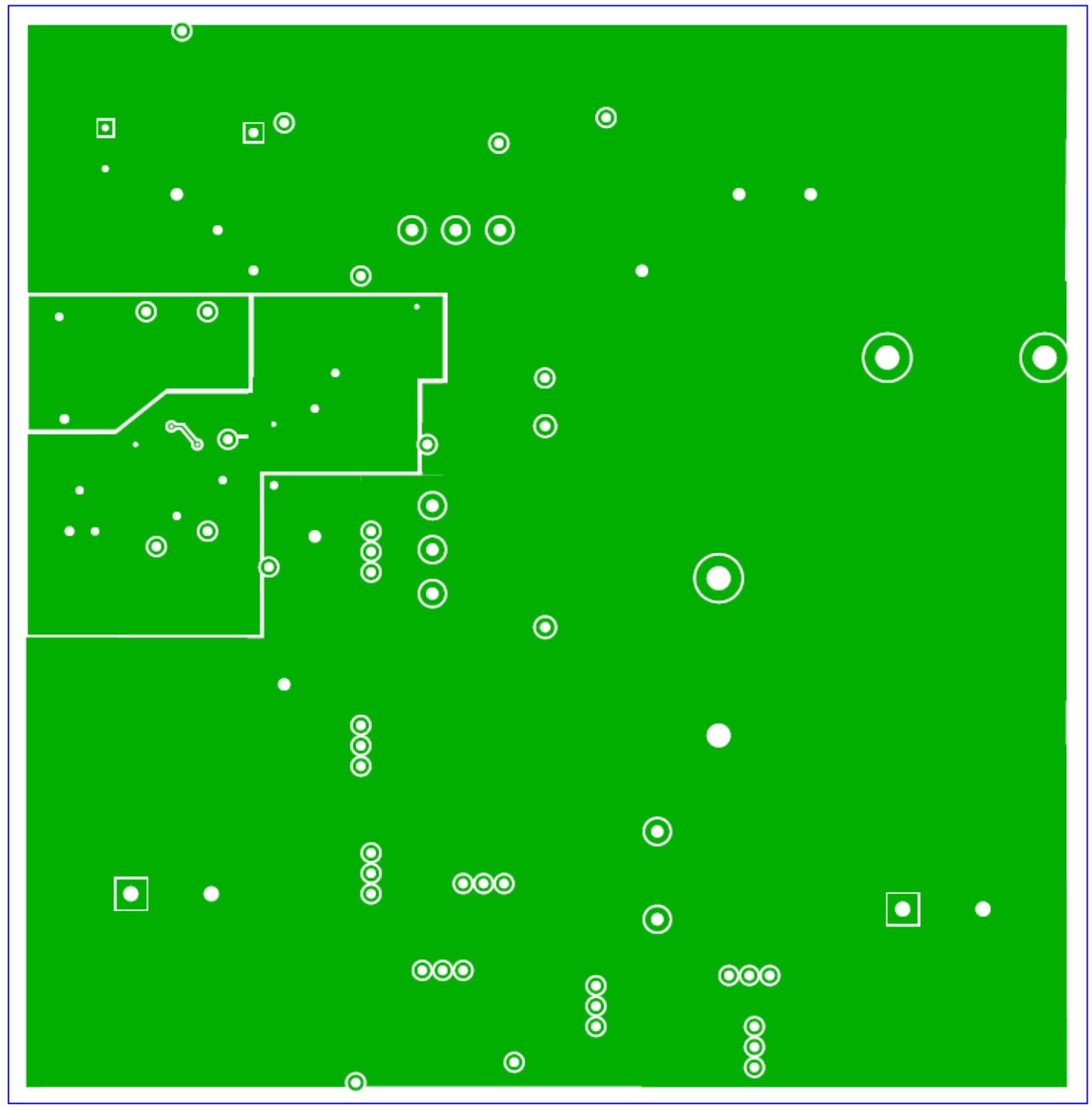

Figure 9.4: SEPIC First Revision PCB Layout Bottom Copper Layer 


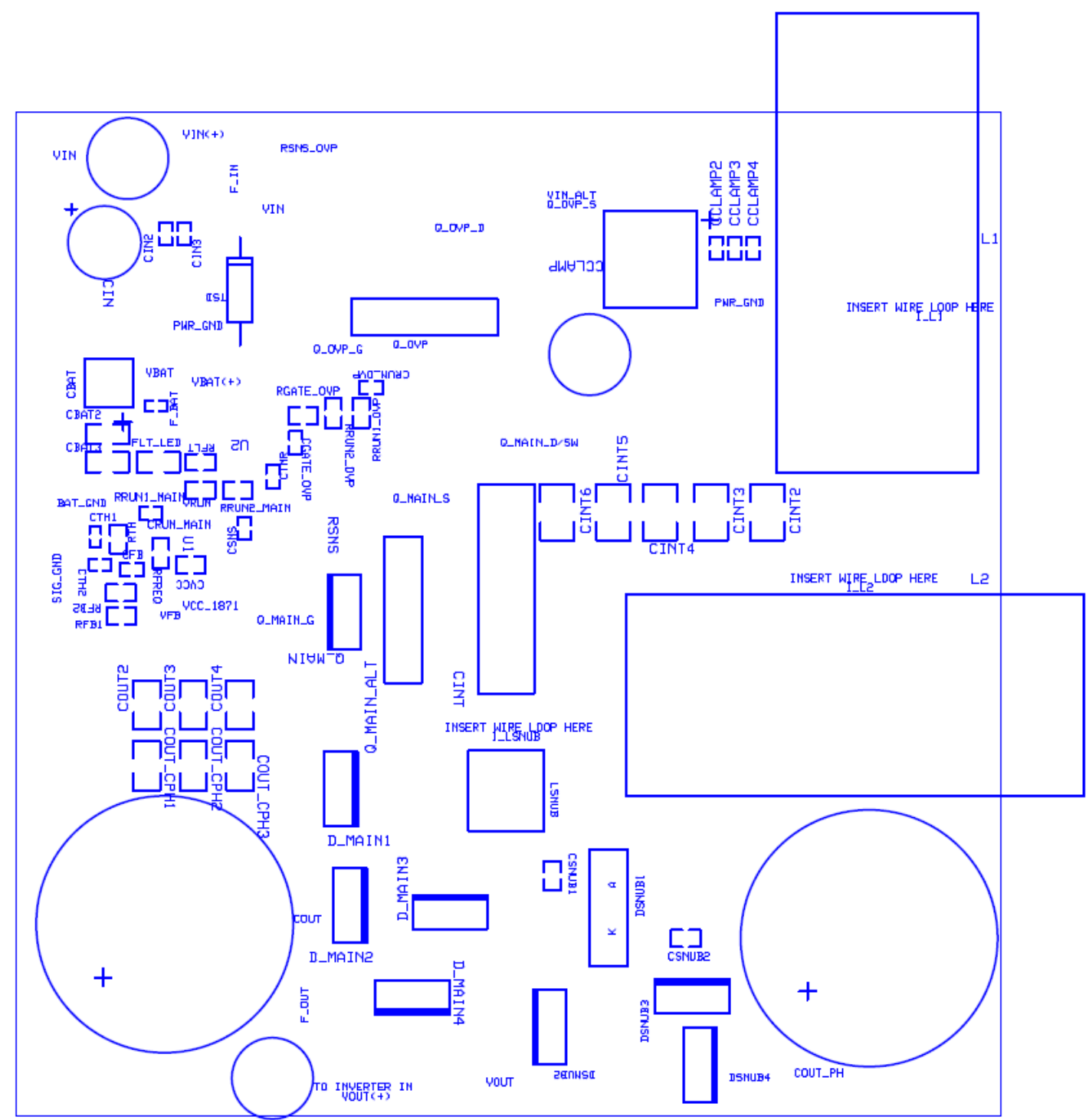

Figure 9.5: SEPIC First Revision PCB Layout Silkscreen Layer

The PCB's bottom copper layer consists almost entirely of solid ground planes, with separate planes for the elliptical battery, low-power control circuit components such as the LTC1871 controller and high power components such as $\mathrm{L}_{2}$. This preliminary layout does not yet have each ground plane bridged together. The top copper layer consists of narrow ( 0.010 " to 0.020 " width) traces for the control circuit components and wide traces (0.200" and wider) for the high power components. The high power components require 
wide traces for effective high current flow capability, namely at the SEPIC's input, output and switching (Q_MAIN and $\mathrm{C}_{\mathrm{INT}}$ connection) nodes. Additionally, the PCB also contains holes for mounting test points so that this project's author can easily measure critical node voltages. The silkscreen layer also labels each test point; for example, Q_MAIN_D corresponds to the test point located at Q_MAIN's drain node. This project, however, would remove these test point mounting holes in a final production version of its SEPIC's PCB, as the test points are only necessary for PCB prototyping and testing purposes. Inductor current paths contain break points for inserting wire loops for easing the inductor current measurement process. This PCB contains wire loop insertion points at $\mathrm{L}_{1}, \mathrm{~L}_{2}$ and $\mathrm{L}_{\mathrm{SNUB}}$ 's inputs. This layout also provides additional placeholder footprints for additional output capacitors. Furthermore, it also provides a placeholder footprint for Q_MAIN_ALT, in case the IPP110N20N3 power MOSFET used for Q_MAIN is not suitable for this SEPIC during hardware testing. The layout also contains break points at the SEPIC's elliptical input, $12 \mathrm{~V}$ battery input and output nodes for soldering fuses, in order to abide by UL, IEEE, PG\&E and NEC safety requirements.

One major flaw with this preliminary layout is that it neglects heatsink spacing as well as chassis mounting holes and voltage source connection points with the proper mechanical dimensions. Furthermore, this layout revision is also missing $\mathrm{R}_{\mathrm{VCC} 1}$ and $\mathrm{R}_{\mathrm{VCC}}$ 's footprints. This project, however, did not consider inserting $\mathrm{R}_{\mathrm{VCC} 1}$ and $\mathrm{R}_{\mathrm{VCC} 2}$ into the SEPIC's schematic until the PCB layout's second revision. The SEPIC's second revision layout addresses these problems by including heatsink footprints in its silkscreen 
layer, $\mathrm{R}_{\mathrm{VCC} 1}$ and $\mathrm{R}_{\mathrm{VCC} 2}$ footprints and chassis mounting holes and holes for inserting banana binding posts to crucial voltage source, load and ground connection points.

\subsection{SEPIC Second Revision PCB Layout Design}

The SEPIC's second revision PCB layout includes all proper heatsink footprints, semiconductor components and chassis mounting holes and device connection points. Present are eight chassis mounting holes located on the PCB corners and edges. Each chassis mounting hole has a $0.125 "$ diameter and supports \#5-40 and \#5-44 size screws [71]. Some other major differences with the SEPIC's second revision PCB layout (compared to its first revision) are that this revision replaces wide power path traces with solid copper planes for trace editing ease, and widens physically long low power (small signal traces) at their power component connection end points. Such traces are 0.030 ” and wider for minimizing parasitic resistance caused by long narrow trace lengths. Figure 9.6 shows the SEPIC's second revision PCB layout. 


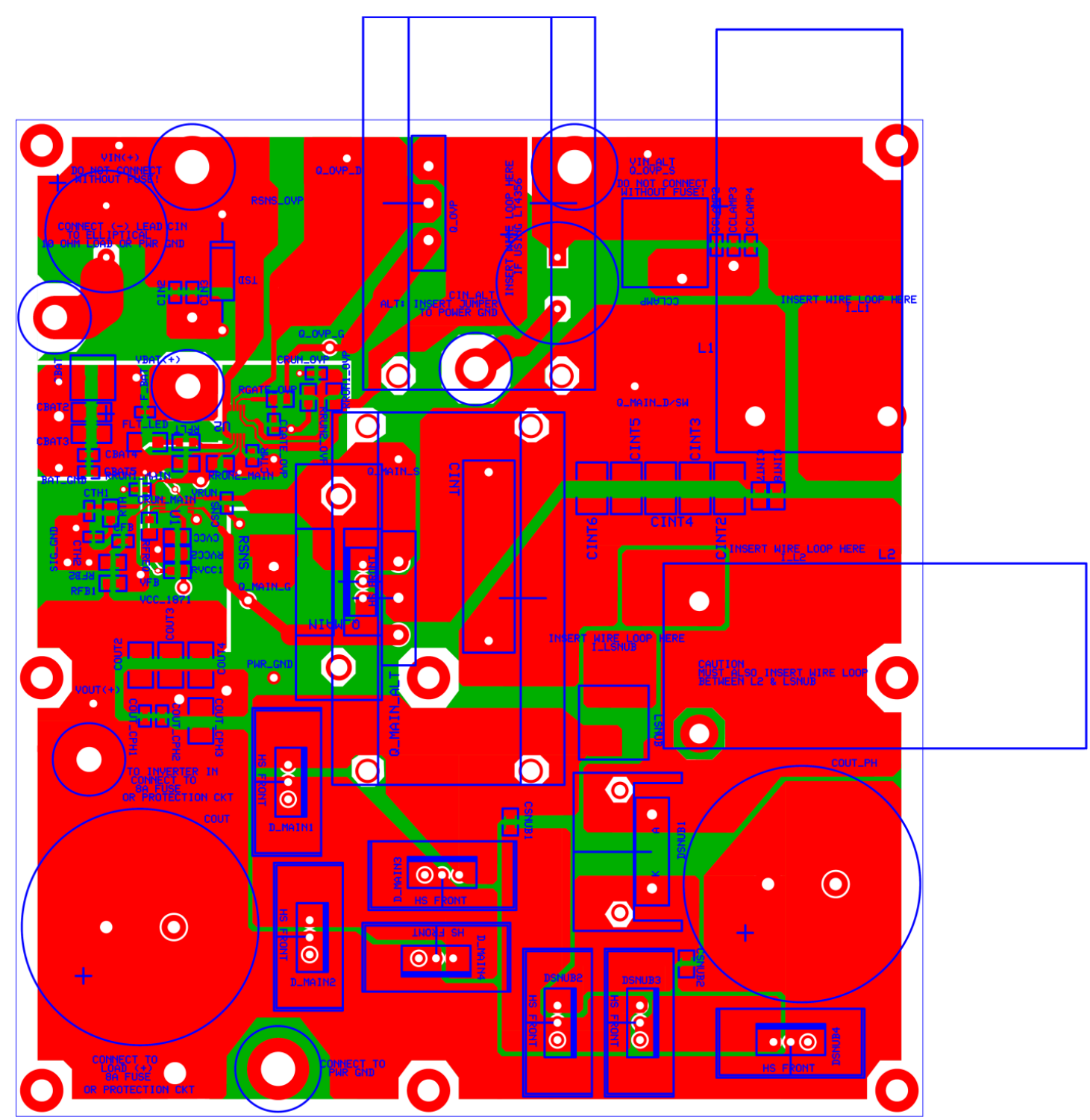

Figure 9.6: SEPIC Second Revision PCB Layout

This layout revision includes footprints for all components from the finalized SEPIC schematic in figure 7.9, as well as heatsink mounting holes and connection points for voltage sources and electronic loads. Additionally, this layout also includes a break point at the LT4356-1 protection circuit's output (as well as an alternate $\mathrm{C}_{\mathrm{IN}}$ connection point) in case it ever requires bypassing for troubleshooting purposes during the hardware testing process. The layout also retains inductor break points for soldering wire loops. 
Furthermore, this layout also contains a connection point for connecting $\mathrm{C}_{\mathrm{IN}}$ in series with the Precor elliptical trainer's $10 \Omega$ resistor coils, for reducing RMS current as this report denotes in section 7.5. For synthetic hardware tests (using a power supply as the SEPIC's main input voltage source instead of the elliptical), this project uses a solderable jumper for connecting $\mathrm{C}_{\mathrm{IN}}$ 's negative terminal to ground. The Q_MAIN and Q_MAIN_ALT heatsink footprints overlap each other because for proper operation, this SEPIC would only use either transistor, but not both simultaneously. The productionlevel PCB layout would obviously remove one of the footprints depending on which transistor yields better hardware test results. This layout revision also removes solderable fuse connection points at the elliptical input and output nodes, as for fuse replacement ease this project decided on using external blade fuses and blade fuse holders connected to those nodes. Therefore, no one should ever connect this board to a voltage source or load without connecting blade fuses to the elliptical input and output nodes. Although the LT4356-1 circuit protects the SEPIC's input, one major flaw that this project realized late into implementation is that there this converter does not have any output protection (such as an output current limiter circuit). Therefore this SEPIC requires fuses, not only to conform to UL, IEEE, PG\&E and NEC safety requirements, but also for end-user safety. This layout still requires a solderable fuse for the $12 \mathrm{~V}$ battery input node, however. Figures 9.7, 9.8 and 9.9 show the second revision SEPIC's individual top and bottom copper and silkscreen layers: 


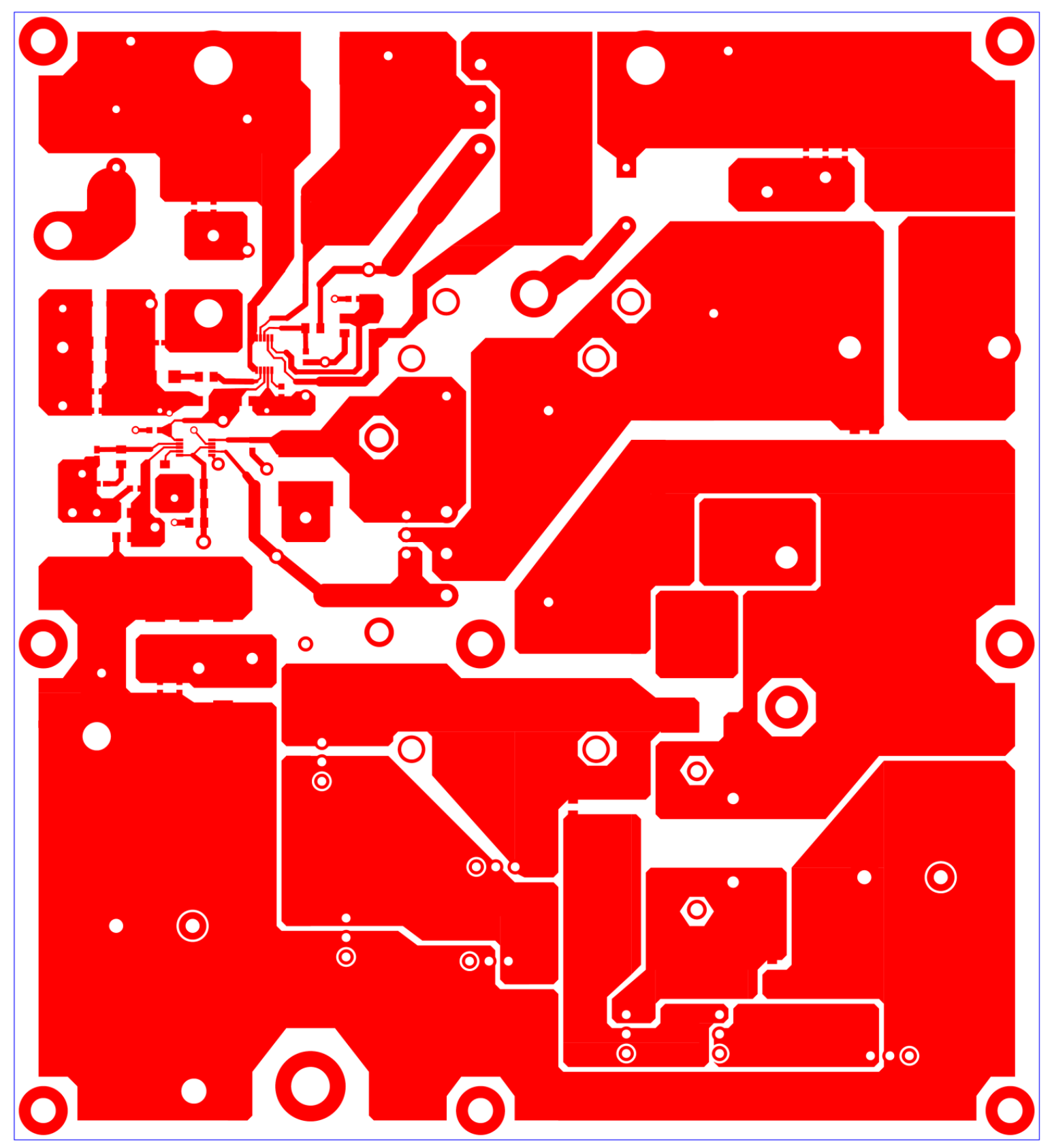

Figure 9.7: SEPIC Second Revision PCB Layout Top Copper Layer 


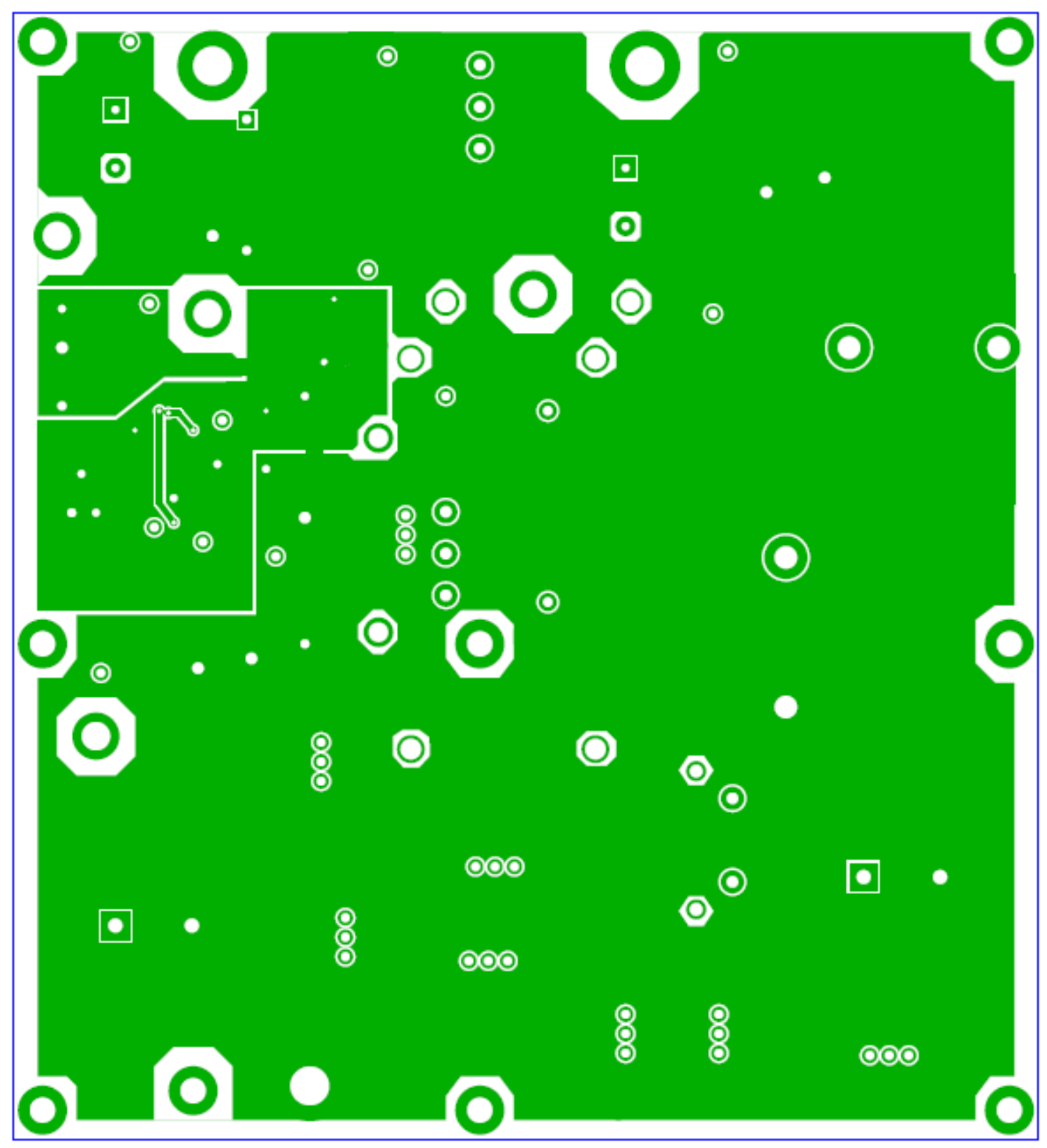

Figure 9.8: SEPIC Second Revision PCB Layout Bottom Copper Layer 


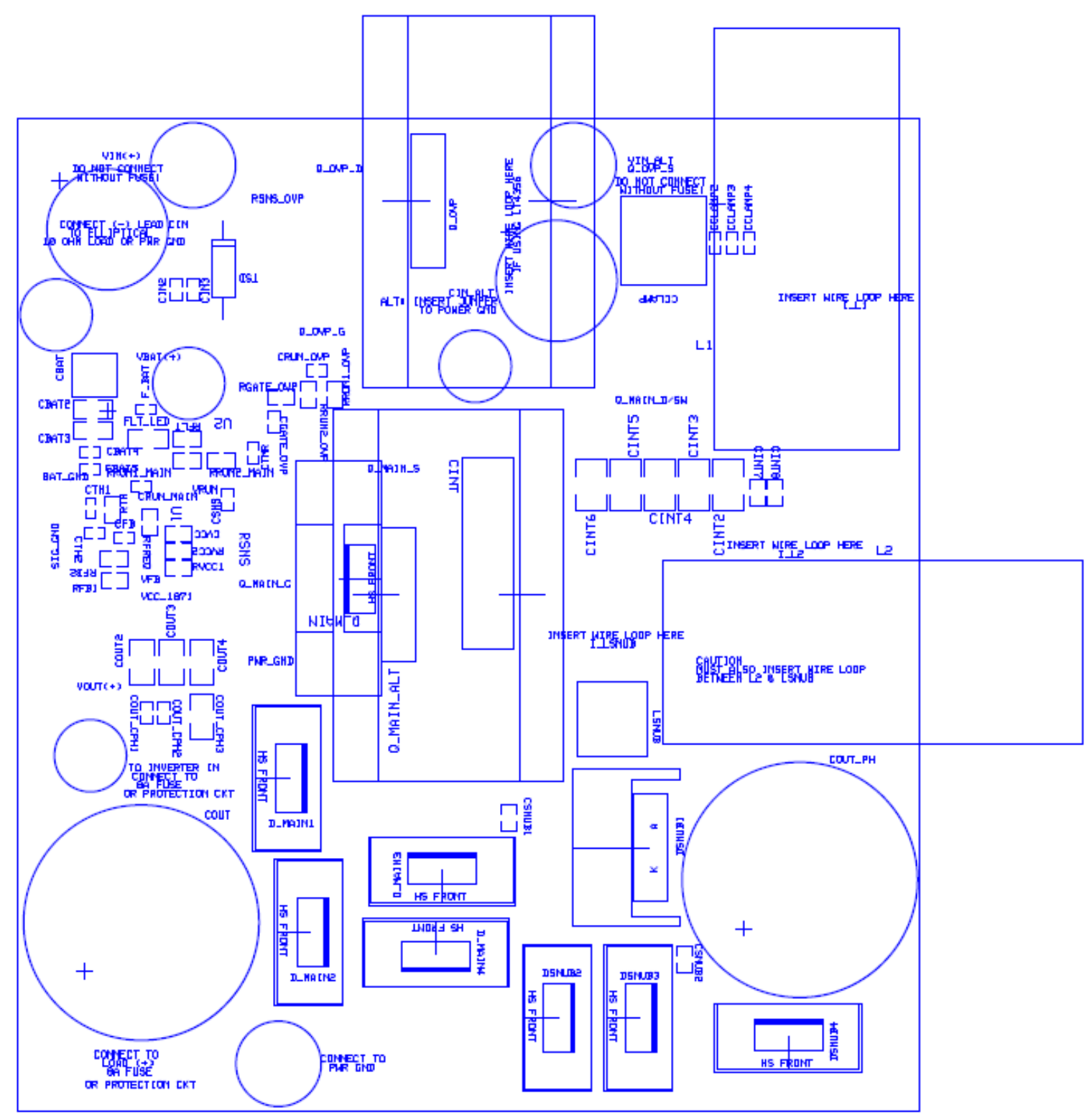

Figure 9.9: SEPIC Second Revision PCB Layout Silkscreen Layer

This second revision PCB layout also bridges each ground plane together, based on component return current flow direction.

Likewise with $\mathrm{L}_{1}$ and $\mathrm{L}_{2}$, Q_OVP's heatsink can freely extend beyond the PCB's physical borders, as it physically stands upward and only requires two mounting holes for 
physical security. Furthermore, as mentioned in section 9.1, the prototype PCB used for this SEPIC's hardware testing does not have the silkscreen layer.

This second revision board also measures 5.275" $\times 5.800 "$, corresponding to a 30.595 in. $^{2}$ area. It is quite large compared to the previously used off-the-shelf Vicor Maxi 28V DC-DC converter (which is $4.60 " \times 2.20$ "), but is more functional with the EHFEM system than the Vicor converter. This PCB also costs $\$ 124.84$ for two boards, with $\$ 84.24$ for a single board. This results in a $\$ 259.07$ total converter cost, as table D-1 shows. Again, it is more expensive even without a chassis when compared to the Vicor Maxi 28V DC-DC converter (which costs \$208.00 [1]), but again, its functionality compensates for its cost. Table D-1 shows this finalized SEPIC's BOM with the PCB and heatsink costs included. 
Table D-1: Finalized SEPIC BOM with PCB and Heatsink Costs

\begin{tabular}{|c|c|c|c|c|c|c|c|}
\hline PCBs & Quantity: & $\begin{array}{l}\text { Unit Price } \\
\text { (\$): }\end{array}$ & Total Price: & Components: & Type: & \begin{tabular}{|c|}
$65 V, 8 A$ Required \\
Rating
\end{tabular} & $5 \mathrm{~V}, 0.5 \mathrm{~A}$ \\
\hline Custom 2-layer 5.8" × 5.275" & 1 & 84.42 & 84.42 & PCB & 2-layer & $\mathrm{N} / \mathrm{A}$ & $\mathrm{N} / \mathrm{A}$ \\
\hline \multicolumn{3}{|c|}{ Total Cost (\$) } & 84.42 & & & & \\
\hline Controller I.C.s: & Quantity: & $\begin{array}{l}\text { Unit Price } \\
\text { (\$): }\end{array}$ & Total Price: & Components: & Type: & $\begin{array}{c}\text { 65V, 8A Required } \\
\text { Rating }\end{array}$ & $5 \mathrm{~V}, 0.5 \mathrm{~A}$ \\
\hline LTC1871HMS (10-MSOP) & 1 & 4.83 & 4.83 & U1 & 10-MSOP & $\mathrm{N} / \mathrm{A}$ & $\mathrm{N} / \mathrm{A}$ \\
\hline LT4356-1HMS (10-MSOP) & 2 & 3.70 & 7.40 & U2 & 10-MSOP & $\mathrm{N} / \mathrm{A}$ & $\mathrm{N} / \mathrm{A}$ \\
\hline \multicolumn{3}{|c|}{ Total Cost (\$) } & 12.23 & & & & \\
\hline Transistors: & Quantity: & $\begin{array}{l}\text { Unit Price } \\
\text { (\$): }\end{array}$ & Total Price: & Components: & Type: & $\begin{array}{c}\text { 65V, 8A Required } \\
\text { Rating }\end{array}$ & $5 \mathrm{~V}, 0.5 \mathrm{~A}$ \\
\hline Infineon IPP110N20N3 & 1 & 8.06 & 8.06 & Q_MAIN & TO-220-3 & $2.45 \mathrm{~W}$ & $4.01 \mathrm{~W}$ \\
\hline IXYS IXFK230N20T & 1 & 9.50 & 9.50 & Q_OVP & TO-264 & $1.625 \mathrm{~kW}$ & $1.625 \mathrm{~kW}$ \\
\hline \multicolumn{3}{|c|}{ Total Cost (\$) } & 17.56 & & & & \\
\hline Diodes: & Quantity: & $\begin{array}{l}\text { Unit Price } \\
\text { (\$): }\end{array}$ & Total Price: & Components: & Type: & $\begin{array}{c}\text { 65V, 8A Required } \\
\text { Rating }\end{array}$ & $5 \mathrm{~V}, 0.5 \mathrm{~A}$ \\
\hline MBR20100CT (Schottky) & 7 & 0.88 & 6.16 & $\begin{array}{c}\text { D_MAIN1, D_MAIN2, D_MAIN3, } \\
\text { D_MAIN4, DSNUB2, DSNUB3, } \\
\text { DSNUB4 }\end{array}$ & TO-220-3 & $31.11 \mathrm{~A}, 110 \mathrm{~V}$ & $27.72 \mathrm{~A}, 56.46 \mathrm{~V}$ \\
\hline Microsemi APT30S20BG (Schottky) & 1 & 3.67 & 3.67 & DSNUB1 & TO-247 & $39.43 \mathrm{~A}, 15.95 \mathrm{~V}$ & $27.89 \mathrm{~A}, 9.58 \mathrm{~V}$ \\
\hline 1N5378B (Zener) & 1 & 0.57 & 0.57 & TSD & Through-Hole & $100 \mathrm{~V}$ & $100 \mathrm{~V}$ \\
\hline SML-LX1206SRC-TR (LED) & 1 & 0.43 & 0.43 & FLT_LED & 1206 & $\begin{array}{c}1.2 \mathrm{~V} \text { min. forward } \\
\text { drop }\end{array}$ & $\begin{array}{c}1.2 \mathrm{~V} \text { min. forward } \\
\text { drop }\end{array}$ \\
\hline \multicolumn{3}{|c|}{ Total Cost (\$) } & 10.83 & & & & \\
\hline Heatsinks: & Quantity: & $\begin{array}{l}\text { Unit Price } \\
\text { (\$): }\end{array}$ & Total Price: & Components: & Type: & $\begin{array}{c}\text { 65V, 8A Required } \\
\text { Rating }\end{array}$ & $5 \mathrm{~V}, 0.5 \mathrm{~A}$ \\
\hline 531202B02500G (TO-220) & 1 & 1.38 & 1.38 & Q_MAIN heatsink & TO-220 & $\mathrm{N} / \mathrm{A}$ & $\mathrm{N} / \mathrm{A}$ \\
\hline MV-102-55E (TO-247 and TO-264) & 1 & 7.39 & 7.39 & Q_OVP heatsink & TO-264 & $\mathrm{N} / \mathrm{A}$ & $\mathrm{N} / \mathrm{A}$ \\
\hline WA-T247-101E (TO-247) & 1 & 2.07 & 2.07 & DSNUB1 heatsink & TO-247 & $\mathrm{N} / \mathrm{A}$ & $\mathrm{N} / \mathrm{A}$ \\
\hline 574502B00000G (TO-220) & 7 & 0.27 & 1.89 & $\begin{array}{c}\text { D_MAIN1, D_MAIN2, D_MAIN3, } \\
\text { D_MAIN4, DSNUB2, DSNUB3, } \\
\text { DSNUB4 }\end{array}$ & TO-220 & $\mathrm{N} / \mathrm{A}$ & $\mathrm{N} / \mathrm{A}$ \\
\hline \multicolumn{3}{|c|}{ Total Cost (\$) } & 12.73 & & & & \\
\hline
\end{tabular}


Table D-1 (Continued)

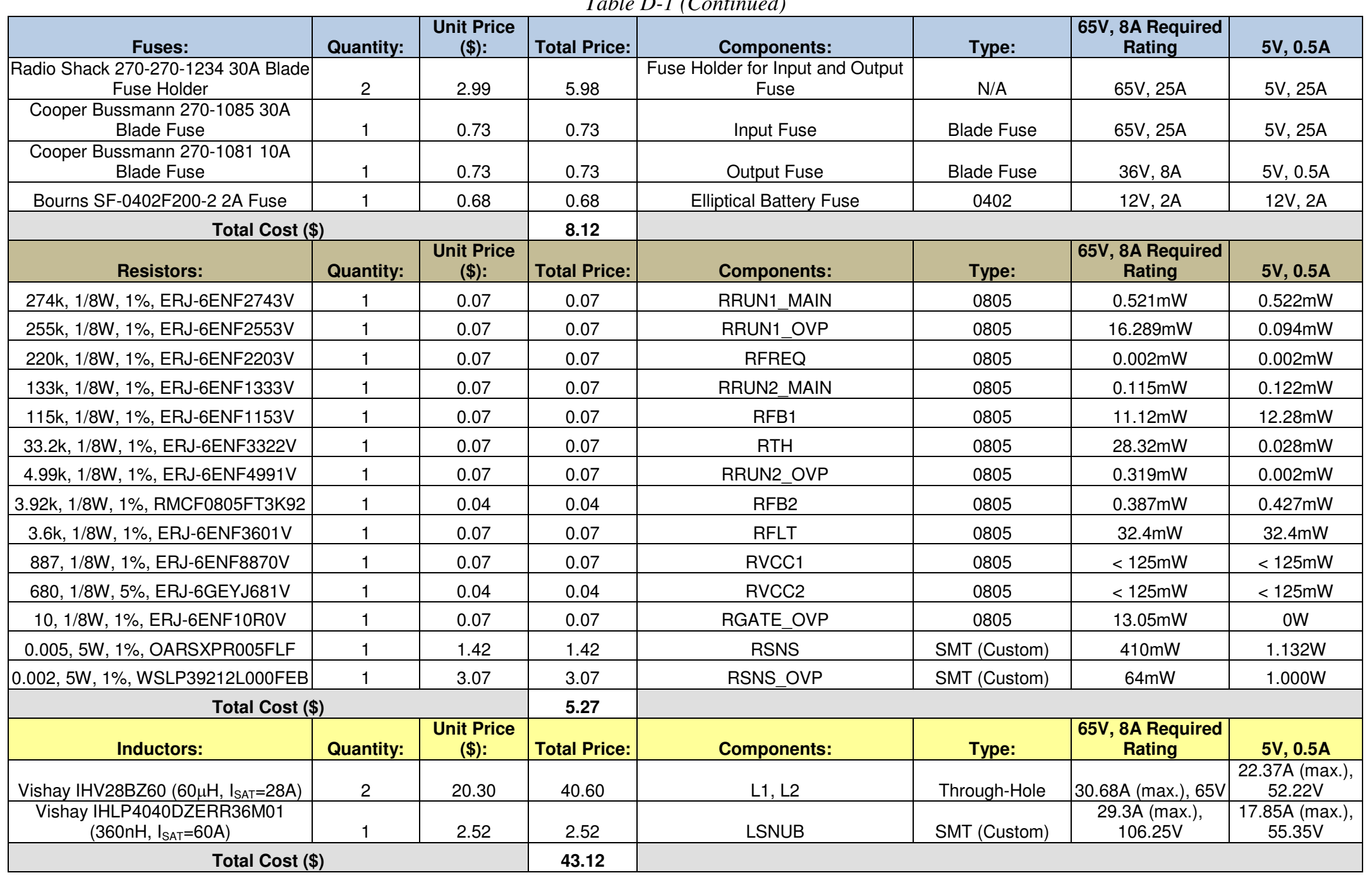


Table D-1 (Continued)

\begin{tabular}{|c|c|c|c|c|c|c|c|}
\hline Capacitors: & Quantity: & $\begin{array}{l}\text { Unit Price } \\
\text { (\$): }\end{array}$ & Total Price: & Components: & Type: & $\begin{array}{c}\text { 65V, 8A Required } \\
\text { Rating }\end{array}$ & $5 \mathrm{~V}, 0.5 \mathrm{~A}$ \\
\hline $\begin{array}{c}470 \mu, \text { Electrolytic, } 400 \mathrm{~V}, \\
\text { B43504A9477M }\end{array}$ & 1 & 13.34 & 13.34 & COUT & Through-Hole & $36 \mathrm{~V}, 5.43 \mathrm{~A}(\mathrm{RMS})$ & $36 \mathrm{~V}, 2.368 \mathrm{~A}(\mathrm{RMS})$ \\
\hline $\begin{array}{c}1000 \mu, \text { Electrolytic, } 100 \mathrm{~V} \\
\text { UPW2A102MHD }\end{array}$ & 1 & 3.03 & 3.03 & $\mathrm{CIN}$ & Through-Hole & $\begin{array}{l}65 \mathrm{~V}, 1.886 \mathrm{pA} \\
\text { (RMS) }\end{array}$ & $5 \mathrm{~V}, 227.67 \mathrm{fA}$ (RMS) \\
\hline $\begin{array}{l}\text { 47 } \mu, \text { Electrolytic, 100V, } \\
\text { AFK476M2AH32T-F }\end{array}$ & 1 & 2.35 & 2.35 & CCLAMP & SMT (Custom) & $\begin{array}{c}\text { 65.68V, } 119.05 \mathrm{~mA} \\
\text { (RMS) }\end{array}$ & $\begin{array}{l}5 \mathrm{~V}, 42.067 \mathrm{~mA} \\
\text { (RMS) }\end{array}$ \\
\hline $\begin{array}{c}33 \mu \text {, Electrolytic, 35V, EEE- } \\
\text { HA1V330WP }\end{array}$ & 1 & 0.63 & 0.63 & CBAT & SMT (Custom) & $\begin{array}{l}\text { 12V, } 206.56 \mathrm{fA} \\
\text { (RMS) }\end{array}$ & $\begin{array}{l}12 \mathrm{~V}, 442.09 \mathrm{fA} \\
\text { (RMS) }\end{array}$ \\
\hline $\begin{array}{l}\text { 10 } \mu \text {, Ceramic, 50V, } \\
\text { UMK325C7106MM-T }\end{array}$ & 3 & 0.96 & 2.88 & COUT2, COUT3, COUT4 & 1210 & $36 \mathrm{~V}, 2.758 \mathrm{~A}(\mathrm{RMS})$ & $36 \mathrm{~V}, 2.016 \mathrm{~A}$ (RMS) \\
\hline $\begin{array}{c}10 \mu \text {, Ceramic, 25V, TMK316B7106KL- } \\
\text { TD }\end{array}$ & 2 & 0.58 & 1.16 & СВАТ2, СВАТЗ & 1206 & $\begin{array}{c}\text { 12V, 963.63fA } \\
\text { (RMS) }\end{array}$ & $12 \mathrm{~V}, 1.211 \mathrm{pA}(\mathrm{RMS})$ \\
\hline $\begin{array}{l}4.7 \mu, \text { Ceramic, } 100 \mathrm{~V} \\
\text { C4532X7S2A475M }\end{array}$ & 5 & 1.75 & 8.75 & $\begin{array}{c}\text { CINT2, CINT3, CINT4, CINT5, } \\
\text { CINT6 }\end{array}$ & 1812 & $\begin{array}{l}\text { 77.05V, } 1.22 \mathrm{~A} \\
\text { (RMS) }\end{array}$ & $\begin{array}{l}21.22 \mathrm{~V}, 1.22 \mathrm{~A} \\
\text { (RMS) }\end{array}$ \\
\hline 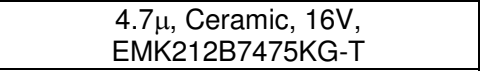 & 1 & 0.38 & 0.38 & CVCC & 0805 & $\begin{array}{c}5.23 \mathrm{~V}, 153.1 \mathrm{~mA} \\
\text { (RMS) }\end{array}$ & $\begin{array}{c}5.23 \mathrm{~V}, 170.84 \mathrm{~mA} \\
\text { (RMS) }\end{array}$ \\
\hline $\begin{array}{l}0.1 \mu \text {, Ceramic, } 100 \mathrm{~V} \\
\text { CGA3E3X7S2A104K }\end{array}$ & 7 & 0.30 & 2.10 & $\begin{array}{c}\text { CIN2, CIN3, CCLAMP2, } \\
\text { CCLAMP3, CCLAMP4, CBAT4, } \\
\text { CBAT5 } \\
\end{array}$ & 0603 & $\begin{array}{l}65 \mathrm{~V}, 1.000 \mathrm{~mA} \\
\text { (RMS) }\end{array}$ & $5 \mathrm{~V}, 0.285 \mathrm{~mA}$ (RMS) \\
\hline $\begin{array}{l}0.1 \mu \text {, Ceramic, } 100 \mathrm{~V} \\
\text { HMK212B7104KG-T }\end{array}$ & 2 & 0.30 & 0.60 & CINT7, CINT8 & 0805 & $\begin{array}{c}77.05 \mathrm{~V}, 47.184 \mathrm{~mA} \\
\text { (RMS) }\end{array}$ & $\begin{array}{l}21.22 \mathrm{~V}, 17.59 \mathrm{~mA} \\
\text { (RMS) }\end{array}$ \\
\hline 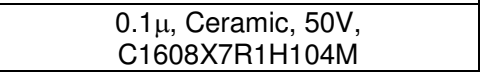 & 2 & 0.16 & 0.32 & COUT_CPH1, COUT_CPH2 & 0603 & $\begin{array}{l}\text { 36V, 63.00mA } \\
\text { (RMS) }\end{array}$ & $\begin{array}{l}36 \mathrm{~V}, 46.00 \mathrm{~mA} \\
\text { (RMS) }\end{array}$ \\
\hline $\begin{array}{l}\text { 10000p, Ceramic, 250V, } \\
\text { C2012X7R2E103K }\end{array}$ & 1 & 0.33 & 0.33 & CSNUB2 & 0805 & $\begin{array}{c}\text { 103.44V, 1.619A } \\
\text { (RMS) }\end{array}$ & $\begin{array}{c}\text { 56.06V, } 756.43 \mathrm{~mA} \\
\text { (RMS) }\end{array}$ \\
\hline $\begin{array}{l}\text { 6800p, Ceramic, 100V, } \\
\text { C1608X8R2A682K }\end{array}$ & 1 & 0.36 & 0.36 & CGATE_OVP & 0603 & $\begin{array}{c}77.47 \mathrm{~V}, 4.796 \mathrm{~mA} \\
\text { (RMS) }\end{array}$ & $\begin{array}{c}11.331 \mathrm{~V}, 0.006 \mathrm{~mA} \\
\text { (RMS) }\end{array}$ \\
\hline $\begin{array}{l}\text { 6800p, Ceramic, 25V, } \\
\text { C1005X7R1E682K }\end{array}$ & 1 & 0.09 & 0.09 & $\mathrm{CTH} 2$ & 0402 & $\begin{array}{c}1.232 \mathrm{~V}, 0.110 \mathrm{~mA} \\
(\mathrm{RMS})\end{array}$ & $\begin{array}{c}1.232 \mathrm{~V}, 0.030 \mathrm{~mA} \\
\text { (RMS) }\end{array}$ \\
\hline $\begin{array}{c}\text { 56p, Ceramic, 10V, } \\
\text { C0402C560J8GACTU }\end{array}$ & 1 & 0.35 & 0.35 & CTH1 & 0402 & $\begin{array}{c}1.232 \mathrm{~V}, 0.390 \mathrm{~mA} \\
\text { (RMS) }\end{array}$ & $\begin{array}{c}1.232 \mathrm{~V}, 0.009 \mathrm{~mA} \\
\text { (RMS) }\end{array}$ \\
\hline $\begin{array}{l}\text { 47p, Ceramic, 10V, } \\
\text { 0603ZA470DAT2A }\end{array}$ & 5 & 0.30 & 1.50 & $\begin{array}{c}\text { CFB, CSNS, CTMR, } \\
\text { CRUN_OVP, CRUN_MAIN }\end{array}$ & 0603 & $\begin{array}{c}1.5 \mathrm{~V}, 0.028 \mathrm{~mA} \\
\text { (RMS) }\end{array}$ & $\begin{array}{c}1.307 \mathrm{~V}, 0.020 \mathrm{~mA} \\
\text { (RMS) }\end{array}$ \\
\hline \multicolumn{3}{|c|}{ Total Cost (\$) } & 64.79 & & & & \\
\hline Component Count & 79 & & & & & & \\
\hline
\end{tabular}


After receiving the PCB via ExpressPCB, this project's author performed an exhaustive continuity test for ensuring that the PCB contained proper connection points and that there were no undesirable or potentially fatal bridge points. As expected, the PCB passed the continuity test upon first receipt. After verifying the PCB, this project's author soldered SEPIC components onto it and began the hardware testing process. 


\section{CHAPTER 10: HARDWARE TESTING}

\subsection{Component Soldering Process}

This SEPIC's component soldering process only took five days. This project's author began by first soldering the physically smaller low-power components such as the LTC1871 controller and gradually moved on to larger, high-power components such as $\mathrm{L}_{1}$ and $\mathrm{L}_{2}$. Lack of fine solder tips in Cal Poly's power electronics laboratory impeded the soldering process, but for the most part it was not difficult because this SEPIC did not have components requiring a reflow oven. Furthermore, during this SEPIC's initial soldering process, this project's author accidentally removed a thin copper trace connecting the LTC1871 controller's FREQ pin, thus the author had to spend an additional three hours re-bridging the connection using a very thin wire. That solution sufficed, however, as the LTC1871's FREQ pin is not very sensitive to wire width, as long as the wire does not greatly modify the resistance the pin senses. Figures 10.1 and 10.2 show this project's SEPIC with all components soldered. 


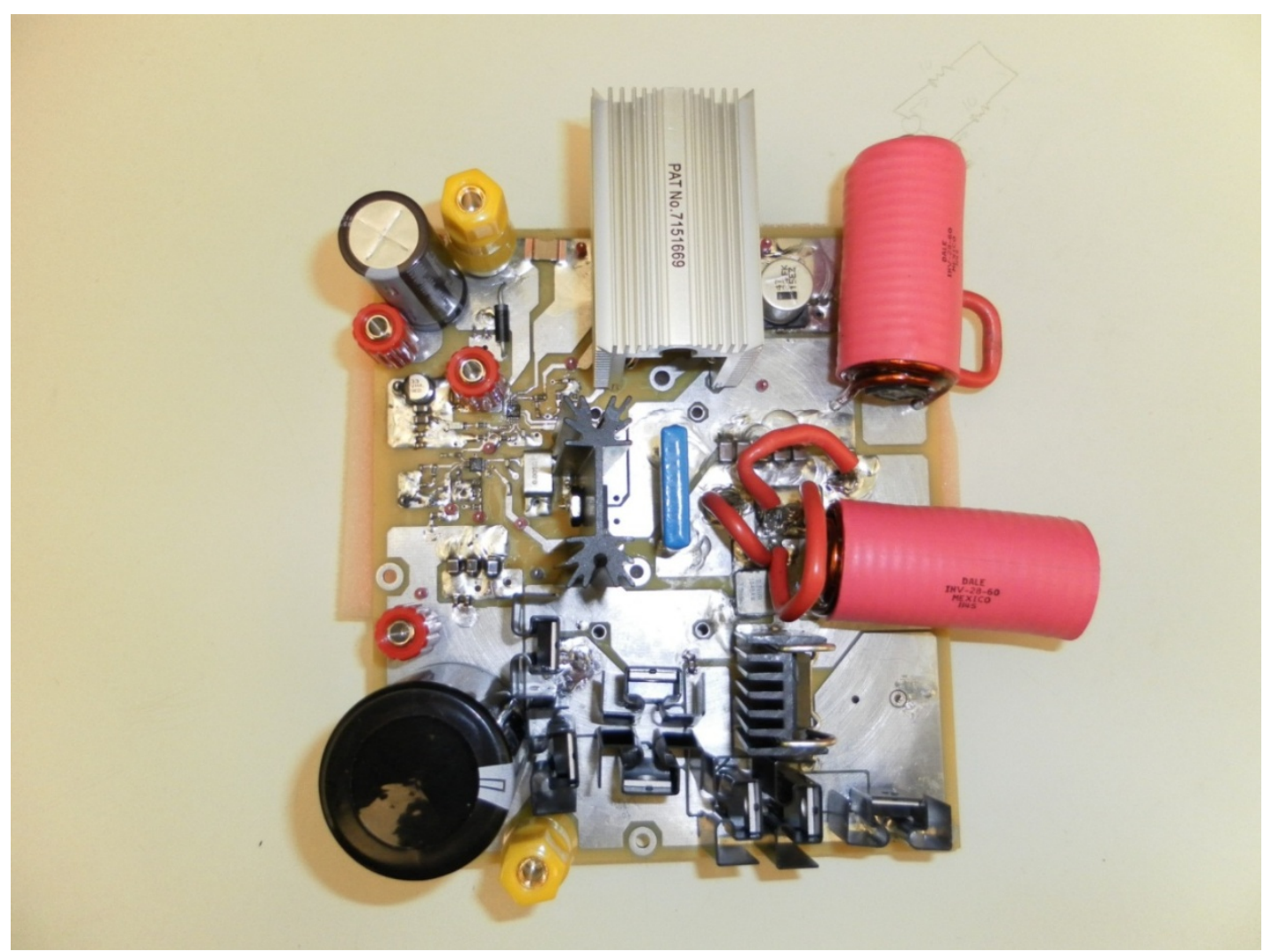

Figure 10.1: First Revision SEPIC, Top View 


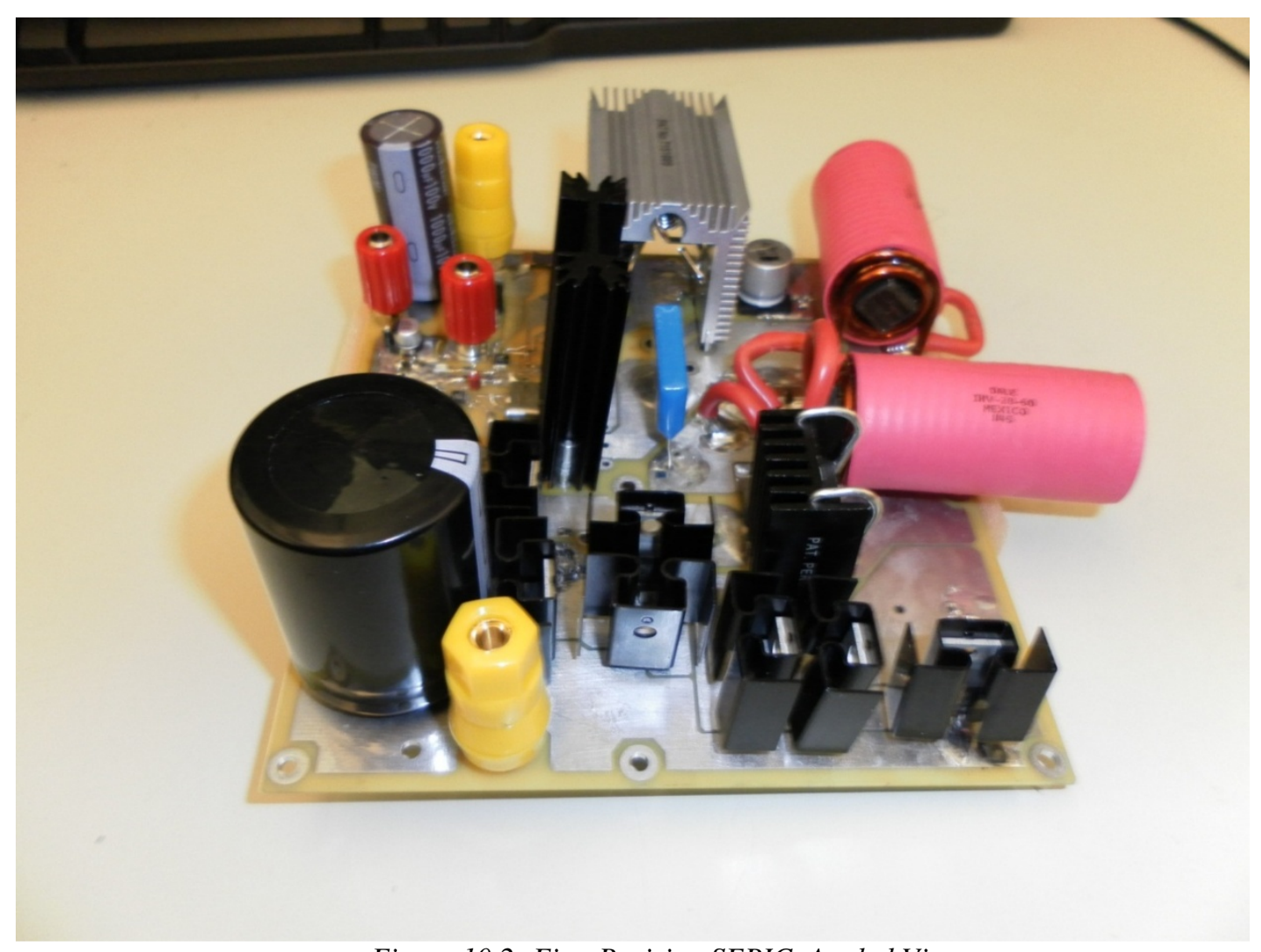

Figure 10.2: First Revision SEPIC, Angled View

After completing the SEPIC's component soldering process, this project begins its first hardware testing round.

\subsection{No-Load Testing}

This project's first set of hardware tests consist of running the SEPIC with no load (open-circuit output). These tests ensure that both the SEPIC's LTC1871 and LT4356-1 controllers function, as well as crucial SEPIC components such as Q_MAIN and $\mathrm{L}_{1}$ and $\mathrm{L}_{2}$. Figure 10.3 shows the block diagram for this project's SEPIC's no-load tests. 


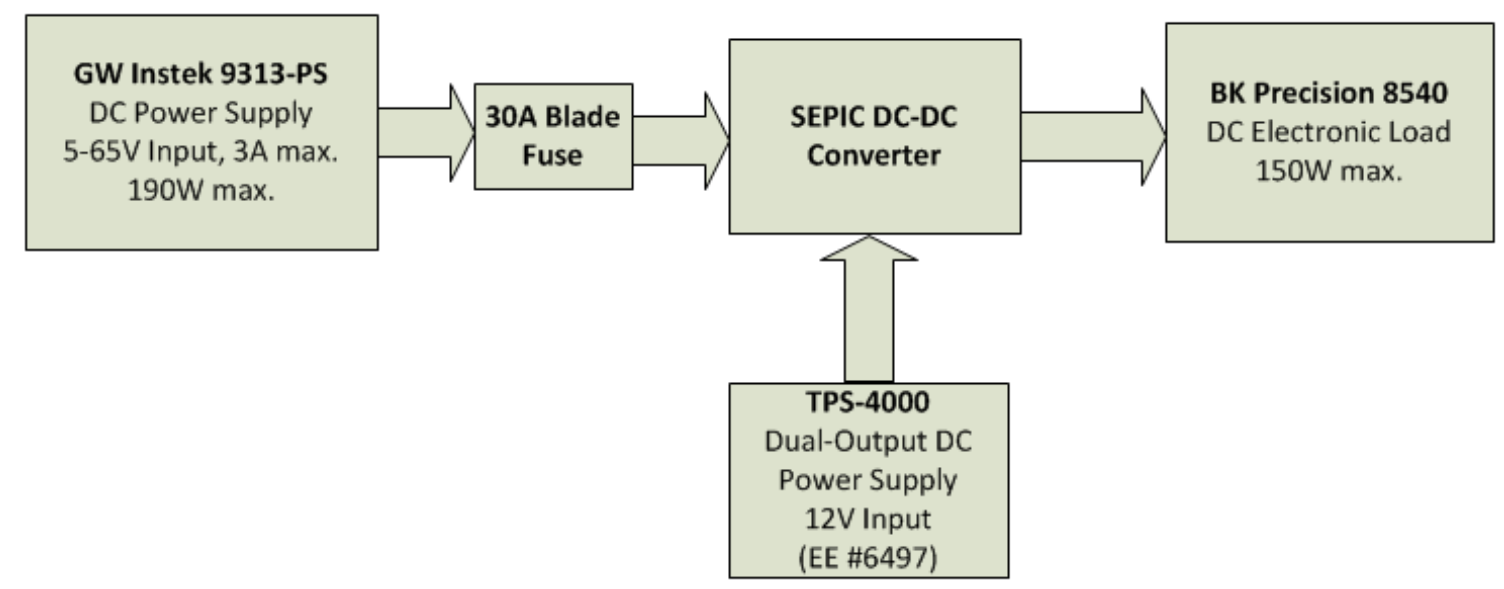

Figure 10.3: SEPIC No-Load Test Block Diagram

These no load tests utilize a TPS-4000 dual rail power supply acting as a $12 \mathrm{~V}$ source for biasing the SEPIC's LTC1871 controller, as well as a GW Instek DC power supply acting as the SEPIC's main input source and a BK Precision $150 \mathrm{~W}$ electronic load for obtaining output voltage measurements. The GW Instek power supply has a $3 \mathrm{~A}$ and 190W limit, however, those pitfalls do not matter for no-load tests. Figure 10.4 shows the SEPIC's no-load test wiring set up. 


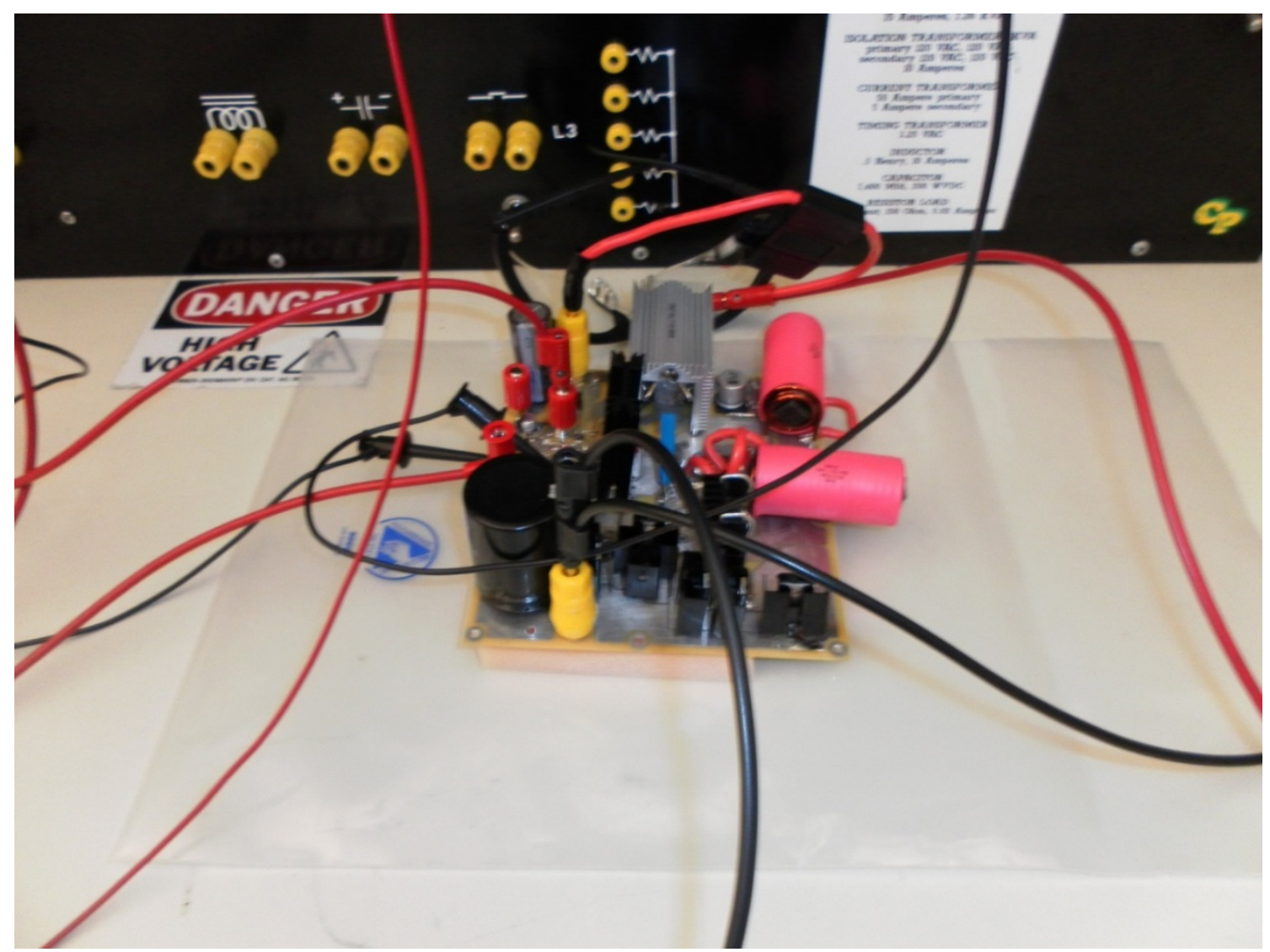

Figure 10.4: SEPIC No-Load Test Wiring

For this project's no-load tests, this project uses a 30A input protection fuse but no output protection fuse. The LT4356-1 protection circuit described in Chapter 7 limits the SEPIC's peak pulsed input current to $25 \mathrm{~A}$, thus the $30 \mathrm{~A}$ input protection fuse suffices.

However, these no-load tests do not require an output protection fuse because these tests do not cause any current flow through the SEPIC's output node. Figure 10.5 shows instrument readouts with all sources energized and the SEPIC running. 


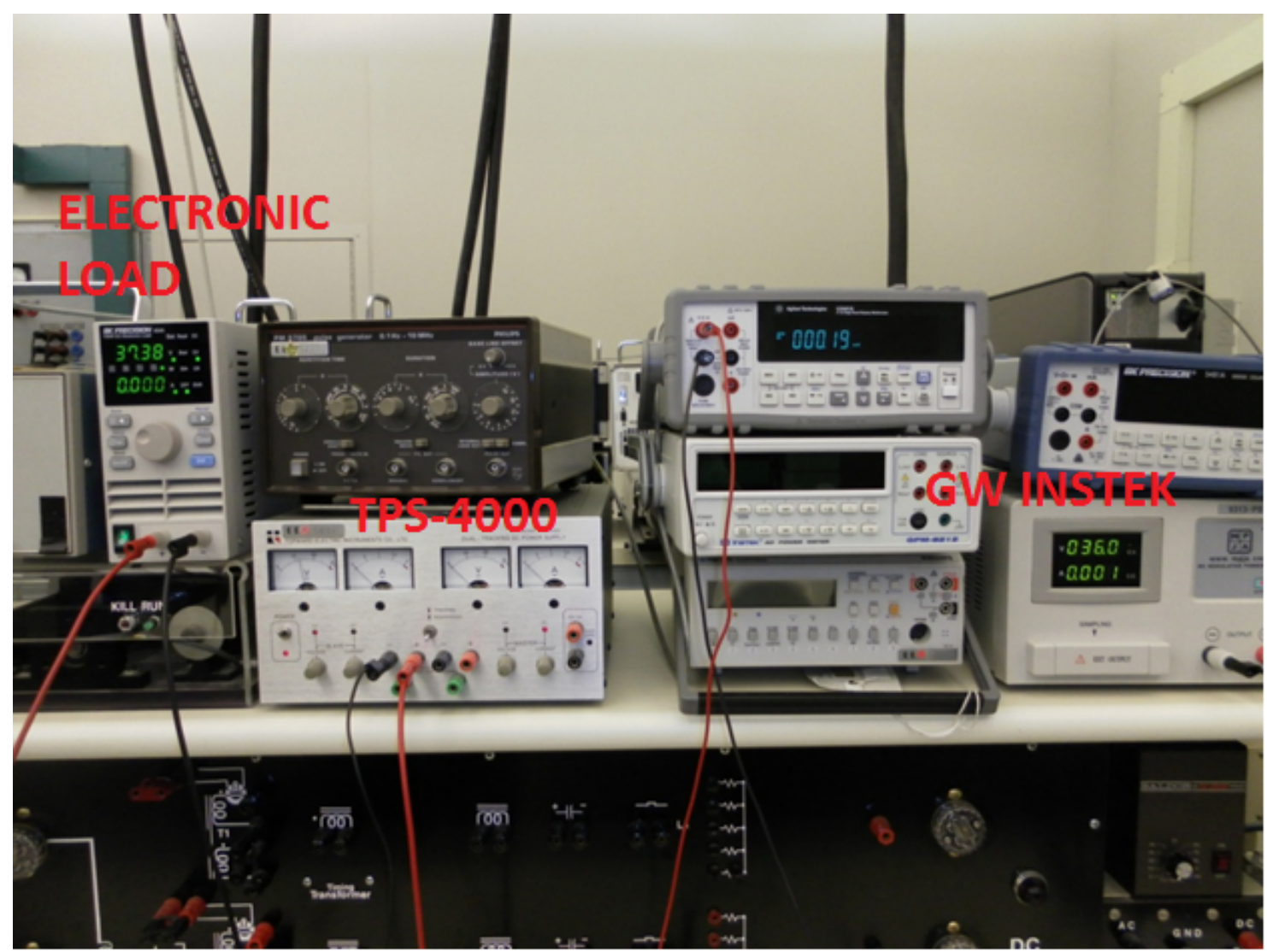

Figure 10.5: SEPIC No-Load Test at 36V Input

From left to right are the BK Precision electronic load, TPS-4000 power supply and the

GW Instek power supply. With no load, the SEPIC outputs 37.38V through the entire 5$65 \mathrm{~V}$ input range from the GW Instek power supply. This is slightly above the $36 \mathrm{~V}$ output requirement described in section 1.2 , but this extra voltage allows for headroom when the SEPIC drives a load, as typically its output voltage should reduce by a small margin when it drives a load. At around 63V input, however, the SEPIC's LT4356-1 protection LED turned on, indicating an overvoltage input condition. The LT4356-1 circuit was very sensitive to input voltage and at $65.1 \mathrm{~V}$ input it shut off voltage and current flow to the SEPIC. When the input voltage reduced to $63 \mathrm{~V}$, the SEPIC functioned again and regulated a $37.38 \mathrm{~V}$ output. This confirmed that the LT4356-1 protection circuit was 
properly functioning. Therefore, this project's SEPIC functions with no load and this project's author properly connected the SEPIC's components during the soldering process.

\subsection{Load Testing}

The next SEPIC hardware testing round determines the SEPIC's load regulation abilities. As mentioned in earlier chapters, this project does not require the SEPIC driving its specified 8A maximum load current at all input voltages - only when it receives the maximum 65V input from the Precor elliptical trainer. The first load test set uses an electronic load in constant current mode for determining the SEPIC's load regulation abilities. Later tests use the electronic load in constant voltage mode for determining the SEPIC's maximum output load current for maintaining 36V output over this project's specified 5-65V input range.

\subsubsection{Electronic Load in Constant Current Mode}

Figure 10.6 shows the SEPIC's initial load test block diagram. 


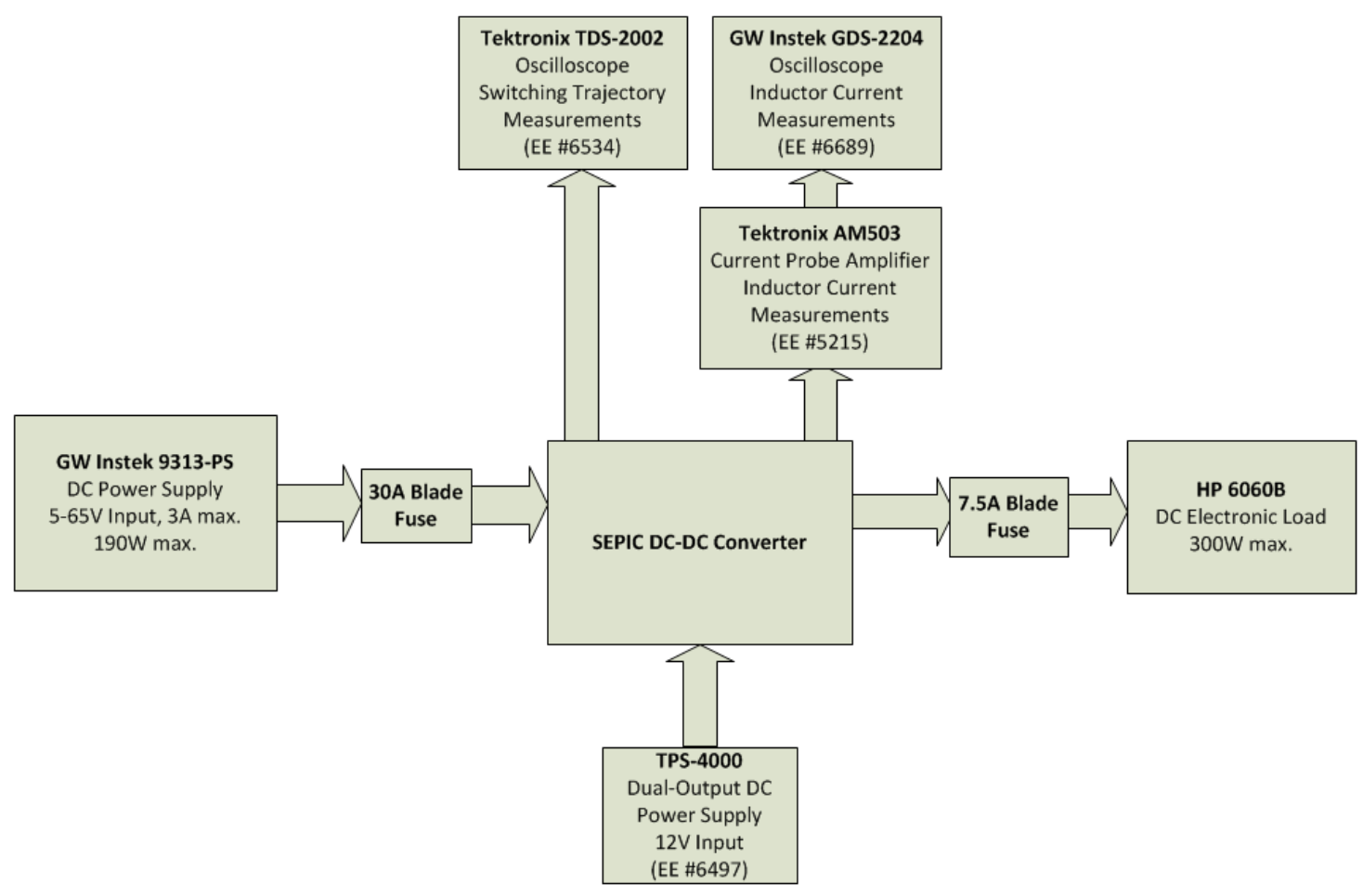

Figure 10.6: SEPIC Initial Load Test Block Diagram

The initial load tests use the same setup as the no-load tests, except this time this project uses a 7.5A blade fuse for protection. No commercially-available $8 \mathrm{~A}$ blade fuses exist (as only rating increments of $0.5 \mathrm{~A}$ exist for such blade fuses) therefore later higher current tests use 10A blade fuses. This project uses the oscilloscopes and current probe amplifiers in the next section for troubleshooting purposes. Because the GW Instek power supply has a $3 \mathrm{~A}$ limit, this first set of load tests only tests loads up to $2.3 \mathrm{~A}$. Furthermore, these tests use an HP 300W electronic load because this SEPIC's 288W maximum output power rating exceeds the BK Precision electronic load's (used for the no-load tests) 150W rating. Tables E-1 to E-16 show these test results at each input voltage and output load current. 
Table E-1: SEPIC Initial Load Tests with 0.2A Constant Current Electronic Load, TPS-4000 and GW Instek Power Supplies

\begin{tabular}{|c|c|c|c|c|c|c|c|}
\hline \multicolumn{10}{|c|}{ 0.2A Load Tests: } \\
\hline $\mathbf{V}_{\text {IN }}(\mathbf{V})$ & $\mathbf{I}_{\text {IN }}(\mathbf{A})$ & $\mathbf{V}_{\text {OUT }}(\mathbf{V})$ & $\mathbf{I}_{\text {OUT }}(\mathbf{A})$ & $\mathbf{P}_{\text {IN }}(\mathbf{W})$ & $\mathbf{P}_{\text {OUT }}(\mathbf{W})$ & $\eta(\%)$ & $\mathbf{R}_{\text {IN }}(\mathbf{\Omega})$ \\
\hline 10 & 1.019 & 29.73 & 0.2 & 10.19 & 5.946 & 58.351 & 9.814 \\
\hline 12 & 1.077 & 37.34 & 0.2 & 12.924 & 7.468 & 57.784 & 11.142 \\
\hline 15 & 0.848 & 37.34 & 0.2 & 12.72 & 7.468 & 58.711 & 17.689 \\
\hline 20 & 0.621 & 37.34 & 0.2 & 12.42 & 7.468 & 60.129 & 32.206 \\
\hline 25 & 0.487 & 37.34 & 0.2 & 12.175 & 7.468 & 61.339 & 51.335 \\
\hline 27 & 0.454 & 37.34 & 0.2 & 12.258 & 7.468 & 60.923 & 59.471 \\
\hline
\end{tabular}

Table E-2: SEPIC Initial Load Tests with 0.25A Constant Current Electronic Load, TPS-4000 and GW Instek Power Supplies

\begin{tabular}{|c|c|c|c|c|c|c|c|}
\hline \multicolumn{10}{|c|}{ 0.25A Load Tests: } \\
\hline $\mathbf{V}_{\text {IN }}(\mathbf{V})$ & $\mathbf{I}_{\text {IN }}(\mathbf{A})$ & $\mathbf{V}_{\text {OUT }}(\mathbf{V})$ & $\mathbf{I}_{\text {OUT }}(\mathbf{A})$ & $\mathbf{P}_{\text {IN }}(\mathbf{W})$ & $\mathbf{P}_{\text {OUT }}(\mathbf{W})$ & $\eta(\%)$ & $\mathbf{R}_{\text {IN }}(\mathbf{\Omega})$ \\
\hline 12 & 1.21 & 33.96 & 0.25 & 14.52 & 8.49 & 58.471 & 9.917 \\
\hline 15 & 1.038 & 37.34 & 0.25 & 15.57 & 9.335 & 59.955 & 14.451 \\
\hline 20 & 0.76 & 37.34 & 0.25 & 15.2 & 9.335 & 61.414 & 26.316 \\
\hline 25 & 0.6 & 37.34 & 0.25 & 15 & 9.335 & 62.233 & 41.667 \\
\hline 27 & 0.56 & 37.34 & 0.25 & 15.12 & 9.335 & 61.739 & 48.214 \\
\hline
\end{tabular}

Table E-3: SEPIC Initial Load Tests with 0.3A Constant Current Electronic Load, TPS-4000 and GW Instek Power Supplies

\begin{tabular}{|c|c|c|c|c|c|c|c|}
\hline \multicolumn{10}{|c|}{ 0.3A Load Tests: } \\
\hline $\mathbf{V}_{\text {IN }}(\mathbf{V})$ & $\mathbf{I}_{\text {IN }}(\mathbf{A})$ & $\mathbf{V}_{\text {OUT }}(\mathbf{V})$ & $\mathbf{I}_{\text {OUT }}(\mathbf{A})$ & $\mathbf{P}_{\text {IN }}(\mathbf{W})$ & $\mathbf{P}_{\text {OUT }}(\mathbf{W})$ & $\eta(\%)$ & $\mathbf{R}_{\text {IN }}(\boldsymbol{\Omega})$ \\
\hline 27 & 0.666 & 33.96 & 0.3 & 17.982 & 10.188 & 56.657 & 40.541 \\
\hline 36 & 0.512 & 37.34 & 0.3 & 18.432 & 11.202 & 60.775 & 70.313 \\
\hline
\end{tabular}

Table E-4: SEPIC Initial Load Tests with 0.35A Constant Current Electronic Load, TPS-4000 and GW Instek Power Supplies

\begin{tabular}{|c|c|c|c|c|c|c|c|}
\hline \multicolumn{10}{|c|}{ 0.35A Load Tests: } \\
\hline $\mathbf{V}_{\text {IN }}(\mathbf{V})$ & $\mathbf{I}_{\text {IN }}(\mathbf{A})$ & $\mathbf{V}_{\text {OUT }}(\mathbf{V})$ & $\mathbf{I}_{\text {OUT }}(\mathbf{A})$ & $\mathbf{P}_{\text {IN }}(\mathbf{W})$ & $\mathbf{P}_{\text {OUT }}(\mathbf{W})$ & $\eta(\%)$ & $\mathbf{R}_{\text {IN }}(\Omega)$ \\
\hline 27 & 0.772 & 33.96 & 0.35 & 20.844 & 11.886 & 57.024 & 34.974 \\
\hline 36 & 0.591 & 37.34 & 0.35 & 21.276 & 13.069 & 61.426 & 60.914 \\
\hline
\end{tabular}

Table E-5: SEPIC Initial Load Tests with 0.4A Constant Current Electronic Load, TPS-4000 and GW Instek Power Supplies

\begin{tabular}{|c|c|c|c|c|c|c|c|}
\hline \multicolumn{10}{|c|}{ 0.4A Load Tests } \\
\hline $\mathbf{V}_{\text {IN }}(\mathbf{V})$ & $\mathbf{I}_{\text {IN }}(\mathbf{A})$ & $\mathbf{V}_{\text {OUT }}(\mathbf{V})$ & $\mathbf{I}_{\text {OUT }}(\mathbf{A})$ & $\mathbf{P}_{\text {IN }}(\mathbf{W})$ & $\mathbf{P}_{\text {OUT }}(\mathbf{W})$ & $\eta(\%)$ & $\mathbf{R}_{\text {IN }}(\boldsymbol{\Omega})$ \\
\hline 27 & 0.876 & 33.96 & 0.4 & 23.652 & 13.584 & 57.433 & 30.822 \\
\hline 36 & 0.672 & 37.34 & 0.4 & 24.192 & 14.936 & 61.739 & 53.571 \\
\hline
\end{tabular}


Table E-6: SEPIC Initial Load Tests with 0.45A Constant Current Electronic Load, TPS-4000 and GW Instek Power Supplies

\begin{tabular}{|c|c|c|c|c|c|c|c|}
\hline \multicolumn{10}{|c|}{ 0.45A Load Tests } \\
\hline $\mathbf{V}_{\text {IN }}(\mathbf{V})$ & $\mathbf{I}_{\text {IN }}(\mathbf{A})$ & $\mathbf{V}_{\text {OUT }}(\mathbf{V})$ & $\mathbf{I}_{\text {OUT }}(\mathbf{A})$ & $\mathbf{P}_{\text {IN }}(\mathbf{W})$ & $\mathbf{P}_{\text {OUT }}(\mathbf{W})$ & $\eta(\%)$ & $\mathbf{R}_{\text {IN }}(\boldsymbol{\Omega})$ \\
\hline 27 & 1 & 33.96 & 0.45 & 27 & 15.282 & 56.600 & 27.000 \\
\hline 36 & 0.751 & 37.34 & 0.45 & 27.036 & 16.803 & 62.150 & 47.936 \\
\hline
\end{tabular}

Table E-7: SEPIC Initial Load Tests with 0.5A Constant Current Electronic Load, TPS-4000 and GW Instek Power Supplies

\begin{tabular}{|c|c|c|c|c|c|c|c|}
\hline \multicolumn{10}{|c|}{ 0.5A Load Tests } \\
\hline $\mathbf{V}_{\text {IN }}(\mathbf{V})$ & $\mathbf{I}_{\text {IN }}(\mathbf{A})$ & $\mathbf{V}_{\text {OUT }}(\mathbf{V})$ & $\mathbf{I}_{\text {OUT }}(\mathbf{A})$ & $\mathbf{P}_{\text {IN }}(\mathbf{W})$ & $\mathbf{P}_{\text {OUT }}(\mathbf{W})$ & $\eta(\%)$ & $\mathbf{R}_{\text {IN }}(\Omega)$ \\
\hline 27 & 1.085 & 33.96 & 0.5 & 29.295 & 16.98 & 57.962 & 24.885 \\
\hline 36 & 0.831 & 37.34 & 0.5 & 29.916 & 18.67 & 62.408 & 43.321 \\
\hline
\end{tabular}

Table E-8: SEPIC Initial Load Tests with 0.6A Constant Current Electronic Load, TPS-4000 and GW Instek Power Supplies

\begin{tabular}{|c|c|c|c|c|c|c|c|}
\hline \multicolumn{10}{|c|}{ 0.6A Load Tests } \\
\hline $\mathbf{V}_{\text {IN }}(\mathbf{V})$ & $\mathbf{I}_{\text {IN }}(\mathbf{A})$ & $\mathbf{V}_{\text {OUT }}(\mathbf{V})$ & $\mathbf{I}_{\text {OUT }}(\mathbf{A})$ & $\mathbf{P}_{\text {IN }}(\mathbf{W})$ & $\mathbf{P}_{\text {OUT }}(\mathbf{W})$ & $\eta(\%)$ & $\mathbf{R}_{\text {IN }}(\boldsymbol{\Omega})$ \\
\hline 27 & 1.325 & 33.96 & 0.6 & 35.775 & 20.376 & 56.956 & 20.377 \\
\hline 36 & 0.99 & 37.34 & 0.6 & 35.64 & 22.404 & 62.862 & 36.364 \\
\hline
\end{tabular}

Table E-9: SEPIC Initial Load Tests with 0.7A Constant Current Electronic Load, TPS-4000 and GW Instek Power Supplies

\begin{tabular}{|c|c|c|c|c|c|c|c|}
\hline \multicolumn{10}{|c|}{ 0.7A Load Tests } \\
\hline $\mathbf{V}_{\text {IN }}(\mathbf{V})$ & $\mathbf{I}_{\text {IN }}(\mathbf{A})$ & $\mathbf{V}_{\text {OUT }}(\mathbf{V})$ & $\mathbf{I}_{\text {OUT }}(\mathbf{A})$ & $\mathbf{P}_{\text {IN }}(\mathbf{W})$ & $\mathbf{P}_{\text {OUT }}(\mathbf{W})$ & $\eta(\%)$ & $\mathbf{R}_{\text {IN }}(\boldsymbol{\Omega})$ \\
\hline 27 & 1.526 & 33.96 & 0.7 & 41.202 & 23.772 & 57.696 & 17.693 \\
\hline 36 & 1.15 & 37.34 & 0.7 & 41.4 & 26.138 & 63.135 & 31.304 \\
\hline
\end{tabular}

Table E-10: SEPIC Initial Load Tests with 0.8A Constant Current Electronic Load, TPS-4000 and GW Instek Power Supplies

\begin{tabular}{|c|c|c|c|c|c|c|c|}
\hline \multicolumn{10}{|c|}{ 0.8A Load Tests } \\
\hline $\mathbf{V}_{\text {IN }}(\mathbf{V})$ & $\mathbf{I}_{\text {IN }}(\mathbf{A})$ & $\mathbf{V}_{\text {OUT }}(\mathbf{V})$ & $\mathbf{I}_{\text {OUT }}(\mathbf{A})$ & $\mathbf{P}_{\text {IN }}(\mathbf{W})$ & $\mathbf{P}_{\text {OUT }}(\mathbf{W})$ & $\eta(\%)$ & $\mathbf{R}_{\text {IN }}(\boldsymbol{\Omega})$ \\
\hline 27 & 1.727 & 33.96 & 0.8 & 46.629 & 27.168 & 58.264 & 15.634 \\
\hline 36 & 1.305 & 37.34 & 0.8 & 46.98 & 29.872 & 63.585 & 27.586 \\
\hline
\end{tabular}

Table E-11: SEPIC Initial Load Tests with 0.9A Constant Current Electronic Load, TPS-4000 and GW Instek Power Supplies

\begin{tabular}{|c|c|c|c|c|c|c|c|}
\hline \multicolumn{7}{|c|}{ 0.9A Load Tests } \\
\hline $\mathbf{V}_{\text {IN }}(\mathbf{V})$ & $\mathbf{I}_{\text {IN }}(\mathbf{A})$ & $\mathbf{V}_{\text {OUT }}(\mathbf{V})$ & $\mathbf{I}_{\text {OUT }}(\mathbf{A})$ & $\mathbf{P}_{\text {IN }}(\mathbf{W})$ & $\mathbf{P}_{\text {OUT }}(\mathbf{W})$ & $\eta(\%)$ & $\mathbf{R}_{\text {IN }}(\boldsymbol{\Omega})$ \\
\hline 27 & 1.923 & 33.96 & 0.9 & 51.921 & 30.564 & 58.866 & 14.041 \\
\hline 36 & 1.458 & 37.34 & 0.9 & 52.488 & 33.606 & 64.026 & 24.691 \\
\hline
\end{tabular}


Table E-12: SEPIC Initial Load Tests with 1A Constant Current Electronic Load, TPS-4000 and GW Instek Power Supplies

\begin{tabular}{|c|c|c|c|c|c|c|c|}
\hline \multicolumn{7}{|c|}{ 1A Load Tests } \\
\hline $\mathbf{V}_{\text {IN }}(\mathbf{V})$ & $\mathbf{I}_{\text {IN }}(\mathbf{A})$ & $\mathbf{V}_{\text {OUT }}(\mathbf{V})$ & $\mathbf{I}_{\text {OUT }}(\mathbf{A})$ & $\mathbf{P}_{\text {IN }}(\mathbf{W})$ & $\mathbf{P}_{\text {OUT }}(\mathbf{W})$ & $\eta(\%)$ & $\mathbf{R}_{\text {IN }}(\mathbf{\Omega})$ \\
\hline 18 & 1.776 & 21.18 & 1 & 31.968 & 21.18 & 66.254 & 10.135 \\
\hline 27 & 2.119 & 33.96 & 1 & 57.213 & 33.96 & 59.357 & 12.742 \\
\hline 36 & 1.61 & 37.34 & 1 & 57.96 & 37.34 & 64.424 & 22.360 \\
\hline 50 & 1.155 & 37.18 & 1 & 57.75 & 37.18 & 64.381 & 43.290 \\
\hline
\end{tabular}

Table E-13: SEPIC Initial Load Tests with 1.5A Constant Current Electronic Load, TPS-4000 and GW Instek Power Supplies

\begin{tabular}{|c|c|c|c|c|c|c|c|}
\hline \multicolumn{10}{|c|}{ 1.5A Load Tests } \\
\hline $\mathbf{V}_{\text {IN }}(\mathbf{V})$ & $\mathbf{I}_{\text {IN }}(\mathbf{A})$ & $\mathbf{V}_{\text {OUT }}(\mathbf{V})$ & $\mathbf{I}_{\text {OUT }}(\mathbf{A})$ & $\mathbf{P}_{\text {IN }}(\mathbf{W})$ & $\mathbf{P}_{\text {OUT }}(\mathbf{W})$ & $\eta(\%)$ & $\mathbf{R}_{\text {IN }}(\Omega)$ \\
\hline 27 & 2.613 & 31.57 & 1.5 & 70.551 & 47.355 & 67.122 & 10.333 \\
\hline 36 & 2.3 & 36.27 & 1.5 & 82.8 & 54.405 & 65.707 & 15.652 \\
\hline 50 & 1.705 & 37.15 & 1.5 & 85.25 & 55.725 & 65.367 & 29.326 \\
\hline
\end{tabular}

Table E-14: SEPIC Initial Load Tests with 2A Constant Current Electronic Load, TPS-4000 and GW Instek Power Supplies

\begin{tabular}{|c|c|c|c|c|c|c|c|}
\hline \multicolumn{10}{|c|}{ 2A Load Tests } \\
\hline $\mathbf{V}_{\text {IN }}(\mathbf{V})$ & $\mathbf{I}_{\text {IN }}(\mathbf{A})$ & $\mathbf{V}_{\text {OUT }}(\mathbf{V})$ & $\mathbf{I}_{\text {OUT }}(\mathbf{A})$ & $\mathbf{P}_{\text {IN }}(\mathbf{W})$ & $\mathbf{P}_{\text {OUT }}(\mathbf{W})$ & $\eta(\%)$ & $\mathbf{R}_{\text {IN }}(\mathbf{\Omega})$ \\
\hline 36 & 2.85 & 34.57 & 2 & 102.6 & 69.14 & 67.388 & 12.632 \\
\hline 50 & 2.24 & 37.04 & 2 & 112 & 74.08 & 66.143 & 22.321 \\
\hline
\end{tabular}

Table E-15: SEPIC Initial Load Tests with 2.2A Constant Current Electronic Load, TPS-4000 and GW Instek Power Supplies

\begin{tabular}{|c|c|c|c|c|c|c|c|}
\hline \multicolumn{7}{|c|}{ 2.2A Load Tests } \\
\hline $\mathbf{V}_{\text {IN }}(\mathrm{V})$ & $\mathbf{I}_{\text {IN }}(\mathrm{A})$ & $\mathbf{V}_{\text {OUT }}(\mathrm{V})$ & $\mathbf{I}_{\text {OUT }}(\mathbf{A})$ & $\mathbf{P}_{\text {IN }}(\mathrm{W})$ & $\mathbf{P}_{\text {OUT }}(\mathrm{W})$ & $\eta(\%)$ & $\mathbf{R}_{\text {IN }}(\boldsymbol{\Omega})$ \\
\hline 50 & 2.204 & 34.39 & 2.2 & 110.2 & 75.658 & 68.655 & 22.686 \\
\hline
\end{tabular}

Table E-16: SEPIC Initial Load Tests with 2.3A Constant Current Electronic Load, TPS-4000 and GW Instek Power Supplies

\begin{tabular}{|c|c|c|c|c|c|c|c|}
\hline \multicolumn{10}{|c|}{ 2.3A Load Tests } \\
\hline $\mathbf{V}_{\text {IN }}(\mathbf{V})$ & $\mathbf{I}_{\text {IN }}(\mathbf{A})$ & $\mathbf{V}_{\text {OUT }}(\mathbf{V})$ & $\mathbf{I}_{\text {OUT }}(\mathbf{A})$ & $\mathbf{P}_{\text {IN }}(\mathbf{W})$ & $\mathbf{P}_{\text {OUT }}(\mathbf{W})$ & $\eta(\%)$ & $\mathbf{R}_{\text {IN }}(\Omega)$ \\
\hline 50 & 2.293 & 32.9 & 2.3 & 114.65 & 75.67 & 66.001 & 21.805 \\
\hline
\end{tabular}

$\mathrm{V}_{\mathrm{IN}}$ denotes the SEPIC's main input voltage, $\mathrm{I}_{\mathrm{IN}}$ denotes its input current (read from the GW Instek power supply), $V_{\text {OUT }}$ denotes its output voltage, IOUT denotes its output current, $\mathrm{P}_{\mathrm{IN}}$ denotes its input power, $\mathrm{P}_{\mathrm{OUT}}$ denotes its output power, $\eta$ denotes its overall 
efficiency, and $\mathrm{R}_{\mathrm{IN}}$ denotes its input resistance at the specified input voltage and output load current. These initial load tests do not take $12 \mathrm{~V}$ battery power dissipation into account because this project's author neglected using a method for accurately measuring the TPS-4000 power supply's current. However, the TPS-4000 power supply's current was very small during these load tests, therefore overall converter efficiency would not change by any significant margin if the battery's power dissipation was included. Therefore, these tests safely neglect the $12 \mathrm{~V}$ battery's power dissipation. These efficiency results, however, are below the project's specifications outlined in section 1.2 and require improvement. Also, at input voltages above $52 \mathrm{~V}$, the converter does not tolerate very high current loads (only up to 0.09A maximum), thus this project discards those results. Therefore this initial converter has load regulation problems at input voltages above $52 \mathrm{~V}$. For the most part, overall converter efficiency increases with increasing input voltage and load current. Furthermore, these varying load currents do not allow for the SEPIC maintaining a constant $10 \Omega$ input resistance, therefore this project does not expect these same results if the electronic load was in constant voltage mode (instead of constant current mode as in this initial load test). However, this project must address the load regulation problem at input voltages above $52 \mathrm{~V}$, otherwise this SEPIC will not function with the entire EHFEM system. The next load test set uses the HP electronic load in constant voltage mode for determining the maximum load current that the SEPIC drives while maintaining 36V output over the SEPIC's specified input range. 


\subsubsection{Electronic Load in Constant Voltage Mode}

Table E-17 shows the SEPIC's output load driving abilities while using the electronic load in constant voltage mode (36V). Table E-18 shows the same test results, except using input voltages (with their corresponding elliptical training resistance levels) that match the 160 strides per minute exercise test results from the previous EHFEM group in [1].

Table E-17: SEPIC Initial Load Tests with Constant Voltage Electronic Load, TPS-4000 and GW Instek Power Supplies

\begin{tabular}{|c|c|c|c|c|c|c|c|}
\hline $\mathbf{V}_{\text {IN }}(\mathbf{V})$ & $\mathbf{I}_{\text {IN }}(\mathbf{A})$ & $\mathbf{V}_{\text {OUT }}(\mathbf{V})$ & $\mathbf{I}_{\text {OUT }}(\mathbf{A})$ & $\mathbf{P}_{\text {IN }}(\mathbf{W})$ & $\mathbf{P}_{\text {OUT }}(\mathbf{W})$ & $\eta(\%)$ & $\mathbf{R}_{\text {IN }}(\Omega)$ \\
\hline 5 & 0.89 & 36.02 & 0.05 & 4.450 & 1.801 & 40.472 & 5.618 \\
\hline 10 & 0.996 & 36.02 & 0.14 & 9.960 & 5.043 & 50.631 & 10.040 \\
\hline 12 & 1.185 & 36.02 & 0.22 & 14.220 & 7.924 & 55.727 & 10.127 \\
\hline 15 & 1.453 & 36.02 & 0.35 & 21.795 & 12.607 & 57.844 & 10.323 \\
\hline 18 & 1.745 & 36.02 & 0.53 & 31.410 & 19.091 & 60.779 & 10.315 \\
\hline 20 & 1.939 & 36.02 & 0.67 & 38.780 & 24.133 & 62.232 & 10.315 \\
\hline 25 & 2.402 & 36.02 & 1.08 & 60.050 & 38.902 & 64.782 & 10.408 \\
\hline 27 & 2.565 & 36.02 & 1.26 & 69.255 & 45.385 & 65.533 & 10.526 \\
\hline 30 & 2.796 & 36.02 & 1.54 & 83.880 & 55.471 & 66.131 & 10.730 \\
\hline 36 & 2.241 & 36.02 & 1.46 & 80.676 & 52.589 & 65.186 & 16.064 \\
\hline $40 *$ & $3.065 *$ & $36.02 *$ & $2.55 *$ & $122.600 *$ & $91.851 *$ & $74.919 *$ & $13.051 *$ \\
\hline 45 & 2.753 & 36.02 & 2.32 & 123.885 & 83.566 & 67.455 & 16.346 \\
\hline 50 & 2.285 & 36.06 & 2.09 & 114.250 & 75.365 & 65.965 & 21.882 \\
\hline 52 & 2.297 & 36.06 & 2.19 & 119.444 & 78.971 & 66.116 & 22.638 \\
\hline 60 & 0.112 & 36.01 & 0.09 & 6.720 & 3.241 & 48.228 & 535.714 \\
\hline$*$ GW-Instek Current Limited & & & & \\
\hline
\end{tabular}


Table E-18: SEPIC Initial Load Tests with 160 Strides/Min. Theoretical Input Voltages from [1], Constant Voltage Electronic Load, TPS-4000 and GW Instek Power Supplies

\begin{tabular}{|c|c|c|c|c|c|c|c|c|}
\hline$V_{\text {IN }}(\mathrm{V})$ & $\mathrm{I}_{\mathrm{IN}}(\mathrm{A})$ & $V_{\text {oUt }}(\mathrm{V})$ & $\mathrm{I}_{\text {OUT }}(\mathrm{A})$ & $P_{\text {IN }}(W)$ & $P_{\text {OUT }}(W)$ & $\eta(\%)$ & $R_{\mathrm{IN}}(\Omega)$ & Training Resistance Level \\
\hline 8.08 & 0.797 & 36.02 & 0.09 & 6.440 & 3.242 & 50.340 & 10.138 & 2 \\
\hline 11.54 & 1.119 & 36.02 & 0.19 & 12.913 & 6.844 & 52.998 & 10.313 & 3 \\
\hline 13.49 & 1.295 & 36.02 & 0.27 & 17.470 & 9.725 & 55.671 & 10.417 & 4 \\
\hline 15.88 & 1.502 & 36.02 & 0.38 & 23.852 & 13.688 & 57.386 & 10.573 & 5 \\
\hline 18.27 & 1.718 & 36.02 & 0.52 & 31.388 & 18.730 & 59.674 & 10.634 & 6 \\
\hline 21 & 1.99 & 36.02 & 0.72 & 41.790 & 25.934 & 62.059 & 10.553 & 7 \\
\hline 23.3 & 2.202 & 36.02 & 0.9 & 51.307 & 32.418 & 63.185 & 10.581 & 8 \\
\hline 26.2 & 2.452 & 36.02 & 1.15 & 64.242 & 41.423 & 64.479 & 10.685 & 9 \\
\hline 29.1 & 2.695 & 36.02 & 1.43 & 78.425 & 51.509 & 65.679 & 10.798 & 10 \\
\hline 31.8 & 2.517 & 36.02 & 1.46 & 80.041 & 52.589 & 65.703 & 12.634 & 11 \\
\hline 34.3 & 2.112 & 36.02 & 1.29 & 72.442 & 46.466 & 64.142 & 16.241 & 12 \\
\hline 35.1 & 2.174 & 36.02 & 1.38 & 76.307 & 49.708 & 65.141 & 16.145 & 13 \\
\hline $39.9 *$ & $3.071 *$ & $36.03 *$ & $2.62^{*}$ & $122.533^{*}$ & 94.399* & 77.039* & $12.993 *$ & 14 \\
\hline 44.8 & 2.738 & 36.04 & 2.3 & 122.662 & 82.892 & 67.577 & 16.362 & 15 \\
\hline 46.4 & 2.138 & 36.04 & 1.81 & 99.203 & 65.232 & 65.756 & 21.703 & 16 \\
\hline 47.6 & 2.197 & 36.02 & 1.91 & 104.577 & 68.798 & 65.787 & 21.666 & 17 \\
\hline 48.3 & 2.232 & 36.06 & 1.96 & 107.806 & 70.678 & 65.560 & 21.640 & 18 \\
\hline 58.2 & 0.11 & 36.02 & 0.09 & 6.402 & 3.242 & 50.637 & 529.091 & 19 \\
\hline 59.8 & 0.112 & 36.02 & 0.09 & 6.698 & 3.242 & 48.402 & 533.929 & 20 \\
\hline
\end{tabular}

This test does not include input voltages above $60 \mathrm{~V}$ because beyond that input level the converter cannot drive sufficient load current (greater than 1A). Asterisks indicate input voltages for which the GW Instek power supply reached or exceeded its current limit, therefore maximum load current and efficiency results for such input voltages are not completely accurate. Overall, as long as the SEPIC maintains a constant 36V output, it maintains a $10 \Omega$ input resistance even at the elliptical trainer's lowest output power level. However, this only holds true up to around 40V input. Beyond that, the SEPIC loses its input resistance maintenance high load current maintenance abilities. Overall converter efficiency at the tested input voltages is also below specification. Running the SEPIC in 
CCM explains its low efficiency at lower input voltage and hence while driving lowcurrent loads, as mentioned in section 4.2. The converter shows decent efficiency levels during the typical workout's 15-30V input range; however, such levels are still below what this project desires. However, these results show that the SEPIC can handle $120 \mathrm{~W}$ input as well as $60 \mathrm{~V}$ input from the elliptical trainer. $60 \mathrm{~V}$ was the maximum voltage that the EHFEM group in [1] obtained from a 160 strides per minute exercise test, and 160 strides per minute falls within the typical pace range at which Cal Poly Recreational Center users exercise. The next section investigates possible faults for causing the SEPIC's poor load regulation at input voltages above $52 \mathrm{~V}$.

\subsubsection{First Troubleshooting Phase}

This project now examines what SEPIC components could cause these faults beginning with the SEPIC's switching inductors. Figures 10.7 to 10.10 show primary inductor $\mathrm{L}_{1}$ 's current waveforms under various input voltages and load levels. The figures' respective captions indicate SEPIC input voltage and load level. Secondary inductor $\mathrm{L}_{2}$ 's current waveforms follow a similar shape (with different magnitudes), therefore this project does not include $\mathrm{L}_{2}$ 's current waveforms in this report. 

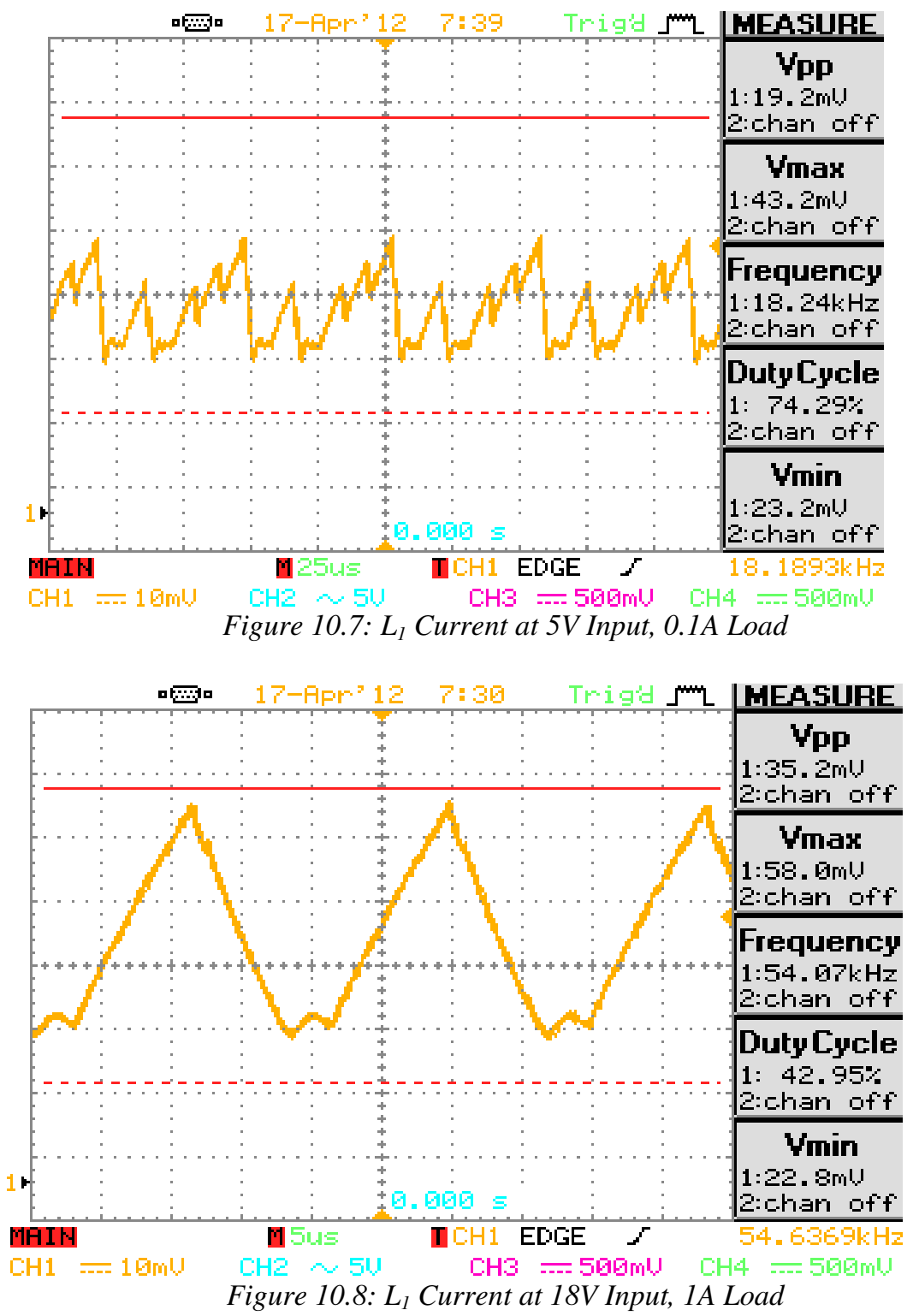

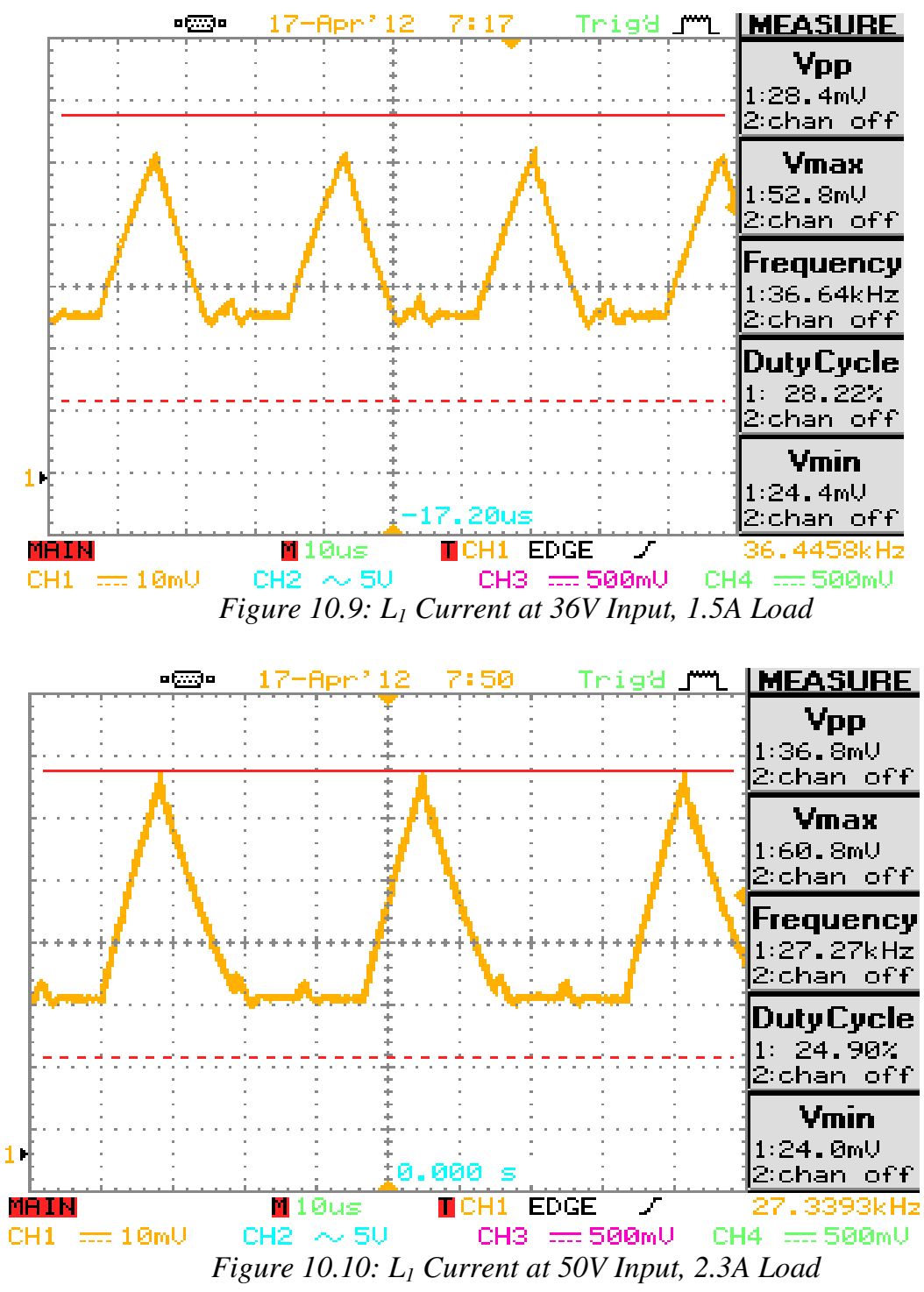
Figure 10.7 shows the SEPIC not regulating a $36 \mathrm{~V}$ output (5V input with a $0.1 \mathrm{~A}$ load) the inductor waveform is distorted. Figures 10.8 to 10.10 are more realistic waveforms, as the SEPIC regulates a $36 \mathrm{~V}$ output with the conditions in the mentioned figures. Furthermore, figures 10.8 to 10.10 show "dead time" between inductor charging cycles. These waveform shapes indicate DCM operation because of the dead time, even though the current levels do not drop to $0 \mathrm{~A}$ for any significant time period relative to the SEPIC's $100 \mathrm{kHz}$ switching frequency. Ideally, the inductor waveforms should not have any dead time in between charging periods. However, because Q_MAIN's switching trajectory affects inductor operation, thus Q_MAIN may not be switching properly. This report's next paragraph addresses and investigates this problem in greater detail.

However, these $\mathrm{L}_{1}$ current waveforms in figures 10.7 to 10.10 also show that $\mathrm{L}_{1}$ never saturates (and hence $\mathrm{L}_{2}$ never saturates, either) for the 5-50V input and the 0.2-2.3A load current range, therefore the SEPIC's $\mathrm{L}_{1}$ and $\mathrm{L}_{2}$ saturation current rating suffices.

This project's next investigation interest area is Q_MAIN's switching trajectory. Figures 10.11 to 10.13 show Q_MAIN's gate voltage waveforms (with respect to signal ground) under various input voltages and load levels. 


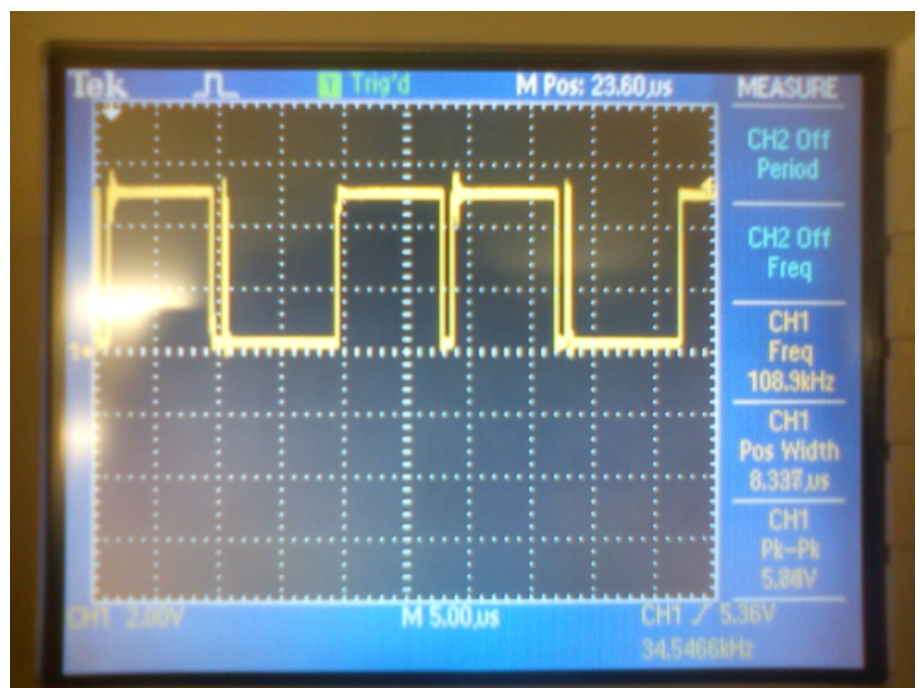

Figure 10.11: Q_MAIN Switching Trajectory at 5V Input and 0.1A Load

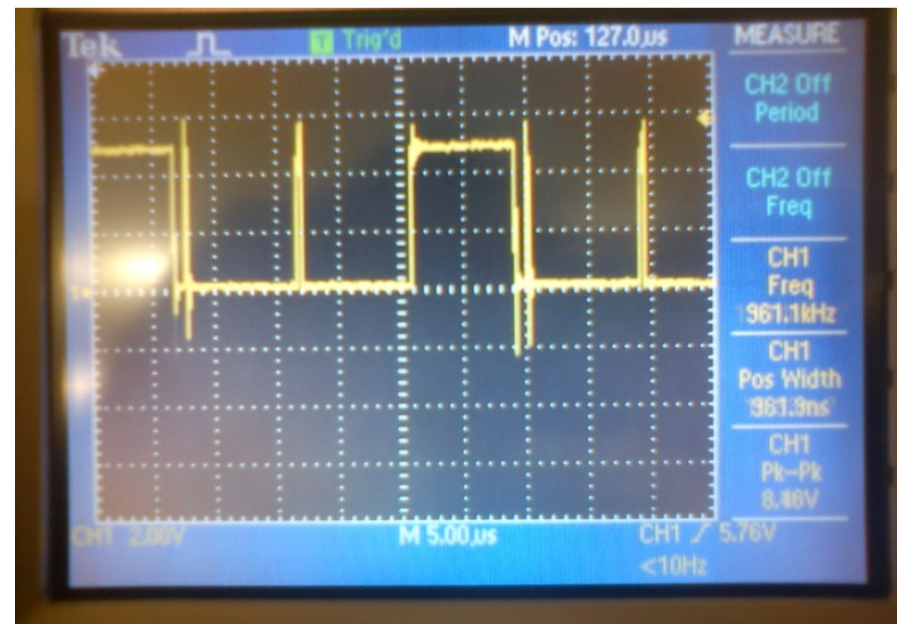

Figure 10.12: Q_MAIN Switching Trajectory at 36V Input and 1.46A Load

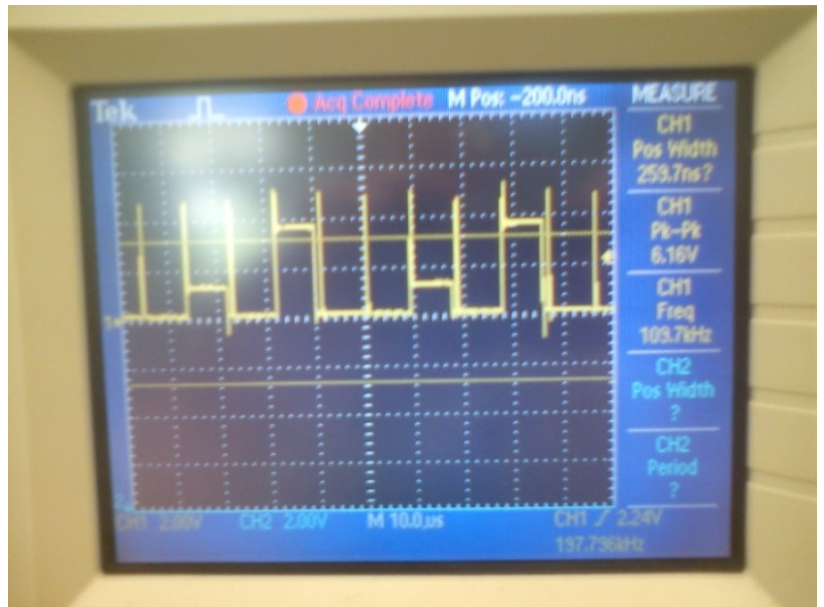

Figure 10.13: Q_MAIN Switching Trajectory at 50V Input and 2.3A Load 
Figures 10.10 to 10.13 show that Q_MAIN's gate switching waveforms are nowhere close to ideal. Although Q_MAIN switches at $110 \mathrm{kHz}$, the LTC1871 controller has a problem maintaining its duty cycle. Figure 10.13 shows that at $50 \mathrm{~V}$ input and $2.3 \mathrm{~A}$ load, Q_MAIN's gate voltage reaches metastable states, resulting in unstable SEPIC operation. Furthermore, figures 10.10 to 10.13 show high frequency harmonics (greater than $100 \mathrm{kHz}$ ) in Q_MAIN's switching trajectories, which may contribute to the SEPIC's abnormal operation at higher input voltages and higher current loads. Ideally Q_MAIN's gate switching waveform should be close to what figure 5.10 shows. This project now revisits the finalized SEPIC converter's (shown in figure 7.9) simulations because unlike the simulation this project performed on the initial basic SEPIC design in chapter 5, the simulations on the finalized SEPIC design in chapter 7 neglected inspecting inductor current waveforms as well as gate switching waveforms with all parasitic elements added to the SEPIC's passive components. Figure 10.14 shows all pertinent waveforms in the finalized SEPIC from figure 7.9, with the SEPIC running under full load and steady-state operation conditions. In figure 10.14, V(out) denotes the SEPIC's output voltage, V(n013) denotes Q_MAIN's gate voltage, I(L1) denotes L_'s current, I(D_main1) denotes the SEPIC's main switching diode D_MAIN1's current, V(sw) denotes Q_MAIN's drain voltage, V(fb) denotes the feedback node's voltage, and V(sense) denotes the sense node and Q_MAIN's source voltage, which is proportional to Q_MAIN's drain current. 


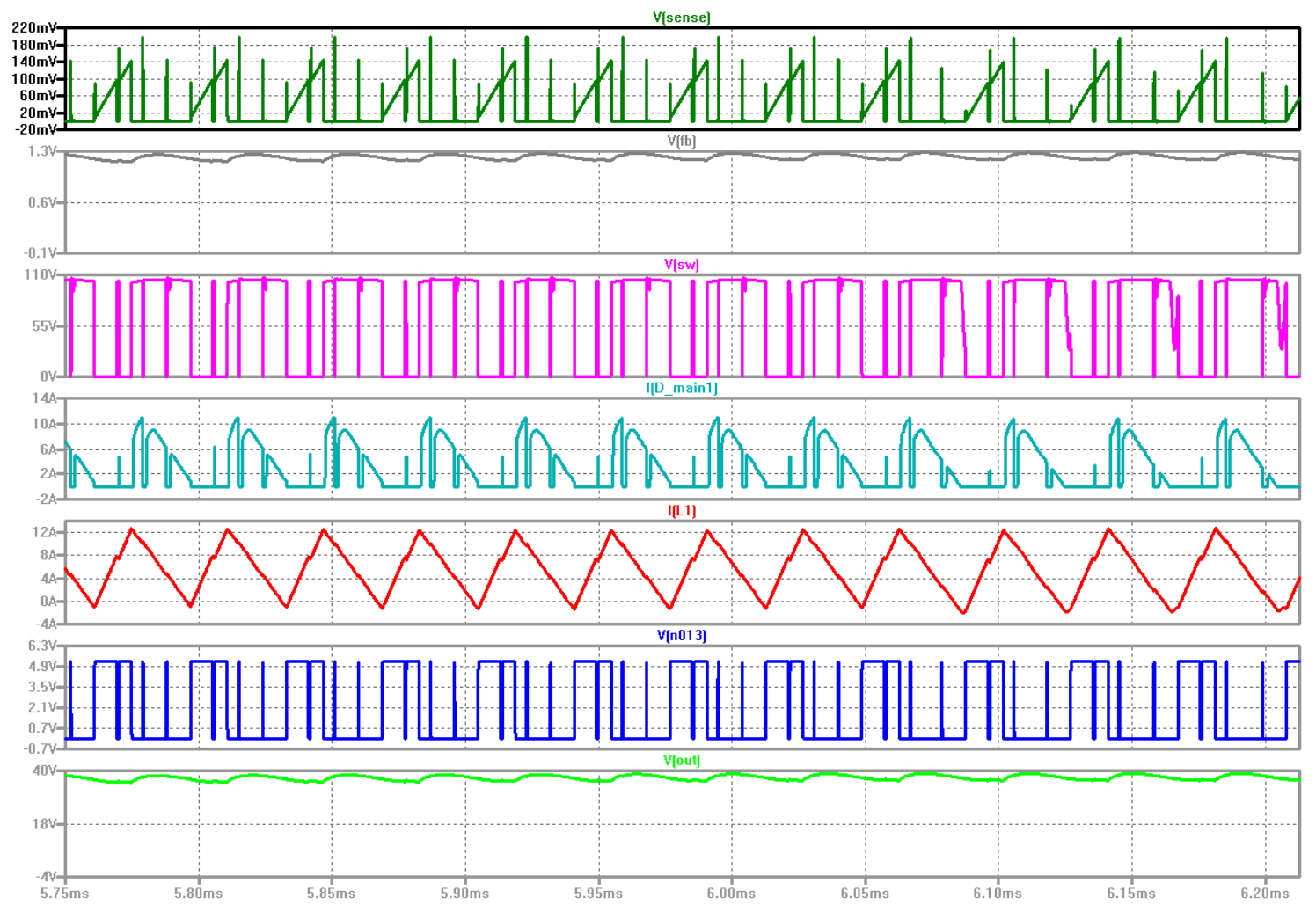

Figure 10.14: Finalized SEPIC Output Voltage, $Q \_M A I N$ Switching Trajectory, $L_{l}$ Current and Feedback and Sense Node Voltages from LTSpice Simulation

As figure 10.14 shows, the same problem with Q_MAIN's switching trajectory shows up in simulation results. Other crucial switching waveforms also do not match their shapes from figure 5.10. Additionally, the SEPIC's feedback voltage also oscillates, indicating that its node filter capacitor $\mathrm{C}_{\mathrm{FB}}$ may not be large enough. This project then determined that Q_MAIN's abnormal gate switching trajectory in turn caused abnormal $\mathrm{L}_{1}$ current (from figures 10.7 to 10.10 and 10.14), D_MAIN1 current and Q_MAIN drain current waveform shapes. Therefore, this project now attempts eliminating the additional high frequency harmonics from Q_MAIN's gate switching trajectory.

One possible solution involves putting additional capacitance between Q_MAIN's gate and ground, along with additional capacitance between the SEPIC's feedback node 
and ground for stabilizing its voltage. Putting additional capacitance between Q_MAIN's gate and ground creates a low pass filter and can help shunt higher frequency harmonics to ground. Similarly, RC low pass filters dampen any oscillation that occurs on the LTC1871's GATE, FB and SENSE pins; however, adding a series resistor to those pins requires physically severing PCB copper traces already connected to them. Therefore, this project first implements purely capacitive low pass filters on the LTC1871's GATE, FB and SENSE pins and then only replaces them with RC low pass filters if necessary. An RC low pass filter, however, eliminates the leading harmonic spike on the current sense resistor voltage that results from the LTC1871 controller sensing Q_MAIN's drain current - figure 10.14 shows this phenomenon [28]. This project begins its signal harmonic suppression task by first placing a $0.01 \mu \mathrm{F}$ capacitor across the LTC1871 controller's FB pin.

Figure 10.15 the result from inserting a $0.01 \mu \mathrm{F}$ capacitor across the LTC1871's FB pin, using the same waveforms as from figure 10.14 above. 


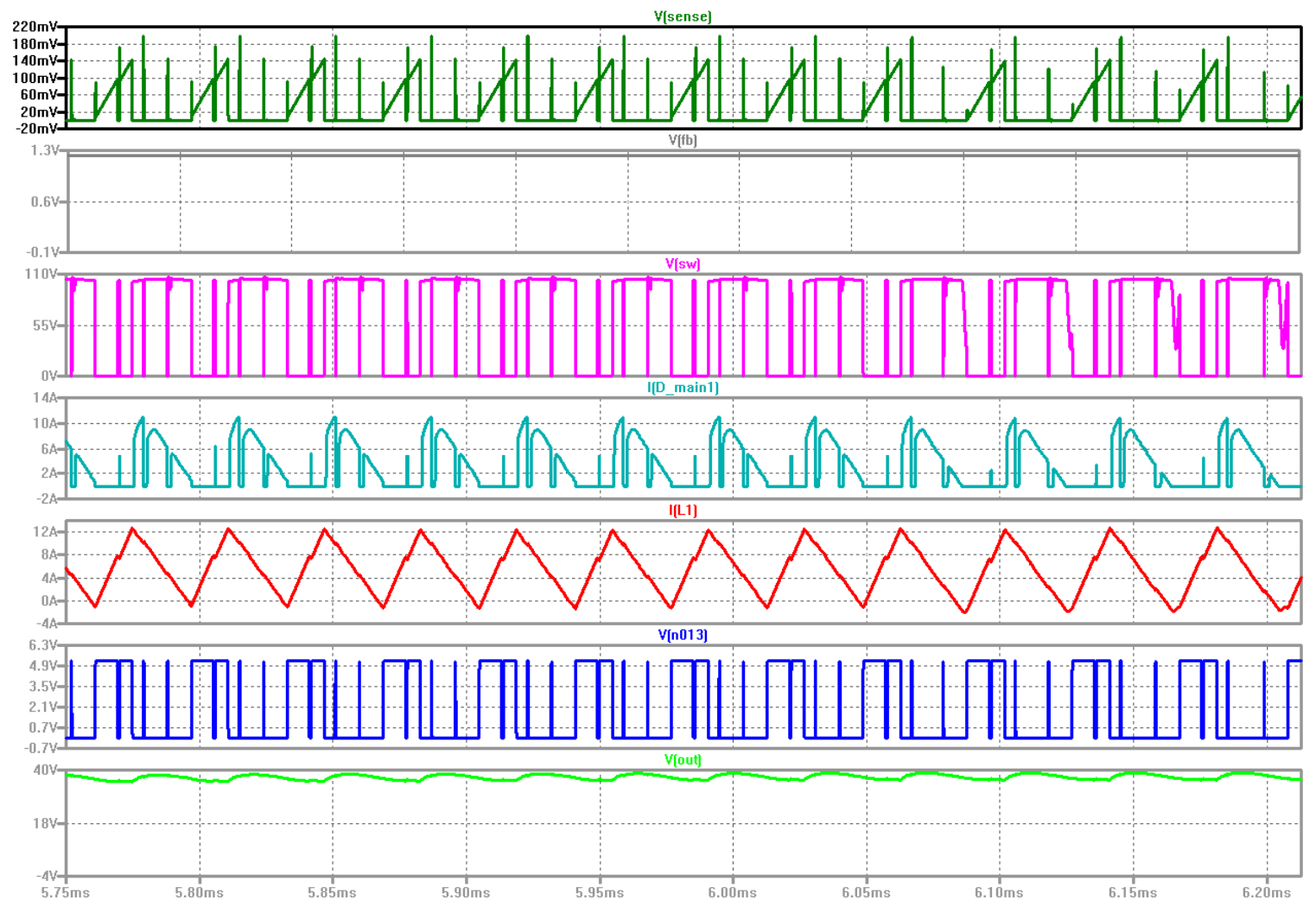

Figure 10.15: Finalized SEPIC with 0.01 $\mu$ F Feedback Capacitor Output Voltage, Q_MAIN Switching

Trajectory, $L_{l}$ Current and Feedback and Sense Node Voltages from LTSpice Simulation

Although adding capacitance across the LTC1871's FB pin cleans the SEPIC's feedback voltage signal, its other waveforms are still nowhere close to their ideal shapes.

Therefore, increasing capacitance across the LTC1871's FB pin alone is not effective for obtaining proper switching waveform shapes and hence proper SEPIC operation. This project next adds a small capacitor across the LTC1871's GATE pin for smoothing out Q_MAIN's gate switching waveform. For Q_MAIN's gate, adding too little capacitance is ineffective for harmonic suppression, while adding too much results in unstable and inefficient converter operation. Large gate capacitances across Q_MAIN result in it expending more energy charging and discharging the gate, resulting in higher switching power dissipation losses and hence less efficient overall converter operation. Therefore 
this project selects $0.01 \mu \mathrm{F}$ as a conservative Q_MAIN gate capacitor filter value, as it is neither too small nor too large.

Figure 10.16 shows the result from inserting a $0.01 \mu \mathrm{F}$ capacitor across the LTC1871's GATE pin (in addition to the $0.01 \mu \mathrm{F}$ capacitor across the LTC1871's FB pin), using the same waveforms as from figure 10.14 and 10.15 .

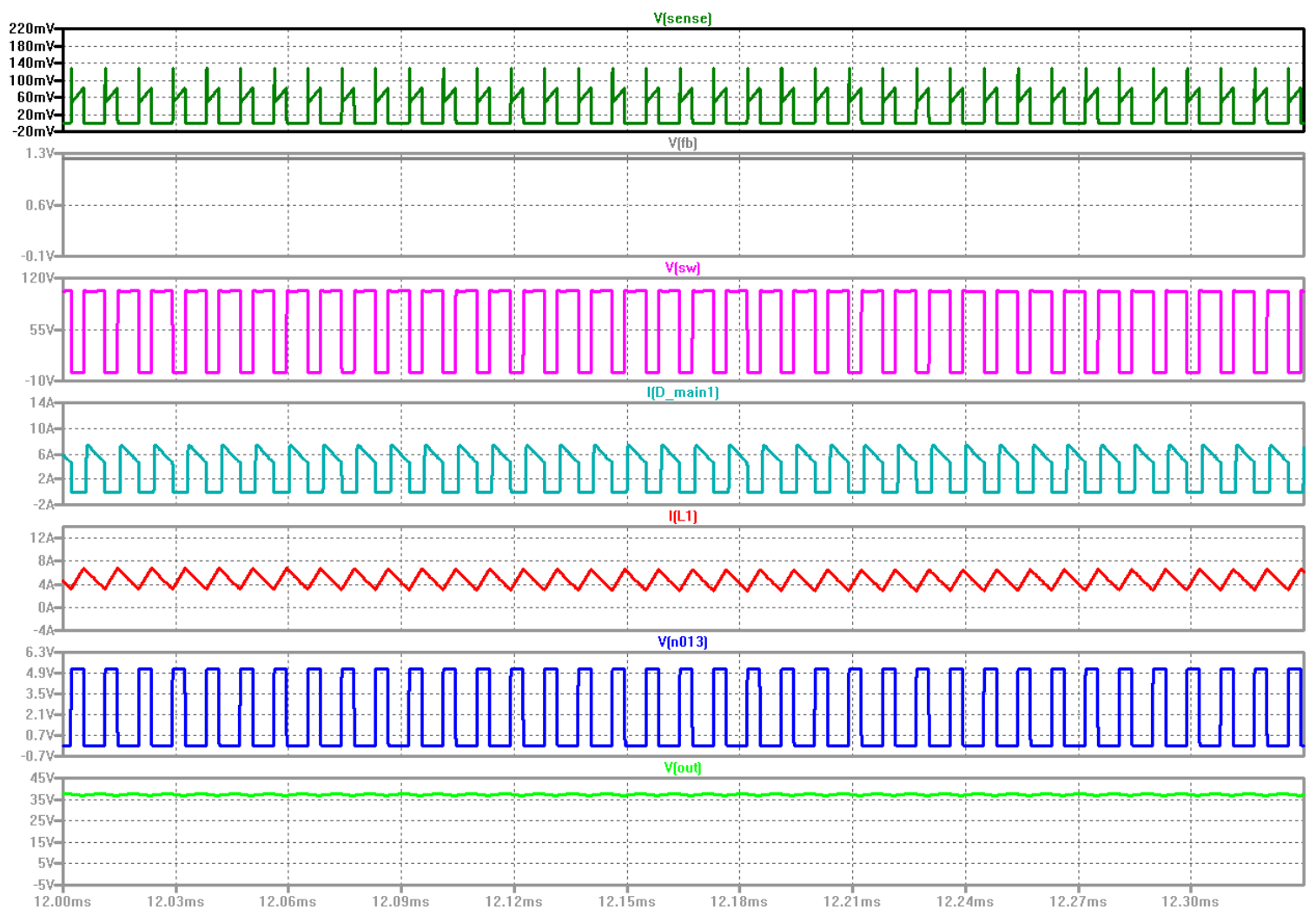

Figure 10.16: Finalized SEPIC with $0.01 \mu F$ Feedback Capacitor and 0.01 $\mu F Q$ QMAIN Gate Capacitor Output Voltage, Q_MAIN Switching Trajectory, $L_{1}$ Current and Feedback and Sense Node Voltages from LTSpice Simulation

Adding a $0.01 \mu \mathrm{F}$ capacitor across the LTC1871's GATE pin helps return Q_MAIN's gate switching waveform as well as its drain voltage waveform to their proper shapes. Likewise, the same occurs with D_MAIN1's and L L $_{1}$ 's current waveforms. Overall Q_MAIN power dissipation also decreases to $1.8 \mathrm{~W}$ (from $2.2 \mathrm{~W}$ in section 7.4 ) after 
making this change. However, the LTC1871's SENSE pin voltage waveform still contains a leading harmonic spike. Regardless, this project performs another hardware test checking if there is any overall converter improvement with the FB and GATE pin changes.

Tables E-19 and E-20 show the results from these modifications, using the same test instrument setup as the results from tables E-17 and E-18 above. This project uses decade capacitance boxes for the additional capacitors across the LTC1871's FB and GATE pins, for prototyping purposes. SEPIC revisions in later sections of this report use discrete ceramic capacitors. Again, likewise with the results from tables E-17 and E-18, Table E-19 shows the SEPIC's output load driving abilities while using the electronic load in constant voltage mode (36V) and Table E-20 shows the same test results, except using input voltages (with their corresponding elliptical training resistance levels) that match the 160 strides per minute exercise test results from the previous EHFEM group in [1]. 
Table E-19: Modified Feedback and Gate Nodes SEPIC Load Tests with Constant Voltage Electronic Load, TPS-4000 and GW Instek Power Supplies

\begin{tabular}{|c|c|c|c|c|c|c|c|}
\hline$V_{\text {IN }}(V)$ & $I_{I N}(A)$ & $\mathrm{V}_{\text {OUT }}(\mathrm{V})$ & $\mathrm{I}_{\text {OUT }}(\mathrm{A})$ & $P_{\text {IN }}(W)$ & $P_{\text {OUT }}(W)$ & $\eta(\%)$ & $R_{I N}(\Omega)$ \\
\hline 5 & 2.463 & 36.01 & 0.17 & 12.315 & 6.122 & 49.709 & 2.030 \\
\hline 10 & $3.067^{*}$ & 36.02 & $0.24^{*}$ & $30.67^{*}$ & $8.645^{*}$ & $28.187^{*}$ & $3.261 *$ \\
\hline 12 & $3.067 *$ & 36.02 & $0.62 *$ & $36.804^{*}$ & $22.332 *$ & $60.679 *$ & 3.913* \\
\hline 15 & $2.946 *$ & 36.02 & $0.72 *$ & $44.19 *$ & $25.934^{*}$ & $58.688^{*}$ & $5.092 *$ \\
\hline 18 & $3.068 *$ & 36.02 & $0.9 *$ & $55.224^{*}$ & $32.418^{*}$ & $58.703 *$ & $5.867 *$ \\
\hline 20 & 2.037 & 36.02 & 0.65 & 40.74 & 23.413 & 57.469 & 9.818 \\
\hline 25 & 2.423 & 36.02 & 1 & 60.575 & 36.020 & 59.463 & 10.318 \\
\hline 27 & 2.594 & 36.02 & 1.18 & 70.038 & 42.504 & 60.686 & 10.409 \\
\hline 30 & 2.851 & 36.02 & 1.48 & 85.53 & 53.310 & 62.329 & 10.523 \\
\hline 36 & $3.067^{*}$ & 36.02 & $1.79 *$ & $110.412^{*}$ & $64.476^{*}$ & $58.396 *$ & $11.738^{*}$ \\
\hline 40 & $3.067^{*}$ & 36.02 & $1.79 *$ & $122.68^{*}$ & $64.476^{*}$ & $52.556^{*}$ & $13.042^{*}$ \\
\hline 45 & $3.067^{*}$ & 36.02 & $1.79 *$ & $138.015^{*}$ & $64.476^{*}$ & $46.717^{*}$ & $14.672 *$ \\
\hline 50 & $3.067^{*}$ & 36.02 & $1.79 *$ & $153.35^{*}$ & $64.476 *$ & $42.045^{*}$ & $16.303 *$ \\
\hline 52 & $3.067^{*}$ & 36.02 & $1.79 *$ & $159.484 *$ & $64.476 *$ & $40.428^{*}$ & $16.955^{*}$ \\
\hline 60 & $3.067^{*}$ & 36.02 & $1.79 *$ & $184.02 *$ & $64.476 *$ & $35.037^{*}$ & $19.563 *$ \\
\hline
\end{tabular}


Table E-20: Modified Feedback and Gate Nodes SEPIC Load Tests with 160 Strides/Min. Theoretical Input Voltages from [1], Constant Voltage Electronic Load, TPS-4000 and GW Instek Power Supplies

\begin{tabular}{|c|c|c|c|c|c|c|c|c|}
\hline$V_{\text {IN }}(\mathrm{V})$ & $\mathrm{I}_{\mathrm{IN}}(\mathrm{A})$ & $\mathrm{V}_{\text {OUT }}(\mathrm{V})$ & $\mathrm{I}_{\text {OUT }}(\mathrm{A})$ & $P_{\text {IN }}(W)$ & $P_{\text {OUT }}(W)$ & $\eta(\%)$ & $R_{I N}(\Omega)$ & Training Resistance Level \\
\hline $8.08^{*}$ & $3.068 *$ & 36 & $0.251^{*}$ & $24.789 *$ & $9.036^{*}$ & $36.451^{*}$ & $2.634 *$ & 2 \\
\hline $11.54^{*}$ & $3.068^{*}$ & 36 & $0.251 *$ & $35.405^{*}$ & $9.036^{*}$ & $25.522 *$ & $3.761 *$ & 3 \\
\hline $13.49 *$ & $3.068^{*}$ & 36 & $0.251^{*}$ & $41.387^{*}$ & $9.036^{*}$ & $21.833^{*}$ & $4.397^{*}$ & 4 \\
\hline $15.88^{*}$ & $3.067^{*}$ & 36 & $0.804 *$ & $48.704^{*}$ & $28.944 *$ & $59.428^{*}$ & $5.178^{*}$ & 5 \\
\hline $18.27^{*}$ & $3.067^{*}$ & 36 & $0.804^{*}$ & $56.034^{*}$ & $28.944 *$ & $51.654 *$ & $5.957^{*}$ & 6 \\
\hline 21 & 2.145 & 36 & 0.748 & 45.045 & 26.928 & 59.780 & 9.790 & 7 \\
\hline 23.3 & 2.289 & 36 & 0.885 & 53.334 & 31.86 & 59.737 & 10.179 & 8 \\
\hline 26.2 & 2.533 & 36 & 1.128 & 66.365 & 40.608 & 61.189 & 10.343 & 9 \\
\hline 29.1 & 2.774 & 36 & 1.397 & 80.723 & 50.292 & 62.302 & 10.490 & 10 \\
\hline 31.8 & 3.032 & 36 & 1.691 & 96.418 & 60.876 & 63.138 & 10.488 & 11 \\
\hline $34.3^{*}$ & $3.069 *$ & 36 & $1.735^{*}$ & $105.267^{*}$ & $62.46^{*}$ & $59.335^{*}$ & $11.176^{*}$ & 12 \\
\hline $35.1^{*}$ & $3.069 *$ & 36 & $1.735^{*}$ & $107.722 *$ & $62.46^{*}$ & 57.983* & $11.437^{*}$ & 13 \\
\hline $39.9 *$ & $3.069 *$ & 36 & $1.735^{*}$ & $122.453^{*}$ & $62.46^{*}$ & $51.007^{*}$ & $13.001 *$ & 14 \\
\hline $44.8^{*}$ & $3.069 *$ & 36 & $1.735^{*}$ & $137.491^{*}$ & $62.46^{*}$ & $45.428 *$ & $14.598^{*}$ & 15 \\
\hline $46.4^{*}$ & $3.069 *$ & 36 & $1.735^{*}$ & $142.402^{*}$ & $62.46^{*}$ & $43.862 *$ & $15.119 *$ & 16 \\
\hline $47.6^{*}$ & $3.069 *$ & 36 & $1.735^{*}$ & $146.084^{*}$ & $62.46^{*}$ & $42.756^{*}$ & $15.510^{*}$ & 17 \\
\hline $48.3^{*}$ & $3.069 *$ & 36 & $1.735^{*}$ & $148.233^{*}$ & $62.46^{*}$ & 42.136* & $15.738^{*}$ & 18 \\
\hline $58.2^{*}$ & $3.069 *$ & 36 & $1.735^{*}$ & $178.616^{*}$ & $62.46^{*}$ & $34.969 *$ & $18.964 *$ & 19 \\
\hline $59.8^{*}$ & $3.069 *$ & 36 & $1.735^{*}$ & $183.526^{*}$ & $62.46^{*}$ & $34.033 *$ & $19.485^{*}$ & 20 \\
\hline
\end{tabular}

Likewise with the results from tables E-17 and E-18, this test does not include input voltages above $60 \mathrm{~V}$ because beyond that input level the converter begins shutting down operation, which the LT4356-1 input protection circuit causes. Asterisks indicate input voltages for which the GW Instek power supply reached or exceeded its current limit, therefore maximum load current and efficiency results for such input voltages are not completely accurate. A problem with load current capacity exists between $15 \mathrm{~V}$ to $20 \mathrm{~V}$ input - the SEPIC's maximum load current driving capability drops after the $18 \mathrm{~V}$ point. The next troubleshooting round in section 10.3.4 addresses this issue. However, this issue is not critical because at $18 \mathrm{~V}$ and lower input voltages, the SEPIC does not maintain a 
$10 \Omega$ input impedance while driving its maximum load current while simultaneously maintaining $36 \mathrm{~V}$ output. Adding a $0.01 \mu \mathrm{F}$ capacitor to the LTC1871's FB pin and a $0.01 \mu \mathrm{F}$ capacitor to its GATE pin shows promising results and improves overall converter efficiency, although the GW Instek power supply's 3A current limit renders most of these results inaccurate. Therefore, this project requires a power supply capable of supplying this converter's specified 6.5A current limit. The next section describes a new instrument setup for removing the input supply current limit from results.

\subsubsection{Constant Voltage Load Tests Using Non-Current Limited Input Supply}

The instrument setup for this section's testing round uses a BK Precision XLN3640 power supply. This power supply can supply up to 40A but it also has a 36V output limit. Therefore this testing round only tests the SEPIC at input voltages up to 36V. This voltage limit suffices for testing the SEPIC when operating in boost mode, as section 3.3 describes the typical elliptical trainer exercise output voltage range as $15-30 \mathrm{~V}$. Subsequent sections in this report use a power supply capable of this SEPIC's required input voltage range as well as current limit. Figure 10.17 shows the instrument setup block diagram for this section's testing round. 


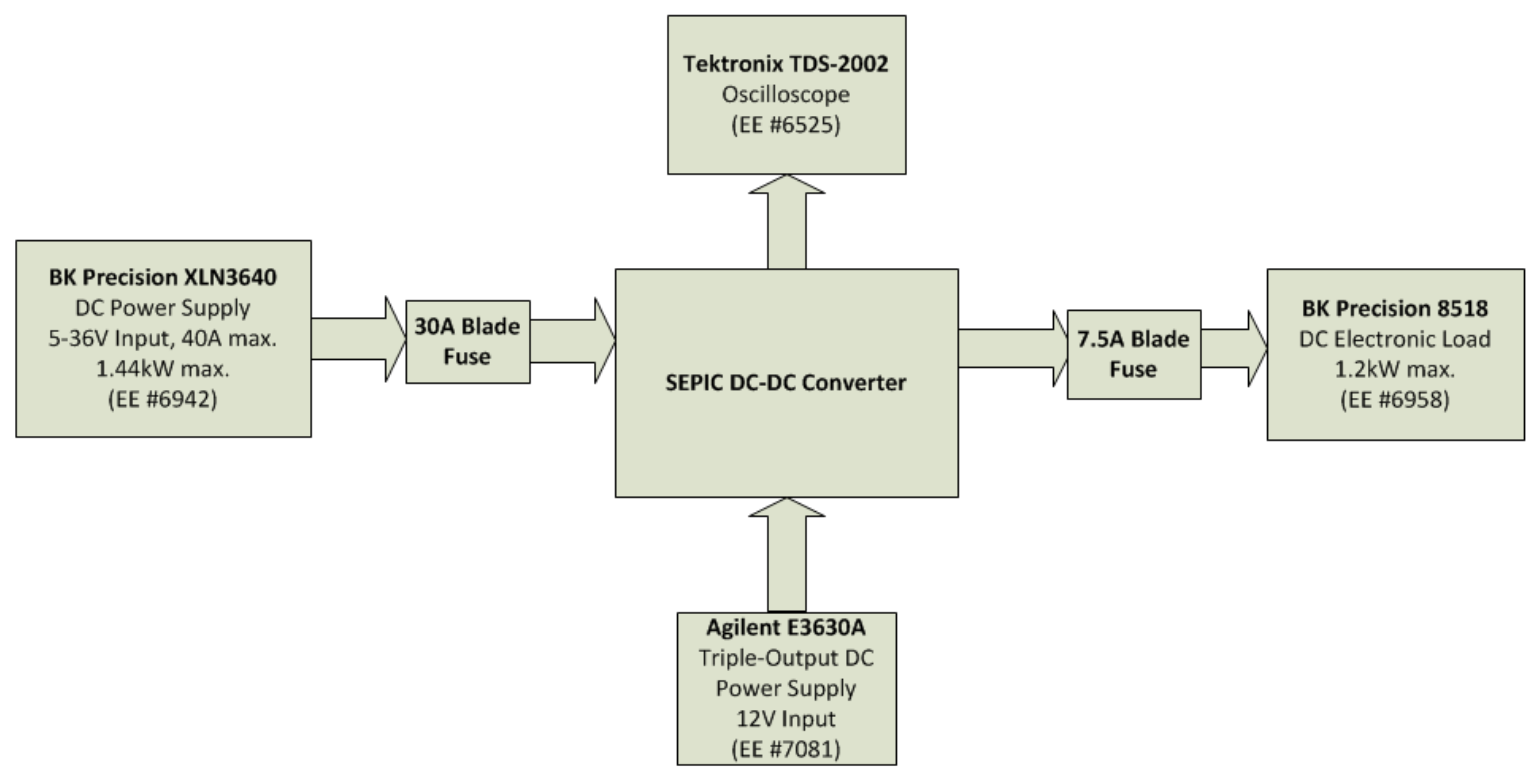

Figure 10.17: Finalized SEPIC with $0.01 \mu F$ Feedback Capacitor and 0.01 $\mu F$ Q_MAIN Gate Capacitor Test Setup Block Diagram

This power supply was in Cal Poly's electrochemical engineering laboratory (room 20-

130), therefore this project performs this testing round using equipment in that laboratory.

Furthermore, this testing round removes the inductor current wire loops from the PCB and replaces those wires with solder bridges because no current probe amplifiers exist in the 20-130 laboratory. This project requires current probe amplifiers in addition to the mentioned wire loops for measuring inductor current. This testing round uses the same Tektronix oscilloscope as the previous one, but a different electronic load. The $12 \mathrm{~V}$ power supply used to simulate the elliptical trainer's onboard battery is also different this time this project uses an Agilent E3630A power supply for simulating the elliptical trainer's battery. This testing round uses a BK Precision $85181.2 \mathrm{~kW}$ electronic load, which more than suffices for this SEPIC's 288W maximum output load driving requirement. Tables E-21 and E-22 show the results from this instrument setup. Likewise 
with the tests from the previous section, this project uses decade capacitance boxes for the additional capacitors across the LTC1871's FB and GATE pins, for prototyping purposes. Also, likewise with the results from the previous section, Table E-21 shows the SEPIC's output load driving abilities while using the electronic load in constant voltage mode (36V) and Table E-22 shows the same test results, except using input voltages (with their corresponding elliptical training resistance levels) that match the 160 strides per minute exercise test results from the previous EHFEM group in [1].

Table E-21: Modified Feedback and Gate Nodes SEPIC Load Tests with Constant Voltage Electronic Load, Agilent E3630A and BK XLN3640 Power Supplies

\begin{tabular}{|c|c|c|c|c|c|c|c|}
\hline $\mathbf{V}_{\text {IN }}(\mathbf{V})$ & $\mathbf{I}_{\text {IN }}(\mathbf{A})$ & $\mathbf{V}_{\text {OUT }}(\mathbf{V})$ & $\mathbf{I}_{\text {OUT }}(\mathbf{A})$ & $\mathbf{P}_{\text {IN }}(\mathbf{W})$ & $\mathbf{P}_{\text {OUT }}(\mathbf{W})$ & $\eta(\%)$ & $\mathbf{R}_{\text {IN }}(\Omega)$ \\
\hline 5 & 3.037 & 36 & 0.25 & 15.185 & 9 & 59.269 & 1.646 \\
\hline 10 & 3.466 & 36 & 0.626 & 34.66 & 22.536 & 65.020 & 2.885 \\
\hline 12 & 3.407 & 36 & 0.687 & 40.884 & 24.732 & 60.493 & 3.522 \\
\hline 15 & 3.029 & 36 & 0.772 & 45.435 & 27.792 & 61.169 & 4.952 \\
\hline 18 & 3.59 & 36 & 1.149 & 64.62 & 41.364 & 64.011 & 5.014 \\
\hline 20 & 2.099 & 36 & 0.697 & 41.98 & 25.092 & 59.771 & 9.528 \\
\hline 25 & 2.445 & 36 & 1.043 & 61.125 & 37.548 & 61.428 & 10.225 \\
\hline 27 & 2.585 & 36 & 1.23 & 69.795 & 44.28 & 63.443 & 10.445 \\
\hline 30 & 2.802 & 36 & 1.553 & 84.06 & 55.908 & 66.510 & 10.707 \\
\hline 36 & 3.513 & 36 & 2.473 & 126.468 & 89.028 & 70.396 & 10.248 \\
\hline
\end{tabular}


Table E-22: Modified Feedback and Gate Nodes SEPIC Load Tests with 160 Strides/Min. Theoretical Input Voltages from [1], Constant Voltage Electronic Load, Agilent E3630A and BK XLN3640 Power Supplies

\begin{tabular}{|c|c|c|c|c|c|c|c|c|}
\hline $\mathbf{V}_{\text {IN }}(\mathbf{V})$ & $\mathbf{I}_{\text {IN }}(\mathbf{A})$ & $\mathbf{V}_{\text {OUT }}(\mathbf{V})$ & $\mathbf{I}_{\text {OUT }}(\mathbf{A})$ & $\mathbf{P}_{\text {IN }}(\mathbf{W})$ & $\mathbf{P}_{\text {OUT }}(\mathbf{W})$ & $\eta(\%)$ & $\mathbf{R}_{\text {IN }}(\Omega)$ & Training Resistance Level \\
\hline 8.08 & 3.445 & 36 & 0.46 & 27.835 & 16.56 & 59.492 & 2.345 & 2 \\
\hline 11.54 & 3.067 & 36 & 0.595 & 35.393 & 21.42 & 60.520 & 3.763 & 3 \\
\hline 13.49 & 2.736 & 36 & 0.62 & 36.908 & 22.32 & 60.474 & 4.931 & 4 \\
\hline 15.88 & 3.183 & 36 & 0.866 & 50.546 & 31.176 & 61.678 & 4.989 & 5 \\
\hline 18.27 & 2.683 & 36 & 0.825 & 49.018 & 29.7 & 60.589 & 6.810 & 6 \\
\hline 21 & 2.163 & 36 & 0.758 & 45.423 & 27.288 & 60.075 & 9.709 & 7 \\
\hline 23.3 & 2.316 & 36 & 0.903 & 53.962 & 32.508 & 60.241 & 10.060 & 8 \\
\hline 26.2 & 2.508 & 36 & 1.158 & 65.709 & 41.688 & 63.443 & 10.447 & 9 \\
\hline 29.1 & 2.736 & 36 & 1.445 & 79.617 & 52.02 & 65.337 & 10.636 & 10 \\
\hline 31.8 & 2.985 & 36 & 1.76 & 94.923 & 63.36 & 66.749 & 10.653 & 11 \\
\hline 34.3 & 3.291 & 36 & 2.152 & 112.881 & 77.472 & 68.631 & 10.422 & 12 \\
\hline 35.1 & 3.402 & 36 & 2.297 & 119.41 & 82.692 & 69.250 & 10.317 & 13 \\
\hline
\end{tabular}

From these non-current limited results, adding a $0.01 \mu \mathrm{F}$ capacitor across the LTC1871's FB pin and across its GATE pin improves the SEPIC's overall efficiency. Furthermore, these modifications also improve the SEPIC's output load driving ability, as the SEPIC now drives more current at its load while maintaining a 36V output. Overall converter efficiency increases with increasing input voltage and output load current, however, the load current capacity problem at input voltages between $15 \mathrm{Vand} 20 \mathrm{~V}$ still exists.

Furthermore, the SEPIC does not maintain a $10 \Omega$ input impedance until the $20 \mathrm{~V}$ input point. This project then hypothesizes that a slightly larger capacitance across the LTC1871's GATE pin may correct the load current capacity problem. This project then increases the LTC1871's new GATE pin capacitance to $0.02 \mu \mathrm{F}$.

Figure 10.18 shows the result from inserting a $0.02 \mu \mathrm{F}$ capacitor across the LTC1871's GATE pin (in addition to the $0.01 \mu \mathrm{F}$ capacitor across the LTC1871's FB pin), using the same waveforms as from figure 10.14 and 10.15 . 


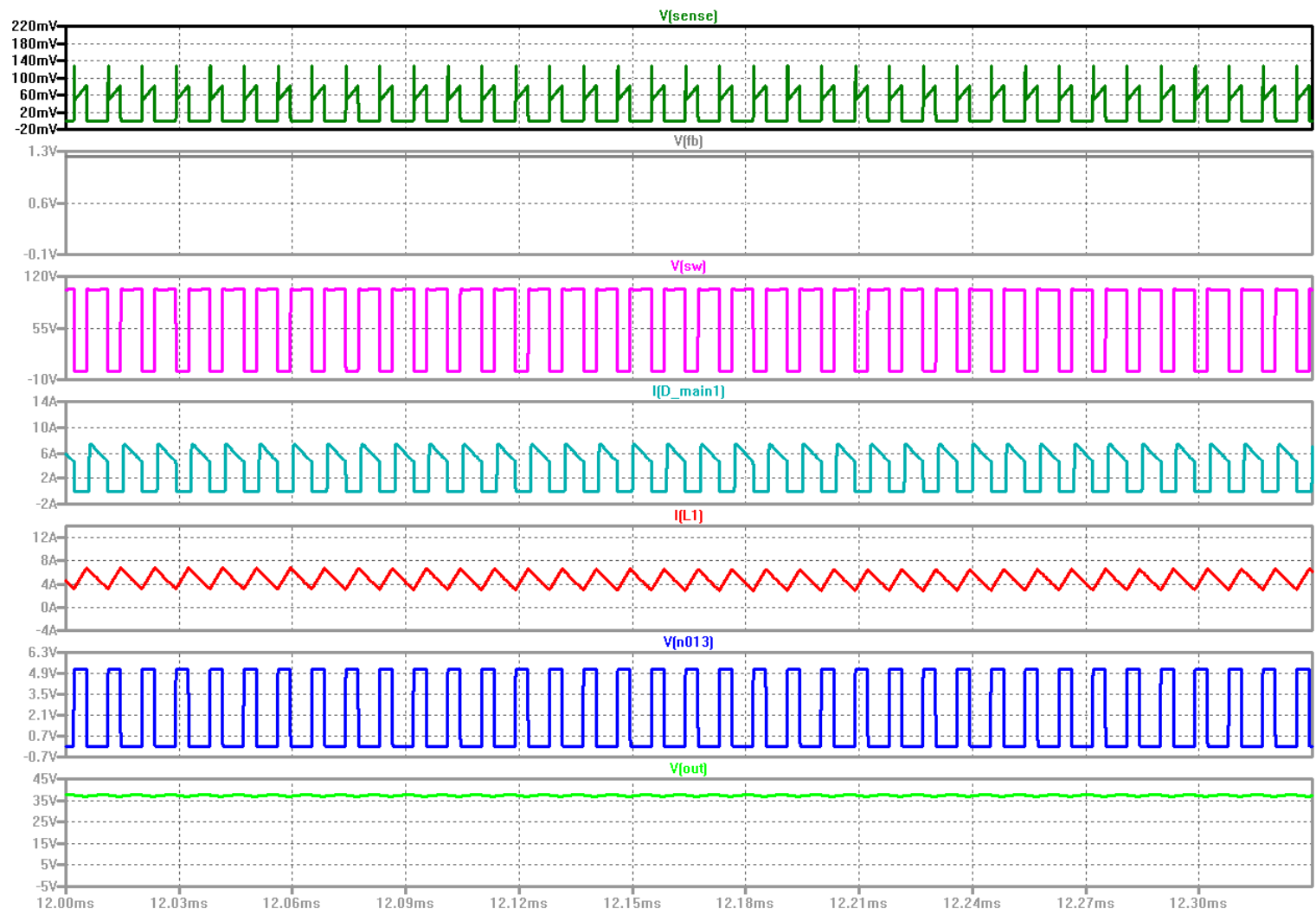

Figure 10.18: Finalized SEPIC with 0.01 $\mathrm{F}$ Feedback Capacitor and 0.02 $\mu F$ Q_MAIN Gate Capacitor Output Voltage, Q_MAIN Switching Trajectory, $L_{1}$ Current and Feedback and Sense Node Voltages from LTSpice Simulation

The results remain unchanged from figure 10.16, when this project used a $0.01 \mu \mathrm{F}$

capacitor across the LTC1871's GATE pin. Power dissipation across Q_MAIN also remains the same as before. However, simulation results do not always necessary translate to physical hardware test results, therefore this project now tests this capacitance change using the same instrument setup described earlier in this section. Tables E-23 and E-24 show these results. Likewise with the tests from earlier in this section, this project uses decade capacitance boxes for the additional capacitors across the LTC1871's FB and GATE pins, for prototyping purposes. Also, likewise with those tests, Table E-23 shows the SEPIC's output load driving abilities while using the electronic load in constant voltage mode (36V) and Table E-24 shows the same test results, except using input 
voltages (with their corresponding elliptical training resistance levels) that match the 160 strides per minute exercise test results from the previous EHFEM group in [1].

Table E-23: SEPIC Load Tests with 0.02 $\mu$ F Gate Capacitor, Constant Voltage Electronic Load, Agilent E3630A and BK XLN3640 Power Supplies

\begin{tabular}{|c|c|c|c|c|c|c|c|}
\hline $\mathbf{V}_{\text {IN }}(\mathbf{V})$ & $\mathbf{I}_{\text {IN }}(\mathbf{A})$ & $\mathbf{V}_{\text {OUT }}(\mathbf{V})$ & $I_{\text {OUT }}(A)$ & $P_{\text {IN }}(\mathbf{W})$ & $P_{\text {OUT }}(W)$ & $\eta(\%)$ & $R_{\text {IN }}(\Omega)$ \\
\hline 5 & 0.486 & 36 & 0.03 & 2.43 & 1.08 & 44.444 & 10.288 \\
\hline 10 & 0.948 & 36 & 0.136 & 9.48 & 4.896 & 51.646 & 10.549 \\
\hline 12 & 1.135 & 36 & 0.211 & 13.62 & 7.596 & 55.771 & 10.573 \\
\hline 15 & 1.406 & 36 & 0.351 & 21.09 & 12.636 & 59.915 & 10.669 \\
\hline 18 & 1.684 & 36 & 0.531 & 30.312 & 19.116 & 63.064 & 10.689 \\
\hline 20 & 1.881 & 36 & 0.677 & 37.62 & 24.372 & 64.785 & 10.633 \\
\hline 25 & 2.347 & 36 & 1.102 & 58.675 & 39.672 & 67.613 & 10.652 \\
\hline 27 & 2.51 & 36 & 1.287 & 67.77 & 46.332 & 68.367 & 10.757 \\
\hline 30 & 2.755 & 36 & 1.591 & 82.65 & 57.276 & 69.299 & 10.889 \\
\hline 36 & 3.479 & 36 & 2.484 & 125.244 & 89.424 & 71.400 & 10.348 \\
\hline
\end{tabular}

Table E-24: SEPIC Load Test with 0.02 $\mu$ F Gate Capacitor Using 160 Strides/Min. Theoretical Input Voltages from [1], Constant Voltage Electronic Load, Agilent E3630A and BK XLN3640 Power Supplies

\begin{tabular}{|c|c|c|c|c|c|c|c|c|}
\hline $\mathbf{V}_{\text {IN }}(\mathbf{V})$ & $\mathbf{I}_{\text {IN }}(\mathbf{A})$ & $\mathbf{V}_{\text {OUT }}(\mathbf{V})$ & $\mathbf{I}_{\text {OUT }}(\mathbf{A})$ & $\mathbf{P}_{\text {IN }}(\mathbf{W})$ & $\mathbf{P}_{\text {OUT }}(\mathbf{W})$ & $\eta(\%)$ & $\mathbf{R}_{\text {IN }}(\boldsymbol{\Omega})$ & Training Resistance Level \\
\hline 8.08 & 0.764 & 36 & 0.08 & 6.173 & 2.88 & 46.654 & 10.576 & 2 \\
\hline 11.54 & 1.088 & 36 & 0.191 & 12.555 & 6.876 & 54.765 & 10.607 & 3 \\
\hline 13.49 & 1.263 & 36 & 0.274 & 17.037 & 9.864 & 57.895 & 10.681 & 4 \\
\hline 15.88 & 1.478 & 36 & 0.396 & 23.47 & 14.256 & 60.740 & 10.744 & 5 \\
\hline 18.27 & 1.703 & 36 & 0.547 & 31.113 & 19.692 & 63.290 & 10.728 & 6 \\
\hline 21 & 1.972 & 36 & 0.752 & 41.412 & 27.072 & 65.372 & 10.649 & 7 \\
\hline 23.3 & 2.189 & 36 & 0.944 & 51.003 & 33.984 & 66.630 & 10.644 & 8 \\
\hline 26.2 & 2.436 & 36 & 1.206 & 63.823 & 43.416 & 68.025 & 10.755 & 9 \\
\hline 29.1 & 2.669 & 36 & 1.488 & 77.667 & 53.568 & 68.971 & 10.903 & 10 \\
\hline 31.8 & 2.92 & 36 & 1.801 & 92.856 & 64.836 & 69.824 & 10.890 & 11 \\
\hline 34.3 & 3.243 & 36 & 2.188 & 111.234 & 78.768 & 70.812 & 10.577 & 12 \\
\hline 35.1 & 3.362 & 36 & 2.33 & 118.006 & 83.88 & 71.081 & 10.440 & 13 \\
\hline
\end{tabular}

Increasing the LTC1871's GATE pin capacitance to $0.02 \mu \mathrm{F}$ improves overall converter efficiency. It also decreases the SEPIC's maximum load current driving capability at input voltages below $25 \mathrm{~V}$, but allows the SEPIC to maintain a $10 \Omega$ input impedance at all tested input voltage levels. These results would lead one believing that increasing the 
LTC1871's GATE pin capacitance further would further increase SEPIC performance, but that is not true. Q_MAIN gate capacitances above $0.02 \mu \mathrm{F}$ result in deteriorated converter performance, and its overall performance at such higher gate capacitances is no different from the original finalized SEPIC design with no additional gate capacitance. Therefore this project does not include results for Q_MAIN gate capacitances above $0.02 \mu \mathrm{F}$. This project hypothesizes that Q_MAIN's switching timing is crucial for proper operation with the SEPIC's snubber, as the snubber component selection process from section 6.3 and figure 6.14 relies on Q_MAIN's gate charging and discharging time periods. Selecting the proper Q_MAIN gate capacitor alters Q_MAIN's charging and discharging time periods such that Q_MAIN effectively exchanges energy with the SEPIC's snubber, therefore Q_MAIN's gate capacitance cannot be too large or small. Furthermore, as mentioned in section 10.3.3, too much capacitance on Q_MAIN's gate increases the energy required for charging and discharging it, therefore this project does not desire larger capacitance across Q_MAIN's gate.

Now this project investigates Q_MAIN's switching trajectory waveforms with the additional $0.02 \mu \mathrm{F}$ capacitor connected across its gate. Figures 10.19 and 10.20 show Q_MAIN's new gate voltage waveforms (with respect to signal ground) under 5V and 36V input, while the SEPIC drives its maximum load current (while maintaining 36V output) under those input conditions. 


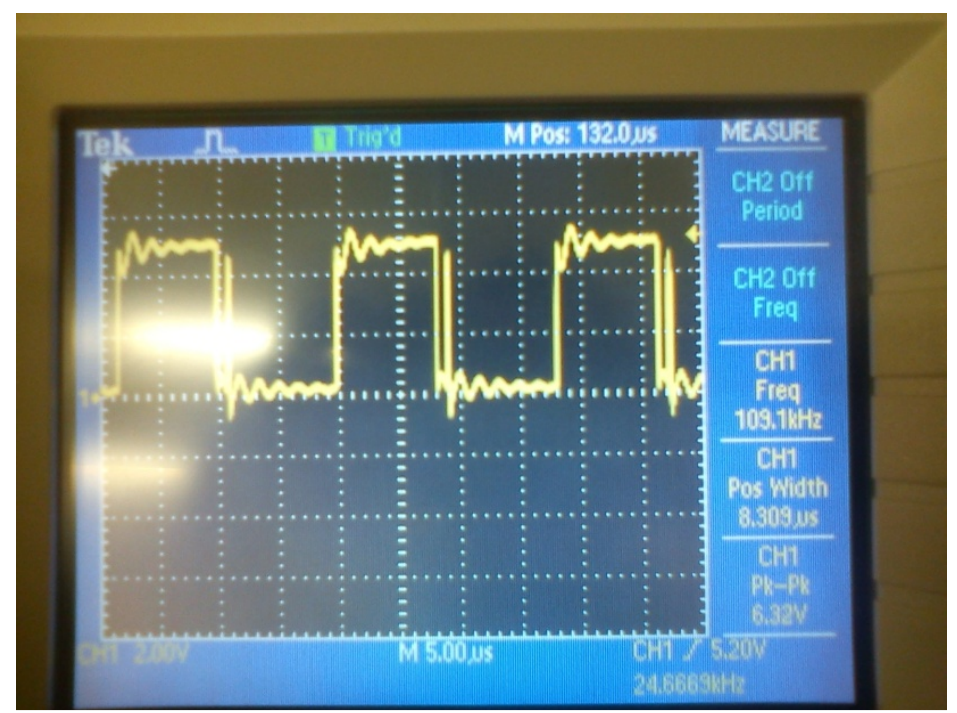

Figure 10.19: Q_MAIN Switching Trajectory with 0.02 $\mu$ F Gate Capacitor at 5 V Input and 0.03A Load

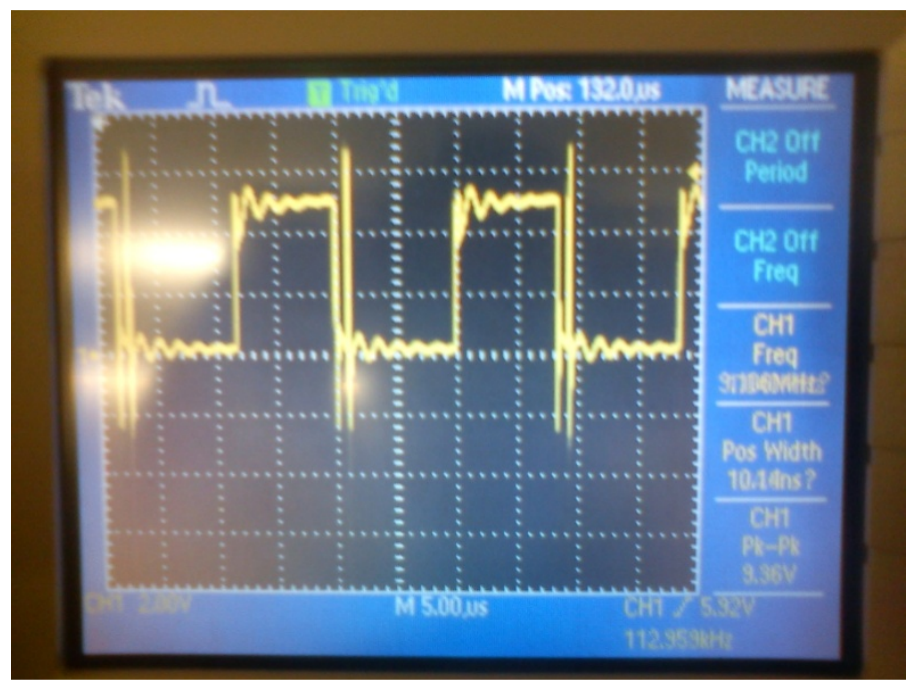

Figure 10.20: $Q \_$MAIN Switching Trajectory with $0.02 \mu F$ at $36 \mathrm{~V}$ Input and 2.484A Load

The decade capacitor boxes used for adding capacitance to the LTC1871's FB and GATE pins introduce oscillations into Q_MAIN's switching trajectory waveform. These oscillations originate from parasitic resistance and inductance in both the decade capacitor boxes as well as the long wire leads that this project uses for connecting them. Furthermore, there are still higher frequency harmonics present in the waveforms. Using discrete ceramic capacitors with low ESR ratings rather than decade capacitor boxes may 
eliminate the parasitic resistance and inductance problem. This project's next test set uses discrete ceramic capacitors for the LTC1871's FB and GATE pins, for checking any differences between the test results from tables E-23 and E-24 as well as the results that using discrete capacitors show.

Tables E-25 and E-26 show the results from when this project uses discrete capacitors instead for the additional capacitors across the LTC1871's FB and GATE pins. Likewise with the tests from earlier in this section, Table E-25 shows the SEPIC's output load driving abilities while using the electronic load in constant voltage mode (36V) and Table E-26 shows the same test results, except using input voltages (with their corresponding elliptical training resistance levels) that match the 160 strides per minute exercise test results from the previous EHFEM group in [1]. 
Table E-25: SEPIC Load Tests with 0.02 $\mu$ F Gate Capacitor, Constant Voltage Electronic Load, Agilent E3630A and BK XLN3640 Power Supplies

\begin{tabular}{|c|c|c|c|c|c|c|c|}
\hline $\mathbf{V}_{\text {IN }}(\mathbf{V})$ & $\mathbf{I}_{\text {IN }}(\mathbf{A})$ & $\mathbf{V}_{\text {OUT }}(\mathbf{V})$ & $\mathbf{I}_{\text {OUT }}(\mathbf{A})$ & $\mathbf{P}_{\text {IN }}(\mathbf{W})$ & $\mathbf{P}_{\text {OUT }}(\mathbf{W})$ & $\eta(\%)$ & $\mathbf{R}_{\text {IN }}(\Omega)$ \\
\hline 5 & 1.037 & 36 & 0.07 & 5.185 & 2.52 & 48.602 & 4.822 \\
\hline 10 & 2.778 & 36 & 0.465 & 27.78 & 16.74 & 60.259 & 3.600 \\
\hline 12 & 2.868 & 36 & 0.585 & 34.416 & 21.06 & 61.192 & 4.184 \\
\hline 15 & 2.646 & 36 & 0.692 & 39.69 & 24.912 & 62.766 & 5.669 \\
\hline 18 & 1.605 & 36 & 0.507 & 28.89 & 18.252 & 63.178 & 11.215 \\
\hline 20 & 1.785 & 36 & 0.634 & 35.7 & 22.824 & 63.933 & 11.204 \\
\hline 25 & 2.237 & 36 & 1.012 & 55.925 & 36.432 & 65.144 & 11.176 \\
\hline 27 & 2.417 & 36 & 1.184 & 65.259 & 42.624 & 65.315 & 11.171 \\
\hline 30 & 2.686 & 36 & 1.471 & 80.58 & 52.956 & 65.719 & 11.169 \\
\hline 36 & 3.239 & 36 & 2.164 & 116.604 & 77.904 & 66.811 & 11.115 \\
\hline
\end{tabular}

Table E-26: SEPIC Load Test with 0.02 $\mu F$ Gate Capacitor Using 160 Strides/Min. Theoretical Input Voltages from [1], Constant Voltage Electronic Load, Agilent E3630A and BK XLN3640 Power Supplies

\begin{tabular}{|c|c|c|c|c|c|c|c|c|}
\hline $\mathbf{V}_{\text {IN }}(\mathbf{V})$ & $\mathbf{I}_{\text {IN }}(\mathbf{A})$ & $\mathbf{V}_{\text {OUT }}(\mathbf{V})$ & $\mathbf{I}_{\text {OUT }}(\mathbf{A})$ & $\mathbf{P}_{\text {IN }}(\mathbf{W})$ & $\mathbf{P}_{\text {OUT }}(\mathbf{W})$ & $\eta(\%)$ & $\mathbf{R}_{\text {IN }}(\Omega)$ & Training Resistance Level \\
\hline 8.08 & 2.75 & 36 & 0.379 & 22.22 & 13.644 & 61.404 & 2.938 & 2 \\
\hline 11.54 & 2.835 & 36 & 0.575 & 32.715 & 20.7 & 63.272 & 4.071 & 3 \\
\hline 13.49 & 2.379 & 36 & 0.568 & 32.092 & 20.448 & 63.715 & 5.670 & 4 \\
\hline 15.88 & 2.901 & 36 & 0.829 & 46.067 & 29.844 & 64.783 & 5.474 & 5 \\
\hline 18.27 & 1.622 & 36 & 0.523 & 29.633 & 18.828 & 63.535 & 11.264 & 6 \\
\hline 21 & 1.867 & 36 & 0.703 & 39.207 & 25.308 & 64.550 & 11.248 & 7 \\
\hline 23.3 & 2.075 & 36 & 0.875 & 48.347 & 31.5 & 65.153 & 11.229 & 8 \\
\hline 26.2 & 2.338 & 36 & 1.115 & 61.255 & 40.14 & 65.529 & 11.206 & 9 \\
\hline 29.1 & 2.599 & 36 & 1.382 & 75.630 & 49.752 & 65.783 & 11.197 & 10 \\
\hline 31.8 & 2.845 & 36 & 1.66 & 90.471 & 59.76 & 66.054 & 11.178 & 11 \\
\hline 34.3 & 3.078 & 36 & 1.946 & 105.575 & 70.056 & 66.356 & 11.144 & 12 \\
\hline 35.1 & 3.153 & 36 & 2.046 & 110.67 & 73.656 & 66.554 & 11.132 & 13 \\
\hline
\end{tabular}

Using discrete ceramic capacitors rather than decade capacitor boxes for the LTC1871

FB and GATE pin capacitors decreases overall converter efficiency, decreased load

driving ability as well as removes the SEPIC's $10 \Omega$ input resistance regulation ability at all tested input voltages. These results, however, are better than the results from tables E-

17 and E-18, when the SEPIC did not have the LTC1871 FB and GATE pin capacitors.

This project later discovered that the discrete capacitors are not the reason for decreased 
converter functionality. Before replacing the decade capacitor boxes across the LTC1871's FB and GATE pins with discrete capacitors, this project swapped out the IPP110N20N3 MOSFET used for Q_MAIN with the IXFK230N20T MOSFET used for Q_MAIN_ALT, for determining whether Q_MAIN_ALT would yield higher overall converter efficiency and functionality. That side-experiment proved disastrous, as the IXFK230N20T yielded poorer overall converter efficiency and failed to function correctly with the SEPIC's snubber. At 36V input its incorrect functionality with the snubber shorted out the BK XLN3640A power supply and the XLN3640A then supplied 30A (average) to the converter, destroying at least one unknown component on the SEPIC's switching node. During testing this project's author enclosed the SEPIC inside a cinderblock container for preventing any physical injury in case any components (particularly electrolytic capacitors) exploded. The author witnessed a spark and a loud pop noise somewhere near the SEPIC's switching node, but could not determine which component it was from because of difficulty looking inside the enclosure. The author does not know if that short circuit condition destroyed any other components. This project replaced the IXFK230N20T with the previously-used IPP110N20N3 MOSFET shortly afterward. This project discarded all test results using the IXFK230N20T MOSFET because overall converter efficiency was worse than with the IPP110N20N3 MOSFET. Therefore, this project's author speculates that such component destruction caused reduced overall SEPIC efficiency, input resistance maintenance and load current driving abilities. Later, this project author's determined that one of the snubber diodes, DSNUB1, was destroyed by the excessive DC current flow, after measuring its forward 
and reverse voltages as 0V. The author replaced DSNUB1 with a working APT30S20BG Schottky diode afterward. After replacing DSNUB1, this project moves on and focuses on other converter efficiency and functionality improvement methods. Although this project made a mistake in replacing the SEPIC's main switching transistor for side-tests before testing with discrete capacitors on the LTC1871's FB and GATE pins, the sidetest did verify that the IXFK230N20T does not function with the SEPIC's snubber, because the SEPIC's snubber relies heavily on proper main switching transistor timing, based on the snubber design equations from section 6.3 as well as figure 6.14 .

The next converter efficiency and functionality improvement method that this project focuses on is decreasing the SEPIC's output voltage ripple. Figure 10.21 shows the SEPIC's output voltage ripple at 36V input, while driving its maximum load current that maintains a $36 \mathrm{~V}$ output (2.164A). This SEPIC includes the $0.01 \mu \mathrm{F}$ LTC1871 FB pin and $0.02 \mu \mathrm{F}$ GATE pin capacitors. 


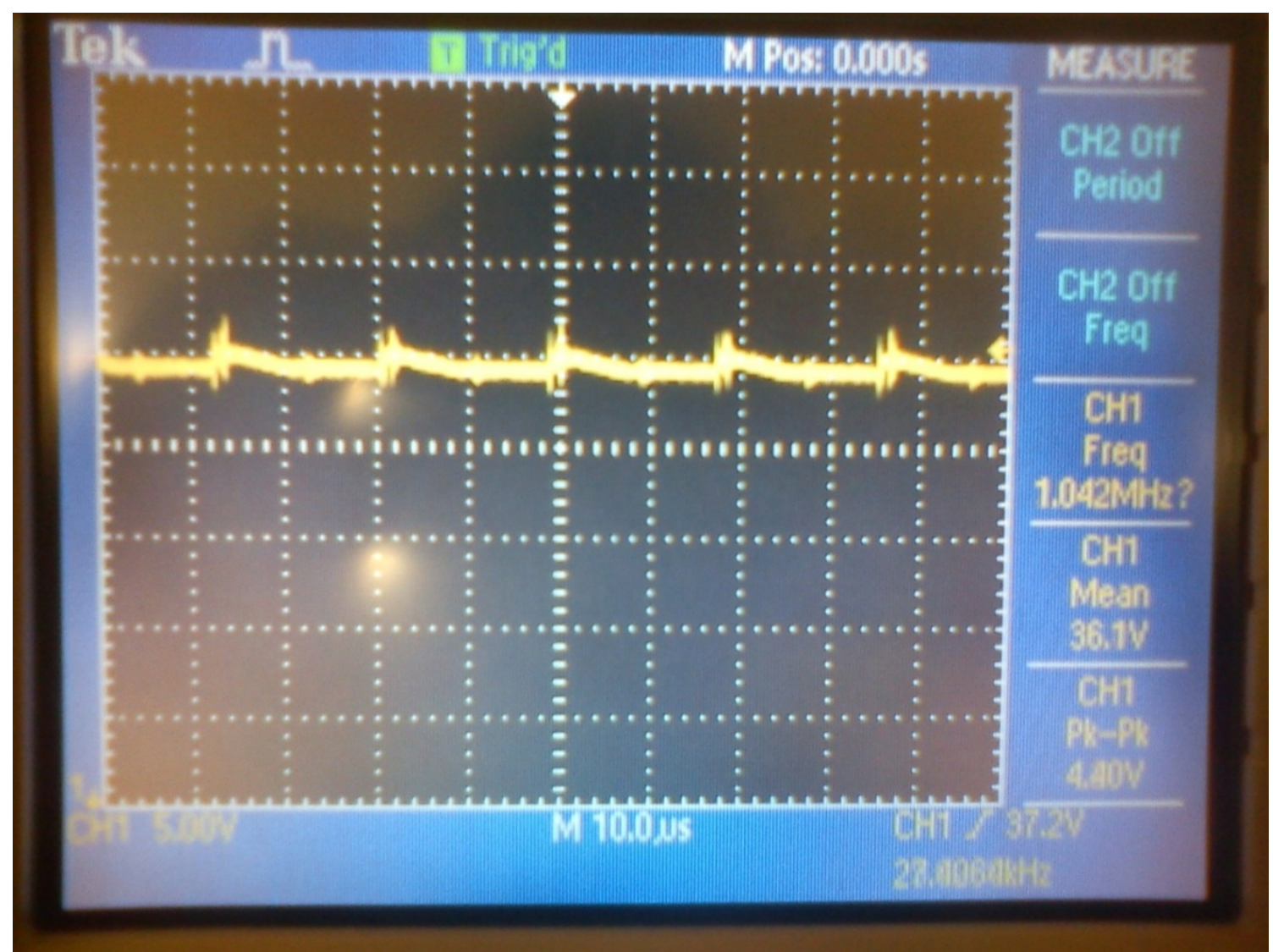

Figure 10.21: SEPIC Output Voltage Ripple at 36V Input and 2.164A Load

This project measured the SEPIC's peak to peak output voltage ripple at 36V input and $2.164 \mathrm{~A}$ load as $4.40 \mathrm{~V}$, which corresponds to a $12.22 \%$ ripple with respect to the SEPIC's 36V average output. This value is higher than the output ripple specification outlined in section 1.2. Therefore this project adds another large capacitor to the SEPIC's output node. The SEPIC's PCB layout in figure 9.6 has a placeholder footprint on the lower right corner for another radial electrolytic capacitor. Therefore, this project adds another $470 \mu \mathrm{F}$ capacitor identical to $\mathrm{C}_{\mathrm{OUT}}$ for testing if the SEPIC's output ripple voltage decreases, as well as if the SEPIC's overall efficiency and load current driving ability improves. This project adds another $470 \mu \mathrm{F}$ Epcos B43504A9477M electrolytic capacitor to the SEPIC's output. This capacitor has $190 \mathrm{~m} \Omega \mathrm{ESR}$, therefore its overall ripple 
reducing performance may not be as ideal as a single $940 \mu \mathrm{F}$ capacitor with a lower ESR value. Tables E-27 and E-28 show the results from when this project uses an additional $470 \mu \mathrm{F}$ electrolytic capacitor at the SEPIC's output, along with the current discrete ceramic capacitors for the LTC1871's FB and GATE pins. Likewise with the tests from earlier in this section, Table E-27 shows the SEPIC's output load driving abilities while using the electronic load in constant voltage mode (36V) and Table E-28 shows the same test results, except using input voltages (with their corresponding elliptical training resistance levels) that match the 160 strides per minute exercise test results from the previous EHFEM group in [1].

Table E-27: SEPIC Load Tests with 0.02 $\mu$ F Gate Capacitor, Additional $470 \mu F$ Output Capacitor, Constant Voltage Electronic Load, Agilent E3630A and BK XLN3640 Power Supplies

\begin{tabular}{|c|c|c|c|c|c|c|c|}
\hline $\mathbf{V}_{\text {IN }}(\mathbf{V})$ & $\mathbf{I}_{\text {IN }}(\mathbf{A})$ & $\mathbf{V}_{\text {OUT }}(\mathbf{V})$ & $\mathbf{I}_{\text {OUT }}(\mathbf{A})$ & $\mathbf{P}_{\text {IN }}(\mathbf{W})$ & $\mathbf{P}_{\text {OUT }}(\mathbf{W})$ & $\eta(\%)$ & $\mathbf{R}_{\text {IN }}(\Omega)$ \\
\hline 5 & 1.216 & 36 & 0.085 & 6.08 & 3.06 & 50.329 & 4.112 \\
\hline 10 & 0.921 & 36 & 0.131 & 9.21 & 4.716 & 51.205 & 10.858 \\
\hline 12 & 1.101 & 36 & 0.194 & 13.212 & 6.984 & 52.861 & 10.899 \\
\hline 15 & 1.366 & 36 & 0.319 & 20.49 & 11.484 & 56.047 & 10.981 \\
\hline 18 & 1.63 & 36 & 0.478 & 29.34 & 17.208 & 58.650 & 11.043 \\
\hline 20 & 1.822 & 36 & 0.608 & 36.44 & 21.888 & 60.066 & 10.977 \\
\hline 25 & 2.284 & 36 & 0.999 & 57.1 & 35.964 & 62.984 & 10.946 \\
\hline 27 & 2.453 & 36 & 1.176 & 66.231 & 42.336 & 63.922 & 11.007 \\
\hline 30 & 2.683 & 36 & 1.46 & 80.49 & 52.56 & 65.300 & 11.182 \\
\hline 36 & 3.302 & 36 & 2.231 & 118.872 & 80.316 & 67.565 & 10.902 \\
\hline
\end{tabular}


Table E-28: SEPIC Load Test with 0.02 $\mu F$ Gate Capacitor and Additional 470 $\mu$ F Output Capacitor Using 160 Strides/Min. Theoretical Input Voltages from [1], Constant Voltage Electronic Load, Agilent E3630A and BK XLN3640 Power Supplies

\begin{tabular}{|c|c|c|c|c|c|c|c|c|}
\hline $\mathbf{V}_{\text {IN }}(\mathbf{V})$ & $\mathbf{I}_{\text {IN }}(\mathbf{A})$ & $\mathbf{V}_{\text {OUT }}(\mathbf{V})$ & $\mathbf{I}_{\text {OUT }}(\mathbf{A})$ & $\mathbf{P}_{\text {IN }}(\mathbf{W})$ & $\mathbf{P}_{\text {OUT }}(\mathbf{W})$ & $\eta(\%)$ & $\mathbf{R}_{\text {IN }}(\Omega)$ & Training Resistance Level \\
\hline 8.08 & 0.741 & 36 & 0.083 & 5.987 & 2.988 & 49.906 & 10.904 & 2 \\
\hline 11.54 & 1.058 & 36 & 0.179 & 12.209 & 6.444 & 52.779 & 10.907 & 3 \\
\hline 13.49 & 1.228 & 36 & 0.251 & 16.565 & 9.036 & 54.546 & 10.985 & 4 \\
\hline 15.88 & 1.421 & 36 & 0.352 & 22.565 & 12.672 & 56.157 & 11.175 & 5 \\
\hline 18.27 & 1.63 & 36 & 0.479 & 29.78 & 17.244 & 57.904 & 11.209 & 6 \\
\hline 21 & 1.846 & 36 & 0.626 & 38.766 & 22.536 & 58.133 & 11.376 & 7 \\
\hline 23.3 & 2.118 & 36 & 0.845 & 49.349 & 30.42 & 61.642 & 11.001 & 8 \\
\hline 26.2 & 2.372 & 36 & 1.093 & 62.146 & 39.348 & 63.315 & 11.046 & 9 \\
\hline 29.1 & 2.595 & 36 & 1.357 & 75.514 & 48.852 & 64.692 & 11.214 & 10 \\
\hline 31.8 & 2.81 & 36 & 1.628 & 89.358 & 58.608 & 65.588 & 11.317 & 11 \\
\hline 34.3 & 3.049 & 36 & 1.93 & 104.58 & 69.48 & 66.437 & 11.250 & 12 \\
\hline 35.1 & 3.143 & 36 & 2.048 & 110.319 & 73.728 & 66.831 & 11.168 & 13 \\
\hline
\end{tabular}

Adding a $470 \mu \mathrm{F}$ capacitor to the SEPIC's output slightly increases converter efficiency as well as load current driving ability at higher input voltages. Furthermore, at all tested SEPIC input voltages except for $5 \mathrm{~V}$ the converter maintains close to a $10 \Omega$ input resistance while driving the maximum load current that it tolerates while maintaining a $36 \mathrm{~V}$ output. The additional capacitor's output voltage ripple reduction may have been responsible for that. Figure 10.22 shows the SEPIC's output voltage ripple at 36V input, while driving its maximum load current that maintains a $36 \mathrm{~V}$ output $(2.231 \mathrm{~A})$, with the additional $470 \mu \mathrm{F}$ output capacitor. 


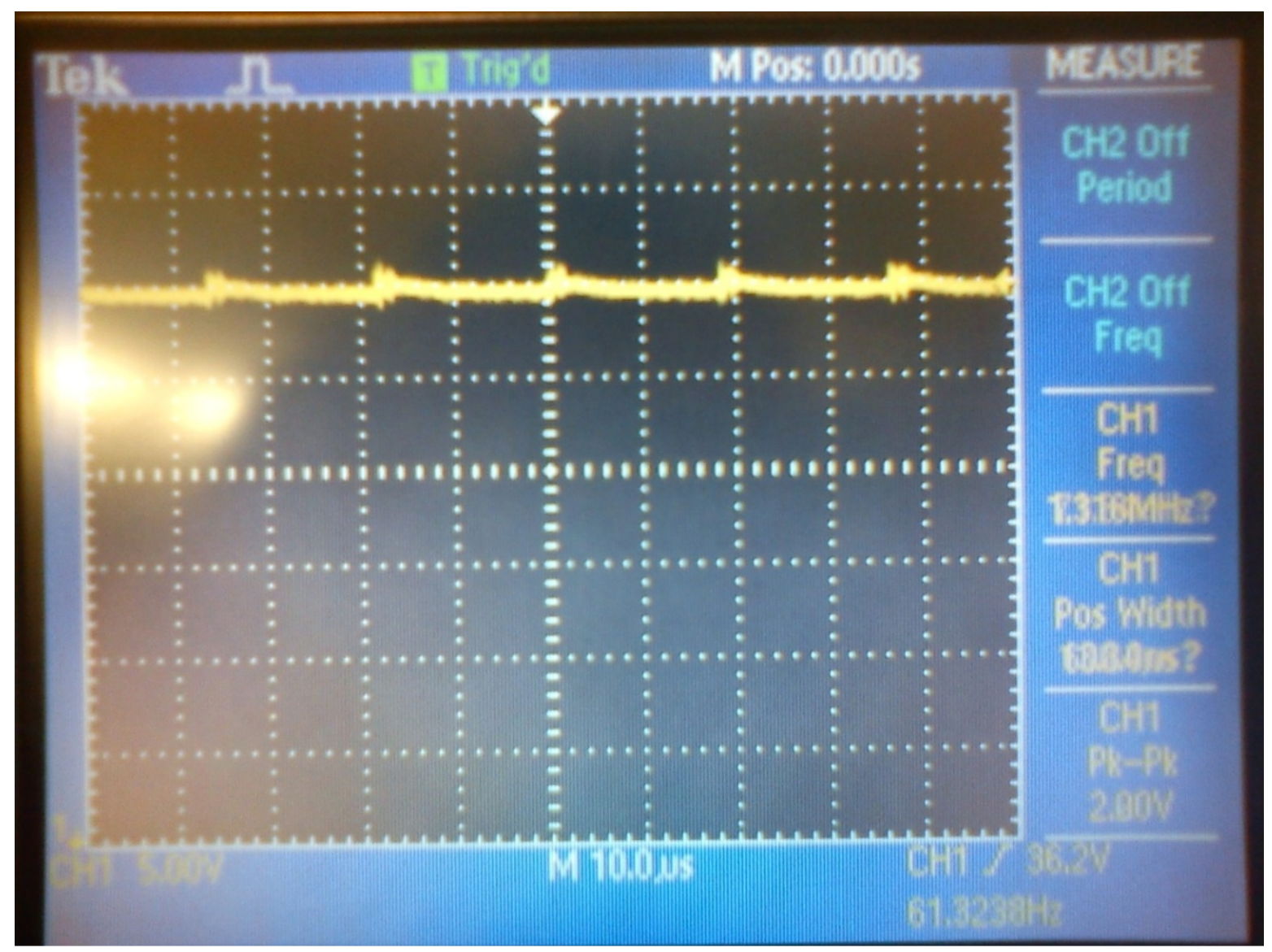

Figure 10.22: SEPIC Output Voltage Ripple at 36V Input and 2.231A Load With Additional 470 $\mu$ F Output Capacitor

Adding a $470 \mu \mathrm{F}$ capacitor to the SEPIC's output reduces its output ripple voltage at 36V input to $2 \mathrm{~V}$, which corresponds to a $5.56 \%$ ripple relative to the $36 \mathrm{~V}$ output. This ripple value suffices from the specifications outlined in section 1.2. Therefore, this project performs all future hardware tests with the additional $470 \mu \mathrm{F}$ capacitor on the SEPIC's output. Figures 10.23 and 10.24 show Q_MAIN's switching trajectory at 5V input and $36 \mathrm{~V}$ input while driving their respective maximum load currents that maintain $36 \mathrm{~V}$ SEPIC output, when this project uses discrete capacitors on the LTC1871's FB and GATE pins. 


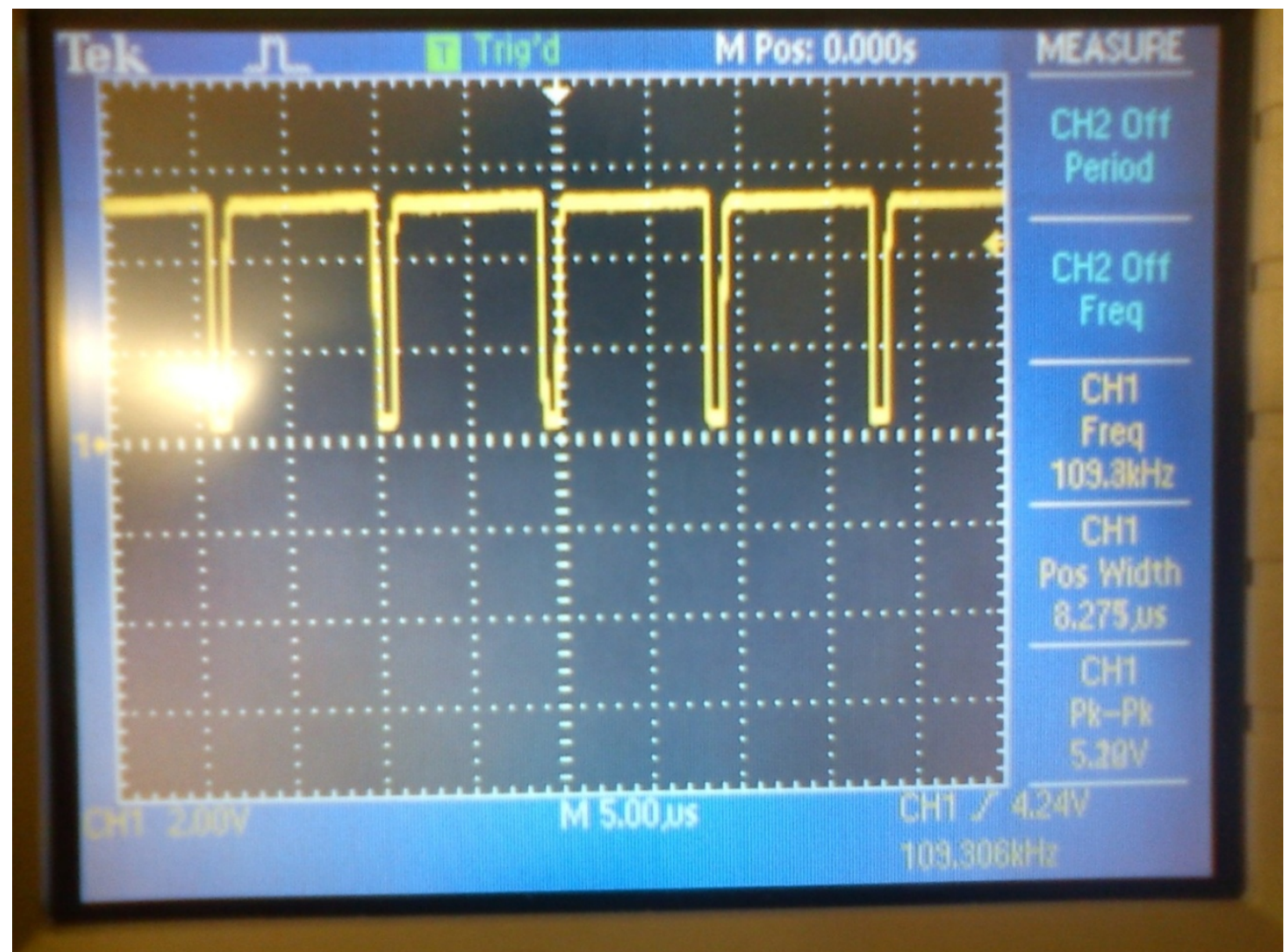

Figure 10.23: Q_MAIN Switching Trajectory with $0.02 \mu F$ Discrete Gate Capacitor and Additional $470 \mu F$ Capacitor at $5 \mathrm{~V}$ Input and $0.085 \mathrm{~A} \mathrm{Load}$ 


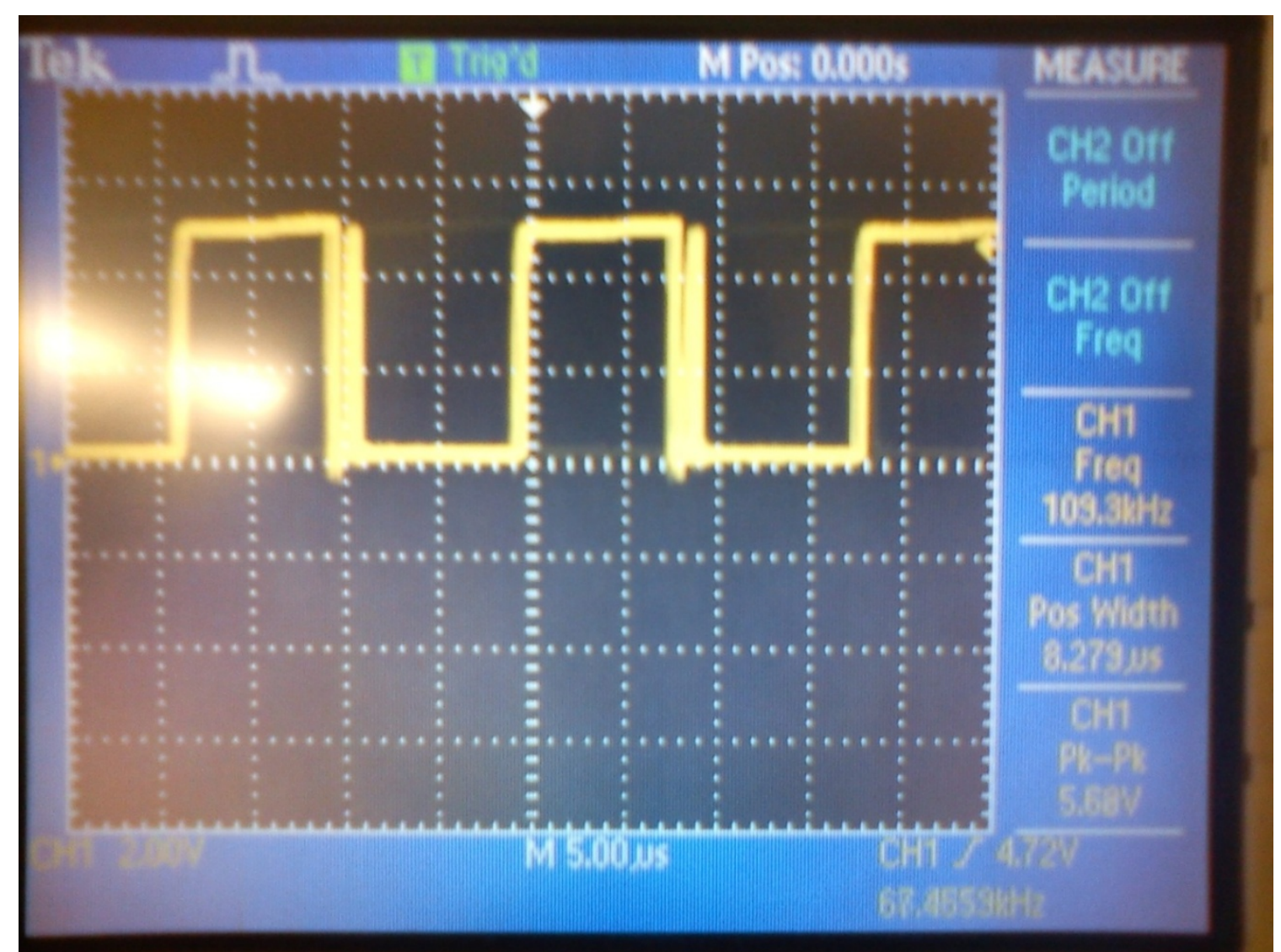

Figure 10.24: $Q \_$MAIN Switching Trajectory with $0.02 \mu F$ Discrete Gate Capacitor and Additional $470 \mu F$ Capacitor at 36V Input and 2.231A Load

Compared to figures 10.19 and 10.20 when this project used decade capacitor boxes for the additional LTC1871 FB and GATE pins, using discrete capacitors on those same pins yields less oscillation, however, high frequency harmonics are still present at $36 \mathrm{~V}$ input (from figure 10.24). In the next section, this project tests whether those high frequency harmonics still greatly affect converter operation. After improving the SEPIC's output voltage ripple performance, this project performs constant voltage load tests at input voltages beyond $36 \mathrm{~V}$. 


\subsubsection{Constant Voltage Load Tests Using Non-Voltage and Current Limited Input}

\section{Supply}

The instrument setup for this section's testing round uses a BK Precision 9153 power supply. This power supply can supply up 60V and 9A. Because of this voltage limitation, this project does not perform any tests beyond $60 \mathrm{~V}$ input voltage. This voltage limit suffices for testing the SEPIC, as it is very close to the $65 \mathrm{~V}$ absolute maximum voltage specification outlined in section 1.2. Furthermore, no-load tests from section 10.2 shows that the SEPIC still outputs its nominal output voltage at $65 \mathrm{~V}$ input. Figure 10.25 shows the instrument setup block diagram for this section's testing round.

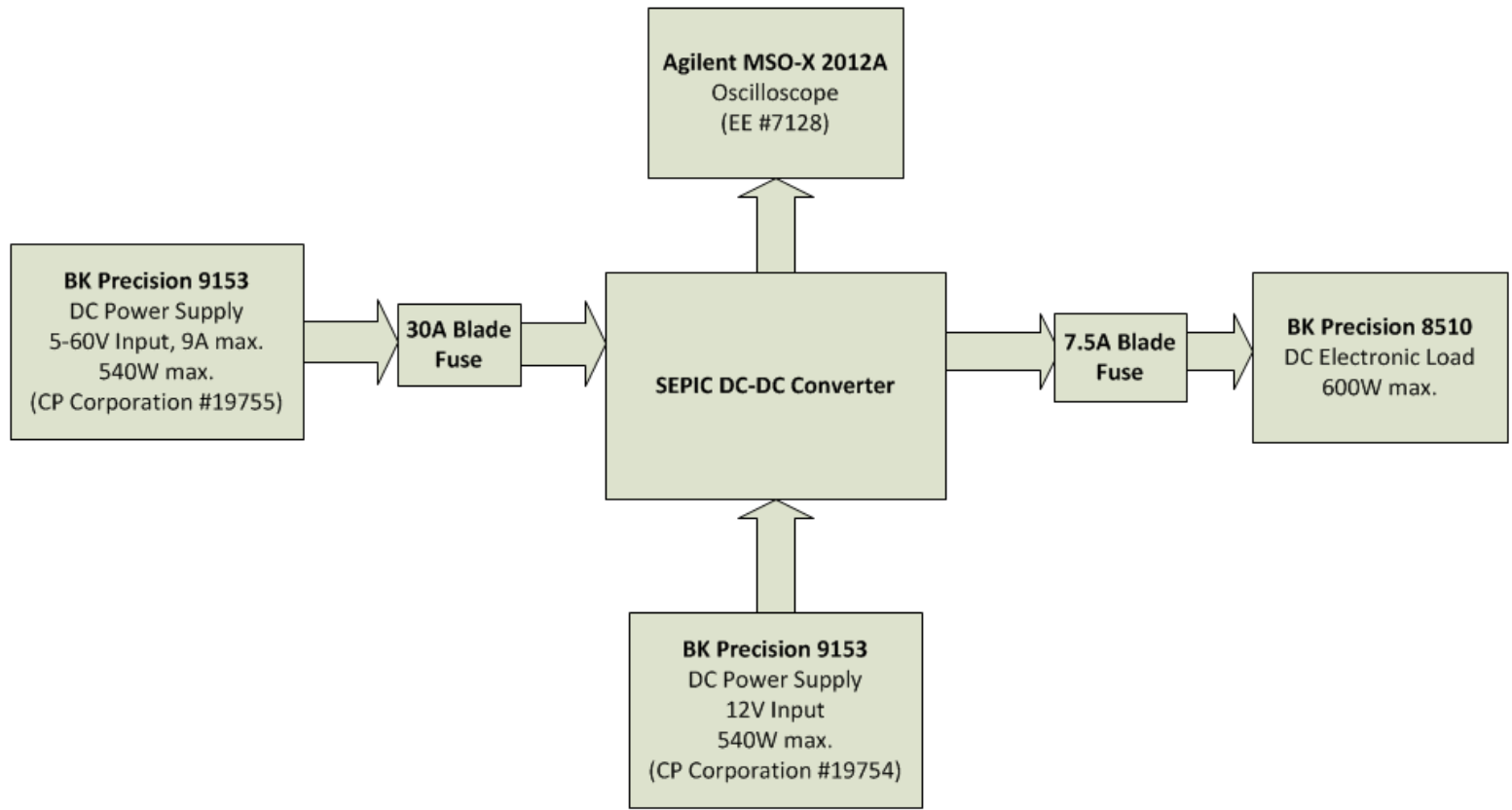

Figure 10.25: Finalized SEPIC with 0.01 $\mu$ F Feedback Capacitor, Additional $470 \mu F$ Output Capacitor and $0.02 \mu F$ Q_MAIN Gate Capacitor Test Setup Block Diagram (Non-Voltage and Current Limited)

This BK Precision power supply was in Cal Poly’s renewable energy laboratory (room 20-150), therefore this project performs this testing round using equipment in that laboratory. Likewise with the previous testing round, this testing round keeps the inductor 
current wire loops removed from the PCB and replaces those wires with solder bridges because no current probe amplifiers exist in the 20-150 laboratory. Unlike the previous testing round, this testing round uses an Agilent MSO-X 2012A oscilloscope. This project also measures the $12 \mathrm{~V}$ source's current during this testing round. Measuring the $12 \mathrm{~V}$ source current allows complete converter efficiency calculations. This testing round also uses a BK Precision 8510 600W electronic load, which more than suffices for this SEPIC's 288W maximum output load driving requirement. Tables E-29 and E-30 show the results from this instrument setup. Likewise with the previous section, Tables E-29 and E-30 show the results from when this project uses an additional $470 \mu \mathrm{F}$ electrolytic capacitor at the SEPIC's output, along with the current discrete ceramic capacitors for the LTC1871's FB and GATE pins. Table E-29 shows the SEPIC's output load driving abilities while using the electronic load in constant voltage mode (36V) and Table E-30 shows the same test results, except using input voltages (with their corresponding elliptical training resistance levels) that match the 160 strides per minute exercise test results from the previous EHFEM group in [1]. 
Table E-29: SEPIC Load Tests with 0.02 $\mu$ F Gate Capacitor, Additional $470 \mu$ F Output Capacitor, Constant Voltage Electronic Load and BK 9153 Power Supplies

\begin{tabular}{|c|c|c|c|c|c|c|c|c|c|}
\hline $\mathbf{V}_{\text {IN }}(\mathbf{V})$ & $\mathbf{I}_{\text {IN }}(\mathbf{A})$ & $\mathbf{V}_{\text {OUT }}(\mathbf{V})$ & $\mathbf{I}_{\text {OUT }}(\mathbf{A})$ & $\mathbf{V}_{\text {BAT }}(\mathbf{V})$ & $\mathbf{I}_{\text {BAT }}(\mathbf{A})$ & $\mathbf{P}_{\text {IN }}(\mathbf{W})$ & $\mathbf{P}_{\text {OUT }}(\mathbf{W})$ & $\eta(\%)$ & $\mathbf{R}_{\text {IN }}(\Omega)$ \\
\hline 5 & 1.237 & 36 & 0.083 & 12 & 0.024 & 6.473 & 2.988 & 46.161 & 4.042 \\
\hline 10 & 0.944 & 36 & 0.131 & 12 & 0.024 & 9.728 & 4.716 & 48.479 & 10.593 \\
\hline 12 & 1.128 & 36 & 0.196 & 12 & 0.023 & 13.812 & 7.056 & 51.086 & 10.638 \\
\hline 15 & 1.396 & 36 & 0.324 & 12 & 0.023 & 21.216 & 11.664 & 54.977 & 10.745 \\
\hline 18 & 1.665 & 36 & 0.486 & 12 & 0.023 & 30.246 & 17.496 & 57.846 & 10.811 \\
\hline 20 & 1.86 & 36 & 0.619 & 12 & 0.023 & 37.476 & 22.284 & 59.462 & 10.753 \\
\hline 25 & 2.34 & 36 & 1.022 & 12 & 0.023 & 58.776 & 36.792 & 62.597 & 10.684 \\
\hline 27 & 2.518 & 36 & 1.207 & 12 & 0.023 & 68.262 & 43.452 & 63.655 & 10.723 \\
\hline 30 & 2.754 & 36 & 1.5 & 12 & 0.023 & 82.896 & 54 & 65.142 & 10.893 \\
\hline 36 & 3.37 & 36 & 2.28 & 12 & 0.023 & 121.596 & 82.08 & 67.502 & 10.682 \\
\hline 40 & 3.838 & 36 & 2.936 & 12 & 0.023 & 153.796 & 105.696 & 68.725 & 10.422 \\
\hline 45 & 4.101 & 36 & 3.566 & 12 & 0.023 & 184.821 & 128.376 & 69.460 & 10.973 \\
\hline 50 & 3.039 & 36 & 2.832 & 12 & 0.023 & 152.226 & 101.952 & 66.974 & 16.453 \\
\hline 52 & 2.3 & 36 & 2.19 & 12 & 0.023 & 119.876 & 78.84 & 65.768 & 22.609 \\
\hline 60 & 0.113 & 36 & 0.089 & 12 & 0.023 & 7.056 & 3.204 & 45.408 & 530.973 \\
\hline
\end{tabular}


Table E-30: SEPIC Load Test with 0.02 $\mu$ F Gate Capacitor and Additional $470 \mu F$ Output Capacitor, Using 160 Strides/Min. Theoretical Input Voltages from [1], Constant Voltage Electronic Load and BK 9153

Power Supplies

\begin{tabular}{|c|c|c|c|c|c|c|c|c|c|c|}
\hline$V_{\text {IN }}(V)$ & $I_{I N}(A)$ & $\mathrm{V}_{\text {out }}(\mathrm{V})$ & I OUT $_{\text {(A) }}$ & $V_{B A T}(V)$ & $I_{B A T}(A)$ & $P_{\text {IN }}(W)$ & $P_{\text {out }}(W)$ & $\eta(\%)$ & $\mathbf{R}_{\mathrm{IN}}(\Omega)$ & Training Resistance Level \\
\hline 8.08 & 0.762 & 36 & 0.082 & 12 & 0.024 & 6.445 & 2.952 & 45.803 & 10.604 & 2 \\
\hline 11.54 & 1.086 & 36 & 0.18 & 12 & 0.023 & 12.808 & 6.48 & 50.592 & 10.626 & 3 \\
\hline 13.49 & 1.264 & 36 & 0.257 & 12 & 0.023 & 17.327 & 9.252 & 53.395 & 10.672 & 4 \\
\hline 15.88 & 1.471 & 36 & 0.367 & 12 & 0.023 & 23.635 & 13.212 & 55.899 & 10.795 & 5 \\
\hline 18.27 & 1.69 & 36 & 0.503 & 12 & 0.023 & 31.152 & 18.108 & 58.127 & 10.811 & 6 \\
\hline 21 & 1.96 & 36 & 0.693 & 12 & 0.023 & 41.436 & 24.948 & 60.209 & 10.714 & 7 \\
\hline 23.3 & 2.185 & 36 & 0.877 & 12 & 0.023 & 51.187 & 31.572 & 61.680 & 10.664 & 8 \\
\hline 26.2 & 2.444 & 36 & 1.132 & 12 & 0.023 & 64.309 & 40.752 & 63.369 & 10.720 & 9 \\
\hline 29.1 & 2.674 & 36 & 1.408 & 12 & 0.023 & 78.089 & 50.688 & 64.910 & 10.883 & 10 \\
\hline 31.8 & 2.894 & 36 & 1.69 & 12 & 0.023 & 92.305 & 60.84 & 65.912 & 10.988 & 11 \\
\hline 34.3 & 3.14 & 36 & 2.006 & 12 & 0.023 & 107.978 & 72.216 & 66.880 & 10.924 & 12 \\
\hline 35.1 & 3.238 & 36 & 2.126 & 12 & 0.023 & 113.930 & 76.536 & 67.178 & 10.840 & 13 \\
\hline 39.9 & 3.82 & 36 & 2.918 & 12 & 0.023 & 152.694 & 105.048 & 68.796 & 10.445 & 14 \\
\hline 44.8 & 4.082 & 36 & 3.533 & 12 & 0.023 & 183.150 & 127.188 & 69.445 & 10.975 & 15 \\
\hline 46.4 & 4.146 & 36 & 3.72 & 12 & 0.023 & 192.650 & 133.92 & 69.515 & 11.192 & 16 \\
\hline 47.6 & 3.351 & 36 & 3.036 & 12 & 0.023 & 159.784 & 109.296 & 68.403 & 14.205 & 17 \\
\hline 48.3 & 3.245 & 36 & 2.949 & 12 & 0.023 & 157.010 & 106.164 & 67.616 & 14.884 & 18 \\
\hline 58.2 & 0.113 & 36 & 0.088 & 12 & 0.023 & 6.853 & 3.168 & 46.231 & 515.044 & 19 \\
\hline 59.8 & 0.113 & 36 & 0.09 & 12 & 0.023 & 7.033 & 3.24 & 46.066 & 529.204 & 20 \\
\hline
\end{tabular}

In tables E-29 and E-30, $\mathrm{V}_{\mathrm{BAT}}$ corresponds to the $12 \mathrm{~V}$ source's voltage and $\mathrm{I}_{\mathrm{BAT}}$

corresponds to the $12 \mathrm{~V}$ source's current. In this section and subsequent hardware testing sections without the elliptical trainer, this project factors in those two parameters into the converter's input power for the final converter efficiency calculation. From tables E-29 and E-30, the SEPIC still experiences the same problems as from section 10.3.2. The SEPIC cannot drive any sufficient load current (greater than $1 \mathrm{~A}$ ) beyond $45 \mathrm{~V}$ input. After adding capacitance to the LTC1871's FB and GATE pins and still achieving less than desirable functionality results, this project hypothesizes that the current sensing pin on the LTC1871 controller (the SENSE pin) requires additional filtering. 


\subsubsection{Second Troubleshooting Phase}

Figure 10.26 shows the LTC1871's SENSE pin switching waveform at 36V input while the SEPIC drives its maximum load current while maintaining 36V output (2.28A), while still using discrete capacitors for the LTC1871's FB and GATE pins.

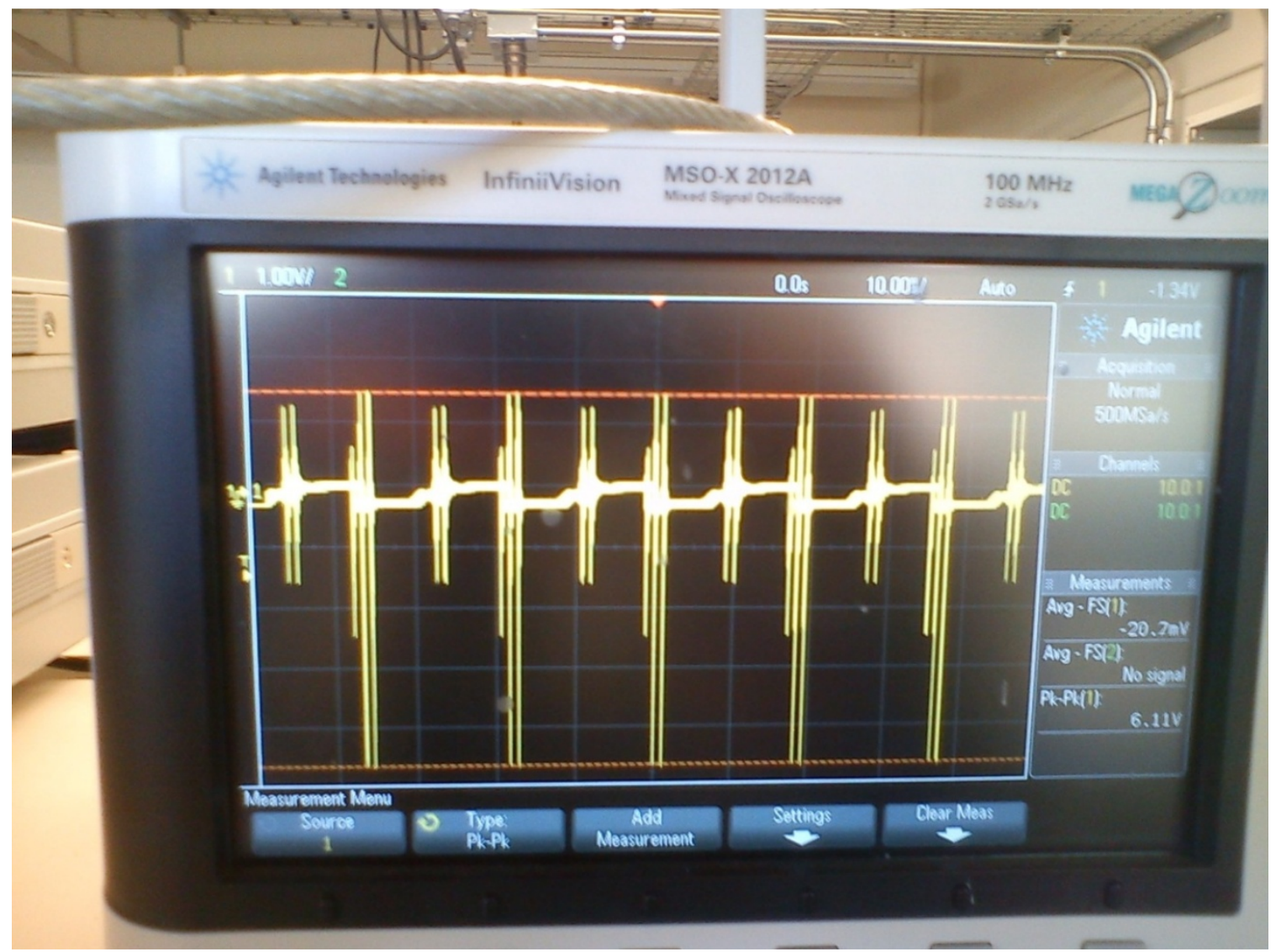

Figure 10.26: LTC1871 Current Sense Pin Switching Waveform at 36V Input and 2.28A Load

From figure 10.26, large voltage spikes exist on the LTC1871's current sense pin, from sensing Q_MAIN's current. These voltage spikes are undesirable and this project hypothesizes that it contributes to the SEPIC's undesirable operation at higher input voltages. One possible solution for this problem involves adding an $\mathrm{RC}$ low pass filter at the LTC1871's SENSE pin input, as an RC low pass filter removes the higher frequency harmonic signals [28]. Figure 10.27 shows this modification to the SEPIC's schematic. 


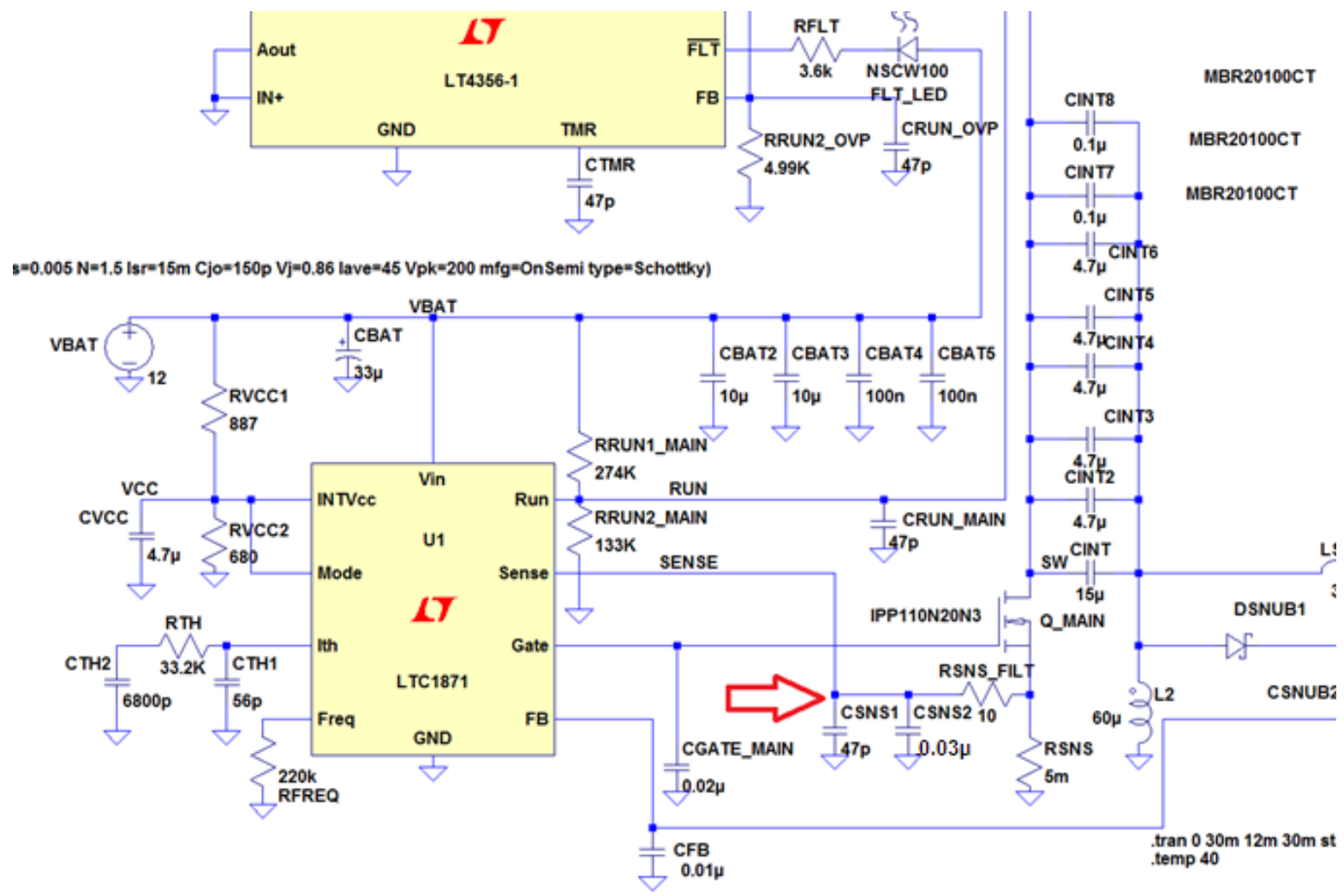

Figure 10.27: LTC1871 Current Sense Pin RC Low Pass Filter

The red arrow in figure 10.27 denotes the SENSE pin filter location. Equation (10.1)

determines the RC low pass filter's attenuation (cut-off) frequency based on values selected for its resistor (RSNS_FILT) and capacitor $\left(\mathrm{C}_{\mathrm{SNS} 2}\right)$. Because the filter consists of only one resistor and one capacitor (this project combines the existing $47 \mathrm{pF}$ capacitor $\mathrm{C}_{\mathrm{SNS} 1}$ on the LTC1871's SENSE pin in parallel with the new filter capacitor, thus adding the two capacitances together), the attenuation after the cut-off frequency only occurs at $20 \mathrm{~dB}$ per decade. This suffices as this project does not desire complex filters for any controller pins, as complex filters increase overall converter cost, complexity, and also require more PCB trace severing.

$$
f_{c}=\frac{1}{2 \pi R_{S N S_{-} F I L T}\left(C_{S N S 1}+C_{S N S 2}\right)}=\frac{1}{2 \pi(10 \Omega)(47 p F+0.03 \mu F)}=531 \mathrm{kHz}
$$


Therefore, the chosen component values for the RC low pass filter attenuate harmonics higher than $531 \mathrm{kHz}$ at $20 \mathrm{~dB}$ per decade. Figure 10.28 shows the LTC1871's current sense pin's waveform from LTSpice simulation results after adding this RC low pass filter to the LTC1871's SENSE pin.

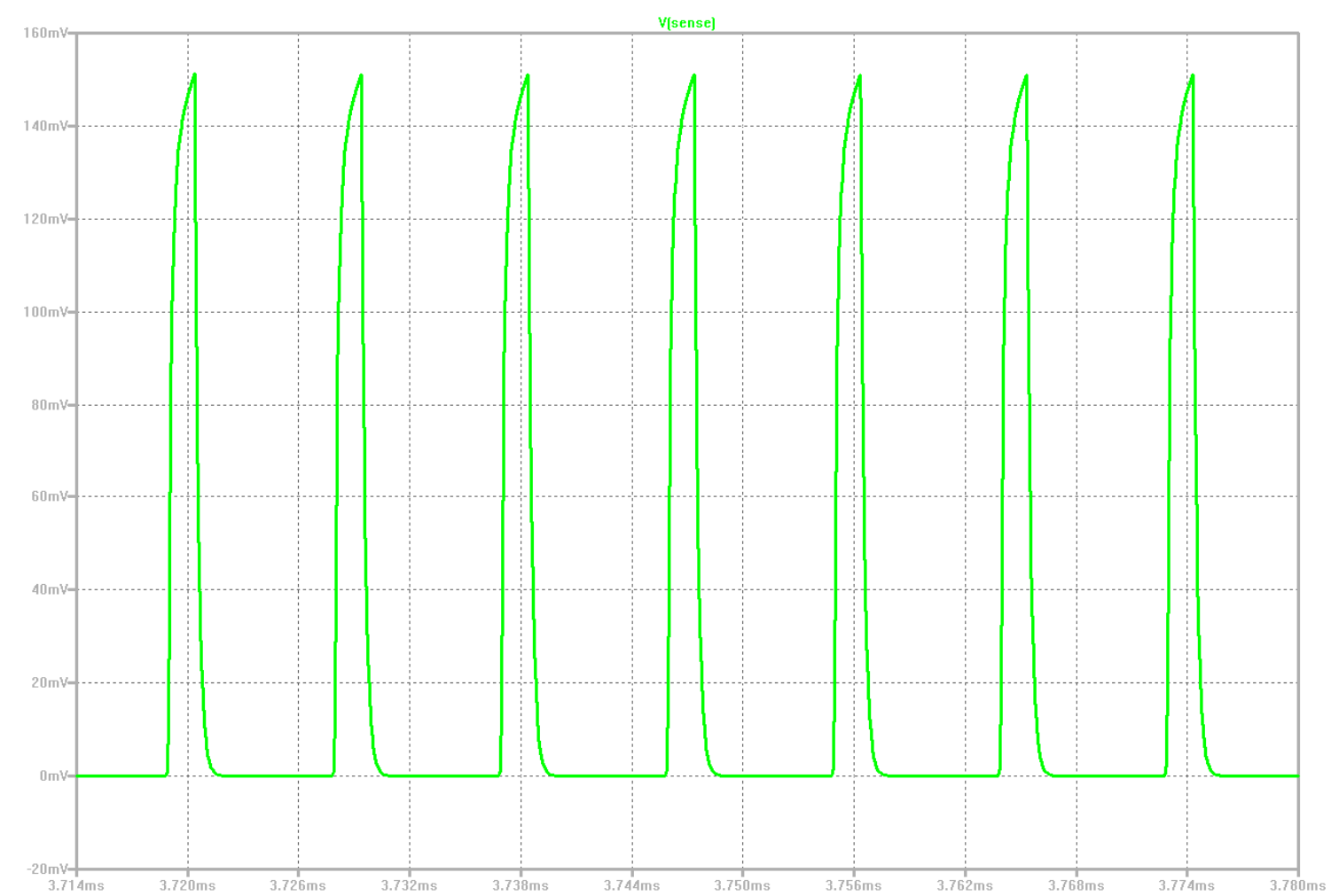

Figure 10.28: LTC1871 Current Sense Pin Waveform With Low Pass RC Filter In Steady-State Operation

Adding this low pass RC filter to the LTC1871's SENSE pin eliminates the leading harmonic spike on the sense resistor's voltage. This current sense signal waveform now appears closer to the ideal Q_MAIN drain current waveform shown in figure 5.9. Thus, the chosen resistor and capacitor values suffice for this filter. Inserting the filter resistor (RSNS_FILT) requires severing an existing PCB trace between $\mathrm{R}_{\text {SNS }}$ and the LTC1871's SENSE pin. This project's author then severed the trace and implemented the RC low 
pass filter on the LTC1871's current sense pin. Tables E-31 and E-32 show the results from adding this RC low pass filter to the LTC1871's current sense pin, while keeping all other SEPIC components intact. Table E-31 shows the SEPIC's output load driving abilities while using the electronic load in constant voltage mode (36V) and Table E-32 shows the same test results, except using input voltages (with their corresponding elliptical training resistance levels) that match the 160 strides per minute exercise test results from the previous EHFEM group in [1].

Table E-31: SEPIC Load Tests with LTC1871 Current Sense Pin Filter and BK 9153 Power Supplies

\begin{tabular}{|c|c|c|c|c|c|c|c|c|c|}
\hline $\mathbf{V}_{\text {IN }}(\mathbf{V})$ & $\mathbf{I}_{\text {IN }}(\mathbf{A})$ & $\mathbf{V}_{\text {OUT }}(\mathbf{V})$ & $\mathbf{I}_{\text {OUT }}(\mathbf{A})$ & $\mathbf{V}_{\text {BAT }}(\mathbf{V})$ & $\mathbf{I}_{\text {BAT }}(\mathbf{A})$ & $\mathbf{P}_{\text {IN }}(\mathbf{W})$ & $\mathbf{P}_{\text {OUT }}(\mathbf{W})$ & $\eta(\%)$ & $\mathbf{R}_{\text {IN }}(\mathbf{\Omega})$ \\
\hline 5 & 0.5 & 37.34 & 0.013 & 12 & 0.024 & 2.788 & 0.485 & 17.411 & 10.000 \\
\hline 10 & 1.099 & 37.34 & 0.148 & 12 & 0.024 & 11.278 & 5.526 & 49.001 & 9.099 \\
\hline 12 & 1.196 & 37.34 & 0.202 & 12 & 0.023 & 14.628 & 7.543 & 51.563 & 10.033 \\
\hline 15 & 1.477 & 37.32 & 0.333 & 12 & 0.023 & 22.431 & 12.428 & 55.404 & 10.156 \\
\hline 18 & 1.76 & 37.31 & 0.498 & 12 & 0.023 & 31.956 & 18.580 & 58.144 & 10.227 \\
\hline 20 & 1.979 & 37.3 & 0.638 & 12 & 0.023 & 39.856 & 23.797 & 59.708 & 10.106 \\
\hline 25 & 2.496 & 37.26 & 1.048 & 12 & 0.023 & 62.676 & 39.048 & 62.302 & 10.016 \\
\hline 27 & 2.721 & 37.24 & 1.248 & 12 & 0.023 & 73.743 & 46.476 & 63.024 & 9.923 \\
\hline 30 & 2.958 & 37.2 & 1.528 & 12 & 0.023 & 89.016 & 56.842 & 63.855 & 10.142 \\
\hline 36 & 3.646 & 36 & 2.4 & 12 & 0.023 & 131.532 & 86.400 & 65.687 & 9.874 \\
\hline 40 & 3.96 & 36 & 2.92 & 12 & 0.023 & 158.676 & 105.120 & 66.248 & 10.101 \\
\hline 45 & 4.24 & 36 & 3.517 & 12 & 0.023 & 191.076 & 126.612 & 66.263 & 10.613 \\
\hline 50 & 4.996 & 36 & 4.73 & 12 & 0.023 & 250.076 & 170.280 & 68.091 & 10.008 \\
\hline 52 & 5.112 & 36 & 5.022 & 12 & 0.023 & 266.1 & 180.792 & 67.941 & 10.172 \\
\hline 60 & 5.253 & 36 & 6.093 & 12 & 0.023 & 315.456 & 219.348 & 69.534 & 11.422 \\
\hline
\end{tabular}


Table E-32: SEPIC Load Test with LTC1871 Current Sense Pin Filter, Using 160 Strides/Min. Theoretical Input Voltages from [1] and BK 9153 Power Supplies

\begin{tabular}{|c|c|c|c|c|c|c|c|c|c|c|}
\hline$V_{\text {IN }}(\mathrm{V})$ & $\mathrm{I}_{\mathrm{IN}}(\mathrm{A})$ & $\mathrm{V}_{\text {OUT }}(\mathrm{V})$ & $\mathrm{I}_{\text {out }}(\mathrm{A})$ & $V_{B A T}(V)$ & $\mathrm{I}_{\mathrm{BAT}}(\mathrm{A})$ & $P_{\text {IN }}(W)$ & $P_{\text {oUt }}(W)$ & $\eta(\%)$ & $R_{I N}(\Omega)$ & Training Resistance Level \\
\hline 8.08 & 0.754 & 37.37 & 0.066 & 12 & 0.024 & 6.380 & 2.466 & 38.657 & 10.716 & 2 \\
\hline 11.54 & 1.111 & 37.35 & 0.168 & 12 & 0.023 & 13.097 & 6.275 & 47.910 & 10.387 & 3 \\
\hline 13.49 & 1.314 & 37.34 & 0.248 & 12 & 0.023 & 18.002 & 9.260 & 51.441 & 10.266 & 4 \\
\hline 15.88 & 1.636 & 37.33 & 0.388 & 12 & 0.023 & 26.256 & 14.484 & 55.165 & 9.707 & 5 \\
\hline 18.27 & 1.814 & 37.32 & 0.518 & 12 & 0.023 & 33.418 & 19.332 & 57.849 & 10.072 & 6 \\
\hline 21 & 2.108 & 37.3 & 0.718 & 12 & 0.023 & 44.544 & 26.781 & 60.123 & 9.962 & 7 \\
\hline 23.3 & 2.327 & 37.29 & 0.898 & 12 & 0.023 & 54.495 & 33.486 & 61.448 & 10.013 & 8 \\
\hline 26.2 & 2.644 & 37.26 & 1.168 & 12 & 0.023 & 69.549 & 43.520 & 62.574 & 9.909 & 9 \\
\hline 29.1 & 2.865 & 37.23 & 1.428 & 12 & 0.023 & 83.648 & 53.164 & 63.558 & 10.157 & 10 \\
\hline 31.8 & 3.115 & 37.14 & 1.718 & 12 & 0.023 & 99.333 & 63.807 & 64.235 & 10.209 & 11 \\
\hline 34.3 & 3.4 & 36.93 & 2.058 & 12 & 0.023 & 116.896 & 76.002 & 65.017 & 10.088 & 12 \\
\hline 35.1 & 3.479 & 36 & 2.242 & 12 & 0.023 & 122.389 & 80.712 & 65.947 & 10.089 & 13 \\
\hline 39.9 & 3.92 & 36 & 2.908 & 12 & 0.023 & 156.684 & 104.688 & 66.815 & 10.179 & 14 \\
\hline 44.8 & 4.196 & 36 & 3.51 & 12 & 0.023 & 188.257 & 126.360 & 67.121 & 10.677 & 15 \\
\hline 46.4 & 4.377 & 36 & 3.803 & 12 & 0.023 & 203.369 & 136.908 & 67.320 & 10.601 & 16 \\
\hline 47.6 & 4.819 & 36 & 4.365 & 12 & 0.023 & 229.660 & 157.140 & 68.423 & 9.878 & 17 \\
\hline 48.3 & 5.103 & 36 & 4.715 & 12 & 0.023 & 246.751 & 169.740 & 68.790 & 9.465 & 18 \\
\hline 58.2 & 5.265 & 36 & 5.924 & 12 & 0.023 & 306.699 & 213.264 & 69.535 & 11.054 & 19 \\
\hline 59.8 & 5.27 & 36 & 6.092 & 12 & 0.023 & 315.422 & 219.312 & 69.530 & 11.347 & 20 \\
\hline
\end{tabular}

From tables E-31 and E-32, adding a RC low pass filter to the LTC1871's current sense pin dramatically improves the SEPIC's functionality. The SEPIC now can drive sufficient load currents at input voltages greater than $45 \mathrm{~V}$. At $60 \mathrm{~V}$ input the SEPIC drives a $220 \mathrm{~W}$ load (6.092A output current), while taking in $315 \mathrm{~W}$ from the input power supply. Therefore, adding filtering to the LTC1871's FB, GATE and SENSE pins is necessary for improving the SEPIC's overall load driving ability. For this set of tests, this project used constant current mode on the electronic load for input voltages less than $36 \mathrm{~V}$ because at those input voltages, the SEPIC draws the absolute maximum current limit (6.5A) when using constant $36 \mathrm{~V}$ output mode on the electronic load. This means that the 
SEPIC can drive higher load currents at those input voltages, but this project only selects current limits that allow the SEPIC to maintain a $10 \Omega$ input resistance. Figure 10.29 shows the SEPIC's maximum load current driving ability at all tested input voltages while still maintaining a 36V output. Figure 10.30 shows the SEPIC's efficiency at all tested input voltages.

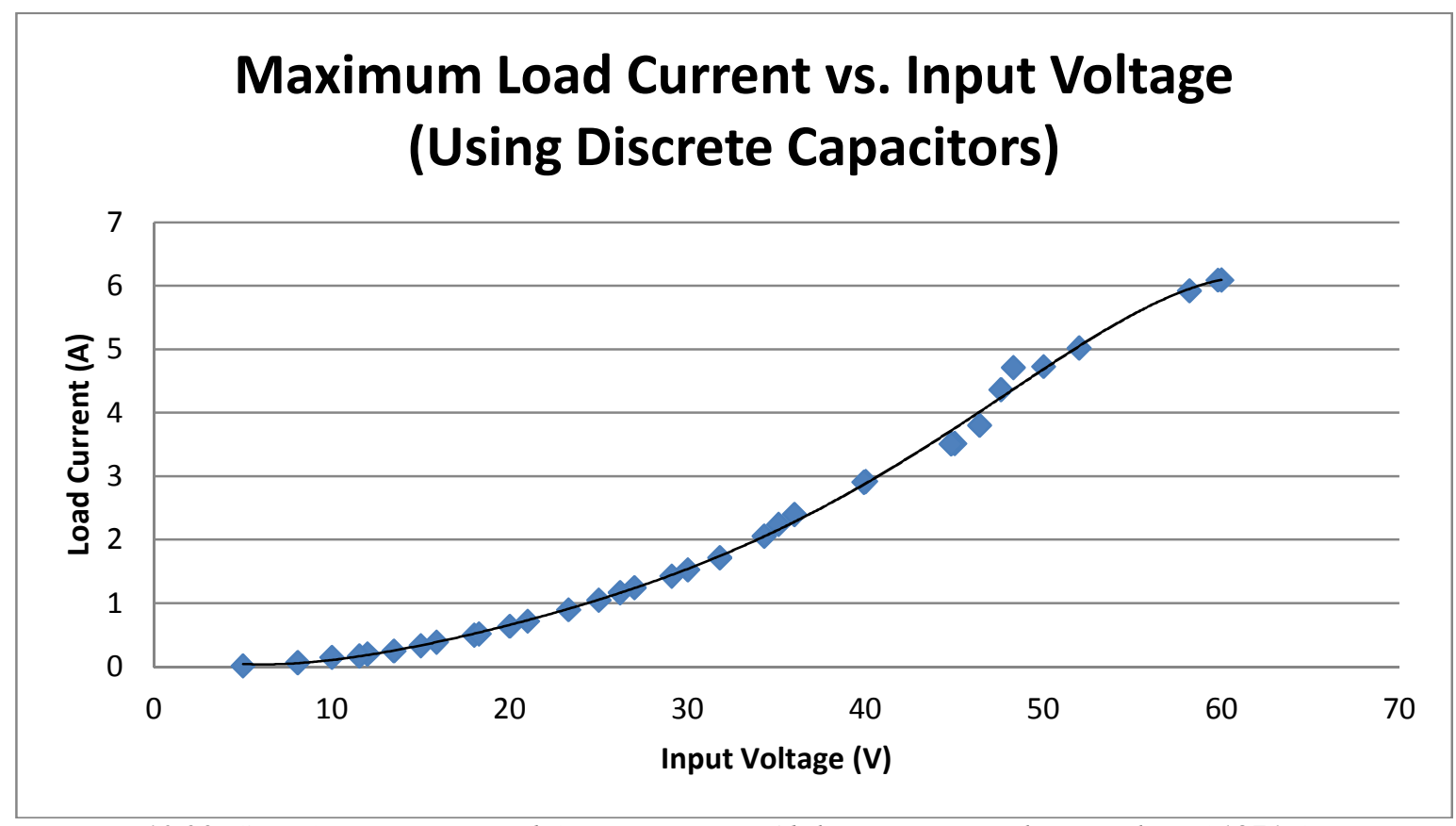

Figure 10.29: SEPIC Maximum Load Current Driving Ability vs. Input Voltage with LTC1871 Current Sense Pin Filtering 


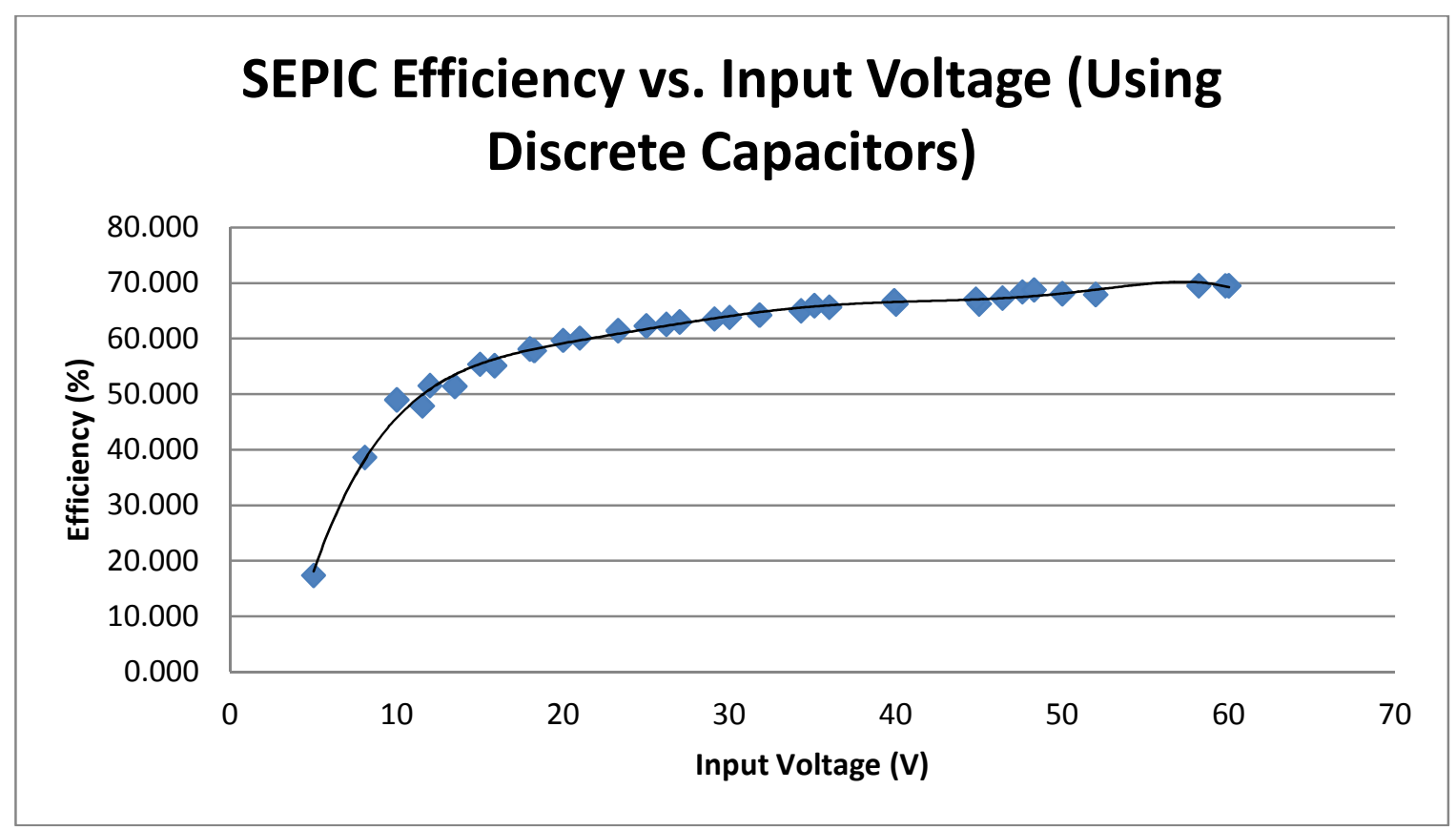

Figure 10.30: SEPIC Efficiency vs. Input Voltage with LTC1871 Current Sense Pin Filtering

The SEPIC drives a 6.092A maximum load at 60V input, and its efficiency peaks at nearly $70 \%$. Maximum load current driving ability and efficiency increase with input voltage. Therefore, adding filtering to the LTC1871's critical control pins (the FB, GATE and SENSE pins) is necessary for proper converter operation. However, its efficiency is still below the $75 \%$ specification outlined in section 1.2 . This project hypothesizes that magnetic losses (such as core losses) in the SEPIC's main switching inductors $\mathrm{L}_{1}$ and $\mathrm{L}_{2}$ are responsible for the low efficiency. Magnetic losses in turn cause switching and conduction losses in Q_MAIN, leading to increased Q_MAIN operating stress. The next section describes a method for improving the converter's overall efficiency. 


\subsubsection{SEPIC Efficiency Improvement}

Magnetic losses are unavoidable for this project's SEPIC because this project selected commercially available inductors for $\mathrm{L}_{1}$ and $\mathrm{L}_{2}$ rather than use custom inductors. Therefore, the efficiency improvement method described in this section focuses on minimizing switching and conduction losses across Q_MAIN. The power supply’s input transient voltage step to the SEPIC affects Q_MAIN's performance. Linear Technology's LT4356-1 datasheet describes a method for improving transistor switching and conduction performance when fast input voltage steps occur at the converter's input. Figure 10.31 shows this method, which involves adding damping circuitry to Q_MAIN $[55]$.

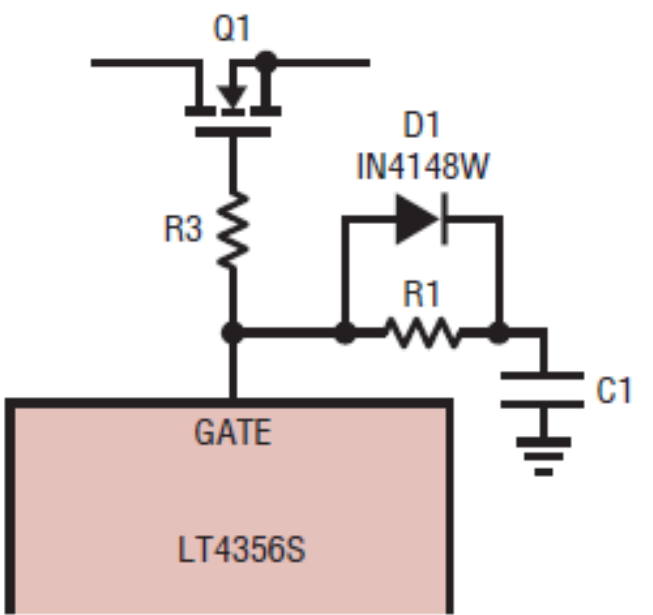

Figure 10.31: Q_MAIN Performance Enhancement Circuit [55]

This modification involves adding a series resistor to the already existing $0.02 \mu \mathrm{F}$ capacitor at the LTC1871's GATE pin, along with a parallel diode with that series resistor. This project omits the additional gate resistor (R3 in figure 10.31) because in LTSpice simulations adding that resistor resulted in increased power dissipation across Q_MAIN. Because the SEPIC's input voltage step may reach faster than 5V per ms from 
the Precor elliptical trainer's output, the $0.02 \mu \mathrm{F}$ capacitor at the LTC1871's GATE pin is necessary for preventing Q_MAIN's self-enhancement. The additional series resistor (R1 in figure 10.31) helps improve Q_MAIN's turn-off time and the parallel diode helps Q_MAIN maintain fast switching trajectories during turn-on. Furthermore, the series RC combination acts as a low pass filter for suppressing high frequency harmonics. Figure 10.32 shows this modification to the SEPIC's schematic.

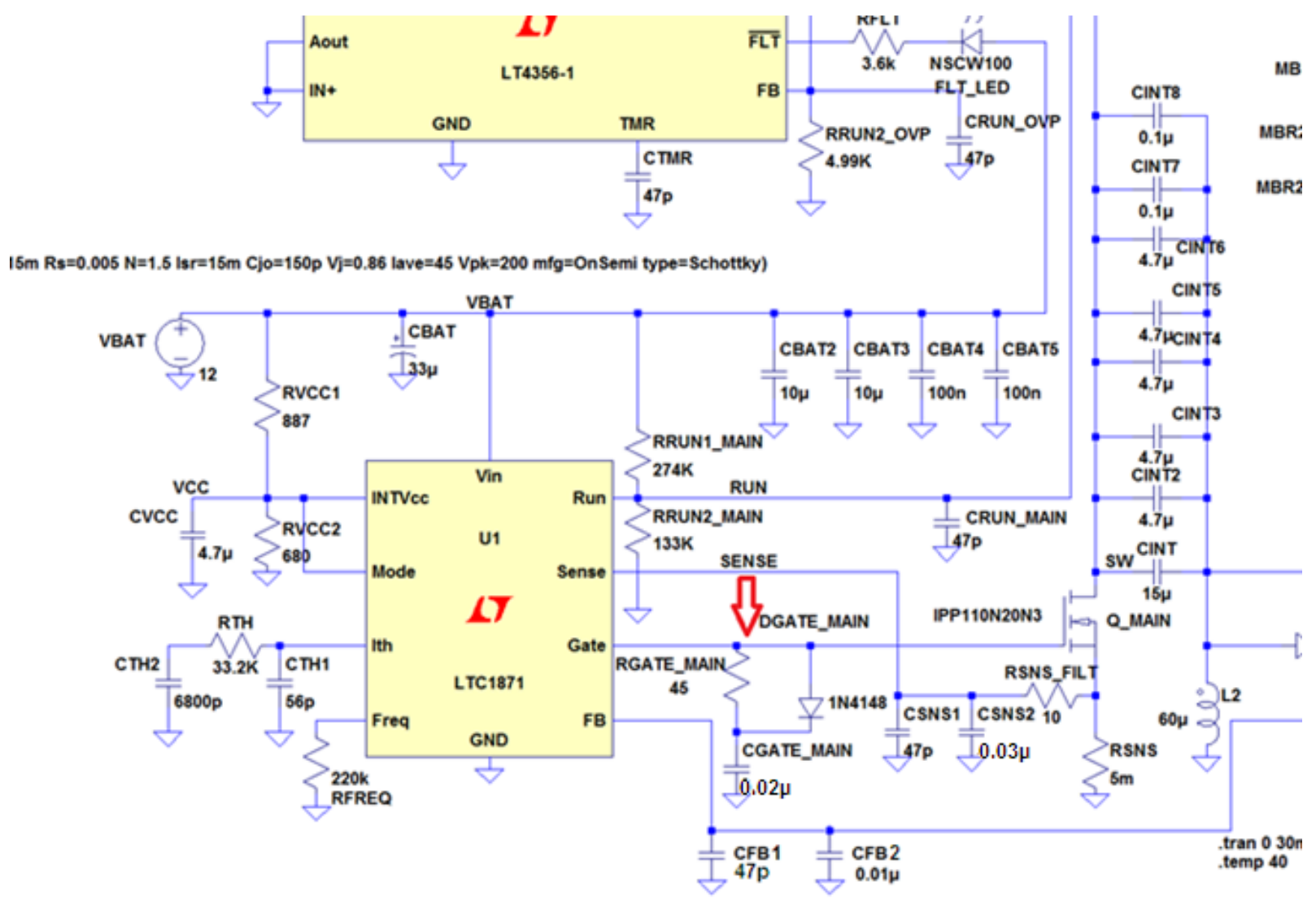

Figure 10.32: LTC1871 GATE Pin Damping Filter

The red arrow in figure 10.27 denotes the GATE pin filter location. Earlier in this chapter, this project used decade capacitor boxes for CGATE_MAIN and $\mathrm{C}_{\mathrm{FB}}$. Those decade capacitor boxes yielded better overall converter performance than using discrete 
capacitors, and that was because of additional series resistance that those decade capacitor boxes had. This project's author measured the ESR of those decade capacitor boxes as roughly $45 \Omega$. Therefore this project selects $45 \Omega$ for the series gate resistor (RGATE_MAIN in figure 10.32) for the LTC1871's GATE Pin damping filter, because it closely matches the decade capacitor box's ESR that helped improve converter functionality in earlier tests. This project uses the same LTC1871 GATE pin capacitor value as before $(0.02 \mu \mathrm{F})$ for the damping filter capacitor (CGATE_MAIN in figure 10.32) because that was the optimal Q_MAIN gate capacitance value determined from previous sections. The LTSpice simulation results with this new damping filter are roughly the same as from the last test run, therefore this project omits those simulation results. Unlike the LTC1871 current sense pin RC low pass filter, this damping filter does not require severing any existing PCB traces leading to Q_MAIN's gate as well as the LTC1871 controller's GATE pin.

Tables E-33 and E-34 show the hardware results from adding this damping filter to the LTC1871's GATE pin, while keeping all other SEPIC components intact. Table E33 shows the SEPIC's output load driving abilities while using the electronic load in constant voltage mode (36V) and Table E-34 shows the same test results, except using input voltages (with their corresponding elliptical training resistance levels) that match the 160 strides per minute exercise test results from the previous EHFEM group in [1]. 
Table E-33: SEPIC Load Tests with LTC1871 Current Sense Pin Filter and GATE Pin Damping Filter and BK 9153 Power Supplies

\begin{tabular}{|c|c|c|c|c|c|c|c|c|c|}
\hline $\mathbf{V}_{\text {IN }}(\mathbf{V})$ & $\mathbf{I}_{\text {IN }}(\mathbf{A})$ & $\mathbf{V}_{\text {OUT }}(\mathbf{V})$ & $\mathbf{I}_{\text {OUT }}(\mathbf{A})$ & $\mathbf{V}_{\text {BAT }}(\mathbf{V})$ & $\mathbf{I}_{\text {BAT }}(\mathbf{A})$ & $\mathbf{P}_{\text {IN }}(\mathbf{W})$ & $\mathbf{P}_{\text {OUT }}(\mathbf{W})$ & $\eta(\%)$ & $\mathbf{R}_{\text {IN }}(\Omega)$ \\
\hline 5 & 0.389 & 37.39 & 0.019 & 12 & 0.024 & 2.233 & 0.710 & 31.814 & 12.853 \\
\hline 10 & 1.002 & 37.37 & 0.163 & 12 & 0.024 & 10.308 & 6.091 & 59.093 & 9.980 \\
\hline 12 & 1.227 & 37.36 & 0.253 & 12 & 0.023 & 15.000 & 9.452 & 63.014 & 9.780 \\
\hline 15 & 1.538 & 37.34 & 0.418 & 12 & 0.023 & 23.346 & 15.608 & 66.856 & 9.753 \\
\hline 18 & 1.83 & 37.33 & 0.618 & 12 & 0.023 & 33.216 & 23.070 & 69.454 & 9.836 \\
\hline 20 & 2.057 & 37.31 & 0.778 & 12 & 0.023 & 41.416 & 29.027 & 70.087 & 9.723 \\
\hline 25 & 2.548 & 37.27 & 1.258 & 12 & 0.023 & 63.976 & 46.886 & 73.286 & 9.812 \\
\hline 27 & 2.69 & 37.25 & 1.448 & 12 & 0.023 & 72.906 & 53.938 & 73.983 & 10.037 \\
\hline 30 & 3.013 & 37.22 & 1.838 & 12 & 0.023 & 90.666 & 68.410 & 75.453 & 9.957 \\
\hline 36 & 3.652 & 37.14 & 2.748 & 12 & 0.023 & 131.748 & 102.061 & 77.467 & 9.858 \\
\hline 40 & 4.05 & 37.07 & 3.418 & 12 & 0.023 & 162.276 & 126.705 & 78.080 & 9.877 \\
\hline 45 & 4.516 & 37 & 4.318 & 12 & 0.023 & 203.496 & 159.766 & 78.511 & 9.965 \\
\hline 50 & 5.075 & 36.89 & 5.418 & 12 & 0.023 & 254.026 & 199.870 & 78.681 & 9.852 \\
\hline 52 & 5.194 & 36.86 & 5.768 & 12 & 0.023 & 270.364 & 212.608 & 78.638 & 10.012 \\
\hline 60 & 5.863 & 36.7 & 7.498 & 12 & 0.023 & 352.056 & 275.177 & 78.163 & 10.234 \\
\hline
\end{tabular}


Table E-34: SEPIC Load Test with LTC1871 Current Sense Pin Filter and GATE Pin Damping Filter, Using 160 Strides/Min. Theoretical Input Voltages from [1] and BK 9153 Power Supplies

\begin{tabular}{|c|c|c|c|c|c|c|c|c|c|c|}
\hline$V_{\text {IN }}(\mathrm{V})$ & $\mathrm{I}_{\mathrm{IN}}(\mathrm{A})$ & $\mathrm{V}_{\text {OUT }}(\mathrm{V})$ & $\mathrm{I}_{\text {oUT }}(\mathrm{A})$ & $V_{B A T}(V)$ & $\mathrm{I}_{\mathrm{BAT}}(\mathrm{A})$ & $P_{\text {IN }}(W)$ & Pout (W) & $\eta(\%)$ & $\mathbf{R}_{\mathrm{IN}}(\Omega)$ & Training Resistance Level \\
\hline 8.08 & 0.792 & 37.38 & 0.088 & 12 & 0.024 & 6.687 & 3.289 & 49.189 & 10.202 & 2 \\
\hline 11.54 & 1.193 & 37.36 & 0.228 & 12 & 0.023 & 14.043 & 8.518 & 60.656 & 9.673 & 3 \\
\hline 13.49 & 1.401 & 37.36 & 0.328 & 12 & 0.023 & 19.175 & 12.254 & 63.905 & 9.629 & 4 \\
\hline 15.88 & 1.629 & 37.34 & 0.468 & 12 & 0.023 & 26.145 & 17.475 & 66.840 & 9.748 & 5 \\
\hline 18.27 & 1.849 & 37.33 & 0.628 & 12 & 0.023 & 34.057 & 23.443 & 68.835 & 9.881 & 6 \\
\hline 21 & 2.163 & 37.31 & 0.868 & 12 & 0.023 & 45.699 & 32.385 & 70.866 & 9.709 & 7 \\
\hline 23.3 & 2.363 & 37.29 & 1.068 & 12 & 0.023 & 55.334 & 39.826 & 71.973 & 9.860 & 8 \\
\hline 26.2 & 2.682 & 37.26 & 1.398 & 12 & 0.023 & 70.544 & 52.089 & 73.839 & 9.769 & 9 \\
\hline 29.1 & 2.919 & 37.23 & 1.718 & 12 & 0.023 & 85.219 & 63.961 & 75.055 & 9.969 & 10 \\
\hline 31.8 & 3.272 & 37.19 & 2.138 & 12 & 0.023 & 104.326 & 79.512 & 76.215 & 9.719 & 11 \\
\hline 34.3 & 3.519 & 37.16 & 2.503 & 12 & 0.023 & 120.978 & 93.011 & 76.883 & 9.747 & 12 \\
\hline 35.1 & 3.577 & 37.15 & 2.618 & 12 & 0.023 & 125.829 & 97.259 & 77.295 & 9.813 & 13 \\
\hline 39.9 & 4.039 & 37.08 & 3.398 & 12 & 0.023 & 161.432 & 125.998 & 78.050 & 9.879 & 14 \\
\hline 44.8 & 4.519 & 37 & 4.298 & 12 & 0.023 & 202.727 & 159.026 & 78.443 & 9.914 & 15 \\
\hline 46.4 & 4.68 & 36.97 & 4.618 & 12 & 0.023 & 217.428 & 170.727 & 78.521 & 9.915 & 16 \\
\hline 47.6 & 4.781 & 36.95 & 4.848 & 12 & 0.023 & 227.852 & 179.134 & 78.619 & 9.956 & 17 \\
\hline 48.3 & 4.883 & 36.93 & 5.028 & 12 & 0.023 & 236.125 & 185.684 & 78.638 & 9.891 & 18 \\
\hline 58.2 & 5.878 & 36.72 & 7.298 & 12 & 0.023 & 342.376 & 267.983 & 78.272 & 9.901 & 19 \\
\hline 59.8 & 5.877 & 36.71 & 7.498 & 12 & 0.023 & 351.721 & 275.252 & 78.259 & 10.175 & 20 \\
\hline
\end{tabular}

Adding the damping filter from figure 10.31 to Q_MAIN's gate dramatically improves SEPIC performance, as the SEPIC now drives higher load currents at all input voltages.

Despite added resistance on the LTC1871's GATE pin, IBAT remains constant relative to previous tests. At $60 \mathrm{~V}$ input the SEPIC drives a 275W load (7.498A output current), while taking in roughly $350 \mathrm{~W}$ from the input power supply. For this set of tests, this project used constant current mode on the electronic load for all input voltages because for all input voltages, the SEPIC now draws the absolute maximum current limit (6.5A) when using constant $36 \mathrm{~V}$ output mode on the electronic load. This means that the SEPIC can drive higher load currents at all input voltages relative to previous tests, but this 
project only selects current limits that allow the SEPIC to maintain a $10 \Omega$ input resistance. Figure 10.33 shows the current SEPIC's maximum load current driving ability at all tested input voltages while still maintaining a 36V output. Figure 10.34 shows the current SEPIC's efficiency at all tested input voltages.

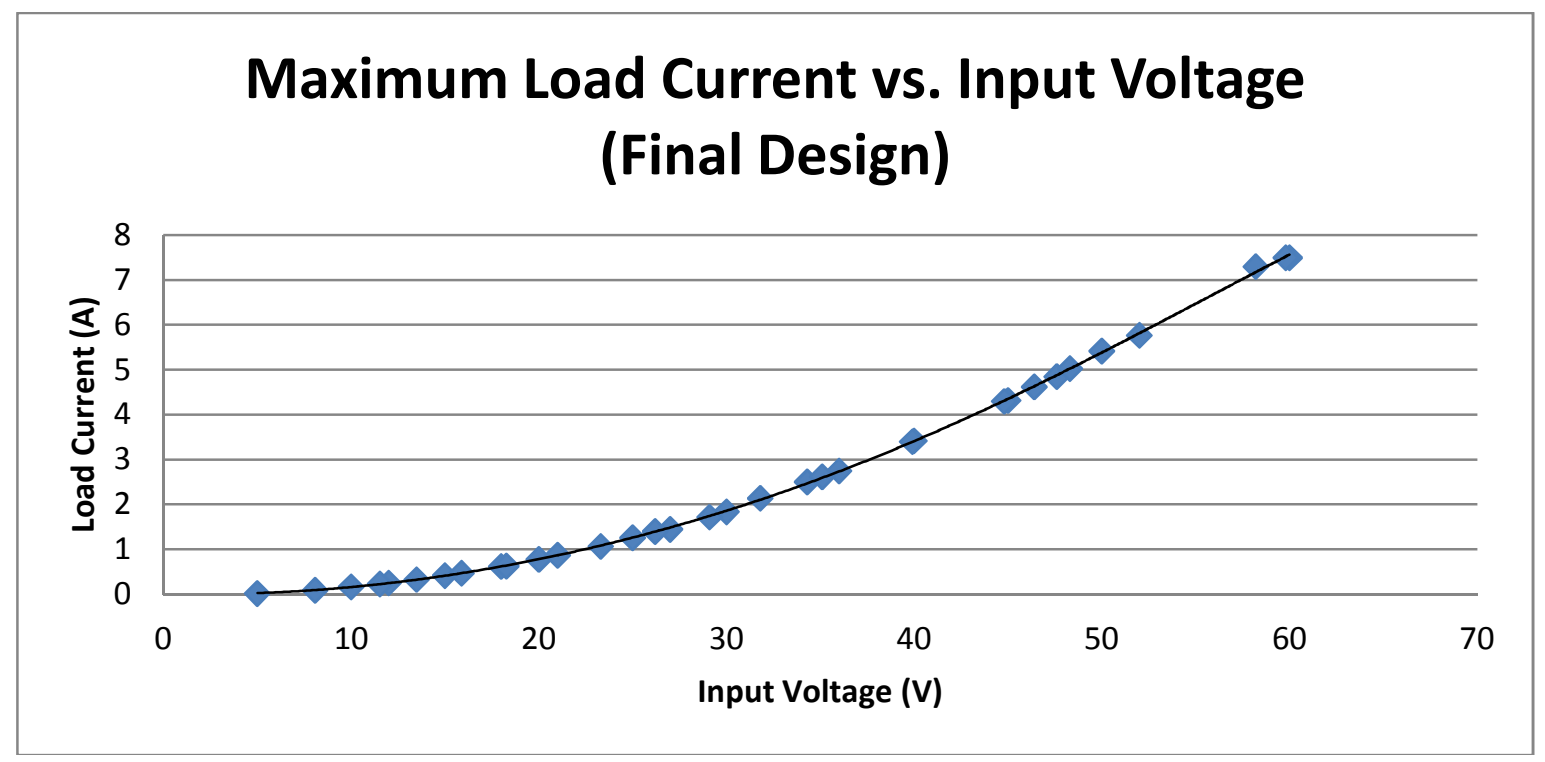

Figure 10.33: SEPIC Maximum Load Current Driving Ability vs. Input Voltage with LTC1871 Current Sense Pin Filtering and GATE Pin Damping Filter 


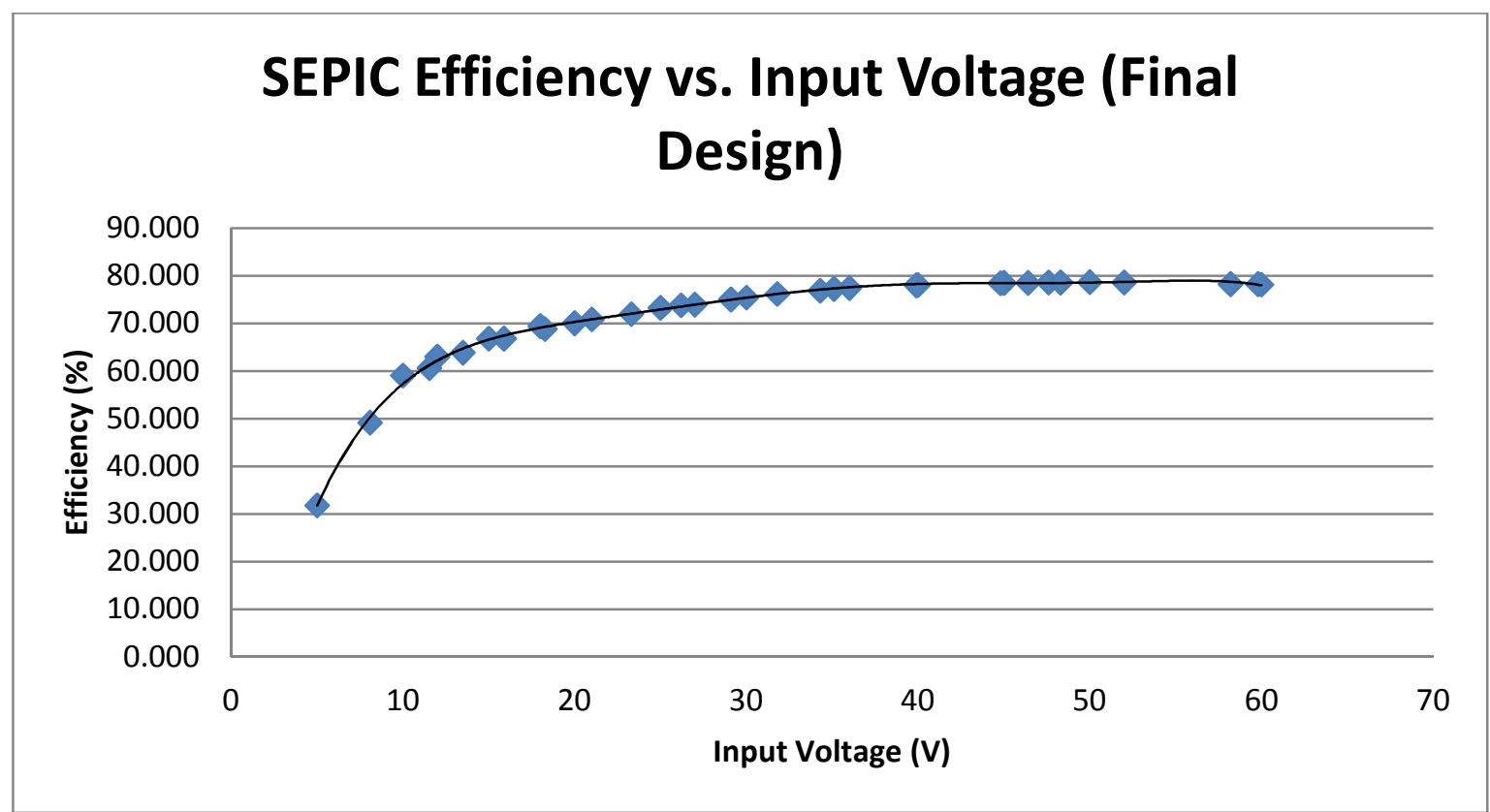

Figure 10.34: SEPIC Efficiency vs. Input Voltage with LTC1871 Current Sense Pin Filtering and GATE Pin Damping Filter

The SEPIC now drives a 7.498A maximum load at 60V input, and its efficiency peaks at nearly $79 \%$, which is an almost $10 \%$ improvement from the previous design.

Theoretically, this project's SEPIC can drive up the 8A maximum specified load current outlined in section 1.2 , but because this converter only uses $7.5 \mathrm{~A}$ output fuses, this project was unable to test with output load currents at the $8 \mathrm{~A}$ level. Therefore, implementing this SEPIC with a Precor elliptical trainer for Cal Poly Recreational Center operation would necessitate a 10A blade fuse, which not only helps the SEPIC supply its specified maximum 8A load current but 10A is also the Enphase M175-24-240 microinverter's absolute maximum input short circuit current rating. Likewise with the previous test results, maximum load current driving ability and efficiency increase with input voltage. Overall, adding filtering to the LTC1871's critical control pins (the FB, GATE and SENSE pins) is necessary for proper converter operation. The SEPIC's 
efficiency now surpasses the $75 \%$ requirement outlined in section 1.2 . Thus, adding a damping circuit to the LTC1871's GATE pin was the crucial key for reducing the SEPIC's switching and conduction losses, as well as decreasing Q_MAIN's operating stresses. From tables E-33 and E-34, overall maximum input power decreased at all input voltages, relative to the results from E-31 and E-32, indicating that the losses incurred $\operatorname{across} \mathrm{L}_{1}, \mathrm{~L}_{2}$ and Q_MAIN decreased as well. Overall SEPIC efficiency is still poor at lower input voltages; however this is because the SEPIC and its PWM controller operate in $\mathrm{CCM}$ for all input voltages, rather than DCM at lower input voltages, resulting in greater magnetic losses in $\mathrm{L}_{1}$ and $\mathrm{L}_{2}$ at lighter output load currents. For the typical Precor elliptical workout output's 15-30V SEPIC input range, the efficiency values suffice. At around $30 \mathrm{~V}$ input, the converter reaches its specified $75 \%$ efficiency. $30 \mathrm{~V}$ is the peak input for a typical workout session on the Precor elliptical trainer. (10.2) calculates this project's SEPIC's line regulation. For calculating line regulation, (10.2) uses the SEPIC's output voltages when the SEPIC operates at $8 \mathrm{~V}$ input and $60 \mathrm{~V}$ input using the maximum load current levels from tables E-33 and E-34.

$$
\text { Line Reg. }=\frac{V_{\text {OUT_8V input }}-V_{\text {OUT_60Vinput }}}{V_{\text {OUT_nominal }}} \times 100 \%=\frac{37.39 \mathrm{~V}-36.7 \mathrm{~V}}{36 \mathrm{~V}} \times 100 \%=1.92 \%
$$

The SEPIC experiences an overall $1.92 \%$ line regulation when its input changes from $8 \mathrm{~V}$ to $60 \mathrm{~V}$. This value is lower than the $5 \%$ requirement outlined in section 1.2 , therefore it suffices. Figures 10.35 and 10.36 show the SEPIC's current sense pin and Q_MAIN switching trajectory waveforms with all the converter enhancement modifications mentioned in this chapter. In both figures the SEPIC runs at $36 \mathrm{~V}$ input along while driving a 2.748 A output load. 


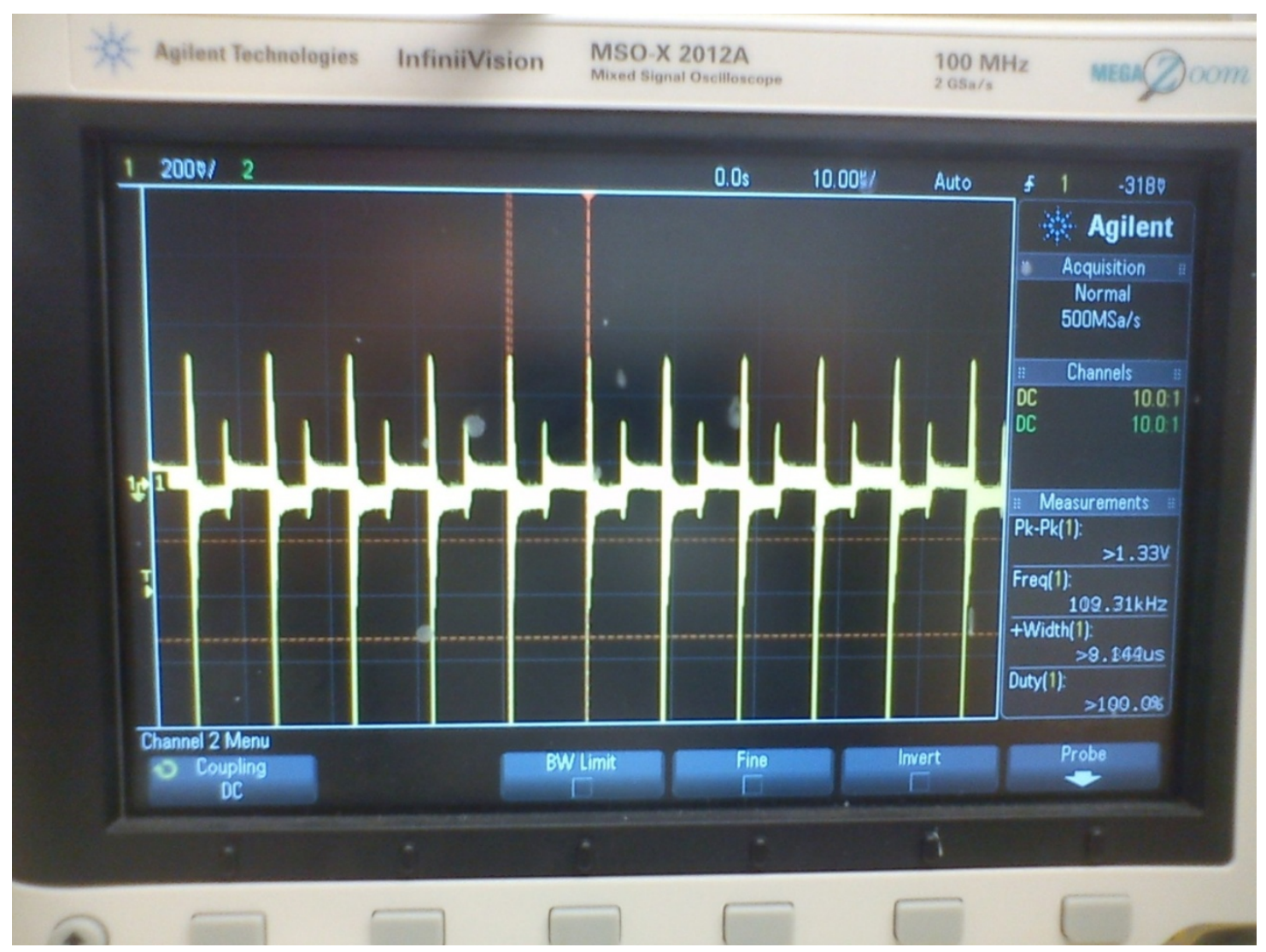

Figure 10.35: LTC1871 Current Sense Pin Switching Waveform with All SEPIC Performance Enhancement Modifications at 36V Input and 2.748A Load 


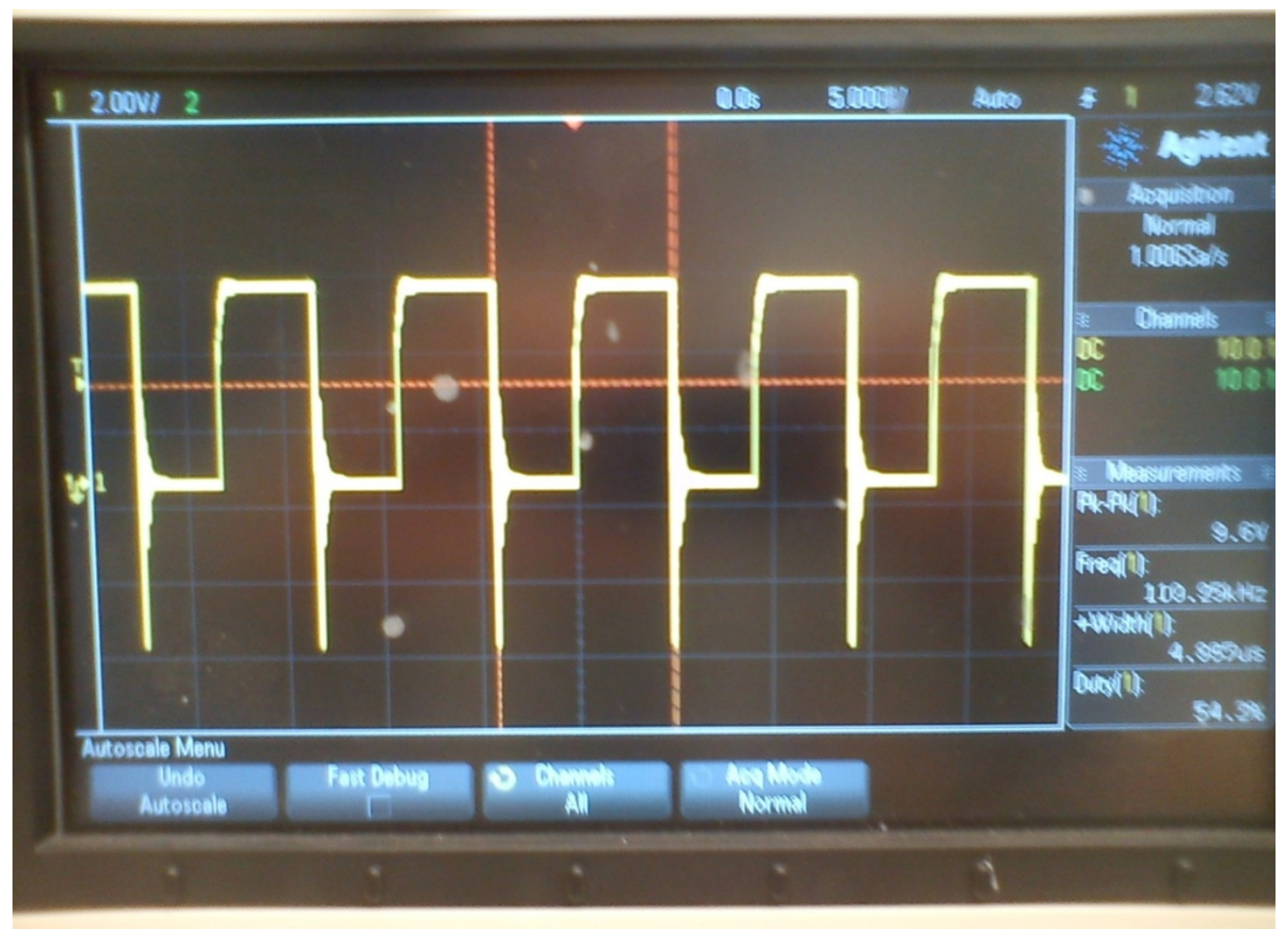

Figure 10.36: Q_MAIN Gate Switching Trajectory with All SEPIC Performance Enhancement Modifications at $36 \mathrm{~V}$ Input and 2.748A Load

Although there are still harmonics present in both waveforms, such harmonics are much lower in magnitude than from previous SEPIC designs and the waveforms look closer to the ideal waveforms in figure 5.9. This project could make further improvements at eliminating those harmonics by tweaking the LTC1871's FB, GATE and SENSE pin filter component values, however, lack of time prevents those improvements from taking place. Figures 10.37 to 10.39 show the SEPIC's output voltage ripple in its three main DC-DC conversion modes - boost, buck-boost and boost. Figure 10.37 uses $30 \mathrm{~V}$ input while driving a 1.838A load for boost mode, figure 10.38 uses $36 \mathrm{~V}$ input while driving a 
2.748A load for buck-boost mode, and figure 10.39 uses $60 \mathrm{~V}$ input while driving a 7.498A load for buck mode.

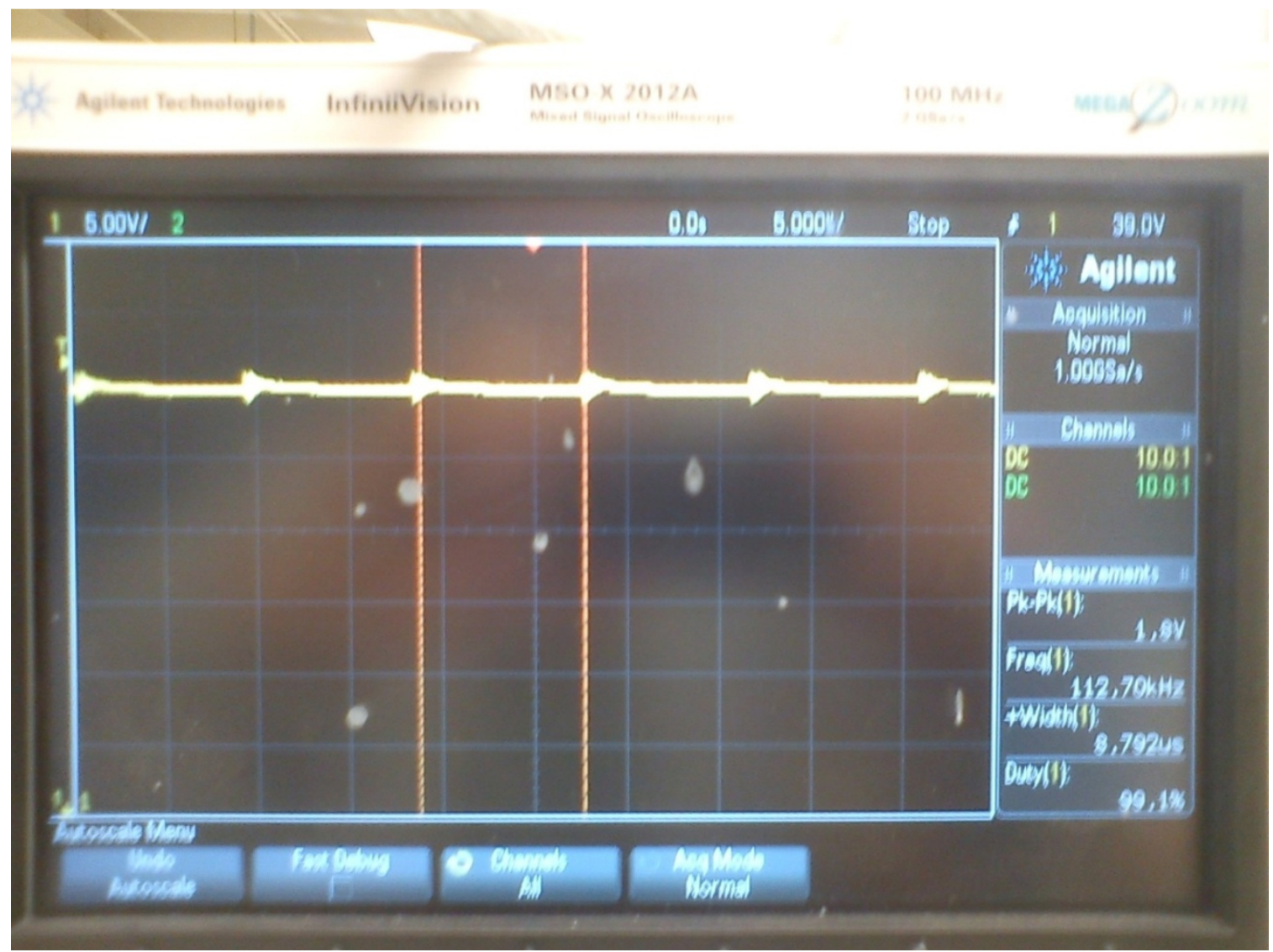

Figure 10.37: SEPIC Output Voltage Ripple at 30V Input and 1.838A Load (Boost Mode) 


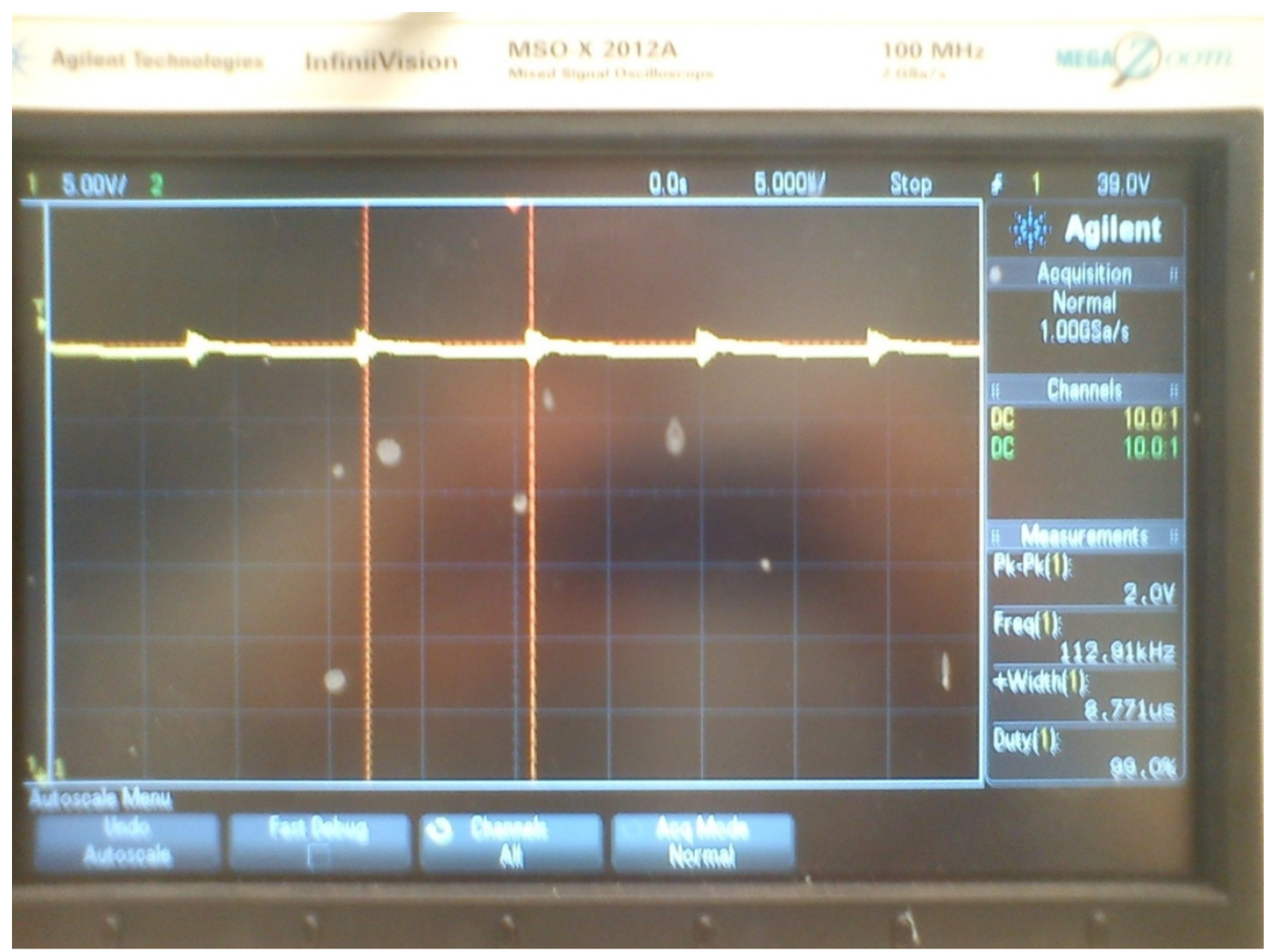

Figure 10.38: SEPIC Output Voltage Ripple at 36V Input and 2.748A Load (Buck-Boost Mode) 


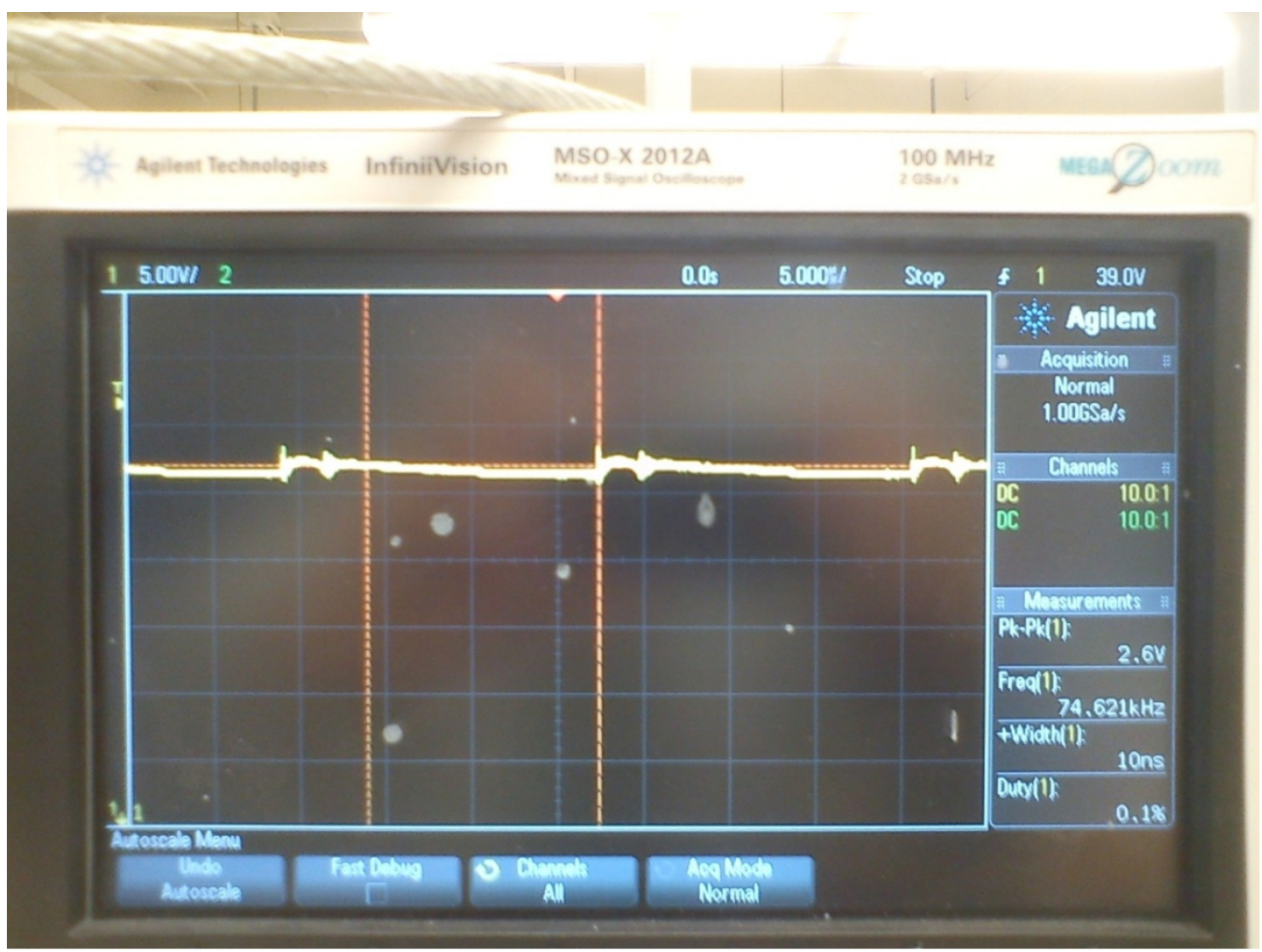

Figure 10.39: SEPIC Output Voltage Ripple at 60V Input and 7.498A Load (Buck Mode)

From figures 10.37 to 10.39 , the SEPIC's peak to peak output voltage ripple worsens with increasing input voltage and output load current. With 30V input, the SEPIC's peak to peak output voltage ripple is $1.8 \mathrm{~V}$. It increases to $2 \mathrm{~V}$ at $36 \mathrm{~V}$ input and in reaches a $2.6 \mathrm{~V}$ maximum at $60 \mathrm{~V}$ input. At worst this corresponds to a $7.08 \%$ ripple at $60 \mathrm{~V}$ input relative to the $36.7 \mathrm{~V}$ output voltage, which is under the maximum $10 \%$ output peak to peak ripple specification outlined in section 1.2. Therefore, the $940 \mu \mathrm{F}$ output capacitance from the two output electrolytic capacitors suffice, although future improvements to this SEPIC design should use a single electrolytic capacitor with low ESR. Also, although during this test the project removed the inductor wire loops from the SEPIC's PCB, Q_MAIN's switching waveform at 36V input from figure 10.36 show no major harmonic 
transients. Therefore inductors $\mathrm{L}_{1}$ and $\mathrm{L}_{2}$ 's current waveforms should not contain any dead time (which those switching harmonics caused) and thus $\mathrm{L}_{1}$ and $\mathrm{L}_{2}$ should run in $\mathrm{CCM}$ at $31 \mathrm{~V}$ input and $2 \mathrm{~A}$ load, as specified in section 1.2. Therefore, this project safely assumes that both SEPIC inductors run in CCM at the specified input voltage and output load levels. The current modifications made to the current SEPIC design already satisfy the specifications requirements outlined in sections 1.1 to 1.3 , therefore no further modifications are necessary because of project time constraints. Figure 10.40 shows this project's SEPIC's final schematic. 


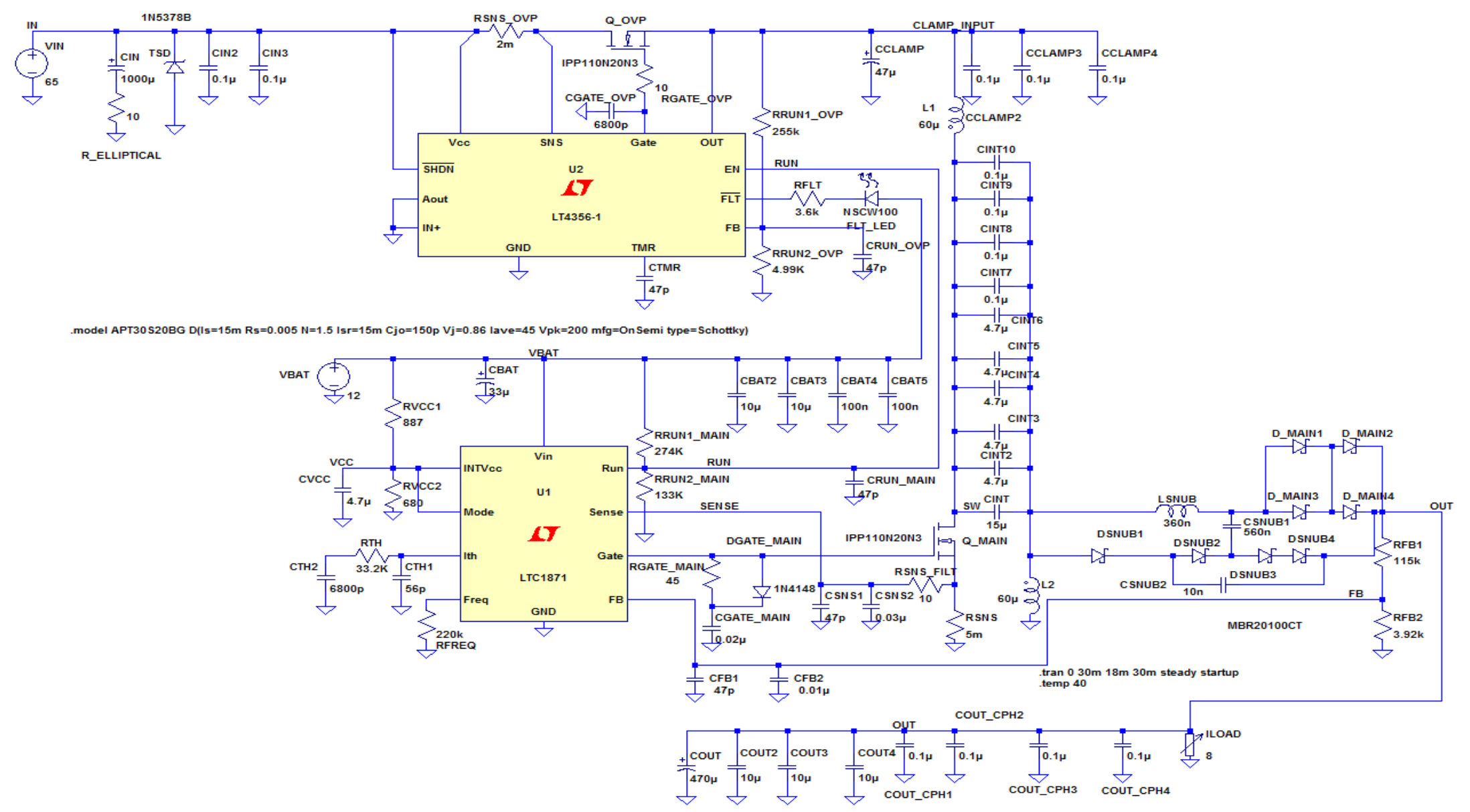

Figure 10.40: Final SEPIC Schematic 
Table E-35 shows this project's SEPIC's final BOM and total cost.

Table E-35: Final SEPIC BOM

\begin{tabular}{|c|c|c|c|c|c|c|c|}
\hline PCBs & Quantity: & $\begin{array}{l}\text { Unit Price } \\
\text { (\$): }\end{array}$ & Total Price: & Components: & Type: & $\begin{array}{c}\text { 65V, 8A Required } \\
\text { Rating }\end{array}$ & $5 \mathrm{~V}, 0.5 \mathrm{~A}$ \\
\hline Custom 2-layer 5.8" × 5.275" & 1 & 84.42 & 84.42 & PCB & 2-layer & $N / A$ & $\mathrm{~N} / \mathrm{A}$ \\
\hline \multicolumn{3}{|c|}{ Total Cost (\$) } & 84.42 & & & & \\
\hline Controller I.C.s: & Quantity: & $\begin{array}{l}\text { Unit Price } \\
(\$):\end{array}$ & Total Price: & Components: & Type: & $\begin{array}{c}\text { 65V, 8A Required } \\
\text { Rating }\end{array}$ & $5 \mathrm{~V}, 0.5 \mathrm{~A}$ \\
\hline LTC1871HMS (10-MSOP) & 1 & 4.83 & 4.83 & U1 & 10-MSOP & $\mathrm{N} / \mathrm{A}$ & $\mathrm{N} / \mathrm{A}$ \\
\hline LT4356-1HMS (10-MSOP) & 2 & 3.70 & 7.40 & U2 & 10-MSOP & $\mathrm{N} / \mathrm{A}$ & $\mathrm{N} / \mathrm{A}$ \\
\hline \multicolumn{3}{|c|}{ Total Cost (\$) } & 12.23 & & & & \\
\hline Transistors: & Quantity: & $\begin{array}{l}\text { Unit Price } \\
\text { (\$): }\end{array}$ & Total Price: & Components: & Type: & $\begin{array}{c}\text { 65V, 8A Required } \\
\text { Rating }\end{array}$ & $5 \mathrm{~V}, 0.5 \mathrm{~A}$ \\
\hline Infineon IPP110N20N3 & 1 & 8.06 & 8.06 & Q_MAIN & TO-220-3 & $2.45 \mathrm{~W}$ & $4.01 \mathrm{~W}$ \\
\hline IXYS IXFK230N20T & 1 & 9.50 & 9.50 & Q_OVP & TO-264 & $1.625 \mathrm{~kW}$ & $1.625 \mathrm{~kW}$ \\
\hline \multicolumn{3}{|c|}{ Total Cost (\$) } & 17.56 & & & & \\
\hline Diodes: & Quantity: & $\begin{array}{l}\text { Unit Price } \\
(\$):\end{array}$ & Total Price: & Components: & Type: & $\begin{array}{c}\text { 65V, 8A Required } \\
\text { Rating }\end{array}$ & $5 \mathrm{~V}, 0.5 \mathrm{~A}$ \\
\hline MBR20100CT (Schottky) & 7 & 0.88 & 6.16 & $\begin{array}{c}\text { D_MAIN1, D_MAIN2, D_MAIN3, } \\
\text { D_MAIN4, DSNUB2, DSNUB3, } \\
\text { DSNUB4 }\end{array}$ & TO-220-3 & $31.11 \mathrm{~A}, 110 \mathrm{~V}$ & 27.72A, 56.46V \\
\hline Microsemi APT30S20BG (Schottky) & 1 & 3.67 & 3.67 & DSNUB1 & TO-247 & $39.43 \mathrm{~A}, 15.95 \mathrm{~V}$ & $27.89 \mathrm{~A}, 9.58 \mathrm{~V}$ \\
\hline 1N5378B (Zener) & 1 & 0.57 & 0.57 & TSD & Through-Hole & $100 \mathrm{~V}$ & $100 \mathrm{~V}$ \\
\hline SML-LX1206SRC-TR (LED) & 1 & 0.43 & 0.43 & FLT_LED & 1206 & $\begin{array}{c}1.2 \mathrm{~V} \text { min. forward } \\
\text { drop }\end{array}$ & $\begin{array}{l}1.2 \mathrm{~V} \text { min. } \\
\text { forward drop }\end{array}$ \\
\hline 1N4148-TR (Silicon) & 1 & 0.06 & 0.06 & DGATE_MAIN & Through-Hole & $\mathrm{N} / \mathrm{A}$ & $\mathrm{N} / \mathrm{A}$ \\
\hline \multicolumn{3}{|c|}{ Total Cost (\$) } & 10.89 & & & & \\
\hline Heatsinks: & Quantity: & $\begin{array}{l}\text { Unit Price } \\
\text { (\$): }\end{array}$ & Total Price: & Components: & Type: & $\begin{array}{c}\text { 65V, 8A Required } \\
\text { Rating }\end{array}$ & $5 \mathrm{~V}, 0.5 \mathrm{~A}$ \\
\hline 531202B02500G (TO-220) & 1 & 1.38 & 1.38 & Q_MAIN heatsink & TO-220 & $\mathrm{N} / \mathrm{A}$ & $\mathrm{N} / \mathrm{A}$ \\
\hline MV-102-55E (TO-247 and TO-264) & 1 & 7.39 & 7.39 & Q_OVP heatsink & TO-264 & $\mathrm{N} / \mathrm{A}$ & $\mathrm{N} / \mathrm{A}$ \\
\hline WA-T247-101E (TO-247) & 1 & 2.07 & 2.07 & DSNUB1 heatsink & TO-247 & $\mathrm{N} / \mathrm{A}$ & $\mathrm{N} / \mathrm{A}$ \\
\hline 574502B00000G (TO-220) & 7 & 0.27 & 1.89 & $\begin{array}{c}\text { D_MAIN1, D_MAIN2, D_MAIN3, } \\
\text { D_MAIN4, DSNUB2, DSNUB3, } \\
\text { DSNUB4 }\end{array}$ & TO-220 & $\mathrm{N} / \mathrm{A}$ & $\mathrm{N} / \mathrm{A}$ \\
\hline \multicolumn{3}{|c|}{ Total Cost (\$) } & 12.73 & & & & \\
\hline
\end{tabular}


Table E-35 (Continued)

\begin{tabular}{|c|c|c|c|c|c|c|c|}
\hline Fuses: & Quantity: & $\begin{array}{l}\text { Unit Price } \\
\text { (\$): }\end{array}$ & Total Price: & Components: & Type: & $\begin{array}{c}\text { 65V, 8A Required } \\
\text { Rating }\end{array}$ & $5 \mathrm{~V}, 0.5 \mathrm{~A}$ \\
\hline $\begin{array}{c}\text { Radio Shack 270-270-1234 30A Blade } \\
\text { Fuse Holder }\end{array}$ & 2 & 2.99 & 5.98 & Fuse Holder for Input and Output Fuse & $\mathrm{N} / \mathrm{A}$ & $65 \mathrm{~V}, 25 \mathrm{~A}$ & $5 \mathrm{~V}, 25 \mathrm{~A}$ \\
\hline $\begin{array}{c}\text { Cooper Bussmann 270-1085 30A } \\
\text { Blade Fuse }\end{array}$ & 1 & 0.73 & 0.73 & Input Fuse & Blade Fuse & $65 \mathrm{~V}, 25 \mathrm{~A}$ & $5 \mathrm{~V}, 25 \mathrm{~A}$ \\
\hline $\begin{array}{c}\text { Cooper Bussmann 270-1081 10A } \\
\text { Blade Fuse }\end{array}$ & 1 & 0.73 & 0.73 & Output Fuse & Blade Fuse & $36 \mathrm{~V}, 8 \mathrm{~A}$ & $5 \mathrm{~V}, 0.5 \mathrm{~A}$ \\
\hline Bourns SF-0402F200-2 2A Fuse & 1 & 0.68 & 0.68 & Elliptical Battery Fuse & 0402 & $12 \mathrm{~V}, 2 \mathrm{~A}$ & $12 \mathrm{~V}, 2 \mathrm{~A}$ \\
\hline \multicolumn{3}{|c|}{ Total Cost (\$) } & 8.12 & & & & \\
\hline 274k, 1/8W, 1\%, ERJ-6ENF2743V & 1 & 0.07 & 0.07 & RRUN1_MAIN & 0805 & $0.521 \mathrm{~mW}$ & $0.522 \mathrm{~mW}$ \\
\hline 255k, 1/8W, 1\%, ERJ-6ENF2553V & 1 & 0.07 & 0.07 & RRUN1_OVP & 0805 & $16.289 \mathrm{~mW}$ & $0.094 \mathrm{~mW}$ \\
\hline 220k, 1/8W, 1\%, ERJ-6ENF2203V & 1 & 0.07 & 0.07 & RFREQ & 0805 & $0.002 \mathrm{~mW}$ & $0.002 \mathrm{~mW}$ \\
\hline 133k, 1/8W, 1\%, ERJ-6ENF1333V & 1 & 0.07 & 0.07 & RRUN2_MAIN & 0805 & $0.115 \mathrm{~mW}$ & $0.122 \mathrm{~mW}$ \\
\hline 115k, 1/8W, 1\%, ERJ-6ENF1153V & 1 & 0.07 & 0.07 & RFB1 & 0805 & $11.12 \mathrm{~mW}$ & $12.28 \mathrm{~mW}$ \\
\hline 33.2k, $1 / 8 \mathrm{~W}, 1 \%$, ERJ-6ENF3322V & 1 & 0.07 & 0.07 & $\mathrm{RTH}$ & 0805 & $28.32 \mathrm{~mW}$ & $0.028 \mathrm{~mW}$ \\
\hline 3.92k, 1/8W, 1\%, RMCF0805FT3K92 & 1 & 0.04 & 0.04 & RFB2 & 0805 & $0.387 \mathrm{~mW}$ & $0.427 \mathrm{~mW}$ \\
\hline 3.6k, 1/8W, 1\%, ERJ-6ENF3601V & 1 & 0.07 & 0.07 & RFLT & 0805 & $32.4 \mathrm{~mW}$ & $32.4 \mathrm{~mW}$ \\
\hline $887,1 / 8 \mathrm{~W}, 1 \%$, ERJ-6ENF8870V & 1 & 0.07 & 0.07 & RVCC1 & 0805 & $<125 \mathrm{~mW}$ & $<125 \mathrm{~mW}$ \\
\hline $680,1 / 8 \mathrm{~W}, 5 \%$, ERJ-6GEYJ681V & 1 & 0.04 & 0.04 & RVCC2 & 0805 & $<125 \mathrm{~mW}$ & $<125 \mathrm{~mW}$ \\
\hline 47, 1/8W, 5\%, CF18JT47R0 & 1 & 0.09 & 0.09 & RGATE_MAIN & Through-Hole & $<125 \mathrm{~mW}$ & $<125 \mathrm{~mW}$ \\
\hline 10, 1/8W, $1 \%$, ERJ-6ENF10R0V & 2 & 0.07 & 0.14 & RGATE_OVP, RSNS_FILT & 0805 & $13.05 \mathrm{~mW}$ & OW \\
\hline $0.005,5 \mathrm{~W}, 1 \%$, OARSXPR005FLF & 1 & 1.42 & 1.42 & RSNS & SMT (Custom) & $410 \mathrm{~mW}$ & $1.132 \mathrm{~W}$ \\
\hline $0.002,5 \mathrm{~W}, 1 \%, \mathrm{WSLP} 39212 \mathrm{~L} 000 \mathrm{FEB}$ & 1 & 3.07 & 3.07 & RSNS_OVP & SMT (Custom) & $64 \mathrm{~mW}$ & $1.000 \mathrm{~W}$ \\
\hline \multicolumn{3}{|c|}{ Total Cost (\$) } & 5.43 & & & & \\
\hline
\end{tabular}


Table E-35 (Continued)

\begin{tabular}{|c|c|c|c|c|c|c|c|}
\hline Inductors: & Quantity: & $\begin{array}{l}\text { Unit Price } \\
(\$) \text { : }\end{array}$ & Total Price: & Components: & Type: & \begin{tabular}{|c|}
$65 \mathrm{~V}$, 8A Required \\
Rating
\end{tabular} & $5 \mathrm{~V}, 0.5 \mathrm{~A}$ \\
\hline Vishay IHV28BZ60 $\left(60 \mu \mathrm{H}, \mathrm{I}_{\mathrm{SAT}}=28 \mathrm{~A}\right)$ & 2 & 20.30 & 40.60 & $\mathrm{~L} 1, \mathrm{~L} 2$ & Through-Hole & $30.68 \mathrm{~A}$ (max.), $65 \mathrm{~V}$ & $\begin{array}{l}\text { 22.37A (max.), } \\
52.22 \mathrm{~V}\end{array}$ \\
\hline $\begin{array}{c}\text { Vishay IHLP4040DZERR36M01 } \\
\left(360 \mathrm{nH}, \mathrm{I}_{\mathrm{SAT}}=60 \mathrm{~A}\right)\end{array}$ & 1 & 2.52 & 2.52 & LSNUB & SMT (Custom) & $\begin{array}{c}29.3 \mathrm{~A} \text { (max.), } \\
106.25 \mathrm{~V}\end{array}$ & $\begin{array}{c}17.85 \mathrm{~A}(\max .), \\
55.35 \mathrm{~V}\end{array}$ \\
\hline \multicolumn{3}{|c|}{ Total Cost (\$) } & 43.12 & & & & \\
\hline
\end{tabular}


Table E-35 (Continued)

\begin{tabular}{|c|c|c|c|c|c|c|c|}
\hline Capacitors: & Quantity: & $\begin{array}{c}\text { Unit } \\
\text { Price } \\
\text { (\$): }\end{array}$ & $\begin{array}{l}\text { Total } \\
\text { Price: }\end{array}$ & Components: & Type: & $\begin{array}{c}\text { 65V, 8A } \\
\text { Required Rating }\end{array}$ & $5 \mathrm{~V}, 0.5 \mathrm{~A}$ \\
\hline $\begin{array}{l}\text { 1000 } \mu \text {, Electrolytic, 63V, } \\
\text { B41695A8108Q7 }\end{array}$ & 1 & 5.15 & 5.15 & COUT & $\begin{array}{l}\text { Through- } \\
\text { Hole }\end{array}$ & $36 \mathrm{~V}, 5.43 \mathrm{~A}(\mathrm{RMS})$ & $\begin{array}{l}\text { 36V, } 2.368 \mathrm{~A} \\
\text { (RMS) }\end{array}$ \\
\hline 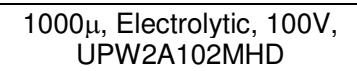 & 1 & 3.03 & 3.03 & $\mathrm{CIN}$ & $\begin{array}{l}\text { Through- } \\
\text { Hole }\end{array}$ & $\begin{array}{l}\text { 65V, } 1.886 \mathrm{pA} \\
\text { (RMS) }\end{array}$ & $\begin{array}{l}5 \mathrm{~V}, 227.67 f \mathrm{~A} \\
\text { (RMS) }\end{array}$ \\
\hline $\begin{array}{l}\text { 47 } \mu \text {, Electrolytic, } 100 \mathrm{~V} \\
\text { AFK476M2AH32T-F }\end{array}$ & 1 & 2.35 & 2.35 & CCLAMP & $\begin{array}{c}\text { SMT } \\
\text { (Custom) }\end{array}$ & $\begin{array}{c}65.68 \mathrm{~V} \\
119.05 \mathrm{~mA}(\mathrm{RMS}) \\
\end{array}$ & $\begin{array}{c}5 \mathrm{~V}, 42.067 \mathrm{~mA} \\
\text { (RMS) }\end{array}$ \\
\hline $\begin{array}{c}\text { 33 } \mu \text {, Electrolytic, 35V, EEE- } \\
\text { HA1V330WP }\end{array}$ & 1 & 0.63 & 0.63 & CBAT & $\begin{array}{c}\text { SMT } \\
\text { (Custom) }\end{array}$ & $\begin{array}{l}12 \mathrm{~V}, 206.56 \mathrm{fA} \\
\text { (RMS) }\end{array}$ & $\begin{array}{l}12 \mathrm{~V}, 442.09 \mathrm{fA} \\
\text { (RMS) }\end{array}$ \\
\hline 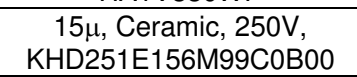 & 1 & 25.78 & 25.78 & CINT & $\begin{array}{l}\text { Through- } \\
\text { Hole }\end{array}$ & $\begin{array}{c}\text { 77.05V, 3.67A } \\
\text { (RMS) }\end{array}$ & $\begin{array}{c}21.22 \mathrm{~V}, 1.404 \mathrm{~A} \\
\text { (RMS) }\end{array}$ \\
\hline $\begin{array}{l}\text { 10 } \mu \text {, Ceramic, 50V, } \\
\text { UMK325C7106MM-T }\end{array}$ & 3 & 0.96 & 2.88 & COUT2, COUT3, COUT4 & 1210 & $\begin{array}{l}\text { 36V, } 2.758 \mathrm{~A} \\
\text { (RMS) }\end{array}$ & $\begin{array}{l}\text { 36V, } 2.016 \mathrm{~A} \\
\text { (RMS) }\end{array}$ \\
\hline $\begin{array}{l}\text { 10 } \mu \text {, Ceramic, 25V, } \\
\text { TMK316B7106KL-TD }\end{array}$ & 2 & 0.58 & 1.16 & СВАТ2, СВАТЗ & 1206 & $\begin{array}{l}\text { 12V, } 963.63 f A \\
\text { (RMS) }\end{array}$ & $\begin{array}{l}12 \mathrm{~V}, 1.211 \mathrm{pA} \\
\text { (RMS) }\end{array}$ \\
\hline 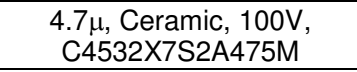 & 5 & 1.75 & 8.75 & CINT2, CINT3, CINT4, CINT5, CINT6 & 1812 & $\begin{array}{c}77.05 \mathrm{~V}, 1.22 \mathrm{~A} \\
\text { (RMS) }\end{array}$ & $\begin{array}{l}21.22 \mathrm{~V}, 1.22 \mathrm{~A} \\
(\mathrm{RMS})\end{array}$ \\
\hline $\begin{array}{l}\text { 4.7 } \mu \text {, Ceramic, } 16 \mathrm{~V}, \\
\text { EMK212B7475KG-T }\end{array}$ & 1 & 0.38 & 0.38 & CVCC & 0805 & $\begin{array}{l}\text { 5.23V, } 153.1 \mathrm{~mA} \\
\text { (RMS) }\end{array}$ & $\begin{array}{l}\text { 5.23V, } 170.84 \mathrm{~mA} \\
\text { (RMS) }\end{array}$ \\
\hline $\begin{array}{l}\text { 0.56 } \mu, \text { Ceramic, } 25 \mathrm{~V} \\
\text { c0805C564K3RACTU }\end{array}$ & 1 & 0.84 & 0.84 & CSNUB1 & 0805 & $\begin{array}{l}\text { 11.33V, 3.629A } \\
\text { (RMS) }\end{array}$ & $\begin{array}{l}\text { 11.00V, } 1.577 \mathrm{~A} \\
\text { (RMS) }\end{array}$ \\
\hline $\begin{array}{l}0.1 \mu \text {, Ceramic, } 100 \mathrm{~V} \\
\text { CGA3E3X7S2A104K }\end{array}$ & 7 & 0.30 & 2.10 & $\begin{array}{l}\text { CIN2, CIN3, CCLAMP2, CCLAMP3, } \\
\text { CCLAMP4, CBAT4, CBAT5 }\end{array}$ & 0603 & $\begin{array}{l}65 \mathrm{~V}, 1.000 \mathrm{~mA} \\
\text { (RMS) }\end{array}$ & $\begin{array}{l}5 \mathrm{~V}, 0.285 \mathrm{~mA} \\
\text { (RMS) }\end{array}$ \\
\hline 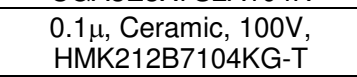 & 4 & 0.30 & 1.20 & CINT7, CINT8, CINT9, CINT10 & 0805 & $\begin{array}{c}77.05 \mathrm{~V} \\
47.184 \mathrm{~mA} \text { (RMS) }\end{array}$ & $\begin{array}{c}21.22 \mathrm{~V}, 17.59 \mathrm{~mA} \\
\text { (RMS) }\end{array}$ \\
\hline $\begin{array}{l}0.1 \mu \text {, Ceramic, 50V, } \\
\text { C1608X7R1H104M }\end{array}$ & 4 & 0.16 & 0.64 & $\begin{array}{c}\text { COUT_CPH1, COUT_CPH2, COUT_CPH3, } \\
\text { COUT_CPH4 }\end{array}$ & 0603 & $\begin{array}{l}\text { 36V, } 63.00 \mathrm{~mA} \\
\text { (RMS) }\end{array}$ & $\begin{array}{l}\text { 36V, } 46.00 \mathrm{~mA} \\
\text { (RMS) }\end{array}$ \\
\hline $\begin{array}{c}\text { 0.033 } \mu, \text { Ceramic, 50V, } \\
\text { RPER71H333K2K1A03A }\end{array}$ & 1 & 0.10 & 0.10 & CSNS2 & $\begin{array}{l}\text { Through- } \\
\text { Hole }\end{array}$ & $\begin{array}{l}1.5 \mathrm{~V}, 0.028 \mathrm{~mA} \\
\text { (RMS) }\end{array}$ & $\begin{array}{l}1.307 \mathrm{~V}, 0.020 \mathrm{~mA} \\
\text { (RMS) }\end{array}$ \\
\hline $\begin{array}{c}\text { 0.02 } \mu \text {, Ceramic, 50V, } \\
\text { RDEF51H103Z0K1C03B }\end{array}$ & 1 & 0.22 & 0.22 & CGATE_MAIN & $\begin{array}{l}\text { Through- } \\
\text { Hole }\end{array}$ & $5 \mathrm{~V}, 50 \mathrm{~mA}(\mathrm{RMS})$ & $5 \mathrm{~V}, 50 \mathrm{~mA}(\mathrm{RMS})$ \\
\hline $\begin{array}{l}\text { 10000p, Ceramic, 16V, } \\
\text { C1005X7R1C103K }\end{array}$ & 1 & 0.10 & 0.10 & CFB2 & 0402 & $\begin{array}{l}1.5 \mathrm{~V}, 0.028 \mathrm{~mA} \\
\text { (RMS) }\end{array}$ & $\begin{array}{l}\text { 1.307V, } 0.020 \mathrm{~mA} \\
\text { (RMS) }\end{array}$ \\
\hline $\begin{array}{l}\text { 10000p, Ceramic, 250V, } \\
\text { C2012X7R2E103K }\end{array}$ & 1 & 0.33 & 0.33 & CSNUB2 & 0805 & $\begin{array}{l}\text { 103.44V, } 1.619 \mathrm{~A} \\
\text { (RMS) }\end{array}$ & $\begin{array}{c}\text { 56.06V, } \\
756.43 \mathrm{~mA} \text { (RMS) }\end{array}$ \\
\hline $\begin{array}{l}\text { 6800p, Ceramic, } 100 \mathrm{~V} \\
\text { C1608X8R2A682K }\end{array}$ & 1 & 0.36 & 0.36 & CGATE_OVP & 0603 & $\begin{array}{l}77.47 \mathrm{~V}, 4.796 \mathrm{~mA} \\
\text { (RMS) }\end{array}$ & $\begin{array}{c}11.331 \mathrm{~V} \\
0.006 \mathrm{~mA} \text { (RMS) }\end{array}$ \\
\hline $\begin{array}{l}\text { 6800p, Ceramic, 25V, } \\
\text { C1005X7R1E682K }\end{array}$ & 1 & 0.09 & 0.09 & CTH2 & 0402 & $\begin{array}{l}1.232 \mathrm{~V}, 0.110 \mathrm{~mA} \\
\text { (RMS) }\end{array}$ & $\begin{array}{l}1.232 \mathrm{~V}, 0.030 \mathrm{~mA} \\
\text { (RMS) }\end{array}$ \\
\hline $\begin{array}{c}\text { 56p, Ceramic, 10V, } \\
\text { C0402C560J8GACTU }\end{array}$ & 1 & 0.35 & 0.35 & CTH1 & 0402 & $\begin{array}{c}1.232 \mathrm{~V}, 0.390 \mathrm{~mA} \\
\text { (RMS) }\end{array}$ & $\begin{array}{l}1.232 \mathrm{~V}, 0.009 \mathrm{~mA} \\
\text { (RMS) }\end{array}$ \\
\hline
\end{tabular}


Table E-35 (Continued)

\begin{tabular}{|c|c|c|c|c|c|c|c|}
\hline Capacitors: & Quantity: & $\begin{array}{c}\text { Unit } \\
\text { Price } \\
\text { (\$): }\end{array}$ & $\begin{array}{l}\text { Total } \\
\text { Price: }\end{array}$ & Components: & Type: & $\begin{array}{c}\text { 65V, 8A } \\
\text { Required Rating }\end{array}$ & $5 \mathrm{~V}, 0.5 \mathrm{~A}$ \\
\hline $\begin{array}{l}\text { 47p, Ceramic, 10V, } \\
\text { 0603ZA470DAT2A }\end{array}$ & 5 & 0.30 & 1.50 & $\begin{array}{c}\text { CFB1, CSNS1, CTMR, CRUN_OVP, } \\
\text { CRUN_MAIN }\end{array}$ & 0603 & $\begin{array}{l}1.5 \mathrm{~V}, 0.028 \mathrm{~mA} \\
\text { (RMS) }\end{array}$ & $\begin{array}{c}1.307 \mathrm{~V}, 0.020 \mathrm{~mA} \\
\text { (RMS) }\end{array}$ \\
\hline \multicolumn{3}{|c|}{ Total Cost (\$) } & 57.94 & & & & \\
\hline Component Count & 88 & & & & & & \\
\hline Total Converter Cost (\$) & 252.44 & & & & & & \\
\hline
\end{tabular}


Because single $940 \mu \mathrm{F}$ electrolytic capacitors were unavailable from Digi-Key, this project changes $\mathrm{C}_{\text {OUT }}$ to a $1000 \mu \mathrm{F}$ capacitor. Using a single $1000 \mu \mathrm{F}$ capacitor for $\mathrm{C}_{\text {OUT }}$ decreases the total converter cost. This final SEPIC costs $\$ 252.44$, including components, heatsinks and a PCB. Overall, the additional parts do not add much to the converter's overall cost and are necessary for proper operation and meeting all guidelines in sections 1.1 to 1.3. Although this SEPIC costs more than the Vicor Maxi 28V DC-DC converter used by the EHFEM group in [1], this SEPIC functions over a wider input voltage range, which satisfies this project's purpose. For the final portions of this report, this project tests this SEPIC design with the full Precor elliptical trainer generation system for functionality, investigates efficiency losses and describes further improvements for this SEPIC design.

\subsection{Precor Elliptical Trainer Full System Hardware Testing}

The final hardware testing round tests this project's final SEPIC design with the Precor elliptical trainer as the SEPIC's input voltage source. This project first tests the SEPIC with a constant $10 \Omega$ output load before using the Enphase micro-inverter as a load. However, this project later could not conduct any tests using the Enphase microinverter as a load because initializing the micro-inverter caused an open load condition at the elliptical trainer's output, causing it to output voltage levels greater than $100 \mathrm{~V}$ and subsequently destroy the SEPIC's input pass transistor (Q_OVP) and main switching transistor (Q_MAIN). Figures 10.41 and 10.42 show this project's SEPIC's test setup with the Precor elliptical trainer. 


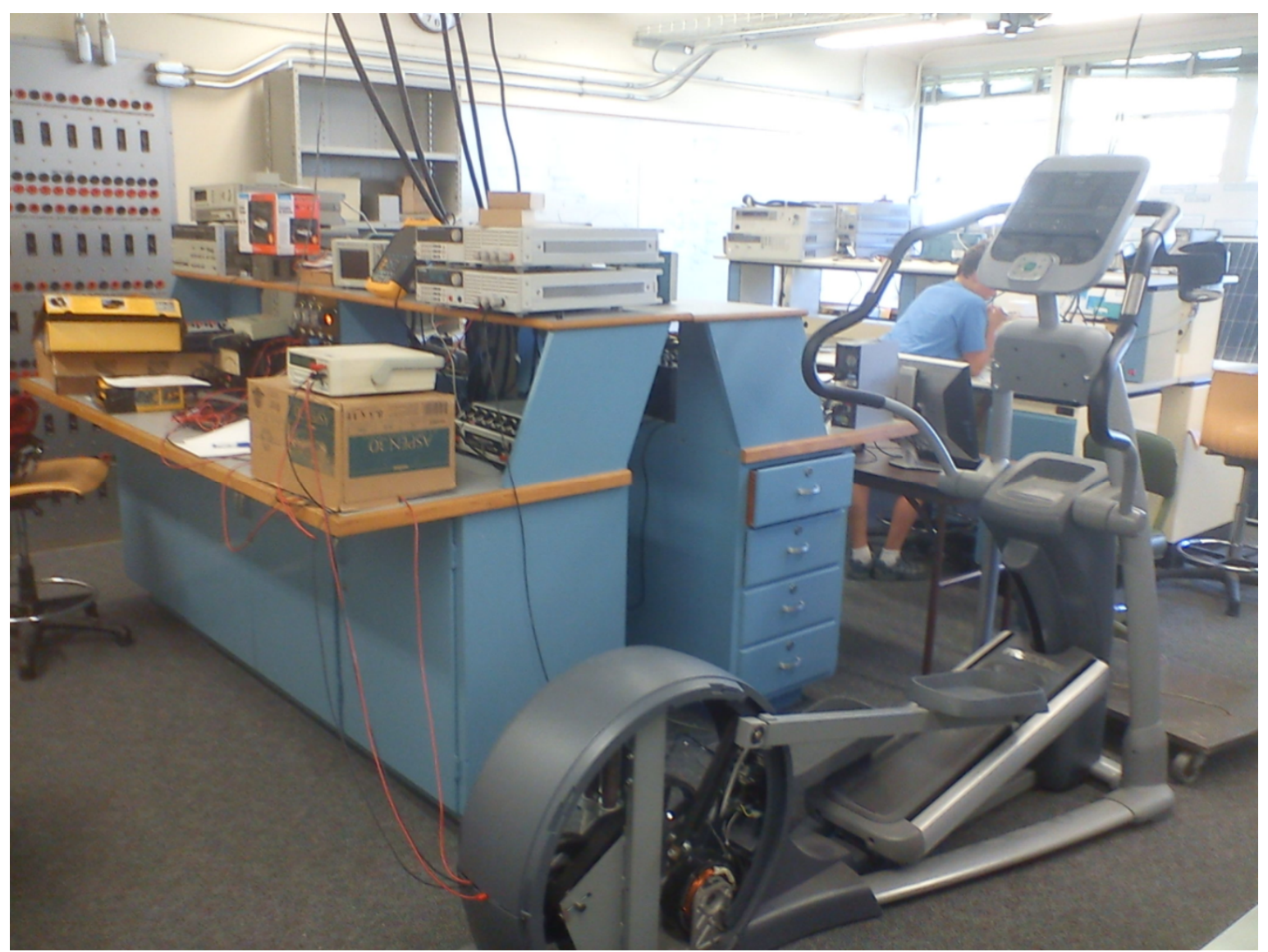

Figure 10.41: SEPIC Testing with Precor Elliptical Trainer, Angled View 


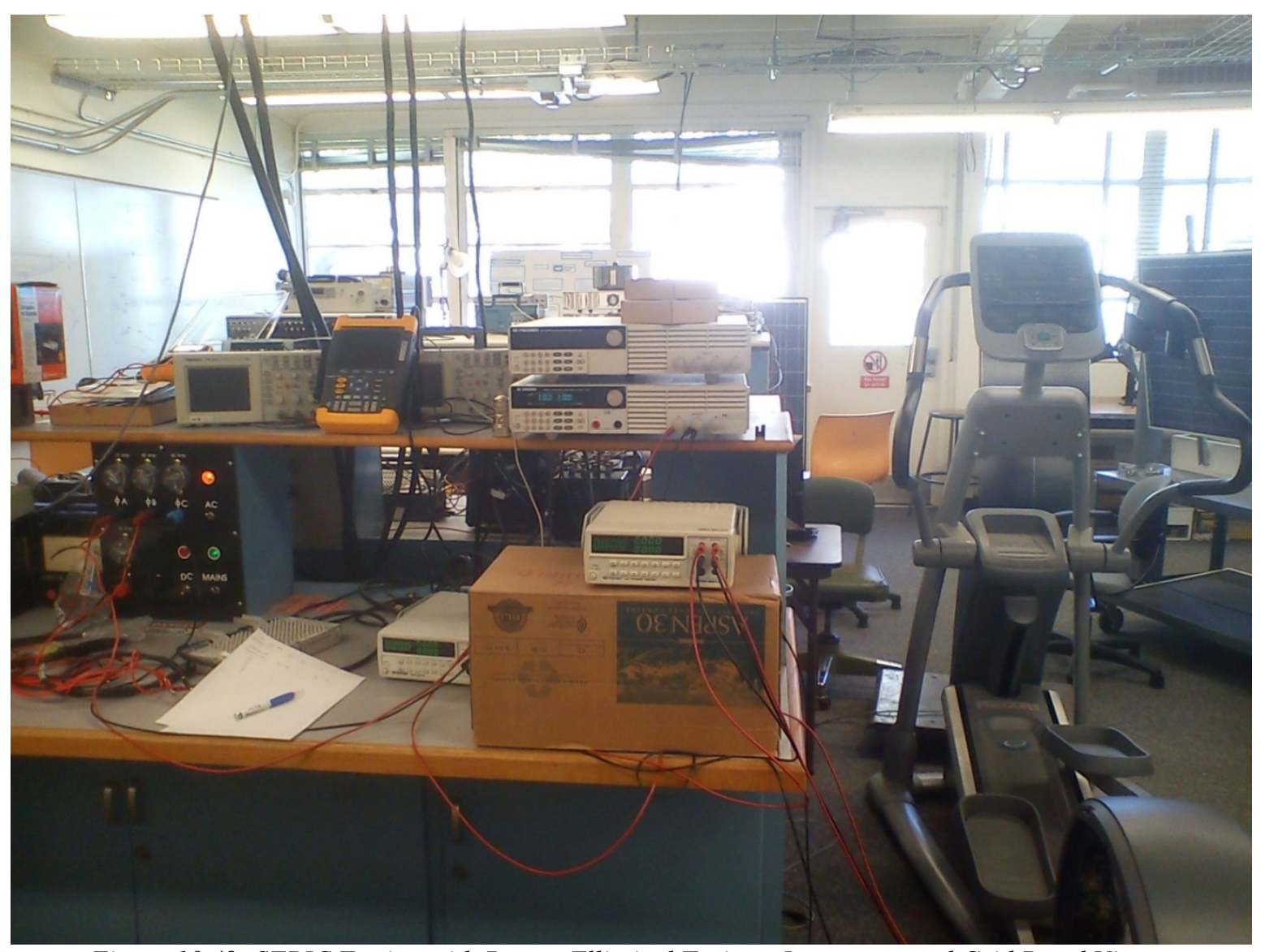

Figure 10.42: SEPIC Testing with Precor Elliptical Trainer, Instrument and Grid Panel View

The first full system test uses a BK Precision 8510 600W electronic load for simulating the constant $10 \Omega$ resistor load connected to the SEPIC's output, because for this test this project connects the SEPIC's main input capacitor $\mathrm{C}_{\mathrm{IN}}$ in series with the elliptical trainer's onboard $10 \Omega$ resistor coils for limiting $\mathrm{C}_{\mathrm{IN}}$ 's RMS current to safe levels $(1.52 \mathrm{~A}$ maximum). Figure 10.40 shows this series resistor connection with $\mathrm{C}_{\mathrm{IN}}$. A BK Precision 9153 DC power supply simulates the elliptical trainer's onboard $12 \mathrm{~V}$ battery because this project's author could not obtain any test leads long enough for connecting the elliptical trainer's battery to the SEPIC. Replacing the elliptical trainer's battery with a $12 \mathrm{~V}$ DC power supply does not affect the SEPIC's performance. This project also sets up the 
testing bench for connecting the Enphase micro-inverter to the 240V (RMS) grid. Also, for safety purposes, this project's author covered the SEPIC with a cardboard box visible in figures 10.41 and 10.42. Table E-36 shows this test's results. 


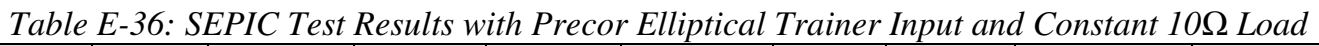

\begin{tabular}{|c|c|c|c|c|c|c|c|c|c|c|c|c|}
\hline Training Resistance Level & $V_{\text {IN }}(V)$ & $I_{I N}(A)$ & $\mathrm{V}_{\text {OUT }}(\mathrm{V})$ & $\mathrm{I}_{\text {OUT }}(\mathrm{A})$ & $P_{\text {IN }}(W)$ & $P_{\text {OUT }}(W)$ & $\eta(\%)$ & $R_{I N}(\Omega)$ & Error $\left(V_{\mathrm{IN}}\right)$ & Error $\left(I_{\text {IN }}\right)$ & Error ( $\left.\mathrm{V}_{\text {OUT }}\right)$ & Error ( $\mathrm{I}_{\text {OUT }}$ ) \\
\hline 2 & 2.5 & 2.319 & 18.04 & 0.166 & 5.80 & 2.99 & 51.65 & 1.08 & 1.5 & 1.5 & 2.5 & 0.8 \\
\hline 3 & 4 & 2.995 & 23.4 & 0.307 & 11.98 & 7.18 & 59.96 & 1.34 & 1.2 & 1.2 & 3.4 & 0.045 \\
\hline 4 & 5.6 & 5 & 28.3 & 0.694 & 28 & 19.64 & 70.14 & 1.12 & 0.5 & 1 & 1 & 0.05 \\
\hline 5 & 11 & 4.2 & 35 & 0.967 & 46.2 & 33.85 & 73.26 & 2.62 & 1 & 0.2 & 0.8 & 0.05 \\
\hline 6 & 13.7 & 4.5 & 37.33 & 1.285 & 61.65 & 47.97 & 77.81 & 3.04 & 1 & 0.2 & 0.5 & 0.05 \\
\hline 7 & 16.7 & 4.734 & 37.33 & 1.72 & 79.06 & 64.21 & 81.22 & 3.53 & 2 & 1 & 0 & 0.5 \\
\hline 8 & 18.8 & 5.2 & 37.44 & 2.23 & 97.76 & 83.49 & 85.40 & 3.62 & 2 & 1.5 & 0 & 0.5 \\
\hline 9 & 23.3 & 5.493 & 37.44 & 2.5 & 127.99 & 93.6 & 73.13 & 4.24 & 2 & 1.5 & 0 & 0.1 \\
\hline
\end{tabular}


Table E-36 shows the Precor elliptical trainer's selected training resistance level as well as the SEPIC's input voltage from the elliptical trainer's output $\left(\mathrm{V}_{\mathrm{IN}}\right)$, input current $\left(\mathrm{I}_{\mathrm{IN}}\right)$, SEPIC output voltage $\left(\mathrm{V}_{\text {OUT }}\right)$, output current $\left(\mathrm{I}_{\mathrm{OUT}}\right)$, elliptical trainer output power $\left(\mathrm{P}_{\mathrm{IN}}\right)$, SEPIC output power $\left(\mathrm{P}_{\text {OUT }}\right)$, efficiency $(\eta)$, SEPIC input resistance $\left(\mathrm{R}_{\mathrm{IN}}\right)$, as well as error values for $\mathrm{V}_{\mathrm{IN}}, \mathrm{I}_{\mathrm{IN}}, \mathrm{V}_{\mathrm{OUT}}$ and $\mathrm{I}_{\mathrm{OUT}}$. The SEPIC's input voltage, input current, output voltage and output current fluctuated as the participant exercised on the Precor elliptical trainer, indicating that the elliptical trainer's unstable, rectified DC output voltage caused those fluctuating values in the SEPIC. However, at training resistance levels 8 and 9 the SEPIC's input voltage and input current were more stable than at lower elliptical machine training resistance levels, resulting in more stable output current levels. The last four columns in table E-36 indicate error values, which indicate the observed deviations from the most frequently occurring values (which this project uses for the $\mathrm{V}_{\mathrm{IN}}, \mathrm{I}_{\mathrm{IN}}, \mathrm{V}_{\mathrm{OUT}}$ and $\mathrm{I}_{\text {OUT }}$ values in table E-36) for $\mathrm{V}_{\mathrm{IN}}, \mathrm{I}_{\mathrm{IN}}, \mathrm{V}_{\text {OUT }}$ and $\mathrm{I}_{\mathrm{OUT}}$. These error values tend to begin decreasing at higher training resistance levels. For safety reasons this project only tests the Precor elliptical trainer up to resistance level 9. Also, the SEPIC begins regulating 36V output around training resistance level 5 from the elliptical trainer. Resistance levels 2 and 3 do not produce the required minimum input threshold voltage (5V) for the SEPIC regulating 36V output. Figures 10.43 and 10.44 show the SEPIC's output load current driving ability as well as efficiency from the results in table E-36. 


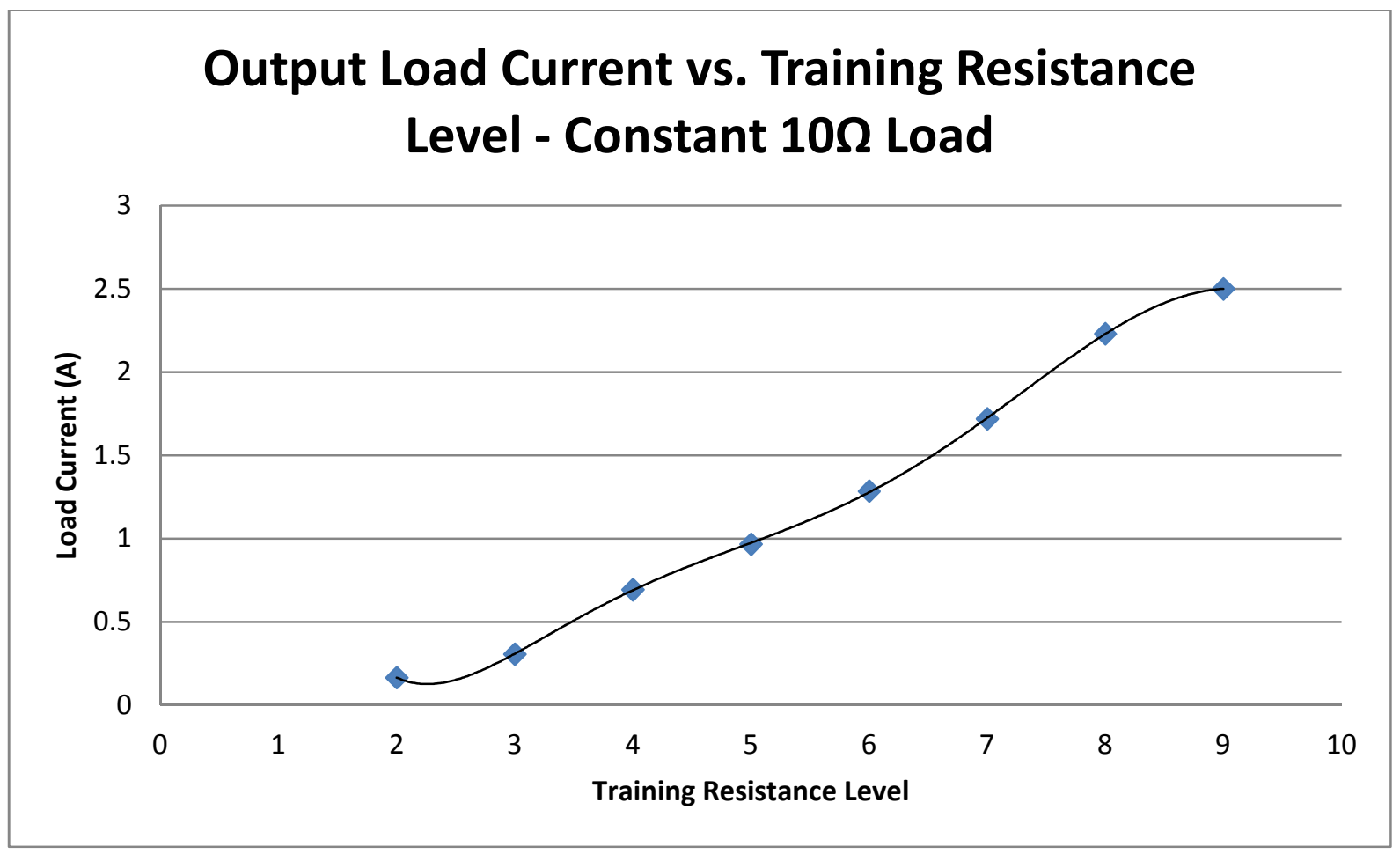

Figure 10.43: SEPIC Load Current Driving Ability vs. Elliptical Training Resistance Level with Precor Elliptical Trainer Input

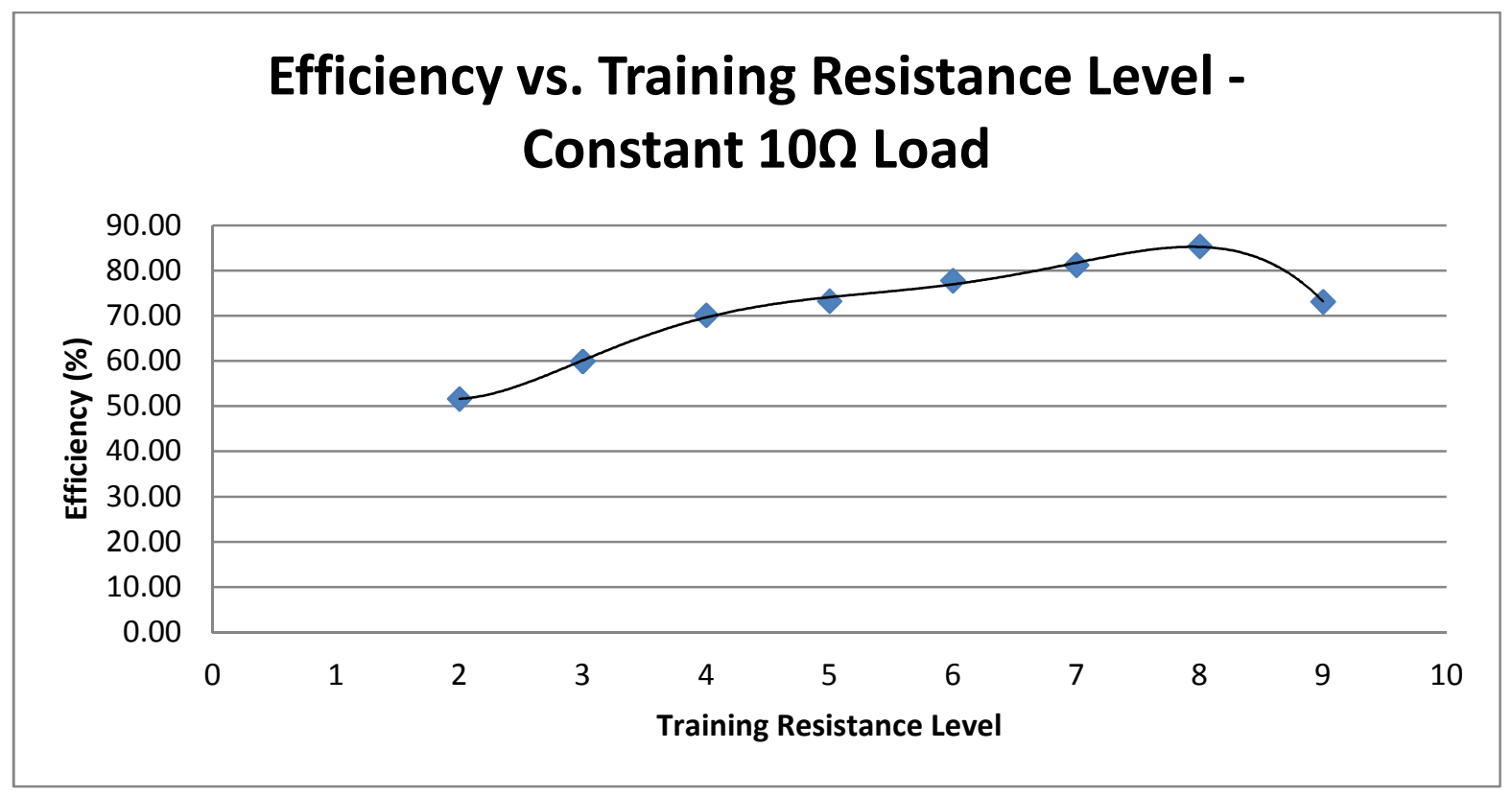

Figure 10.44: SEPIC Efficiency vs. Elliptical Training Resistance Level with Precor Elliptical Trainer Input 
From figure 10.43 , this project's SEPIC outputs $2.5 \mathrm{~A}$ to the $10 \Omega$ electronic load at elliptical training resistance level 9. From figure 10.44, the SEPIC reaches $85 \%$ peak efficiency at elliptical training resistance level 8 and 73\% efficiency at training level 9. The $85 \%$ efficiency figure may be inaccurate because of fluctuating input voltages and currents from the elliptical trainer. Although loading the SEPIC with a constant resistive load and using the elliptical trainer as its input voltage source doesn't maintain the $10 \Omega$ input resistance required for keeping the elliptical trainer's eddy current braking system intact, the participant during this test did not report any deviation in proper elliptical trainer user experience as he changed the elliptical trainer's training resistance level - in other words, higher resistance levels were more difficult for him to exercise at and lower resistance levels were easier. However, this test shows that this project's SEPIC functions with the Precor elliptical trainer, provided that its output load resistance remains constant. Unfortunately, this project could not test the entire EHFEM system with the Enphase micro-inverter. One characteristic that this project's author did not know about the Enphase micro-inverter is that its energy monitoring unit (EMU) has a roughly 20 minute warm-up period for detecting if the micro-inverter is connected to the grid. Afterwards, the Enphase micro-inverter requires an additional 5 minute initialization period before it functions with a DC input source. During this entire time period, the SEPIC had an open load (i.e. no load), causing the Precor elliptical trainer to sense its output as having an open load. One other Precor elliptical trainer aspect that this project did not characterize was how it behaved with no load (i.e. by removing its $10 \Omega$ resistor coils). As the participant exercised on the elliptical trainer during the Enphase micro- 
inverter's warm-up period, the elliptical trainer outputted voltages exceeding $100 \mathrm{~V}$. The participant then slowed down the workout pace such that the elliptical trainer outputted $60 \mathrm{~V}$, but that voltage soon increased back to $100 \mathrm{~V}$. The SEPIC's LT4356-1 input overvoltage and overcurrent protection circuit has a $100 \mathrm{~V}$ input limit, as well as the input zener diode. The output voltage exceeding $100 \mathrm{~V}$ from the elliptical trainer then destroyed the SEPIC's input zener diode, as well as its Q_OVP and Q_MAIN transistors. Those voltages also physically destroyed the LT4356-1 IC. This project's author theorizes that the LT4356-1 input circuit did not respond fast enough to the changes between the $60 \mathrm{~V}$ and $100 \mathrm{~V}$ output voltages from the elliptical trainer and allowed excess current flowing through the SEPIC. That excess current first destroyed Q_MAIN, causing a short across it, and that in turn caused excessive drain-source voltage across and drain current through Q_OVP, resulting in excessive power dissipation and thermal runaway across Q_OVP, destroying it in a chain reaction. Lastly, the zener diode could not tolerate the excessive inrush current and hence was destroyed as well. Therefore, this project could not test the SEPIC's functionality with the Enphase micro-inverter because excessive output levels from the Precor elliptical trainer destroyed crucial SEPIC components. However, this test proved that the Precor elliptical trainer cannot function properly with an open load and that it should only output power once the micro-inverter finishes initializing.

However, as speculated from [10], this project's SEPIC would behave in the same manner as the designed DC-DC converter from [10] when loaded with the Enphase micro-inverter. The author in [10] speculates that the Enphase micro-inverter implements a hill-climbing maximum power point tracking (MPPT) algorithm for maximum power 
transfer. As observed in [10], the Enphase micro-inverter begins power tracking at its input voltage level (output voltage from the DC-DC converter) and then slowly increased its input current (output current from the connected DC-DC converter) provided that it could still receive power from the connected DC-DC converter. When the Enphase micro-inverter increased its input current, its input resistance decreased, decreasing the DC-DC converter's load resistance. Furthermore, when the Enphase micro-inverter increased its input current beyond a level that the DC-DC converter could tolerate while regulating $36 \mathrm{~V}$ at its output, the DC-DC converter began de-regulating and hence the DC-DC converter's output voltage dropped below 36V. This behavior is certainly not desirable for this project's EHFEM system application and furthermore, it does not maintain $10 \Omega$ input resistance across the DC-DC converter's input. This behavior functions properly with a photovoltaic panel, as Enphase designed its M175-24-240 micro-inverter for connecting to a photovoltaic panel. However, this project designed its SEPIC for increasing its output load current with input voltage, which is the opposite behavior of a photovoltaic panel. Overall, from the results in [10], this project's SEPIC would produce the same behavior as the DC-DC converter in [10] when loaded with the Enphase micro-inverter - the Enphase micro-inverter would draw as much current as possible from the SEPIC until either its input or output fuse blew. Therefore, this project's SEPIC would not function as this project desires when loaded with the Enphase micro-inverter. Getting this project's SEPIC to function properly with the Enphase microinverter, however, requires an additional control mechanism for the Enphase microinverter such that it limits the micro-inverter's input current based on the SEPIC's input 
voltage (for maintaining $10 \Omega$ resistance at the SEPIC's input). Such control mechanisms require additional research and are best suited for a separate project, but for the most part, this project's SEPIC met its design requirements, specifications and constraints outlined in sections 1.1 to 1.3 when tested with a DC power supply as its input. Furthermore, this project's SEPIC functions with the Precor elliptical trainer while loaded with a constant resistance. 


\section{CHAPTER 11: CONCLUSION, FUTURE IMPROVEMENTS AND FUTURE EHFEM PROJECTS}

\subsection{Conclusion}

Overall, this project's SEPIC satisfied the design requirements, specifications and constraints outlined in sections 1.1 to 1.3 . At $60 \mathrm{~V}$ input this project's SEPIC outputs $275 \mathrm{~W}$ while taking a $352 \mathrm{~W}$ input. This corresponds to $78.3 \%$ converter efficiency at $60 \mathrm{~V}$ input. The SEPIC's peak efficiency level is $78.7 \%$, and it reaches this level at $50 \mathrm{~V}$ input. Based on these results, this project's SEPIC can also tolerate $65 \mathrm{~V}$ input and can drive an 8A load. However this project did not test the SEPIC under those conditions because of test equipment electrical limitations. This project's SEPIC also runs in CCM at $31 \mathrm{~V}$ input and $2 \mathrm{~A}$ load as specified in section 1.2. Furthermore, its line regulation when its input changes from $8-60 \mathrm{~V}$ (and while driving a load under that input voltage range) is $1.89 \%$, which is well below the 5\% maximum level specified in section 1.2. Although this project's SEPIC did not meet its $\$ 120$ price limit for achieving a 10-year EHFEM system payback period, as mentioned in section 2.3.1, implementing this project's EHFEM system in a frequently-used gym such as Cal Poly's Recreation Center can also raise electricity conservation awareness and reduce overall energy consumption in the city of San Luis Obispo. Furthermore, it can also draw attention from potential donors and sponsors that may frequent Cal Poly's recreation Center, leading to reduced overall system lifecycle costs. Proper SEPIC functionality with this project's selected Enphase M175-24-240 micro-inverter, however, requires additional control mechanisms for the inverter. However, this project did prove that the SEPIC functions with the Precor EFX 
546i elliptical trainer as its input as well as with a constant output load. Therefore, this project's SEPIC would function properly with an inverter that maintains a constant load or a variable load such that it maintains $10 \Omega$ input resistance. The latter case, however, is more desirable for the EHFEM system and as mentioned already it requires an additional control mechanism for the Enphase micro-inverter. This chapter later describes such possible mechanisms. However, this project proved that a PWM-switching SEPIC topology provides a functional DC-DC converter for a dynamic DC voltage generation system such as the Precor elliptical trainer as well as inverter interfacing, given that the inverter takes in 36V and limits its input current based on the Precor elliptical trainer's output voltage. Therefore, based on its test results, this project proves that a PWMswitching SEPIC topology provides a functional DC-DC converter design for DC power generation and inverter interfacing from a dynamic input voltage generator because of its wide input voltage range, high power driving capability and inherent voltage step-up and step-down functions. The next sections describe possible improvements for this project's SEPIC design, as well as future projects for the EHFEM project as a whole.

\subsection{Future SEPIC Improvements}

Despite the fact that this project's SEPIC satisfied all design requirements, specifications and constraints, numerous modifications can improve its functionality and efficiency performance, as well as reduce its cost. Such improvements include adding output current limiting circuitry, eliminating unnecessary PCB footprints and reducing the size of PCB copper planes in the SEPIC's power path, as well as adding modifications for conserving elliptical trainer battery use, and using better input 
protection methods than the LT4356-1 protection circuit used in this project.

Furthermore, this project's SEPIC design also creates important EHFEM projects for future senior project or master thesis students. As mentioned earlier, one such important project is a control mechanism for the currently used Enphase M175-24-240 microinverter for helping maintain a constant $10 \Omega$ input resistance to this project's SEPIC. This project's SEPIC could also use a single custom coupled inductor for housing $\mathrm{L}_{1}$ and $\mathrm{L}_{2}$ rather than two discrete inductors, potentially decreasing converter cost as well as creating another project for future EHFEM project students. Lastly, another potential project includes designing a grid-tie inverter that properly controls its input current for proper functionality with this project's SEPIC.

\subsubsection{SEPIC Output Current Limiting}

The first improvement that this project's SEPIC necessitates is output current limiting. Output current limiting is a standard feature in most offline DC power supplies. This feature prevents power supply components from exceeding their electrical limits as well as provides electrical safety to its end-user. Although this project's SEPIC's output fuses already provide overcurrent protection, the EHFEM system's owner must replace them every time an overcurrent fault occurs at the SEPIC's output. This causes inconvenience as well as brings added operation costs (for replacing the fuses) for the EHFEM system's owner. Furthermore, if the fuses do not react quickly enough to an overcurrent fault, then crucial SEPIC components can be destroyed, adding further component replacement costs for the EHFEM system's owner. Therefore, output current limiting greatly benefits this project's SEPIC in eliminating those mentioned problems. A 
relatively simple current limiting circuit that future EHFEM project students can expand on is a crowbar circuit, or simply known as a crowbar. A crowbar detects an overcurrent or overvoltage fault at the SEPIC's output (typically through a current sense resistor) and shorts the SEPIC's output to a low voltage, typically via a silicon-controlled rectifier (SCR) or thyristor [72]. SCRs typically can tolerate very high current levels while maintaining low forward voltages, resulting in very low power dissipation during an overcurrent fault. Using an active crowbar (rather than a passive one) returns the SEPIC's output to normal operation once the fault disappears. Figure 11.1 shows an active overvoltage crowbar (which can be used at the SEPIC's input as an alternative to the LT4356-1) and figure 11.2 shows an overcurrent crowbar [72, 73].

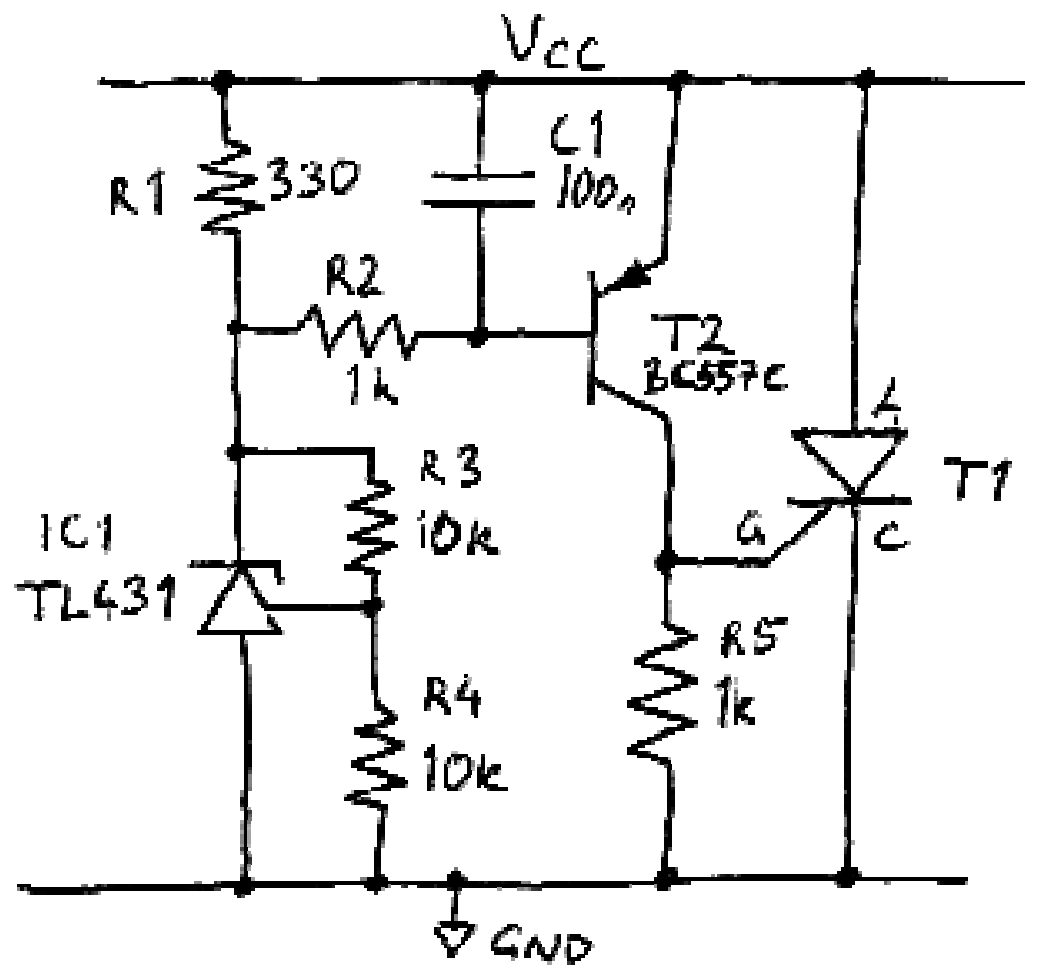

Figure 11.1: Active Overvoltage Crowbar [72] 


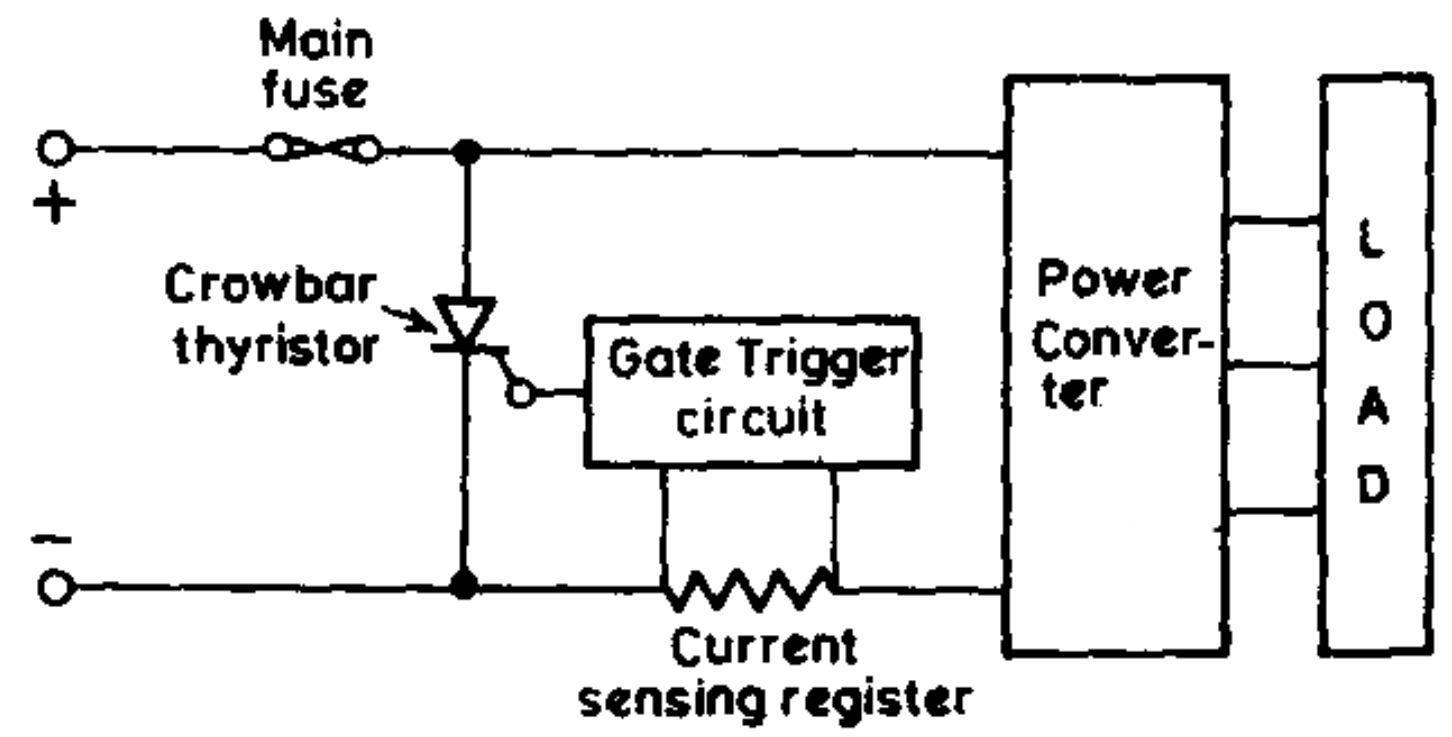

Figure 11.2: Overcurrent Crowbar [73]

Using a crowbar for current limiting requires an additional current sense resistor.

However, the main flaw with using a crowbar as an output current limiter for this project's SEPIC is that a crowbar drops the SEPIC's output voltage to a level below the Enphase micro-inverter's input voltage range during an overcurrent fault. Ideally this project desires keeping the SEPIC's output voltage at 36V for optimal EHFEM system operation, even during an overcurrent fault. However, future projects can make modifications to the default overcurrent crowbar for limiting SEPIC output current while maintaining 36V output voltage. In Spring Quarter 2011, EHFEM team member Greg Hollister attempted implementing a DC-DC converter output current limiter that maintained 36V output voltage for his DC-DC converter [53]. Greg Hollister's implementation uses a multiplier circuit that limits the DC-DC converter's output current proportionally to the squared value of the converter's input voltage. This implementation 
would thus let a DC-DC converter maintain a constant $10 \Omega$ input resistance. Figure 11.3 shows his current limiter circuit [53].

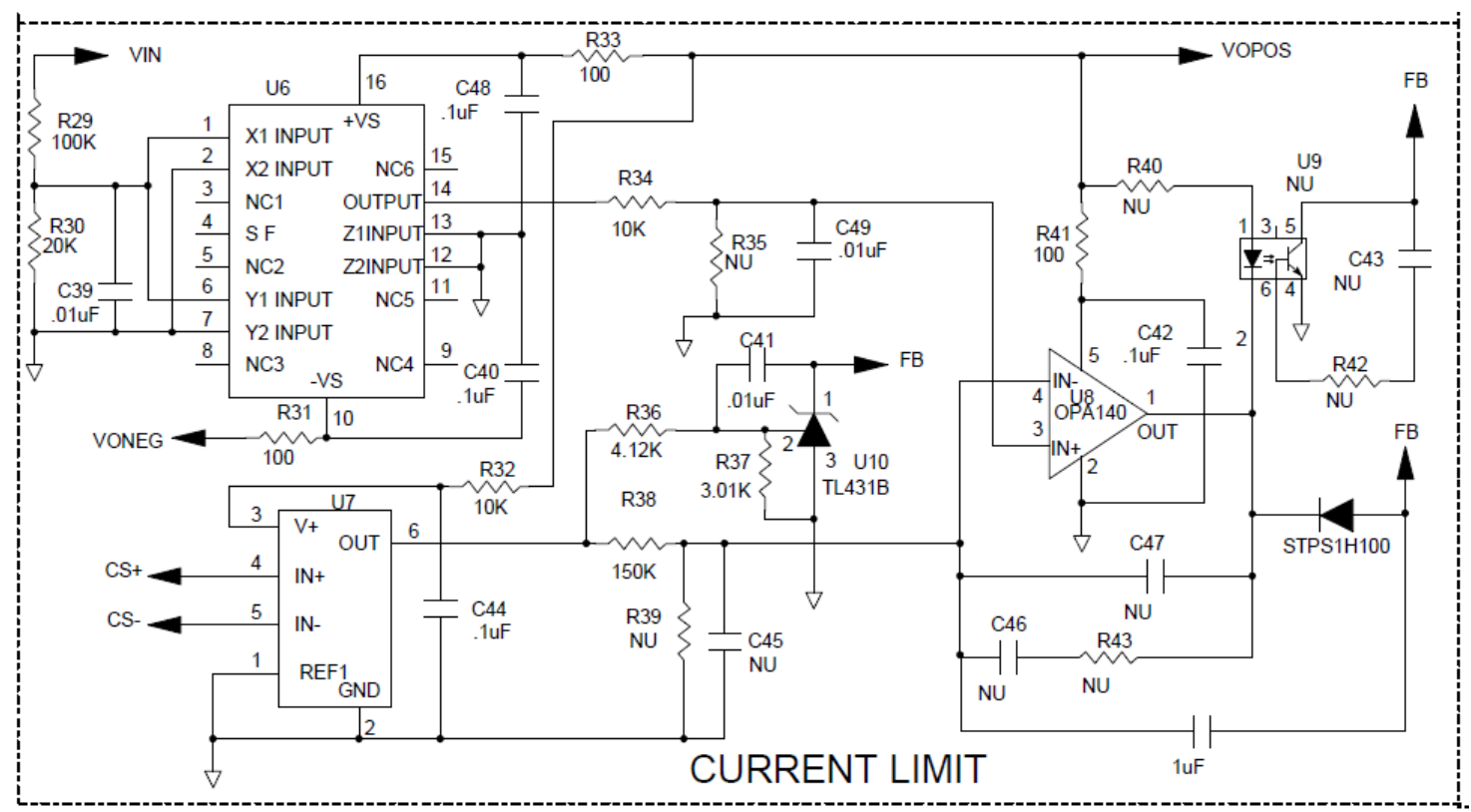

Figure 11.3: DC-DC Converter Output Current Limiter Using Multiplier [53]

This current limiting implementation connects to and controls a DC-DC converter's input, feedback, current sense and output nodes. It has a very small PCB footprint and dissipates very little power. However, it ultimately did not function as desired because the Enphase micro-inverter kept increasing its input current level, ultimately deregulating the DC-DC converter's output voltage. Pass transistor current limiting topologies may also function but typically require more PCB space and dissipate very large amounts of power, requiring very effective and potentially expensive cooling methods. Therefore, while such current limiting circuits (as well as crowbars) may be feasible for this project's SEPIC, they require additional modifications for functional designs that overcome the Enphase micro-inverter's hill-climbing MPPT algorithm. The 
next section describes another further improvement for this project's SEPIC - reducing its PCB size.

\subsubsection{SEPIC PCB Size Reduction}

Reducing the SEPIC's PCB size can reduce its overall cost, as typically PCB manufacturers determine PCB cost by its surface area. Final production-level PCBs of this project's SEPIC can eliminate unnecessary footprints such as ones for Q_MAIN_ALT, or the additional footprint for adding another large electrolytic capacitor at the SEPIC's output. Such footprints were only necessary for prototyping and hardware testing and a final production-level PCB would not require them. Furthermore, this project's SEPIC's power path PCB copper planes can also be reduced in size such that

they tolerate just the average required current levels and not the peak current levels. This project originally sized those planes for peak current tolerance levels from design calculations and computer simulations. Reducing the size of those copper planes reduces PCB size as well as plane capacitance. The next section describes a method for helping conserve elliptical trainer battery use when the elliptical trainer idles.

\subsubsection{Elliptical Trainer Battery Conservation}

One major flaw with this project's SEPIC is that the Precor elliptical trainer's onboard $12 \mathrm{~V}$ battery still supplies power to both the SEPIC's LTC1871 PWM controller and LT4356-1 surge stopper IC even when the elliptical trainer idles with no user exercising on it. This drains the battery's charge and the battery supplies power to both ICs until it completely discharges. Discharging the elliptical trainer's battery results in extra energy required from the next elliptical trainer user (from exercise) for charging the 
battery to its nominal $12 \mathrm{~V}$ level. A method that helps eliminate this problem involves connecting the LTC1871 and LT4356-1's RUN and EN pins instead to the elliptical trainer's output (the SEPIC's input) rather than the battery terminals. Using this method, the battery only transfers power to the LTC1871 and LT4356-1 ICs if the elliptical trainer outputs its minimum threshold voltage - in the case of this project, that threshold is $5 \mathrm{~V}$. Because each mentioned pin also has maximum voltage limits, the existing resistor voltage dividers at the mentioned pins may also require modification. Figure 11.4 shows such an example, from the designed DC-DC converter in [10].

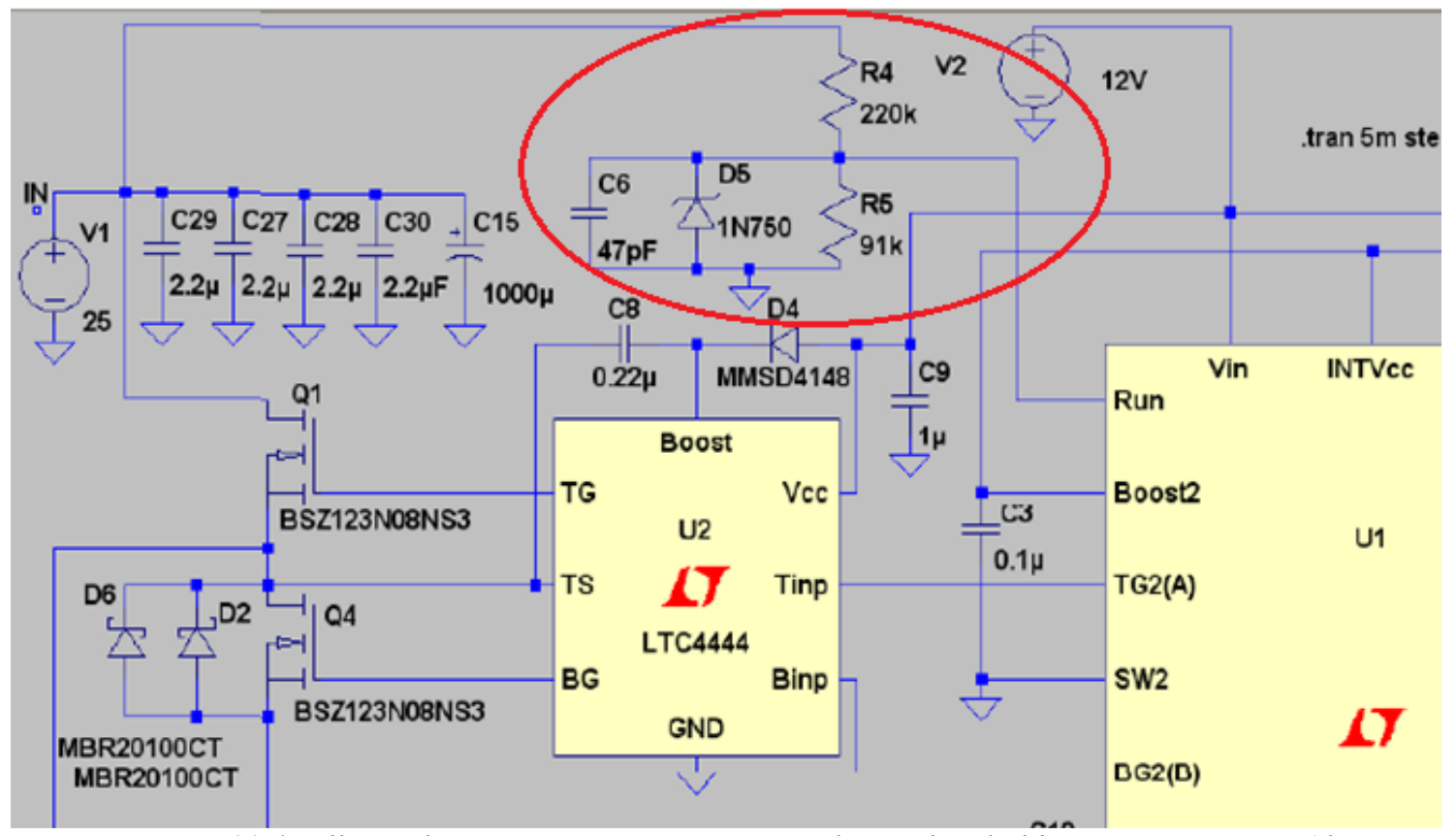

Figure 11.4: Elliptical Trainer Battery Minimum Voltage Threshold Detection Circuit [10]

Implementing this modification requires modifying the SEPIC's PCB layout, however. Designing a new PCB layout for this project's SEPIC while using the same SEPIC components as this project suffices as a potential senior project. Implementing this modification, however, results in large potential energy savings for this project's EHFEM 
system. The next section describes possible alternatives for the SEPIC's LT4356-1 input protection circuit.

\subsubsection{Improved Input Protection Schemes}

As mentioned in section 10.4, the Precor elliptical trainer outputs voltages exceeding $100 \mathrm{~V}$ when it detects an open load at its output. This problem occurs during the Enphase micro-inverter's initialization phase. The LT4356-1 input protection circuit in this project's SEPIC does not adequately safeguard against that previously unforeseen problem and adds complexity as well as additional required PCB space for the converter, mainly because of its pass transistor (Q_OVP). Furthermore, any catastrophic event that destroys Q_MAIN destroys Q_OVP as well, because of the series connection path to ground between both transistors. This design flaw results in additional component replacement costs for the EHFEM system's owner when such an event occurs. This project's SEPIC's current PCB has an alternate connection point for the elliptical trainer's output, located at VIN_ALT in figures 9.6 and 9.9. This connection point bypasses the LT4356-1 input protection circuit. However, while bypassing the LT4356-1 input protection circuit eliminates the transistor destruction problem, the SEPIC still requires inrush current limiting at start up. An additional soft-start circuit for the LTC1871 controller would eliminate that problem, but does not address the mentioned overvoltage problem. Therefore, this project's SEPIC necessitates more efficient input protection schemes that do not utilize pass transistors. Metal oxide varistors (MOVs) and transient voltage suppression (TVS) diodes at the SEPIC's input can help alleviate overvoltage and overcurrent problems, however, they must tolerate high voltage and 
current levels during a fault. Future EHFEM projects can research this topic in greater detail for improving this project's SEPIC design. Section 11.3 discusses future projects that can further improve this project's SEPIC design, previously designed DC-DC converters for the EHFEM project as well the whole EHFEM system.

\subsection{Future EHFEM Projects}

This section discusses future projects that can further improve this project's SEPIC design, previously designed DC-DC converters for the EHFEM project as well the whole EHFEM system. These projects include SEPIC inductor design, control schemes for the Enphase M175-24-240 micro-inverter and grid-tie inverter design.

\subsubsection{SEPIC Inductor Design}

Along with PCB size reduction, using a single magnetically coupled inductor for housing $\mathrm{L}_{1}$ and $\mathrm{L}_{2}$ in this project's SEPIC may potentially reduce the SEPIC's cost. Furthermore, using a coupled inductor can also reduce this project's SEPIC's physical PCB size. However, using a coupled inductor with this project's SEPIC requires a custom inductor design because as mentioned earlier in this report, this project's author could not find a suitable commercially available coupled inductor or switching transformer that satisfied $\mathrm{L}_{1}$ and $\mathrm{L}_{2}$ 's saturation current rating requirements. A custom coupled inductor requires using commercially available magnetic cores, which are typically expensive. However, using a custom, coupled inductor that produces the required inductance at 100 $\mathrm{kHz}$ switching frequency may result in greatly improved efficiency, offsetting the magnetic core costs while maintaining a 10 year system payback period. One possible source of efficiency loss in this project's SEPIC may have resulted from this project's 
author's failure in recognizing that the $60 \mu \mathrm{H}$ inductance and $85 \mathrm{~m} \Omega$ ESR values stated in Vishay's IHV28BZ60 datasheet was the nominal $\mathrm{L}_{1}$ and $\mathrm{L}_{2}$ inductance value at $1 \mathrm{kHz}$ frequency (and not this project's SEPIC's $100 \mathrm{kHz}$ switching frequency) [36]. After noticing that caveat, this project's author then measured $\mathrm{L}_{1}$ 's inductance and ESR at 1 $\mathrm{kHz}$ and $100 \mathrm{kHz}$ frequencies using a GW Instek LCR meter. This project's author did not measure $\mathrm{L}_{2}$ 's inductance because $\mathrm{L}_{2}$ is the exact same physical inductor as $\mathrm{L}_{1}$. Figures 11.5 and 11.6 show $\mathrm{L}_{1}$ 's inductance and ESR at $1 \mathrm{kHz}$ frequency, while figures 11.7 and 11.8 show $\mathrm{L}_{1}$ 's inductance and ESR at $100 \mathrm{kHz}$ frequency.

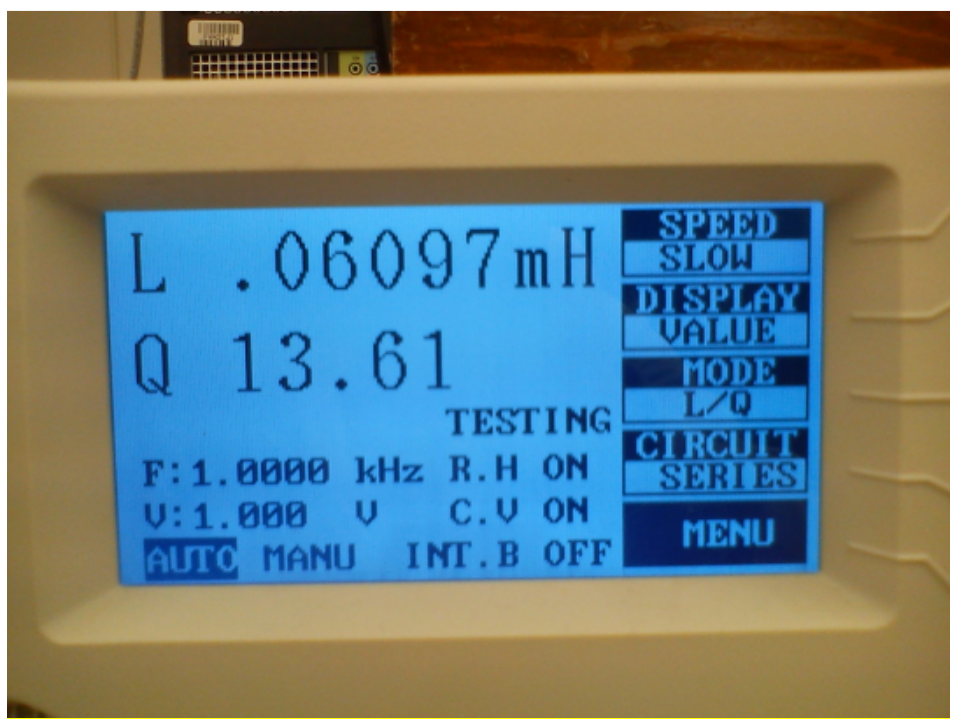

Figure 11.5: $L_{1}$ Measured Inductance at $1 \mathrm{kHz}$ 


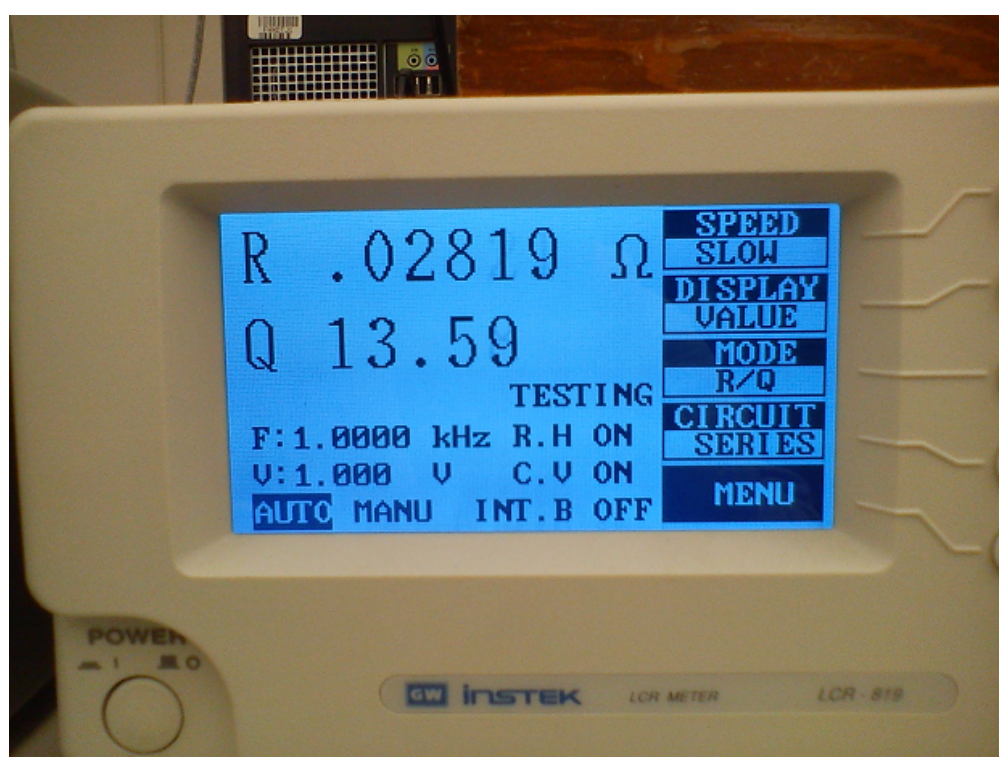

Figure 11.6: $L_{1}$ Measured ESR at $1 \mathrm{kHz}$

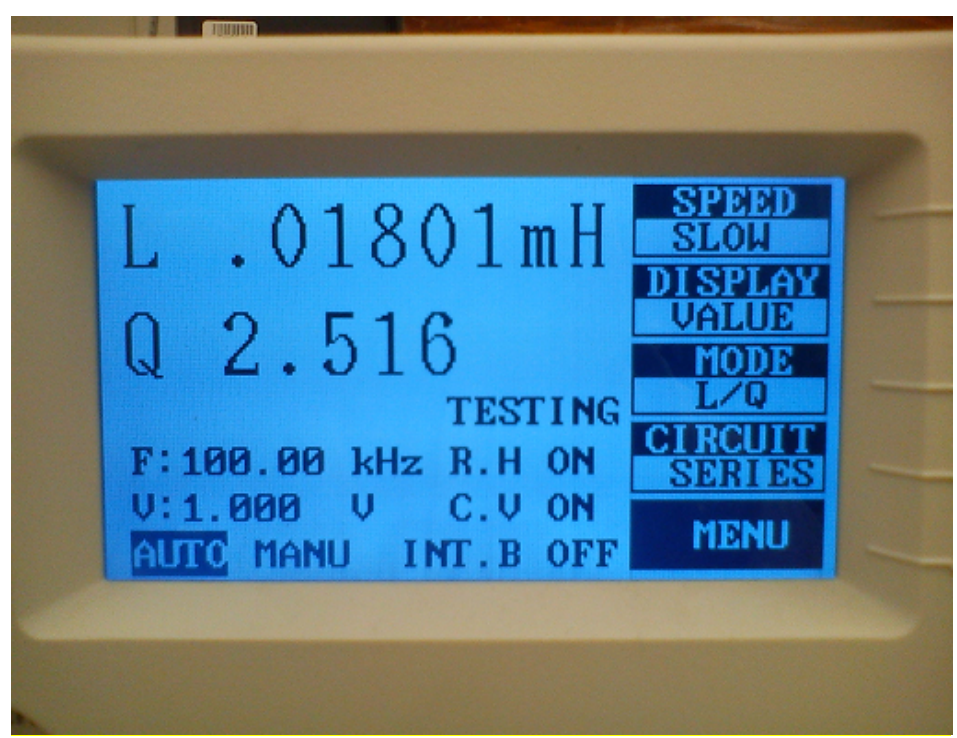

Figure 11.7: $L_{1}$ Measured Inductance at $100 \mathrm{kHz}$ 


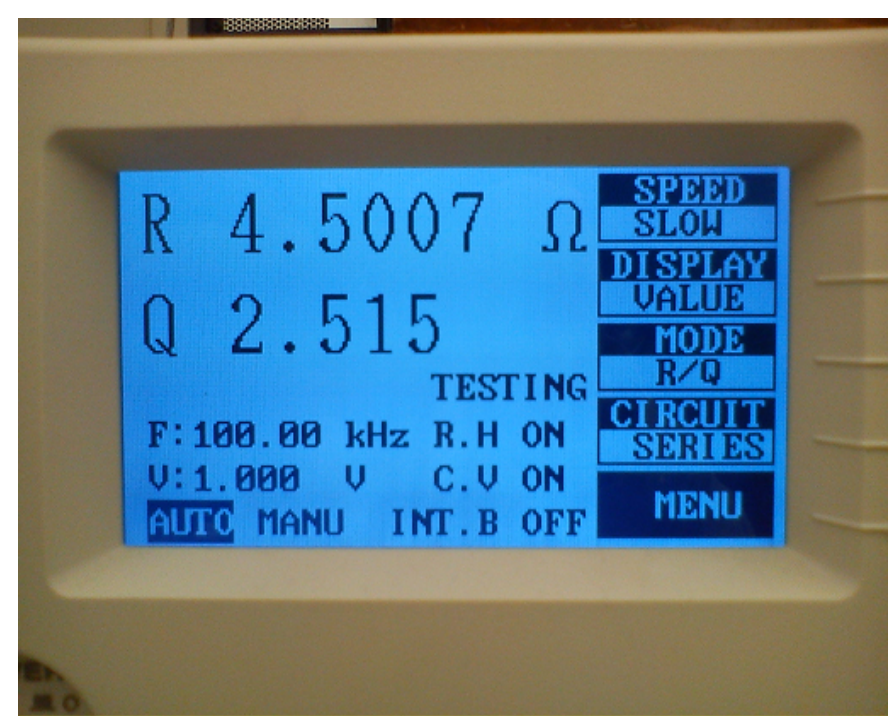

Figure 11.8: $L_{1}$ Measured ESR at $100 \mathrm{kHz}$

Figures 11.6 to 11.8 show that as the LCR meter's test frequency increases changes from $1 \mathrm{kHz}$ to $100 \mathrm{kHz}, \mathrm{L}_{1}$ 's inductance drops from $60 \mu \mathrm{H}$ to $18 \mu \mathrm{H}$, and its ESR increases to 4.5 . This inductance drop and ESR increase at $100 \mathrm{kHz}$ switching frequency in both $\mathrm{L}_{1}$ and $\mathrm{L}_{2}$ may have caused the majority of the efficiency losses in this project's SEPIC. The $18 \mu \mathrm{H}$ inductance value is lower than the SEPIC's required critical inductance for both $\mathrm{L}_{1}$ and $\mathrm{L}_{2}$, and may have caused either DCM operation at lower input voltages or inductor saturation, resulting in greater stresses across Q_MAIN and those stresses across Q_MAIN in turn may have contributed to the SEPIC's efficiency loss. Vishay Siliconix manufactures similar inductors to the IHV28BZ60 used in this project's SEPIC, with higher inductance values and saturation current ratings. However, those inductors are more expensive than the IHV28BZ60. Therefore, a future EHFEM project could first resimulate this project's SEPIC with the mentioned inductance and ESR values for $\mathrm{L}_{1}$ and $\mathrm{L}_{2}$ and note how much inductance drop and ESR increase at $100 \mathrm{kHz}$ affects overall SEPIC performance. Afterwards, that project could then design a custom coupled 
inductor that maintains $60 \mu \mathrm{H}$ and low ESR (close to the Vishay IHV28BZ60's specified $85 \mathrm{~m} \Omega \mathrm{ESR}$ ) at $100 \mathrm{kHz}$, for maintaining compatibility with this project's snubber. That project is suitable for students who have completed a magnetic component design course.

\subsubsection{Inverter Control Mechanism}

So far, each DC-DC converter from each EHFEM project (including this project's SEPIC) functioned properly as DC-DC converters. However, the Enphase microinverter's behavior is undesirable for EHFEM system operation. As mentioned earlier in this report, Enphase designed the M175-24-240 micro-inverter for operation with a photovoltaic panel input. This project's SEPIC behaves in the opposite manner from a photovoltaic panel; maintaining a $10 \Omega$ input resistance requires its output current increasing with input voltage rather than outputting as much current as possible at any given input voltage. Therefore, a proper control mechanism for the Enphase microinverter is necessary for proper EHFEM system functionality and deployment. A proper control mechanism for the Enphase micro-inverter allows any chosen DC-DC converter to maintain a constant $10 \Omega$ input resistance for keeping the Precor ellipticial trainer's eddy current braking system intact, while regulating the DC-DC converter with the proper $36 \mathrm{~V}$ output voltage. Maintaining a constant $10 \Omega$ resistance at the DC-DC converter's input requires a controller limiting the Enphase micro-inverter's input current based on input voltage and power levels at the DC-DC converter. Figure 11.9 shows a potentially feasible control scheme, taken from [10]. 


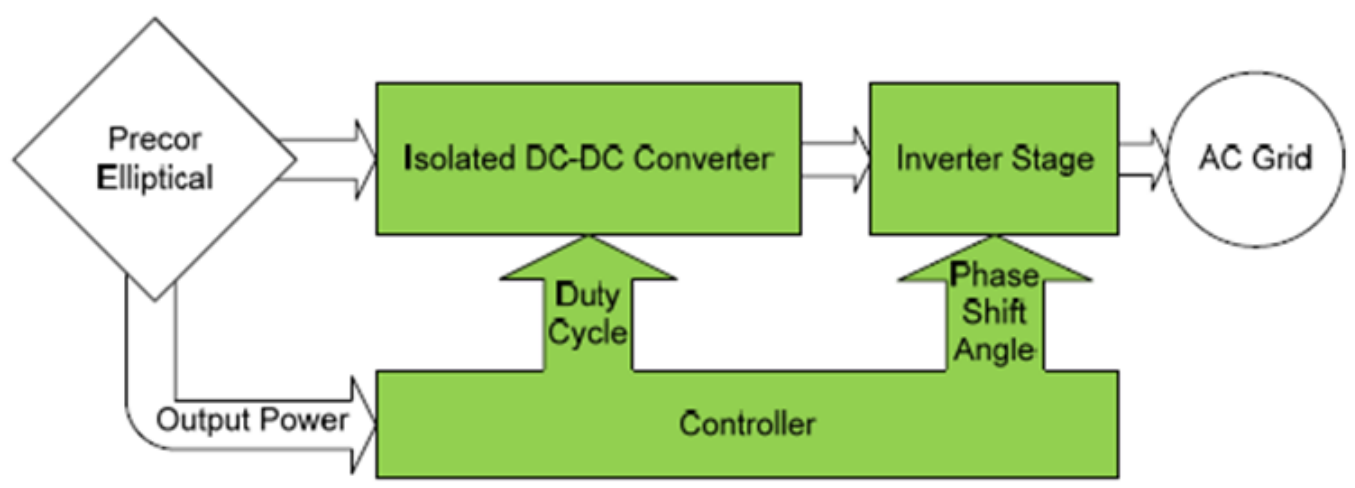

Figure 11.9: Possible Control Scheme for Enphase M175-24-240 Micro-Inverter [10]

This control scheme only requires an external controller for varying the DC-DC converter's duty cycle as well as the inverter's phase shift angle relative to that of the electrical grid, based on the elliptical trainer's output power. Modifying the DC-DC converter's duty cycle and inverter's phase shift angle based on the elliptical trainer's output power should help limit the DC-DC converter's output current and help maintain $10 \Omega$ resistance at the DC-DC converter's input. This control scheme may or may not be costly for implementation. In any case, possible control mechanisms for the Enphase micro-inverter require further research and are beyond this project's purpose and scope.

\subsubsection{Inverter Design}

An alternative project to implementing an input current controller for this EHFEM system's existing Enphase M175-24-240 micro-inverter involves designing a custom grid-tie inverter that optimally takes in 36V DC voltage (within a small error range) and at least $8 \mathrm{~A}$ current. However, the custom inverter would still require a mechanism for limiting its input current based on the DC-DC converter's input voltage. In other words, the custom inverter would need to behave in a similar fashion to a 
variable current electronic load. This project may also produce an inverter design that costs more than designing an external controller for both a selected DC-DC converter and the Enphase micro-inverter as well as the Enphase micro-inverter itself, but further research in this area may turn up feasible designs that function with DC-DC converters requiring constant input resistance. In any case, proper inverter control mechanisms are the highest priority for future projects, as inverter control mechanisms are crucial for both EHFEM system functionality and deployment. 


\section{APPENDIX A: ĆUK CONVERTER DESIGN}

Appendices A and B document initially selected DC-DC converter topologies and designs for this project that either did not function in computer simulations with the project requirements and specifications outlined in sections 1.1 to 1.3 or had exorbitantly high component costs that such designs would not produce a reasonable payback time period for the EHFEM system. In spring quarter 2010 this project began by selecting a

non-inverting Ćuk DC-DC converter topology for its DC-DC converter design because of its voltage boost and buck capabilities and inherently good input and output current characteristics (i.e. it produces non-pulsating input and output current) because of inductors connected directly to its input and output nodes. However, finding a PWM controller that suited a non-inverting Ćuk DC-DC converter proved difficult because such a design required two switches and using two separate PWM controllers for switch synchronization added such difficulties. This project then explored variations of the buckboost DC-DC converter for its design, culminating with an interleaved, multi-phase, noninverting buck-boost converter design. However, that interleaved, multi-phase, noninverting buck-boost converter design had a very high component cost (much greater than that of the SEPIC design used in this project) and its overall efficiency level could not justify its high cost. Simulating those mentioned designs that were unfeasible for this project led to selecting the SEPIC design used for this project. The following appendix subsections document the initial converter topology selection and design for this project, beginning with selecting an appropriate PWM controller for the initially selected Ćuk DC-DC converter design. 


\section{A1.1 PWM Controller Decision Matrix}

Once this project's author drafted the initial specifications for this project's DCDC converter, this project's author then made a decision on which PWM controller IC to use based on the input extremes set in the specifications. To select the PWM controller IC, this project's author made a decision matrix that weighs each of the following PWM controller ICs' characteristics: 
- Input voltage range (the greater the range, the better, however it must support the $5-65 \mathrm{~V}$ or $60 \mathrm{~V}$ range required for this project converter; the $60 \mathrm{~V}$ maximum suffices without the maximum voltage headroom specification)

- Current or voltage mode sensing (this project prefers current-mode sensing because of its ability for sensing both voltage and current to ensure that they both don't reach values out of the desired electrical ranges specified in sections 1.1 to 1.3 )

- Turn-on threshold voltage

- Reference voltage

- Power dissipation (the lower the better)

- Duty cycle (must support a high enough duty cycle for stepping up an minimum input voltage of $8 \mathrm{~V}$ to the nominal $36 \mathrm{~V}$ that the Enphase micro-inverter requires)

- Switching frequency (higher range is better)

- Package size / number of pins (smaller package size is better)

This project then set absolute requirements for the PWM controller's supported duty cycle levels and switching frequencies. This project instantly disqualified any controller IC that failed one of these requirements:

- $81.82 \%$ duty cycle required for regulating $8 \mathrm{~V}$ input to $36 \mathrm{~V}$ output

- Switching frequency capability must be high to minimize ripple ( $\geq 50 \mathrm{kHz}$ )

This project calculated the $81.82 \%$ duty cycle requirement based on the non-inverting Ćuk converter and SEPIC's voltage transfer function in continuous conduction mode operation, which (A1.1) describes:

$$
\frac{V_{O U T}}{V_{I N}}=\frac{D}{1-D}
$$

D denotes the controller's switching duty cycle. This project then researched possible PWM controllers to use with the DC-DC converter: 
- Leadtrend LD7576J/K

- ON Semiconductor NCP1219

- Fairchild SG6741

- Fairchild FAN6300A

- National LM5022

- National LM3429

- Maxim MAX5003

- National LM5025A

- National LM5020

- National LM3421

- National LM3423

This project then assigned a weight to each characteristic for determining a final rating (based on a 0-100\% scale) for deciding which PWM controller IC to use:

- Input voltage range: $15 \%$

- Sensing mode: $5 \%$

- Turn-on threshold voltage: $20 \%$

- Reference voltage: $5 \%$

- Power dissipation: $10 \%$

- Duty cycle: $20 \%$

- Switching frequency: $10 \%$

- Package size: $15 \%$

The quantitative total adds to $100 \%$. Tables A.A-1 through A.A-3 below show the decision matrix in tabular form. This project disqualifies and marks in pink any controller scoring $0 \%$ in any category (if it does not meet this project's absolute requirements). This project marks in yellow the two highest scoring controller ICs. 
Table A.A-1: PWM Controller Decision Matrix - Characteristics of Each Controller IC

\begin{tabular}{|c|c|c|c|c|c|c|c|c|c|}
\hline $\begin{array}{c}\text { PWM } \\
\text { Controller }\end{array}$ & $\begin{array}{c}\text { Input Voltage } \\
\text { Range (V) }\end{array}$ & $\begin{array}{l}\text { Sense } \\
\text { Mode }\end{array}$ & $\begin{array}{c}\text { Min. Turn-On } \\
\text { Voltage (V) }\end{array}$ & $\begin{array}{l}\text { Reference } \\
\text { Voltage (V) }\end{array}$ & $\begin{array}{c}\text { Continuous } \\
\text { Power } \\
\text { Dissipation }\end{array}$ & $\begin{array}{l}\text { Duty Cycle } \\
\text { Range (\%) }\end{array}$ & $\begin{array}{c}\text { Switching } \\
\text { Frequency } \\
\text { Range }\end{array}$ & Package & $\begin{array}{l}\text { \# of } \\
\text { Pins }\end{array}$ \\
\hline $\begin{array}{l}\text { Leadtrend } \\
\text { LD7576J/K }\end{array}$ & $-0.3-600$ & 1 & 15 & 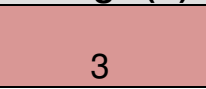 & $400-650 \mathrm{~mW}$ & $0-75$ & $94-106 \mathrm{kHz}$ & $\begin{array}{l}\text { DIP8 or } \\
\text { SOP8 }\end{array}$ & 8 \\
\hline $\begin{array}{c}\text { ON } \\
\text { NCP1219 }\end{array}$ & $-0.3-500$ & 1 & 11.2 & 2 & $920 \mathrm{~mW}$ & 85 (Max.) & $\begin{array}{c}65 \mathrm{kHz} \text { or } 100 \\
\mathrm{kHz}\end{array}$ & SOIC7 & 7 \\
\hline $\begin{array}{l}\text { Fairchild } \\
\text { SG6741 }\end{array}$ & $0-500$ & 1 & 15.5 & 2.3 & $400 \mathrm{~mW}$ & 80 (Max.) & $47-109 \mathrm{kHz}$ & SOP8 & 8 \\
\hline $\begin{array}{c}\text { Fairchild } \\
\text { FAN6300A }\end{array}$ & $0-500$ & 1 & 15 & 2.5 & $400-800 \mathrm{~mW}$ & 85 (Max.) & 100 kHz (Max.) & $\begin{array}{l}\text { DIP8 or } \\
\text { SOP8 }\end{array}$ & 8 \\
\hline $\begin{array}{l}\text { National } \\
\text { LM5022 }\end{array}$ & $6-60$ & I & 6.6 & 1.25 & Internally Limited & $0-95$ & $170-1115 \mathrm{kHz}$ & MSOP-10 & 10 \\
\hline $\begin{array}{l}\text { National } \\
\text { LM3429 }\end{array}$ & $4.5-75$ & I & 6.3 & 1.235 & Internally Limited & Programmed & $2 \mathrm{MHz}$ (Max.) & TSSOP14 & 14 \\
\hline $\begin{array}{c}\text { MAXIM } \\
\text { MAX5003 }\end{array}$ & $11-110$ & V & 25 & 3 & $667-762 \mathrm{~mW}$ & 75 (Max.) & $200-1200 \mathrm{kHz}$ & QSOP-16 & 16 \\
\hline $\begin{array}{l}\text { National } \\
\text { LM5020 }\end{array}$ & $-0.3-100$ & 1 & 7.4 & 1.25 & Internally Limited & $0-80$ & $175-700 \mathrm{kHz}$ & MSOP-10 & 10 \\
\hline $\begin{array}{l}\text { National } \\
\text { LM3421 }\end{array}$ & $4.5-75$ & 1 & 6.3 & 1.235 & Internally Limited & $0-95$ & Programmable & TSSOP16 & 16 \\
\hline $\begin{array}{l}\text { National } \\
\text { LM3423 }\end{array}$ & $4.5-75$ & 1 & 6.3 & 1.235 & Internally Limited & $0-95$ & Programmable & TSSOP20 & 20 \\
\hline
\end{tabular}


Table A.A-2: PWM Controller Decision Matrix - Preference Ratings for Each Controller IC

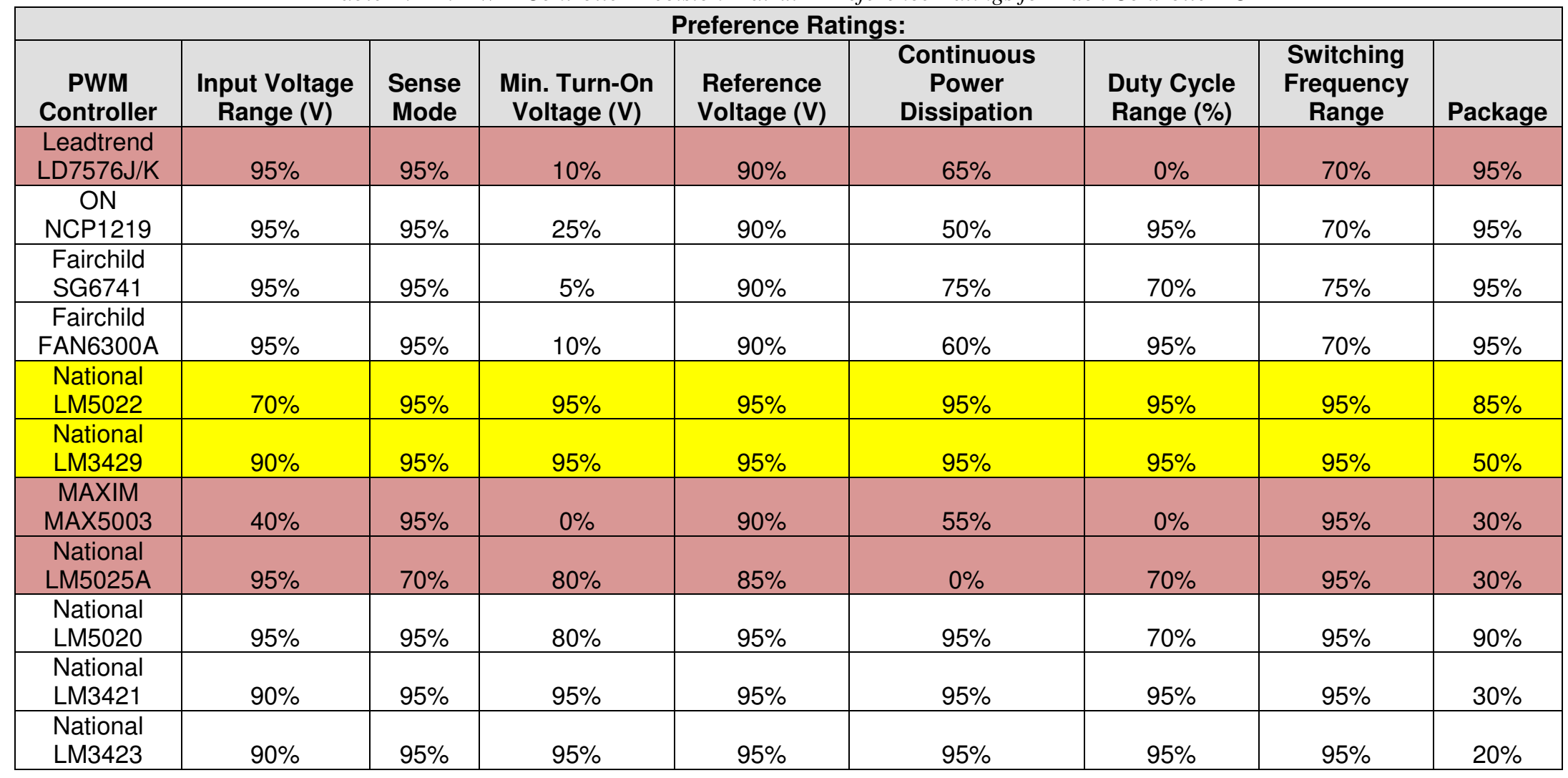


Table A.A-3: PWM Controller Decision Matrix - Weighted Final Preference Ratings for Each Controller IC

\begin{tabular}{|c|c|c|c|c|c|c|c|c|c|}
\hline $\begin{array}{c}\text { PWM } \\
\text { Controller }\end{array}$ & $\begin{array}{c}\text { Input Voltage } \\
\text { Range (V) }\end{array}$ & $\begin{array}{l}\text { Sense } \\
\text { Mode }\end{array}$ & $\begin{array}{l}\text { Min. Turn-On } \\
\text { Voltage (V) }\end{array}$ & $\begin{array}{l}\text { Reference } \\
\text { Voltage (V) }\end{array}$ & $\begin{array}{c}\text { Continuous } \\
\text { Power } \\
\text { Dissipation }\end{array}$ & $\begin{array}{l}\text { Duty Cycle } \\
\text { Range (\%) }\end{array}$ & $\begin{array}{c}\text { Switching } \\
\text { Frequency } \\
\text { Range } \\
\end{array}$ & Package & \begin{tabular}{|c} 
Total \\
Rating
\end{tabular} \\
\hline $\begin{array}{c}\text { ON } \\
\text { NCP1219 }\end{array}$ & $14.25 \%$ & $4.75 \%$ & $5.00 \%$ & $4.50 \%$ & $5.00 \%$ & $19.00 \%$ & $7.00 \%$ & $14.25 \%$ & $73.75 \%$ \\
\hline $\begin{array}{l}\text { Fairchild } \\
\text { SG6741 }\end{array}$ & $14.25 \%$ & $4.75 \%$ & $1.00 \%$ & $4.50 \%$ & $7.50 \%$ & $14.00 \%$ & $7.50 \%$ & $14.25 \%$ & $67.75 \%$ \\
\hline $\begin{array}{l}\text { Fairchild } \\
\text { FAN6300A }\end{array}$ & $14.25 \%$ & $4.75 \%$ & $2.00 \%$ & $4.50 \%$ & $6.00 \%$ & $19.00 \%$ & $7.00 \%$ & $14.25 \%$ & $71.75 \%$ \\
\hline $\begin{array}{l}\text { National } \\
\text { LM5022 }\end{array}$ & $10.50 \%$ & $4.75 \%$ & $19.00 \%$ & $4.75 \%$ & $9.50 \%$ & $19.00 \%$ & $9.50 \%$ & $12.75 \%$ & $89.75 \%$ \\
\hline $\begin{array}{l}\text { National } \\
\text { LM3429 }\end{array}$ & $13.50 \%$ & $4.75 \%$ & $19.00 \%$ & $4.75 \%$ & $9.50 \%$ & $19.00 \%$ & $9.50 \%$ & $7.50 \%$ & $87.50 \%$ \\
\hline $\begin{array}{l}\text { National } \\
\text { LM5020 }\end{array}$ & $14.25 \%$ & $4.75 \%$ & $16.00 \%$ & $4.75 \%$ & $9.50 \%$ & $14.00 \%$ & $9.50 \%$ & $13.50 \%$ & $86.25 \%$ \\
\hline $\begin{array}{l}\text { National } \\
\text { LM3421 }\end{array}$ & $13.50 \%$ & $4.75 \%$ & $19.00 \%$ & $4.75 \%$ & $9.50 \%$ & $19.00 \%$ & $9.50 \%$ & $4.50 \%$ & $84.50 \%$ \\
\hline $\begin{array}{l}\text { National } \\
\text { LM3423 }\end{array}$ & $13.50 \%$ & $4.75 \%$ & $19.00 \%$ & $4.75 \%$ & $9.50 \%$ & $19.00 \%$ & $9.50 \%$ & $3.00 \%$ & $83.00 \%$ \\
\hline
\end{tabular}


From Table A.A-3, the two best PWM controller chips based on this project's characteristic selection matrix are the LM5022 and LM3429, both from National Semiconductor. This project disqualified Leadtrend L7576J/K and Maxim MAX5003 because they did not support the minimum duty cycle required for the converter, and this project disqualified the National LM5025A IC for National not specifying its power consumption. From this controller decision matrix this project then constructed in Intusoft ICAP a non-inverting Ćuk converter using the National LM5022 PWM controller IC.

\section{A1.2 Non-Inverting Ćuk Converter Design using National LM5022 PWM}

\section{Controller}

Figure A-1 shows this project's initial non-inverting Ćuk converter schematic using the National LM5022 PWM controller. 


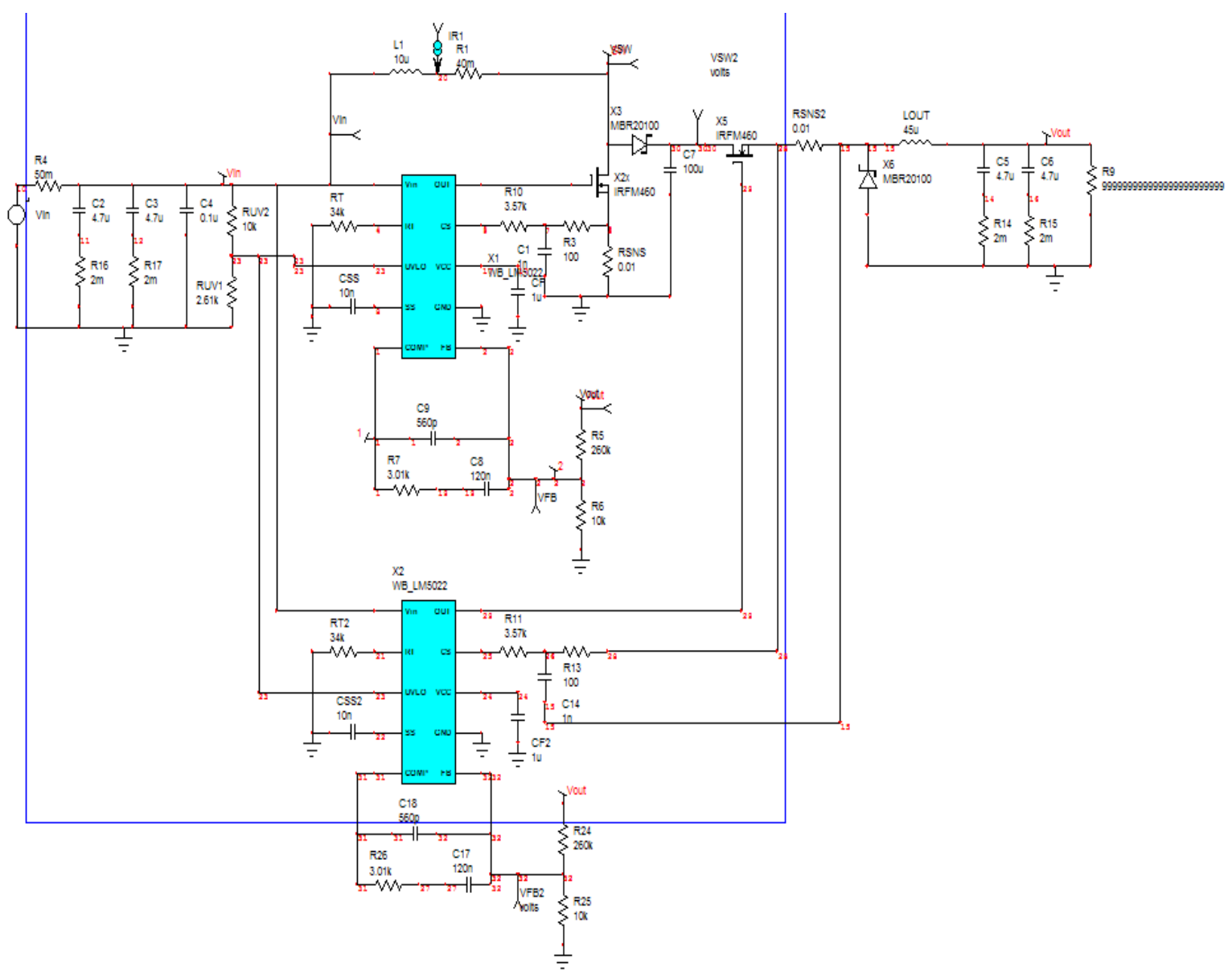

Figure A-1: Non-Inverting Cuk Converter Using National LM5022 PWM Controller

Obtaining a non-inverted voltage (relative to the converter's input) at the converter's output requires an additional switch and diode for the converter. Each switch (power MOSFETs) requires an individual PWM controller IC, hence this implementation requires two National LM5022 ICs. The National LM5022 has a nominal 1.25V feedback voltage, and its feedback network influences the converter's output voltage. At the converter's output this project selected a feedback resistor network that would obtain the $36 \mathrm{~V}$ that the converter requires (resistance values of $10 \mathrm{k} \Omega$ and $260 \mathrm{k} \Omega$ for the bottom and top feedback resistors). Resistor $\mathrm{R}_{\mathrm{T}}$ also sets the PWM controller's switching frequency; this project set it to $34 \mathrm{k} \Omega$ for obtaining a $500 \mathrm{kHz}$ switching frequency, which is high 
enough for minimizing any undesirable ripple at the converter's output. This project also selected a MOSFET and diode (International Rectifier's IRFM460 N-channel power MOSFET and the MBR20100 diode) that tolerated the wide input voltage range of the DC-DC converter. This project then simulated this converter using Intusoft ICAP (as the only simulation model for the LM5022 that National Semiconductor offered was for Intusoft ICAP/ISpice) using a 50ms simulation time. Figure A-2 shows the converter's performance with the PWM controller's 60V maximum input voltage with no load:

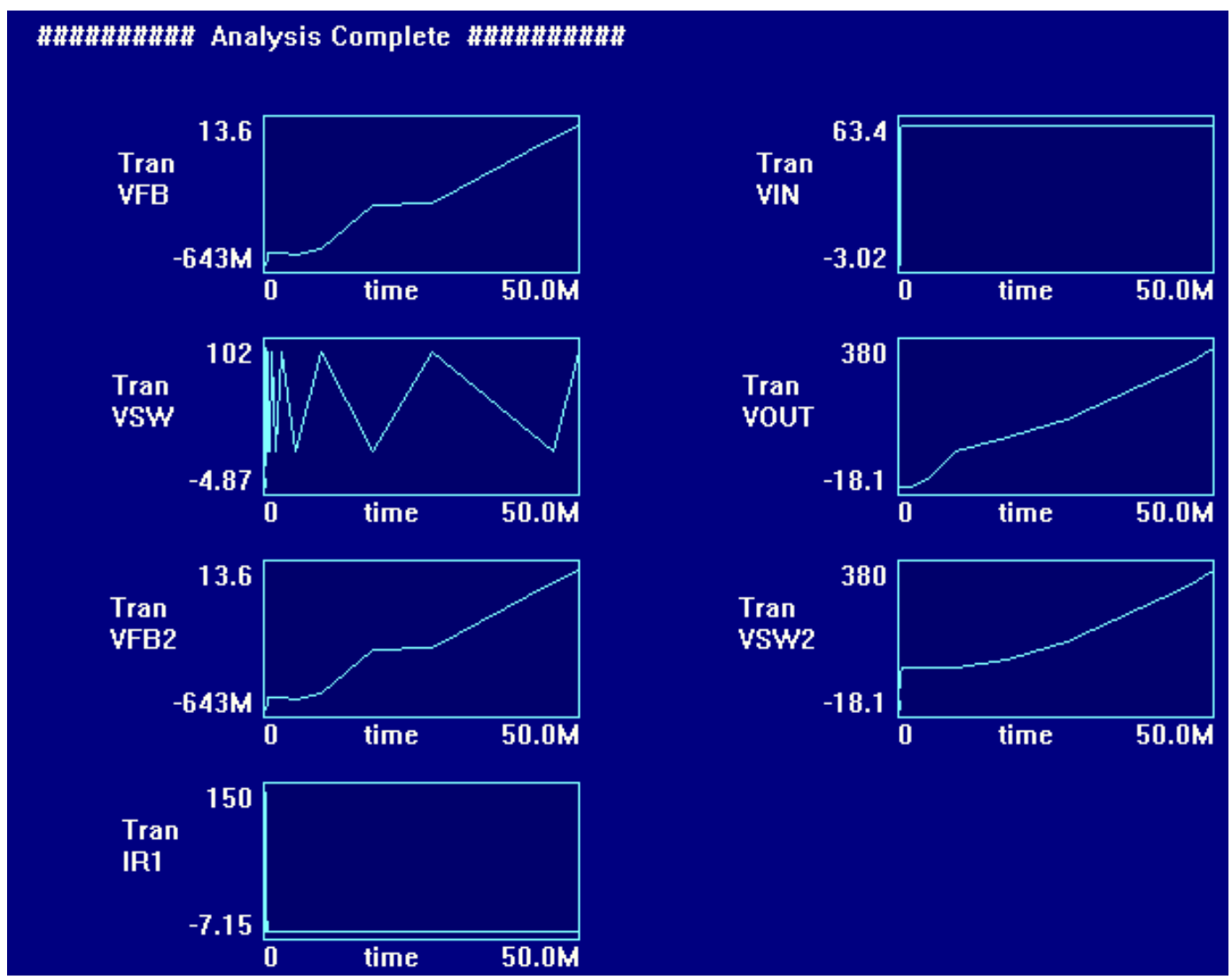

Figure A-2: Non-Inverting Cuk Converter Performance Characteristics at 60V Input, No Load, Using National LM5022 PWM Controller

VFB denotes the voltage across first PWM controller's feedback pin, VFB2 denotes the voltage across the second PWM controller's feedback pin, VSW denotes the converter's input MOSFET's drain voltage, VSW2 denotes the converter's output MOSFET's drain 
voltage, IR1 denotes the current through the input inductor, and VOUT denotes the converter's output voltage.

At $60 \mathrm{~V}$ input and no load, not only does the converter not output the correct voltage, but both PWM controller feedback voltages also rise sharply above the $1.25 \mathrm{~V}$ nominal value. The drain voltages across each MOSFET also rise to dangerous levels. In a real-world situation, this converter would be instantly destroyed. This project hypothesizes that the reason for this abnormal converter behavior may be the way that this project configured the two PWM controller ICs. This project next examines how the converter behaves with 60V input and an 8A full load current (by Ohm's Law, the required load resistance at the converter's output is $4.5 \Omega$ with $36 \mathrm{~V}$ output). Figure A-3 shows the converter's performance in this situation: 


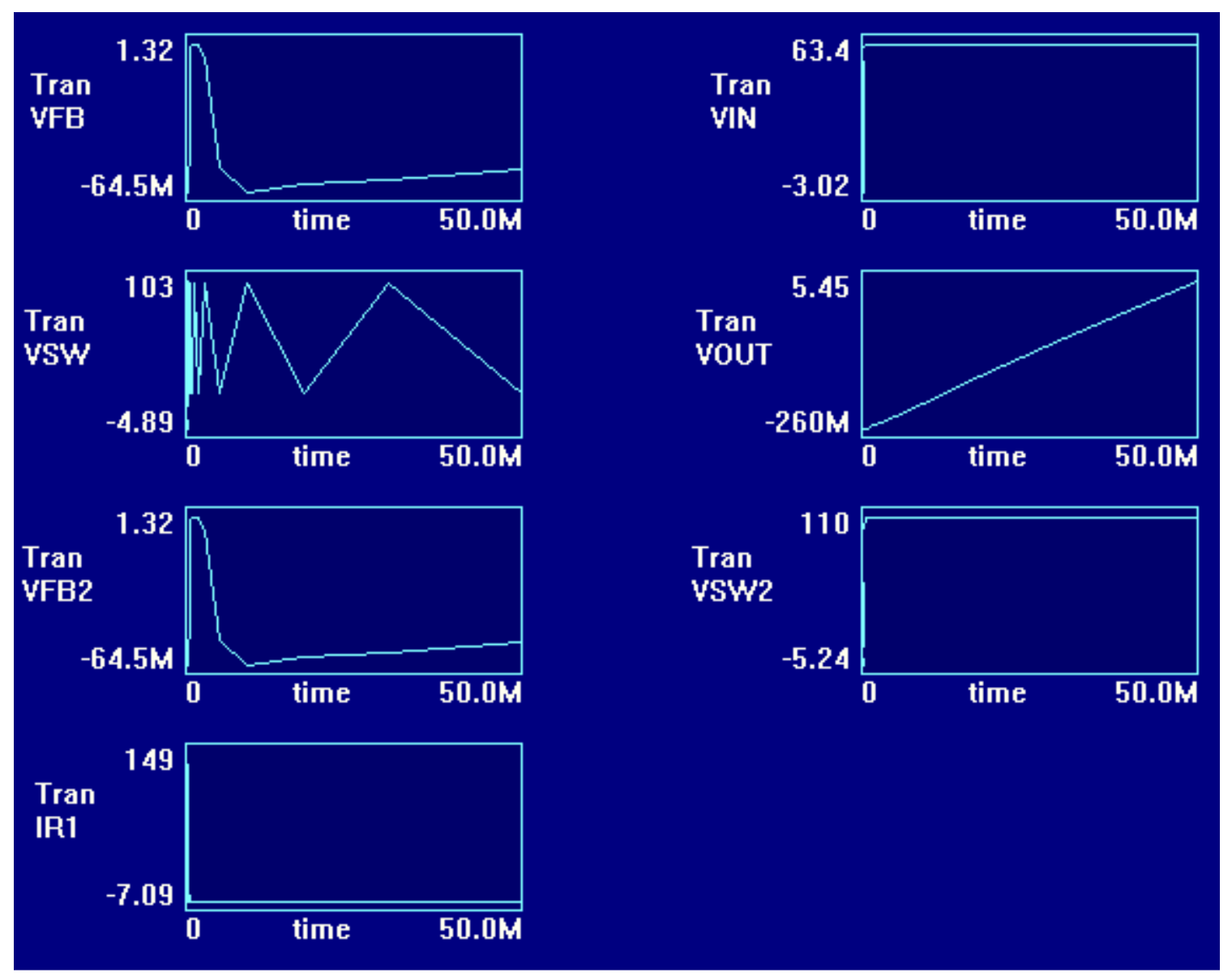

Figure A-3: Non-Inverting Cuk Converter Performance Characteristics at 60V Input, Full Load, Using National LM5022 PWM Controller

Both PWM controllers' feedback voltages drop significantly below the nominal 1.25V and the PWM controllers fail to regulate. This is the opposite effect of what this project observed earlier with no load. Thus, the non-inverting Ćuk converter design may not work properly with this particular PWM controller. The same results occur with $8 \mathrm{~V}$ input.

From these preliminary simulation test results, the National LM5022 is not a feasible PWM controller for use with this project's DC-DC converter. Despite its wide input voltage range, this design has very poor line and load regulation, and these two characteristics are very crucial for the DC-DC converter functioning with the rest of the 
elliptical system because of the elliptical machine's wide input voltage range and the Enphase micro-inverter's wide load range for DC-to-AC power conversion. Thus, after this first phase design process, this project concludes that a non-inverting, PWM switching Ćuk DC-DC converter is not feasible for functioning with the EHFEM system in this project. Appendix B describes a more potentially suitable DC-DC converter design for this project's EHFEM system using an interleaved, multi-stage non-inverting buckboost topology. 


\section{APPENDIX B: INTERLEAVED BUCK-BOOST CONVERTER DESIGN}

\section{B1.1 Interleaved, Multi-Stage Buck-Boost Converter Design Using LT3845 and LTC3862 PWM Controllers}

After unsuccessfully designing a DC-DC converter based on this project's original non-inverting, PWM switching Ćuk topology selection, this project designed a new converter based on an interleaved, multi-stage buck-boost topology. However, this project still retained its original DC-DC converter operation requirements and specifications from sections 1.1 to 1.3. Likewise the Ćuk converter topology, a basic buck-boost DC-DC converter outputs a DC voltage either greater or less than its input voltage, though with its polarity inverted with respect to its input voltage. Because of the buck-boost converter's inherently low power density, achieving greater stability under larger loads (> 100W) requires a multi-stage, multi-phase brute-force approach: this project designs a buck-boost converter with its input stage as a multi-phase, non-isolated PWM switching buck converter that steps down the input voltage. Its output stage is a multi-phase, non-isolated PWM switching boost converter that takes that stepped-down voltage and steps it up to the $36 \mathrm{~V}$ that the Enphase micro-inverter requires at its input. This approach also results in a non-inverted output voltage polarity with respect to the converter's input voltage. This project initially began with a single phase design for each stage; however its simulation results show that using a single phase for each stage does not supply enough current needed for adequately driving the converter under high power loads. Hence, this project later moved to a multi-phase approach after receiving dismal results from its single-phase converter design. By adding more phases to each stage, the 
converter increases its current-carrying capacity for driving high power loads at high switching frequencies. Finding a suitable PWM controller for implementing this design was perhaps the most difficult part of this design phase. The majority of the PWM controllers that this project's author researched could not tolerate the switching current (30A maximum) required for driving a $288 \mathrm{~W}$ load for this design. However, this project's author eventually selected suitable PWM controllers for its input buck and output boost stages. For the buck stage design this project selected an LT3845A PWM controller from Linear Technology. For the boost stage design this project selected an LTC3862, also from Linear Technology. This project lists the LT3845A features below (based on the PWM controller selection matrix this project specified in appendix A1.1):

- $4 \mathrm{~V}$ to $65 \mathrm{~V}$ input voltage range

- Current-mode sensing

- 4V turn-on threshold

- $1.231 \mathrm{~V}$ reference (feedback) voltage

- Internally limited power dissipation

- $0-90 \%$ duty cycle operation

- $100 \mathrm{kHz}$ to $500 \mathrm{kHz}$ switching frequency operation

- TSSOP-16 package

The $4 \mathrm{~V}$ to $65 \mathrm{~V}$ input range is suitable for this project's EHFEM system application, as this project's DC-DC converter specifications require a 5-65V input range. This project lists the LTC3862's controller's features below:

- $-0.3 \mathrm{~V}$ to $40 \mathrm{~V}$ input voltage range

- Current-mode sensing

- $3.3 \mathrm{~V}$ turn-on threshold

- $1.223 \mathrm{~V}$ reference (feedback) voltage

- Internally limited power dissipation

- $96 \%$ maximum duty cycle operation

- $50 \mathrm{kHz}$ to $650 \mathrm{kHz}$ switching frequency operation

- $\quad$ TSSOP-24 or 24-lead QFN packages 
For the output boost converter stage, the LTC3862's 40V input ceiling more than suffices. This project designs the buck converter stage such that it steps down the input to a much lower voltage than $40 \mathrm{~V}$. The next section describes this buck-boost converter's design process.

\section{B1.2 Single-Phase Input and Output Stage Implementation}

Using the two selected PWM controller ICs, this project initially designed a multi-stage, single-phase buck boost converter. Figure B-1 shows its schematic. 


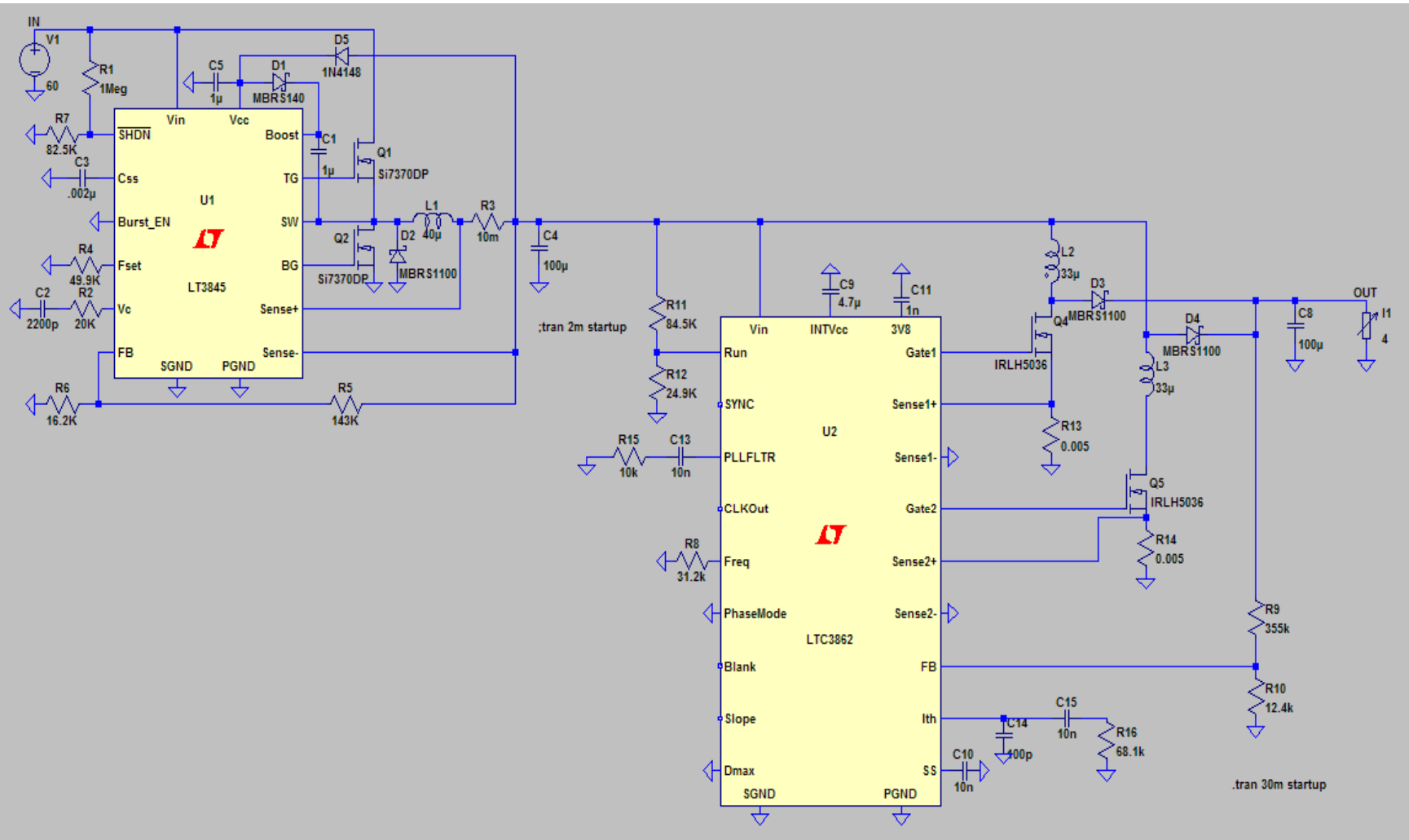

Figure B-1: Single-Phase, Multi-Stage Buck-Boost Converter Design 
This converter is essentially a buck converter and boost converter connected in cascade.

For the purpose of all testing in this design phase, this project uses $12 \mathrm{~V}$ as the voltage that the input buck converter stage steps the input voltage down to. This means that this converter cannot use the full input voltage range down to $8 \mathrm{~V}$; however, using $12 \mathrm{~V}$ as the buck stage's output provides this project with ideas for modifications in its next design phases. Section 3.2 indicates that any power generated from the Precor EFX 546i elliptical trainer is insignificant for any generated output voltage below $12 \mathrm{~V}$; however this project aims its final design at using its original specified input voltage range from section 1.2. The input stage in the schematic in figure B-1 has a $12 \mathrm{~V}$ output voltage, rated at $6.25 \mathrm{~A}$ (per phase). The input stage runs at a $300 \mathrm{kHz}$ switching frequency while the output stage runs at a $500 \mathrm{kHz}$ switching frequency. Selecting components required for the input buck converter stage requires the following parameters:

$V_{I N}=$ Buck stage input voltage

$V_{\text {OUT }}=$ Buck stage output voltage $=12 \mathrm{~V}$

$V_{O R}=$ Maximum amount of desired output voltage ripple $=10 \%$

$D=$ Duty cycle

$f_{s}=$ Buck stage switching frequency $=300 \mathrm{kHz}$

$I_{\text {OUT }}=$ Buck stage average output current $=6.25 \mathrm{~A}$ (maximum)

$C_{O}=$ Output filter capacitor

$L_{C}=$ Minimum inductor size required to maintain constant conduction mode (CCM)

$V_{S W}=$ Switching MOSFET drain - source voltage rating

$I_{D}=$ Switching MOSFET average drain current rating

$V_{R}=$ Diode maximum reverse voltage rating

$I_{F}=$ Diode maximum average forward current rating

A buck converter's voltage transfer function determines its duty cycle. Equation (B1.1) shows the buck converter's transfer function.

$$
D=\frac{V_{\text {OUT }}}{V_{I N}}
$$


$\mathrm{V}_{\mathrm{IN}}$ ranges from 12 to $65 \mathrm{~V}$. However, a $12 \mathrm{~V}$ input requires $100 \%$ duty cycle operation, and the LT3845A can only handle 90\% maximum duty cycle. Thus the minimum input voltage required for regulating the buck converter stage is $13.3 \mathrm{~V}$. For a $13.3-65 \mathrm{~V}$ input range, this translates to a $5.42 \%$ to $90 \%$ duty cycle range for the input buck converter stage. Equation (B1.2) determines the buck stage's output filter capacitor $\left(\mathrm{C}_{\mathrm{O}}\right)$ size.

$$
C_{O}=65 \times 10^{-6}\left(\frac{0.2 I_{O U T}}{V_{O R}}\right)=65 \times 10^{-6}\left(\frac{0.2(6.25 A)}{0.10}\right)=813 \mu F
$$

$\mathrm{V}_{\mathrm{OR}}$ denotes the converter's output ripple voltage. Because the boost converter output stage regulates the voltage outputted by the buck converter input stage, it doesn't require a completely clean input voltage, just as long as it is high enough (at least $5 \mathrm{~V}$ minimum) in order for the converter to output $36 \mathrm{~V}$. This project arbitrarily selected $100 \mu \mathrm{F}$ as a baseline for the purpose of testing. Equation (B1.3) determines this buck stage's required inductor size, using duty cycle and input voltage parameters. For the duty cycle parameter, this project assumes the $90 \%$ maximum condition, and for the input voltage parameter, this project assumes the $65 \mathrm{~V}$ maximum condition.

$$
L_{C}=\frac{\left(V_{I N}-V_{\text {OUT }}\right) D}{2 I_{\text {OUT }} f_{S}}=\frac{(65-12) 0.9}{2(6.25)(300 \mathrm{kHz})}=12.7 \mu \mathrm{H}
$$

$\mathrm{f}_{\mathrm{S}}$ denotes the input buck stage's switching frequency. This project selects $40 \mu \mathrm{H}$ for allowing for extra headroom in case the output stage requires more current than expected on the physical converter design. Equations (B1.4) and (B1.5) determine the buck stage's switching MOSFET drain-source voltage and drain current ratings.

$$
\begin{gathered}
V_{D S}=V_{I N}(\text { maximum })=65 \mathrm{~V} \\
I_{D}=I_{\text {OUT }}=6.25 \mathrm{~A}
\end{gathered}
$$


For testing this project used a MOSFET with a $60 \mathrm{~V}$ maximum $\mathrm{V}_{\mathrm{DS}}$ rating (as $60 \mathrm{~V}$ is the absolute maximum that any typical Precor EFX 546i elliptical trainer user generates according to [1]). Later designs used MOSFETs with higher $\mathrm{V}_{\mathrm{DS}}$ ratings to account for the extra 5V headroom. Equations (B1.6) and (B1.7) determine the buck stage's diode ratings.

$$
\begin{gathered}
V_{R}=V_{I N}(\text { maximum })=65 \mathrm{~V} \\
I_{F}=I_{\text {OUT }}=6.25 \mathrm{~A}
\end{gathered}
$$

This project's author later realized that the selected diode for the input buck stage had only a 1A average forward current rating. Later designs the MBR20100CT Schottky diode, which tolerates up to $10 \mathrm{~A}$ forward average current.

Likewise with the input buck converter stage, selecting the components required for the output boost converter stage requires the following parameters:

$V_{I N}=$ Boost stage input voltage

$V_{\text {OUT }}=$ Boost stage output voltage $=36 \mathrm{~V}$

$V_{O R}=$ Maximum amount of desired output voltage ripple $=10 \%$

$D=$ Duty cycle

$f_{s}=$ Boost stage switching frequency $=500 \mathrm{kHz}$

$I_{\text {OUT }}=$ Boost stage average output current $=8 \mathrm{~A}$ (maximum)

$P_{\text {OUT }}=$ Boost stage output power $=288 \mathrm{~W}$ (maximum)

$C_{O}=$ Output filter capacitor

$L_{C}$

$=$ Minimum inductor size required to maintain constant conduction mode (CCM)

$V_{S W}=$ Switching MOSFET drain - source voltage rating

$I_{D}=$ Switching MOSFET average drain current rating

$V_{R}=$ Diode maximum reverse voltage rating

$I_{F}=$ Diode maximum average forward current rating

A boost converter's voltage transfer function determines its duty cycle. Equation (B1.8)

determines the boost stage's duty cycle.

$$
D=1-\frac{V_{I N}}{V_{O U T}}
$$


$\mathrm{V}_{\mathrm{IN}}$ ideally is $12 \mathrm{~V}$. However, the filter capacitor this project selected for the buck input stage can cause $\mathrm{V}_{\mathrm{IN}}$ shifting as low as $5 \mathrm{~V}$. Even then, that $5 \mathrm{~V}$ minimum input voltage translates to $86 \%$ duty cycle operation, and the LTC3862 PWM controller can handle up to $96 \%$ duty cycle operation. For a $5-12 \mathrm{~V}$ input range to the boost converter stage, the boost stage's duty cycle ranges from $67 \%$ to $86 \%$. Equation (B1.9) determines the boost stage's output filter capacitor size, assuming the maximum $86 \%$ duty cycle operation.

$$
C_{O}=\frac{D V_{\text {OUT }}}{\left(\frac{V_{\text {OUT }}}{\left.I_{\text {OUT }}\right) f_{S}\left(V_{\text {OUT }}\right)\left(V_{\text {OR }}\right)}\right.}=\frac{(0.86)(36)}{\left(\frac{36}{8}\right)(500 \mathrm{kHz})(36)(0.10)}=3.82 \mu \mathrm{F}
$$

In reality, the minimum capacitance value is usually not enough for regulating the converter at the desired voltage under the maximum load condition. For this output stage, this project requires a clean output voltage for ensuring that the Enphase micro-inverter receives its desired optimal input voltage. Therefore this project boosted the output filter capacitor value from its calculated minimum to a reasonable $100 \mu \mathrm{F}$. Equation (B1.10) determines the boost stage's inductor size, assuming 67\% duty cycle operation for obtaining the largest possible minimum value.

$$
L_{C}=\frac{D(1-D)^{2}\left(\frac{V_{\text {OUT }}}{I_{\text {OUT }}}\right)}{2 f_{S}}=\frac{0.67(1-0.67)^{2}\left(\frac{36}{8}\right)}{2(500 \mathrm{kHz})}=0.33 \mu \mathrm{H}
$$

This project selects $33 \mu \mathrm{H}$ (which is 100 times the minimum calculated value) for extra headroom in case the physical converter's output stage requires more current than expected. Equations (B1.11) and (B1.12) determine the boost stage's switching MOSFET drain-source voltage and drain current ratings.

$$
\begin{gathered}
V_{D S}=V_{\text {OUT }}(\text { maximum })=36 \mathrm{~V} \\
I_{D}=\frac{2 P_{\text {OUT }}}{V_{I N}(\text { minimum })}=\frac{2(288)}{5}=115.2 \mathrm{~A}
\end{gathered}
$$


The output stage requires power MOSFETs that can tolerate $>120 \mathrm{~A}$. For simulation this project uses a MOSFET with a $60 \mathrm{~V}$ maximum $\mathrm{V}_{\mathrm{DS}}$ rating. Equations (B1.13) and (B1.14) determine the boost stage's diode ratings.

$$
\begin{gathered}
V_{R}=V_{I N}(\operatorname{maximum})=12 \mathrm{~V} \\
I_{F}=I_{O U T}=8 \mathrm{~A}
\end{gathered}
$$

This project's author realized the mistake during testing that the selected diode used for the input buck stage only had a $1 \mathrm{~A}$ average forward current rating. Later designs use the MBR20100CT and MBRB2545CT Schottky diodes, which can tolerate up to 10A and 25A forward average current.

This design is now ready for simulation. Figure B-2 shows a 3ms simulation operation of the designed single-phase buck-boost design using $60 \mathrm{~V}$ input and a $1 \mathrm{~A}$ load. The green waveform denotes boost stage's output voltage and blue denotes the buck stage's output voltage.

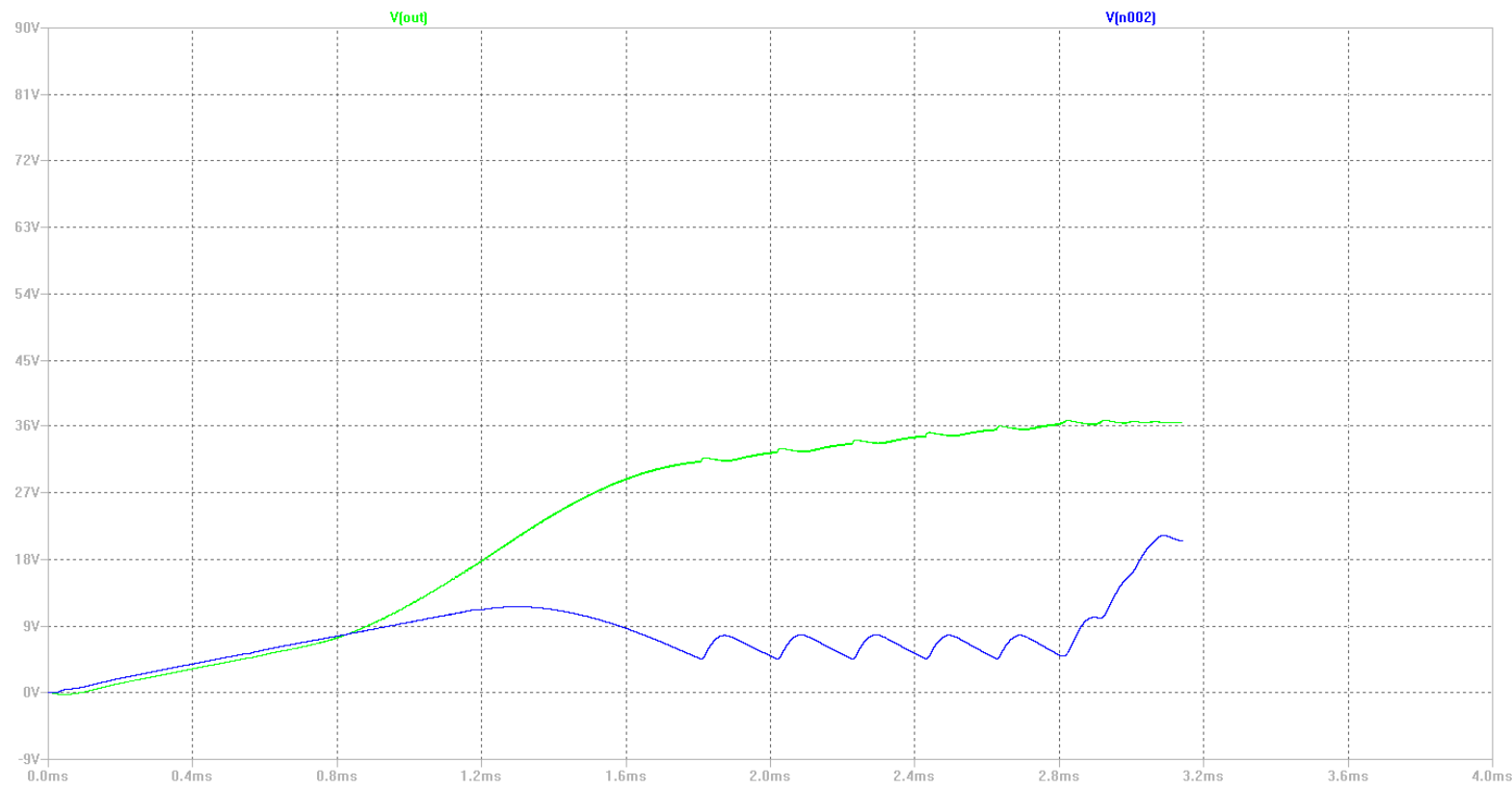

Figure B-2: Single-Phase Buck-Boost Converter Individual Stage Output Voltages at 60V Input, 1A Load 
The converter appears to regulate the required $36 \mathrm{~V}$ output without too much problem. This project then boosts the load to $2 \mathrm{~A}$ and simulates the converter design. Figure B-3 shows these results. The green waveform denotes the boost stage's output voltage V(out) and blue denotes the buck stage's output voltage V(n002).

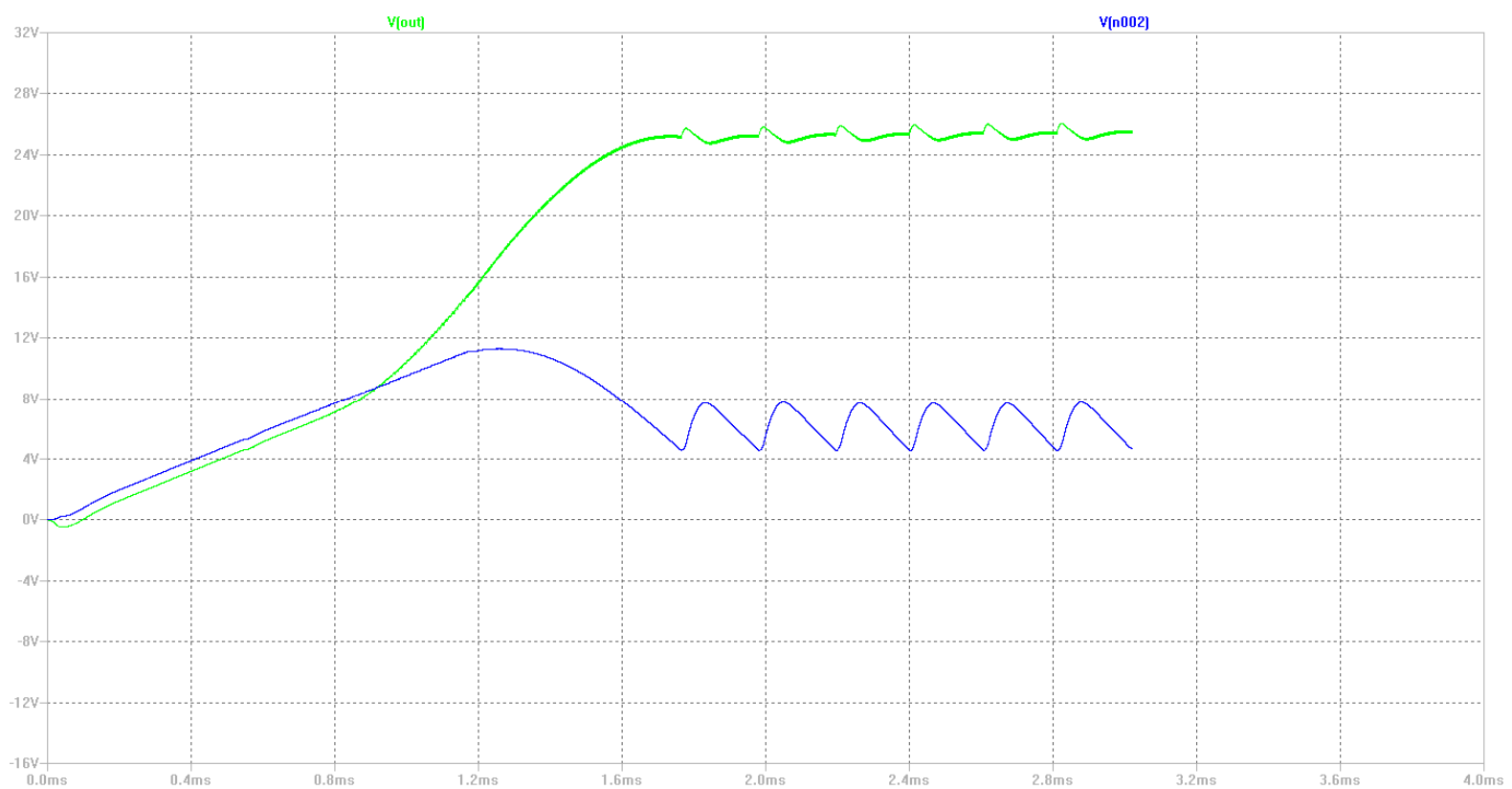

Figure B-3: Single-Phase Buck-Boost Converter Individual Stage Output Voltages at 60V Input, 2A Load

These results show a noticeable load regulation problem for this converter, as the output voltage begins dropping to $25 \mathrm{~V}$ (which is below the minimum input operating voltage required for the Enphase micro-inverter). Figure B-4 shows what happens when the simulation increases the load current to $4 \mathrm{~A}$. 


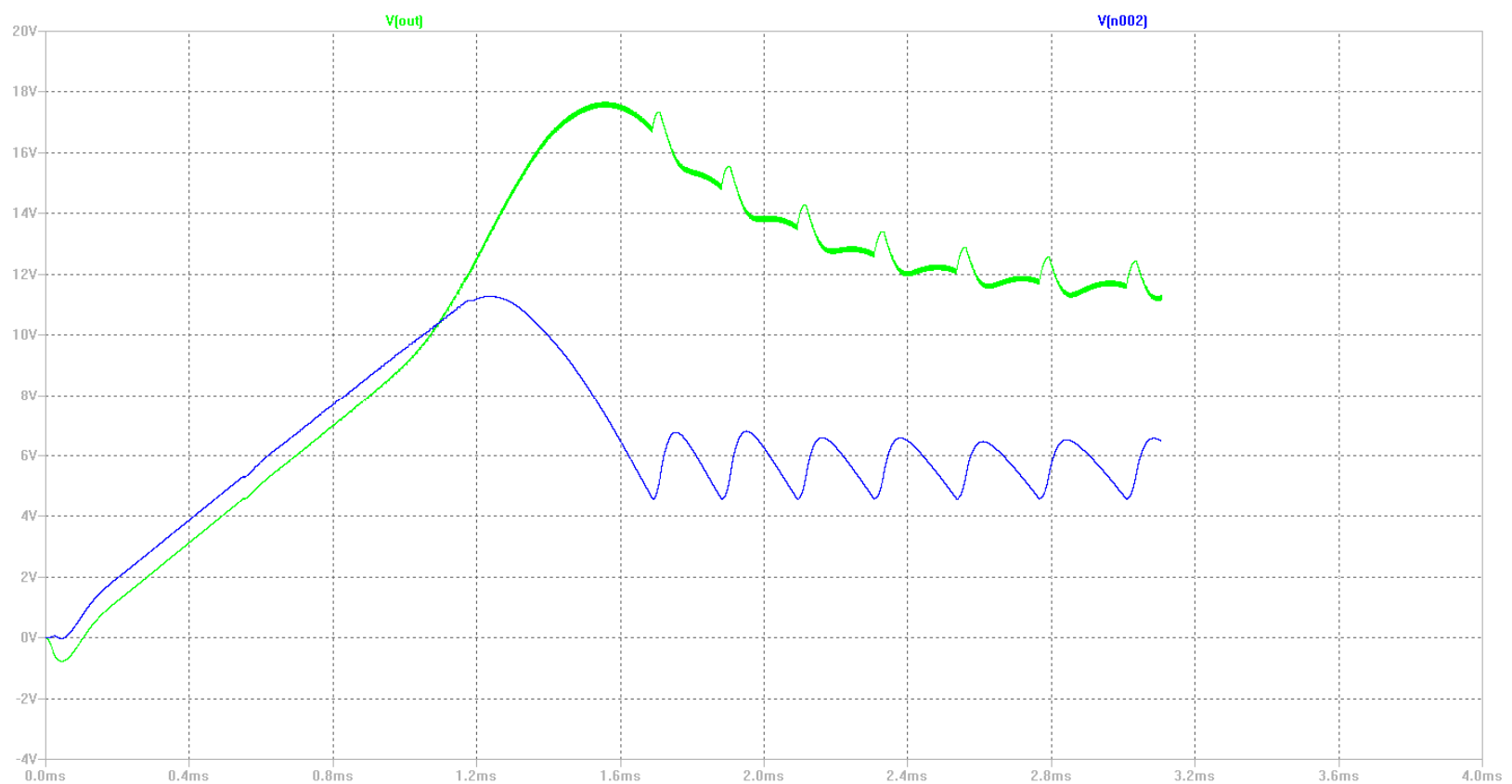

Figure B-4: Single-Phase Buck-Boost Converter Individual Stage Output Voltages at 60V Input, 4A Load

The load regulation problem worsens. Figure B-5 shows converter operation with a $5 \mathrm{~A}$ load.

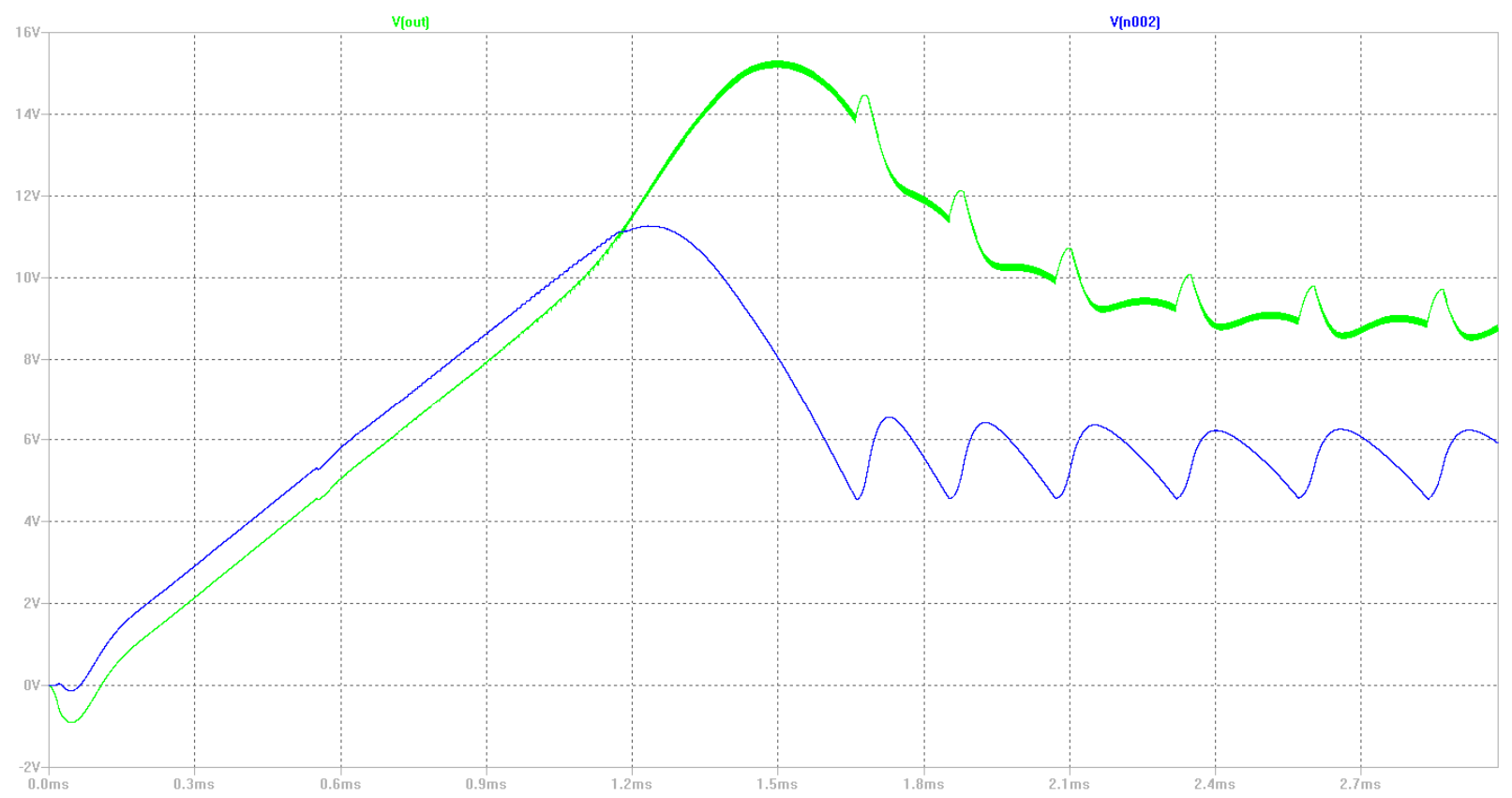

Figure B-5: Single-Phase Buck-Boost Converter Individual Stage Output Voltages at 60V Input, 5A Load

Figure B-6 shows converter operation with the maximum 8 A load. 


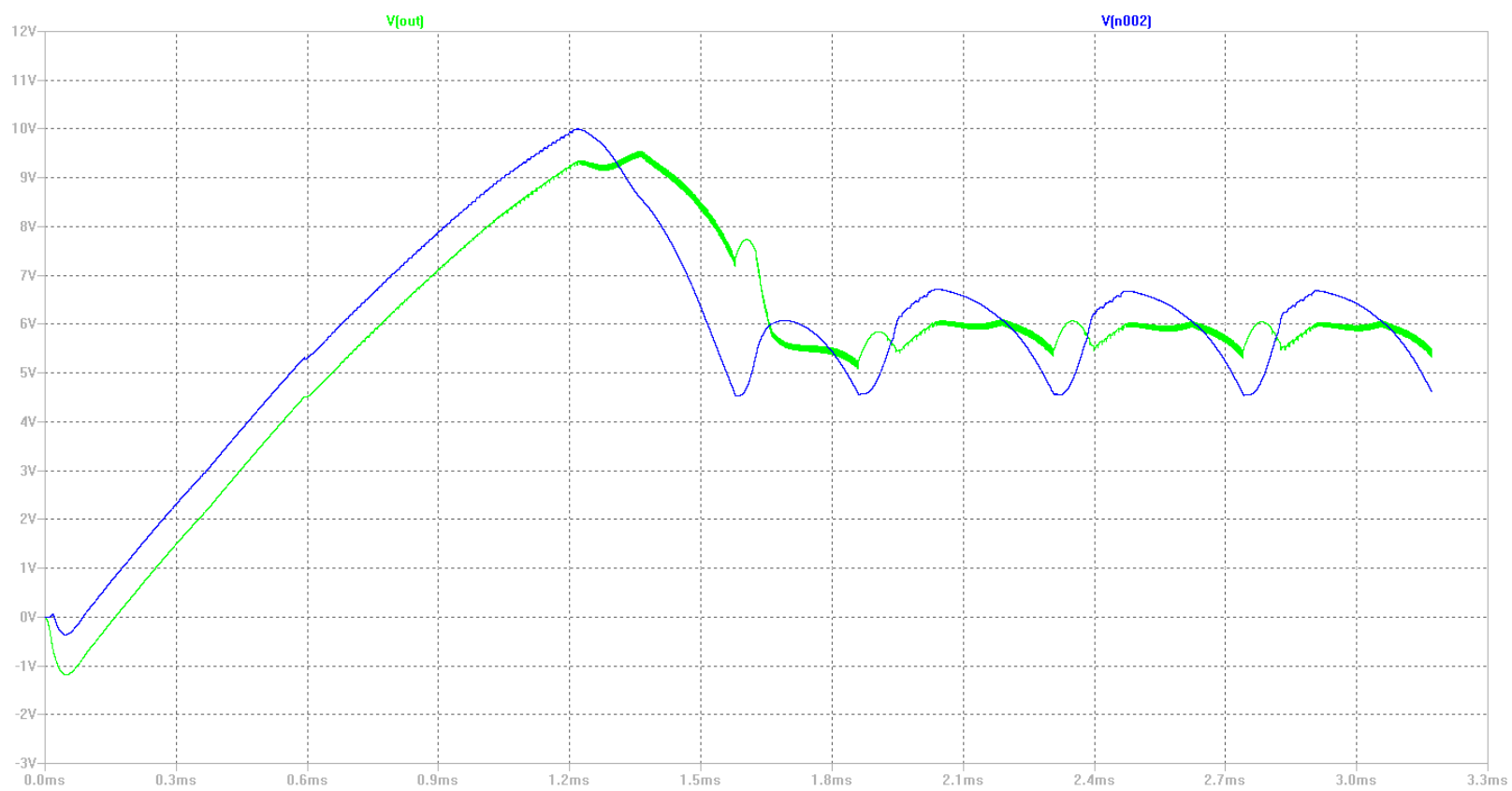

Figure B-6: Single-Phase Buck-Boost Converter Individual Stage Output Voltages at 60V Input, 8A Load

From these simulation results, the converter has an obvious load regulation problem. The input buck stage may not be providing enough average current under higher load conditions to the output boost stage for the boost stage to properly regulate the required 36V. Furthermore this load regulation problem can also be attributed to the switching frequencies on both stages not being exactly the same as each other (causing each PWM controller to operate out of sync) as well as using components that are not rated for this converter's ideal maximum values. Using a multi-phase design for the converter's input buck stage can mitigate the input current supply problem. The next section describes turning this single-phase cascaded buck-boost converter design into a multi-phase implementation. 


\section{B1.3 Two-Phase Input Stage and Single-Phase Output Stage Implementation}

This project attempts improving the design from appendix B1.2 by adding another phase to both the input buck stage, while maintaining the same components as with the previous design. Adding another phase to the input buck stage doubles its currentcarrying capacity, now to $12.5 \mathrm{~A}$. A multi-phase design further requires an additional LTC6908-1 external oscillator (also available from Linear Technology) tied to the SYNC pin of each PWM controller for every two phases for properly synchronizing each controller. Because LTSpice's LT3845A model does not contain a SYNC pin, the LTC6908 is not shown for any of the design schematics. However, it is necessary for the final physical converter design. The input stage still outputs $12 \mathrm{~V}$ to the boost-converter output stage. Furthermore, the component ratings remain the same for each phase added, hence this project uses the same components for each multi-phase design iteration. Figure B-7 shows the first multi-phase design realization, a two-phase buck, single-phase boost converter. 


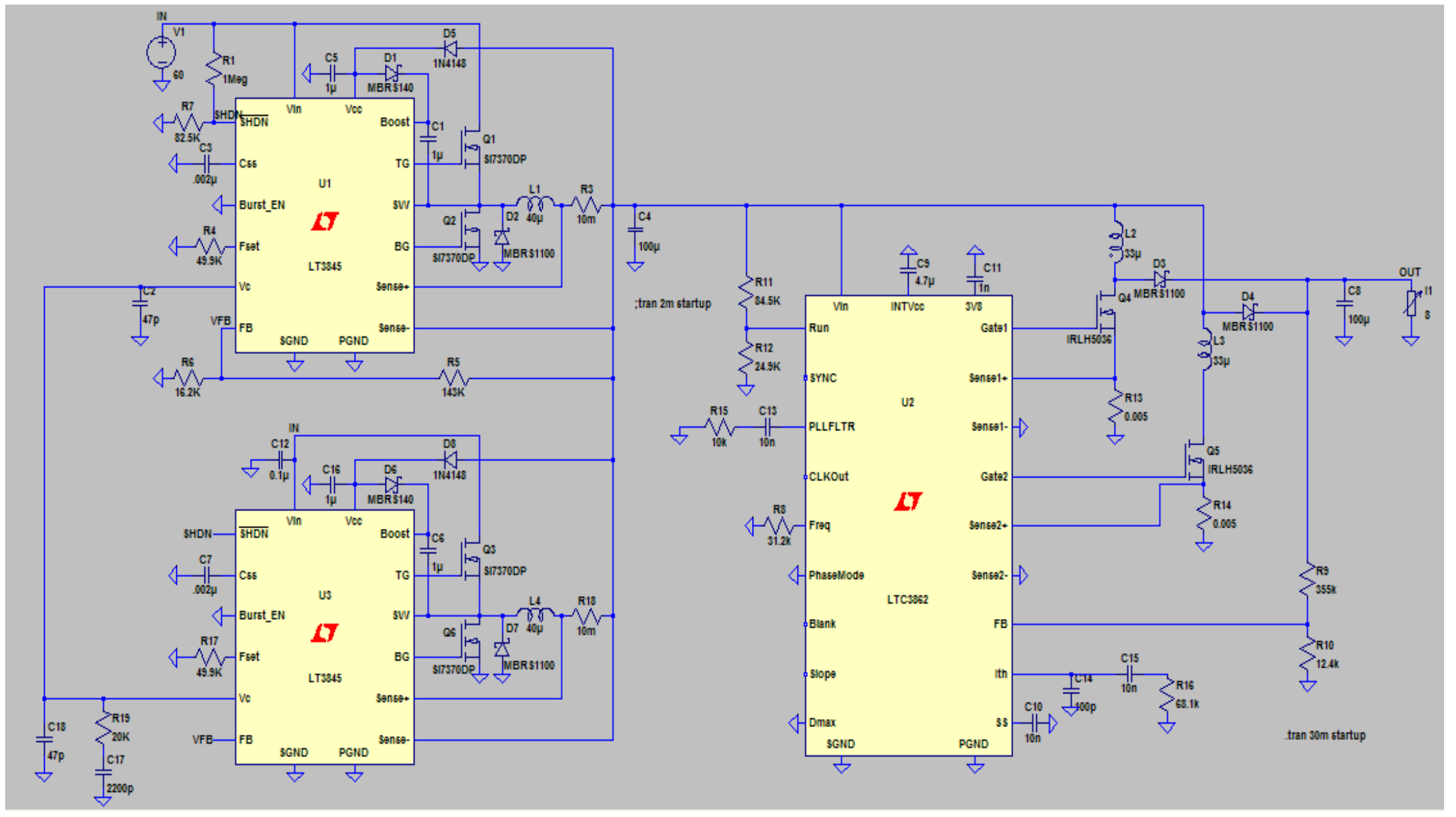

Figure B-7: Two-Phase Buck, Single-Phase Boost, Multi-Stage Buck-Boost Converter Design 
This project then tests this design using the maximum nominal stress condition with $60 \mathrm{~V}$ input and an 8A load. Figure B-8 shows the converter's operation for a 30ms simulation time window. The green waveform denotes the boost stage's output voltage and blue denotes the buck stage's output voltage.

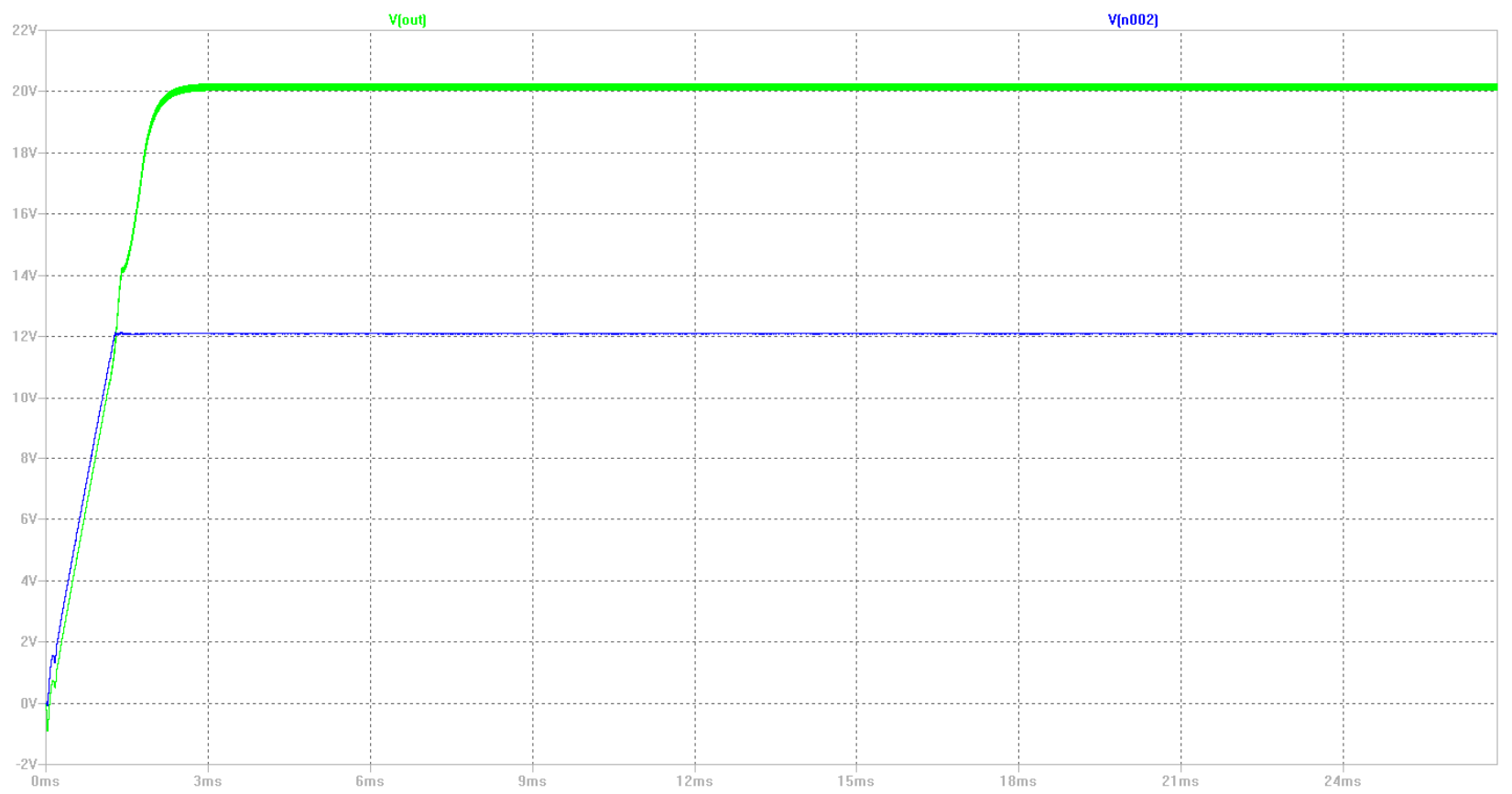

Figure B-8: Two-Phase Input, Single-Phase Output Buck-Boost Converter Individual Stage Output Voltages at 60V Input, 8A Load

Strangely enough, the boost stage's maximum output voltage only reaches $20 \mathrm{~V}$, which is not enough for the Enphase micro-inverter to properly regulate and deliver power. Figure B-9 below shows what happens if the simulation decreases the load to $6 \mathrm{~A}$, using the same 30ms time simulation window. 


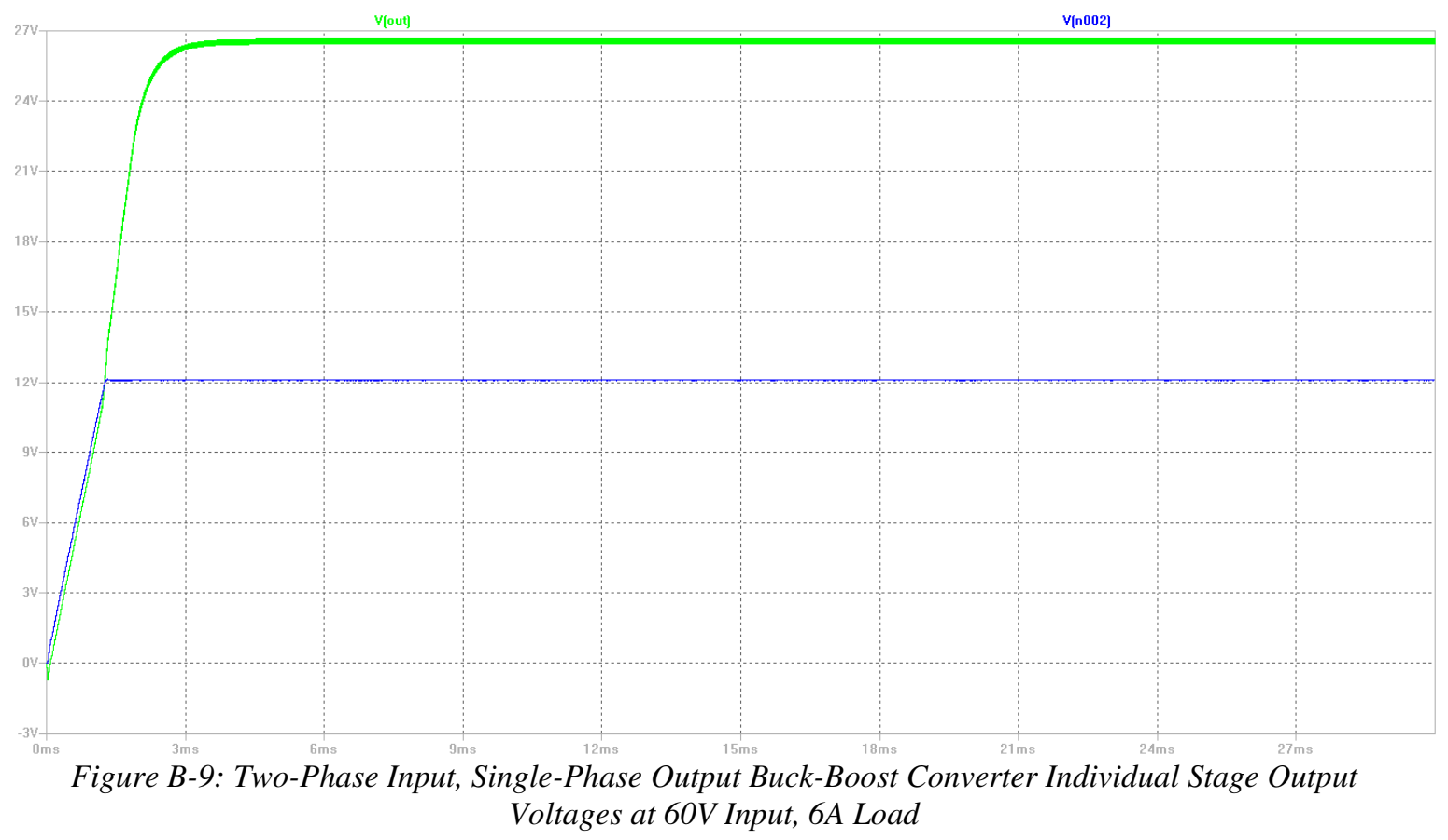

The result looks better, as the converter outputs $27 \mathrm{~V}$ - enough for the Enphase microinverter to properly deliver power. However, it is still far from the ideal $36 \mathrm{~V}$ that allows for maximum efficiency from the Enphase micro-inverter. Figure B-10 shows the converter's operation with a 4.75A load over the same $30 \mathrm{~ms}$ simulation period (This project selected $4.75 \mathrm{~A}$ as an intermediate point between 4 and $6 \mathrm{~A}$, with $4 \mathrm{~A}$ being the midpoint relative to the $8 \mathrm{~A}$ full load condition). 


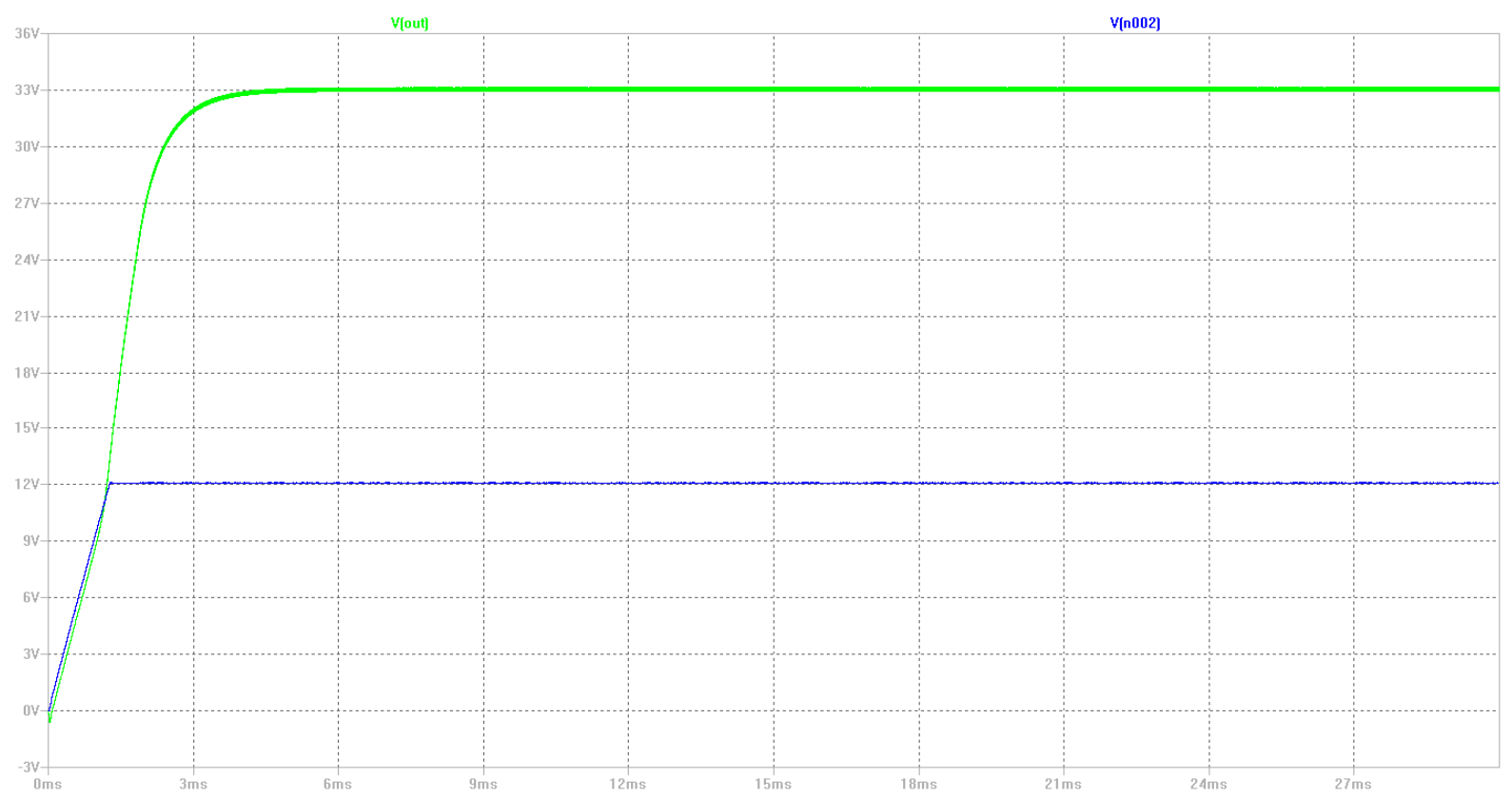

Figure B-10: Two-Phase Input, Single-Phase Output Buck-Boost Converter Individual Stage Output Voltages at $60 \mathrm{~V}$ Input, $4.75 \mathrm{~A} \mathrm{Load}$

Now the converter reaches $33 \mathrm{~V}$ output. However, this project desires that the converter properly regulates $36 \mathrm{~V}$ output under the maximum stress condition (60V input, $8 \mathrm{~A}$ load). This two-phase input design, however, shows much better results than with the previous purely single-phase design. The next section descrbes a further improved iteration of this design using four phases for the input buck stage.

\section{B1.4 Four-Phase Input Stage and Single-Phase Output Stage Implementation}

This project now determines how a 4-phase input design would theoretically perform (theoretical current carrying capacity of the input stage then becomes 20A). Again, this project uses the same components as with the previous designs. This project adds two more phases to the input (while maintaining the same number of phases at the output), resuting in a converter with a 4-phase buck input and single-phase boost output. Figure B-11 shows this design. 


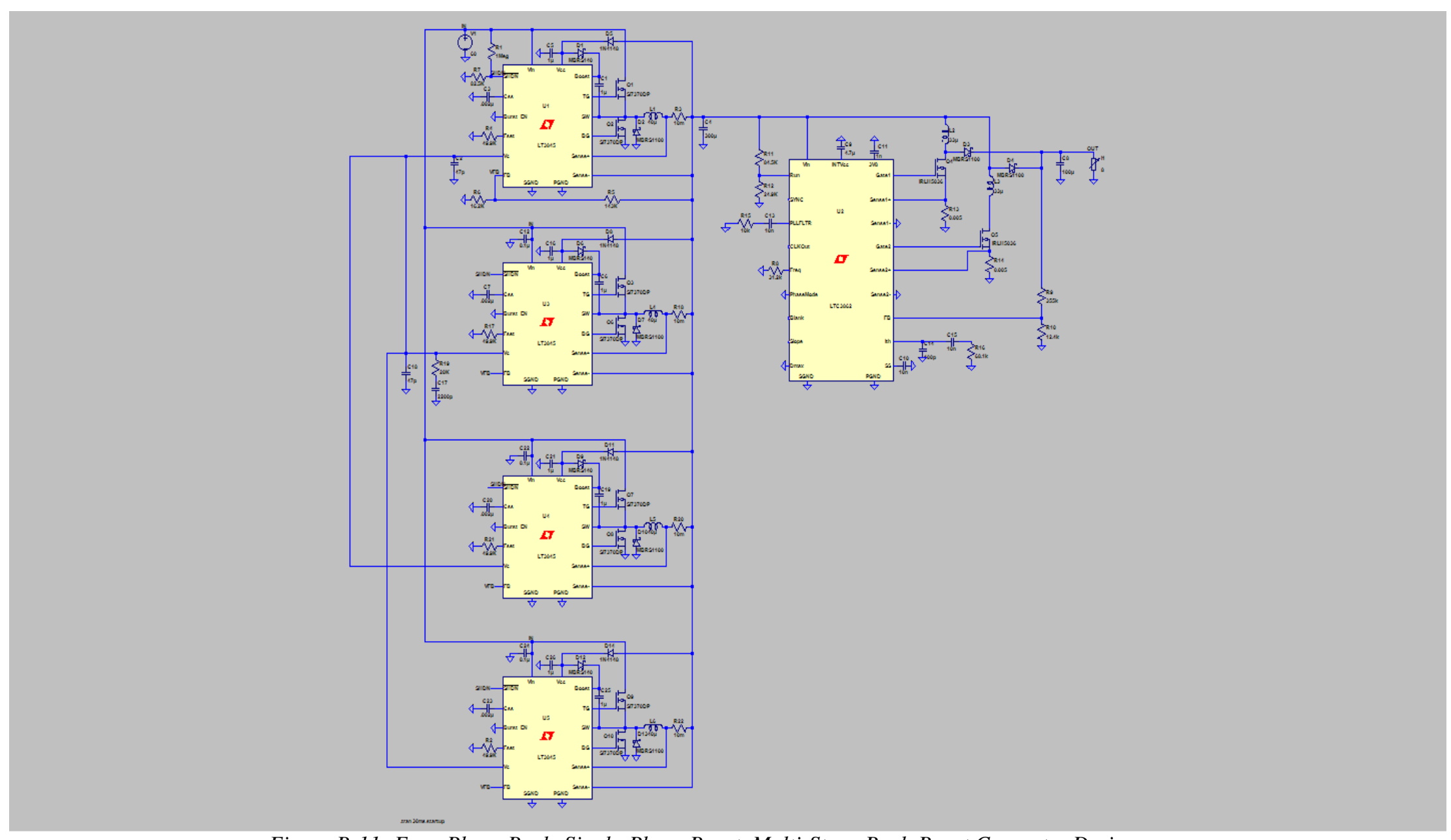

Figure B-11: Four-Phase Buck, Single-Phase Boost, Multi-Stage Buck-Boost Converter Design 
This project then tests this design using the nominal maximum stress condition with $60 \mathrm{~V}$ input and an 8A load. Figure B-12 shows the converter's operation from start-up to steady-state. The green waveform denotes the boost stage's output voltage and blue denotes the buck stage's output voltage.

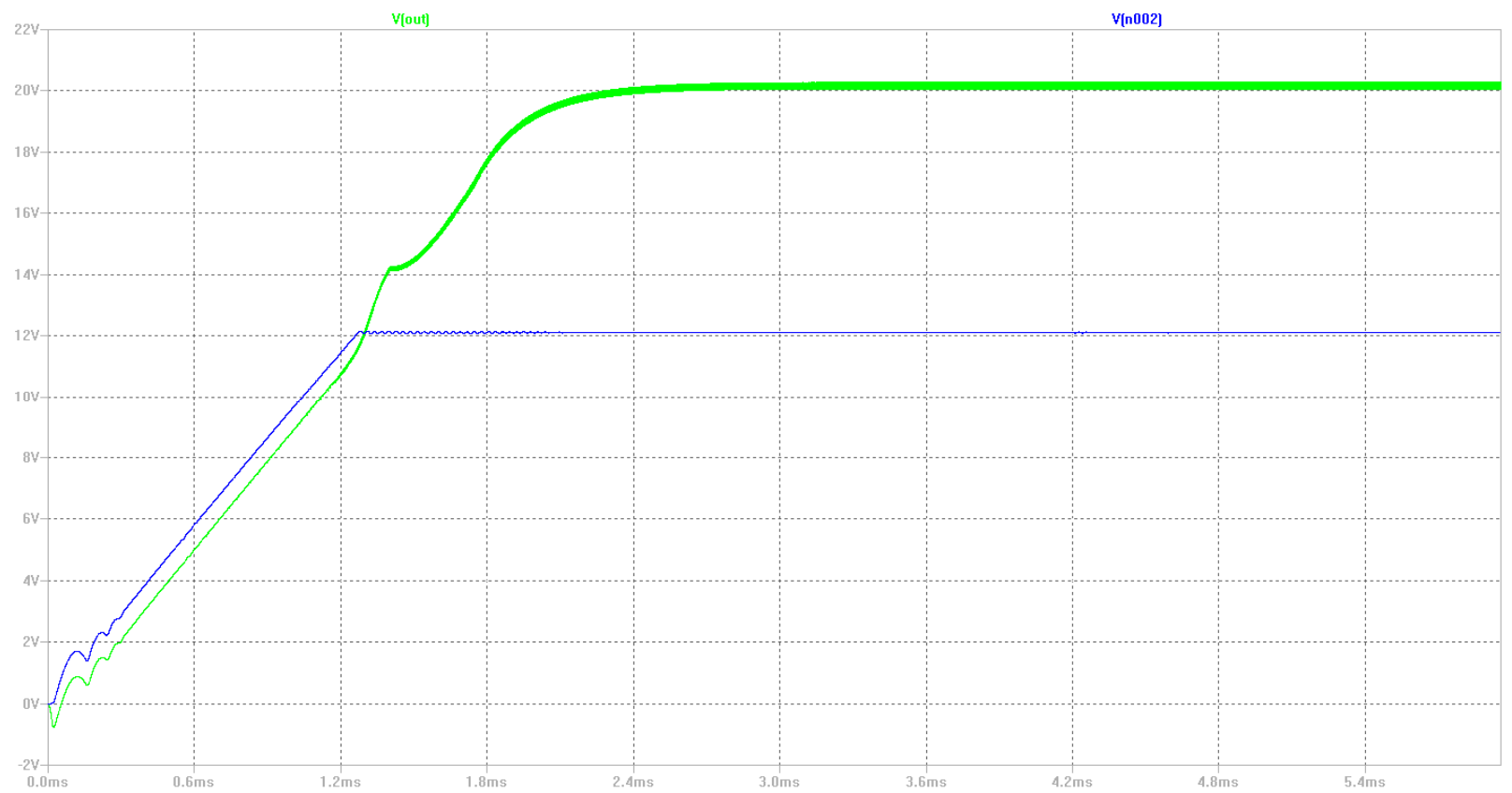

Figure B-12: Four-Phase input, Single-Phase Output Buck-Boost converter Individual Stage Output Voltages at 60V Input, 8A Load

The results are nearly identical to that of the previous 2-phase-input design. It is possible that the output stage reaches a current limit because of the increased number of phases at the input stage in this design and the output stage cannot handle the current fed in by the input stage in order to properly regulate its output. This project further enhances this design by adding an additional phase for the output boost converter for greater current carrying capacity for this stage. This results in a converter with a four-phase buck input and two-phase boost output. Figure B-13 shows this design. 


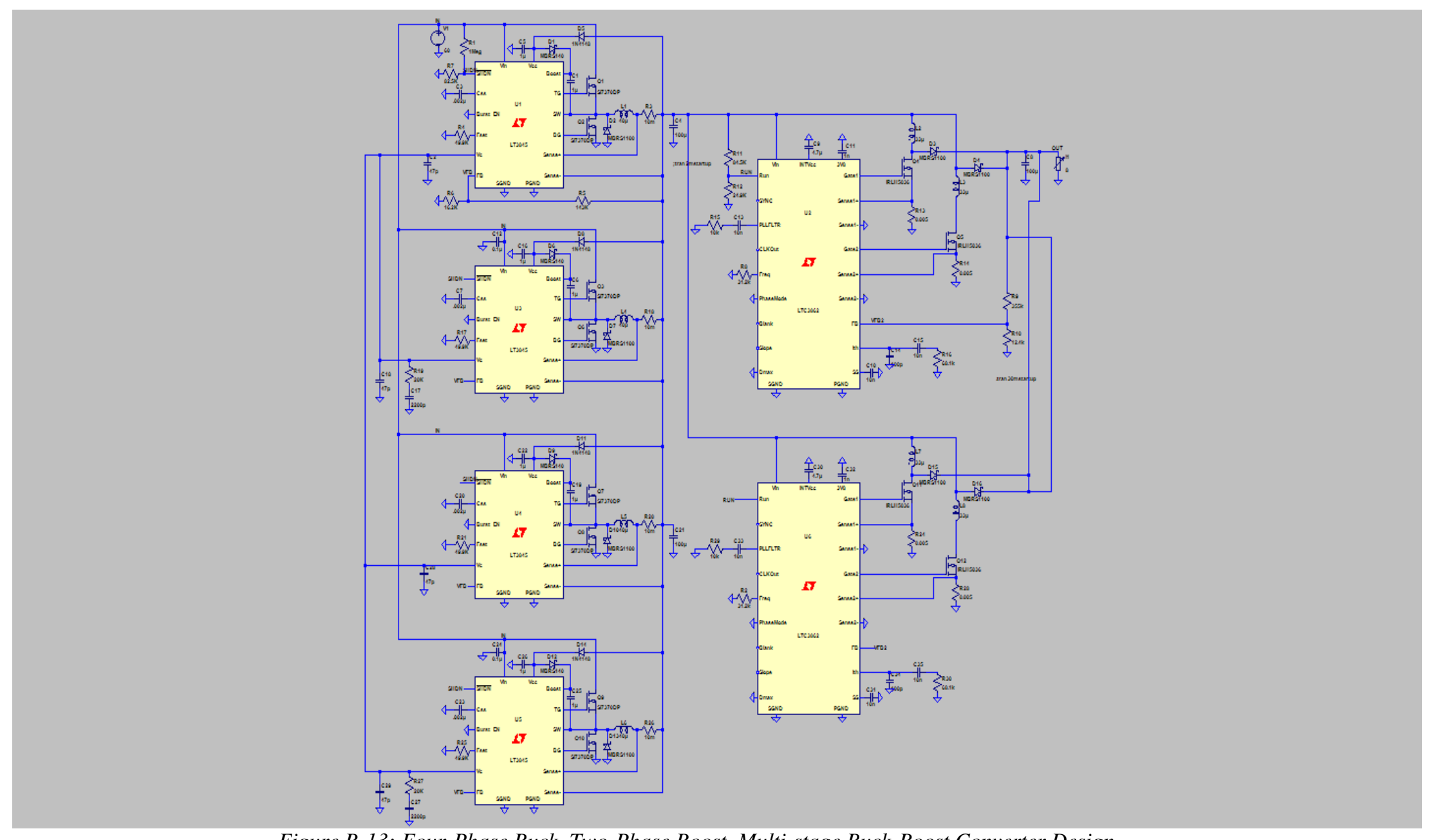

Figure B-13: Four-Phase Buck, Two-Phase Boost, Multi-stage Buck-Boost Converter Design 
This project then tests this design using the nominal maximum stress condition with $60 \mathrm{~V}$ input and an 8A load. Figure B-14 shows the converter's operation from start-up to steady-state. The green waveform denotes the boost stage's output voltage and blue denotes the buck stage's output voltage.

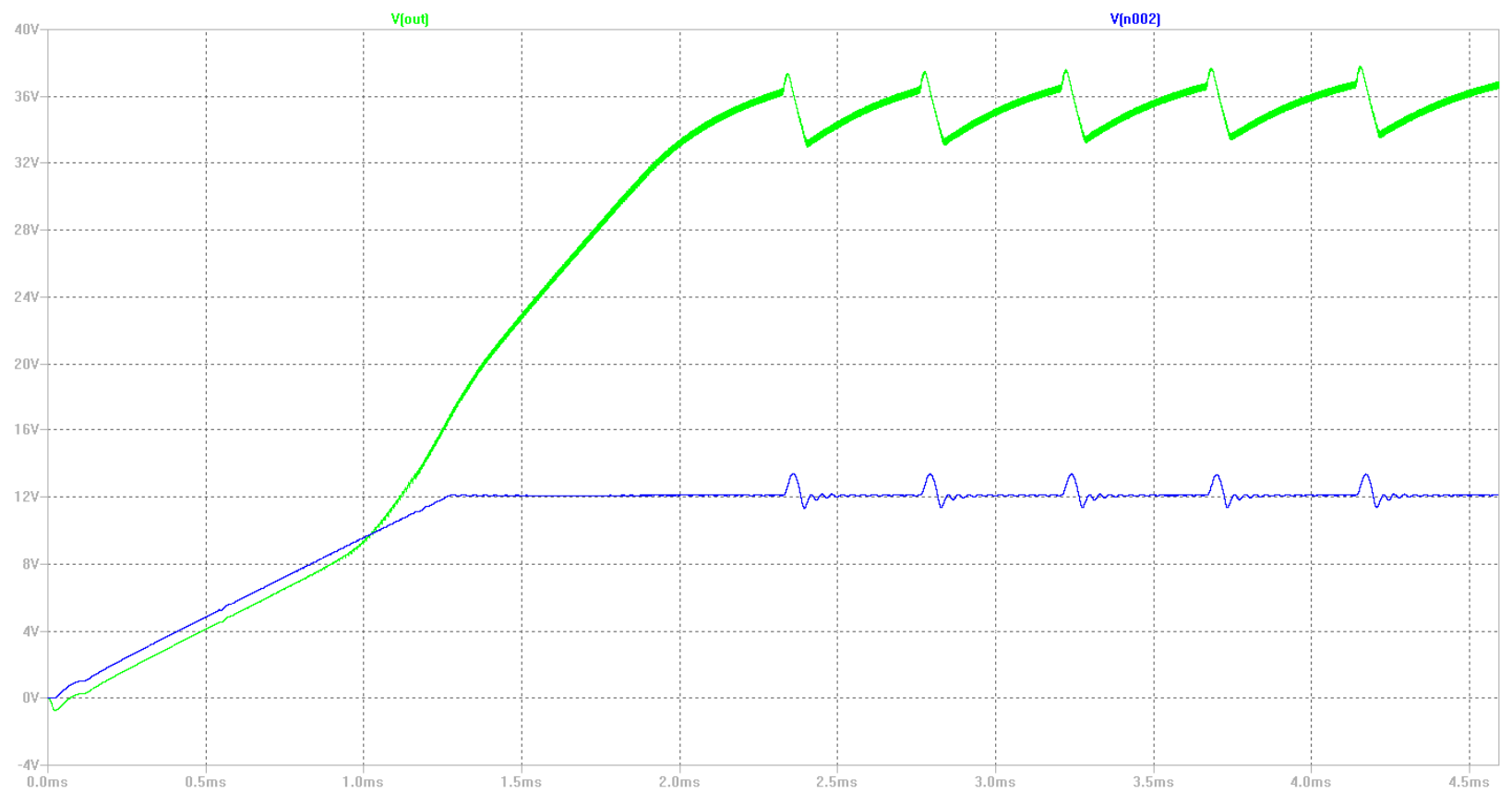

Figure B-14: Four-Phase Input, Two-Phase Output Buck-Boost Converter Individual Stage Output Voltages at 60V Input, 8A Load

The results are greatly improved from previous designs, although in steady state the voltage swings between 33 and $37 \mathrm{~V}$, which is quite a huge ripple (approximately $11 \%$, though this is under the maximum stress condition with a $60 \mathrm{~V}$ input and $8 \mathrm{~A}$ load), but this converter's output voltage reaches the optimal level, and stays within the voltage range that the Enphase micro-inverter desires. The efficiency for this time window is roughly $72.6 \%$ (Using LTSpice's math functions this project measured a $25.6 \mathrm{~V}$ average output voltage with $8 \mathrm{~A}$ average output current, along with $60 \mathrm{~V}$ average input voltage with 4.7A average input current). The only major problem with this design is the large 
number of components it uses (as well as the large physical space that it would consume). Because of the large number of components that this design utilizes, this design may end up too costly and take up too much space, considering the large number of inductors and MOSFETs in the system (which comprise most of the PCB's real estate), despite meeting almost all of this project's original required specifications. Perhaps this project can use a more simplified design instead, with 2 phases at both the input and output stages. The next section describes a such a $2 \times 2$ converter design.

\section{B1.5 Two-Phase Input and Output Stage Implementation}

Although reducing the number of phases on the input stage theoretically reduces the input stage's maximum current-carrying capacity, this project now analyzes how maintaining a large currenty-carrying capacity on the output stage affects converter performance. Figure B-15 shows the simplified, two-phase buck input and two-phase boost output design. 


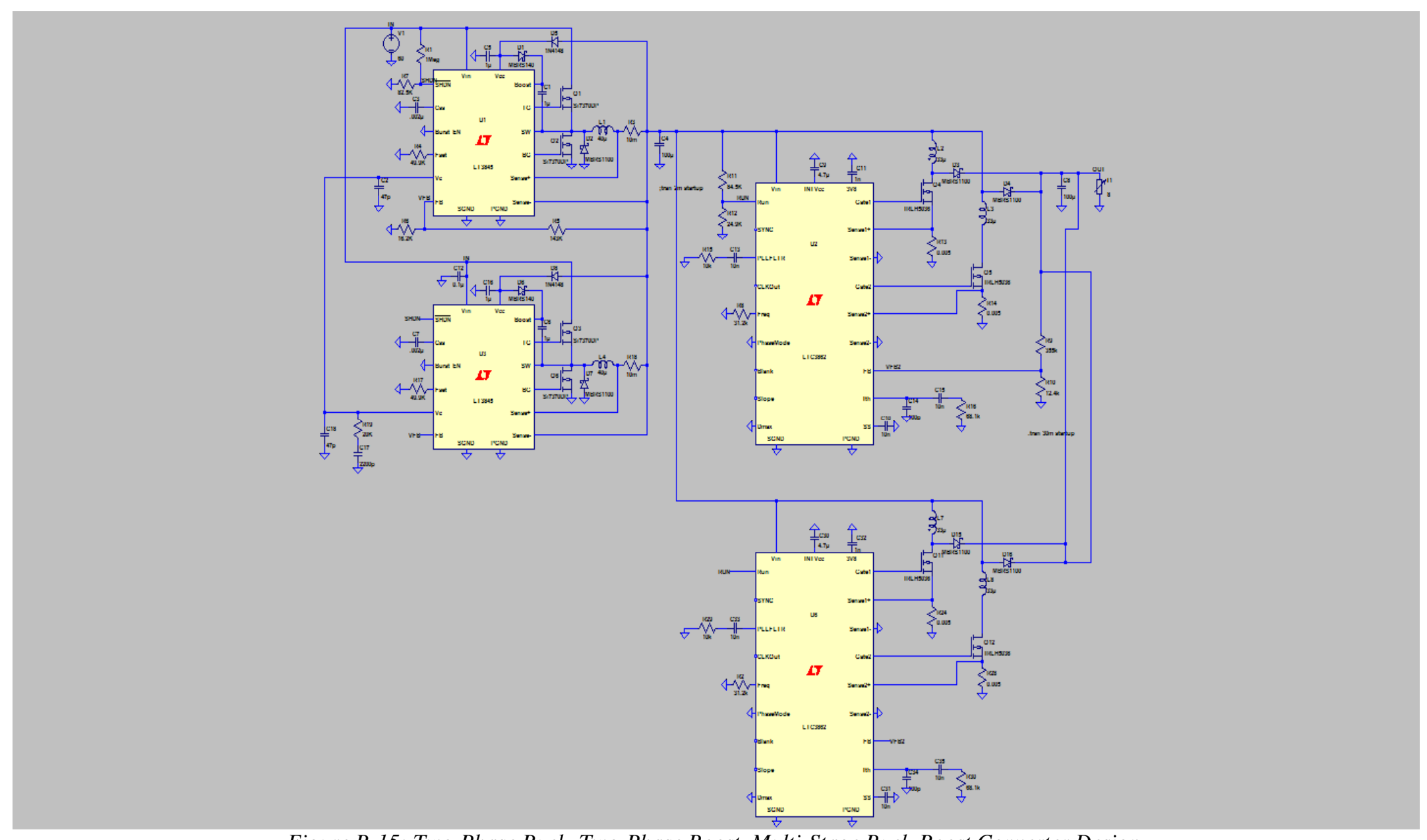

Figure B-15: Two-Phase Buck, Two-Phase Boost, Multi-Stage Buck-Boost Converter Design 
This project now tests this design using the nominal maximum stress condition with $60 \mathrm{~V}$ input and an 8A load. Figure B-16 shows the converter's operation from start-up to steady-state. The green waveform denotes the boost stage's output voltage and blue denotes the buck stage's output voltage.

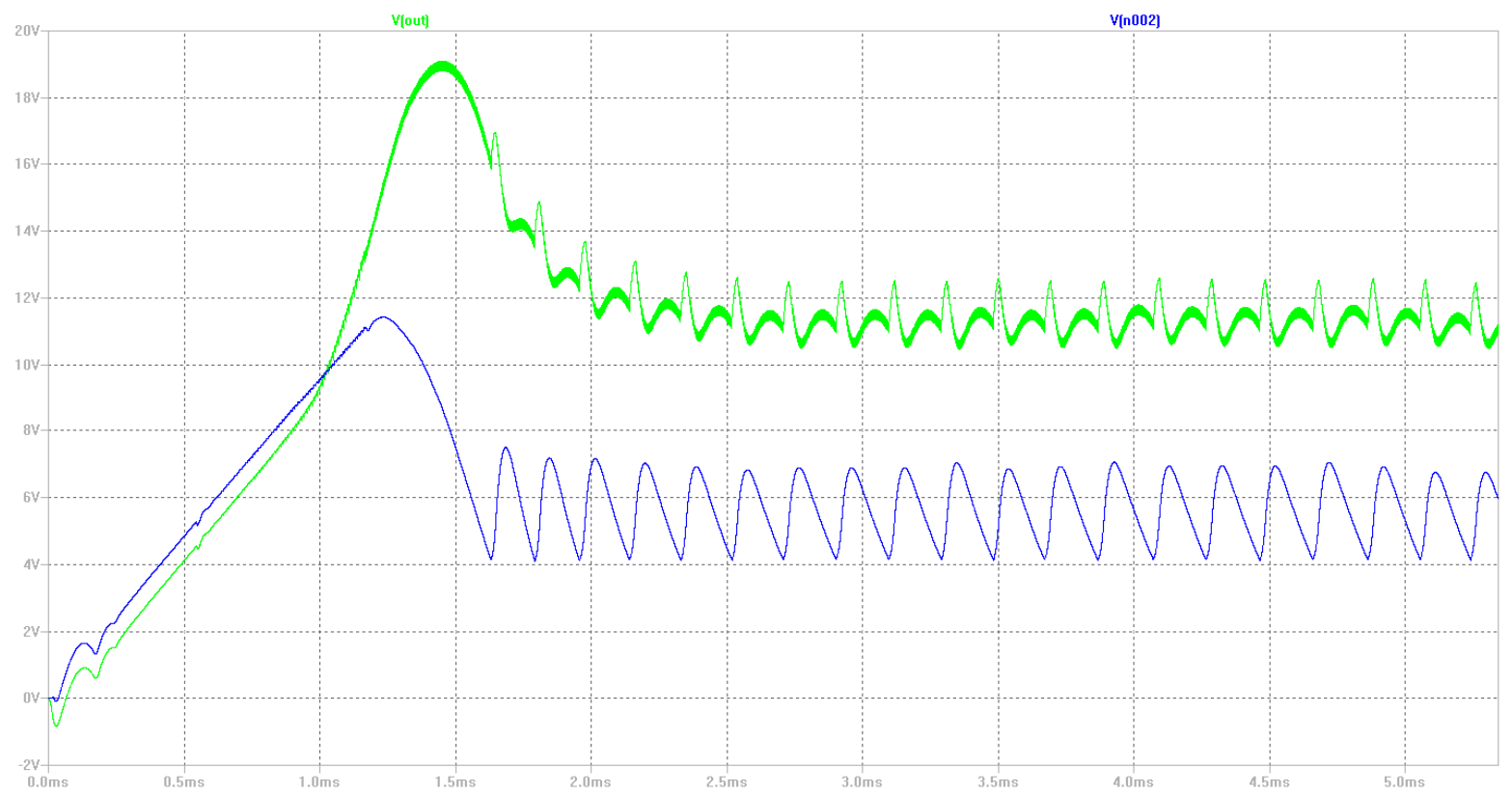

Figure B-16: Two-Phase Input, Two-Phase Output Buck-Boost Converter Individual Stage Output Voltages at 60V Input, $8 \mathrm{~A}$ Load

The ripple at the output is very high and undesirable, and overall performance is worse than that of this project's previous two-phase-input, single-phase-output design.

This project now addresses one crucial design flaw mentioned earlier - the asynchronous switching frequencies for both the input and output stages. This project then revises the two-phase design described in this section, which finally corrects the asynchronous frequency problem by increasing the switching frequency of the input stage to $500 \mathrm{kHz}$, as well as changing the inductor and filter capacitor values at each stage (in order to account for this switching frequency increase). Increasing the switching 
frequency to $500 \mathrm{kHz}$ on each stage only required changing the switching frequency resistor on the LT3845A PWM controller to $29.2 \mathrm{k} \Omega$. Now this project tests this revised two-phase design using the nominal maximum stress condition with $60 \mathrm{~V}$ input and an $8 \mathrm{~A}$ load. Figure B-17 shows the revised converter's operation from start-up to steady-state. The green waveform denotes the boost stage's output voltage and blue denotes the buck stage's output voltage.

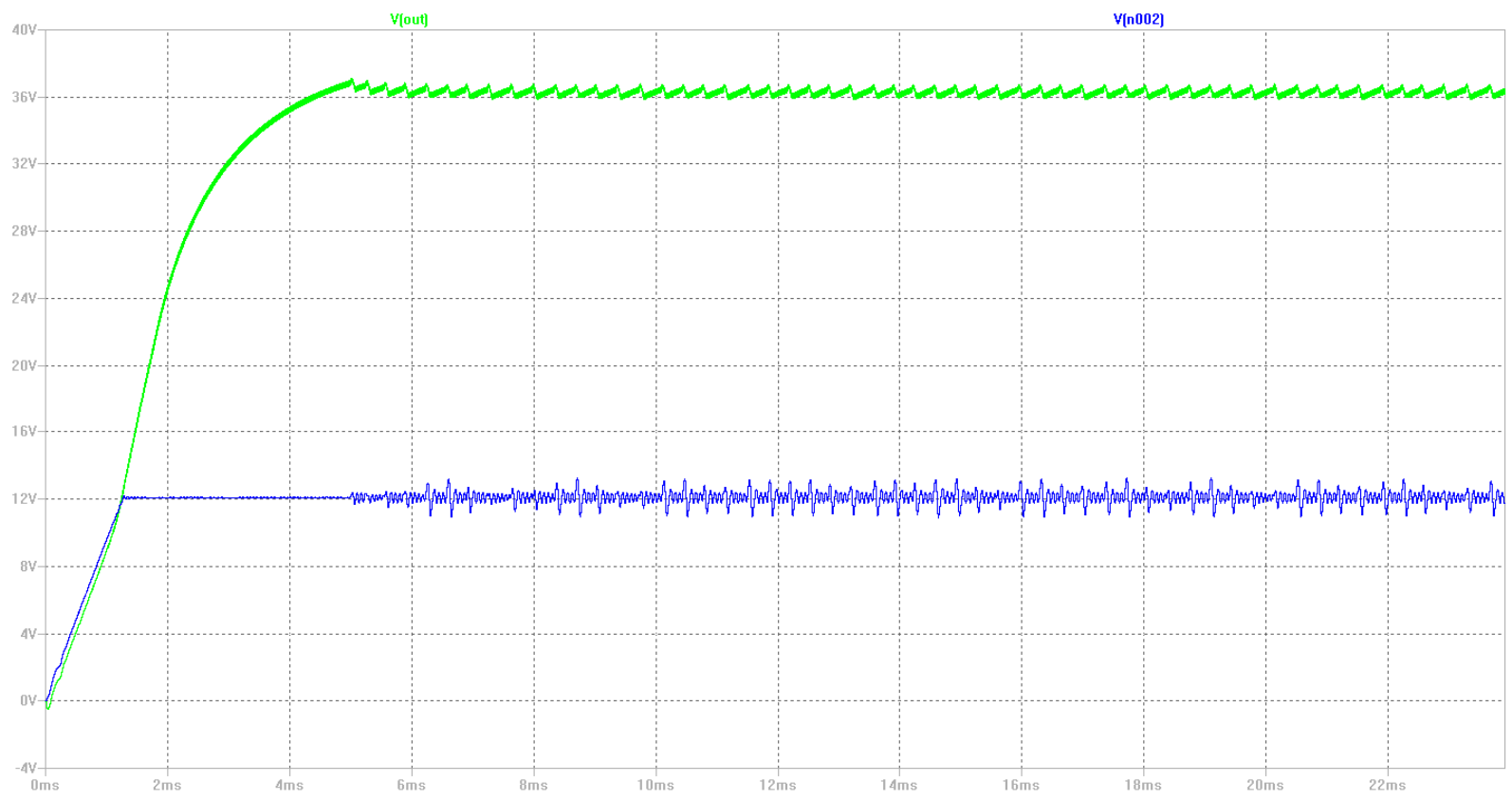

Figure B-17: Two-Phase Input, Two-Phase Output Buck-Boost Converter Individual Stage Output Voltages at 60V Input, 8A Load, Using $500 \mathrm{kHz}$ Switching Frequencies for Each Stage

This result is greatly improved from previous designs, and now the output reaches the 36V optimal voltage for the Enphase micro-inverter. Output ripple voltage is approximately $0.75 \mathrm{~V}$, which is about $2.08 \%$ with respect to the $36 \mathrm{~V}$ output voltage. The output ripple shown in figure B-17 is more desirable than that in this project's original four-phase-input, two-phase-output design from appendix B1.4 because of the higher and synchronous switching frequency for both stages. Average output voltage is $36.236 \mathrm{~V}$ and 
average output current is $8 \mathrm{~A}$. Average input voltage is $60 \mathrm{~V}$ and average input current is 6.14A. This translates to $78.6 \%$ efficiency. However, there is still noticeable oscillation at the input stage, though again, this project does not require a completely clean buck input stage output voltage. This converter design is feasible for this project's purpose, but the next section attempts shrinking the design further in terms of component count and physical space.

B1.6 Single-Phase Input and Output Stage Implementation at $500 \mathrm{kHz}$ Switching Frequency

Figure B-18 shows this project's pure single-phase converter design, but using a $500 \mathrm{kHz}$ switching frequency for both the input and output stages. 


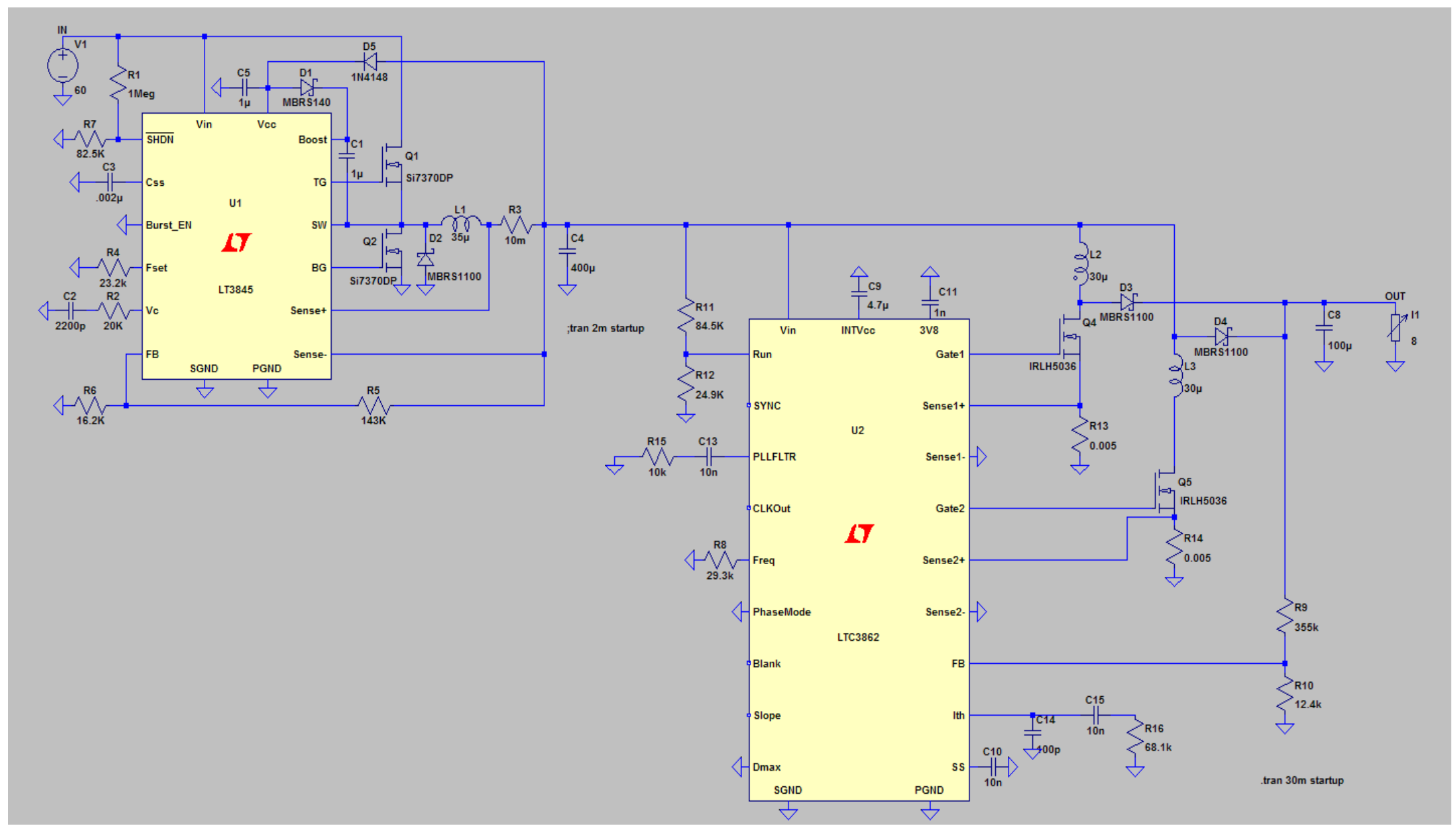

Figure B-18: Single-Phase, Multi-Stage Buck-Boost Converter Design with 500 kHz Switching Frequency for Each Stage 
This project now tests this design using the nominal maximum stress condition with $60 \mathrm{~V}$ input and an 8A load. Figure B-19 shows the converter's operation from start-up to steady-state. The green waveform denotes the boost stage's output voltage and blue denotes the buck stage's output voltage.

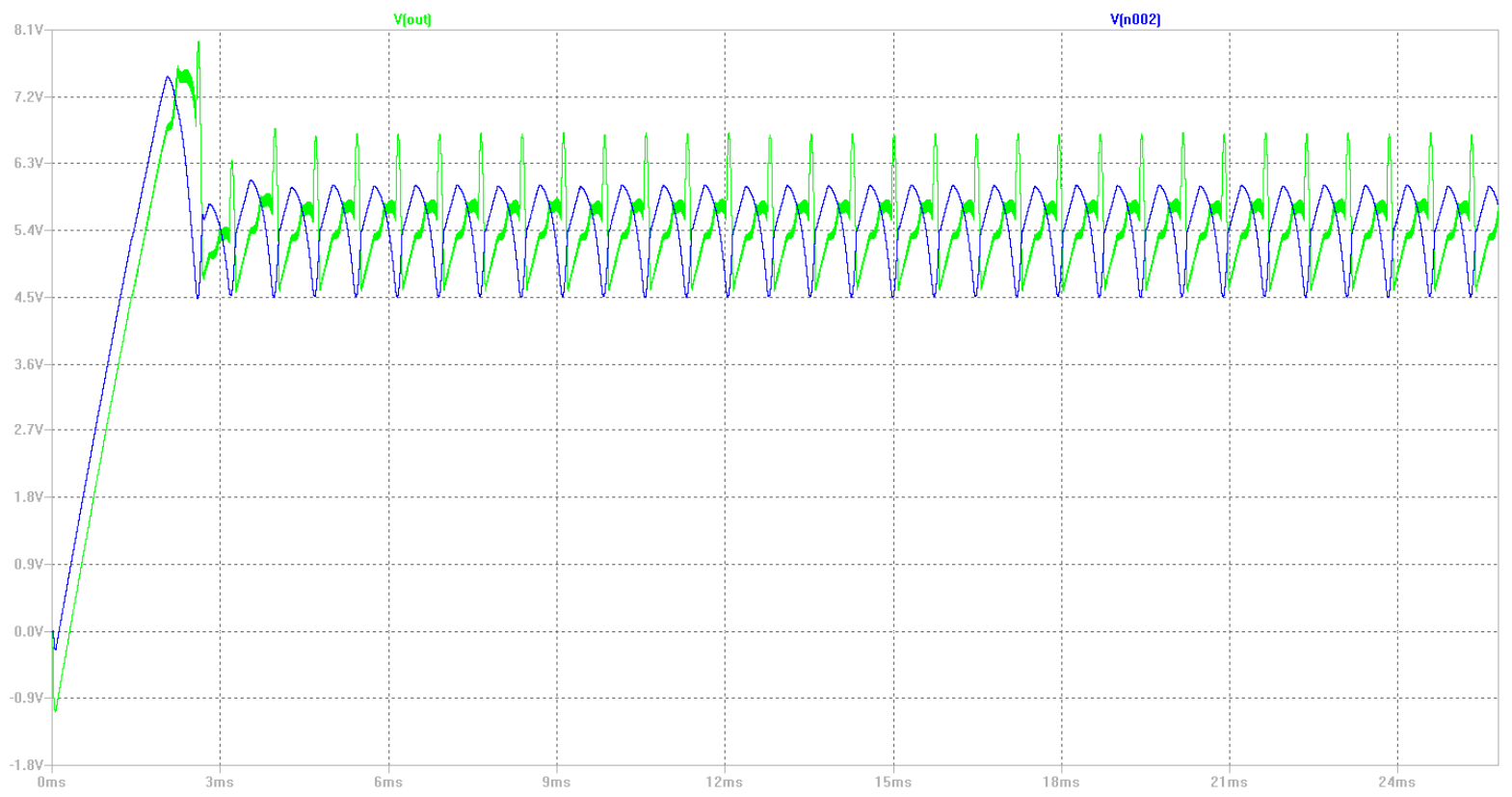

Figure B-19: Single-Phase, Multi-Stage Buck-Boost Converter Individual Stage Output Voltages at 60V Input, 8A Load, Using $500 \mathrm{kHz}$ Switching Frequencies for Each Stage

Even when using the same switching frequencies for both the input and output stages, the output stage still cannot draw the current required that the input stage requires under maximum load. The final output voltage hovers between $4.5 \mathrm{~V}$ to $7 \mathrm{~V}$, with heavy ripple. Therefore, using a purely single-phase design for this converter is completely unfeasible. The next section describes using a single-phase input stage and two-phase output stage variation of this design. 
B1.7 Single-Phase Input Stage and Two-Phase Output Stage Implementation at 500 kHz Switching Frequency

This project now determines if a single-phase-input, two-phase-output design can produce close to its desired electrical values before falling back to the pure two-phase design. Figure B-20 shows this design, again using the same components as with previous designs and a $500 \mathrm{kHz}$ switching frequency for each stage. 


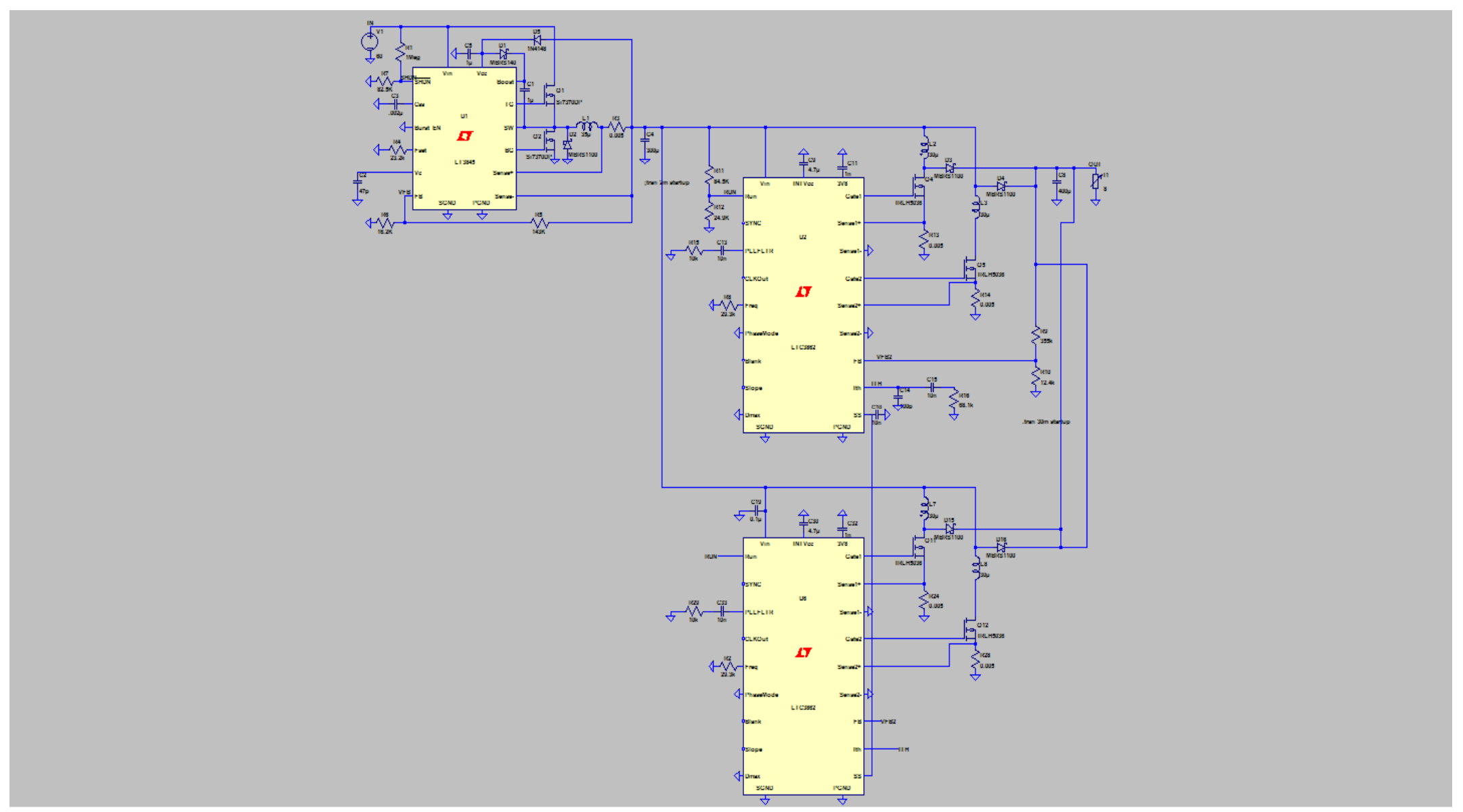


This project now tests this design using the nominal maximum stress condition with $60 \mathrm{~V}$ input and an 8A load. Figure B-21 shows the converter's operation from start-up to steady-state. The green waveform denotes the boost stage's output voltage and blue denotes the buck stage's output voltage.

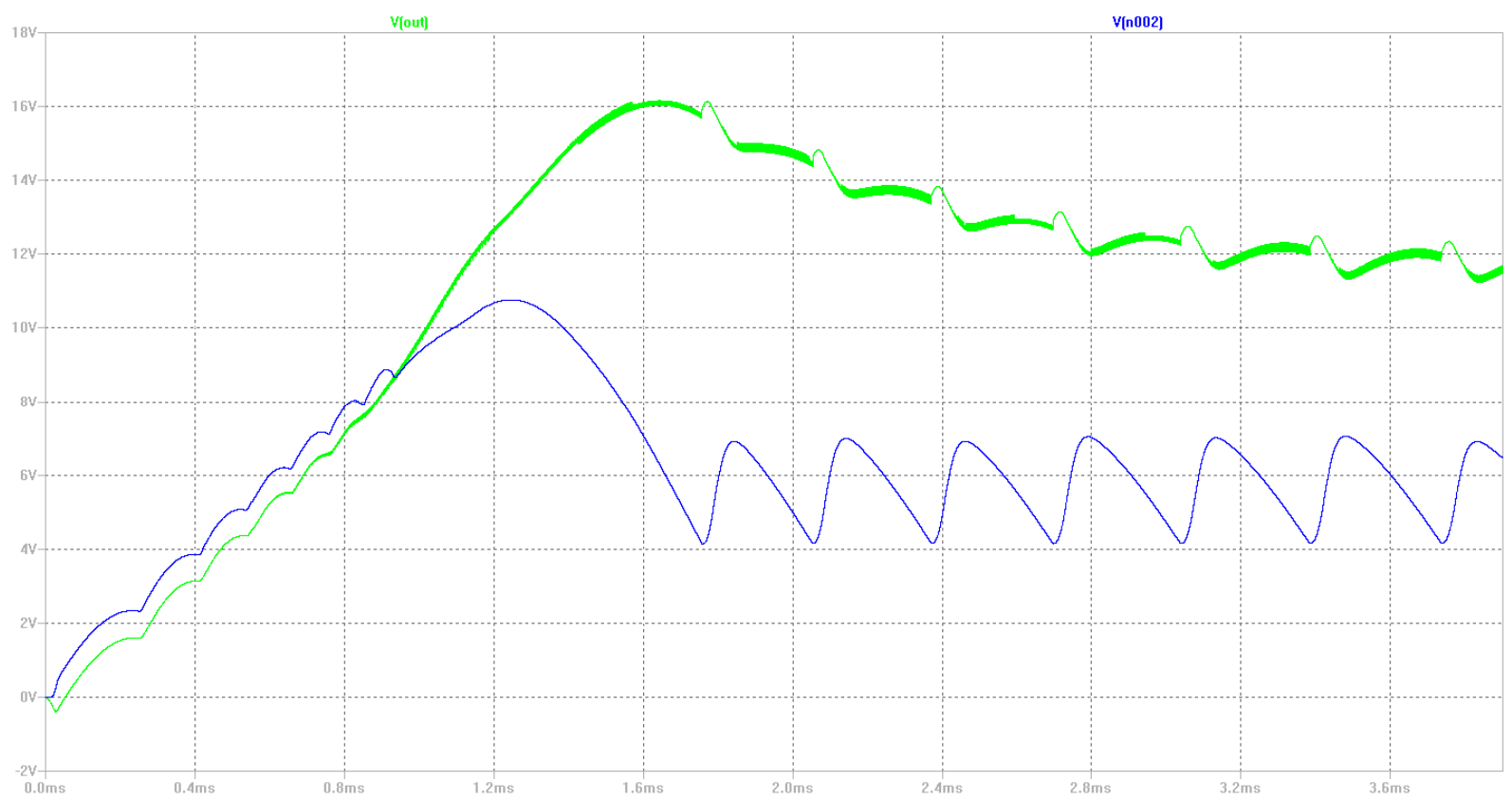

Figure B-21: Single-Phase Buck, Two-Phase Boost, Multi-Stage Buck-Boost Converter Output Voltage with $60 \mathrm{~V}$ Input and 8 A Load

The results are very similar to the purely single-phase design. The input stage isn't pushing enough current to the output stage for regulating the proper output power level. Thus for this project uses the dual-phase configuration from figure B-15. In the next section this project attempts using a lower switching frequency for the dual-phase converter from figure B-15 for determining whether such a change improves overall converter operation and efficiency. 


\section{B1.8 Two-Phase Input and Output Stage Implementation Analysis at $500 \mathrm{kHz}$ and}

\section{0 kHz Switching Frequencies}

This project now examines the individual inductor currents of the dual-phase converter configuration and make sure that the converter itself is not operating in discontinuous conduction mode (DCM). Figure B-22 shows inductor $\mathrm{L}_{1}$ 's current (from the input stage, denoted by the red waveform) under maximum input voltage and load. The green waveform denotes the boost stage's output voltage and blue denotes the buck stage's output voltage.

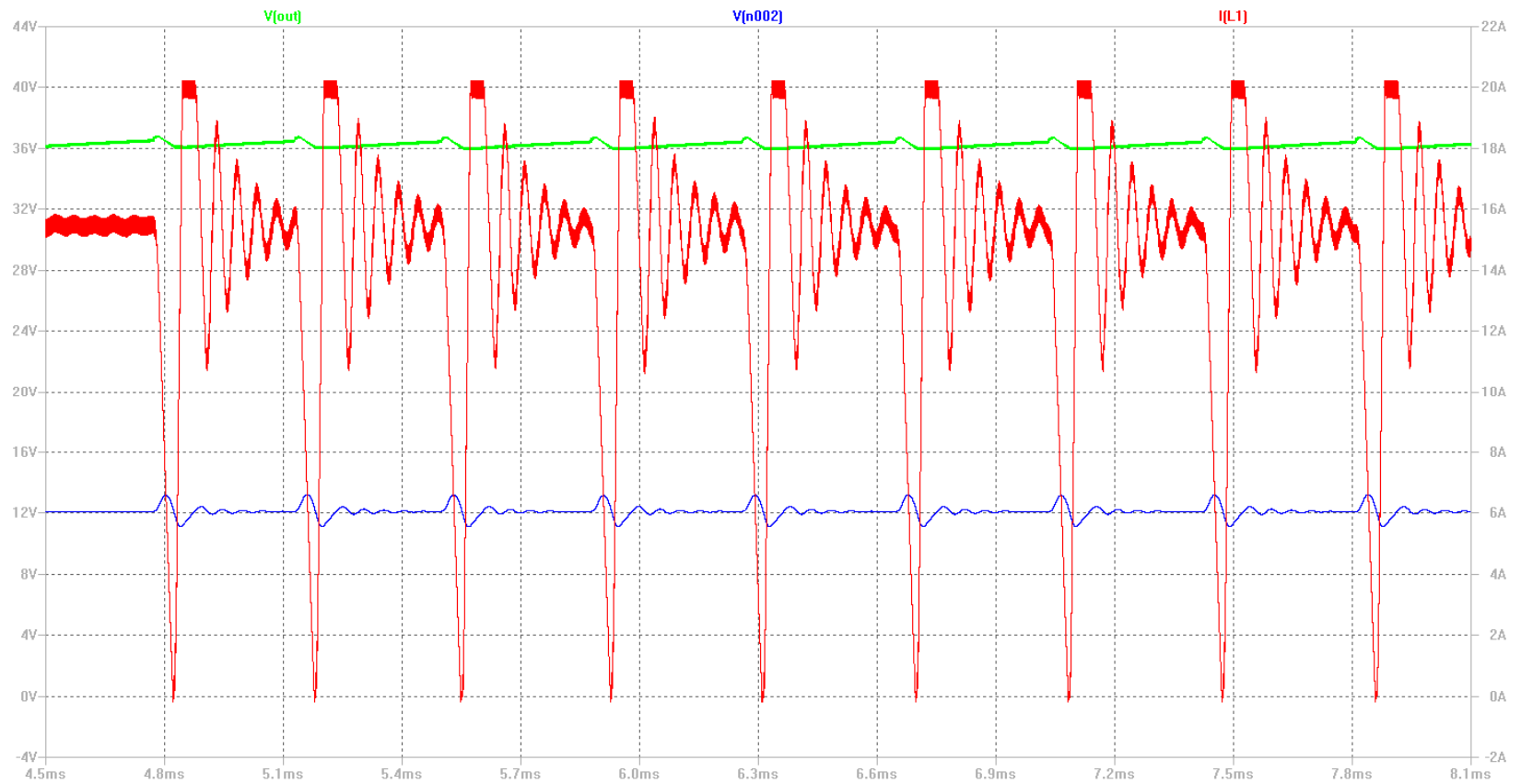

Figure B-22: Inductor Current of $L_{1}$ (Input Stage) of Dual-Phase Buck-Boost Converter Under Maximum Input and Load

$\mathrm{L}_{1}$ 's current reaches $0 \mathrm{~A}$, but not for a significant time interval. Thus the converter operates in BCM. While this condition isn't ideal, it does still allow the converter to properly regulate its output, though it operates dangerously close to DCM. The inductor required for the physical converter requires 30A current-carrying capacity. Figure B-23 
shows inductor L4's (located in the buck stage's second phase, denoted by the red waveform) current under maximum input and load. The green waveform denotes the boost stage's output voltage and blue denotes the buck stage's output voltage.

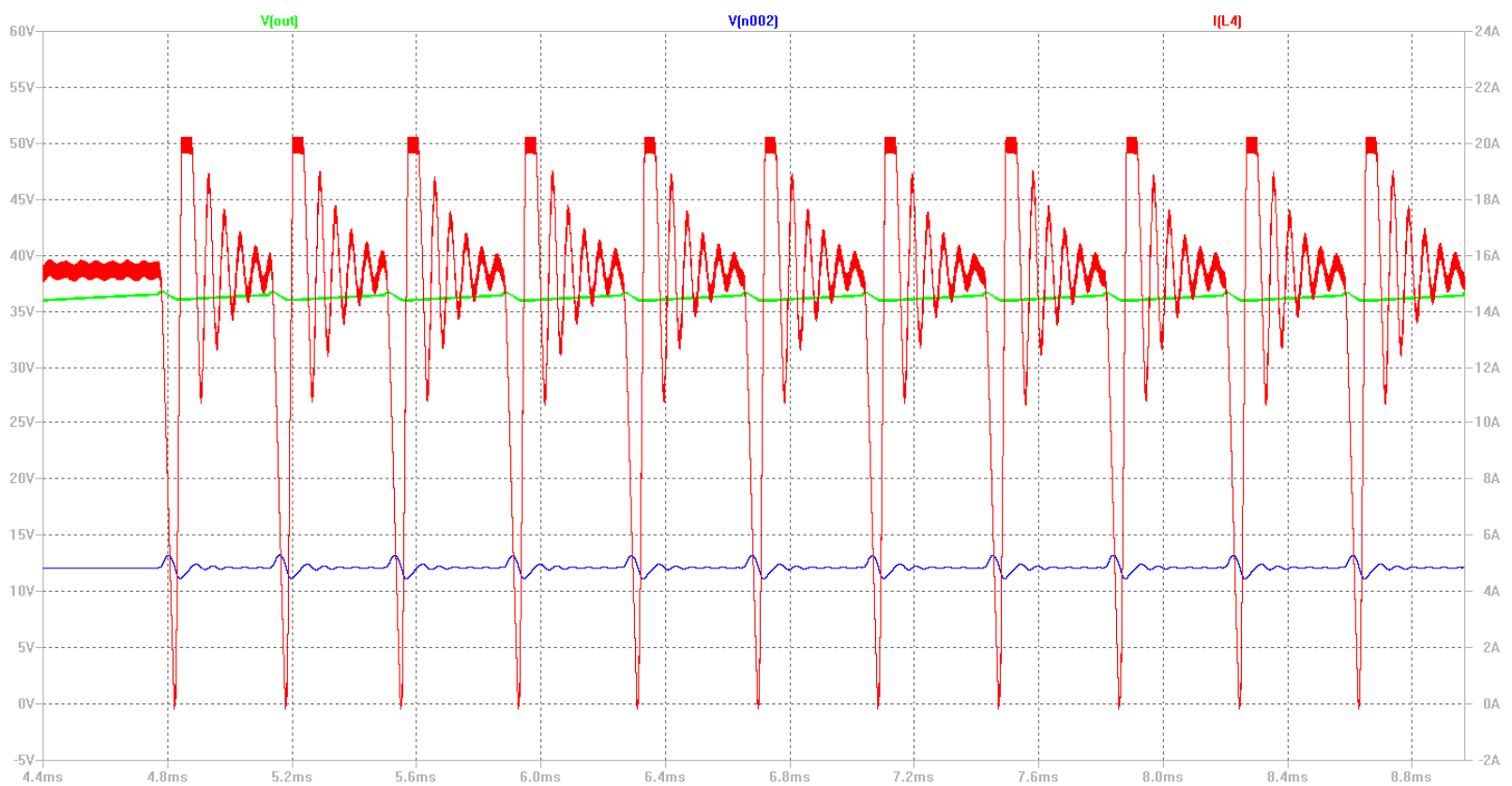

Figure B-23: Inductor Current of $L_{4}$ (Input Stage) of Dual-Phase Buck-Boost Converter Under Maximum Input and Load

The result is identical to that of $\mathrm{L}_{1}$ 's. These results show that each phase operates synchronously with the other. Now this project examines the inductor currents at the output boost stage. This project first examines $\mathrm{L}_{2}$, which is the output stage's primary (high-side) inductor. Figure B-24 shows $\mathrm{L}_{2}$ 's current under maximum input and load (denoted by the red waveform). The green waveform denotes the boost stage's output voltage and blue denotes the buck stage's output voltage. 


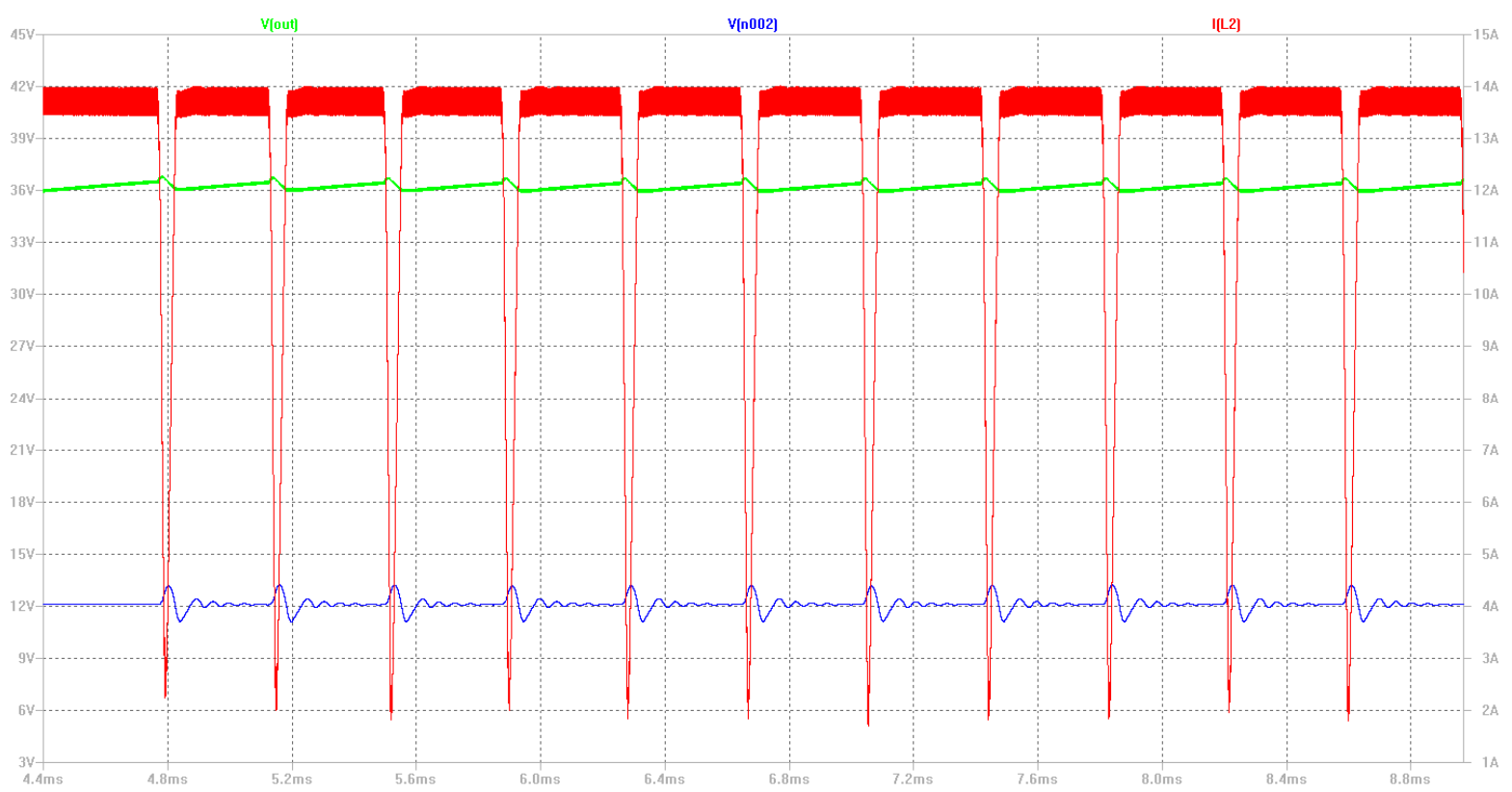

Figure B-24: Inductor Current of $L_{2}$ (Output Stage) of Dual-Phase Buck-Boost Converter Under Maximum Input and Load

No problems occur with $\mathrm{L}_{2}$, as it operates in $\mathrm{CCM}\left(\mathrm{L}_{2}\right.$ 's current never reaches $0 \mathrm{~A}$ for any time period). Next this project examines $\mathrm{L}_{3}$, which is the output stage's secondary (lowside) inductor. Figure B-25 shows $\mathrm{L}_{3}$ 's current under maximum input and load (denoted by the red waveform). The green waveform denotes the boost stage's output voltage and blue denotes the buck stage's output voltage. 


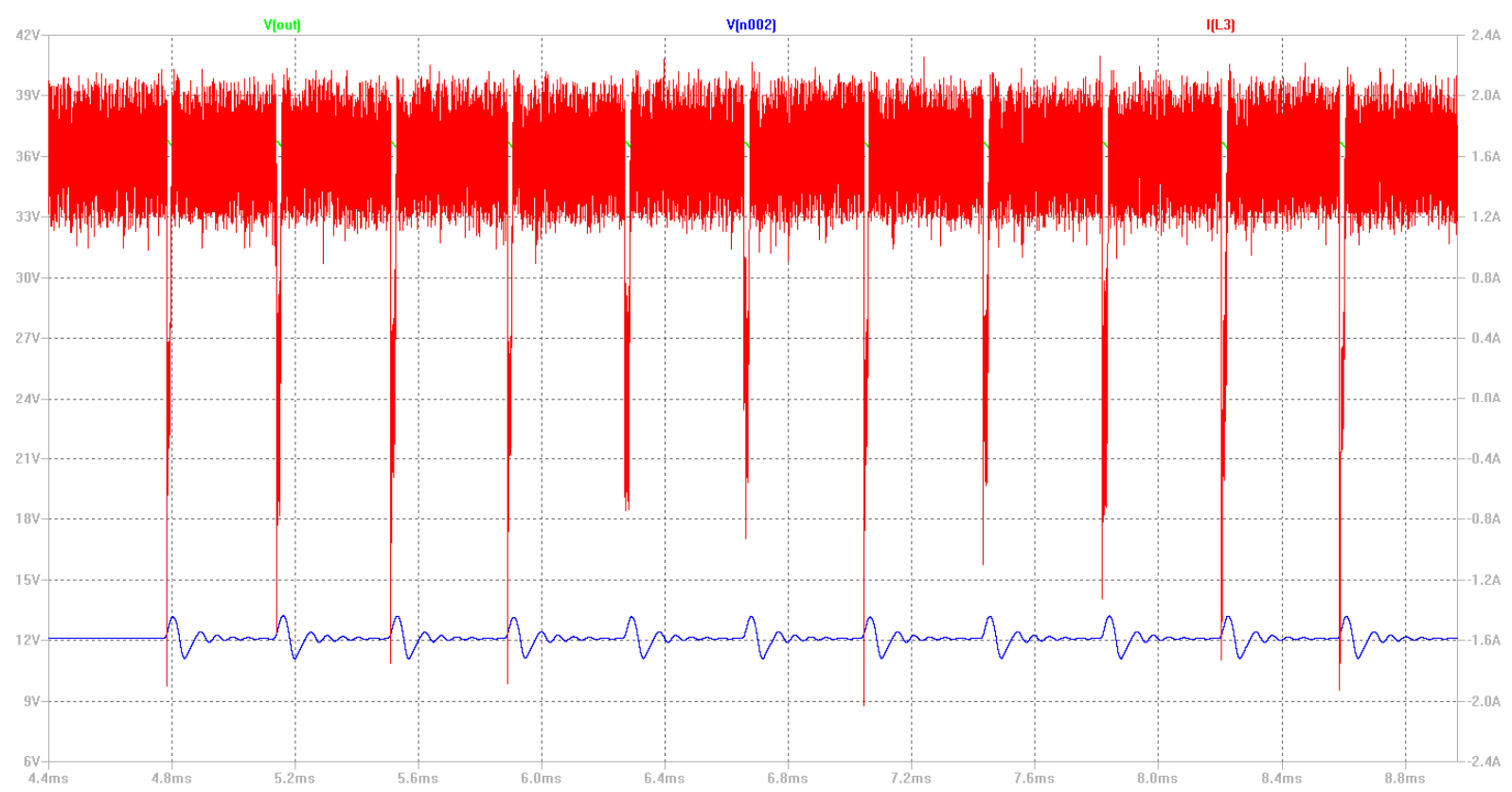

Figure B-25: Inductor Current of $L_{3}$ (Output Stage) of Dual-Phase Buck-Boost Converter Under Maximum Input and Load

$\mathrm{L}_{3}$ 's overall current is much lower than $\mathrm{L}_{2}$ 's, hence the more noticeable ripple with respect to the current scale that this simulation uses. Like $\mathrm{L}_{2}$, its current never reaches $0 \mathrm{~A}$, assuring that converter still operates in CCM. Next this project examines $\mathrm{L}_{7}$, which is the output stage's primary (high-side) inductor located in the output stage's second phase. Figure B-26 hows L7's current under maximum input and load (denoted by the red waveform). The green waveform denotes the boost stage's output voltage and blue denotes the buck stage's output voltage. 


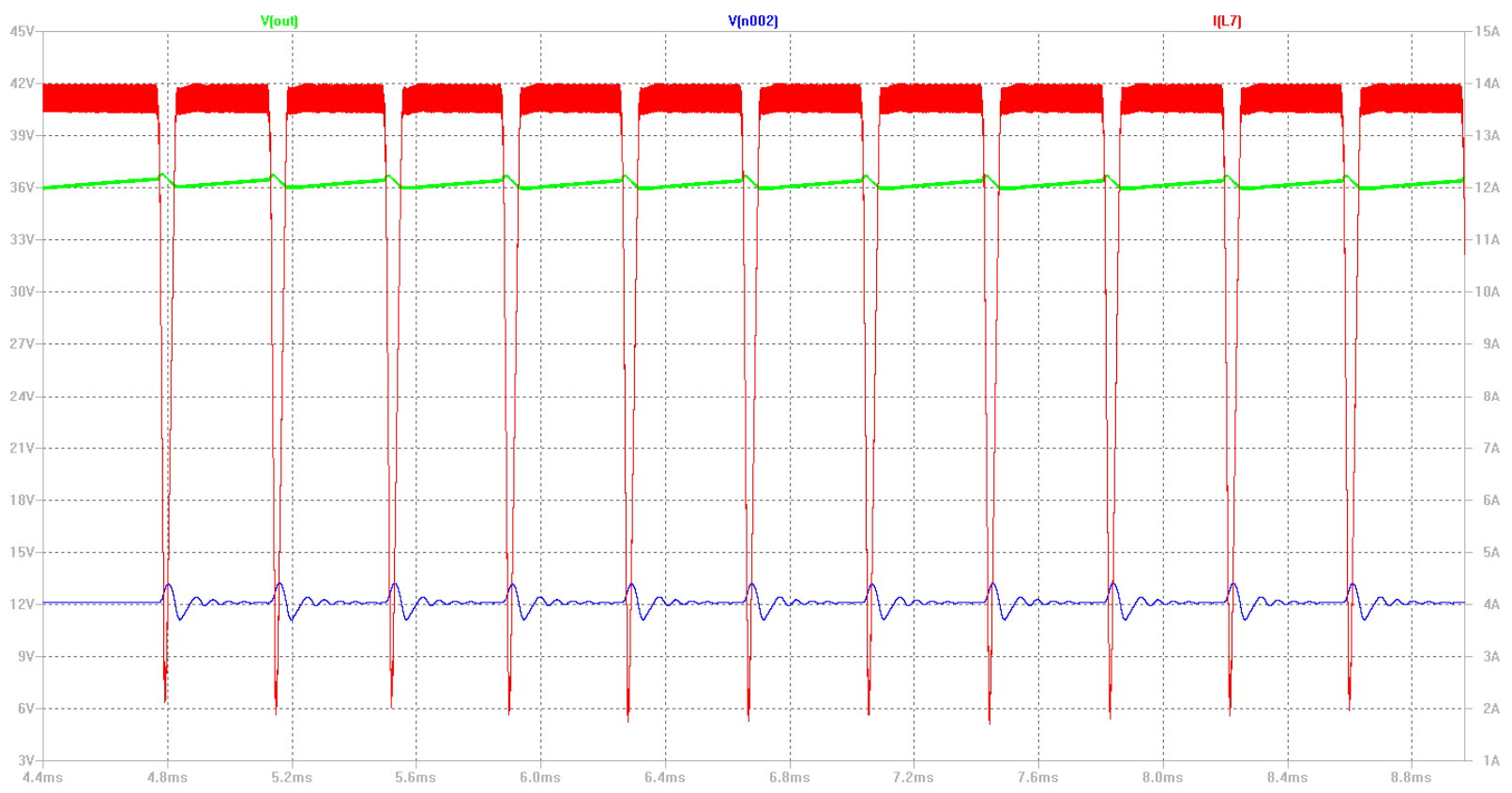

Figure B-26: Inductor Current of $L_{3}$ (Output Stage) of Dual-Phase Buck-Boost Converter Under Maximum Input and Load

$\mathrm{L}_{7}$ 's current is identical to that of $\mathrm{L}_{2}$ 's, and does not stay at $0 \mathrm{~A}$ for any significant time period. This project next examines $\mathrm{L}_{8}$, which is the output stage's secondary (low-side) inductor located in the output stage's second phase. Figure B-27 shows L8's current under maximum input and load (denoted by the red waveform). The green waveform denotes the boost stage's output voltage and blue denotes the buck stage's output voltage. 


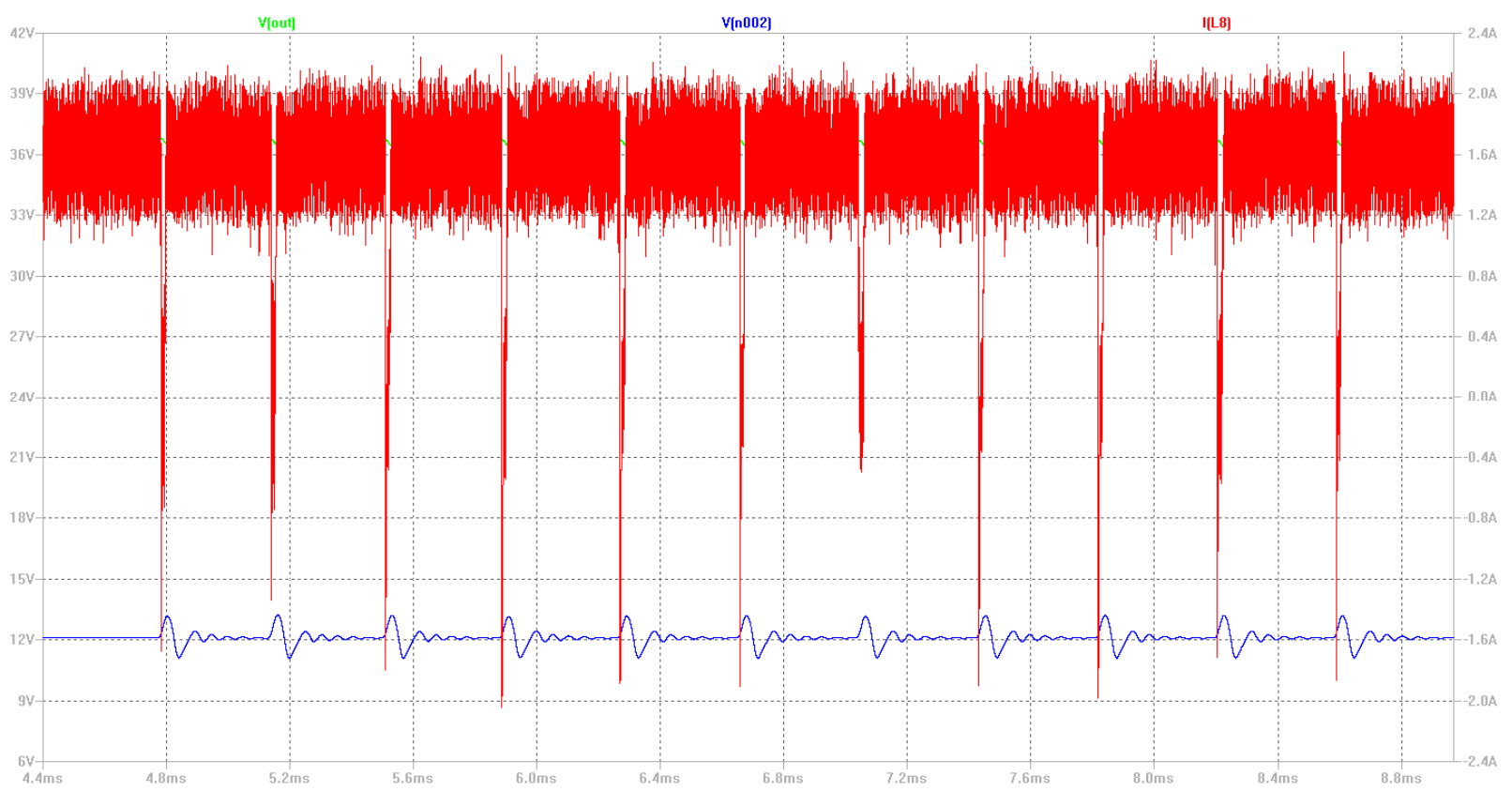

Figure B-27: Inductor Current of $L_{8}$ (Output Stage) of Dual-Phase Buck-Boost Converter Under Maximum Input and Load

$\mathrm{L}_{8}$ 's current is identical to that of $\mathrm{L}_{3}$ 's; because of the simulation's current scale there is more noticeable ripple than with the current through the high-side inductors. Like all of the other inductors, its current never reaches $0 \mathrm{~A}$, assuring that the converter still operates in CCM. Furthermore, because the currents through each high-side and low-side inductor are identical to their respective counterparts, each phase in the output boost stage operates synchronously.

This project next examines the drain currents through each switching MOSFET and ensures that they are within a reasonable operation level. This project first examines Q1's (the primary, or high-side MOSFET in the buck stage's first phase) drain current. Figure B-28 shows $\mathrm{Q}_{1}$ 's drain current under maximum input and load (denoted by the red waveform). The green waveform denotes the boost stage's output voltage and blue denotes the buck stage's output voltage. 


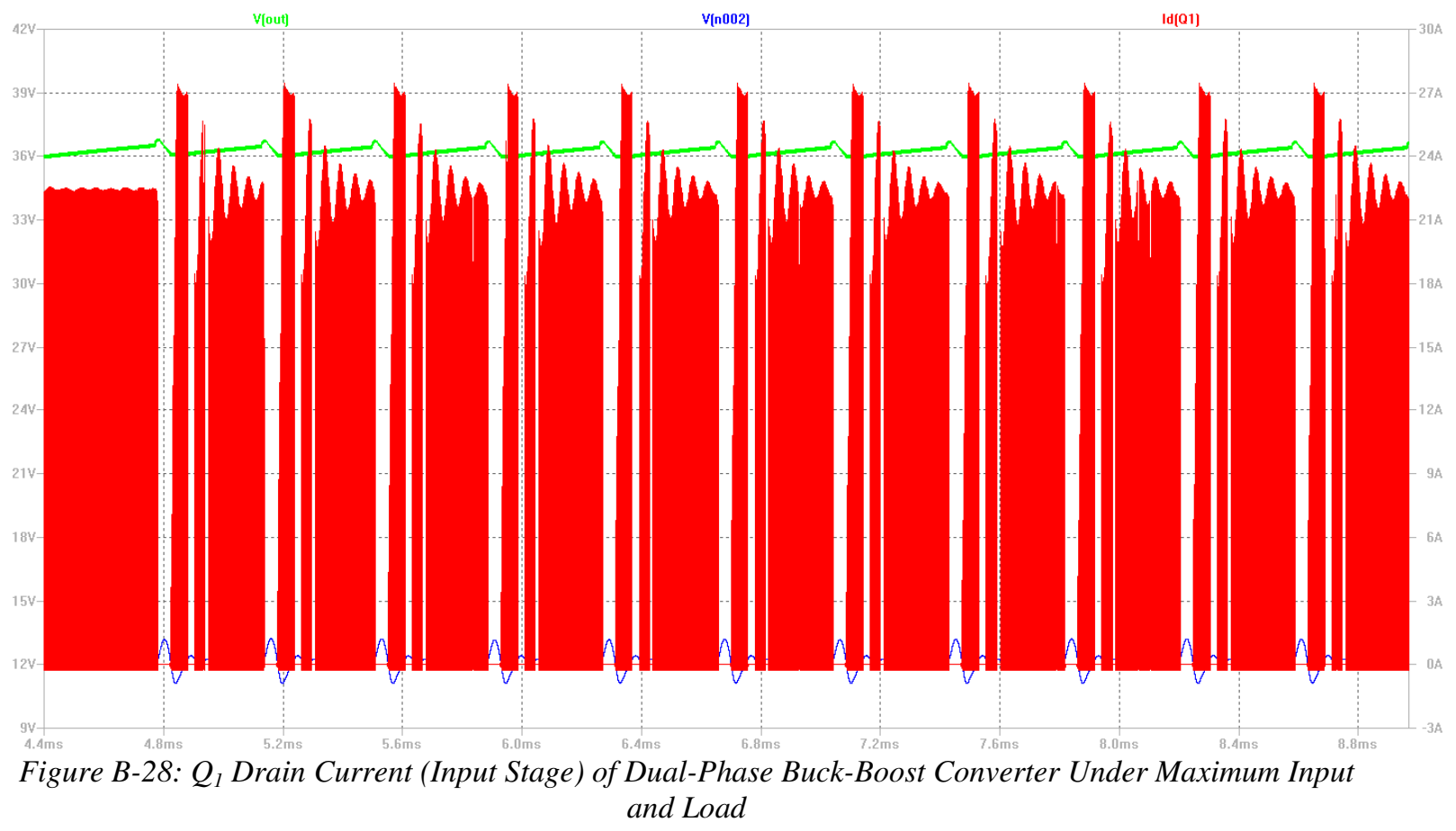

$\mathrm{Q}_{1}$ 's drain current reaches a $27.5 \mathrm{~A}$ peak, and the average drain current for this simulation time window is $3.13 \mathrm{~A}$. This result is not unreasonable, however, this project must ensure that its converter MOSFETs withstand at least 30A for their drain currents. Overall average drain current for $\mathrm{Q}_{1}$, however, is lower than the 6.25A (per phase) drain current current rating that this project established when determining the buck stage's component ratings earlier. This project next examines $\mathrm{Q}_{2}$ 's drain current. $\mathrm{Q}_{2}$ is the secondary (lowside) switching MOSFET located in the input buck stage's first phase. Figure B-29 shows $\mathrm{Q}_{2}$ 's drain current (in red) under maximum input and load (denoted by the red waveform). The green waveform denotes the boost stage's output voltage and blue denotes the buck stage's output voltage. 


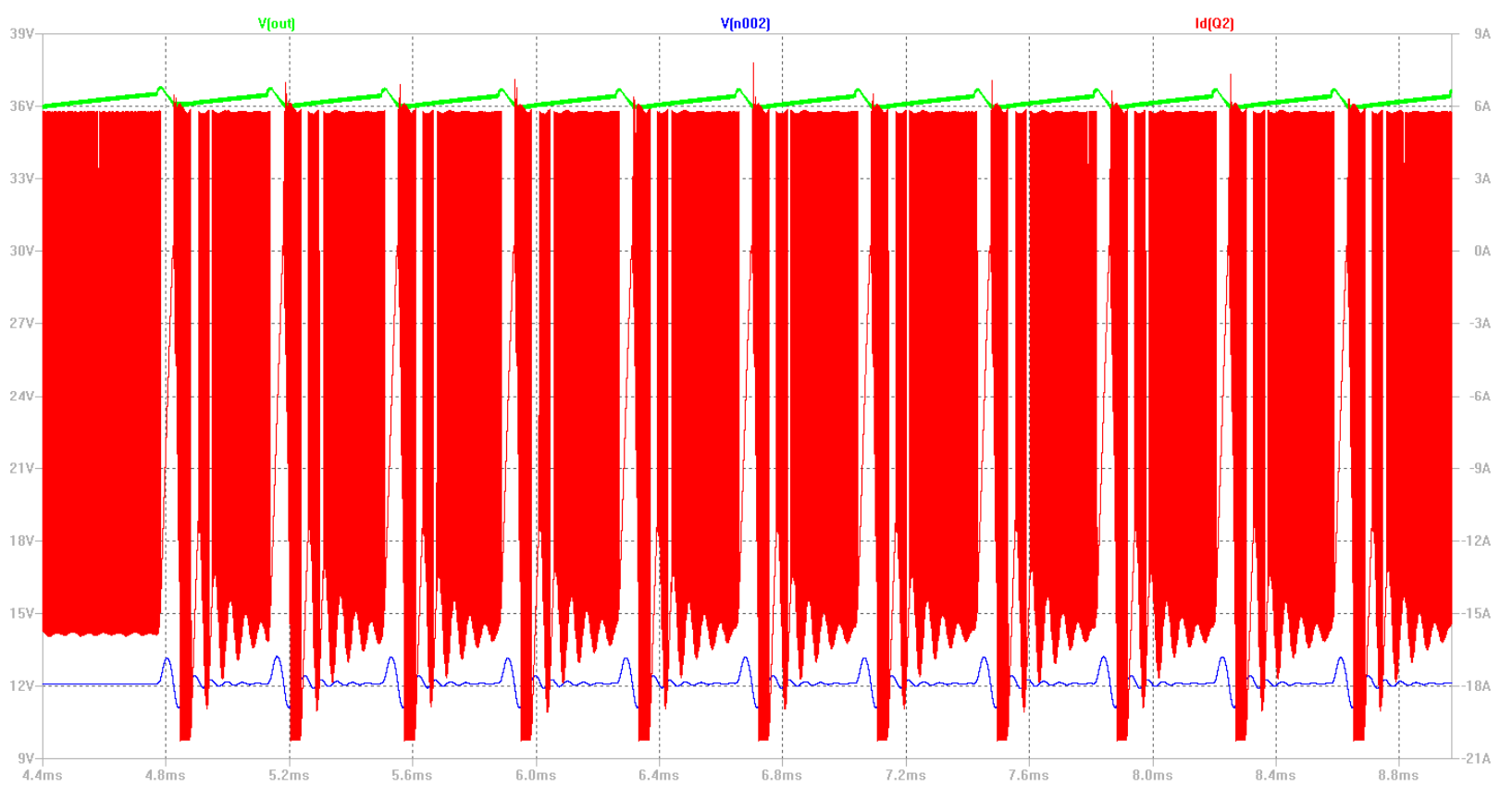

Figure B-29: $Q_{2}$ Drain Current (Input Stage) of Dual-Phase Buck-Boost Converter Under Maximum Input and Load

$\mathrm{Q}_{2}$ 's drain current ranges from $6 \mathrm{~A}$ to $-20.5 \mathrm{~A}$ (total peak amplitude is $26.5 \mathrm{~A}$ ). Overall average drain currrent for this simulation time window is -11A. This result is still within reasonable operation levels, as MOSFETs that can handle average currents up to 20A exist on the commercial market. From the inductor current results, $\mathrm{Q}_{3}$ 's drain current characteristics are the same that of $\mathrm{Q}_{1}$ 's (as both are buck stage high-side MOSFETs, just located in different phases), and Q6's drain current characteristics are the same as that of $\mathrm{Q}_{2}$ 's (both of these are buck stage low-side MOSFETs located in different phases), because of the converter's synchronous nature for each individual phase. Thus this project does not require any drain current plots for $\mathrm{Q}_{3}$ and $\mathrm{Q}_{6}$, as that data is redundant. However, this project now examines the drain currents for the MOSFETs in the output boost stage. This project begins its examination with with $\mathrm{Q}_{4}$, which is the high side MOSFET located in the output boost stage's primary phase. Figure B-30 shows $\mathrm{Q}_{4}$ 's 
drain current (denoted by the red waveform) at maximum input voltage and load. The green waveform denotes the boost stage's output voltage and blue denotes the buck stage's output voltage.

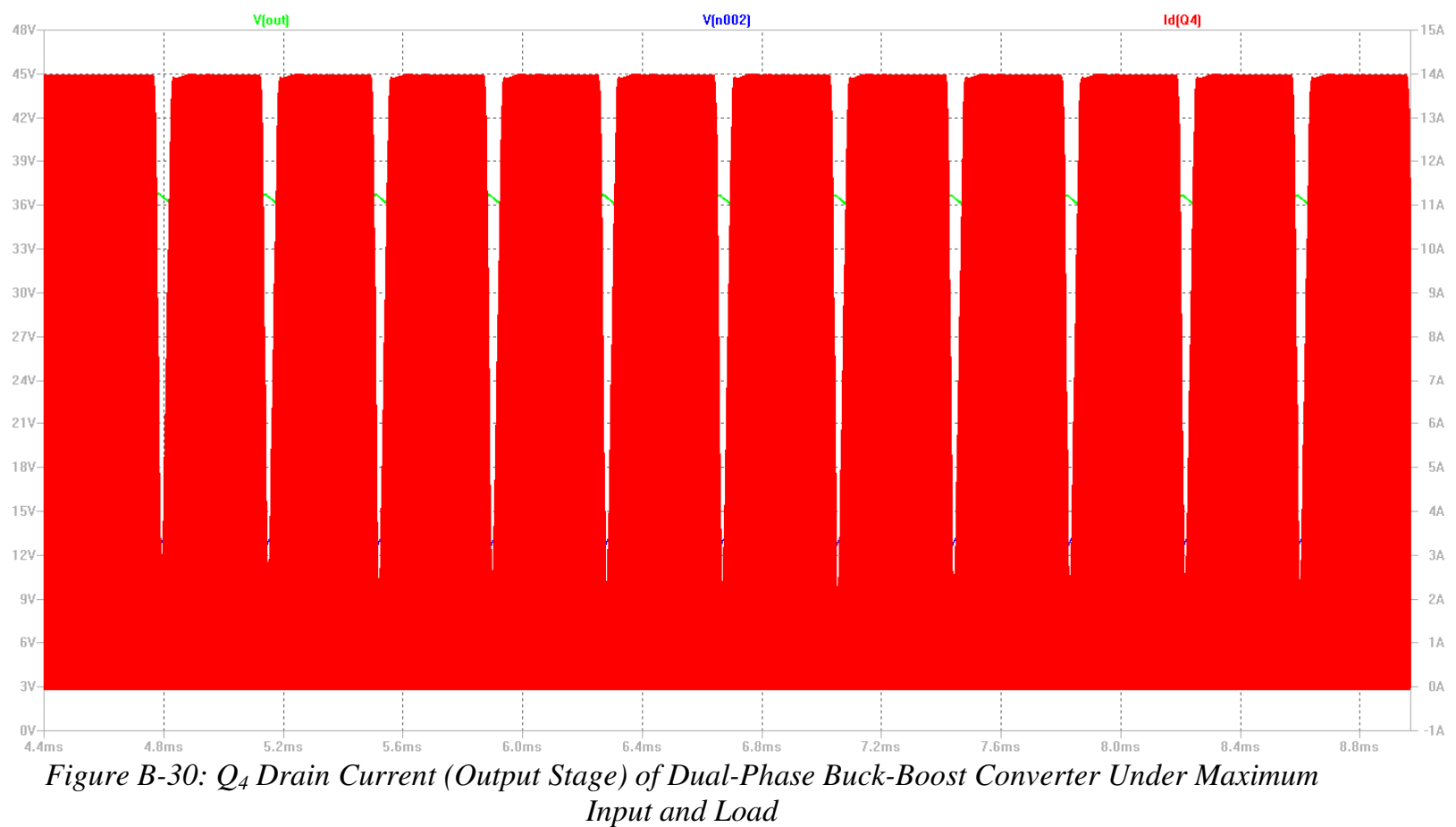

$\mathrm{Q}_{4}$ 's drain current reaches a 14A peak, and the average drain current for this simulation time window is $8.86 \mathrm{~A}$. The original converter design equations required for the MOSFETs in the output boost stage handling at least $112.5 \mathrm{~A}$, however, this simulation result is far from the original specified minimum drain current rating. The MOSFET switches at the desired $500 \mathrm{kHz}$ frequency, which explains the proximity of each individual rise and fall in its drain current. This project now examines $\mathrm{Q}_{5}$, the low-side MOSFET located in the output boost stage's primary phase. Figure B-31 shows Q5's drain current (denoted by the red waveform) at maximum input voltage and load. The 
green waveform denotes the boost stage's output voltage and blue denotes the buck stage's output voltage.

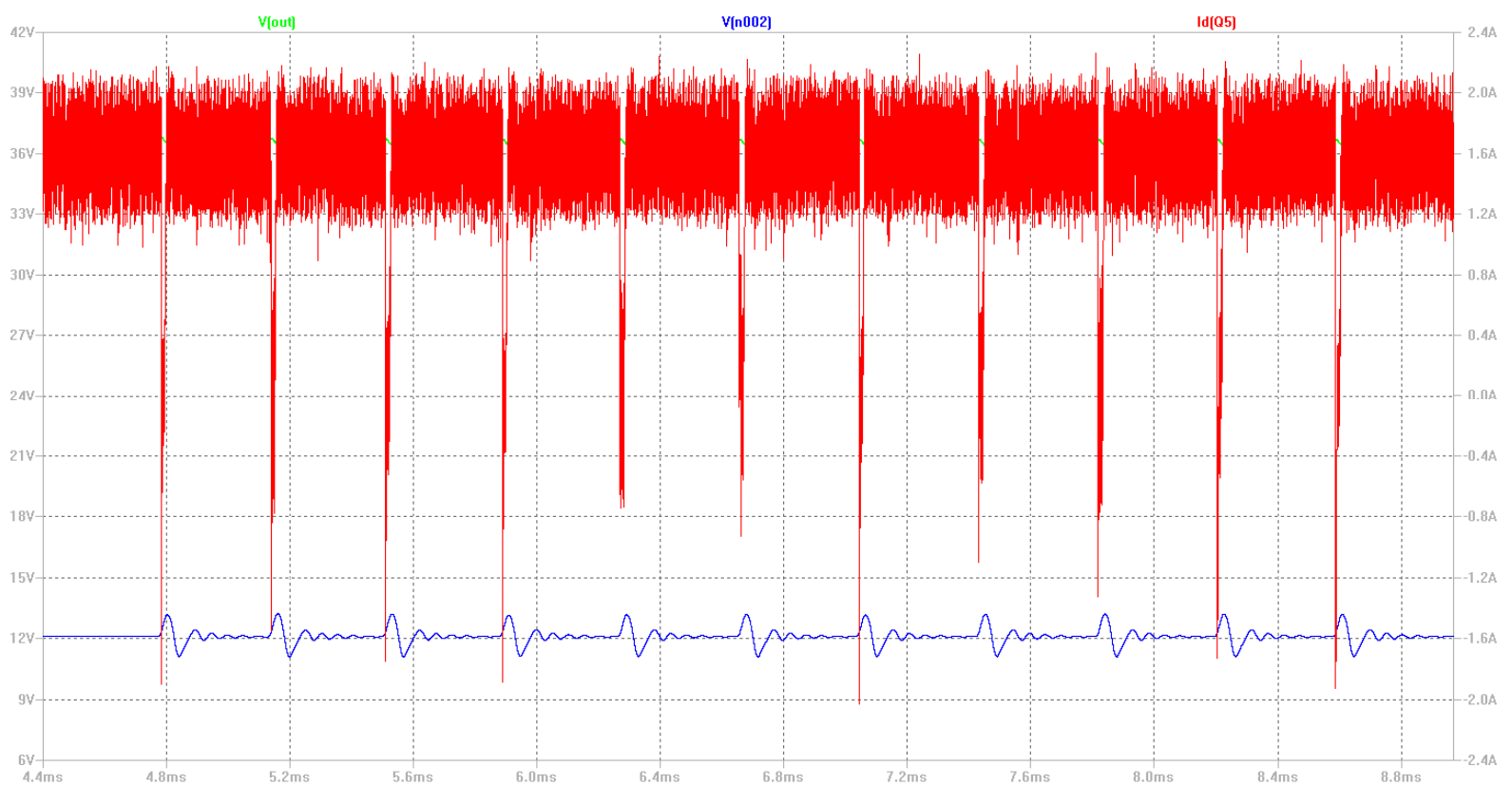

Figure B-31: $Q_{5}$ Drain Current (Output Stage) of Dual-Phase Buck-Boost Converter Under Maximum Input and Load

Q5's drain current ranges from -1.8A to 2.2A (total peak amplitude is 4A). Overall average drain currrent for this simulation time window is 1.54A. As expected, the lowside MOSFET does not conduct as much current as its high-side counterpart. Again, because of the converter's synchronous nature for each phase, $\mathrm{Q}_{11}$ 's (the high-side MOSFET on the output boost stage's secondary phase) drain current characteristics is identical to that of $\mathrm{Q}_{4}$ 's, and $\mathrm{Q}_{12}$ 's (the low-side MOSFET on the output boost stage's secondary phase) drain current characteristics is identical to that of $\mathrm{Q}_{5}$ 's. Thus, showing any plots for $\mathrm{Q}_{11}$ and $\mathrm{Q}_{12}$ is redundant.

The next operation chracteristic that this project investigates in this converter design is the power dissipation across each switching MOSFET. MOSFET switching 
power losses are the primary source of inefficiency in most DC-DC converter topologies. Improving overall converter design to maximize overall converter efficiency requires this project investigating any major power losses imposed across the switching MOSFETs in its converter. Furthermore, investigating power losses imposed across switching MOSFETs allows for noticing any lethal power transients that may occur during MOSFET switching transitions. A real MOSFET does not switch instantaneously. For example, when a MOSFET turns on and begins conducting current, there is usually a short delay between when its drain-source voltage decays to zero and when current begins to flow through its drain. The drain current usually begins flowing long before the drain-source voltage decays to $0 \mathrm{~V}$. By Ohm's Law, the power imposed across the MOSFET during that switching transition would be a non-zero value, and an especially large value in this converter's design because of its high switching currents, as well as high input voltages. This project begins its investigation with $\mathrm{Q}_{1}$ and $\mathrm{Q}_{2}$. Figure B-32 shows $\mathrm{Q}_{1}$ 's power dissipation (denoted by the red waveform) and $\mathrm{Q}_{2}$ 's power dissipation (denoted by the teal waveform) during start-up and steady-state under maximum input voltage and output load. 


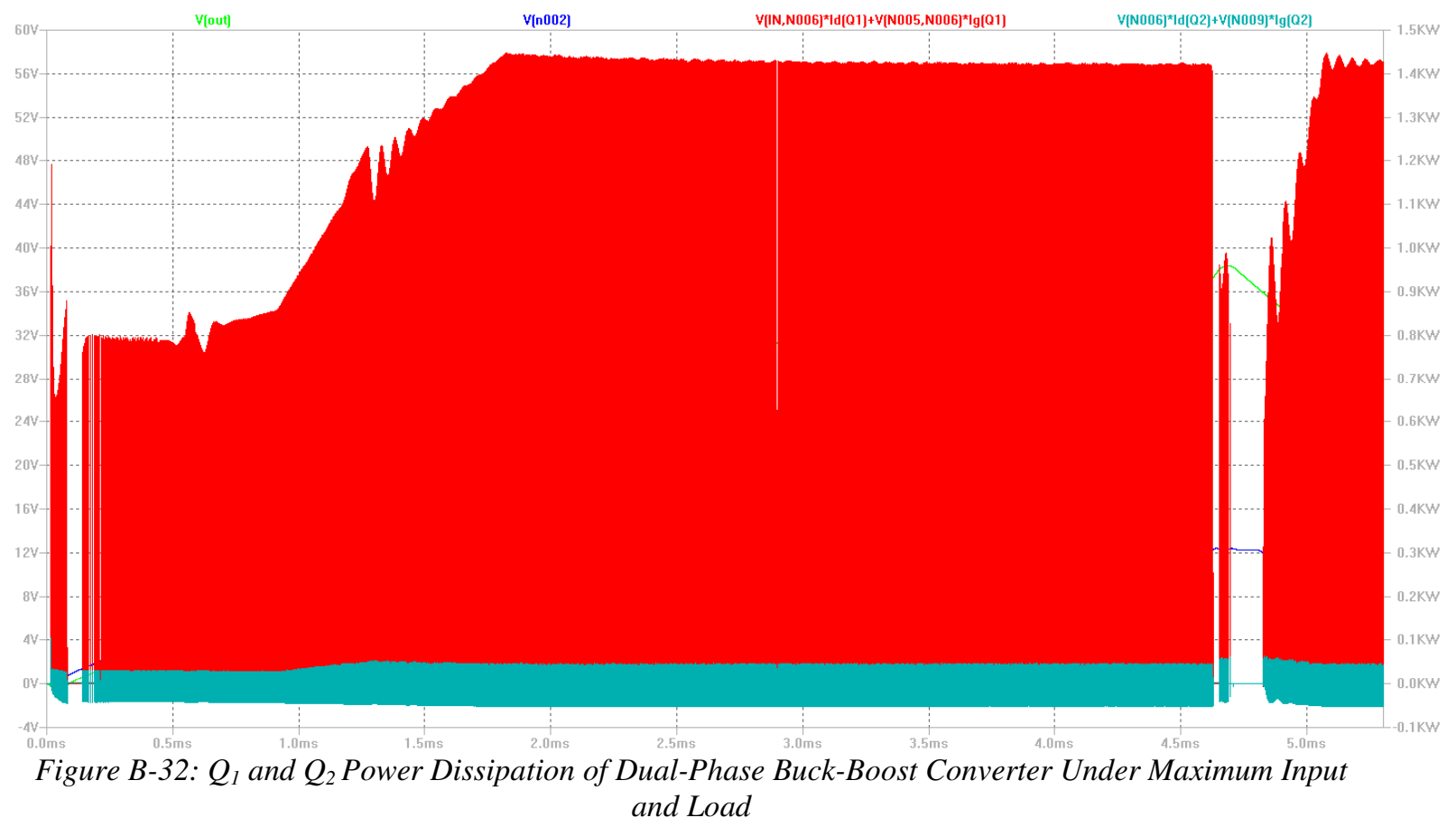

During steady-state, $\mathrm{Q}_{1}$ 's average power dissipation is $11.9 \mathrm{~W}$ and $\mathrm{Q}_{2}$ 's average power dissipation is $1.49 \mathrm{~W}$. However, the transients across $\mathrm{Q}_{1}$ reach $1.4 \mathrm{~kW}$. This shows that $\mathrm{Q}_{1}$ does not switch quickly enough. Unless $Q_{1}$ tolerates such a large power level within a short time period, the high power transients would destroy it. Additionally, such high power transients may cause arcing across other components within the MOSFET's proximity. This project later devises a few solutions for mitigating this switching power transient problem. Again, because of this converter's synchronous nature for each individual phase, $\mathrm{Q}_{3}$ 's power dissipation characteristics are identical to that of $\mathrm{Q}_{1}$ 's and Q6's power dissipation characteristics are identical to that of $\mathrm{Q}_{2}$ 's. Therefore the total dissipated power across the MOSFETs at the input stage is $26.78 \mathrm{~W}$. This project now examines MOSFET power dissipation at the output stage. Figure B-33 shows $\mathrm{Q}_{4}$ 's power dissipation (denoted by the red waveform) during start-up and steady-state. The green 
waveform denotes the boost stage's output voltage and blue denotes the buck stage's output voltage.

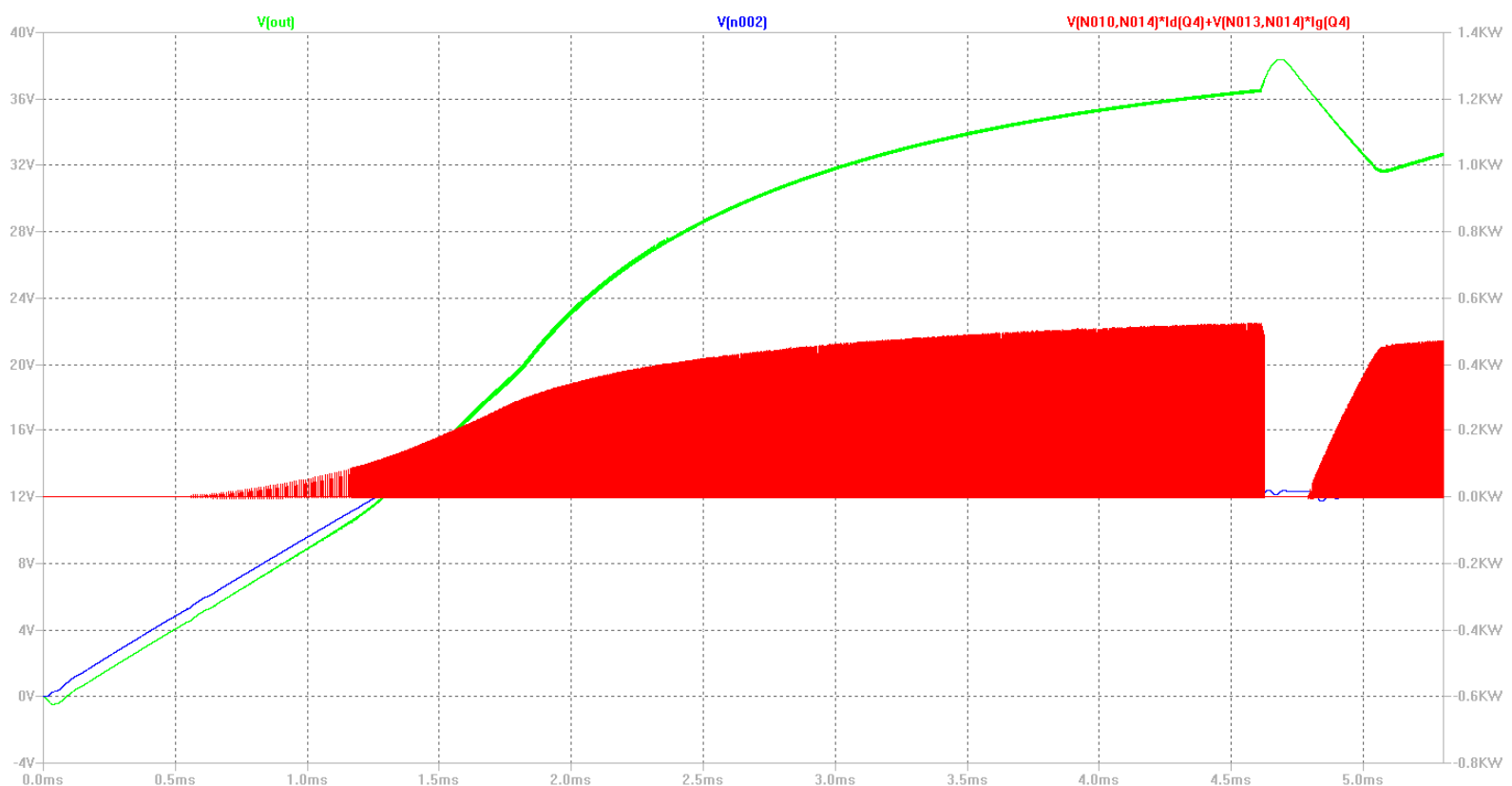

Figure B-33: $Q_{4}$ Power Dissipation of Dual-Phase Buck-Boost Converter Under Maximum Input and Load

Q4's average power dissipation in steady state is $3.23 \mathrm{~W}$; however, its power dissipation transients reach 500W. While it's not as lethal as $\mathrm{Q}_{1}$ 's power transients, this project nonetheless addresses this problem as not all MOSFETs can tolerate such high power levels (i.e. simultaneous high drain-source voltage and high current) for even a time period in the microsecond range. This project now examines the low-side MOSFET in the output stage. Figure B-34 shows $\mathrm{Q}_{5}$ 's power dissipation (denoted by the teal waveform). The green waveform denotes the boost stage's output voltage and blue denotes the buck stage's output voltage. 


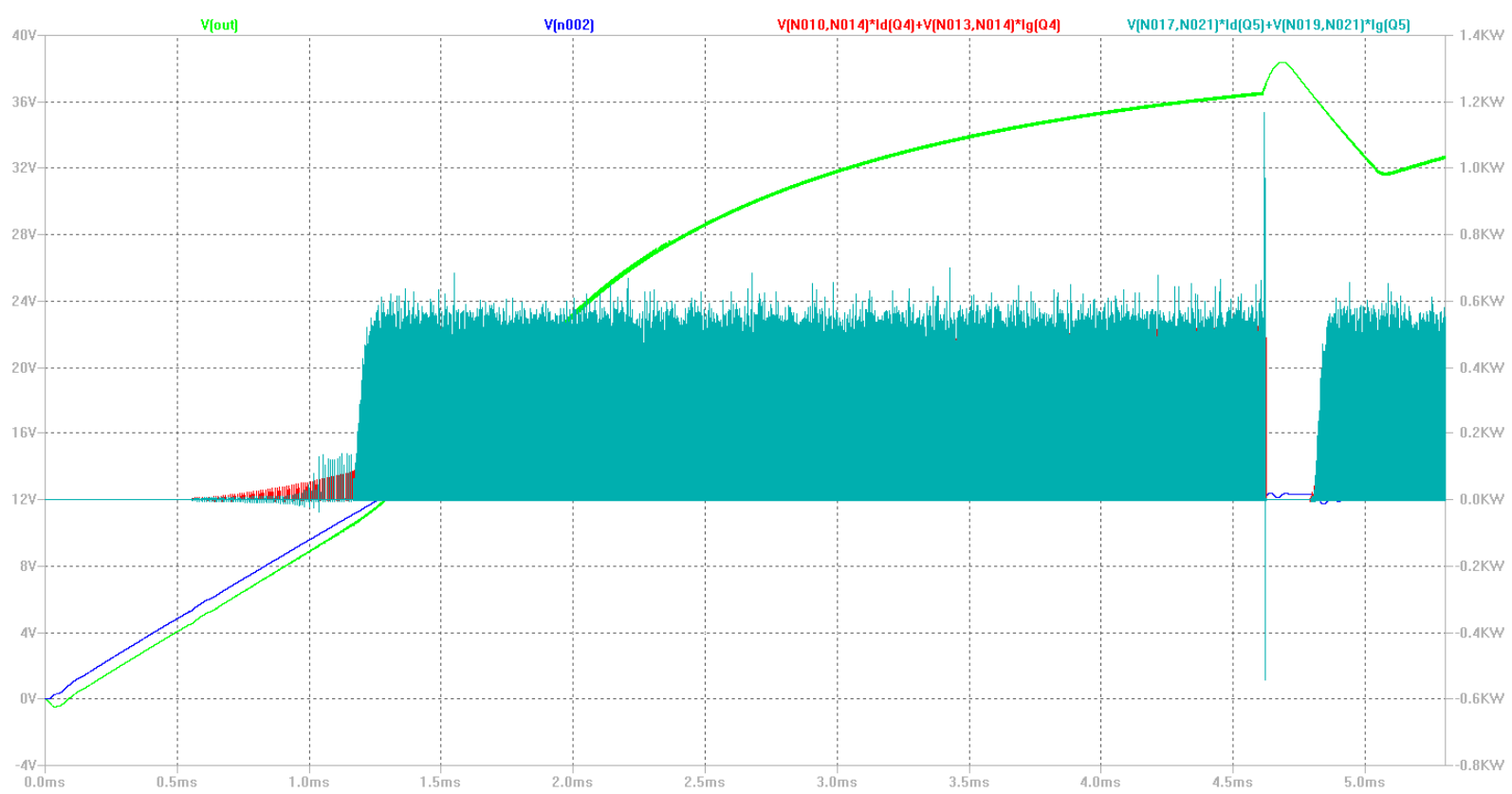

Figure B-34: $Q_{5}$ Power Dissipation of Dual-Phase Buck-Boost Converter Under Maximum Input and Load

Average power dissipation across $\mathrm{Q}_{5}$ is $16.79 \mathrm{~W}$; much higher than that of its high-side counterpart. $\mathrm{Q}_{11}$ 's power dissipation characteristics are identical to that of $\mathrm{Q}_{4}$ 's and $\mathrm{Q}_{12}$ 's power dissipation characteristics are identical to that of $\mathrm{Q}_{5}$ 's. Therefore the total wasted power on the MOSFETs at the output stage is $40.04 \mathrm{~W}$. After summing together the power dissipation losses at both the input and output stages, the total power dissipated power on the MOSFETs in the converter is $66.82 \mathrm{~W}$, which is mostly responsible for the converter's inefficiency.

This project then made changes to the converter's components for mitigating the power dissipation problem (as well as the lethal power transient problem). This project changed all transistors in the input stage to Siliconix Si4980DYs. According to LTSpice, the Si4980DY's maximum drain-source voltage rating is $80 \mathrm{~V}$, its drain-source onresistance is $0.095 \Omega$ and it has a $15 \mathrm{nC}$ gate charge. This project selected this transistor because if a strong athlete generates more than $60 \mathrm{~V}$ at the input from the elliptical, this 
project's design's previous transistor configuration would not handle such high drainsource voltages (previously, the transistors that this project used at the input stage only had a $60 \mathrm{~V}$ drain-source voltage rating). Selecting the Si4980DY accounts for the original $5 \mathrm{~V}$ input headroom problem. This project also changes the output boost stage transistors to Si4850EYs. According to LTSpice, the Si4850EY has a 60V drain-source voltage rating, a $0.031 \Omega$ drain-source on resistance and $18 \mathrm{nC}$ gate charge. This alone may not affect transistor power consumption, so as another improvement attempt this project finally addresses the diode forward current rating problem mentioned earlier in this appendix. This project then changes all diodes at the input stage to MBR20100CTs, which as mentioned earlier, has a $100 \mathrm{~V}$ reverse breakdown voltage of $100 \mathrm{~V}$ (plenty enough for both the input and output stages, like the previously-used MBRS1100s) and can tolerate 10A average forward current. Furthermore, this project changes the converter's output stage diodes to MBRB2545CTs, which have a 45V reverse breakdown voltage (much lower than that of the MBR20100CTs, however, this converter's output stage diodes require reverse voltage ratings for as high as $\mathrm{V}_{\mathrm{OUT}}-$ which is $36 \mathrm{~V}$ ) and tolerates $25 \mathrm{~A}$ average forward current. The schematic in figure B-35 reflects these applied changes. 


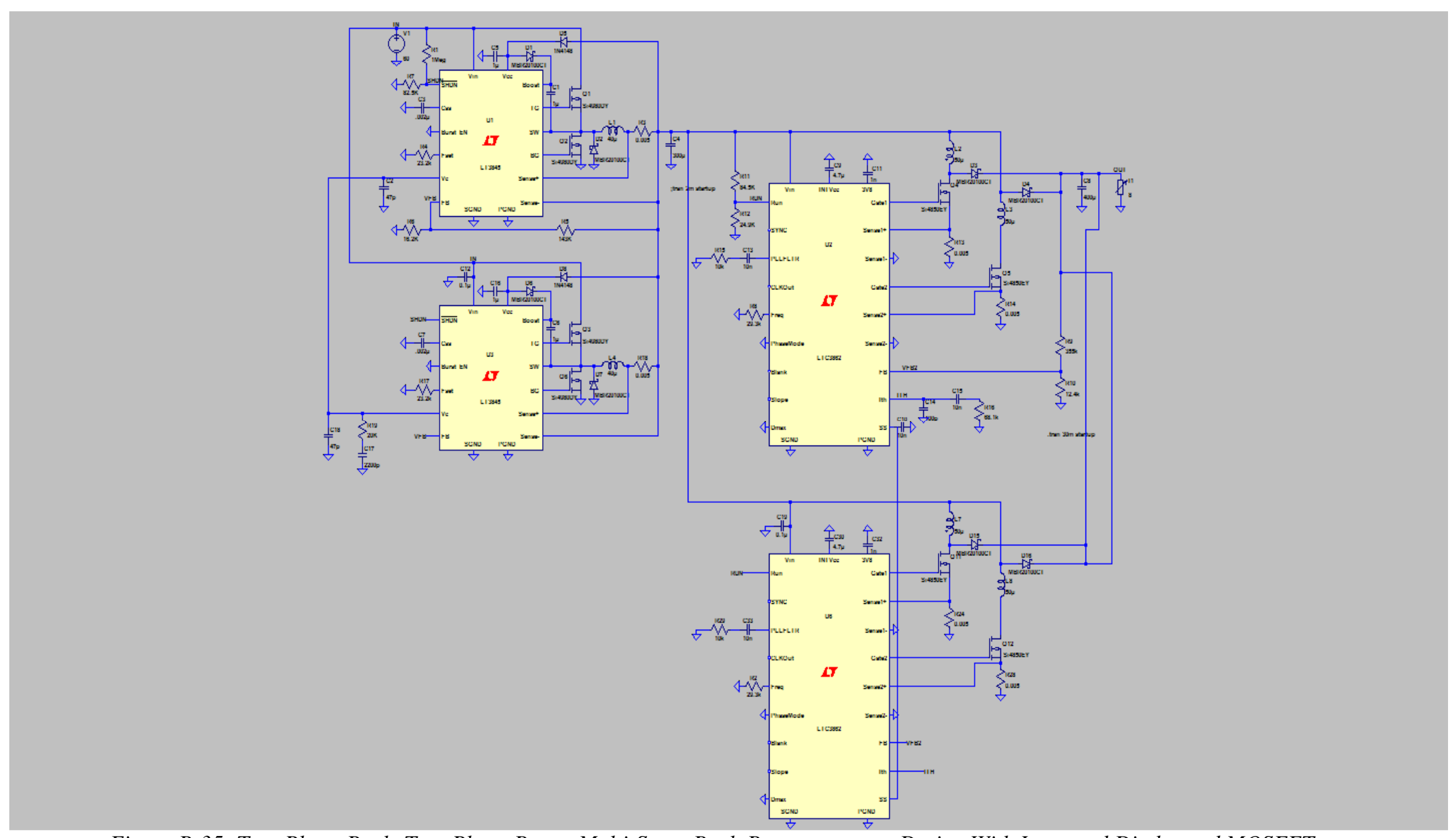

Figure B-35: Two-Phase Buck, Two-Phase Boost, Multi-Stage Buck-Boost converter Design With Improved Diodes and MOSFETs 
This project now examines how these applied changes affect the converter's output characteristics as well as transistor power consumption characteristics. The plot in figure B-36 displays the improved converter's output voltage (denoted by the green waveform) and $\mathrm{Q}_{1}$ 's power dissipation (denoted by the red waveform) in steady-state operation, under maximum input and load. The blue waveform denotes the buck stage's output voltage.

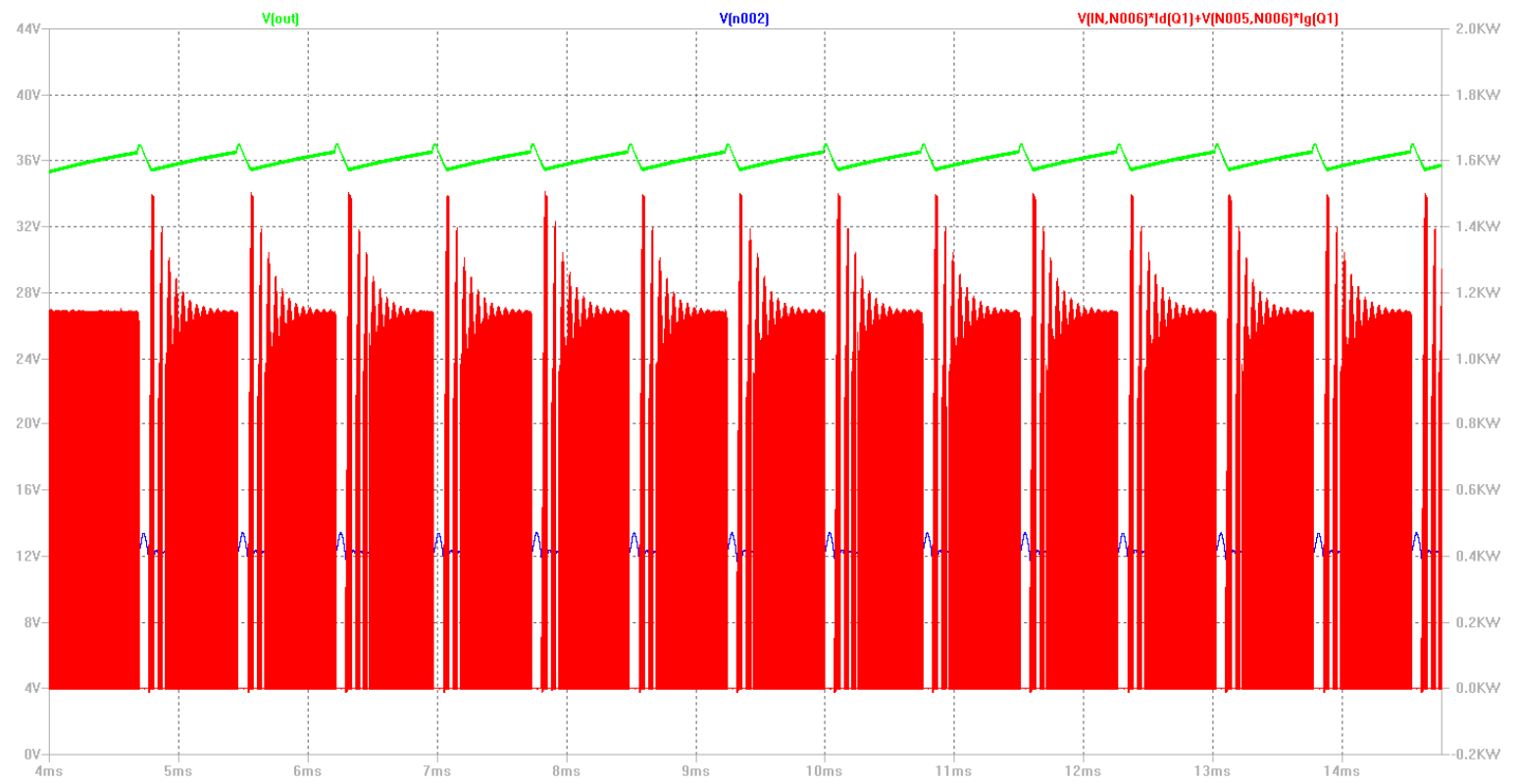

Figure B-36: Output Voltage and $Q_{1}$ Power Dissipation of Dual-Phase Buck-Boost Converter Under Maximum Input and Load, Using Improved Diodes and MOSFETs

$\mathrm{Q}_{1}$ 's average power consumption during steady-state is $5.5 \mathrm{~W}$. Using diodes that can handle a higher average forward current helps sink the additional current that previously flowed through the transistors. Thus there is converter improvement in terms of average power dissipation (it is half of what it was before), but power transients across $\mathrm{Q}_{1}$ still occur in the $1.5 \mathrm{~kW}$ to $1.6 \mathrm{~kW}$ range. $\mathrm{V}_{\text {OUT }}$ swings between 35.5 to $37 \mathrm{~V}$, denoting a $4.2 \%$ ripple relative to the converter's ideal $36 \mathrm{~V}$ output. This ripple level is tolerable for this project's application. Average output voltage for this time window is $36.089 \mathrm{~V}$, average 
output current is $8 \mathrm{~A}$, average input voltage is $60 \mathrm{~V}$ and average input current is $-5.8402 \mathrm{~A}$.

This results in an overall $82.4 \%$ system efficiency, which exceeds the $75 \%$ requirement specified in section 1.2.

The plot in figure B-37 shows a zoomed-in view of $\mathrm{Q}_{1}$ 's switching power transients.

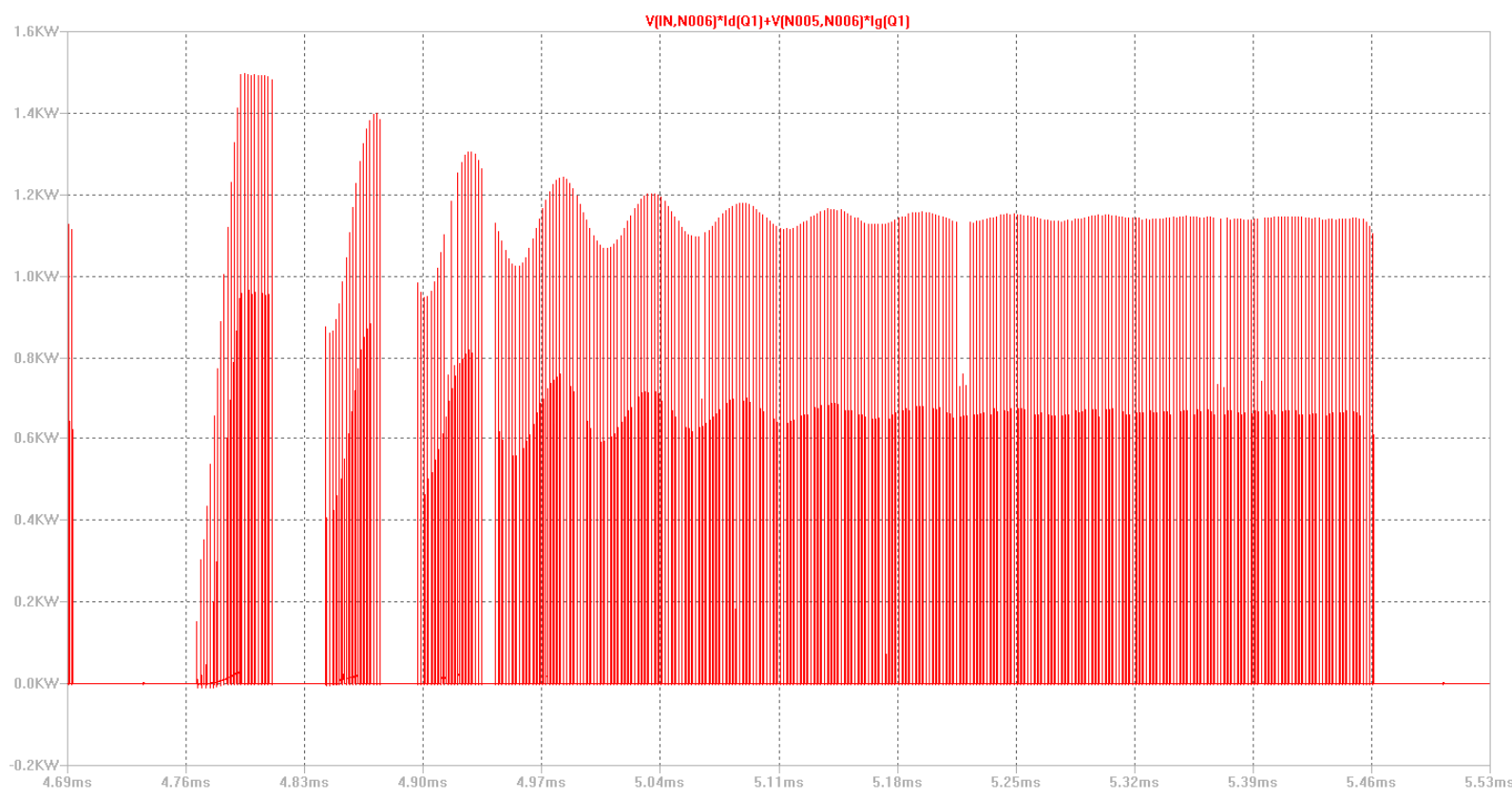

Figure B-37: Zoomed-in View of $Q_{1}$ Power Dissipation Transients of Dual-Phase Buck-Boost Converter Under Maximum Input and Load

The plot in figure B-38 shows the highest power transient width. 


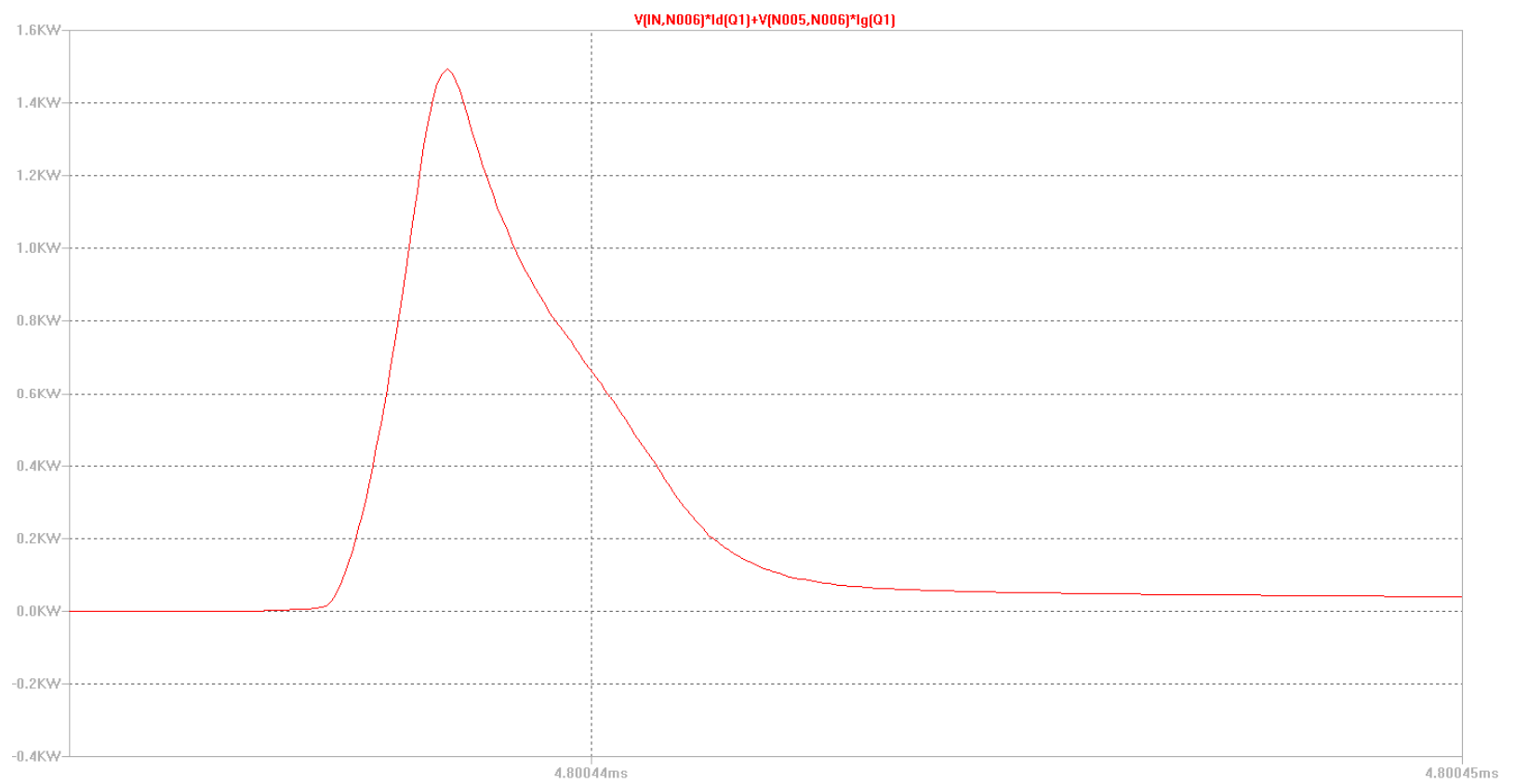

Figure B-38: Width of Largest $Q_{1}$ Power Dissipation Transient Under Maximum Input and Load

This transient's width is approximately $(4.8004442 \mathrm{~ms}-4.8004369 \mathrm{~ms})$, or $7.3 \mathrm{~ns}$. The MOSFETs that this project selected tolerate 1.5 to $1.6 \mathrm{~kW}$ in such a short time period as this, however, such high power transients still poses a danger to other components within the MOSFETs' physical proximity.

This project now investigates $\mathrm{Q}_{1}$ 's drain-source voltage and drain current under maximum input voltage and load. Figure B-39 shows $\mathrm{Q}_{1}$ 's drain source-voltage (denoted by the teal waveform) and drain current (denoted by the blue waveform) under steadystate operation. 


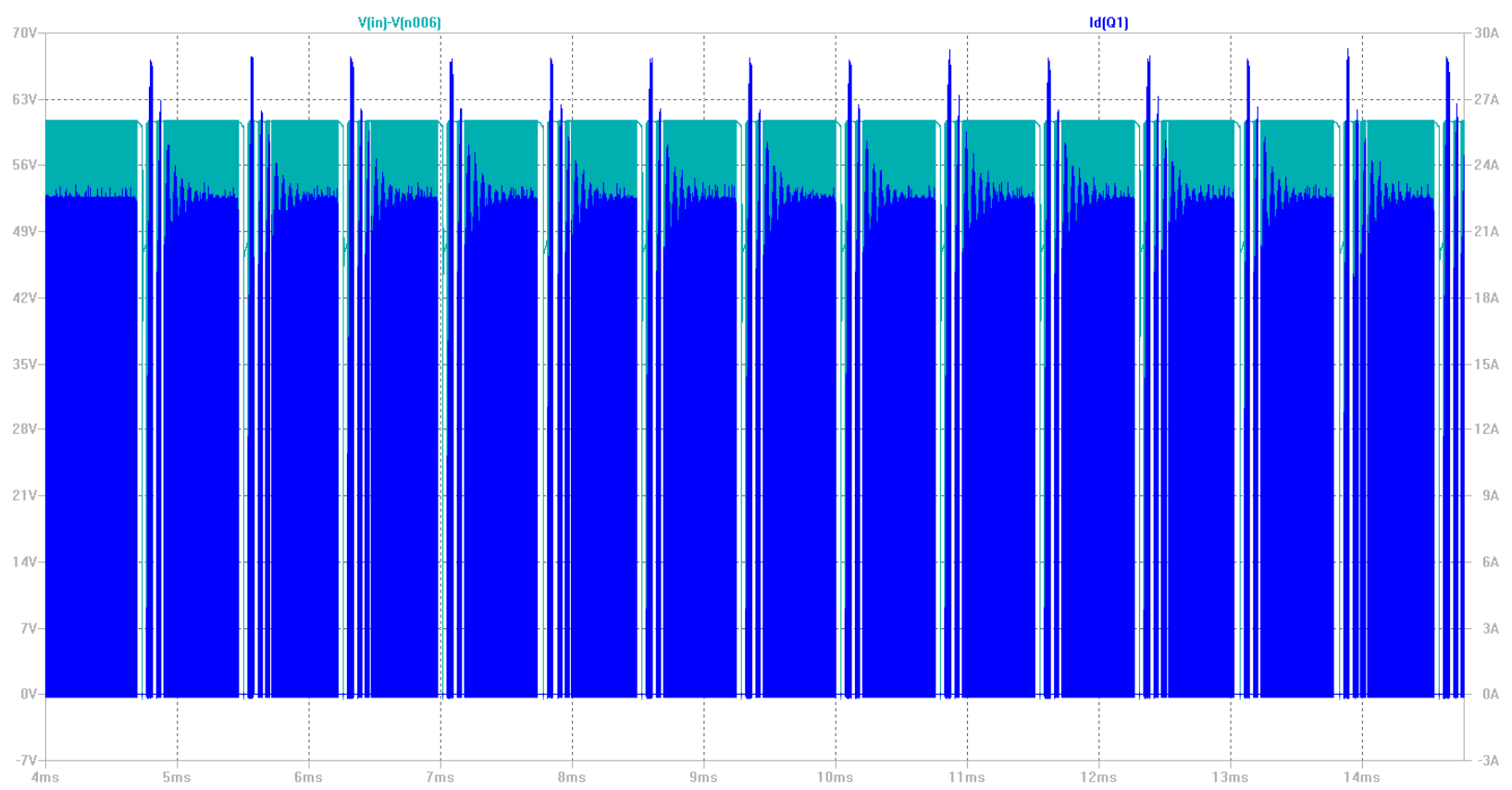

Figure B-39: $Q_{1}$ Drain-Source Voltage and Drain Current Under Maximum Input and Load

$\mathrm{Q}_{1}$ 's average drain-source voltage during steady-state is $47.772 \mathrm{~V}$. $\mathrm{Q}_{1}$ 's average drainsource current during steady-state is $2.92 \mathrm{~A}$. These average values; however, do not provide an accurate average power dissipation representation across this transistor. Instead, this project then investigates what occurs when $\mathrm{Q}_{1}$ 's drain current reaches its highest level (roughly 28.9A), that occurs during $\mathrm{Q}_{1}$ 's turn-on transition. Figure B-40 shows a zoomed in plot of $\mathrm{Q}_{1}$ 's turn-on transition (with the teal waveform denoting drainsource voltage and blue waveform denoting drain current). 


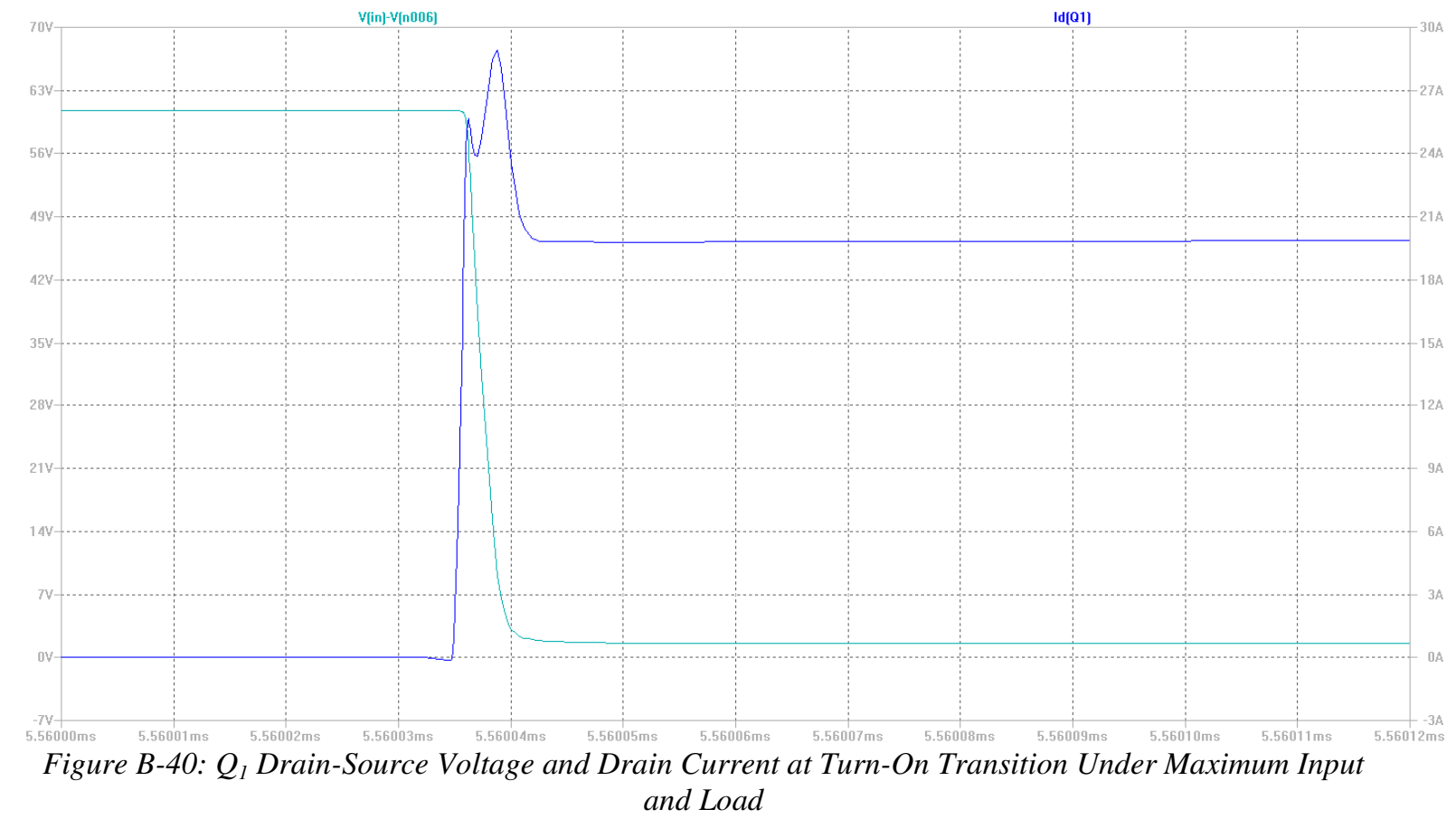

At $\mathrm{Q}_{1}$ 's drain current's highest point, $\mathrm{Q}_{1}$ 's drain-source voltage equals approximately 13.54V. This translates to a $391.3 \mathrm{~W}$ power dissipation at that instant, when the MOSFET is under a transition state. The maximum power dissipation period (i.e. the largest power transient), however, occurs when the drain current and drain-source voltage plots intersect. This occurs when $\mathrm{Q}_{1}$ 's drain-source voltage is $59.02 \mathrm{~V}$ and $\mathrm{Q}_{1}$ 's drain current is 25.29A. This translates to $1.493 \mathrm{~kW}$ power dissipation at that very instant, which explains the $\sim 1.5 \mathrm{~kW}$ power transients visible from earlier figures. From the plot in figure B-40, this project also confirms that $\mathrm{Q}_{1}$ does not switch instantaneously; there is approximately a 7.3ns delay before $\mathrm{Q}_{1}$ 's drain-source voltage fully decays to $0 \mathrm{~V}$ when $\mathrm{Q}_{1}$ turns on, which also explains the $\sim 1.5 \mathrm{~kW}$ power transient lasting approximately $7.3 \mathrm{~ns}$. 
The plot in figure $\mathrm{B}-41$ shows $\mathrm{Q}_{1}$ 's gate at the same instant (the green waveform denotes gate voltage and the red waveform denotes gate-source voltage, the teal waveform denotes drain-source voltage and the blue waveform denotes drain current).

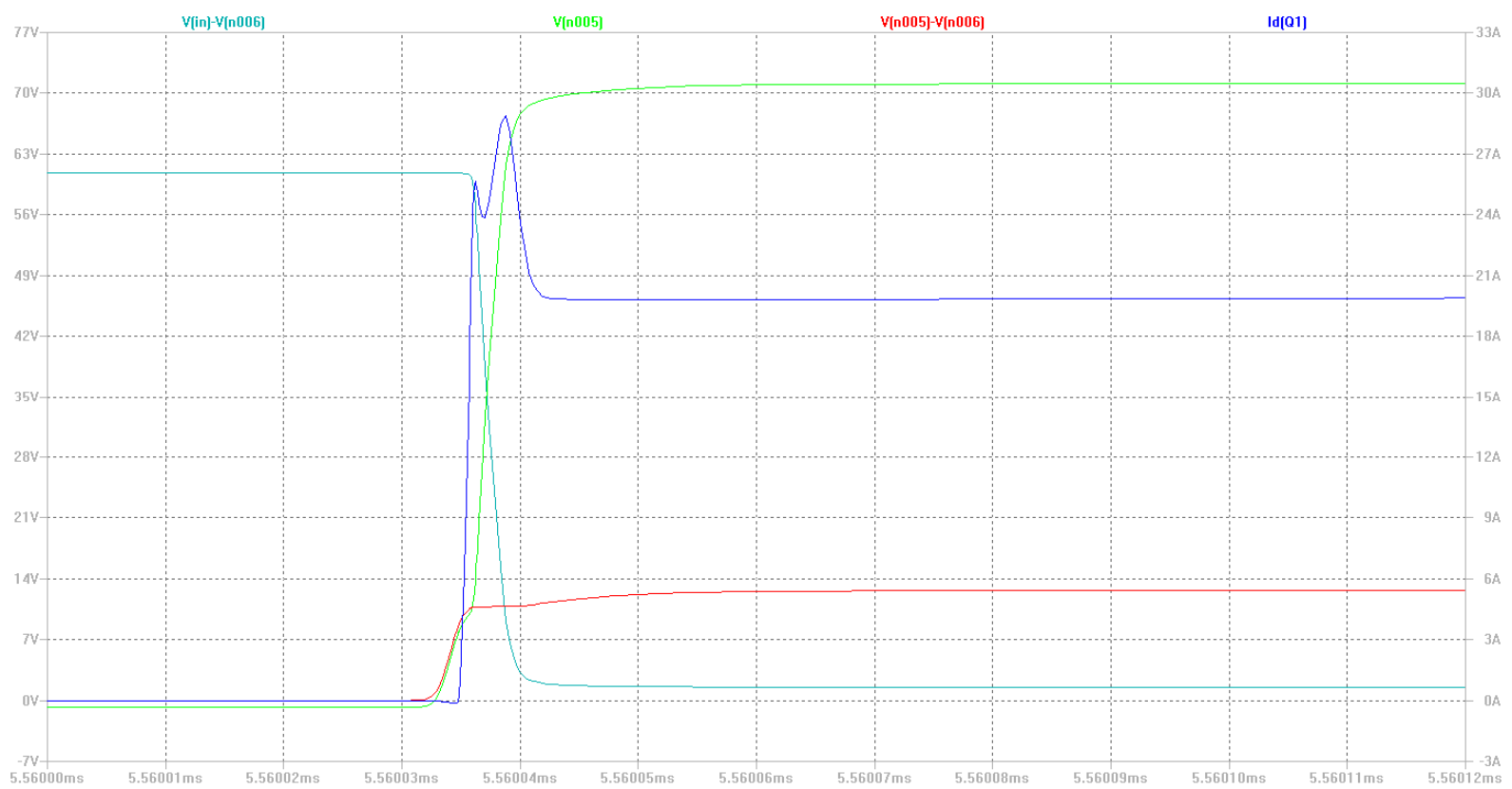

Figure B-41: $Q_{1}$ Drain-Source Voltage, Gate Voltage, Gate-Source Voltage and Drain Current at Turn-On Transition Under Maximum Input and Load

$\mathrm{Q}_{1}$ 's gate-source voltage shows the MOSFET's turn-on transition in greater detail. From this plot, this project concludes that when operating at this maximum stress level, turning on transistor $\mathrm{Q}_{1}$ results in a large and potentially lethal power spike. The transistor's junction capacitance as well as drain-source resistance might be culprits for this delay, as those two items form a series RC circuit, in which both of those items affect the time constant for charging the capacitance portion. This project then devises two possible solutions for the switching power transient problem: either speed up transistor drainsource voltage decay at MOSFET turn-on (as well as slow down its rise during turn-off), or slow down transistor drain current rise at turn-on (as well as speed up its decay during 
turn-off). However, there is a compromise involved for these two methods, because slowing down or speeding up the transistor's switching transition by too large of a margin results in worsening the power dissipation problem.

Figure B-42 shows $\mathrm{Q}_{2}$ 's power dissipation (denoted by the blue waveform) in steady state, using the new diodes and MOSFETs for the converter.

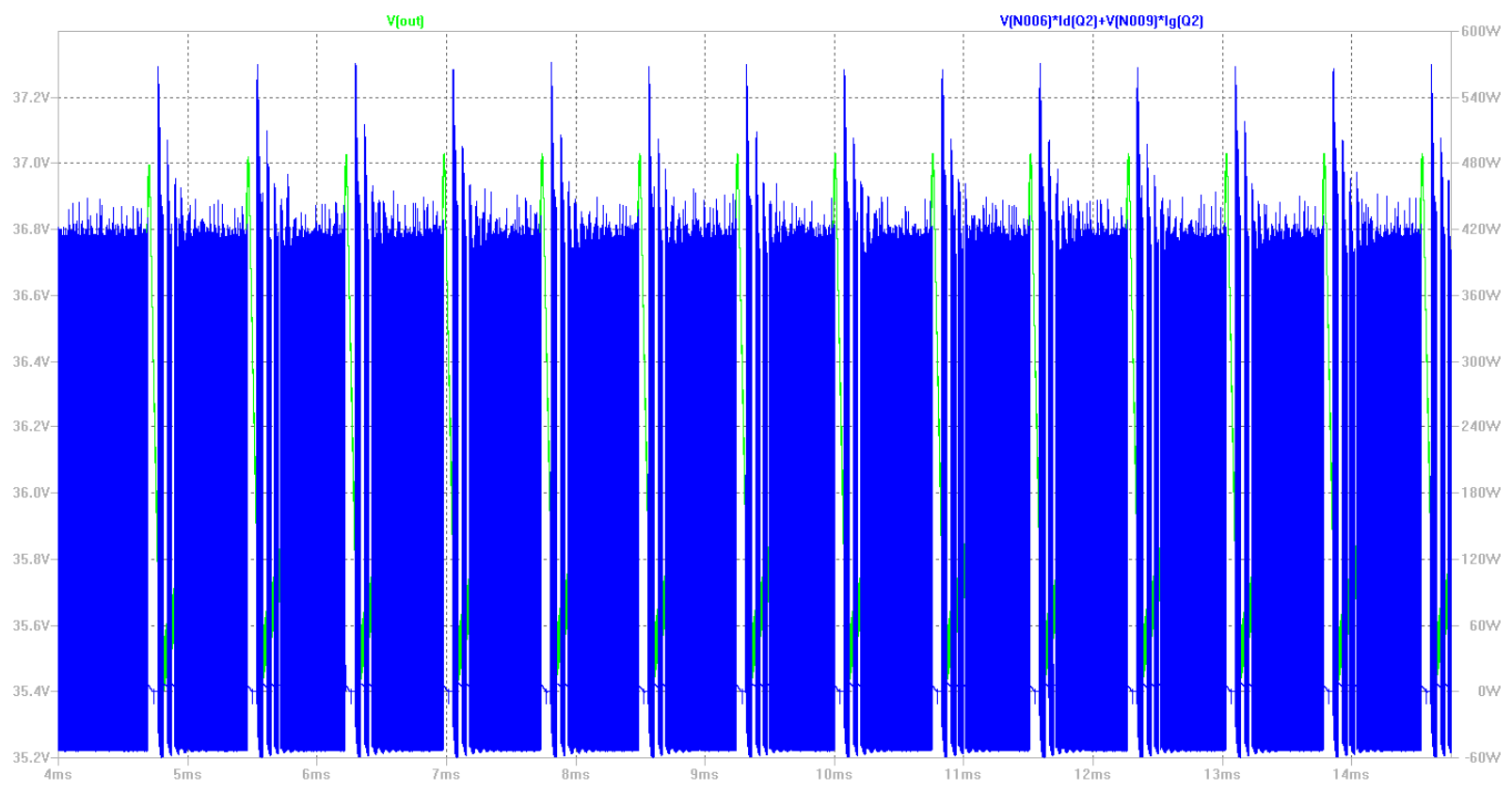

Figure B-42: $Q_{2}$ Power Dissipation of Dual-Phase Buck-Boost Converter Under Maximum Input and Load, Using Improved Diodes and MOSFETS

$\mathrm{Q}_{2}$ 's average power dissipation in steady state is $4.039 \mathrm{~W}$, which is larger than before.

This increased power dissipation may have resulted from the new diode this project selected for $\mathrm{Q}_{1} \cdot \mathrm{Q}_{3}$ and $\mathrm{Q}_{6}$ 's power dissipation characteristics are similar to those of $\mathrm{Q}_{1}$ and $\mathrm{Q}_{2}$. The total power dissipated across the transistors at the input stage is $19.078 \mathrm{~W}$. Thus, using higher voltage and current-rated diodes and MOSFET reduces the total power dissipated across the MOSFETs at the input stage by roughly $7 \mathrm{~W}$. 
This project now investigates MOSFET power dissipation at the boost output stage. Figure B-43 shows $\mathrm{Q}_{4}$ 's power dissipation (denoted by the blue waveform) in steady state, using the new diodes and MOSFETs for the converter.

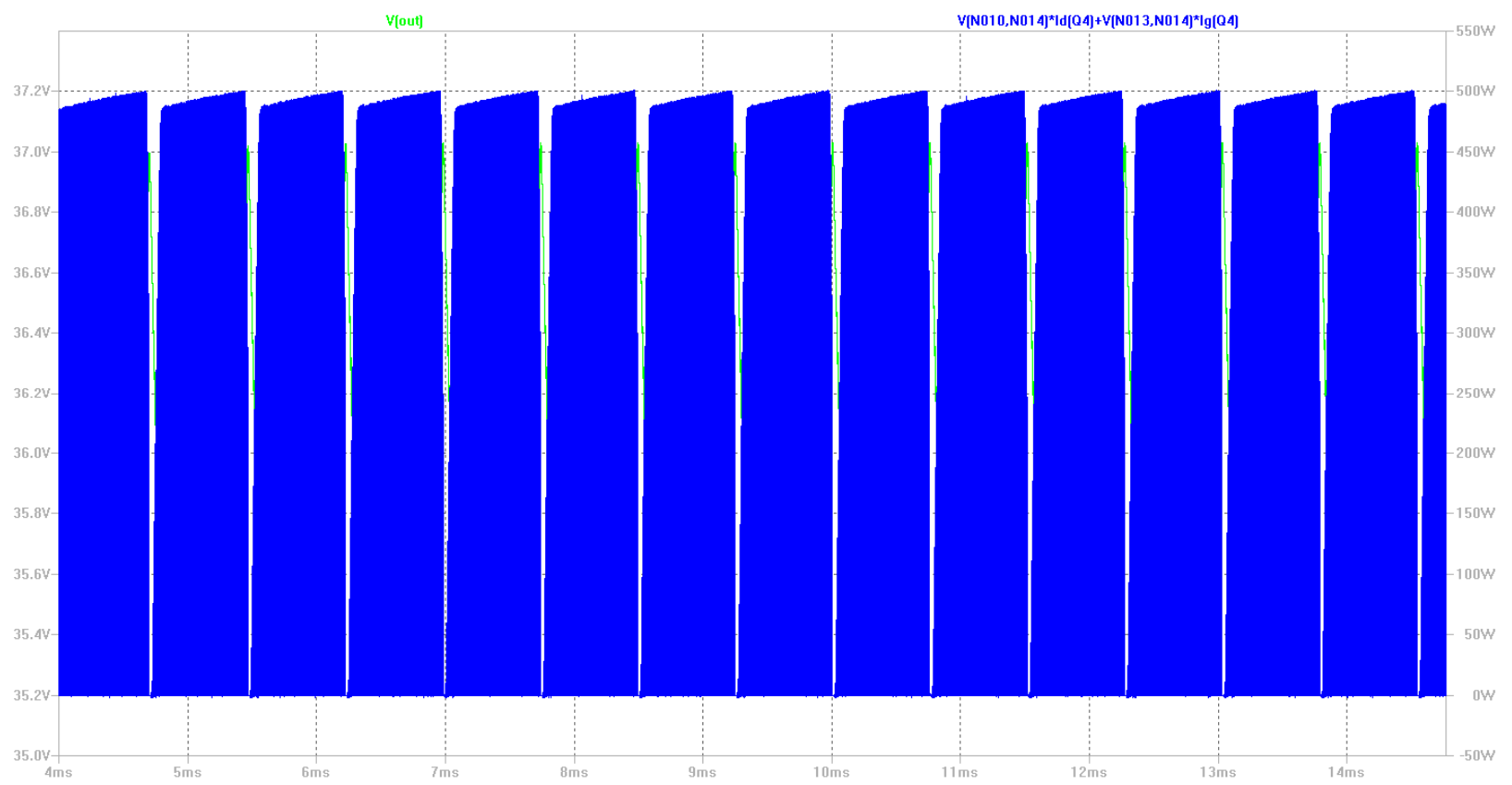

Figure B-43: $Q_{4}$ Power Dissipation of Dual-Phase Buck-Boost Converter Under Maximum Input and Load, Using Improved Diodes and MOSFETs

Strangely enough, $\mathrm{Q}_{4}$ 's average power dissipation increases to 5.922W. This project then determines if that power dissipation increase compensates for significantly reducing $\mathrm{Q}_{5}$ 's power dissipation. Figure B-44 shows $\mathrm{Q}_{5}$ 's power dissipation (denoted by the green waveform) in steady state, using the new diodes and MOSFETs for the converter. 


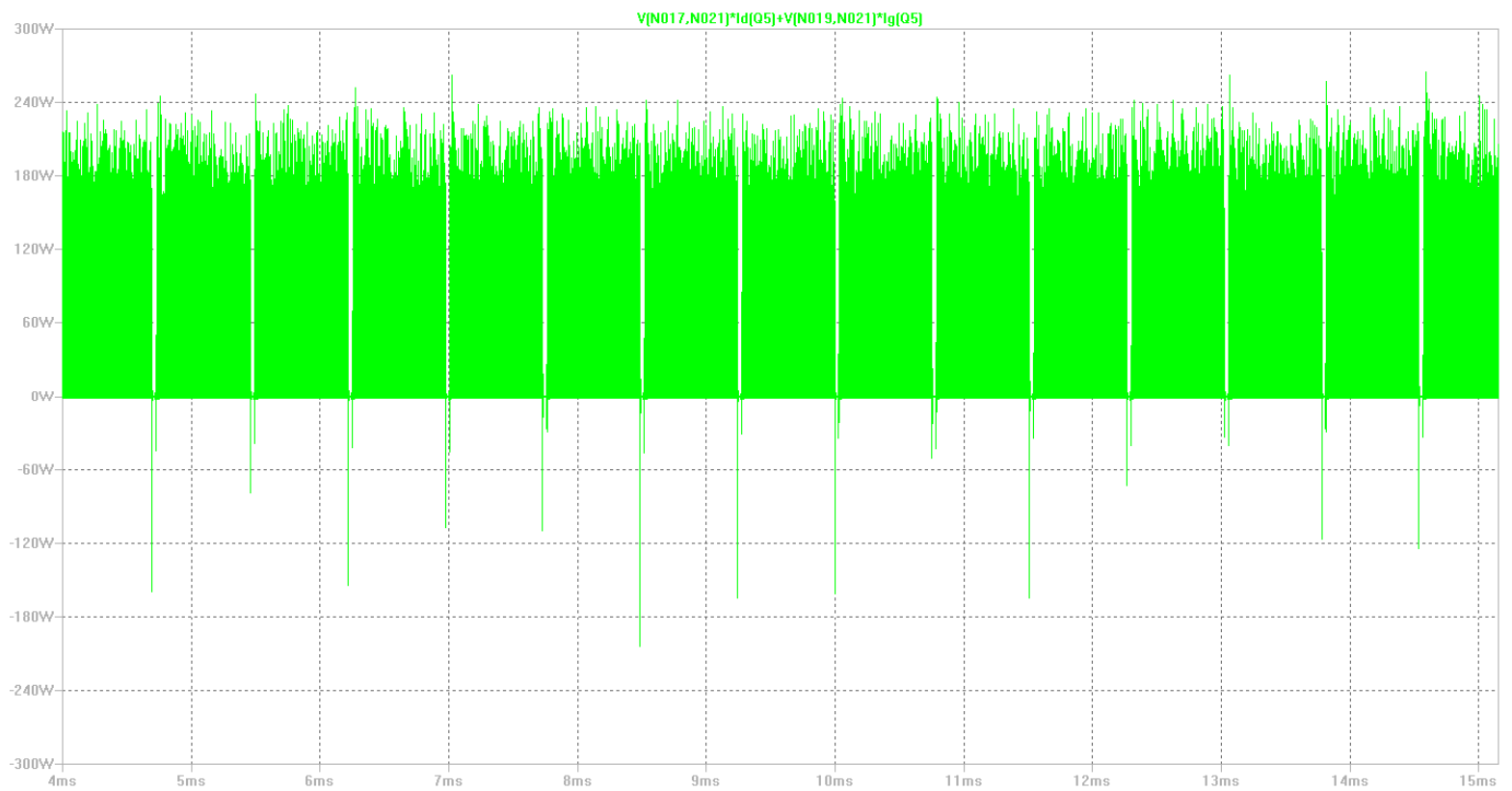

Figure B-44: $Q_{5}$ Power Dissipation of Dual-Phase Buck-Boost Converter Under Maximum Input and Load, Using Improved Diodes and MOSFETs

Using higher-rated voltage and current diodes and MOSFETs reduces $\mathrm{Q}_{5}$ 's average power dissipation to $5.8 \mathrm{~W}$ (from $16.79 \mathrm{~W}$ with the previous converter design). This is an overall converter performance improvement, even if it slightly increases $\mathrm{Q}_{4}$ 's power dissipation. $\mathrm{Q}_{11}$ and $\mathrm{Q}_{12}$ 's power dissipation characteristics are similar to those of $\mathrm{Q}_{4}$ and Q5. Thus, the total power dissipated across the transistors at the output stage is $23.444 \mathrm{~W}$. The total power that each transistor consumes in the entire converter is $42.522 \mathrm{~W}$, a $24 \mathrm{~W}$ overall reduction from the previous converter design.

This project now determines if all the MOSFETs in the converter switch at the proper frequency $(500 \mathrm{kHz})$. Figure B-45 shows a zoomed-in plot of $\mathrm{Q}_{1}$ 's gate switching trajectory. 


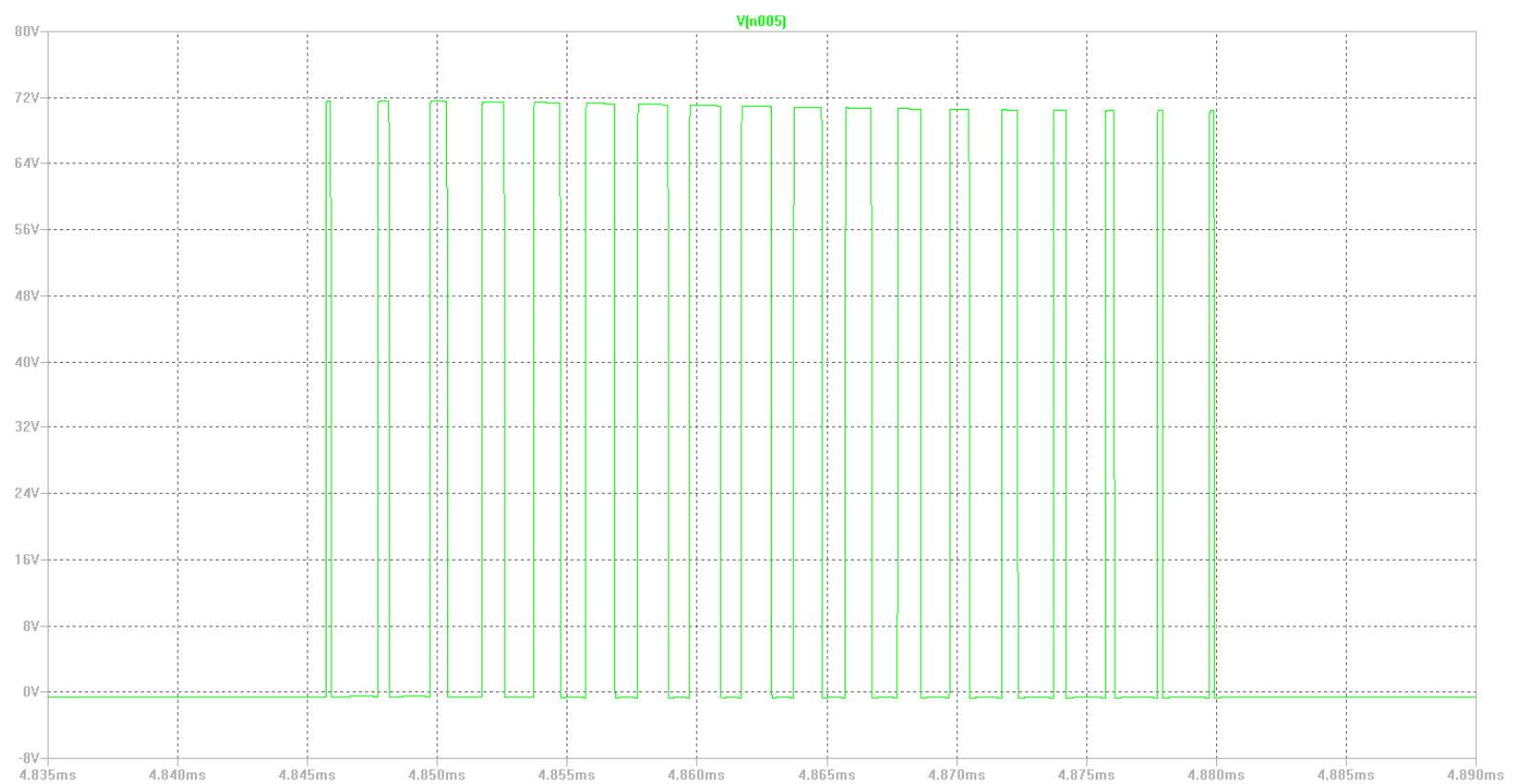

Figure B-45: $Q_{1}$ Gate Voltage of Dual-Phase Buck-Boost Converter Under Maximum Input and Load

This project measures the switching period of the middle rise-fall-rise transition cycle as $2.04 \mu \mathrm{s}$, which corresponds with the $500 \mathrm{kHz}$ switching frequency selected for the input stage. This project now examines one of the output stage MOSFETs's switching trajectory. Figure B-46 shows a zoomed-in plot of $\mathrm{Q}_{4}$ 's gate switching rate. 


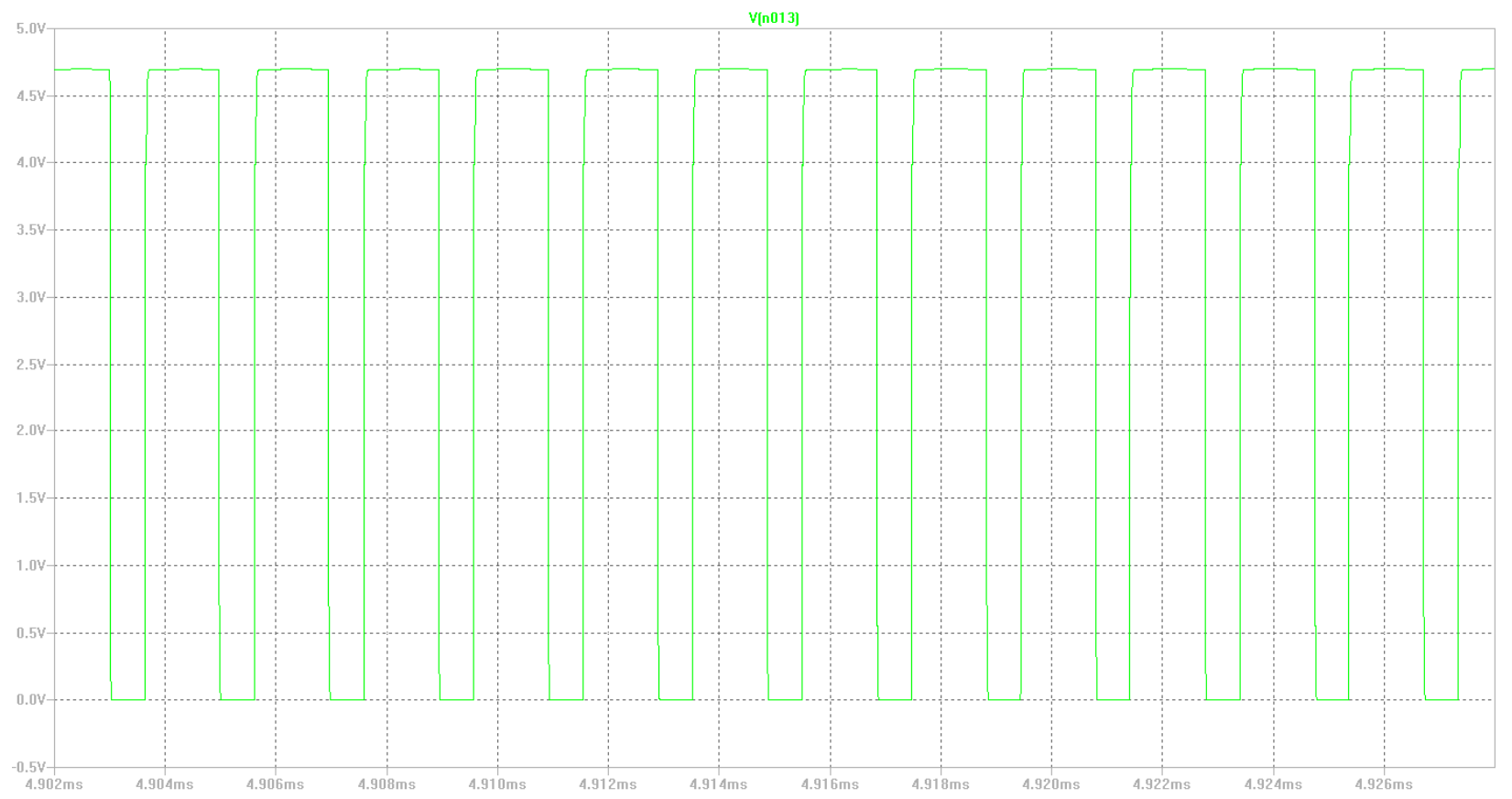

Figure B-46: $Q_{4}$ Gate Voltage of Dual-Phase Buck-Boost Converter Under Maximum Input and Load

Again, this project the measures switching period of the middle rise-fall-rise transition cycle, and it equals $1.98 \mu$ s. This value corresponds with the $500 \mathrm{kHz}$ switching frequency selected for the output stage. From figures B-45 and B-46 this project concludes that its converter switching frequency resistors are the correct values and that the converter synchronizes both its input and output stages at the same switching frequency.

This project now address the lethal switching power transient problem. As mentioned earlier, mitigating the switching delay problem requires either speeding up the drain-source voltage decay at MOSFET turn-on (as well as slow down its rise during turn-off), or slow down the drain current rise at turn-on (as well as speed up its decay during turn-off). This project can use transistors with faster switching speeds, however switching delays always occur regardless of transistor switching speed and cause large power dissipation transients. 
Engineers at National Semiconductor listed numerous MOSFET selection design

notes on their website, which aim at reducing switching power losses (as well as reducing

lethal power transients, which are partially responsible for those switching power losses).

Those design notes are as follows: [74]

1. Switching losses increase for larger gate and drain capacitance and these capacitances are inversely proportional to the on-resistance. FETs with the lowest onresistance inevitably have the higher capacitance hindering $H S$ (high side - primary FET) switching speed.

2. Reducing the switching clock frequency reduces switching losses; that is, at lower frequencies the losses during on/off transitions become a diminishing proportion of the total on-time of the FET causing conduction losses to increasingly dominate.

3. For higher input voltages relative to the output voltage the duty cycle of the HS FET decreases causing the switching losses to increasingly dominate.

4. In order to further reduce conduction losses, multiple, parallel, LS (low sidesynchronous or secondary FET) FETs are often employed. The number of parallel FETs is determined ultimately by cost, the gate driver's ability to drive them, and the point of diminishing returns.

The engineer should be aware that in most POL applications, especially for input voltages higher than $12 \mathrm{~V}$, the switching losses will likely dominate all other losses. Under these circumstances the lowest overall losses in the HS FET are not necessarily achieved by using a device with the lowest on-resistance. The FET must be selected to minimize the sum of all the losses. The FET's on-resistance must be optimized at a higher value to achieve reduced capacitance and so reduce the switching losses. The major MOSFET vendors now provide "reduced charge, fast switching" MOSFETs which are optimized in this way for high-side buck applications.

If optimizing the FETs does not enable high enough efficiency in a system, the switching frequency can be reduced to decrease the switching losses and improve the efficiency. This, however, can result in a physically larger system.

The foregoing discussions have made clear that to achieve maximum efficiency in a high input voltage buck converter, the high side MOSFET must be carefully selected to minimize the sum of the switching and conduction losses. 
From National Semiconductor's suggestions, this project can further reduce switching power losses by using MOSFETs with a higher on-state drain-source resistance (which tend to have lower junction capacitance, allowing for faster switching transitions). Furthermore, this project can also decrease the switching frequency, which in turn decreases switching losses, leaving only MOSFET conduction losses. However, employing multiple MOSFETs in parallel can further reduce conduction losses, though this project refrains from implementing such a design because this project desires to keeping its converter design simple. Furthermore, the converter's PWM controllers may have synchronization problems while each driving more than two MOSFETs.

This project then applies National's MOSFET selection suggestions to its converter design. This process involves changing the converter's input stage and output stage MOSFETs once again. For the new input stage MOSFETs this project selects International Rectifier's IRF510. According to LTSpice the IRF510 has a 100V maximum drain-source voltage rating and a $0.54 \Omega$ on-state drain-source resistance. For the output MOSFETs this project selects Fairchild's HUFA76413. According to LTSpice the HUFA76413 has a 60V maximum drain-source voltage rating and a $0.056 \Omega$ on-state drain-source resistance. This project carefully selected these MOSFETs based on their on-state drain-source resistance value. If the on-state drain-source resistance of the output MOSFETs is too high, the converter may not regulate at the optimal $36 \mathrm{~V}$ that this project requires because of drain current reductions involved with increasing such resistance. Furthermore, this project also reduces the switching frequency to $350 \mathrm{kHz}$ on both stages 
as a further attempt for mitigating the switching losses. Figure B-47 shows the mentioned changes to the converter design. 


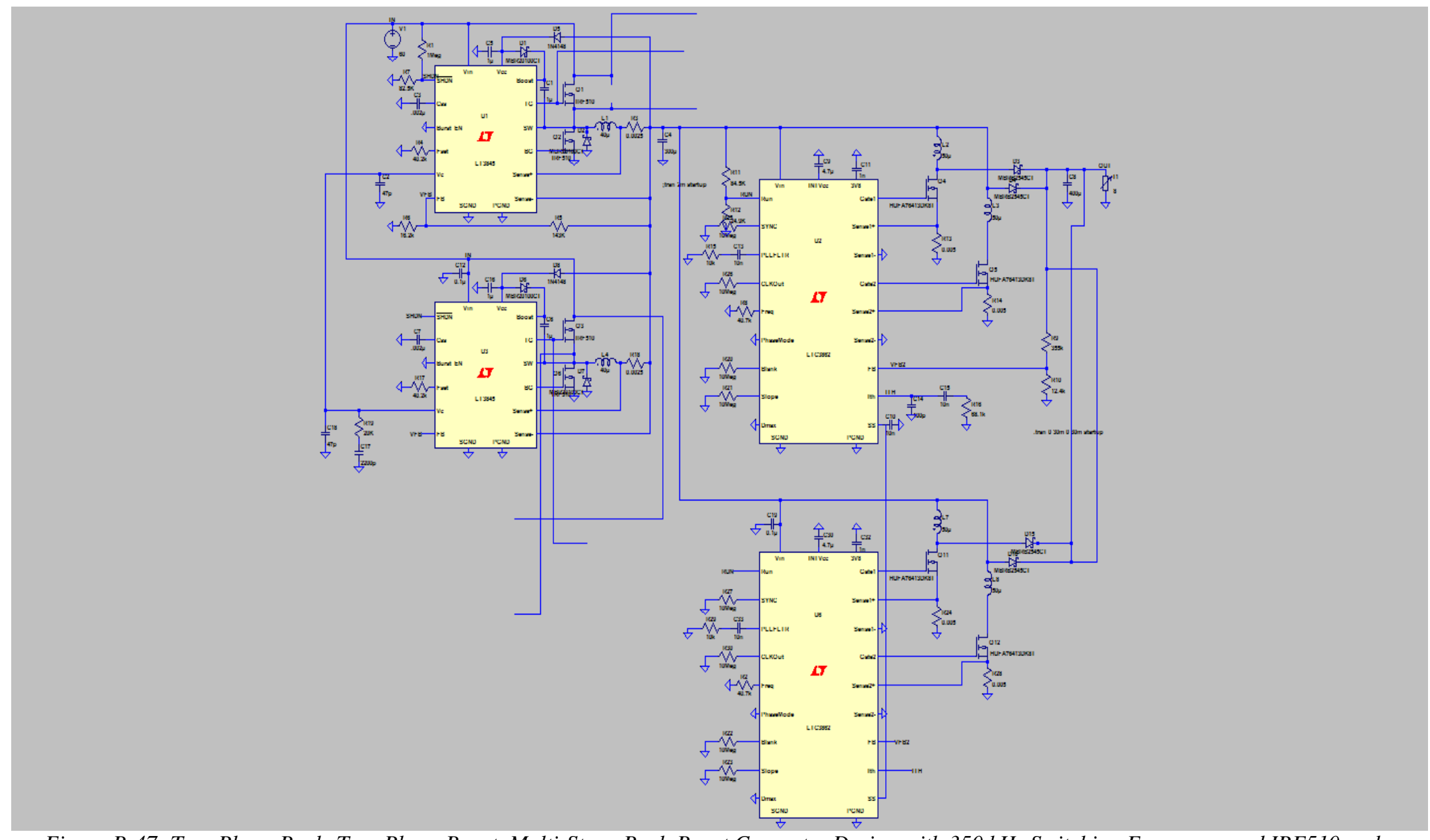

Figure B-47: Two-Phase Buck, Two-Phase Boost, Multi-Stage Buck-Boost Converter Design with 350 kHz Switching Frequency and IRF510 and HUFA76413 MOSFETS 
This project then examines this new converter's performance. The plot in figure B-48 shows the new converter's output voltage (denoted by the green waveform), buck stage output voltage (denoted by the blue waveform), and power dissipated across $\mathrm{Q}_{1}$ (denoted by the red waveform).

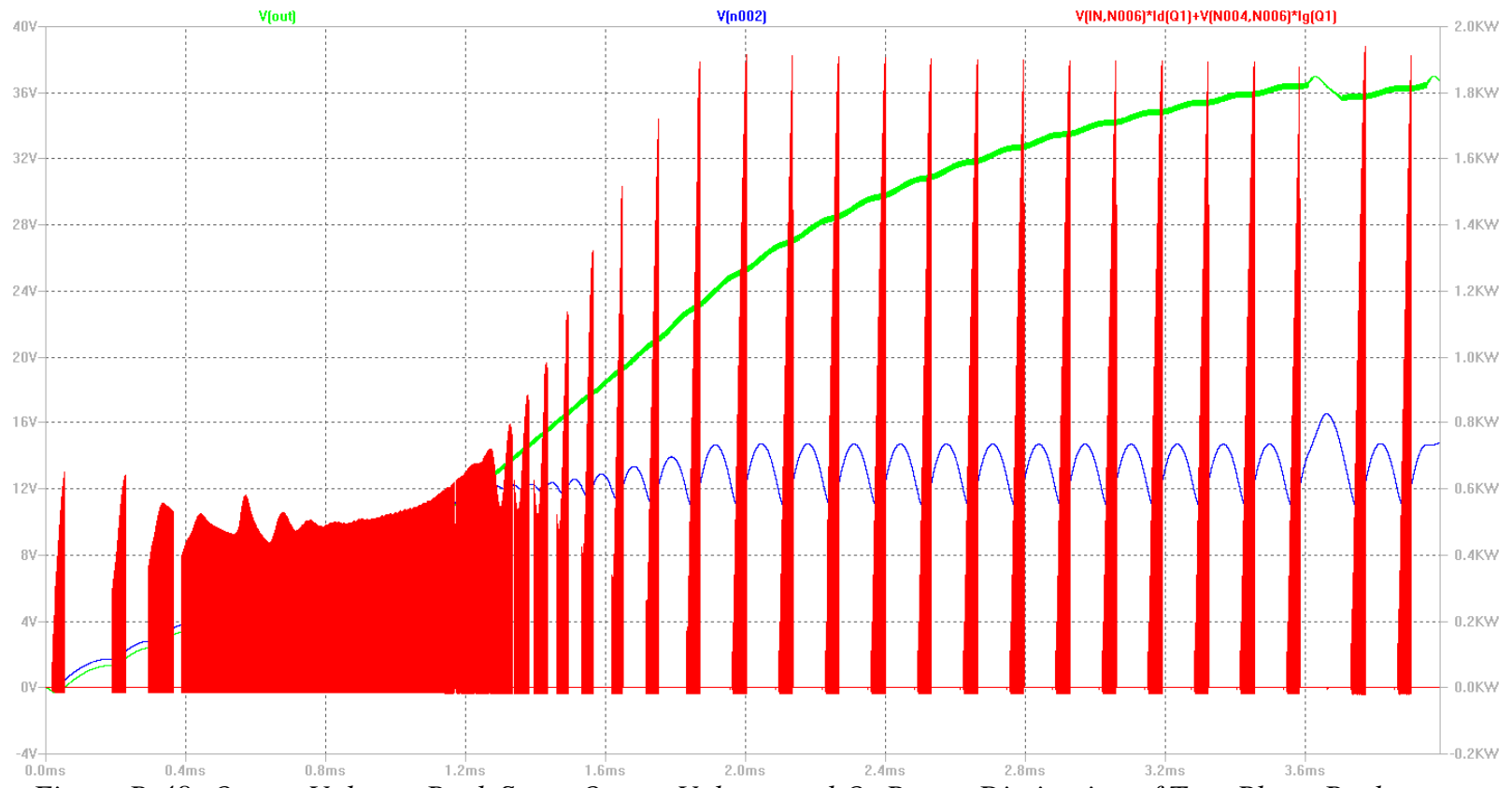

Figure B-48: Output Voltage, Buck Stage Output Voltage and $Q_{1}$ Power Dissipation of Two-Phase Buck, Two-Phase Boost, Multi-Stage Buck-Boost Converter Design with $350 \mathrm{kHz}$ Switching Frequency and IRF510 and HUFA76413 MOSFETs

This result is still undesirable (there are still very lethal power transients across $\mathrm{Q}_{1}$ ), and the switching power transients across $\mathrm{Q}_{1}$ appear to be larger than those from previous designs. From these inherent power dissipation problems, this project deems this dualphase buck-boost converter design unfeasible and in the next section it moves to a fourphase input variation of this design.

\section{B1.9 Four-Phase Input Stage and Two-Phase Output Stage Implementation}

This project now returns to the concept of adding more phases to each stage for increasing current-carrying capacity, and determines if doing so is beneficial for reducing 
switching power losses and switching power transient magnitudes. For this new 4-phase converter design, this project reduced the switching frequency to $300 \mathrm{kHz}$ as a method of decreasing switching losses. Figure B-49 shows this new four-phase-input buck-boost converter. 


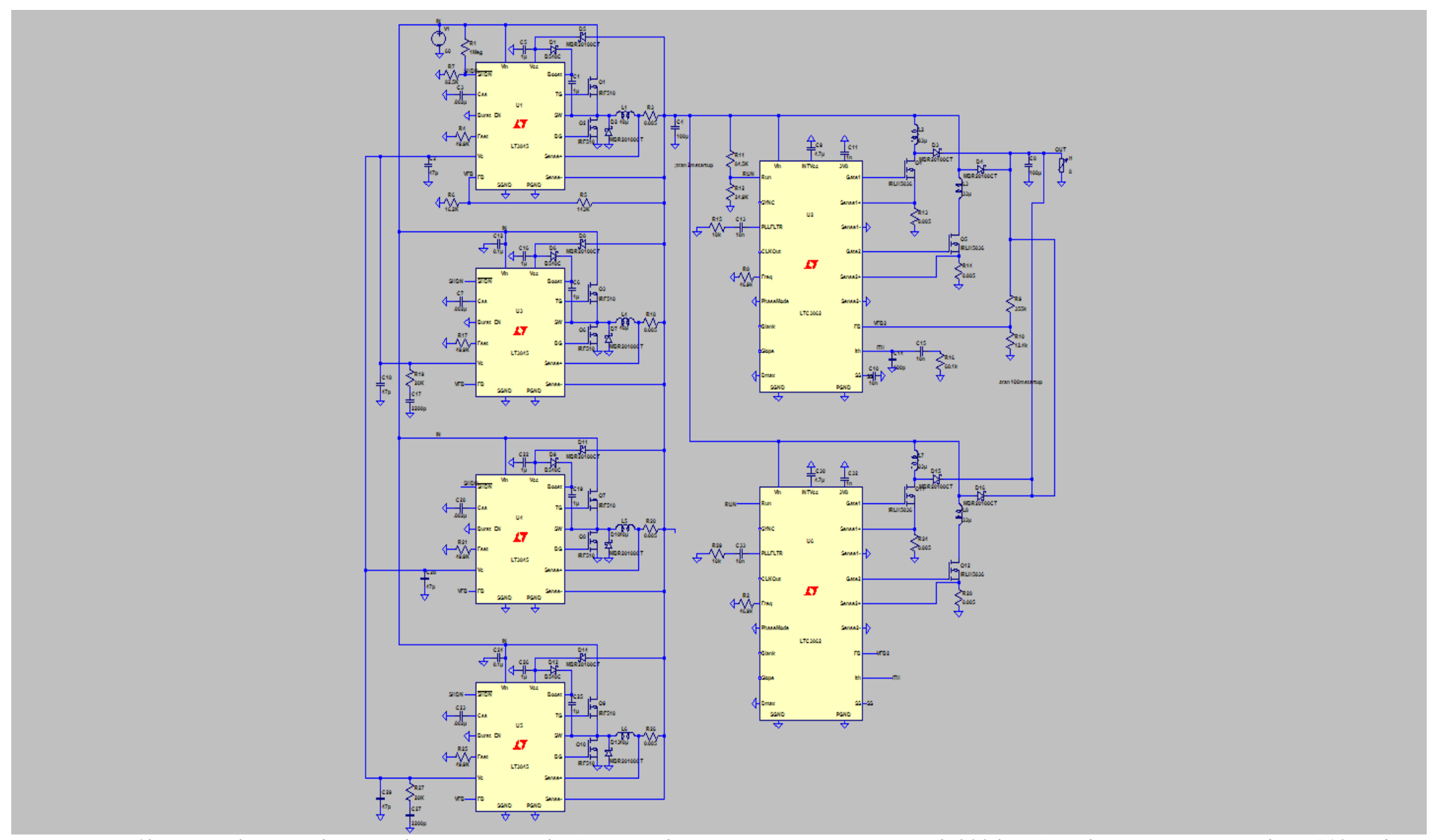

Figure B-49: Four-Phase Buck, Two-Phase Boost, Multi-Stage Buck-Boost Converter Design with 300 kHz Switching Frequency and IRF510 and HUFA76413 MOSFETS 
This project then tests this design's peformance. Again, this project uses the maximum stress condition using $60 \mathrm{~V}$ input voltage with an $8 \mathrm{~A}$ load. Figure B-50 shows this converter's output voltage (denoted by the green waveform), buck stage output voltage (denoted by the blue waveform) and $\mathrm{Q}_{1}$ power dissipation (denoted by the red waveform) in start-up and steady-state operation modes.

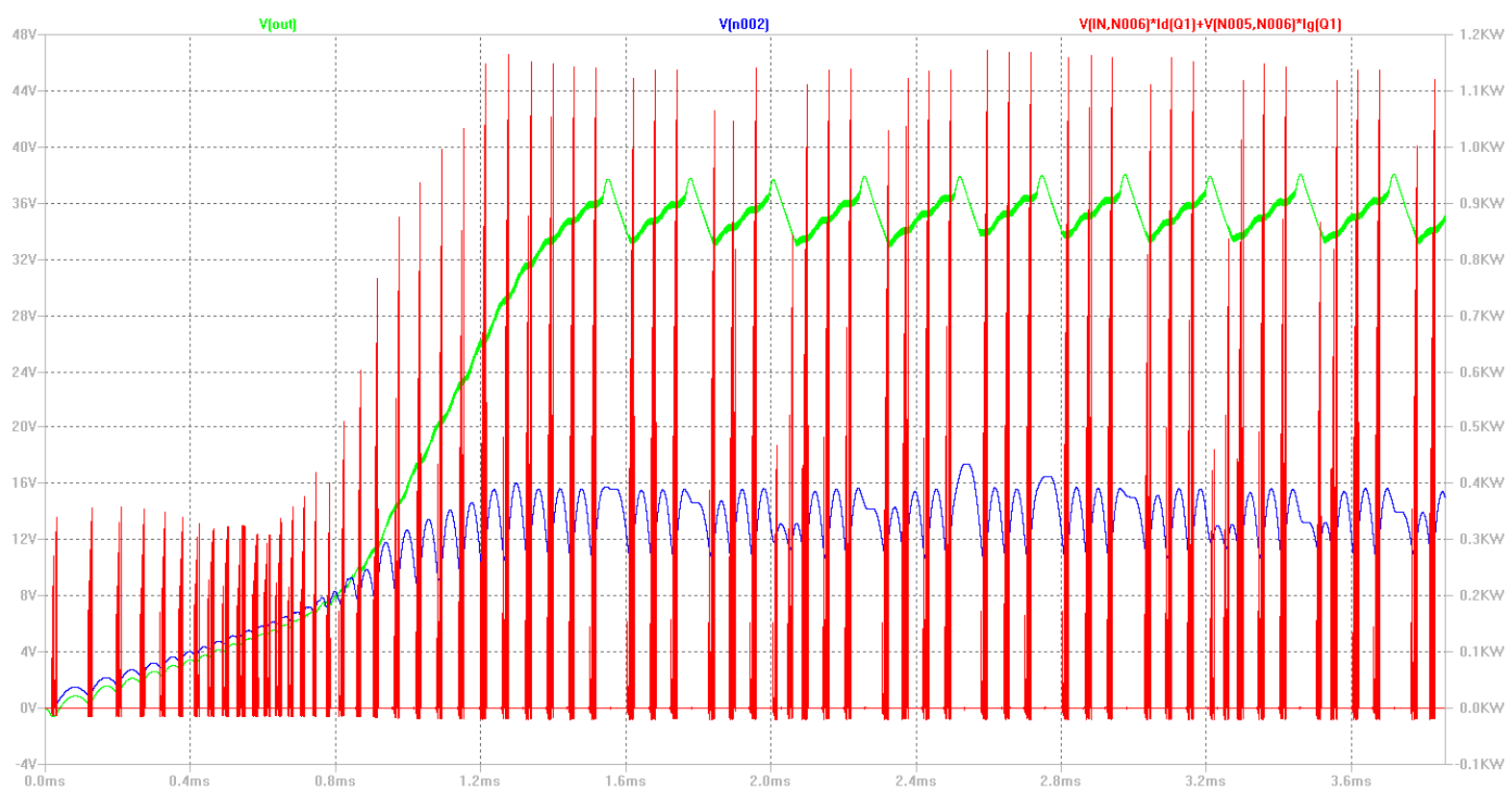

Figure B-50: Output Voltage, Buck Stage Output Voltage and $Q_{1}$ Power Dissipation of Four-Phase Buck, Two-Phase Boost, Multi-Stage Buck-Boost Converter Design with 300 kHz Switching Frequency and IRF510 and HUFA76413 MOSFETS

Figure B-51 shows the same plot, but in converter steady-state operation. 


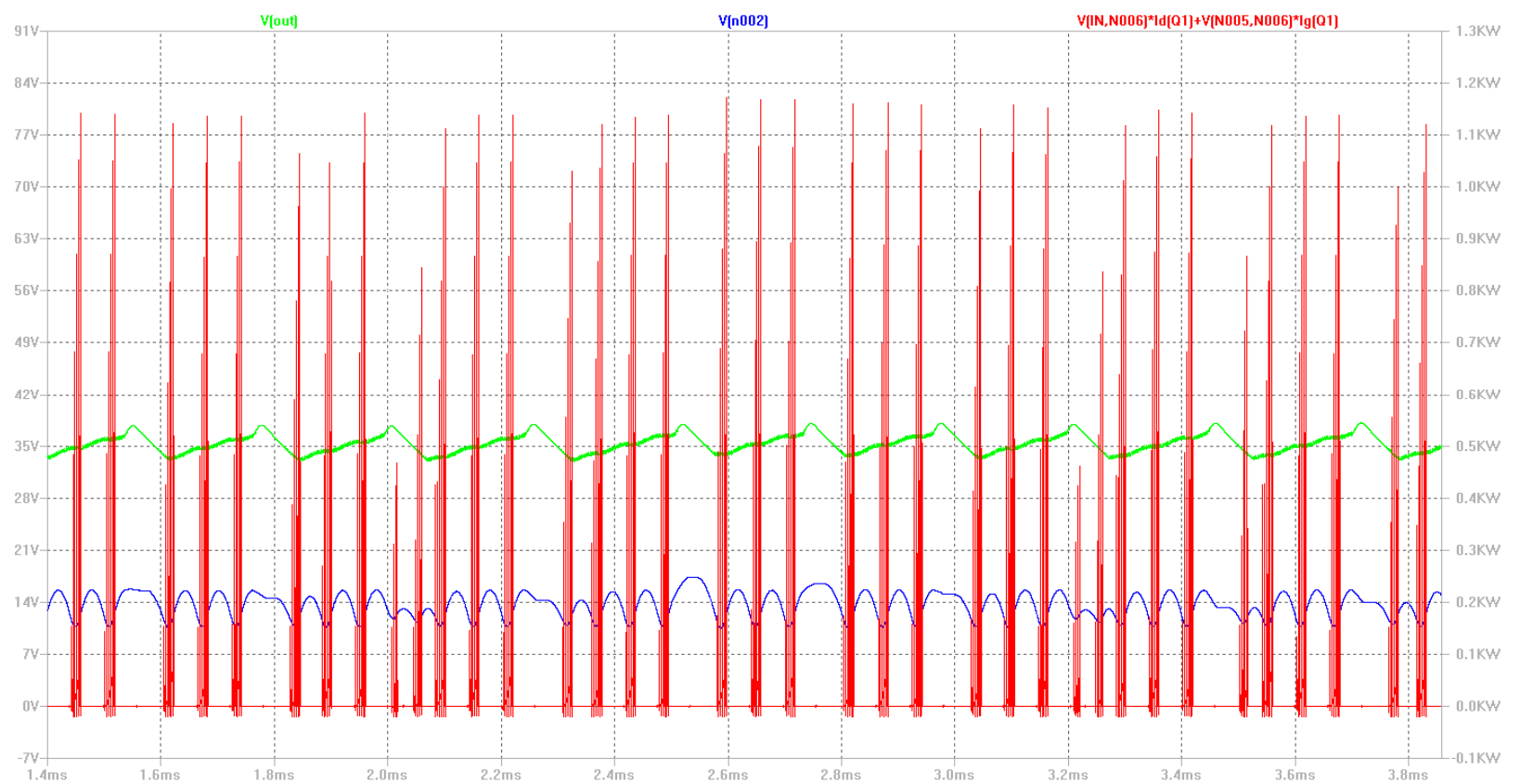

Figure B-51: Output Voltage, Buck Stage Output Voltage and $Q_{1}$ Power Dissipation of Four-Phase Buck, Two-Phase Boost, Multi-Stage Buck-Boost Converter Design in Steady-State Operation with $300 \mathrm{kHz}$ Switching Frequency and IRF510 and HUFA76413 MOSFETS

For steady-state operation, the average power dissipated across $\mathrm{Q}_{1}$ is $4.7982 \mathrm{~W}$. Average output voltage is $35.268 \mathrm{~V}$, average output current is $8 \mathrm{~A}$, average input voltage is $60 \mathrm{~V}$, and average input current is $-5.7403 \mathrm{~A}$. This corresponds to an overall $81.9 \%$ system efficiency. The maximum power transient is $1.17 \mathrm{~kW}$. Likewise with the two-phase converter design from the previous section, lethal switching power transients still exist.

This project then further reduces switching frequency on both stages to $100 \mathrm{kHz}$, as an attempt at reducing the switching power transient magnitudes. The only modifications required for this switching frequency change are changing the buck input stage switching frequency resistors to $191 \mathrm{k} \Omega$ and the boost output stage switching frequency resistors to $129.9 \mathrm{k} \Omega$. This project then measures power dissipated across $\mathrm{Q}_{1}$ using this project's maximum stress condition. Figure B-52 shows in steady-state operation the output voltage (denoted by the green waveform), buck stage output voltage 
(denoted by the blue waveform) and $\mathrm{Q}_{1}$ power dissipation (denoted by the red waveform) for the new converter 4-phase design running at $100 \mathrm{kHz}$ switching frequency.

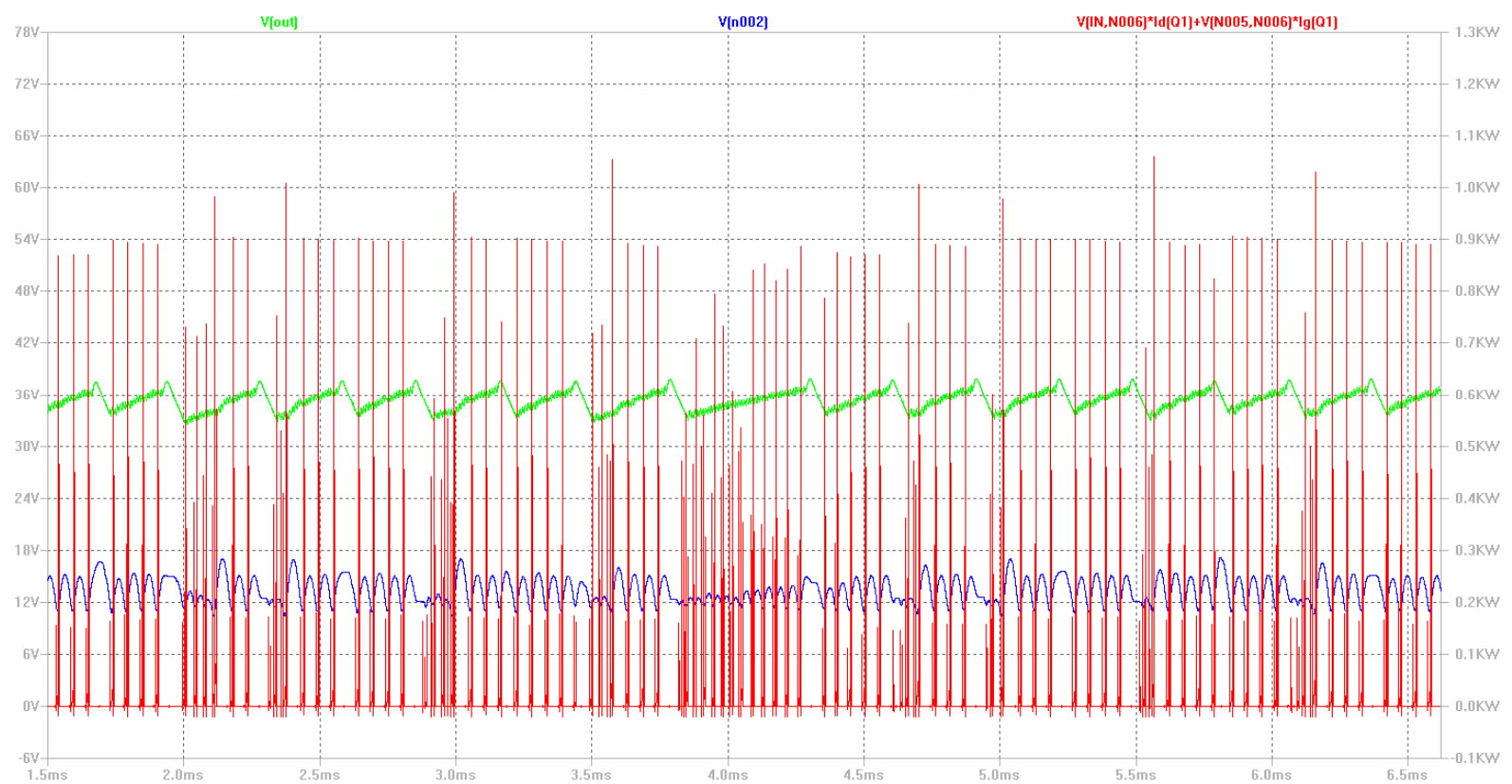

Figure B-52: Output Voltage, Buck Stage Output Voltage and $Q_{1}$ Power Dissipation of Four-Phase Buck, Two-Phase Boost, Multi-Stage Buck-Boost Converter Design in Steady-State Operation with $100 \mathrm{kHz}$ Switching Frequency and IRF510 and HUFA76413 MOSFETs

During steady-state operation, the average power dissipated across $\mathrm{Q}_{1}$ is $3.9228 \mathrm{~W}$.

Average output voltage is $35.321 \mathrm{~V}$, average output current is $8 \mathrm{~A}$, average input voltage is $60 \mathrm{~V}$, and average input current is $-5.412 \mathrm{~A}$. This corresponds to an overall $87 \%$ system efficiency, which suffices. However, from figure B-52, the maximum power transient is $1.055 \mathrm{~kW}$. This project then examines $\mathrm{Q}_{1}$ 's switching trajectory waveforms. Figure B-53 is a zoomed in switching trajectory plot showing $\mathrm{Q}_{1}$ 's drain-source voltage (in magenta) and drain current (in teal), as well as $\mathrm{Q}_{1}$ 's maximum switching power transient (in red) at the maximum power transient's instant. 


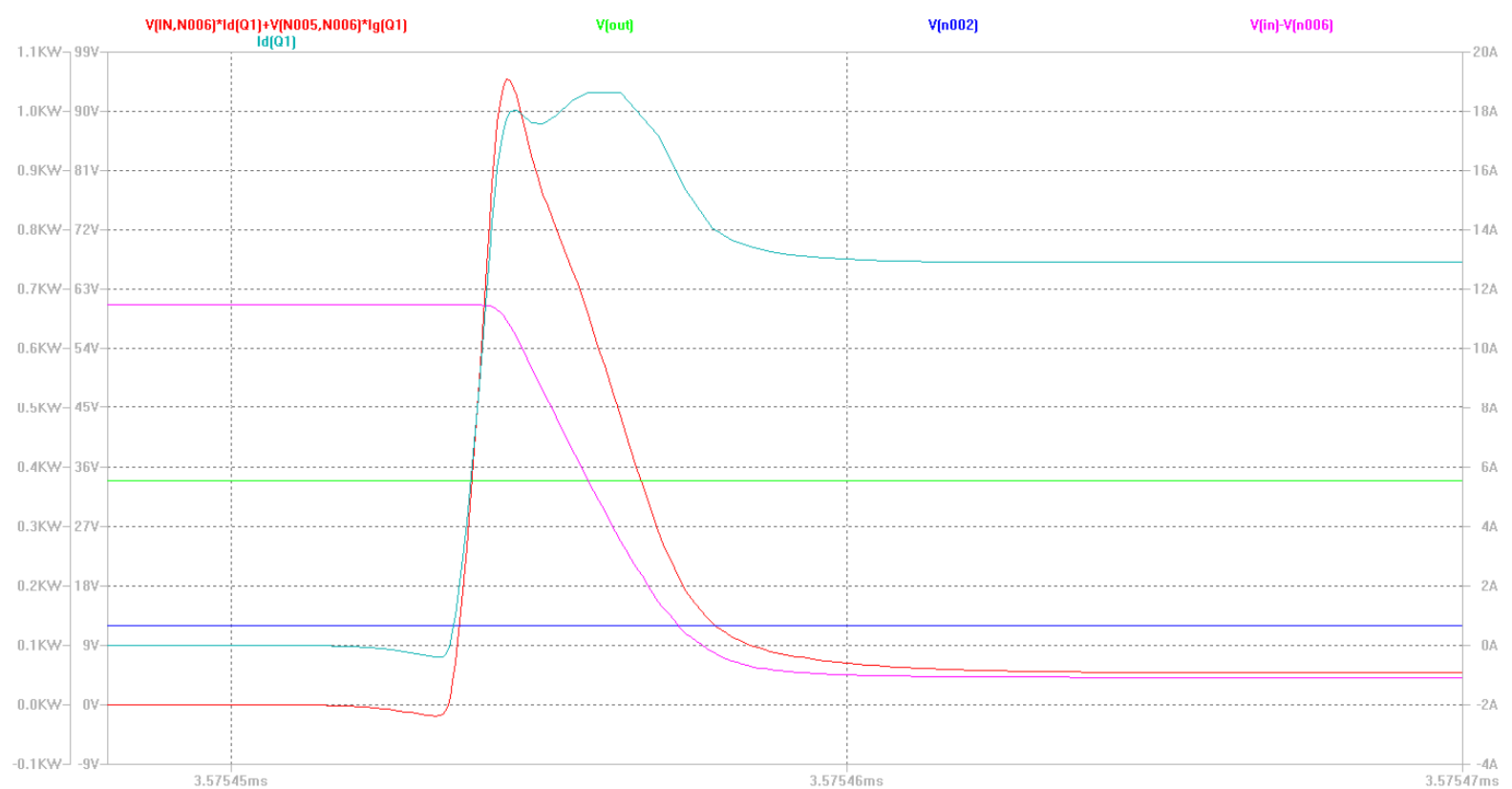

Figure B-53: $Q_{1}$ Drain-Source Voltage, Gate Voltage, Power Dissipation and Drain Current at Turn-On Transition Under Maximum Input and Load

$\mathrm{Q}_{1}$ 's drain-source voltage still does not decay quickly enough as $\mathrm{Q}_{1}$ turns on (when current begins flowing through its drain). The transient lasts approximately $0.923 \mu$ s. This project then examines other solutions for mitigating this power transient problem, as using MOSFETs with higher on-state drain-source resistance and decreasing the switching frequency alone doesn't eliminate it. A soft-switching technique may not function properly with this multi-phase design, and increasing gate-drain capacitance as well as gate resistance on $\mathrm{Q}_{1}$ only make matters worse, as doing so results in the transistor spending more time in the switching region.

A possible viable solution for the switching power loss and switching power transient problems is implementing a snubber. A snubber is a discrete circuit designed for altering a MOSFET's switching trajectory and in the end reduce its switching power loss. By reducing the switching power loss, a snubber in turn reduces the overall power loss in 
a DC-DC converter, thus improving converter efficiency. There are two types of snubbers: turn-on and turn-off snubbers. As their names suggest, they are designed to alter switching trajectory during MOSFET turn-on and turn-off transistions, respectively. A snubber may in fact be the solution that this project desires for reducing its converter power loss problems, as depending on their component values, they slow down current rise or speed up drain-source voltage decay at MOSFET turn-on, or speed up current decay or slow down drain-source voltage rise at turn-off.

Figure B-54 shows a basic turn-off snubber, and it consists of a resistor, capacitor and two diodes.

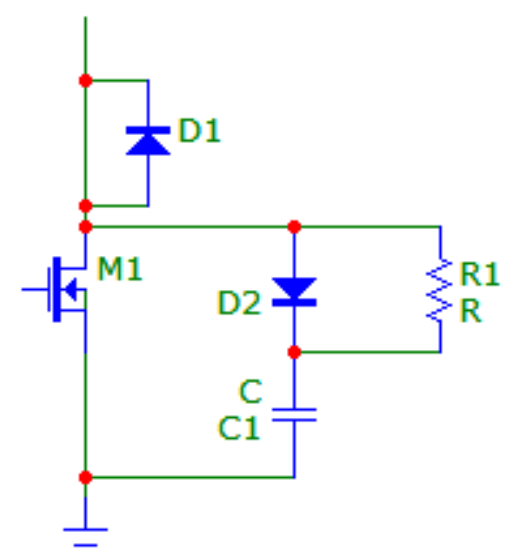

Figure B-54: Basic Turn-Off Snubber Circuit

Diode $\mathrm{D}_{1}$ allows for a drain current flow path when the MOSFET turns off - in which case the MOSFET's drain current flows into the snubber circuit.

There are some design issues with using a snubber, however, according to [13]:

- A smaller snubber capacitor allows for a quicker drain-source voltage rise before its drain current decays to zero, while a larger snubber capacitor slows down drain-source voltage rise and decay (the latter is undesirable during MOSFET turn-on).

- Energy absorbed by the MOSFET is inversely proportional to the snubber capacitor size. 
- The most desirable situation is having the drain current decay to zero before the snubber capacitor voltage rises.

- Increasing the snubber capacitor size results in greater power losses across the snubber circuit itself.

There are numerous compromises that this project must make when designing a snubber.

Designing a snubber requires carefully selecting component sizes because the fast-

switching nature of this converter's MOSFETs results in snubber components sensitivity (especially if this project selects the incorrect component sizes) [13].

Figure B-55 shows a basic turn-on snubber, and consists of an inductor, a resistor and two diodes.

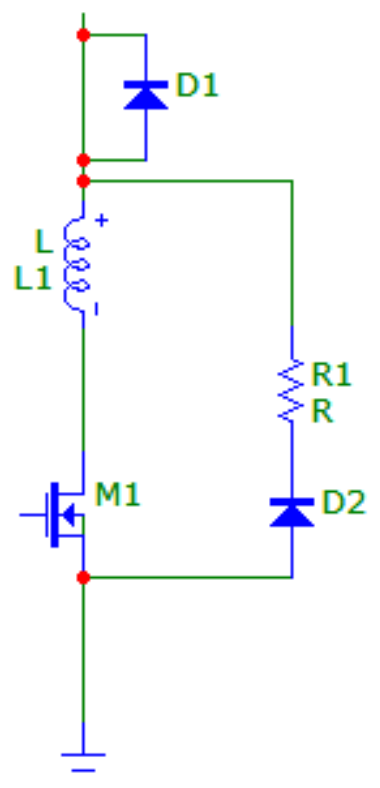

Figure B-55: Basic Turn-On Snubber Circuit

The inductor is a turn-on snubber's most crucial component. Because inductors oppose changes in current, connecting an inductor in series with a MOSFET allows for drain current rise slowdown during the MOSFET's turn-on transition.

In either case, adding a resistive snubber results in some power loss across the snubber (and hence, overall efficiency loss in the converter itself). However, as long as 
this project sizes the snubber components appropriately the losses from the snubber are almost negligible, while at the same time provides the potential for greatly reducing overall power loss across the converter's switching MOSFETs.

This project then returns to the pure two-phase converter design from appendix B1.3 and determines if using both a turn-on and turn-off snubber for the buck input stage MOSFETs improves converter performance. Likewise with the most recent 4-phase design, this project also reduces the switching frequency of both stages to $100 \mathrm{kHz}$.

This project then designs a turn-off snubber for the buck stage for optimal operation (such that the MOSFET's drain-source voltage reaches its final value at the same time the drain current reaches zero, while at the same time minimizing power loss across the snubber resistor).

First, we this project defines the turn-off snubber parameters:

$V_{I N}=$ Input Voltage

$V_{\text {OUT }}=$ Output Voltage

$I_{S W}=$ Maximum Switch (MOSFET)Drain - Source Current

$f_{s}=$ Switching Frequency

$D=$ Duty Cycle

$t_{f}=I_{S W}$ fall time (from $90 \%$ to $10 \%$ of its maximum value)

$C=$ Snubber Capacitor Value

$C_{\text {opt }}=$ Optimal Snubber Capacitor Value

$C_{\text {dis }}=$ Capacitor Discharge Time

$R=$ Snubber Resistor Value

Equations (B1.15) and (B1.16) determines the buck stage's duty cycle under this project's maximum stress level.

$$
\begin{gathered}
V_{\text {OUT }}=D V_{I N} \\
V_{\text {OUT }}=12 \mathrm{~V}, V_{I N}=60 \mathrm{~V}
\end{gathered}
$$

Therefore: 


$$
D=\frac{V_{O U T}}{V_{I N}}=\frac{12}{60}=0.2, \text { or } 20 \%
$$

For the converter operating under the maximum stress level:

$$
V_{I N}=60 \mathrm{~V}, I_{S W}=27 \mathrm{~A}, f_{s}=100 \mathrm{kHz}, D=0.2, t_{f}=5 n \text { ( taken from earlier plots) }
$$

Equation (B1.19) calculates the turn-off snubber capacitor value:

$$
C=\frac{I_{S W} t_{f}}{2 V_{S}}=\frac{27(5 n s)}{2(60)}=1.125 n F
$$

Equation (B1.20) calculates the turn-off snubber resistor value assuming 5 time constants for the capacitor discharge time:

$$
R=\frac{\left(\frac{D}{f_{S}}\right)}{C_{d i s} C}=\frac{\left(\frac{0.2}{100 \mathrm{kHz}}\right)}{(5)(1.125 n F)}=355.556 \Omega
$$

This project rounds this resistor value up to $356 \Omega$. Equation (B1.21) calculates the turnoff snubber's optimum capacitor value associated with the switching power losses:

$$
C_{o p t}=\frac{I_{S W} t_{f}}{\sqrt{12} V_{S}}=\frac{(27)(5 n s)}{\sqrt{12}(60)}=0.6495 n F
$$

This project rounds this capacitor value up to $0.65 \mathrm{nF}$.

This project now designs its converter turn-on snubber. As mentioned before, connecting an inductor in series with the transistor slows down the transistor's drainsource current during the turn-on transition. Because of this project's converter's MOSFETs' fast switching abilities, it is crucial that the turn-on snubber inductor isn't too large (keeping it in the $\mathrm{nH}$ range is optimal). This project also adds a parallel resistor to the inductor. Designing the turn-on snubber first requires knowing the time constant of an RL circuit, as (B1.22) denotes.

$$
\tau=\frac{L}{R}
$$


$\mathrm{L}$ denotes the inductance and $\mathrm{R}$ is the resistance. The inductor current in the snubber also must decay close to zero (but not exactly to zero) for the maximum snubber effectiveness during the next time cycle that the transistor turns on. However, as mentioned earlier the inductor value cannot be too large, otherwise the MOSFET's drain-source current rises and decays too slowly when the drain-source voltage increases, resulting in larger switching power losses. Equation (B1.23) begins the inductor selection process, and it assumes that the snubber requires 3 time constants for inductor discharge.

$$
t_{\text {off-state }}>\frac{3 L}{R}
$$

$\mathrm{t}_{\text {(off-state) }}$ is the time period that the drain current is zero. This project determined $\mathrm{t}_{\text {off-state }}$ as $1.2 \mu$ s from earlier plots. This project selects $40 \mathrm{nH}$ (a reasonably small value, but not too small) for its converter's snubber inductor. From that inductance value, (B1.24) calculates the turn-on snubber resistor value.

$$
R>\frac{3 L}{t_{\text {off-state }}}=\frac{3(40 \mathrm{nH})}{1.2 \mu \mathrm{s}}=0.1 \Omega
$$

Because the resistor value must be greater than $0.1 \Omega$, this project selects $0.125 \Omega$ for the turn-on snubber resistor.

Now this project adds both its designed turn-on and turn-off snubbers to its converter. Figure B-56 shows the snubber additions to this project's earlier pure twophase converter design. 


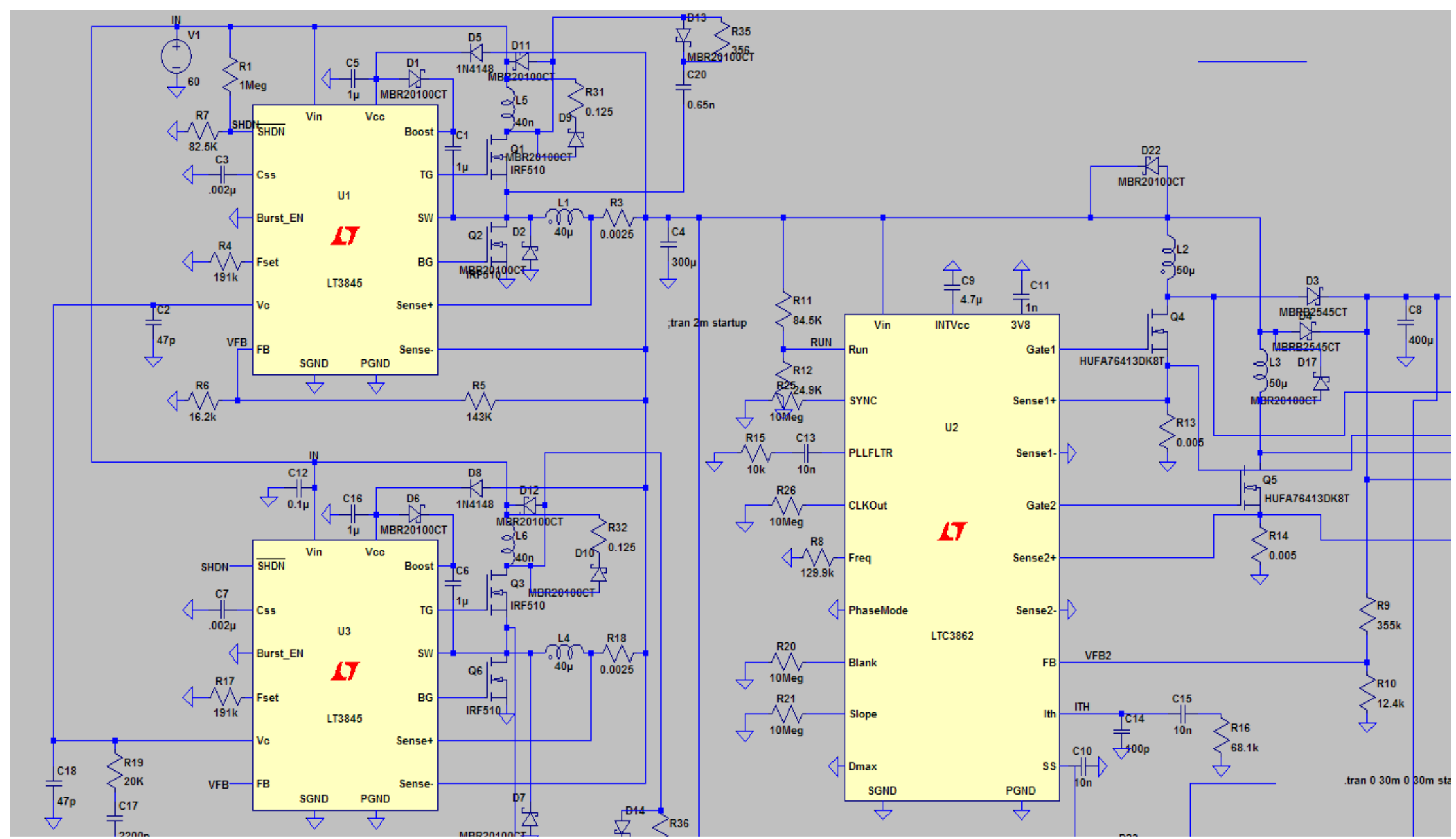

Figure B-56: Two-Phase Buck, Two-Phase Boost, Multi-Stage Buck-Boost Converter Design with 100 kHz Switching Frequency and Turn-On and Turn-Off Snubbers 
This project uses Schottky diodes for the snubber diodes for minimizing snubber response time during MOSFET turn-on and turn-off transitions. This project also increased the input buck stage's current sense resistor to $6.8 \mathrm{~m} \Omega$ (from $1 \mathrm{~m} \Omega$ prior) and decreased the buck stage's output capacitor to $40 \mu \mathrm{F}$ (from $100 \mu \mathrm{F}$ prior) for further decreasing the maximum current allowed through the input buck stage (and hence decreasing overall drain current through the MOSFETs in the buck stage). This project does not require a stable buck stage's output voltage - just as long as it is high enough for the converter's output boost stage to regulate the proper $36 \mathrm{~V}$ output.

This project now simulates the refreshed two-phase converter and checks the power dissipation across $\mathrm{Q}_{1}$. Figure B-57 shows the new two-phase converter's output voltage (in green), buck stage output voltage (in blue), and $\mathrm{Q}_{1}$ power dissipation (in red).

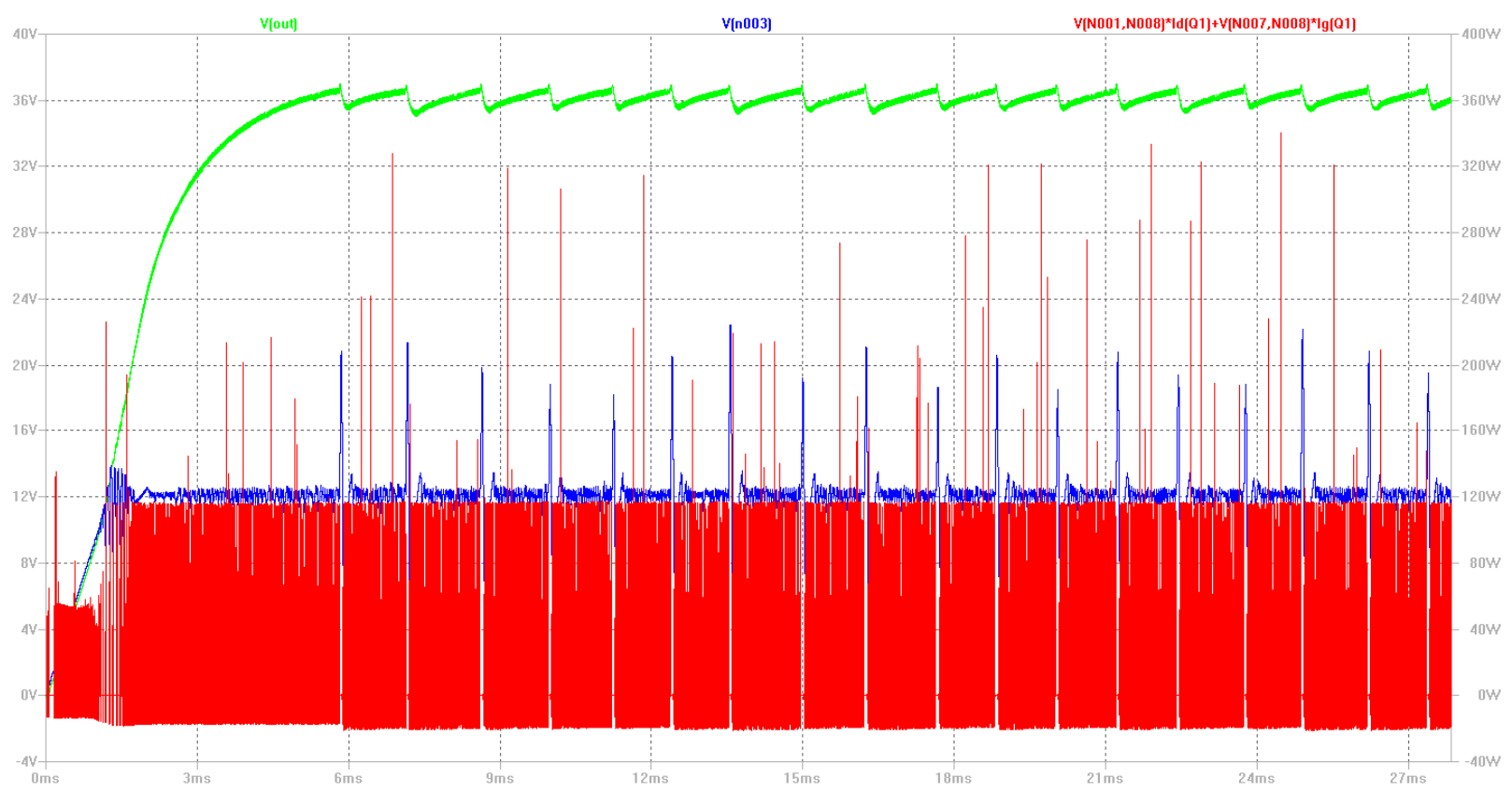

Figure B-57: Output Voltage, Buck Stage Output Voltage and $Q_{1}$ Power Dissipation of Two-Phase Buck, Two-Phase Boost, Multi-Stage Buck-Boost Converter Design with $100 \mathrm{kHz}$ switching frequency and TurnOn and Turn-Off Snubbers 
The buck stage's output voltage is noticeably noisier after adding the snubber (affected mostly by the output capacitor value reduction - as the output capacitor acts as a low pass filter). However, the buck stage's output voltage doesn't need to be completely clean, as the output boost stage regulates that buck voltage to a stable value. The maximum power transient further reduces in magnitude; it is now 340W. Other smaller transients still occur, however. The majority of the transients are closer to $120 \mathrm{~W}$. The converter itself still regulates at $36 \mathrm{~V}$ overall average output. While these power transients aren't too lethal (it is certainly much improved from before), this project can still further improve on this design. Figure B-58 is a zoomed in plot of figure B-57 showing the converter in steady-state operation.

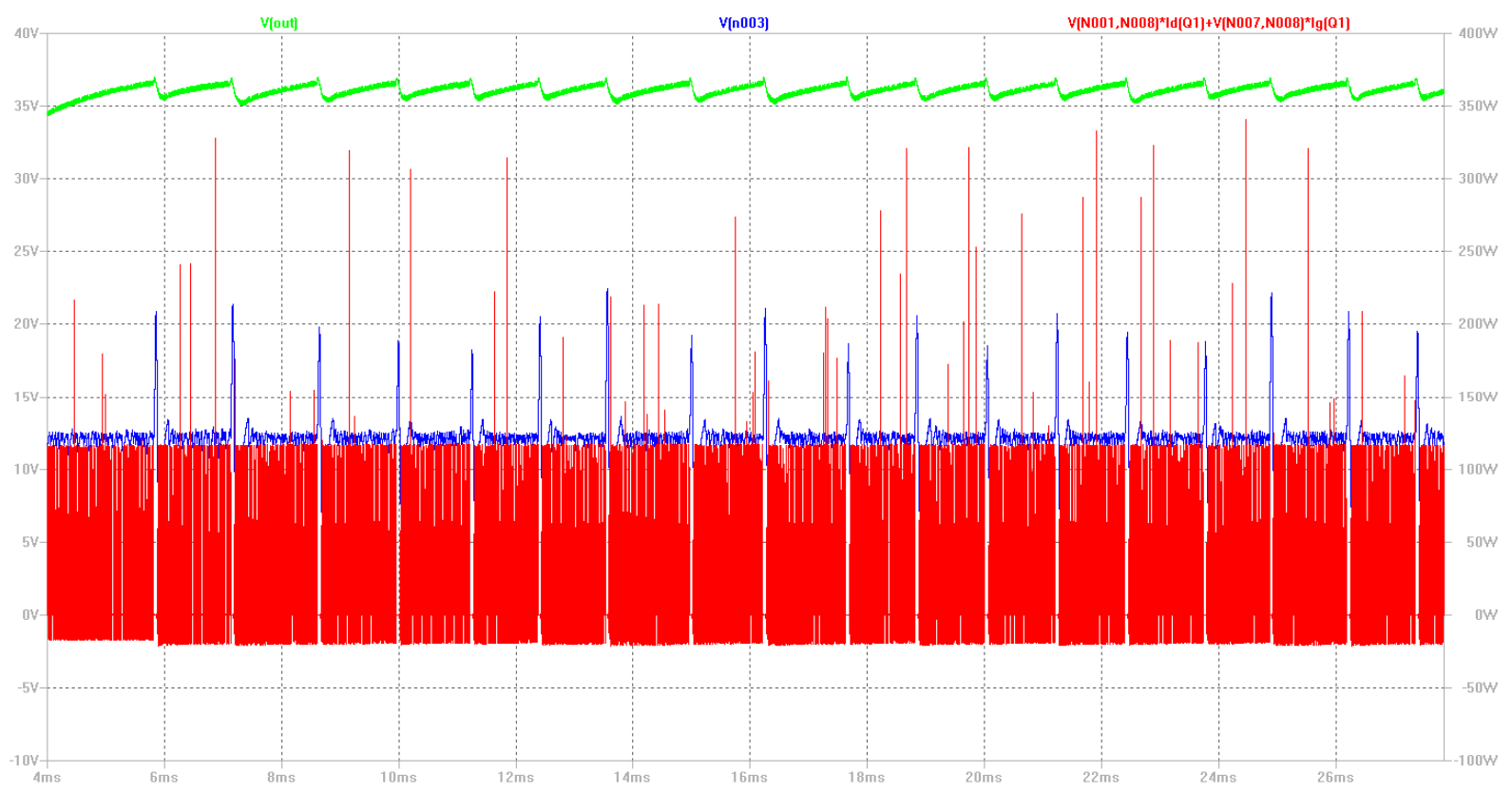

Figure B-58: Output Voltage, Buck Stage Output Voltage and $Q_{1}$ Power Dissipation of Two-Phase Buck, Two-Phase Boost, Multi-Stage Buck-Boost Converter Design in Steady-State Operation with $100 \mathrm{kHz}$ Switching Frequency and Turn-On and Turn-Off Snubbers 
Average power dissipated across $\mathrm{Q}_{1}$ for this time window is $12.66 \mathrm{~W}$, which is actually worse than the previous converter designs that did not employ a snubber. Figure B-59 is a plot that includes the converter's input current (denoted by the teal waveform).

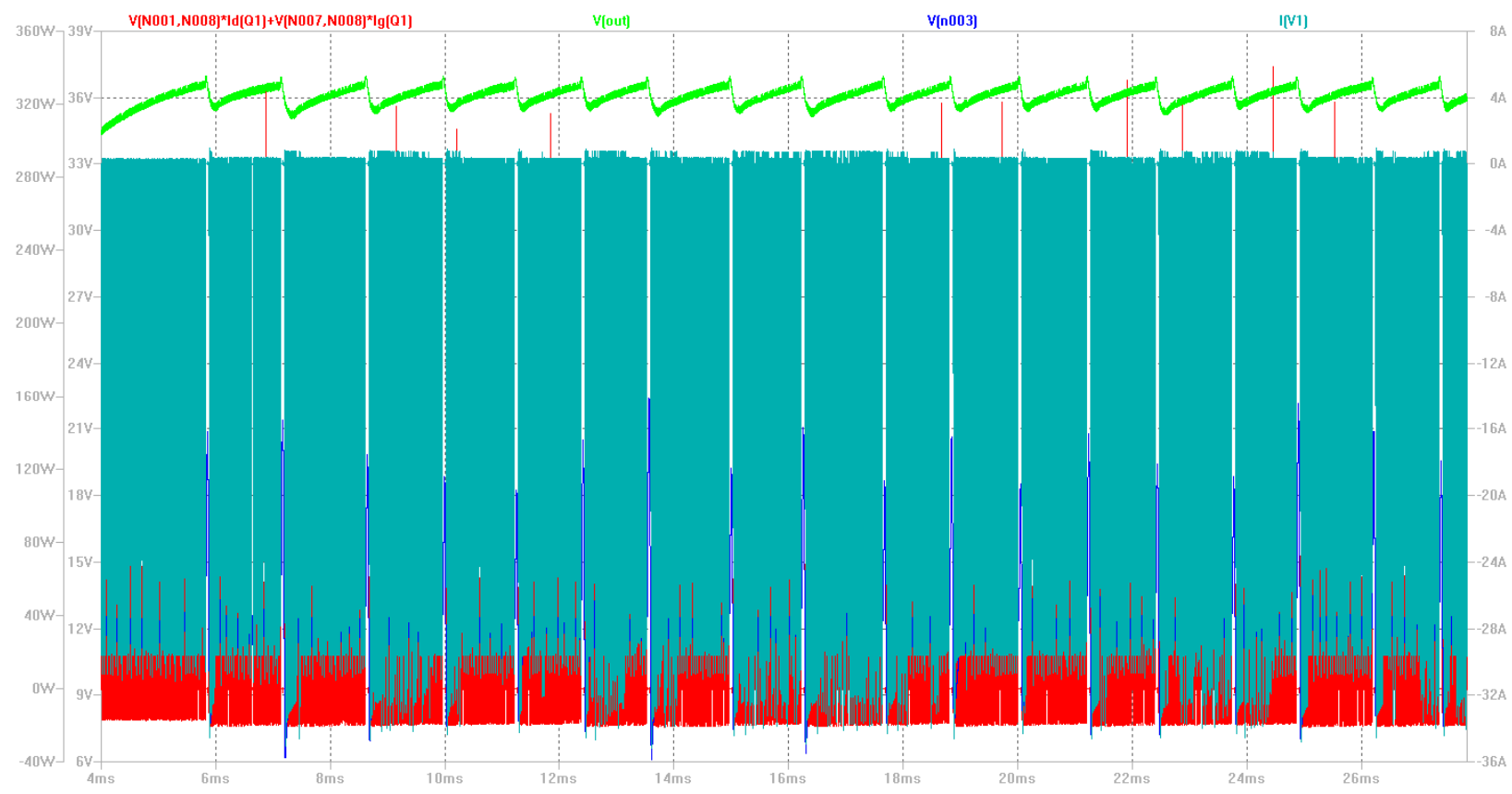

Figure B-59: Output Voltage, Input Current and $Q_{1}$ Power Dissipation of Two-Phase Buck, Two-Phase Boost, Multi-Stage Buck-Boost Converter Design in Steady-State Operation with $100 \mathrm{kHz}$ Switching Frequency and Turn-On and Turn-Off Snubbers

Overall, the maximum input current decreased with respect to the previous design. For this simulation time window, average output voltage is $36.053 \mathrm{~V}$, average output current is $8 \mathrm{~A}$, average input voltage is $60 \mathrm{~V}$, average input current is $-5.94 \mathrm{~A}$. This corresponds to an $80.9 \%$ overall system efficiency. Figure B-60 is a plot showing $\mathrm{Q}_{1}$ 's switching trajectory during the highest measured power transient. The teal waveform denotes $\mathrm{Q}_{1}$ 's drain current while the magenta waveform denotes drain to source voltage. The overall maximum drain current through $\mathrm{Q}_{1}$ during this entire simulation period is $15.6 \mathrm{~A}$. 


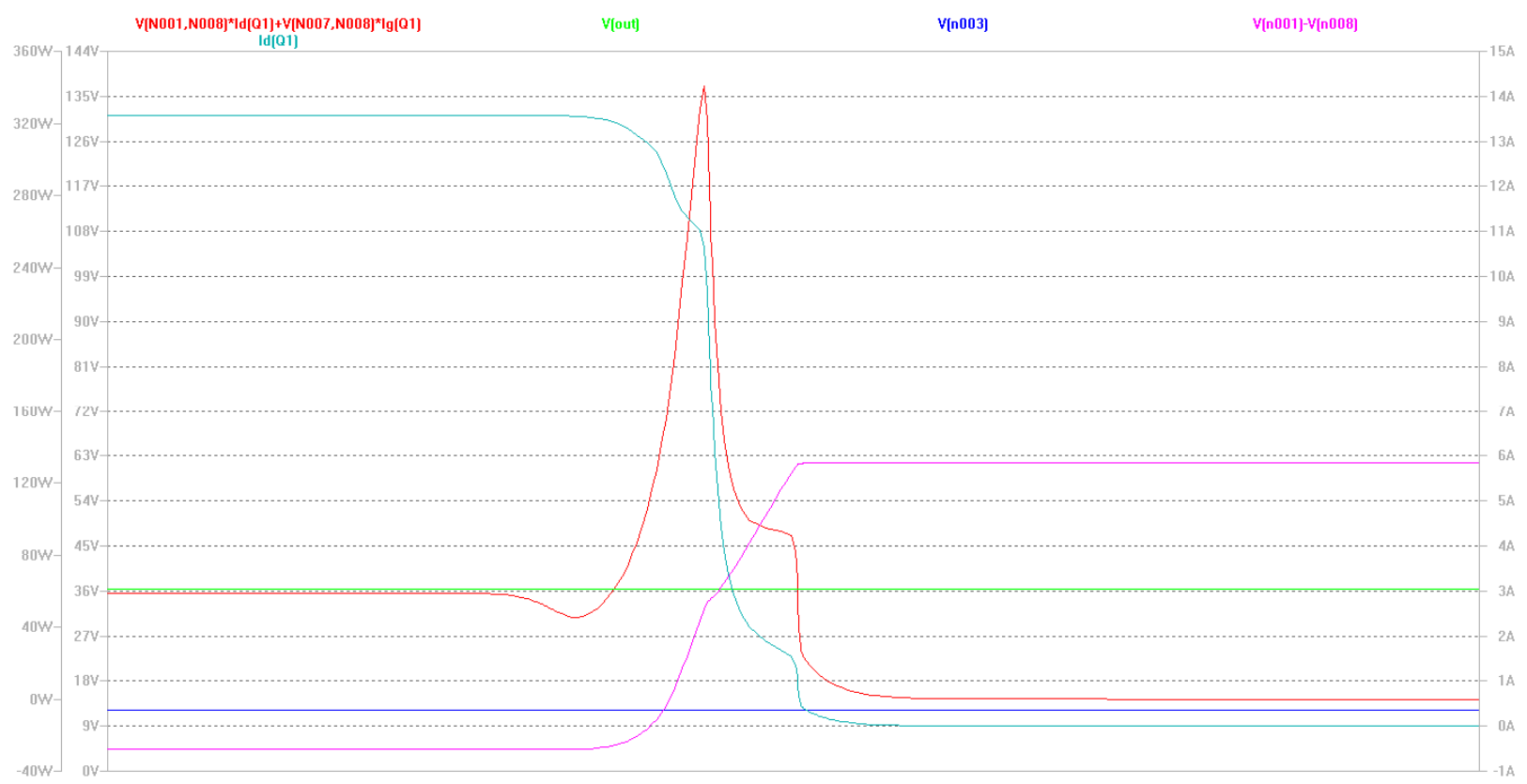

Figure B-60: $Q_{1}$ Power Dissipation, Drain-Source Voltage and Drain Current of Two-Phase Buck, TwoPhase Boost, Multi-Stage Buck-Boost Converter Design in Steady-State Operation with 100 kHz Switching Frequency and Turn-On and Turn-Off Snubbers

Making the power transient levels more acceptable requires severely reducing the current through each transistor. This project accomplishes this task by adding more phases to either the buck or boost stage. While browsing through the LTC3862 datasheet, this project's author noticed that one of the application notes had a simplified method for creating a two-phase boost converter (for our boost stage) while using only one PWM controller IC [75]. This project then adopted that design from the LTC3862 datasheet and modified it for withstanding a $12 \mathrm{~V}$ input (from the converter's input buck stage), 36V output and an 8A load, while using the same transistors and diodes from the previous converter design. Figure B-61 shows this simplified two-phase output boost stage realization. 


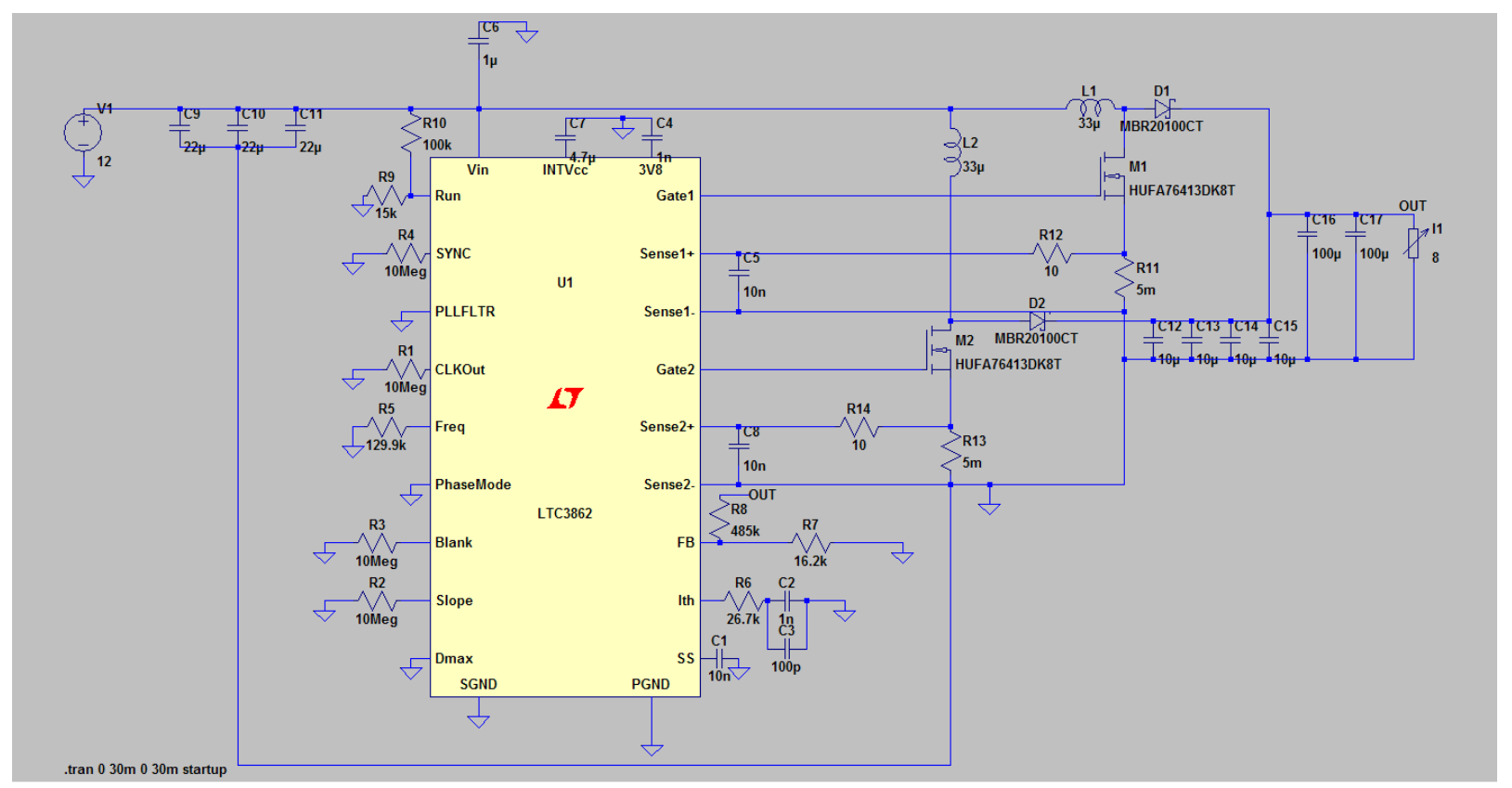

Figure B-61: Simplified Two-Phase Output Boost Converter Stage

This simplified design was certainly a breakthrough, as now this project can add more phases to the converter's input stage without adding too much complexity. This means that a 4-phase input, 2-phase output buck-boost converter may now be more physically feasible DC-DC converter design. Adding more phases to the input buck stage reduces the current through each transistor at each phase, thereby reducing the power transients occurring across those transistors. Now this project revisits its old 4-phase design. Figure B-62 shows this newly revised 4-phase input, 2-phase output design, with turn-on and turn-off snubbers still in place on the buck stage and switching frequency maintained at $100 \mathrm{kHz}$ for both stages. For this design this project changed the current sense resistors on the boost stage to $6 \mathrm{~m} \Omega$, further reducing the maximum current through each output stage MOSFET. 


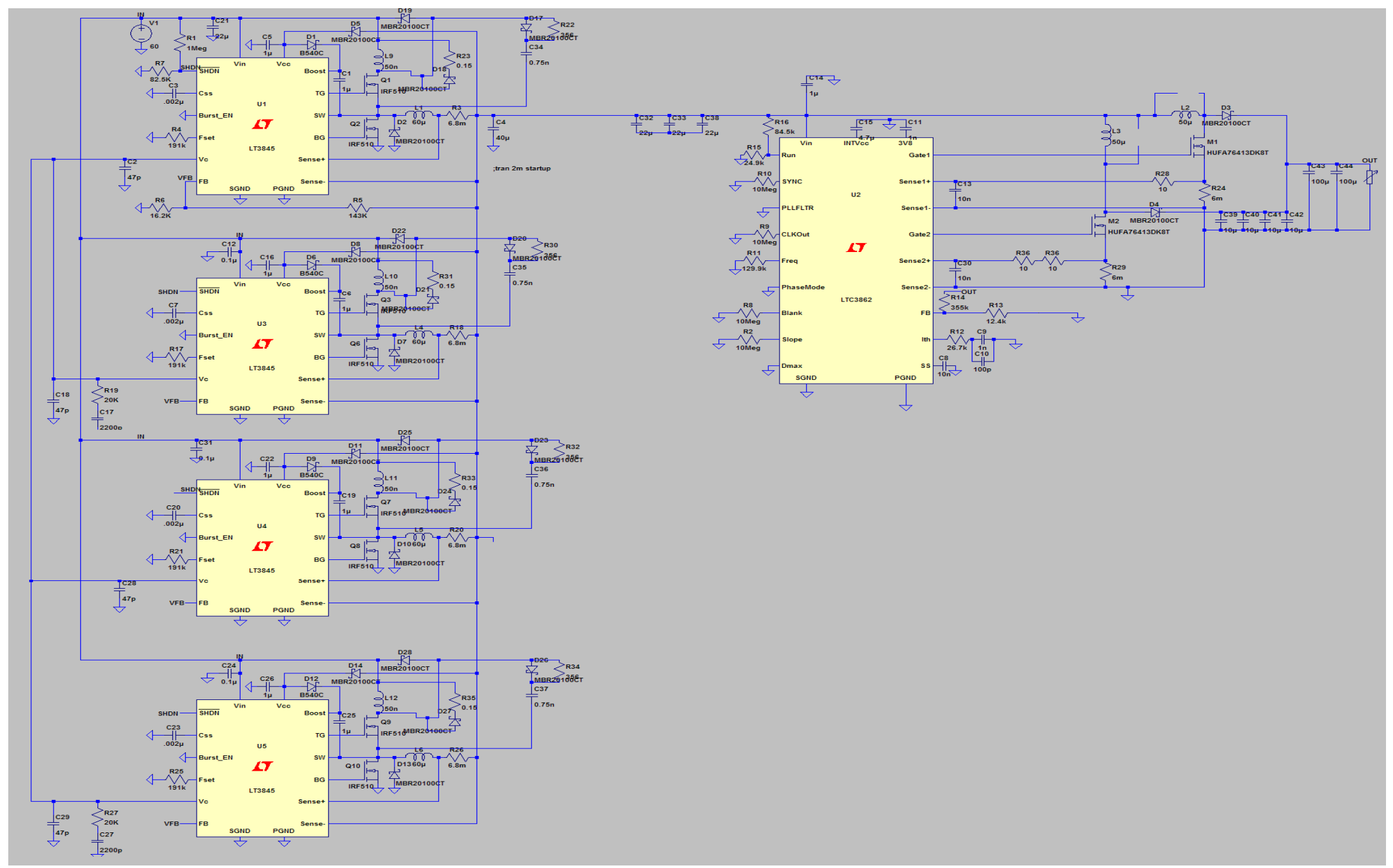

Figure B-62: Four-Phase Buck, Two-Phase Boost, Multi-Stage Buck-Boost Converter Design with 100 kHz Switching Frequency and Turn-On and Turn-Off Snubbers 
This project now examines the power dissipation across $\mathrm{Q}_{1}$. Figure B-63 is a plot showing the newly-redesigned converter's performance under our maximum stress condition (converter output voltage is in green, buck stage output voltage is in blue, and power dissipated across $\mathrm{Q}_{1}$ is in red).

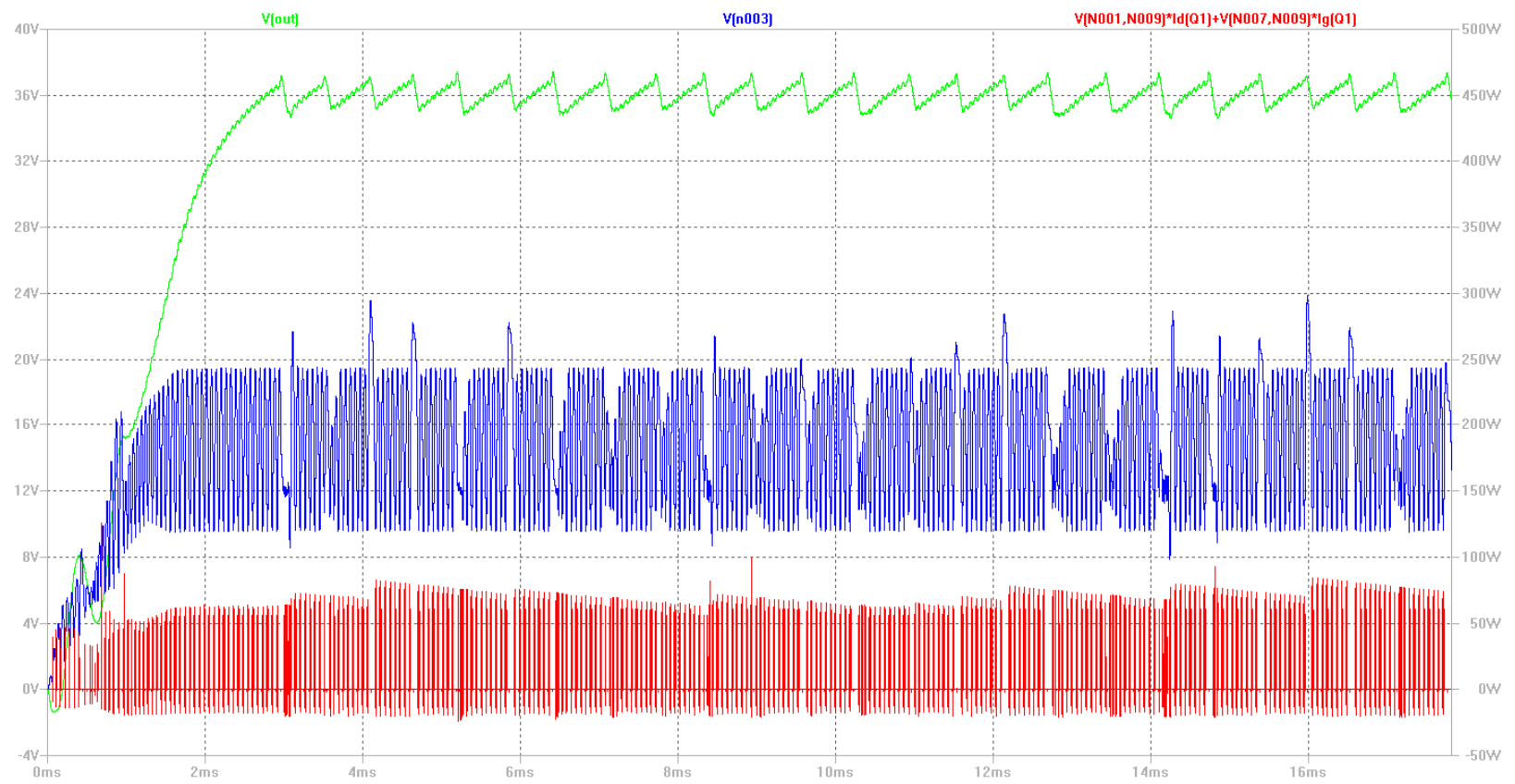

Figure B-63: Output Voltage, Buck Stage Output Voltage and $Q_{1}$ Power Dissipation of Four-Phase Buck, Two-Phase Boost, Multi-Stage Buck-Boost Converter Design with 100 kHz Switching Frequency and TurnOn and Turn-Off Snubbers

The buck stage's output voltage is still noisy. However, for this converter design, that noise is tolerable as long as the voltage is high enough for the boost stage to regulate. The major improvement here is that the majority of $\mathrm{Q}_{1}$ 's switching power transients reduce to the $80 \mathrm{~W}$ range, and is certainly a significant step forward from the $1.5 \mathrm{~kW}$ transients from this project's original converter designs. Converter output voltage swings between 34.8 and $37.4 \mathrm{~V}$, which corresponds to a $7.2 \%$ ripple with respect to a $36 \mathrm{~V}$ average output voltage, which suffices based on the specifications from section 1.2. Figure B-64 shows a zoomed-in plot of figure B-63 in steady-state converter operation: 


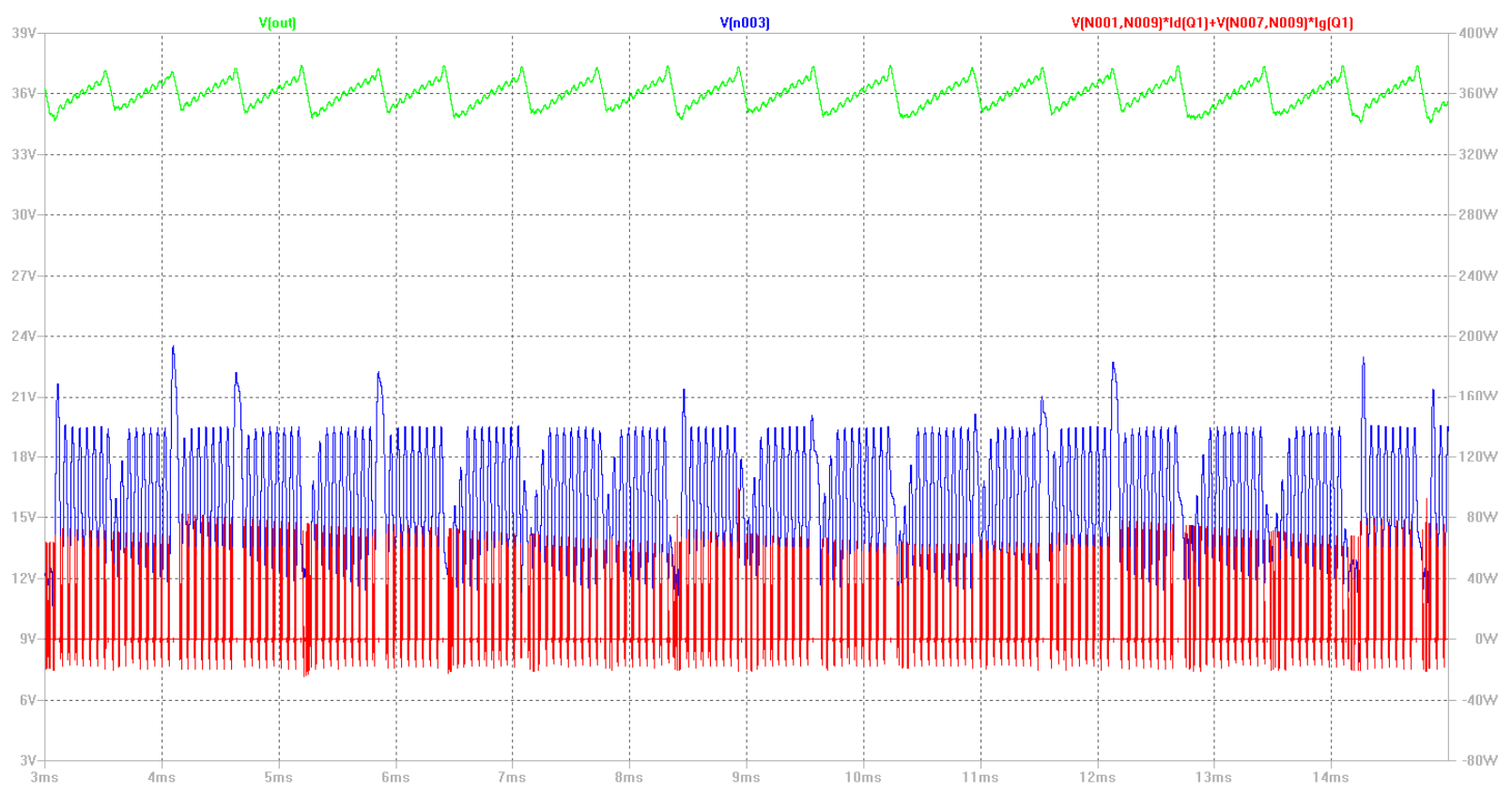

Figure B-64: Output Voltage, Buck Stage Output Voltage and $Q_{1}$ Power Dissipation of Four-Phase Buck, Two-Phase Boost, Multi-Stage Buck-Boost Converter Design in Steady-State Operation with $100 \mathrm{kHz}$ Switching Frequency and Turn-On and Turn-Off Snubbers

For this overall converter the maximum power transient occurs at $125.4 \mathrm{~W}$ (during startup), but once the converter reaches steady-state the majority of the power transients lie near $80 \mathrm{~W},+/-5 \mathrm{~W}$. These transient values are acceptable for the selected transistors used in this design. Average power dissipation across $\mathrm{Q}_{1}$ for this simulation time window (3 to $15 \mathrm{~ms}$ ) is $3.24 \mathrm{~W}$. The converter's average output voltage is $35.994 \mathrm{~V}$, average output current is $8 \mathrm{~A}$, average input voltage is $60 \mathrm{~V}$, and average input current $-5.477 \mathrm{~A}$. This corresponds to an overall $87.6 \%$ system efficiency, which more than suffices against the 75\% efficiency requirement outlined in section 1.2. Figure B-65 shows $\mathrm{Q}_{1}$ 's switching trajectory with drain current in teal and drain-source voltage in magenta. The red waveform denotes $\mathrm{Q}_{1}$ power dissipation, the green waveform denotes converter output voltage and the blue waveform denotes the buck stage's output voltage. 


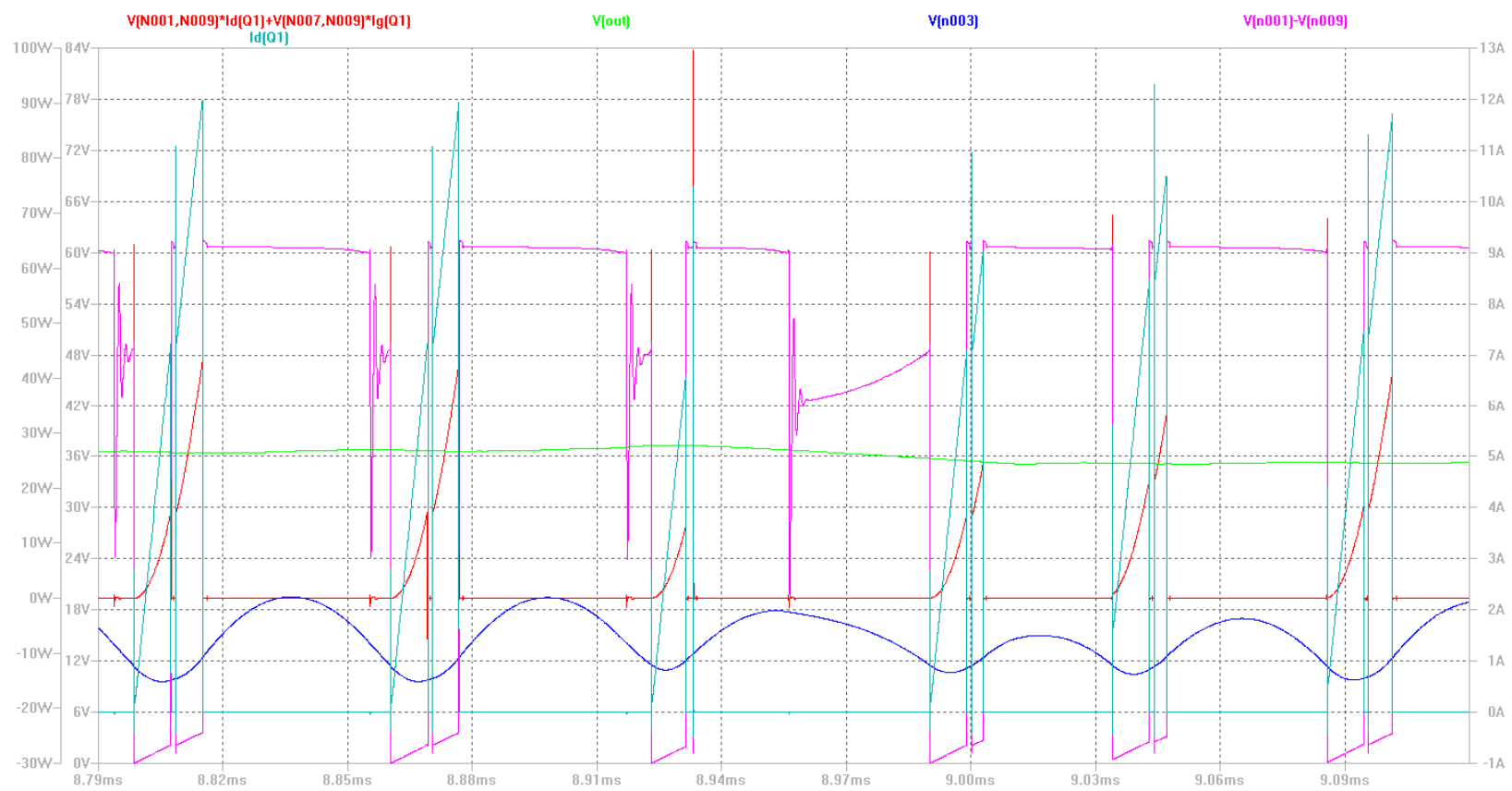

Figure B-65: $Q_{1}$ Power Dissipation, Drain-Source Voltage and Drain Current of Four-Phase Buck, TwoPhase Boost, Multi-Stage Buck-Boost Converter Design in Steady-State Operation with $100 \mathrm{kHz}$ Switching Frequency and Turn-On and Turn-Off Snubbers

Figure B-66 shows a zoomed-in plot of $\mathrm{Q}_{1}$ 's switching trajectory during the highest power transient occurrence in steady-state. 


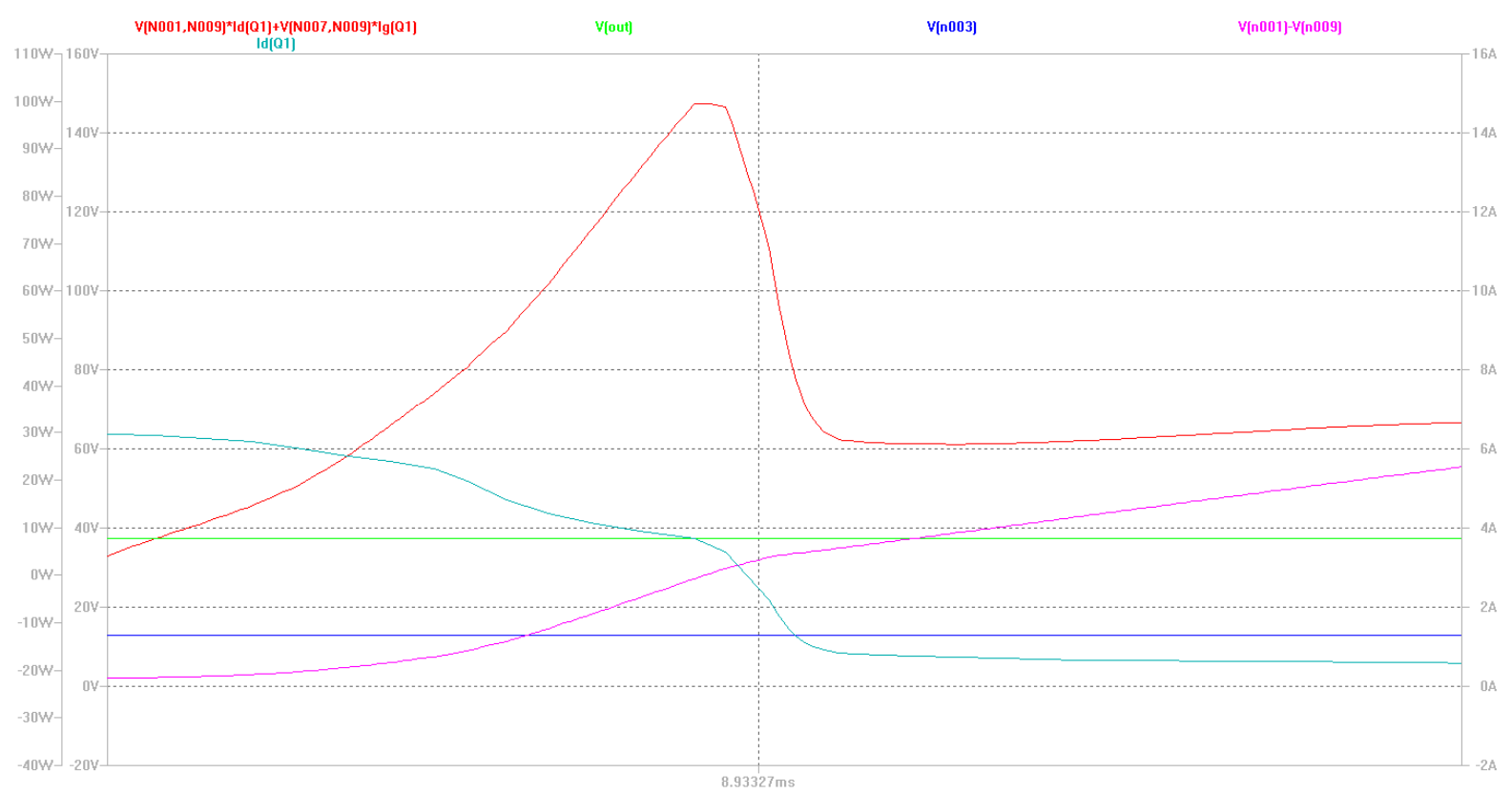

Figure B-66: $Q_{1}$ Switching Trajectory at Maximum Power Dissipation of Four-Phase Buck, Two-Phase Boost, Multi-Stage Buck-Boost Converter Design in Steady-State Operation with $100 \mathrm{kHz}$ Switching Frequency and Turn-On and Turn-On Snubbers

The same transistor turn-on issue from previous designs still exists. However, for now, this power transient level is acceptable for the selected transistors in this converter.

For the next 4-phase converter revision this project reduces the turn-on snubber resistor to $5 \mathrm{~m} \Omega$ and increases the snubber inductor to $75 \mathrm{nH}$, for determining any possible benefits with slightly increasing the inductor size and reducing the resistor size. Figure B67 shows the converter's performance in steady-state operation with these changes (with output voltage in green, buck stage output voltage in blue, and $\mathrm{Q}_{1}$ power dissipation in red). 


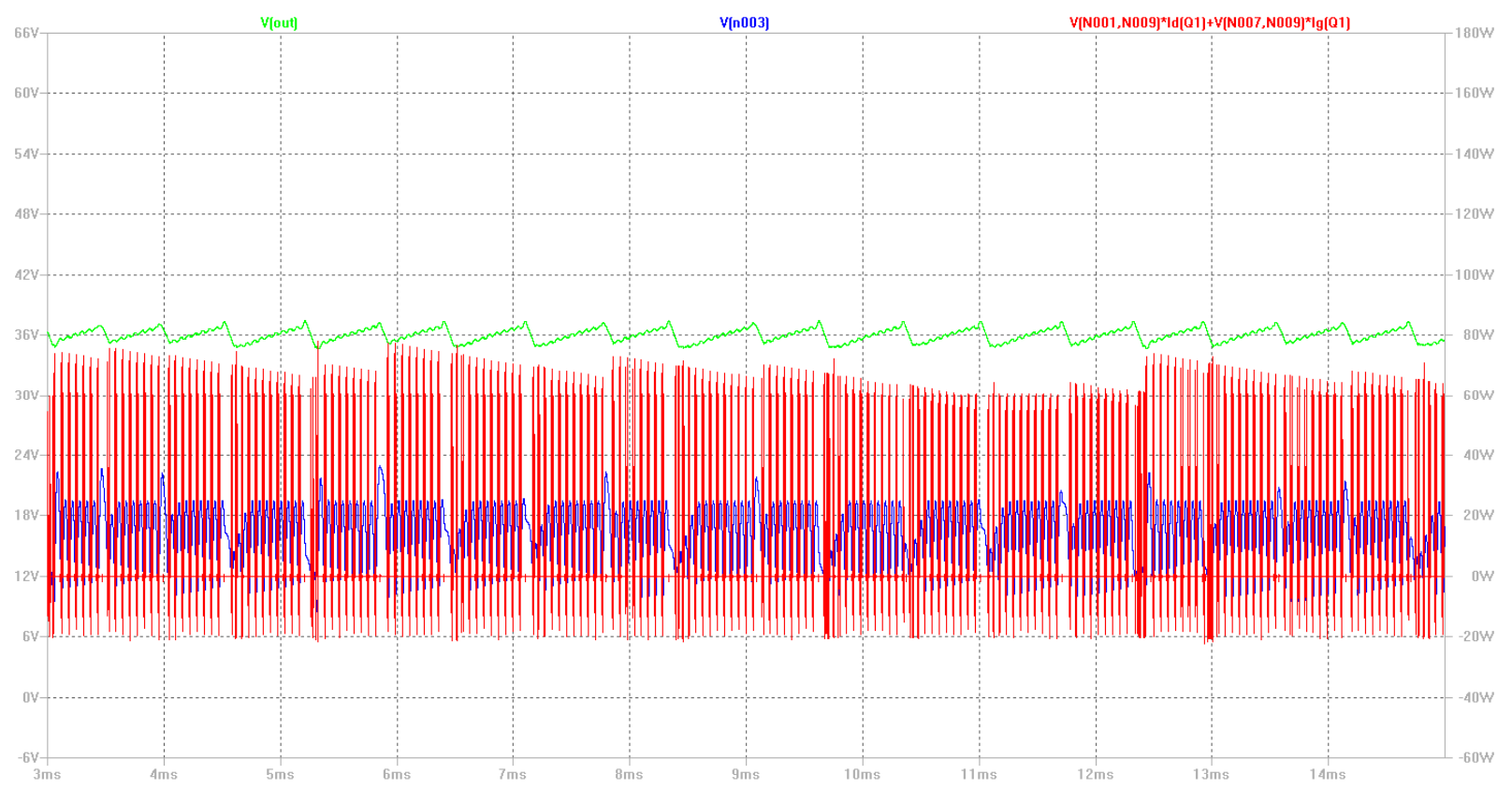

Figure B-67: Output Voltage, Buck Stage Output Voltage and $Q_{1}$ Power Dissipation of Four-Phase Buck, Two-Phase Boost, Multi-Stage Buck-Boost Converter Design with 100 kHz Switching Frequency and TurnOn and Turn-Off Snubbers

Overall, for this refreshed converter, $\mathrm{Q}_{1}$ 's average power dissipation in steady-state is $3.24 \mathrm{~W}$, which remains unchanged from the previous design. However, the majority of $\mathrm{Q}_{1}$ 's switching power transients now lie below $80 \mathrm{~W}$, and only one small $118.5 \mathrm{~W}$ transient occurs during start-up. For any higher or lower inductor values, this result becomes worse. Thus it appears that $75 \mathrm{nH}$ is the optimal turn-on snubber inductor size under maximum converter stress conditions. Even with a small 3.24W dissipation across $\mathrm{Q}_{1}$, however, all converter MOSFETs require adequate heatsinking. This project investigates how much power dissipates in the snubbers. Figure B-68 below shows the power dissipated across the turn-off snubber (in teal), and across the turn-on snubber (in magenta) in steady-state. The green waveform denotes the converter's output voltage. 


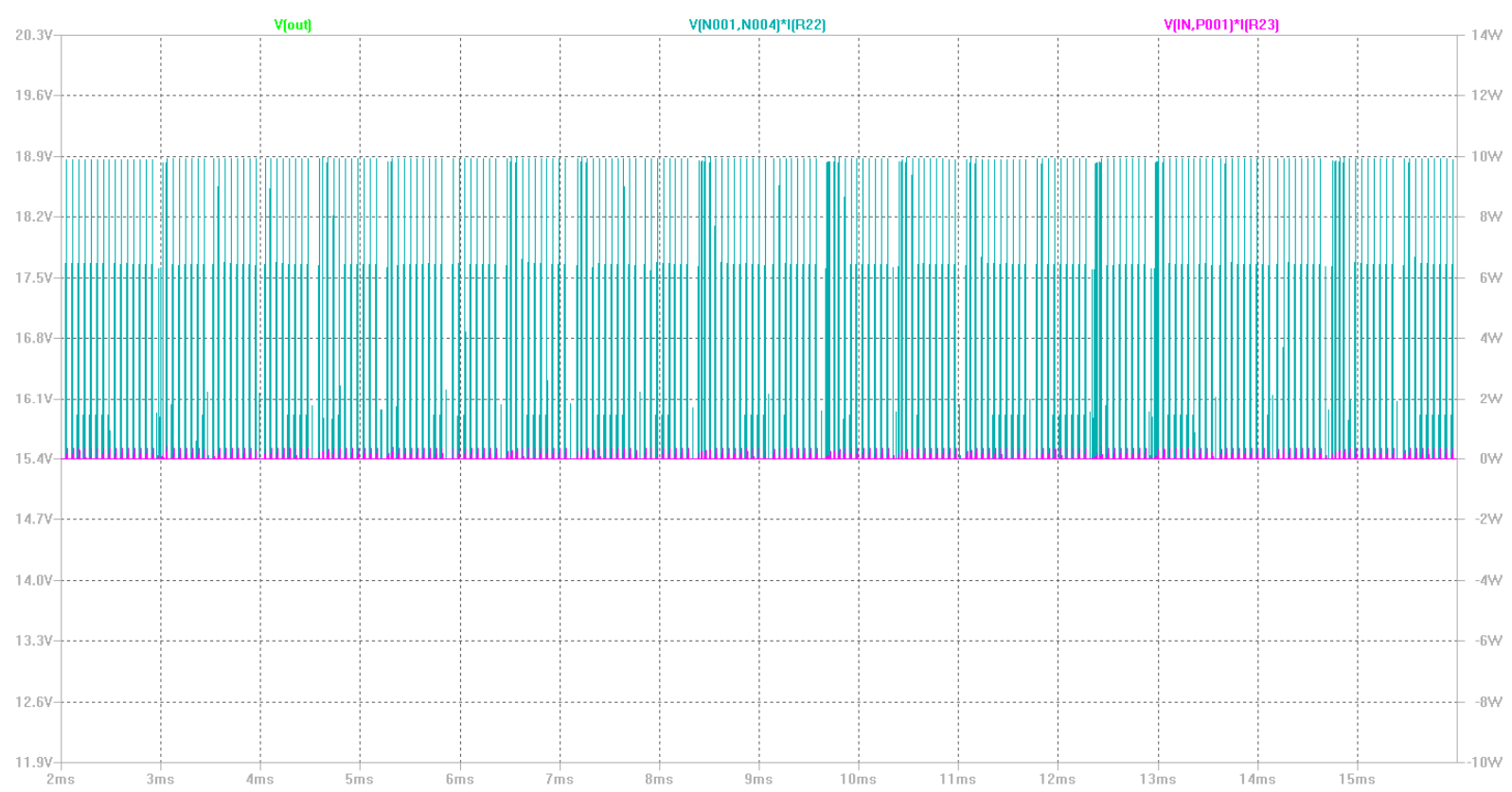

Figure B-68: Turn-On and Turn-Off Snubber Resistor Power Dissipation of Four-Phase Buck, Two-Phase Boost, Multi-Stage Buck-Boost Converter Design

Average power dissipation across turn-on snubber resistor in steady-state is $4.24 \mathrm{~mW}$, and dissipation across each turn-off snubber resistor is $41.6 \mathrm{~mW}$. For all four phases, this corresponds to a $183.36 \mathrm{~mW}$ (average) total power dissipation across the entire snubber implementation, which is still less than a $0.25 \mathrm{~W}$, and is a very reasonable sacrifice for reducing switching power transients across the converter's input buck stage MOSFETs. Figure B-69 shows the overall input current (from the voltage source, in which case is the Precor EFX 546i elliptical trainer) for the converter in steady state (in blue). The green waveform denotes the converter's output voltage. 


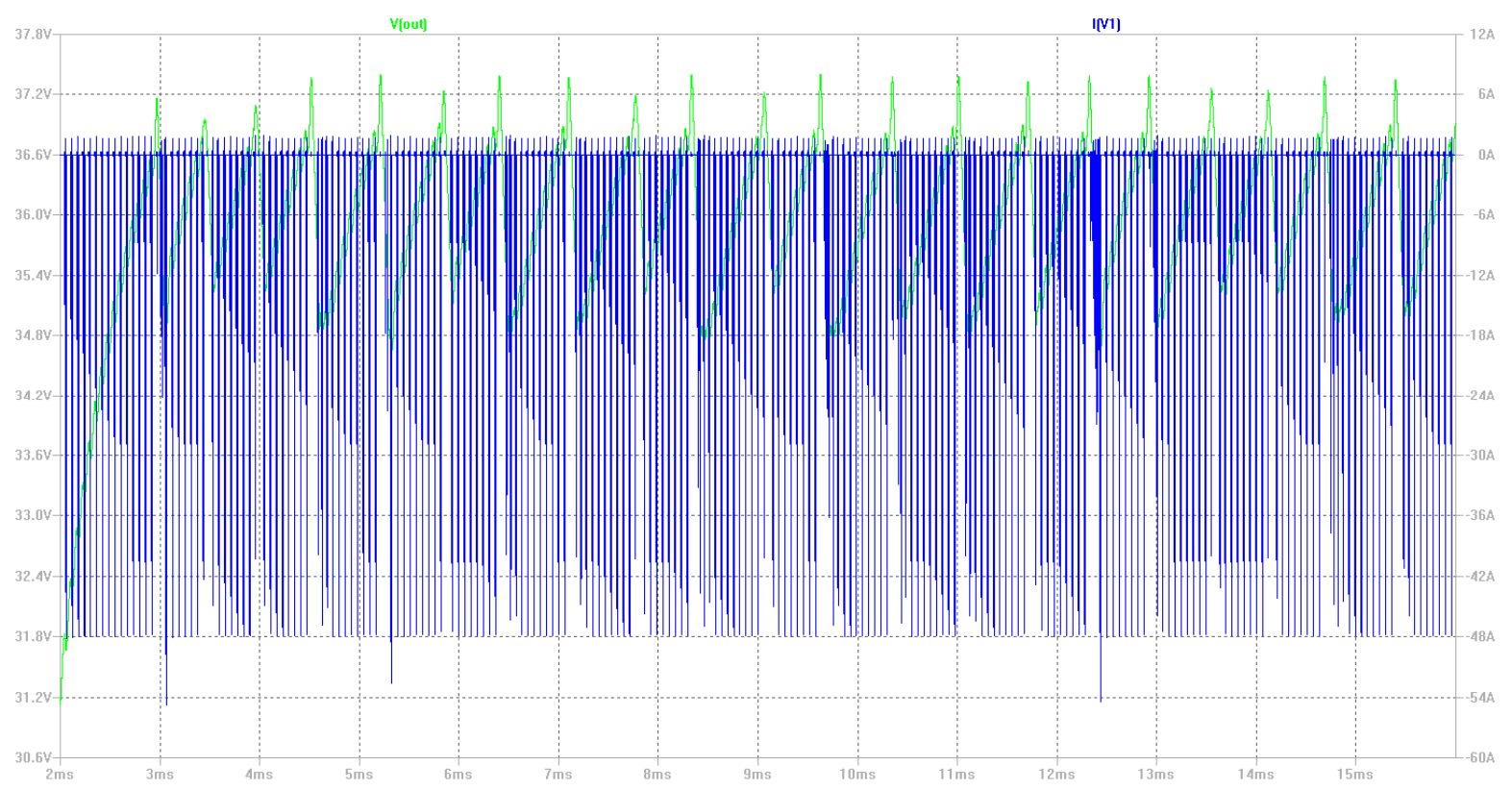

Figure B-69: Input Current of Four-Phase Buck, Two-Phase Boost, Multi-Stage Buck-Boost Converter Design

The converter's average input voltage in steady-state is $60 \mathrm{~V}$, average input current is $5.5 \mathrm{~A}$, average output voltage is $35.893 \mathrm{~V}$ and average output current is $8 \mathrm{~A}$. This translates to an overall $87 \%$ system efficiency, which is the same efficiency level as that of the most recent design's. Therefore this project maximized the converter's snubber's utility for the buck input stage.

The next major step involves correcting power transient issues with the boost stage transistors. Figure B-70 shows the power dissipation across $\mathrm{M}_{1}$, the primary-phase boost stage transistor (in red) with the converter operating in steady-state. The green waveform denotes converter output voltage and the blue waveform denotes the buck stage's output voltage. 


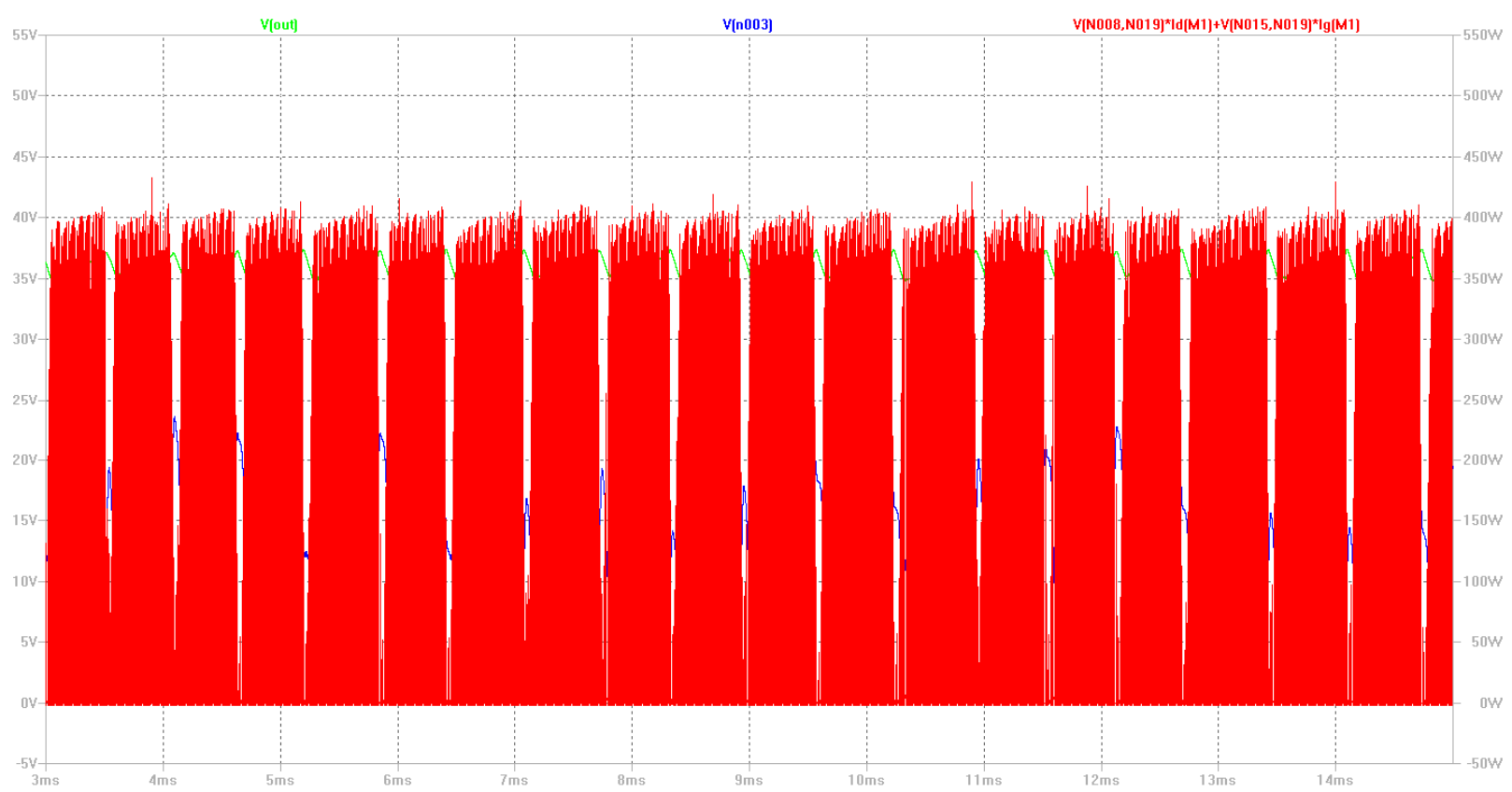

Figure B-70: Output Voltage, Boost Stage Output Voltage and $M_{1}$ Power Dissipation of Four-Phase Buck, Two-Phase Boost, Multi-Stage Buck-Boost Converter Design with 100 kHz Switching Frequency and TurnOn and Turn-Off Snubbers

Despite the large power transients, the overall average power dissipation across $\mathrm{M}_{1}$ (for this time window from 3 to $15 \mathrm{~ms}$ ) is only $3.45 \mathrm{~W}$. However, this project does not desire power transients exceeding $400 \mathrm{~W}$ across its converter components. A similar situation occurs with $\mathrm{M}_{2}$ (the secondary phase transistor on our boost stage) in the plot in figure B71. 


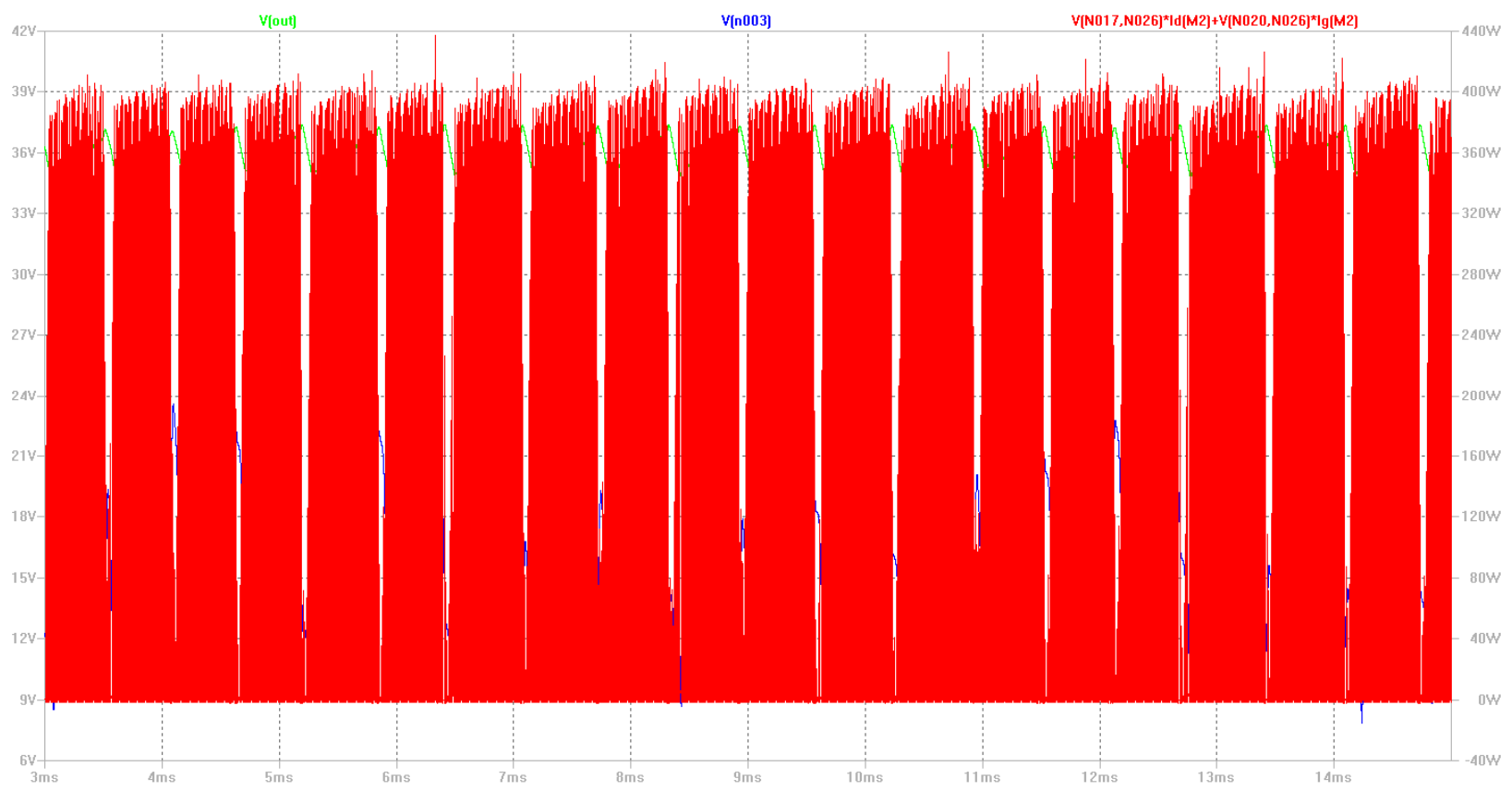

Figure B-71: Output Voltage, Boost Stage Output Voltage and $M_{2}$ Power Dissipation of Four-Phase Buck, Two-Phase Boost, Multi-Stage Buck-Boost Converter Design with 100 kHz Switching Frequency and TurnOn and Turn-Off Snubbers

Figure B-72 shows the output boost stage's operation characteristics in steady state, with a $60 \mathrm{~V}$ input and $8 \mathrm{~A}$ load. Converter output voltage is in light green, buck stage output voltage is in dark blue, inductor $\mathrm{L}_{3}$ 's current is in red, inductor $\mathrm{L}_{2}$ 's current is in teal, $\mathrm{M}_{1}$ 's drain current is in magenta, $\mathrm{M}_{2}$ 's drain current is in gray, diode $\mathrm{D}_{3}$ 's current is in dark green and diode $\mathrm{D}_{4}$ 's current is in dark blue. 


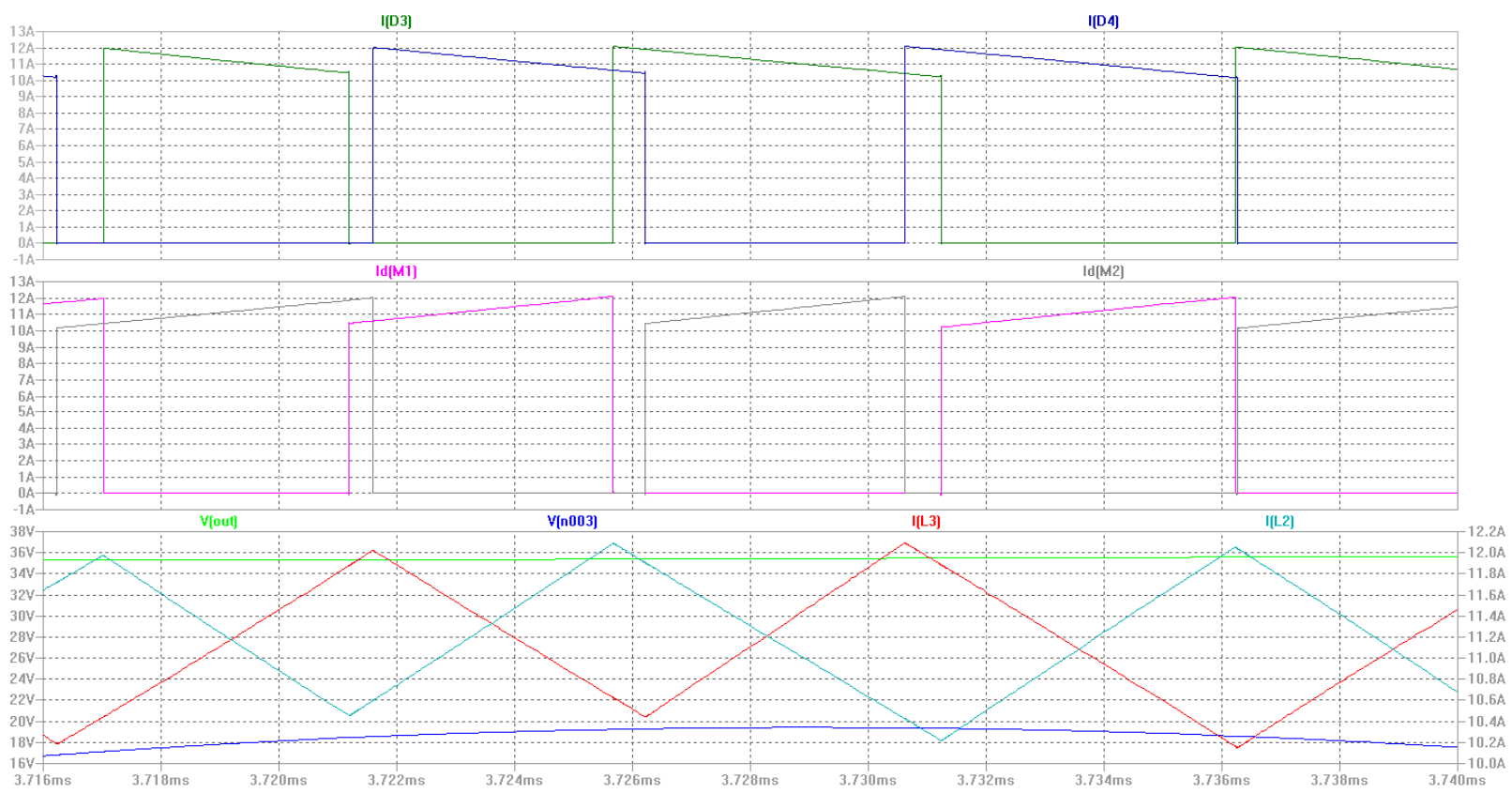

Figure B-72: Output Voltage, Boost Stage Output Voltage, Inductor Currents, Diode Currents and $M_{1}$ and $\mathrm{M}_{2}$ Drain Currents Four-Phase Buck, Two-Phase Boost, Multi-Stage Buck-Boost Converter Design with $100 \mathrm{kHz}$ Switching Frequency and Turn-On and Turn-Off Snubbers

The two transistors for the converter's boost stage operate out of phase with each other. While both boost stage inductors operate in continuous conduction mode, their current values increase as their corresponding series-connected transistors turn on. $\mathrm{L}_{2}$ 's current increases when $M_{1}$ turns on, and $L_{3}$ 's current increases when $M_{2}$ turns on, and each inductor's current decreases when their corresponding transistors turn off. Each diode also behaves in a similar manner. $\mathrm{D}_{3}$ turns on when $\mathrm{M}_{1}$ turns off, and $\mathrm{D}_{4}$ turns on when $\mathrm{M}_{2}$ turns off. The diodes provide a path to ground for the transistors' drain current when their corresponding transistors switch off.

The two boost stage transistors also must switch at the proper frequency. Transistor $\mathrm{M}_{1}$ 's measured switching period (using the drain current plot from figure B72) occurs from $3.14645 \mathrm{~ms}$ to $3.1565 \mathrm{~ms}$, which corresponds to a $10 \mu \mathrm{s}$ period, which in turn corresponds to a $100 \mathrm{kHz}$ switching frequency. Likewise, $\mathbf{M}_{2}$ 's switching period 
occurs from $3.15148 \mathrm{~ms}$ to $3.16153 \mathrm{~ms}$, which corresponds to a $10 \mathrm{~ms}$ period and $100 \mathrm{kHz}$ switching frequency. Thus, both boost stage transistors switch at the proper frequency.

A snubber is also necessary for the output boost stage. Figure B-73 shows $\mathrm{M}_{1}$ 's and $\mathrm{M}_{2}$ 's drain-source voltage turn-off trajectories, in red and teal. The green waveform denotes converter output voltage and the blue waveform denotes the buck stage's output voltage.

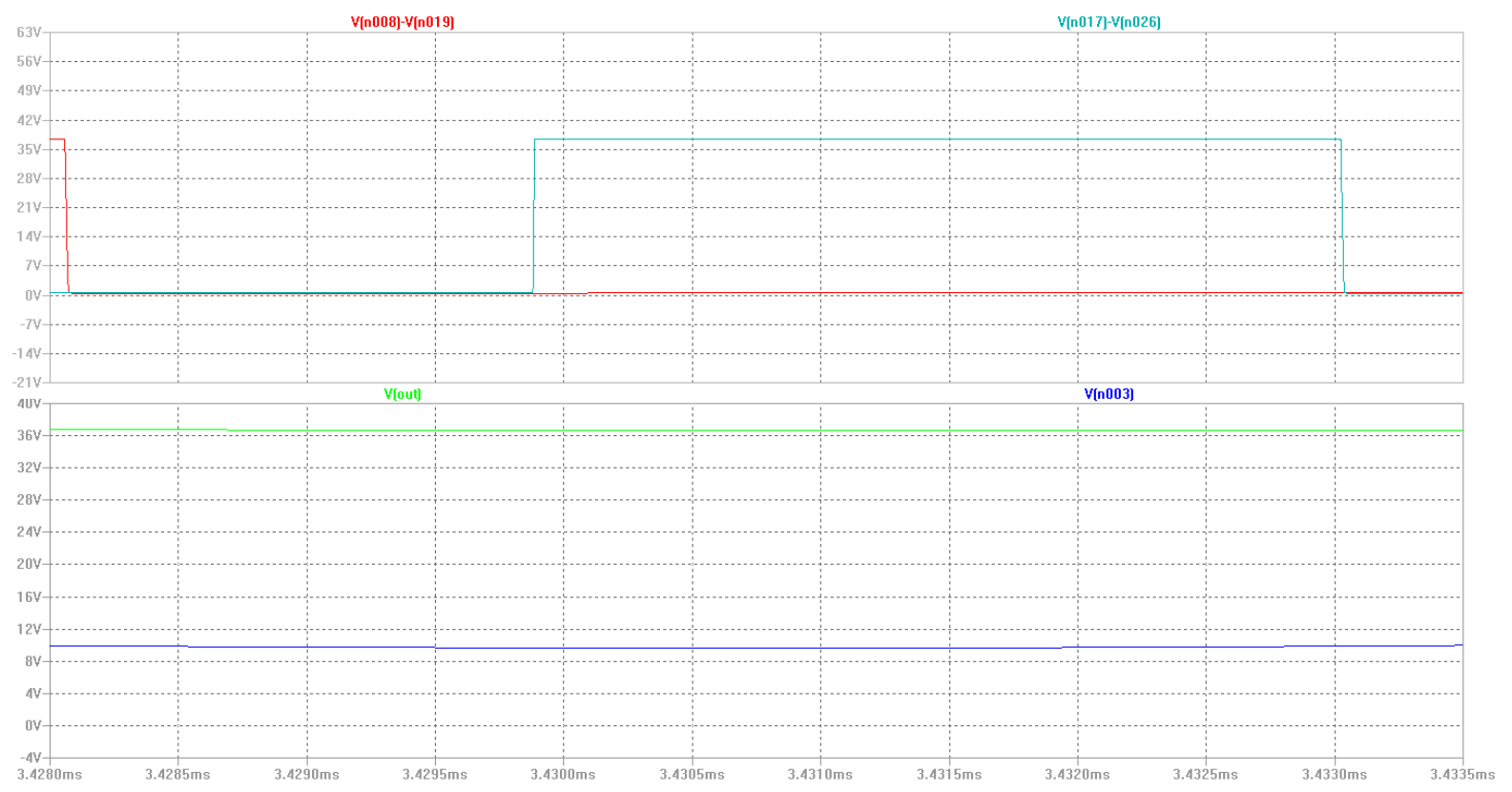

Figure B-73: Output Voltage, Boost Stage Output Voltage and $M_{1}$ and $M_{2}$ Drain-Source Voltages for Four-

Phase Buck, Two-Phase Boost, Multi-Stage Buck-Boost Converter Design with 100 kHz Switching Frequency and Turn-On and Turn-Off Snubbers

$\mathrm{M}_{1}$ 's measured drain-source turn-off time is $14.7 \mathrm{~ns}$, and $\mathrm{M}_{2}$ 's measured drain-source turn-off time is $14.3 \mathrm{~ns}$.

This project now designs the converter's boost output stage's snubber using the exact same methodology as with that of the converter's input buck stage, however, that method is slightly more difficult because the boost output stage already has inductors connected in series with its transistors, and furthermore, the diodes in the output boost 
stage already provide a path for current flow to the load when the transistors switch off.

Likewise with the input buck stage, however, this project can design the snubber for the converter's output boost stage transistors using purely passive components, which do not add a significant amount of PCB real estate to the overall converter design. Working around the two mentioned issues with the snubber design for the output boost stage requires adding a series inductor, capacitor and diode (connected in parallel with each other) for the boost stage's turn-on snubber. This project also implements a turn-off snubber using the same method as with that of the converter's input buck stage.

Designing a turn-off snubber for the converter's output boost stage requires the following parameters:

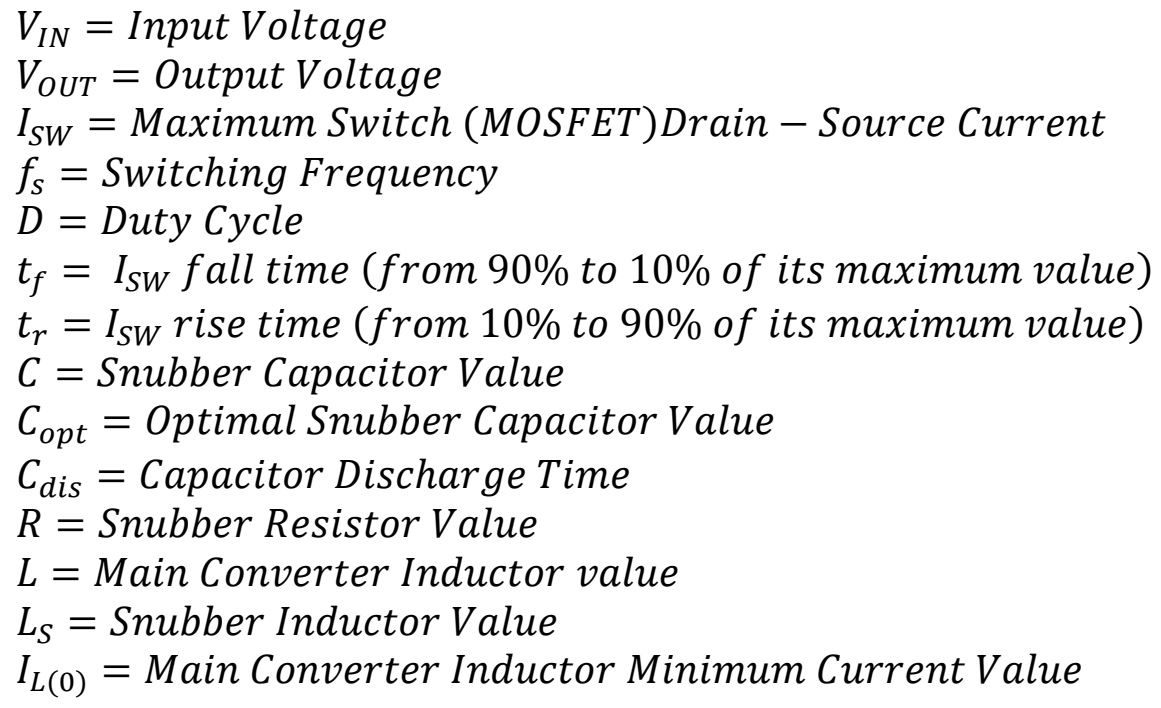

Equations (B1.25) to (B1.27) determine the boost stage's duty cycle under this project's maximum converter stress level.

$$
\begin{gathered}
V_{\text {OUT }}=\frac{V_{I N}}{1-D} \\
V_{\text {OUT }}=36 \mathrm{~V}, V_{I N}=12 \mathrm{~V}(\text { average })
\end{gathered}
$$


Therefore:

$$
D=1-\frac{V_{O U T}}{V_{I N}}=1-\frac{12}{36}=0.667, \text { or } 67 \%
$$

For the converter operating under the maximum stress level:

$$
V_{I N}=12 \mathrm{~V}, I_{S W}=12 \mathrm{~A}, f_{s}=100 \mathrm{kHz}, D=0.67, t_{f}=14.3 \mathrm{~ns}(\text { taken from earlier plots })
$$

Equation (B1.29) calculates the turn-off snubber capacitor value.

$$
C=\frac{I_{S W} t_{f}}{2 V_{S}}=\frac{12(14.3 n s)}{2(12)}=7.15 n F
$$

Equation (B1.30) calculates the turn-off snubber resistor value assuming 5 time constants for the capacitor discharge time,

$$
R=\frac{\left(\frac{D}{f_{s}}\right)}{C_{d i s} C}=\frac{\left(\frac{0.67}{100 \mathrm{kHz}}\right)}{(5)(7.15 n F)}=187.41 \Omega
$$

This project rounds this resistor value down to $185 \Omega$. Equation (B1.31) calculates the turn-off snubber's optimum capacitor value associated with the switching power losses.

$$
C_{o p t}=\frac{I_{S W} t_{f}}{\sqrt{12} V_{S}}=\frac{(12)(14.3 n s)}{\sqrt{12}(12)}=4.1281 n F
$$

This project rounds this capacitor value up to $4.3 \mathrm{nF}$.

Now this project designs the turn-on snubber for the boost stage. Again, likewise with the buck stage, the turn-on snubber inductor cannot be too large (keeping it in the $\mathrm{nH}$ range is optimal). This project selects the snubber inductor as $90 \mathrm{nH}$, which is reasonably small but not so small that it loses its effectiveness in slowing down $\mathrm{M}_{1}$ 's drain current rise.

The converter then underwent several further modifications for safe operation under all of the previously simulated electrical characteristics and conditions. The 
modifications include changing the main inductors to $40 \mu \mathrm{H}$ and changing one of the output capacitors to $500 \mu \mathrm{F}$. Another modification consists of changing the boost stage transistors from Fairchild HUFA76413DKs to Infineon IPB600N25N3s. The IPB600N25N3 has a 250V maximum drain-source voltage rating, 18-25A maximum continuous drain current rating and $136 \mathrm{~W}$ power dissipation at $25^{\circ} \mathrm{C}$ rating. This project selected the IPB600N25N3 as its new boost stage transistors because the previously selected HUFA76413DK's 2.5W continuous power dissipation and 4.8A contnuous drain current rating. The IPB600N25N3 has a much lower drain-source on-resistance ( $21 \mathrm{~m} \Omega$ compared to the HUFA76413DK's $55 \mathrm{~m} \Omega$ ), thus it may be less efficient. Figure B-74 shows the converter's new boost stage with the snubbers installed, along with the mentioned modifications. 


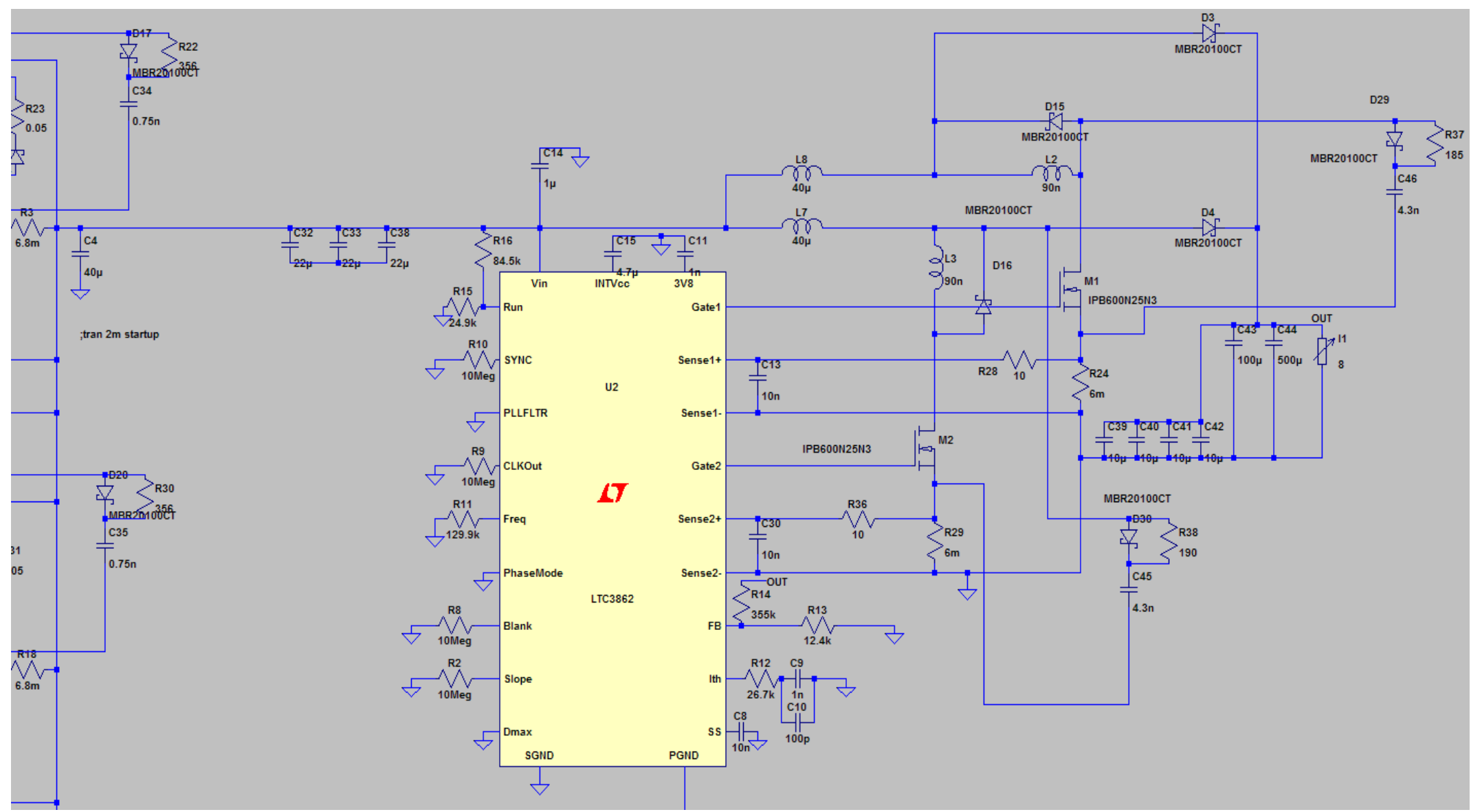

Figure B-74: Output Stage for Four-Phase Buck, Two-Phase Boost, Multi-Stage Buck-Boost Converter Design with 100 kHz, Switching Frequency and Turn-On and Turn-Off Snubbers 
Figure B-75 shows the converter's output voltage (in green), buck stage output voltage (in blue), and power dissipation across $\mathrm{M}_{1}$ (in red) at maximum load stress, with the converter's input voltage set to $60 \mathrm{~V}$ and load set to $8 \mathrm{~A}$.

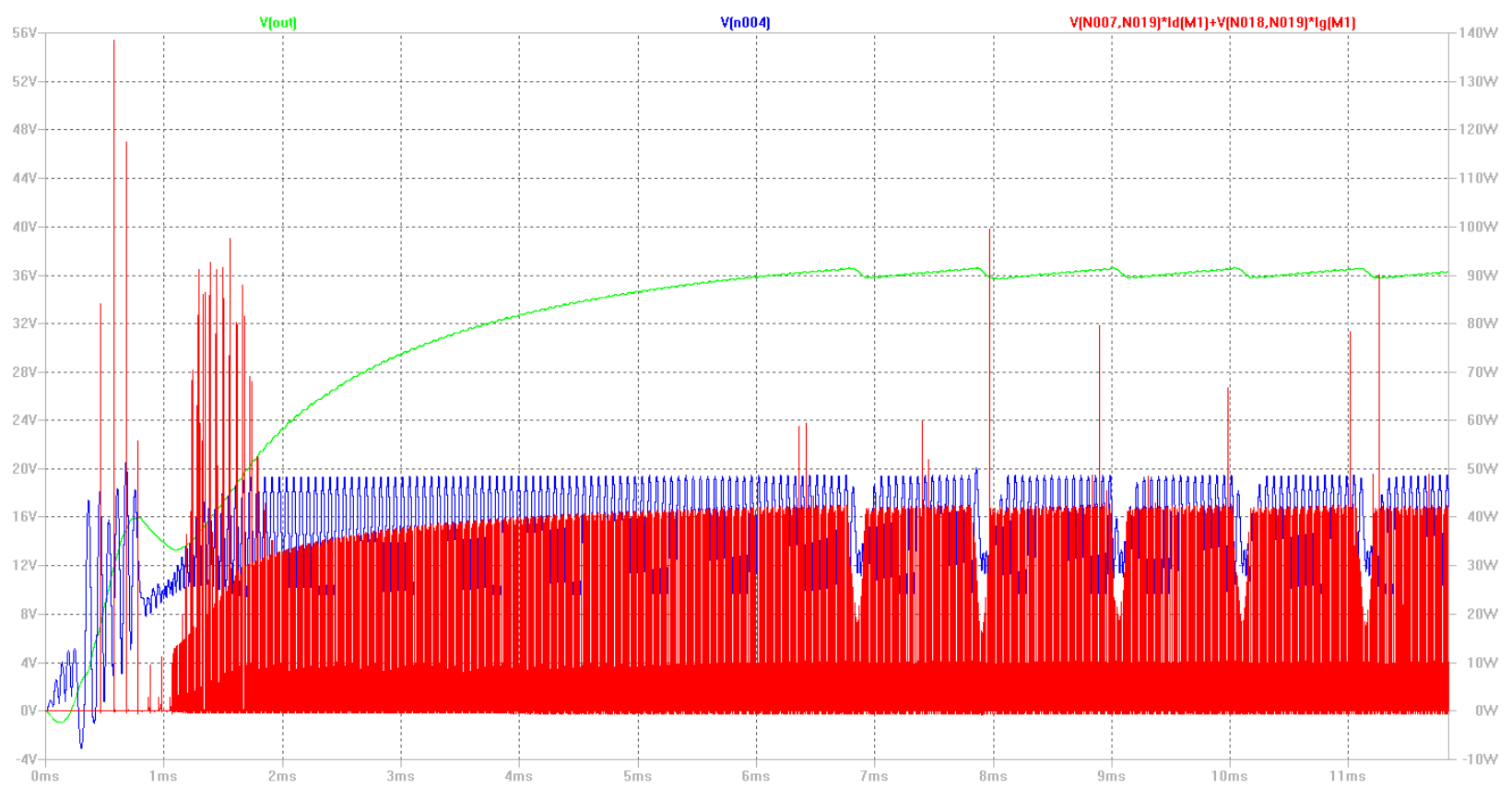

Figure B-75: Output Voltage, Buck Stage Output Voltage and M $M_{1}$ Power Dissipation of Modified $100 \mathrm{kHz}$ Two-Phase Boost, Multi-Stage Buck-Boost Converter Design

Figure B-76 shows the converter's operation in steady state. The green waveform denotes converter output voltage, the blue waveform denotes buck stage output voltage and the red waveform denotes power dissipation across $\mathrm{M}_{1}$. 


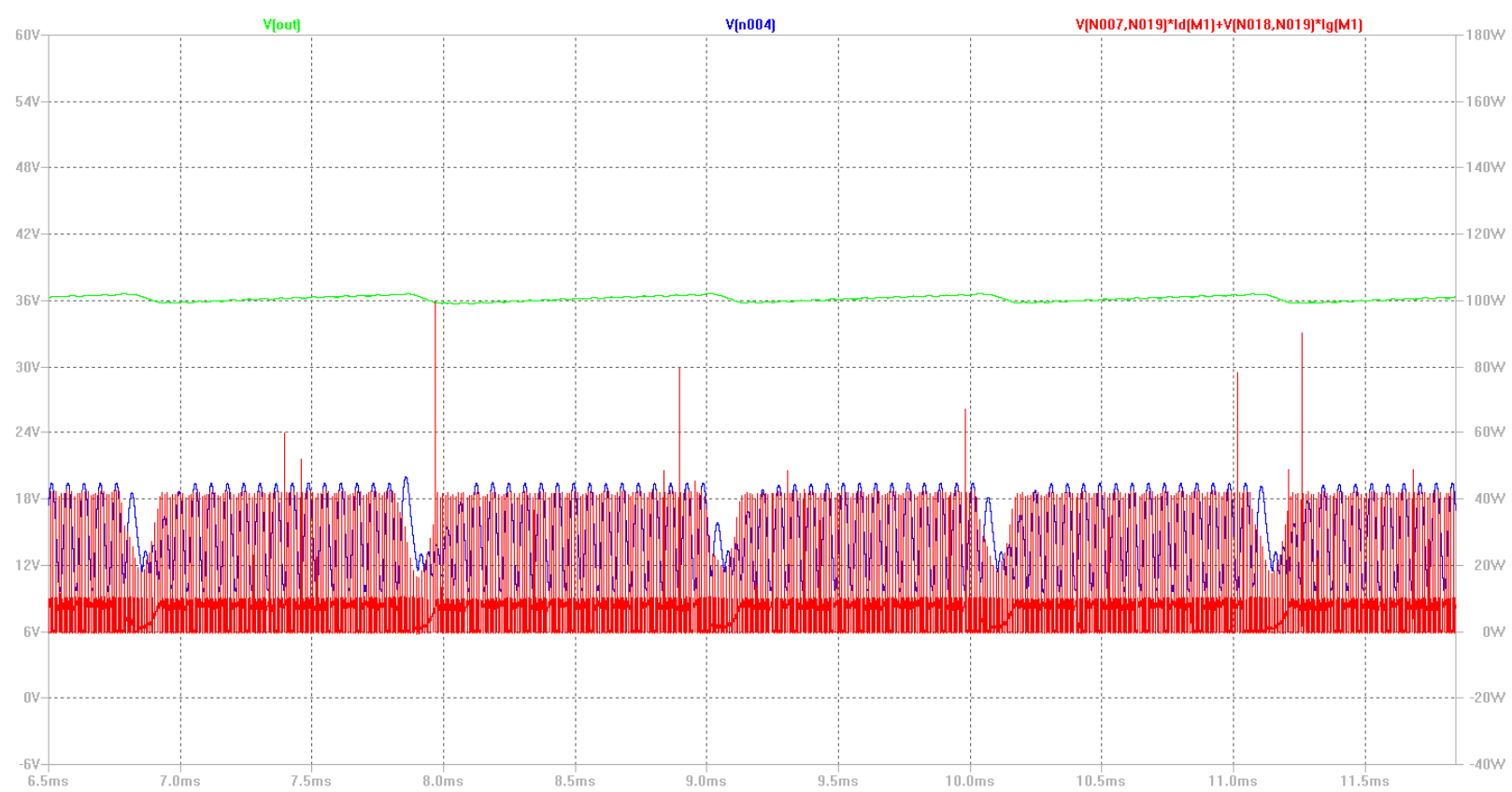

Figure B-76: Steady-State Output Voltage, Buck Stage Output Voltage and $M_{1}$ power Dissipation of Modified 100 kHz Two-Phase Boost, Multi-Stage Buck-Boost Converter Design

The average power dissipation across $\mathrm{M}_{1}$ in steady-state is $4.562 \mathrm{~W} . \mathrm{M}_{2}$ behaves in a similar manner. Thus both boost stage transistors dissipate $9.124 \mathrm{~W}$ in steady-state. The highest power transient occurs at $140 \mathrm{~W}$, however, that occurs at converter start-up (before the converter begins regulating). A few high power transients in the 90-100W range still occur in steady-state. However, those transients are far and few in between. This characteristic is acceptable for now. Figure B-77 shows steady-state converter waveforms including the converter's input current (denoted by the teal waveform). 


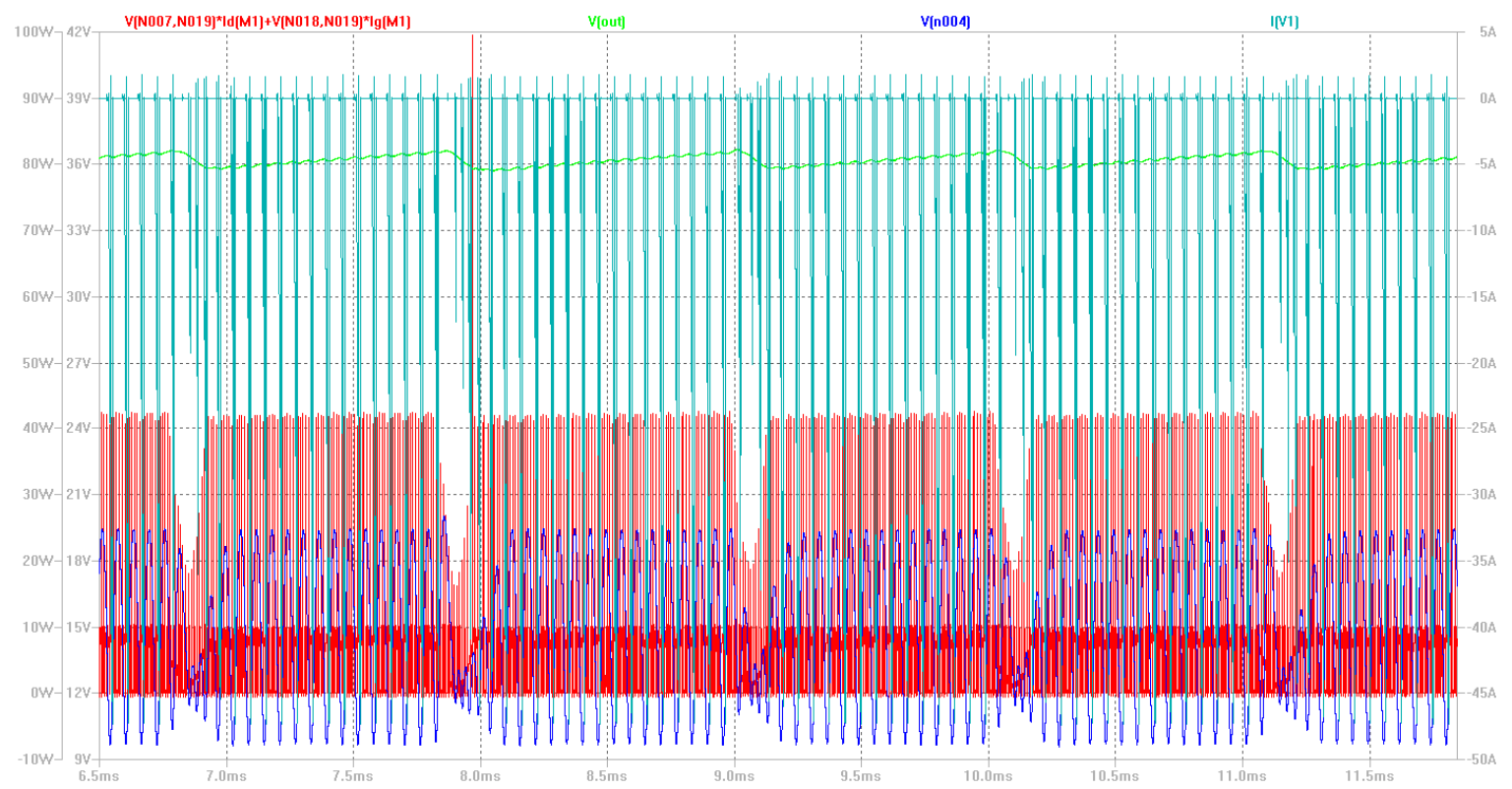

Figure B-77: Steady-State Input Current, Output Voltage, Buck Stage Output Voltage and $M_{1}$ Power Dissipation of Modified 100 kHz Two-Phase Boost, Multi-Stage Buck-Boost Converter Design

During steady state, the converter's average input voltage is $60 \mathrm{~V}$, average input current is $-5.5402 \mathrm{~A}$, average output voltage is $36.185 \mathrm{~V}$, and average output current is $8 \mathrm{~A}$. This translates to an overall $87.1 \%$ system efficiency, which is roughly the same overall efficiency level as with previous implementations of this converter; however, the power transients across each transistor are drastically lower in this implementation because of the additional snubber circuits.

Figure B-78 is a zoomed-in plot showing $\mathrm{M}_{1}$ 's drain current switching trajectory (in teal) during the occurrence of a $100 \mathrm{~W}$ transient dissipated across it (in red): 


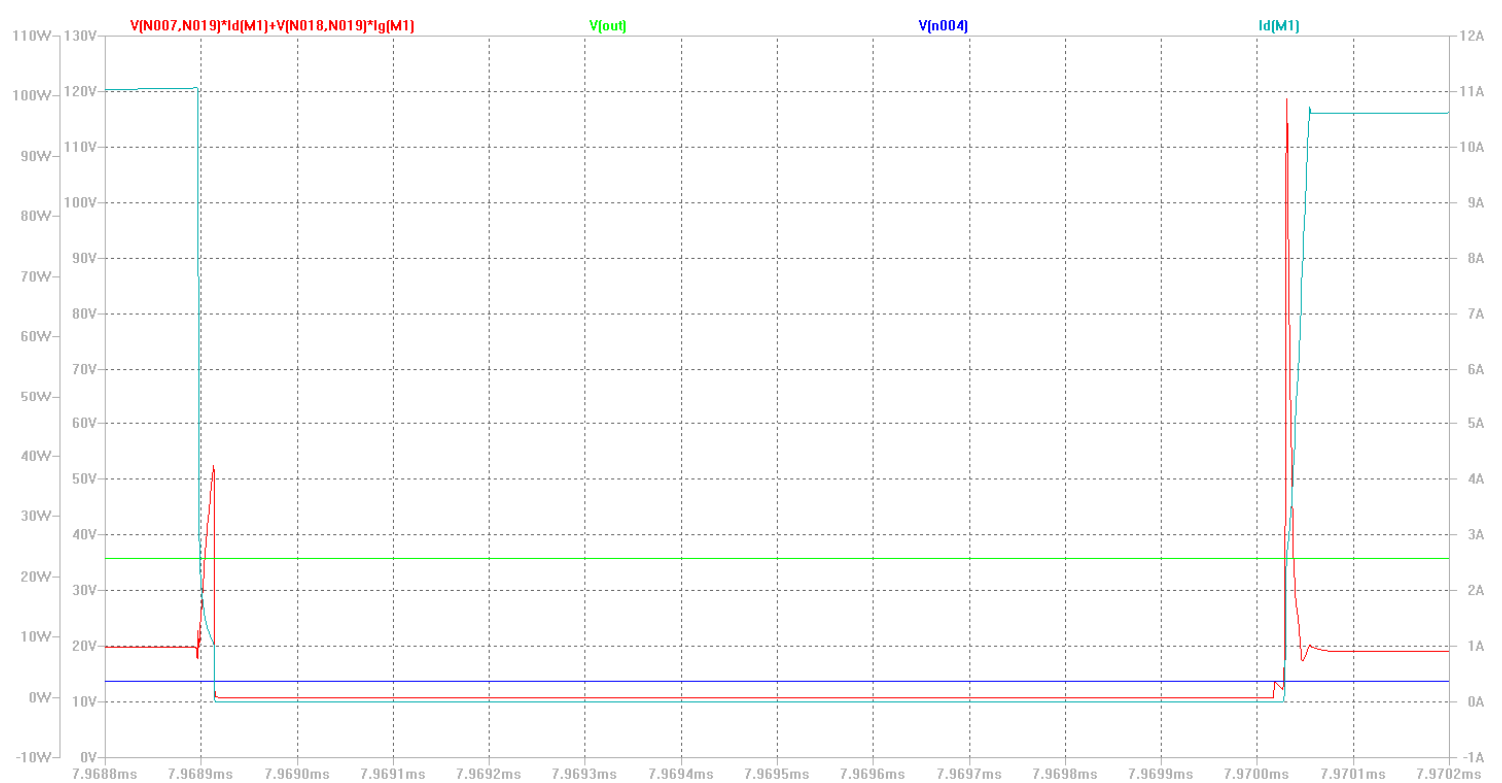

Figure B-78: Output Voltage, Buck Stage Output Voltage, $M_{1}$ Drain Current and $M_{1}$ Power Dissipation of Modified 100 kHz Two-Phase Boost, Multi-Stage Buck-Boost Converter Design

The power transients during transistor turn-off are not as lethal as during turn-on. Thus the converter's turn-on snubber requires further optimization. Additional, these transients occur only once the current through inductor $\mathrm{L}_{8}$ (which is the switching inductor for $\mathrm{M}_{1}$ ) reaches its minimum (i.e. when $\mathrm{L}_{8}$ releases its stored energy). Appendix B1.10 describes the turn-on snubber optimization process.

\section{B1.10 Component Downsizing and Optimization Process}

Minimizing the converter's physical size and cost as much as possible as well as guaranteeing physical component availability required the following further changes for this project's converter:

- All primary switching transistors in the input buck stage $\left(\mathrm{Q}_{1}, \mathrm{Q}_{3}, \mathrm{Q}_{7}\right.$ and $\left.\mathrm{Q}_{9}\right)$ changed to STMicroelectronics STW11NM80 (800V V $\mathrm{V}_{\text {DS }}$ capability, $0.35 \Omega$ $\mathrm{R}_{\mathrm{DS}-\mathrm{ON}}$ ) for handling the $27 \mathrm{~A}$ pulses (and $80 \mathrm{~W}$ power transients) during turnon states. 
- All input buck stage snubber diodes changed to $1 \mathrm{~N} 914$ silicon diodes $(75 \mathrm{~V}$ reverse voltage capability, $0.2 \mathrm{~A}$ average forward current, $4 \mathrm{~A}$ maximum pulsed current).

- All output boost stage snubber diodes changed to MBRS140 Schottky diodes (40V reverse voltage capability, $1 \mathrm{~A}$ average forward current, $40 \mathrm{~A}$ maximum pulsed current), available in SMB (surface mount package).

- All input buck stage stage $\mathrm{V}_{\mathrm{CC}}$ input diodes changed to $1 \mathrm{~N} 914$ silicon diodes (75V reverse voltage capability, 200mA average forward current, 1-4A maximum pulsed current).

- All primary input buck stage inductors reduced to $39 \mu \mathrm{H}$, as no commercial $60 \mu \mathrm{H}$ inductor exists (as of writing) that meets the converter's 15A saturation current requirement. As later simulation tests show, the converter still maintains CCM operation at full load.

- The physical converter design will use Fairchild FDB14N30s (100V V VS capability, $0.29 \Omega \mathrm{R}_{\mathrm{DS}-\mathrm{ON}}$ ) for the primary transistors in the input buck stage changed to for offsetting the costs from the higher-priced 800V STM transistors. The drain-source on resistance is nearly identical to that of the $800 \mathrm{~V}$ STM transistors and the FDB14N30s can handle 14A continuous drain current, as well as 56A maximum pulsed current. No simulation model exists for the Fairchild FDB14N30 transistors, thus further simulations continue using the 800V STM transistors.

Figure B-79 shows the converter schematic with all the mentioned changes. 


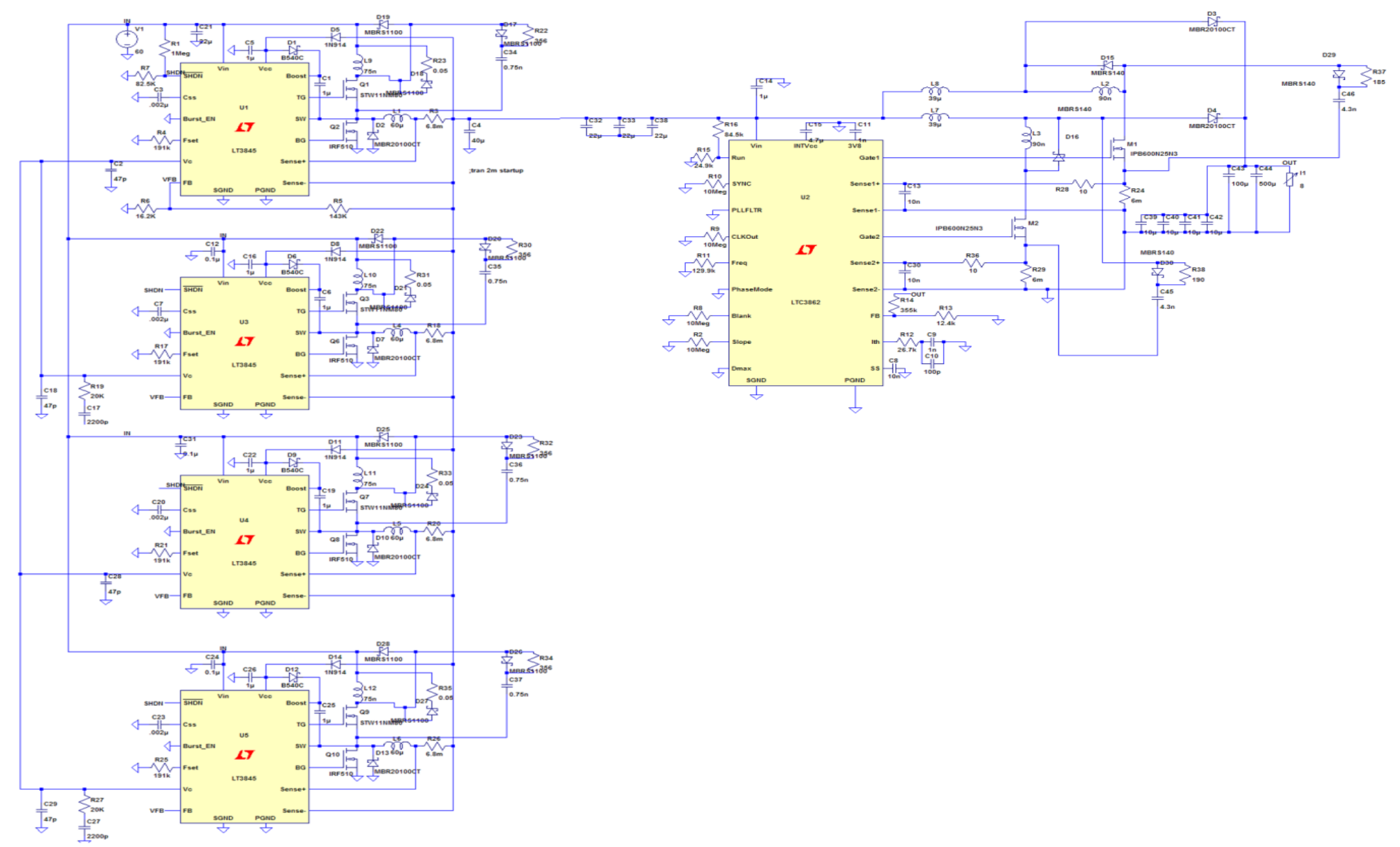

Figure B-79: Optimized 100 kHz Two-Phase Boost, Multi-Stage Buck-Boost Converter Design 
Figure B-80 is a plot showing converter output voltage in green, input buck stage voltage in blue, and power dissipated across $\mathrm{Q}_{1}$ (in red), the primary buck input stage transistor, in steady-state operation with a $60 \mathrm{~V}$ input and $8 \mathrm{~A}$ load.

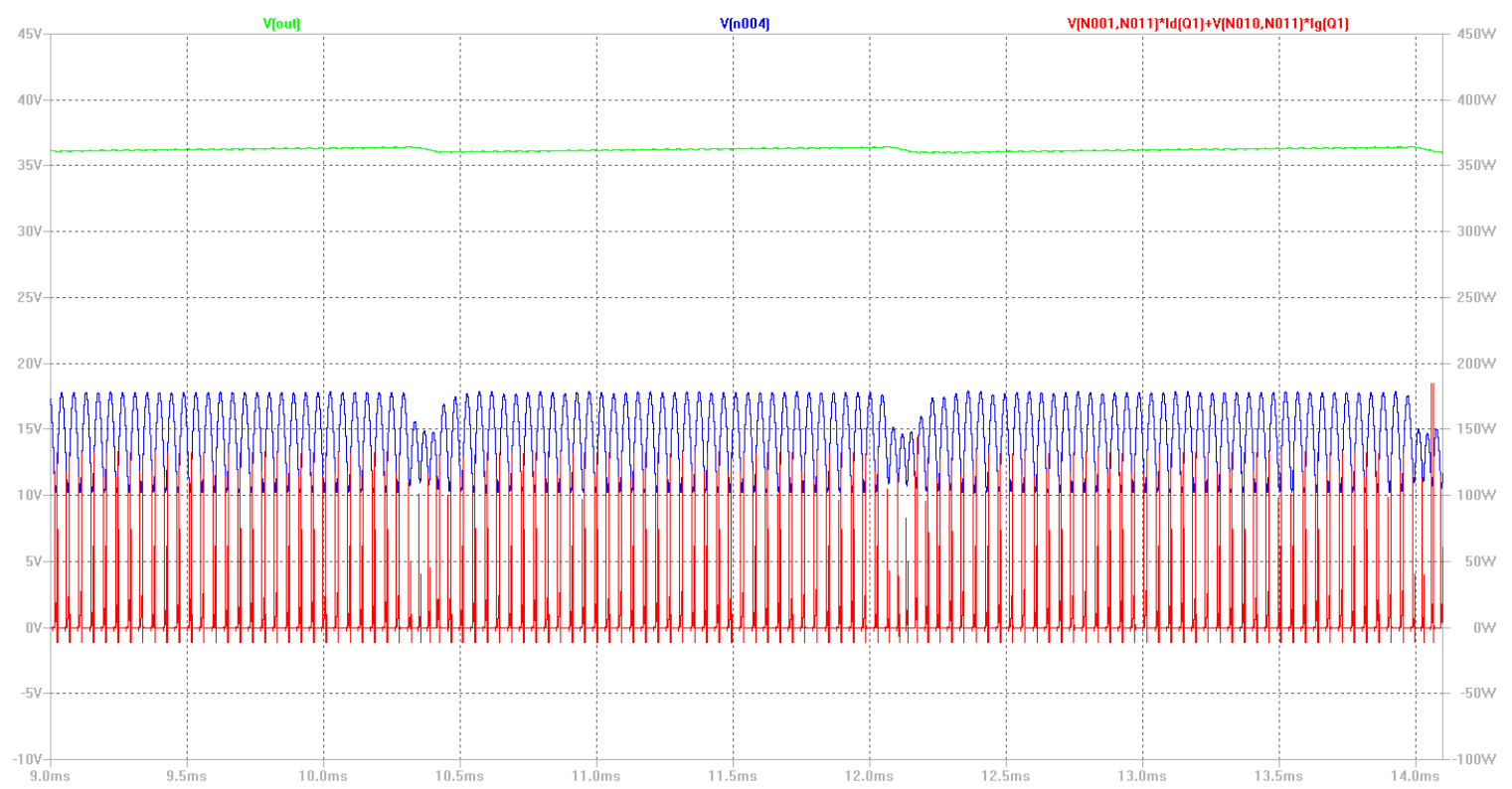

Figure B-80: Output Voltage, Buck Stage Output Voltage and $Q_{1}$ Power Dissipation of Optimized $100 \mathrm{kHz}$ Two-Phase Boost, Multi-Stage Buck-Boost Converter Design

The power transients increase into the 120-160W range; however, these power levels are still acceptable for the selected transistor. The average power dissipated across each of these primary transistors in steady-state operation is $2.79 \mathrm{~W}$, which is still acceptable, however these transistors would still require adequate heatsinking.

Figure B-81 is the same plot as figure B-80 above, except it now denotes in red the power dissipation across $\mathrm{M}_{1}$, the primary output boost-stage transistor, in steady-state operation. 


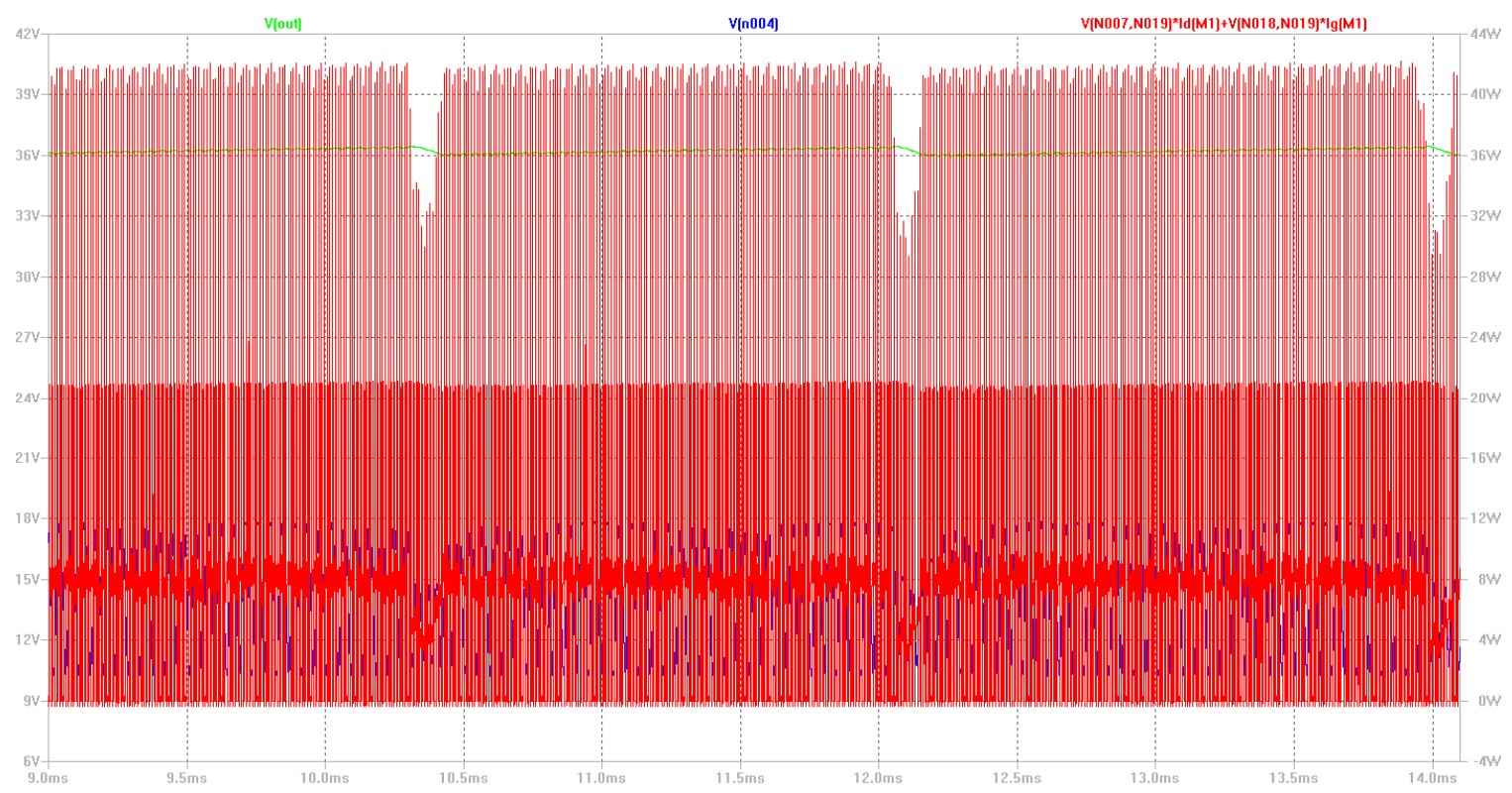

Figure B-81: Output Voltage, Buck Stage Output Voltage, and $M_{1}$ Power Dissipation of Optimized $100 \mathrm{kHz}$ Two-Phase Boost, Multi-Stage Buck-Boost Converter Design

The power transients across $\mathrm{M}_{1}$ still lie in the $40-45 \mathrm{~W}$ range (maximum) in steady-state operation. Average power dissipation across $\mathrm{M}_{1}$ in steady state is roughly $4.94 \mathrm{~W}$ (thus, still near 5W), which means that the converter does not need any further changes to its boost output stage; however, $5 \mathrm{~W}$ average dissipation means that these boost output stage transistors will require good heatsinking.

The converter still has one lingering problem, however - the input buck stage snubber diodes' electrical capabilities. Initially, before using the 1N914 silicon diodes, this project selected MBRS1100 Schottky diodes as the converter's buck stage snubber diodes. Unfortunately those diodes drastically increased the power transient magnitudes across $Q_{1}$ under full load. The root of that problem is that the MBRS1100 Schottky diode has a much higher junction capacitance (roughly 10 times larger) than that of the MBR20100CT Schottky diodes used in earlier simulations, according to LTSpice. The 1N914 silicon diodes have reduced junction capacitance; however, its maximum pulsed 
current rating is only $4 \mathrm{~A}$ and the converter requires a diode that tolerates at least $6 \mathrm{~A}$ pulsed current through the snubber circuits. This project then changed the 1N914 diodes to ES1Ds, allowing for the snubber to tolerate the simulated maximum pulsed current requirements. The ES1D is a silicon diode with very low junction capacitance (roughly $10.5 \mathrm{pF}$, about the same as that of the MBR20100CT), and according to LTSpice it can tolerate up to $200 \mathrm{~V}$ reverse voltage, $1 \mathrm{~A}$ average (continuous) forward current, as well as 30A pulsed current (provided that the current pulse is $8.3 \mathrm{~ms}$ or less). Figure B-82 shows the converter circuit with the new ES1D snubber diodes. 


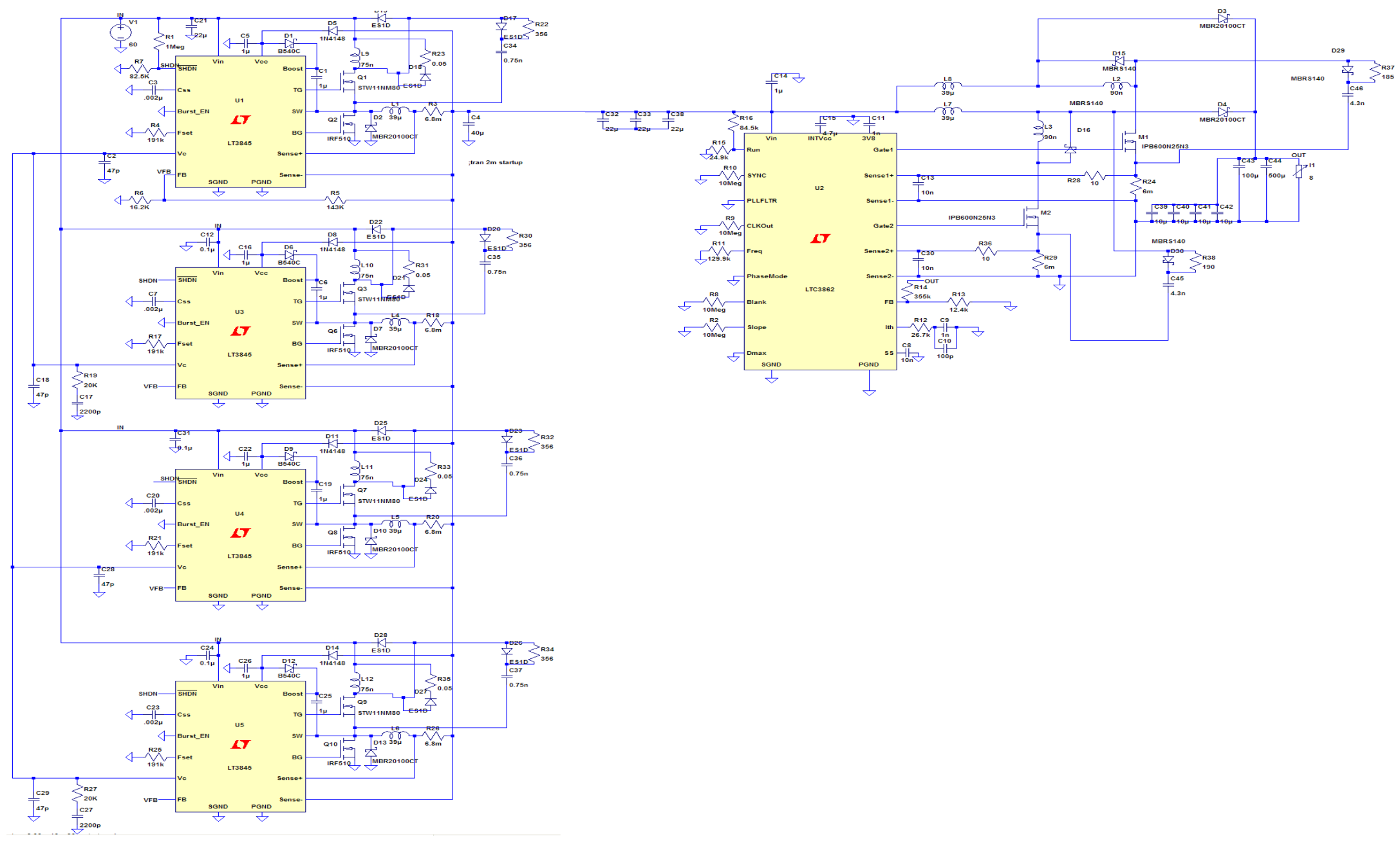

Figure B-82: Optimized 100 kHz Two-Phase Boost, Multi-Stage Buck-Boost Converter Design with ES1D Snubber Diodes 
Figure B-83 shows this modified converter's operation in steady-state, with a $60 \mathrm{~V}$ input and 8A load. Converter output voltage is in green and buck stage output voltage is in blue. The red waveform denotes the power dissipation across $\mathrm{Q}_{1}$.

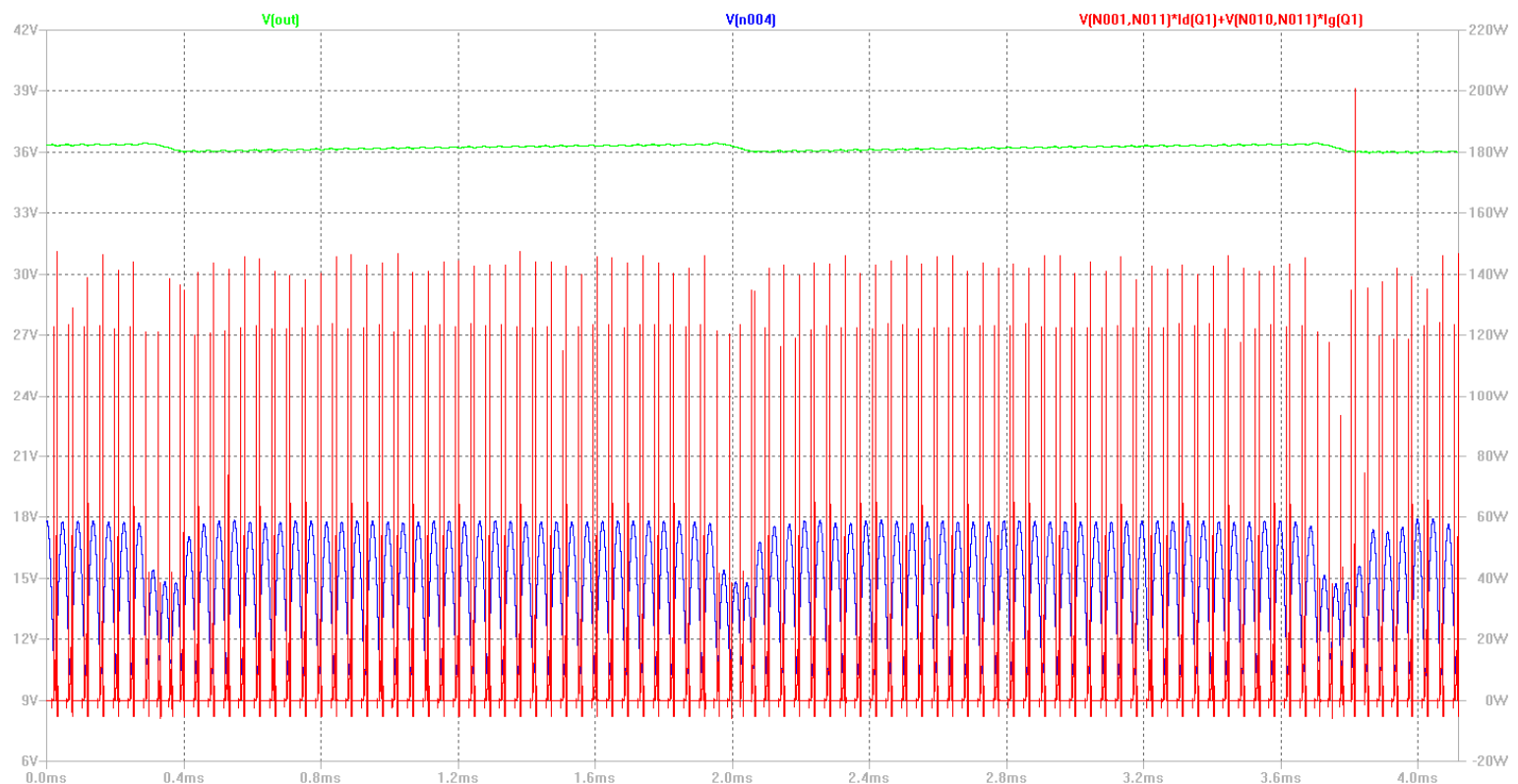

Figure B-83: Steady-State Output Voltage, Buck Stage Output Voltage and $Q_{1}$ Power Dissipation of Optimized 100 kHz Two-Phase boost, Multi-Stage Buck-Boost Converter Design with ESID Snubber Diodes

With the ES1D diodes in place, the power transient magnitudes across $\mathrm{Q}_{1}$ lie between 140-160W, which is still acceptable for this converter. Overall average power dissipation across $\mathrm{Q}_{1}$ in steady state at full load is $2.77 \mathrm{~W}$, which is nearly unchanged from the last circuit design iteration. Figure B-84 is the same plot as Figure B-83, except now the red waveform denotes the power dissipated across $\mathrm{M}_{1}$, the primary output boost stage transistor. 


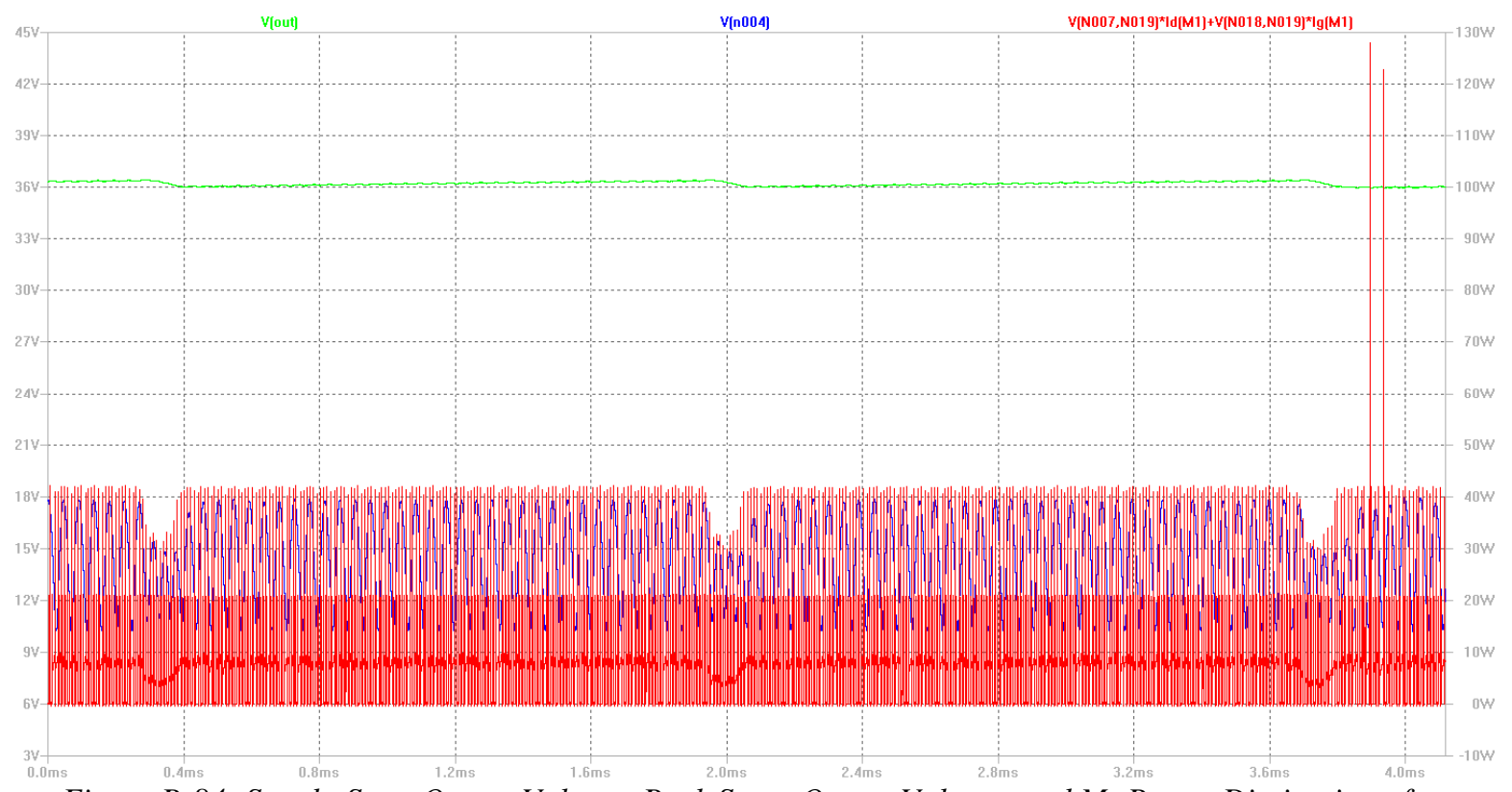

Figure B-84: Steady-State Output Voltage, Buck Stage Output Voltage, and $M_{1}$ Power Dissipation of Optimized 100 kHz Two-Phase Boost, Multi-Stage Buck-Boost Converter Design with ES1D Snubber Diodes

This power dissipation characteristic is very much unchanged from figure B-81, with the exception of the two stray transients in the 120-130W magnitude range. However, this does not pose a problem, as those transients are very brief (roughly $1 \mathrm{~ns}$ ) and operation with such power levels is still within the output boost stage transistors' safe operating areas. Overall average power dissipation across $\mathrm{M}_{1}$ in steady state at full load is roughly $4.9 \mathrm{~W}$, again, nearly unchanged from the previous circuit design iterations. The next section describes additional circuitry required for this circuit for proper stage and phase synchronization.

\section{B1.11 Linear LTC6908-1 External Oscillator Configuration}

Figure B-85 shows the additional circuitry required for synchronizing each PWM controller (per phase) on the converter's buck input stage, using an LTC6908-1 external oscillator IC. 


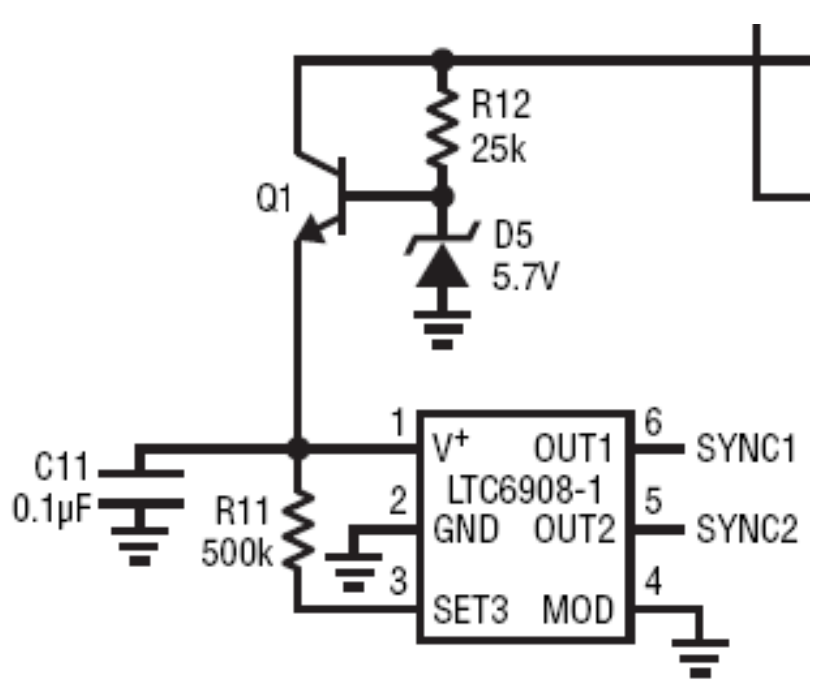

Figure B-85: LTC6908-1 Configuration for use with the LT3845A Controllers

The resistor tied to the SET3 pin sets the output frequency, as determined by the formula $f_{S E T}=10 \mathrm{MHz} \cdot \frac{10 \mathrm{k}}{\left(R_{S E T}\right)}$, where $\mathrm{f}_{\mathrm{SET}}$ is the converter's switching frequency $(100 \mathrm{kHz})$. For $100 \mathrm{kHz}$ switching frequency, $\mathrm{R}_{\mathrm{SET}}$ must be $1 \mathrm{M} \Omega$. The $\mathrm{V}^{+}$pin also requires $2.7-5.5 \mathrm{~V}$ input for maintaining the proper oscillation frequency, and a zener diode in conjunction with an NPN BJT generates that regulated voltage. The $\mathrm{V}^{+}$pin also must be coupled to the LTC6908-1's signal ground using a $0.1 \mu \mathrm{F}$ capacitor. $\mathrm{Q}_{1}$ 's collector and $\mathrm{R}_{12}$ are both connect to the $\mathrm{V}_{\mathrm{CC}}$ pin on the LT3845A PWM controller at the buck input stage. Figure B-86 shows this converter's final design, with the LTC6908-1 external oscillator ICs included. 


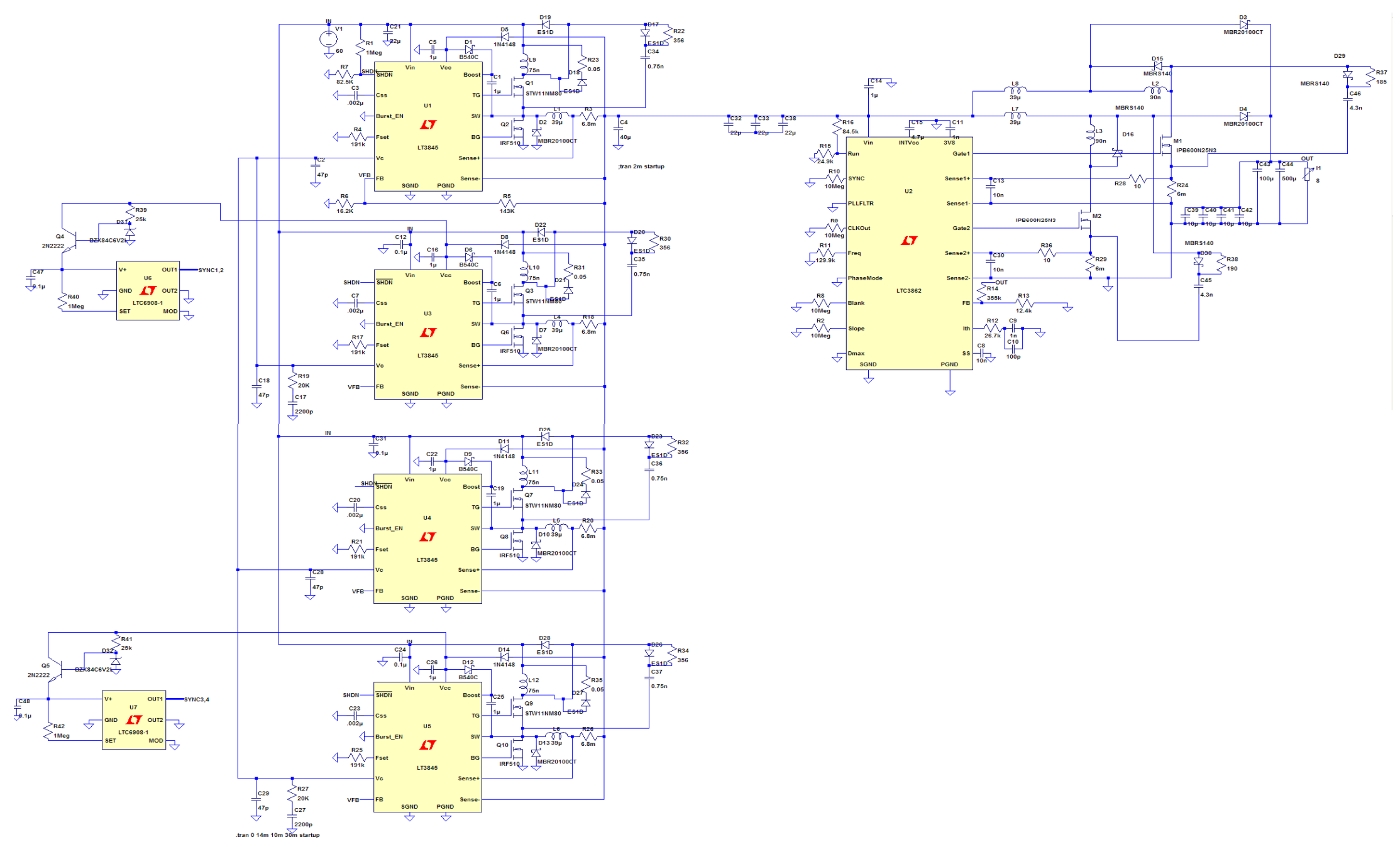

Figure B-86: Complete 100 kHz Two-Phase Boost, Multi-Stage Buck-Boost Converter Design with LTC6908-1 External Oscillators 
Each OUT1 pin on the LTC6908-1 IC connects to the SYNC pins (not shown in the schematic in figure B-86) of its respective LTC3845A controllers; with 2 phases connected per OUT1 pin. This project leaves the OUT2 unused thus it connects to ground. The next section describes setting up the LTC6908-1 external oscillator IC and why this project leaves its OUT2 pin unused.

\section{B1.12 Test Circuit for LTC6908-1 External Oscillator}

Ensuring that the LTC6908-1 external oscillator produces the proper switching signal for synchronizing the input stage's four phases first requires a test simulation circuit for the chip. Figure B-87 shows the test circuit for the LTC6908-1 external oscillator's output.

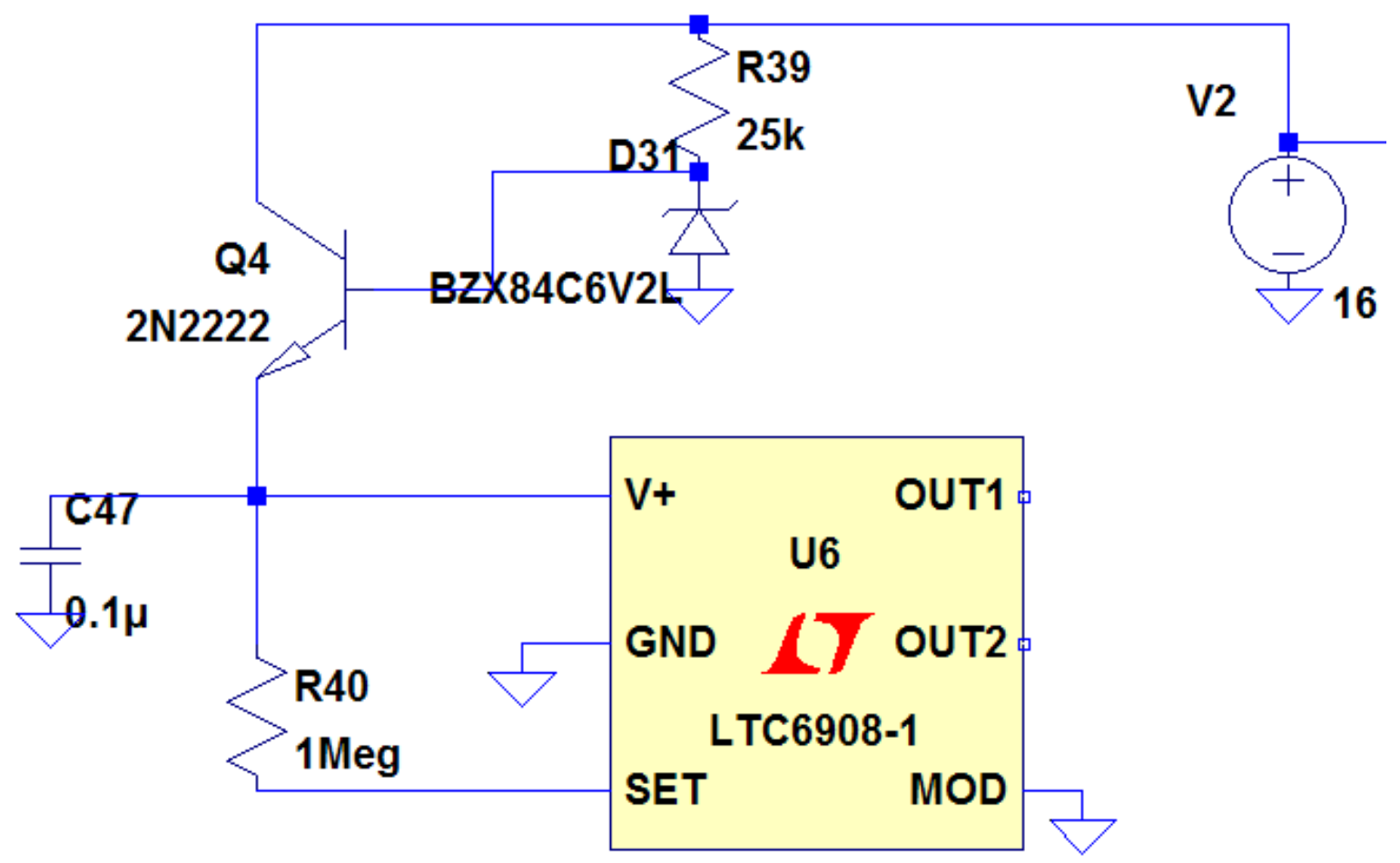

Figure B-87: Test Circuit for LTC6908-1 External Oscillator 
The OUT2 pin on the LTC6908-1 produces a signal $180^{\circ}$ out of phase relative to the signal the chip outputs on its OUT1 pin. Thus, this project only uses the OUT1 pin on the chip. This test circuit determines if a single LTC6908-1 IC can drive all four of the converter's input buck stage stage's phases without any oscillator signal degradation, or if this project's converter requires LTC6908-1 chips. If this converters requires two LTC6908-1 chips, this test will must ensure that both chips' OUT1 output signals are completely in phase. In the final converter, a wire to the converter's LT3845A's PWM controllers' $\mathrm{V}_{\mathrm{CC}}$ pins (which supplies roughly the same $16 \mathrm{~V}$ from each pin) replaces voltage source $\mathrm{V}_{2}$ in figure $\mathrm{B}-87$. The $\mathrm{BZX} 84 \mathrm{C} 6 \mathrm{~V} 2 \mathrm{~L}$ is a $6.2 \mathrm{~V}$ zener diode that helps step down the $\mathrm{V}_{\mathrm{CC}}$ pin voltage to the 2.7-5.5V required for the LTC6908-1's $\mathrm{V}^{+}$pin. After selecting the parts for this project's buck-boost converter design, this project next determines the converter's cost.

\section{B1.13 Converter Cost Analysis}

Tables B.B-1 to B.B-6 (each separated by component type) show the converter's component cost, excluding the additional required cost for PCB fabrication as well as safety components such as fuses. As a prototype, the converter also requires test points (a negligible additional cost relative to the overall converter cost) placed on its PCB for easy problem diagnosis; however, the below tables show the cost for the converter in its final incarnation.

Table B.B-1: Controller IC Components Cost for Converter

\begin{tabular}{|c|c|c|c|c|c|}
\hline Controller IC & Quantity & Unit Price (\$) & Total Price (\$) & Components & Type \\
\hline LT3845A & 4 & 7.13 & 28.52 & U1, U3, U4, U5 & SMT \\
\hline LTC3862 & 1 & 7.00 & 7.00 & U2 & SMT \\
\hline LTC6908-1 & 2 & 3.92 & 7.84 & U6, U7 & SMT \\
\hline Total Cost (\$) & & $\mathbf{4 3 . 3 6}$ & \multicolumn{2}{l}{} \\
\hline
\end{tabular}


Table B.B-2: Transistor Components Cost for Converter

\begin{tabular}{|c|c|c|c|c|c|}
\hline Transistor & Quantity & $\begin{array}{l}\text { Unit Price } \\
\text { (\$) }\end{array}$ & $\begin{array}{l}\text { Total Price } \\
\text { (\$) }\end{array}$ & Components & Type \\
\hline $\begin{array}{c}\text { Fairchild } \\
\text { FDB14N30 }\end{array}$ & 4 & 1.38 & 5.52 & Q1, Q3, Q7, Q9 & SMT \\
\hline IRF510 & 4 & 1.51 & 6.04 & $\begin{array}{c}\text { Q2, Q6, Q8, } \\
\text { Q10 }\end{array}$ & $\begin{array}{c}\text { Through- } \\
\text { Hole }\end{array}$ \\
\hline IPB600N25N3 & 2 & 2.50 & 5.00 & $\mathrm{M} 1, \mathrm{M} 2$ & SMT \\
\hline 2N2222 & 2 & 0.53 & 1.06 & Q4, Q5 & $\begin{array}{c}\text { Through- } \\
\text { Hole }\end{array}$ \\
\hline Total Cost (\$) & & & 17.62 & & \\
\hline
\end{tabular}

Table B.B-3: Diode components Cost for Converter

\begin{tabular}{|c|c|c|c|c|c|}
\hline Diode & Quantity & $\begin{array}{c}\text { Unit Price } \\
\mathbf{( \$ )}\end{array}$ & $\begin{array}{c}\text { Total Price } \\
\mathbf{( \$ )}\end{array}$ & Components & \begin{tabular}{c} 
Type \\
\hline MBR20100CT
\end{tabular} \\
\hline MBRS140 & 4 & 0.91 & 5.46 & D2, D3, D4, D7, D10, & $\begin{array}{c}\text { Through- } \\
\text { Hole }\end{array}$ \\
\hline B540C & 4 & 0.55 & 2.20 & D15, D16, D29, D30 & SMT \\
\hline ES1D & 12 & 0.75 & 3.00 & D1, D6, D9, D12 & SMT \\
\hline 1N4148 & 4 & 0.53 & 6.36 & D17 to D28 & SMT \\
\hline BZX84C6V2L & 2 & 0.46 & 0.92 & D5, D8, D11, D14 & SMT \\
\hline $\begin{array}{c}\text { Total Cost } \\
\text { (\$) }\end{array}$ & & & 1.32 & D31, D32 & SMT \\
\hline
\end{tabular}


Table B.B-4: Resistor Components Cost for Converter

\begin{tabular}{|c|c|c|c|c|c|}
\hline Resistor & Quantity & $\begin{array}{l}\text { Unit Price } \\
\text { (\$) }\end{array}$ & $\begin{array}{c}\text { Total Price } \\
(\$)\end{array}$ & Components & Type \\
\hline $1 \mathrm{M}, 1 / 8 \mathrm{~W}, 1 \%$ & 3 & 0.07 & 0.21 & $\mathrm{R} 1, \mathrm{R} 40, \mathrm{R} 42$ & SMT \\
\hline $357 \mathrm{k}, 1 / 8 \mathrm{~W}, 1 \%$ & 1 & 0.04 & 0.04 & $\mathrm{R} 14$ & SMT \\
\hline $191 \mathrm{k}, 1 / 8 \mathrm{~W}, 1 \%$ & 4 & 0.07 & 0.28 & R4, R17, R21, R25 & SMT \\
\hline $143 \mathrm{k}, 1 / 8 \mathrm{~W}, 1 \%$ & 1 & 0.07 & 0.07 & R5 & SMT \\
\hline $130 \mathrm{k}, 1 / 8 \mathrm{~W}, 1 \%$ & 1 & 0.07 & 0.07 & R11 & SMT \\
\hline $84.5 \mathrm{k}, 1 / 8 \mathrm{~W}, 1 \%$ & 1 & 0.04 & 0.04 & $\mathrm{R} 16$ & SMT \\
\hline $82.5 \mathrm{k}, 1 / 8 \mathrm{~W}, 1 \%$ & 1 & 0.04 & 0.04 & $\mathrm{R} 7$ & SMT \\
\hline $26.7 \mathrm{k}, 1 / 8 \mathrm{~W}, 1 \%$ & 1 & 0.07 & 0.07 & $\mathrm{R} 12$ & SMT \\
\hline $25.5 \mathrm{k}, 1 / 8 \mathrm{~W}, 1 \%$ & 2 & 0.04 & 0.08 & R39, R41 & SMT \\
\hline $24.9 \mathrm{k}, 1 / 8 \mathrm{~W}, 1 \%$ & 1 & 0.04 & 0.04 & $\mathrm{R} 15$ & SMT \\
\hline $20 \mathrm{k}, 1 / 8 \mathrm{~W}, 1 \%$ & 2 & 0.04 & 0.08 & $\mathrm{R} 19, \mathrm{R} 27$ & SMT \\
\hline $16.2 \mathrm{k}, 1 / 8 \mathrm{~W}, 1 \%$ & 1 & 0.04 & 0.04 & $\mathrm{R} 6$ & SMT \\
\hline $12.4 \mathrm{k}, 1 / 8 \mathrm{~W}, 1 \%$ & 1 & 0.05 & 0.05 & R13 & SMT \\
\hline $360,2 W, 5 \%$ & 4 & 0.35 & 1.40 & R22, R30, R32, R34 & $\begin{array}{c}\text { Through- } \\
\text { Hole }\end{array}$ \\
\hline $200,2 W, 5 \%$ & 2 & 0.54 & 1.08 & R37, R38 & $\begin{array}{c}\text { Through- } \\
\text { Hole }\end{array}$ \\
\hline $10,1 / 2 \mathrm{~W}, 1 \%$ & 2 & 0.23 & 0.46 & R28, R36 & $\begin{array}{c}\text { Through- } \\
\text { Hole }\end{array}$ \\
\hline $0.05,1 / 2 \mathrm{~W}, 5 \%$ & 4 & 0.50 & 2.00 & R23, R31, R33, R35 & SMT \\
\hline $0.006,1 \mathrm{~W}, 1 \%$ & 6 & 0.56 & 3.36 & $\begin{array}{c}\text { R3, R18, R20, R24, R26, } \\
\text { R29 }\end{array}$ & SMT \\
\hline $\begin{array}{c}\text { Total Cost } \\
(\$)\end{array}$ & & & 9.41 & & \\
\hline
\end{tabular}

Table B.B-5: Inductor Components Cost for Converter

\begin{tabular}{|c|c|c|c|c|c|}
\hline Inductor & Quantity & $\begin{array}{l}\text { Unit Price } \\
\text { (\$) }\end{array}$ & $\begin{array}{c}\text { Total Price } \\
\text { (\$) }\end{array}$ & Components & Type \\
\hline $\begin{array}{c}39 \mu \mathrm{H}, \\
\mathrm{I}_{\mathrm{SAT}}=17 \mathrm{~A}\end{array}$ & 6 & 7.06 & 42.36 & $\begin{array}{l}\text { L1, L4, L5, L6, L7, } \\
\text { L8 }\end{array}$ & $\begin{array}{c}\text { Through- } \\
\text { Hole }\end{array}$ \\
\hline $\begin{array}{c}90 \mathrm{nH}, \\
\mathrm{I}_{\mathrm{SAT}}=17 \mathrm{~A}\end{array}$ & 2 & 2.45 & 4.90 & L2, L3 & SMT \\
\hline $\begin{array}{c}75 \mathrm{nH}, \\
\mathrm{I}_{\mathrm{SAT}}=17 \mathrm{~A}\end{array}$ & 4 & 2.36 & 9.44 & L9, L10, L11, L12 & SMT \\
\hline Total Cost (\$) & & & 56.70 & & \\
\hline
\end{tabular}


Table B.B-6: Capacitor Components Cost for Converter

\begin{tabular}{|c|c|c|c|c|c|}
\hline Capacitor & Quantity & $\begin{array}{c}\text { Unit Price } \\
\text { (\$) }\end{array}$ & $\begin{array}{c}\text { Total Price } \\
(\$)\end{array}$ & Components & Type \\
\hline $560 \mu$, Electrolytic, $100 \mathrm{~V}$ & 1 & 2.13 & 2.13 & C44 & $\begin{array}{l}\text { Through- } \\
\text { Hole }\end{array}$ \\
\hline $100 \mu$, Electrolytic, $100 \mathrm{~V}$ & 4 & 1.16 & 4.64 & $\begin{array}{l}\text { C21, Additional input filter } \\
\text { capacitors }\end{array}$ & $\begin{array}{l}\text { Through- } \\
\text { Hole }\end{array}$ \\
\hline $100 \mu$, Electrolytic, $50 \mathrm{~V}$ & 1 & 0.81 & 0.81 & C43 & SMT \\
\hline $40 \mu$, Electrolytic, 35V & 1 & 0.55 & 0.55 & C4 & SMT \\
\hline $22 \mu$, Electrolytic, $50 \mathrm{~V}$ & 3 & 2.25 & 6.75 & C32, C33, C38 & SMT \\
\hline $10 \mu$, Ceramic, $50 \mathrm{~V}$ & 4 & 0.69 & 2.76 & C39, C40, C41, C42 & SMT \\
\hline $4.7 \mu$, Ceramic, $10 \mathrm{~V}$ & 1 & 0.30 & 0.30 & C15 & SMT \\
\hline $1 \mu$, Ceramic, $50 \mathrm{~V}$ & 9 & 0.24 & 2.16 & $\begin{array}{c}\text { C1, C5, C6, C14, C16, } \\
\text { C19, C22, C25, C26 }\end{array}$ & SMT \\
\hline $0.1 \mu$, Ceramic, $100 \mathrm{~V}$ & 3 & 0.40 & 1.20 & C12, C24, C31 & SMT \\
\hline $0.1 \mu$, Ceramic, $10 \mathrm{~V}$ & 2 & 0.05 & 0.10 & $\mathrm{C} 47, \mathrm{C} 48$ & SMT \\
\hline 10000p, Ceramic, 6.3V & 3 & 0.08 & 0.24 & $\mathrm{C} 8, \mathrm{C} 13, \mathrm{C} 30$ & SMT \\
\hline 4700p, Ceramic, 50V & 2 & 0.08 & 0.16 & $\mathrm{C} 45, \mathrm{C} 46$ & SMT \\
\hline 2200p, Ceramic, 10V & 6 & 0.08 & 0.48 & $\begin{array}{c}\text { C3, C7, C17, C20, C23, } \\
\text { C27 }\end{array}$ & $\mathrm{SMT}$ \\
\hline 1000p, Ceramic, $10 \mathrm{~V}$ & 2 & 0.36 & 0.72 & $\mathrm{C} 9, \mathrm{C} 11$ & SMT \\
\hline 750p, Ceramic, 100V & 4 & 0.47 & 1.88 & C34, C35, C36, C37 & $\begin{array}{c}\text { Through- } \\
\text { Hole }\end{array}$ \\
\hline 100p, Ceramic, 10V & 1 & 0.40 & 0.40 & C10 & SMT \\
\hline 47p, Ceramic, 25V & 4 & 0.09 & 0.36 & $\mathrm{C} 2, \mathrm{C} 10, \mathrm{C} 28, \mathrm{C} 29$ & SMT \\
\hline Total Cost (\$) & & & 25.64 & & \\
\hline
\end{tabular}


Total component cost for this converter is $\$ 171.99$. The converter also has 152 total base components, exlcuding any safety components or test points. Because the estimated size of the required PCB is fairly large, ExpressPCB typically requires an additional $\$ 233$ as a base price [76] for fabricating a custom-sized PCB such as what this converter requires (resulting in a minimum total cost of $\$ 404.99$ for a final production-level PCB). These costs are unfeasible if the EHFEM elliptical trainer system requires a 10-year payback period. Thus, this converter design must be downsized in order to reduce component and overall converter cost. The next section describes a possible converter downsizing proposal.

\section{B1.14 Converter Downsize Proposal}

One way to reduce this project's converter's component count and overall cost involves using only one LT3845A PWM controller IC to drive all four input stage phases. All the required pin connections on all four phases connect to a single LT3845A controller. This configuration also requires only one current sense resistor (located on the converter's primary phase); however the disadvantage with this design is that the LT3845A controller will not protect the other phases from any over-current conditions, as the current through each phase can become unbalanced if such an event occurs. Another advantage, however, is that this design does not require an additional LTC6908-1 external oscillator IC, as the frequency-set resistor tied to the LT3845A's Fset pin suffices. Figure B-88 shows this reduced-part converter design. 


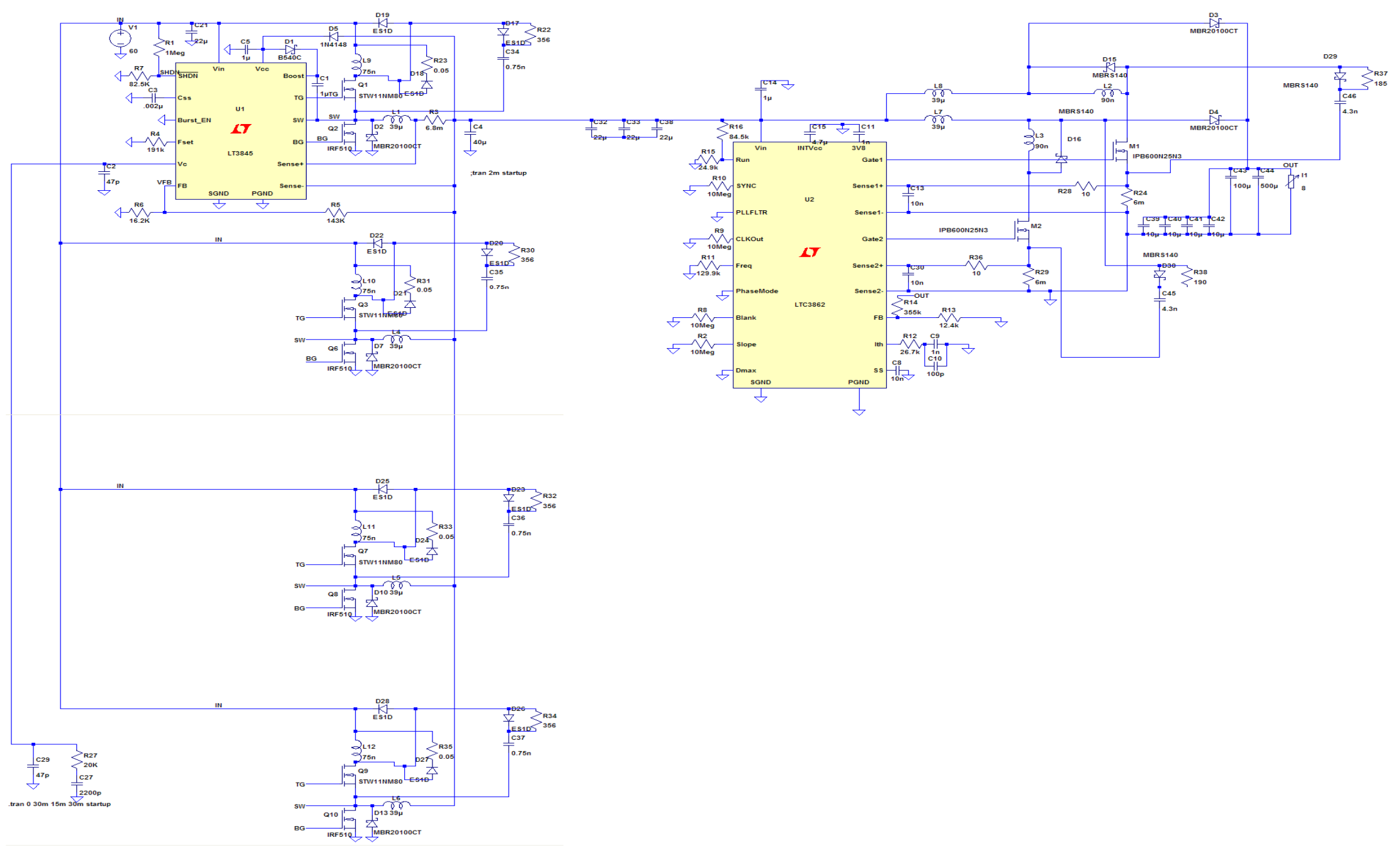

Figure B-88: Downsized 100 kHz Two-Phase Boost, Multi-Stage Buck-Boost Converter Design with LTC6908-1 External Oscillators 
Figure B-89 shows the converter operating at maximum stress conditions (60V input, 8A load) in steady state operation, with converter output voltage in green, input buck stage output voltage in blue, and power dissipation across $\mathrm{Q}_{1}$ (which remains our primary switching transistor for the primary input phase) in red.

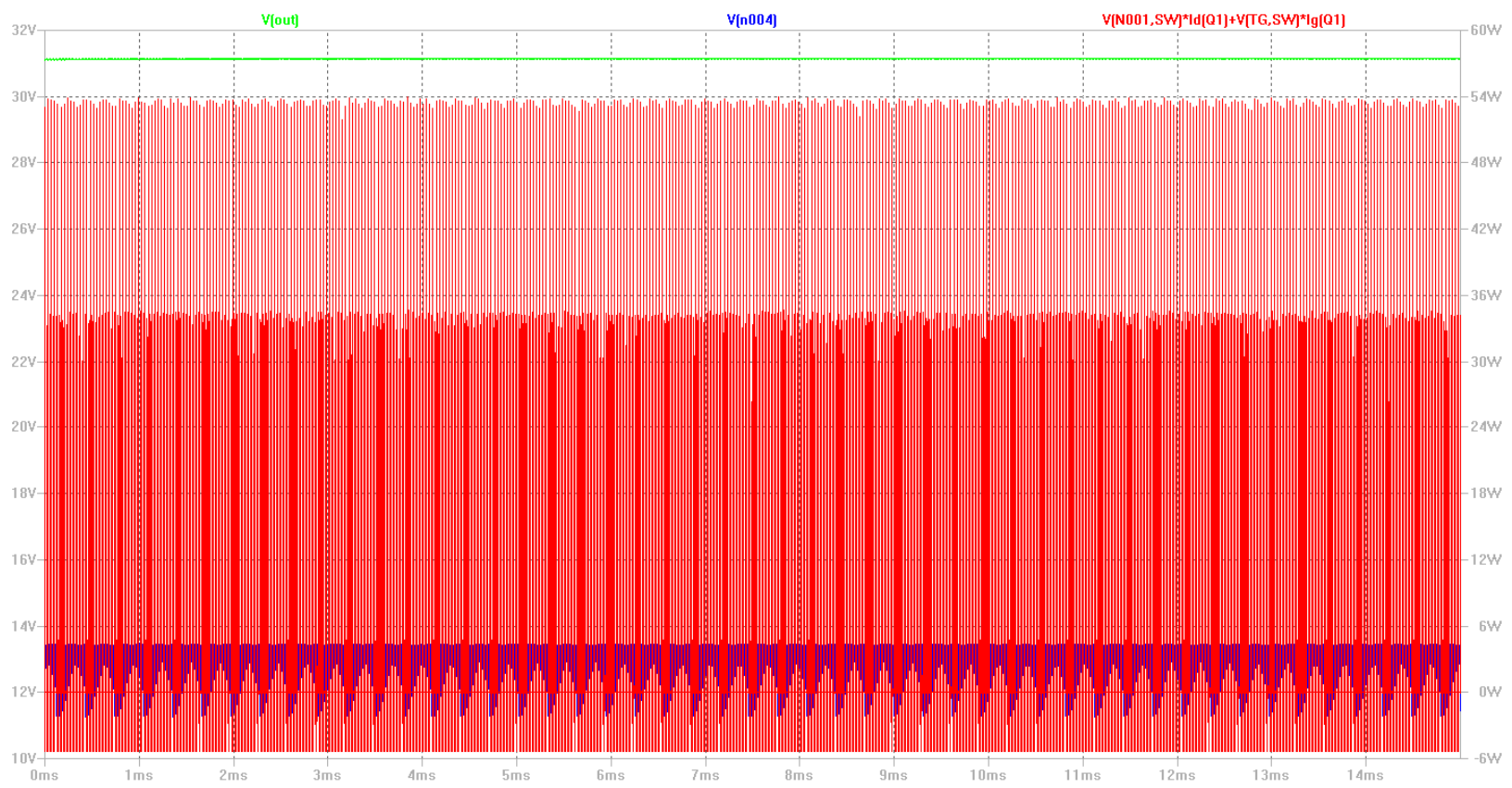

Figure B-89: Output Voltage, Buck Stage Output Voltage and $Q_{1}$ Power Dissipation of Downsized $100 \mathrm{kHz}$

Two-Phase Boost, Multi-stage Buck-Boost Converter Design 
Figure B-90 is same plot as figure B-89, but with the input buck stage ouput voltage in a separate plot pane.

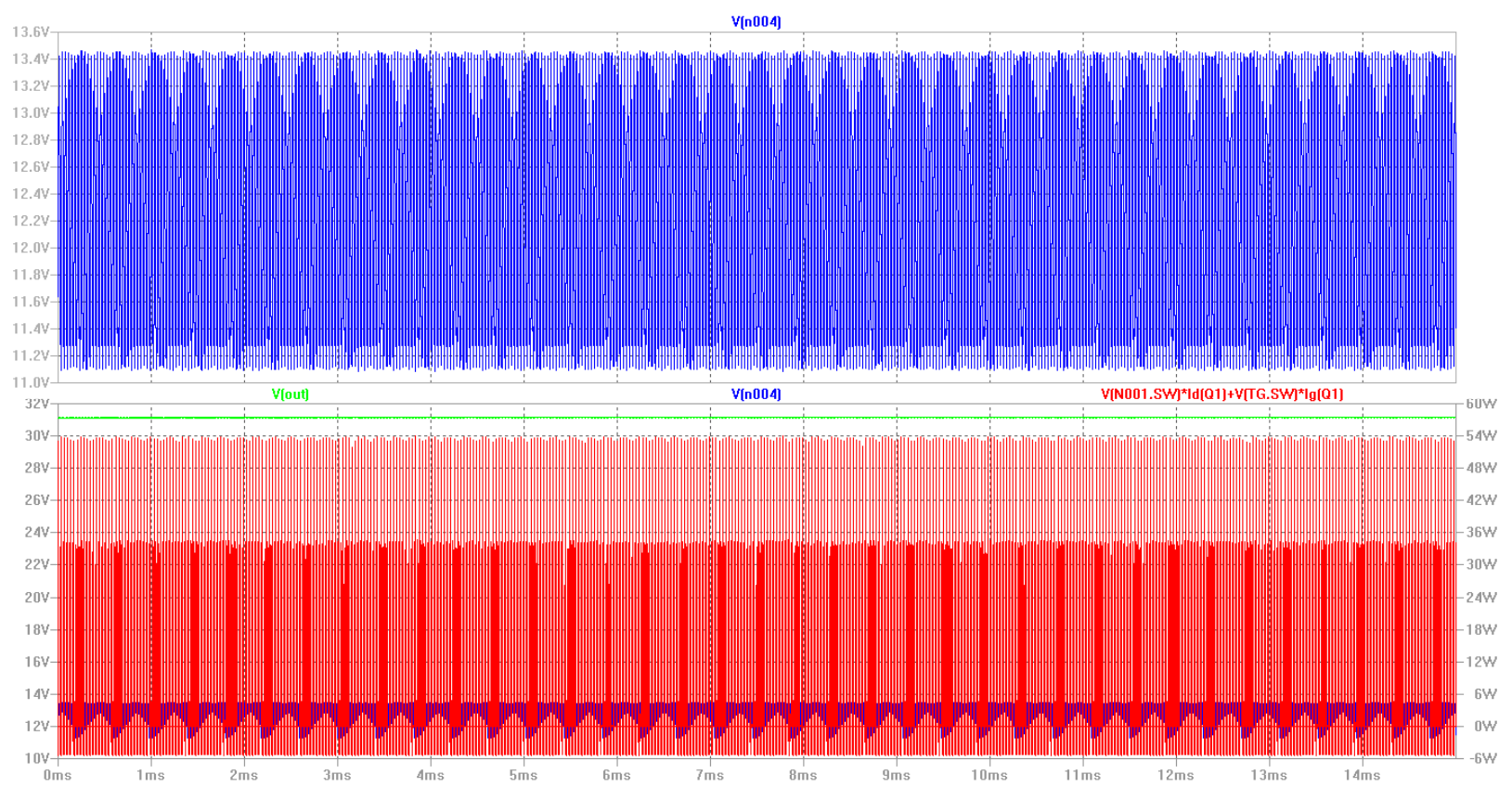

Figure B-90: Output Voltage, Buck Stage Output Voltage and $Q_{1}$ Power Dissipation of Downsized $100 \mathrm{kHz}$ Two-Phase Boost, Multi-Stage Buck-Boost Converter Design

$\mathrm{Q}_{1}$ 's average power dissipation drastically reduces to $1.85 \mathrm{~W}$. The other primary

switching transistors in the other phases follow a similar trend as long as the current through each phase is balanced (i.e. equal through each phase). Figure B-91 shows in teal the power dissipation across $\mathrm{Q}_{3}$, the primary switching transistor on the input stage's second phase. 


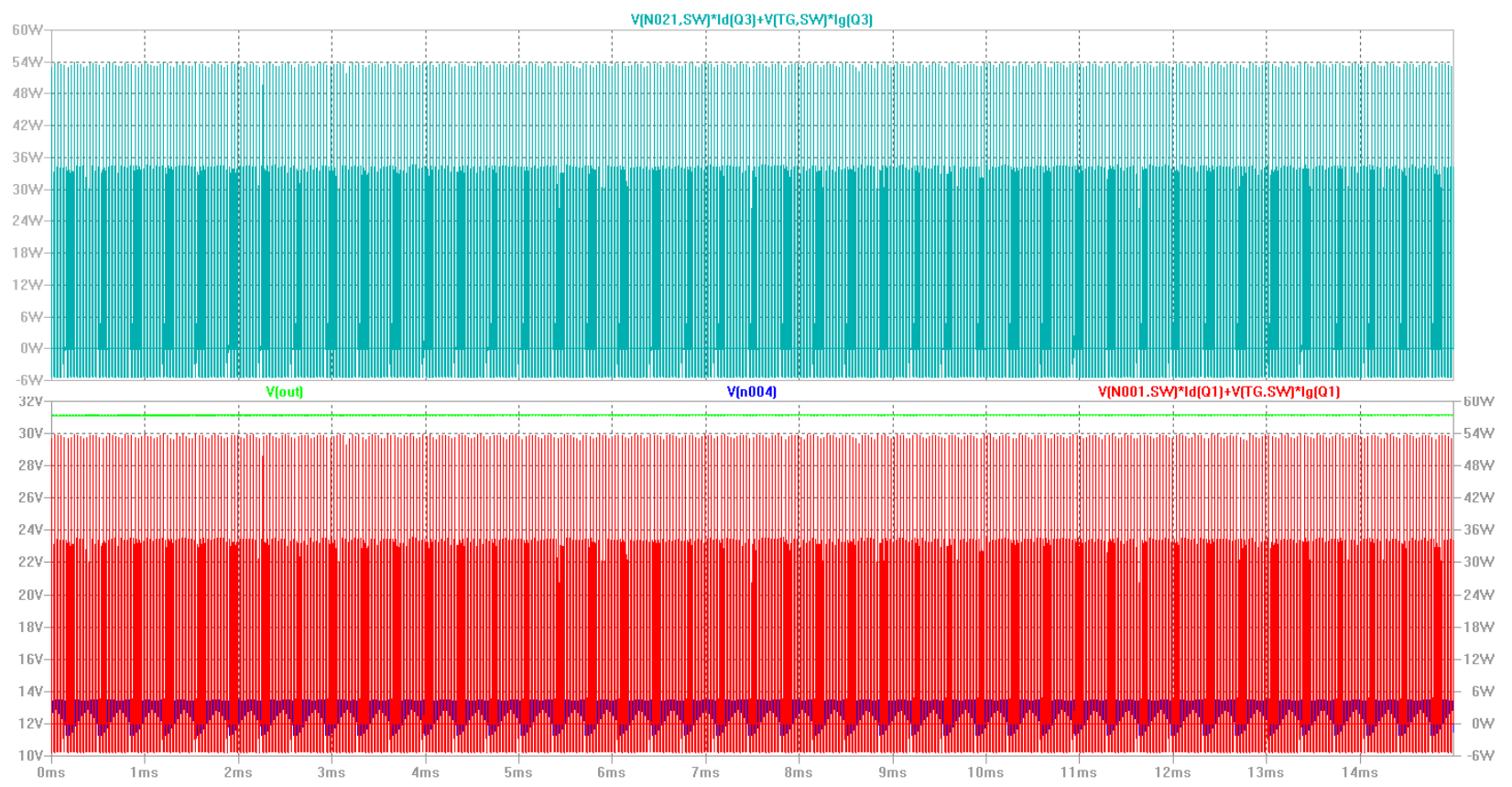

Figure B-91: Output Voltage, Buck Stage Output Voltage and $Q_{1}$ and $Q_{3}$ Power Dissipation of Downsized 100 kHz Two-Phase Boost, Multi-Stage Buck-Boost Converter Design

$\mathrm{Q}_{3}$ 's power dissipation waveform is identical to that of $\mathrm{Q}_{1}$ 's. From figure B-91, the converter's average output voltage only reaches a $31.14 \mathrm{~V}$, which is significantly below the $36 \mathrm{~V}$ from the converter's original specifications. Thus this converter configuration cannot provide the Enphase micro-inverter with the optimal input voltage that requires for maximizing its efficiency. In reality, a single LT3845A controller may also have problems driving more than one parallel phase, which may have resulted in the converter's reduced output voltage in simulation. The next section describes this converter's costs.

\section{B1.15 Downsized Converter Cost Analysis}

Likewise with appendix B1.14, the component cost for this downsized converter does not include additional costs for PCB fabrication as well as safety components and 
test points. Tables B.B-7 to B.B-12 (each separated by component type) show this converter's component cost.

Table B.B-7: Controller IC Components Cost for Downsized Converter

\begin{tabular}{|c|c|c|c|c|c|}
\hline Controller IC & Quantity & Unit Price (\$) & Total Price (\$) & Components & Type \\
\hline LT3845A & 1 & 7.13 & 7.13 & U1 & SMT \\
\hline LTC3862 & 1 & 7.00 & 7.00 & U2 & SMT \\
\hline Total Cost (\$) & & $\mathbf{1 4 . 1 3}$ & \multicolumn{3}{l}{} \\
\hline
\end{tabular}

Table B.B-8: Transistor Components Cost for Downsized Converter

\begin{tabular}{|c|c|c|c|c|c|}
\hline Transistor & Quantity & $\begin{array}{c}\text { Unit Price } \\
\mathbf{( \$ )}\end{array}$ & $\begin{array}{c}\text { Total Price } \\
\mathbf{( \$ )}\end{array}$ & Components & Type \\
\hline $\begin{array}{c}\text { Fairchild } \\
\text { FDB14N30 }\end{array}$ & 4 & 1.38 & 5.52 & Q1, Q3, Q7, Q9 & SMT \\
\hline IRF510 & 4 & 1.51 & 6.04 & Q2, Q6, Q8, & $\begin{array}{c}\text { Through- } \\
\text { Hole }\end{array}$ \\
\hline IPB600N25N3 & 2 & 2.50 & 5.00 & M1, M2 & SMT \\
\hline Total Cost (\$) & & $\mathbf{1 6 . 5 6}$ & & \\
\hline
\end{tabular}

Table B.B-9: Diode Components Cost for Downsized Converter

\begin{tabular}{|c|c|c|c|c|c|}
\hline Diode & Quantity & $\begin{array}{c}\text { Unit Price } \\
\mathbf{( \$ )}\end{array}$ & $\begin{array}{c}\text { Total Price } \\
\mathbf{( \$ )}\end{array}$ & Components & \begin{tabular}{c} 
Type \\
\hline MBR20100CT
\end{tabular} \\
\hline MBRS140 & 4 & 0.91 & 5.46 & $\begin{array}{c}\text { D2, D3, D4, D7, D10, } \\
\text { D13 }\end{array}$ & $\begin{array}{c}\text { Through- } \\
\text { Hole }\end{array}$ \\
\hline B540C & 1 & 0.55 & 2.20 & D15, D16, D29, D30 & SMT \\
\hline ES1D & 12 & 0.75 & 0.75 & D1 & SMT \\
\hline 1N4148 & 1 & 0.33 & 6.36 & D17 to D28 & SMT \\
\hline $\begin{array}{c}\text { Total Cost } \\
\text { (\$) }\end{array}$ & & 0.33 & D5 & SMT \\
\hline
\end{tabular}


Table B.B-10: Resistor Components Cost for Downsized Converter

\begin{tabular}{|c|c|c|c|c|c|}
\hline Resistor & Quantity & $\begin{array}{l}\text { Unit Price } \\
(\$)\end{array}$ & $\begin{array}{c}\text { Total Price } \\
\text { (\$) }\end{array}$ & Components & Type \\
\hline $1 \mathrm{M}, 1 / 8 \mathrm{~W}, 1 \%$ & 1 & 0.07 & 0.07 & $\mathrm{R} 1$ & SMT \\
\hline $357 \mathrm{k}, 1 / 8 \mathrm{~W}, 1 \%$ & 1 & 0.04 & 0.04 & R14 & SMT \\
\hline $191 \mathrm{k}, 1 / 8 \mathrm{~W}, 1 \%$ & 1 & 0.07 & 0.07 & R4 & SMT \\
\hline $143 \mathrm{k}, 1 / 8 \mathrm{~W}, 1 \%$ & 1 & 0.07 & 0.07 & $\mathrm{R} 5$ & SMT \\
\hline 130k, 1/8W, 1\% & 1 & 0.07 & 0.07 & $\mathrm{R} 11$ & SMT \\
\hline $84.5 \mathrm{k}, 1 / 8 \mathrm{~W}, 1 \%$ & 1 & 0.04 & 0.04 & R16 & SMT \\
\hline $82.5 \mathrm{k}, 1 / 8 \mathrm{~W}, 1 \%$ & 1 & 0.04 & 0.04 & $\mathrm{R} 7$ & SMT \\
\hline $26.7 \mathrm{k}, 1 / 8 \mathrm{~W}, 1 \%$ & 1 & 0.07 & 0.07 & $\mathrm{R} 12$ & SMT \\
\hline $24.9 \mathrm{k}, 1 / 8 \mathrm{~W}, 1 \%$ & 1 & 0.04 & 0.04 & $\mathrm{R} 15$ & SMT \\
\hline $16.2 \mathrm{k}, 1 / 8 \mathrm{~W}, 1 \%$ & 1 & 0.04 & 0.04 & R6 & SMT \\
\hline $12.4 \mathrm{k}, 1 / 8 \mathrm{~W}, 1 \%$ & 1 & 0.05 & 0.05 & $\mathrm{R} 13$ & SMT \\
\hline $360,2 W, 5 \%$ & 4 & 0.35 & 1.40 & $\begin{array}{l}\text { R22, } R 30, \text { R32, } \\
\text { R34 }\end{array}$ & $\begin{array}{l}\text { Through- } \\
\text { Hole }\end{array}$ \\
\hline $200,2 W, 5 \%$ & 2 & 0.54 & 1.08 & R37, R38 & $\begin{array}{l}\text { Through- } \\
\text { Hole }\end{array}$ \\
\hline $10,1 / 2 W, 1 \%$ & 2 & 0.23 & 0.46 & $\mathrm{R} 28, \mathrm{R} 36$ & $\begin{array}{l}\text { Through- } \\
\text { Hole }\end{array}$ \\
\hline $0.05,1 / 2 W, 5 \%$ & 4 & 0.50 & 2.00 & $\begin{array}{l}\text { R23, R31, R33, } \\
\text { R35 }\end{array}$ & SMT \\
\hline $0.006,1 \mathrm{~W}, 1 \%$ & 3 & 0.56 & 1.68 & R3, R24, R29 & SMT \\
\hline $\begin{array}{l}\text { Total Cost } \\
(\$)\end{array}$ & & & 7.22 & & \\
\hline
\end{tabular}


Table B.B-11: Inductor Components Cost for Downsized Converter

\begin{tabular}{|c|c|c|c|c|c|}
\hline Inductors & Quantity & $\begin{array}{c}\text { Unit } \\
\text { Price (\$) }\end{array}$ & $\begin{array}{l}\text { Total Price } \\
(\$)\end{array}$ & Components & Type \\
\hline $\begin{array}{c}39 \mu \mathrm{H}, \\
\mathrm{I}_{\mathrm{SAT}}=17 \mathrm{~A}\end{array}$ & 6 & 7.06 & 42.36 & $\begin{array}{c}\mathrm{L1}, \mathrm{L} 4, \mathrm{~L} 5, \mathrm{~L} 6, \mathrm{~L} 7, \\
\mathrm{~L} 8\end{array}$ & $\begin{array}{l}\text { Through- } \\
\text { Hole }\end{array}$ \\
\hline $\begin{array}{c}90 \mathrm{nH}, \\
\mathrm{I}_{\mathrm{SAT}}=17 \mathrm{~A}\end{array}$ & 2 & 2.45 & 4.90 & L2, L3 & SMT \\
\hline $\begin{array}{c}75 \mathrm{nH}, \\
\mathrm{I}_{\mathrm{SAT}}=17 \mathrm{~A}\end{array}$ & 4 & 2.36 & 9.44 & L9, L10, L11, L12 & SMT \\
\hline Total Cost (\$) & & & 56.70 & & \\
\hline
\end{tabular}

Table B.B-12: Capacitor Components Cost for Downsized Converter

\begin{tabular}{|c|c|c|c|c|c|}
\hline Capacitor & Quantity & $\begin{array}{c}\text { Unit } \\
\text { Price } \\
\mathbf{( \$ )}\end{array}$ & $\begin{array}{c}\text { Total } \\
\text { Price } \mathbf{( \$ )}\end{array}$ & Components & C44 \\
\hline $\begin{array}{c}560 \mu, \text { Electrolytic, } \\
100 \mathrm{~V}\end{array}$ & 1 & 2.13 & 2.13 & $\begin{array}{c}\text { Through- } \\
\text { Hole }\end{array}$ \\
\hline $\begin{array}{c}100 \mu \text {, Electrolytic, } \\
\text { 100V }\end{array}$ & 4 & 1.16 & 4.64 & $\begin{array}{c}\text { C21, Additional input } \\
\text { filter capacitors }\end{array}$ & $\begin{array}{c}\text { Through- } \\
\text { Hole }\end{array}$ \\
\hline $100 \mu$, Electrolytic, 50V & 1 & 0.81 & 0.81 & C43 & SMT \\
\hline $40 \mu$, Electrolytic, 35V & 1 & 0.55 & 0.55 & C4 & SMT \\
\hline $22 \mu$, Electrolytic, 50V & 3 & 2.25 & 6.75 & C32, C33, C38 & SMT \\
\hline $10 \mu$, Ceramic, 50V & 4 & 0.69 & 2.76 & C39, C40, C41, C42 & SMT \\
\hline $4.7 \mu$, Ceramic, 10V & 1 & 0.30 & 0.30 & C15 & SMT \\
\hline $1 \mu$, Ceramic, 50V & 3 & 0.24 & 0.72 & C1, C5, C14 & SMT \\
\hline $10000 p$, Ceramic, 6.3V & 3 & 0.08 & 0.24 & C8, C13, C30 & SMT \\
\hline $4700 p$, Ceramic, 50V & 2 & 0.08 & 0.16 & C45, C46 & SMT \\
\hline $2200 p$, Ceramic, 10V & 2 & 0.08 & 0.16 & C3, C27 & SMT \\
\hline $1000 p$, Ceramic, 10V & 2 & 0.36 & 0.72 & C9, C11 & SMT \\
\hline $750 p$, Ceramic, 100V & 4 & 0.47 & 1.88 & C34, C35, C36, C37 & Through- \\
\hline $100 p$, Ceramic, 10V & 1 & 0.40 & 0.40 & C10 & SMT \\
\hline $47 p$, Ceramic, 25V & 2 & 0.09 & 0.18 & C2, C29 & SMT \\
\hline Total Cost (\$) & & & $\mathbf{2 2 . 4 0}$ & & \\
\hline
\end{tabular}


Using only one LT3845A controller for driving all four input stage phases, reduces the total converter component cost to $\$ 132.11$. The converter's component count also reduces to a 108 base component total, excluding any safety components or test points. However, this downsized converter still requires a custom-sized PCB because of converter's inductors' physical sizes. The PCB size that this downsized converter requires is still larger than what PCBExpress's mini-board option offers (a 2.5" $\times 3.8$ " PCB). Again, likewise with the previous converter design, because the required PCB's estimated size is fairly large, ExpressPCB typically requires an additional $\$ 233$ as a base price [76] for fabricating a custom-sized PCB such as what this downsized converter requires. This fabrication cost results in a $\$ 365.11$ minimum total cost for a final production-level PCB. These costs are still unfeasible if the EHFEM elliptical trainer system requires a 10-year payback period. Even then, a $31.14 \mathrm{~V}$ output from this converter design is unacceptable, as the Enphase micro-inverter requires $36 \mathrm{~V}$ input for operating at maximum efficiency. Even with the previous converter design, its overall simulated converter efficiency was roughly $87.1 \%$. If that design was physically implemented, parasitic characteristics of various components, such as ESR on capacitors, would still exist between PCB traces. Thus, the actual converter efficiency will be significantly lower than the results from simulations. The overall converter cost is not worth the converter's efficiency loss (relative to the rest of the EHFEM elliptical trainer system). Furthermore, if designs and functionality problems occur during the physical testing phase, troubleshooting the converter would be very time-consuming and difficult because of the large number of components and intricate circuit complexity. 
Although the interleaved, multi-stage buck-boost converter described in Appendices A and B initially seemed like a feasible design for the EHFEM project after running simulations on it and mitigating its switching transistor power transient issues, its overall cost was too high for justifying its maximum efficiency level. Its exorbitantly high cost also results in an unreasonable payback period for the EHFEM system's life cycle. Furthermore, the converter's intricate complexity would make troubleshooting very difficult if it were ever physically fabricated onto a PCB. For such a complex design, anything could go wrong during testing. After discovering that the Precor EFX $546 \mathrm{i}$ elliptical trainer's onboard $12 \mathrm{~V}$ battery could be used to supply a bias voltage for a converter's PWM controllers, this project decided on the SEPIC topology described in chapter 3 for its DC-DC converter design. The basic SEPIC design from chapter 3 yields a fairly low component count, which results in a smaller PCB required for the converter, as well as a low overall converter cost.

Therefore, this project scrapped entire downsized interleaved buck-boost converter design and its previous design iterations in favor of a new converter design that minimizes component count and overall cost. Chapter 3 details selecting the new converter design used for defending this report's thesis statement. 


\section{APPENDIX C: FINAL SEPIC NETLIST}

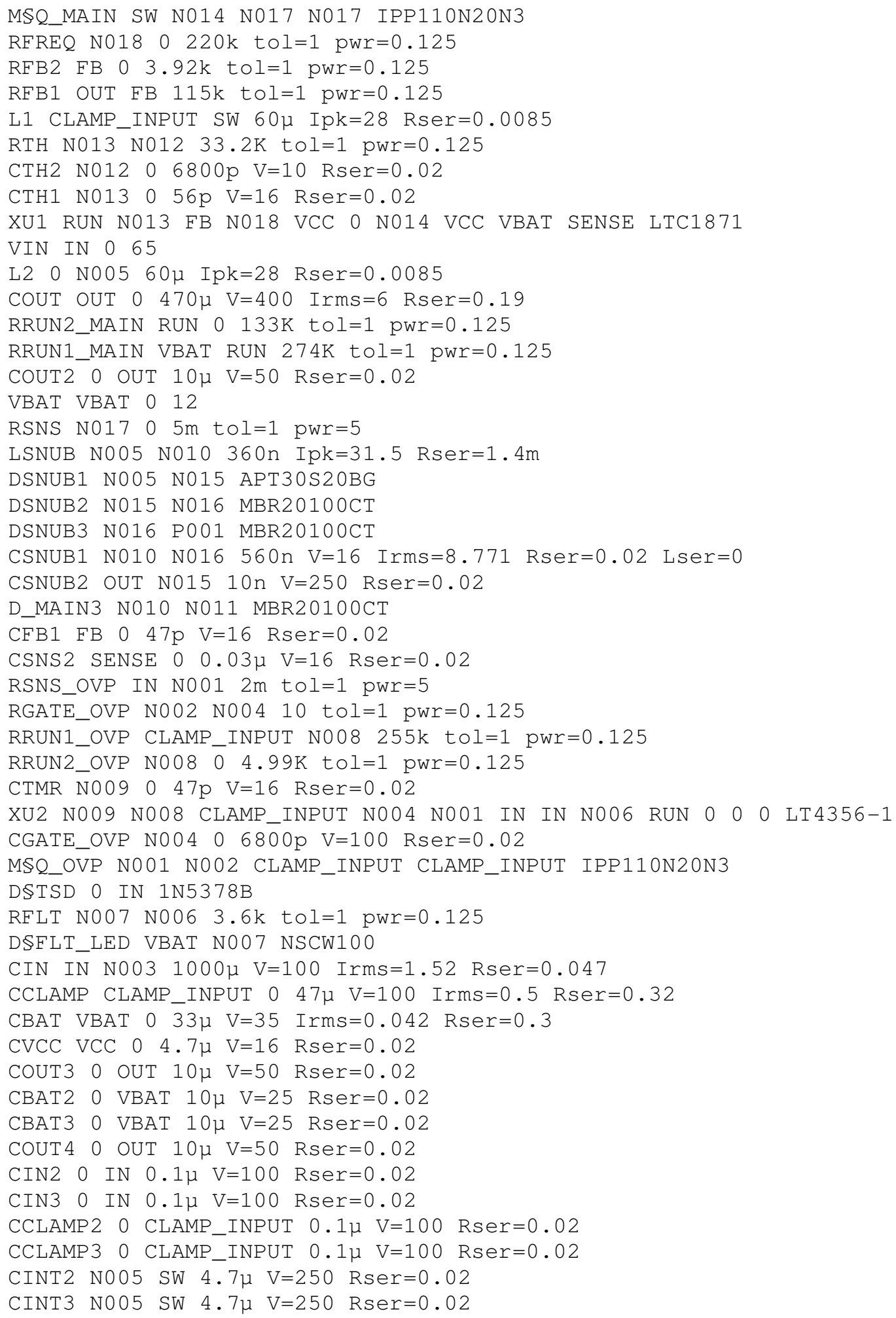


CCLAMP4 0 CLAMP_INPUT $0.1 \mu \quad \mathrm{V}=100$ Rser $=0.02$

CINT N005 SW $15 \mu \quad$ V=250 Irms $=4$ Rser $=0.02$

CINT4 N005 SW $4.7 \mu \quad V=250$ Rser $=0.02$

CINT5 N005 SW $4.7 \mu \quad V=250$ Rser $=0.02$

CINT6 N005 SW 4.7 $\mathrm{V}=250$ Rser $=0.02$

D_MAIN1 N010 N011 MBR20100CT

D_MAIN2 N011 OUT MBR20100CT

D_MAIN 4 N011 OUT MBR20100CT

DSNUB4 P001 OUT MBR20100CT

CRUN_OVP N008 $0 \quad 47 \mathrm{p}$

CRUN_MAIN RUN $0 \quad 47 \mathrm{p}$

CBAT4 0 VBAT $100 \mathrm{n} \quad \mathrm{V}=25$ Rser $=0.02$

CBAT5 0 VBAT $100 \mathrm{n} \quad \mathrm{V}=25$ Rser $=0.02$

COUT_CPH1 0 OUT $0.1 \mu \quad \mathrm{V}=50$ Rser $=0.02$

COUT_CPH2 O OUT $0.1 \mu \quad \mathrm{V}=50$ Rser $=0.02$

CINT7 N005 SW $0.1 \mu$

CINT8 N005 SW $0.1 \mu$

ILOAD OUT 08

RVCC1 VBAT VCC 887

RVCC2 VCC $0 \quad 680$

CSNS1 SENSE $0 \quad 47 \mathrm{p} \quad \mathrm{V}=16$ Rser $=0.02$

RSNS_FILT N017 SENSE 10

RGATE_MAIN N014 N019 45

CGATE_MAIN N019 $00.02 \mu$ Rser $=0.02$

DGATE_MAIN N014 N019 1N4148

R_ELLIPTICAL N003 010

CFB2 FB $00.01 \mu \quad V=16$ Rser $=0.02$

CINT9 N005 SW $0.1 \mu$

CINT10 N005 SW $0.1 \mu$

COUT_CPH3 0 OUT $0.1 \mu \quad \mathrm{V}=50$ Rser $=0.02$

COUT_CPH4 O OUT $0.1 \mu \mathrm{V}=50$ Rser $=0.02$

. model D D

. lib $\mathrm{C}: \backslash \mathrm{PROGRA} \sim 2 \backslash \mathrm{LTC} \backslash \mathrm{LTSPIC} \sim 1 \backslash 1$ ib $\backslash \mathrm{cmp} \backslash$ standard.dio

. model NMOS NMOS

.model PMOS PMOS

. lib $\mathrm{C}: \backslash \mathrm{PROGRA} \sim 2 \backslash \mathrm{LTC} \backslash \mathrm{LTSPIC} \sim 1 \backslash 1 \mathrm{ib} \backslash \mathrm{cmp} \backslash$ standard.mos

.tran $030 \mathrm{~m} 18 \mathrm{~m} 30 \mathrm{~m}$ steady startup

. temp 40

. model APT30S20BG D (Is=15m Rs=0.005 N=1.5 Isr=15m Cjo=150p Vj=0.86

Iave $=45 \mathrm{Vpk}=200 \mathrm{mfg}=0 \mathrm{nSemi}$ type=Schottky)

. lib LT4356-1. sub

.lib LTC1871.sub

. backanno

. end 


\section{REFERENCES}

[1] J. Yuen; M. Lum; C. Cinkornpumin; J. Chan. "Energy Harvesting From Exercise Machines (EHFEM) Self-generator Elliptial Machine," Cal Poly Digital Commons. 2008. [Online]. http://digitalcommons.calpoly.edu/eesp/12

[2] Precor, "EFX® 546i Elliptical Fitness Crosstrainer ${ }^{\mathrm{TM}}$ Specifications" Precor EFX 546i product page, 2010. [Online].

http://www.precor.com/products/en/efx-546i-elliptical-fitness-crosstrainer-1\#

[3] Enphase Energy, "Enphase Micro-Inverter," M175-24-240 datasheet. [Online]. http://enphase.com/downloads/8261_Datasheet_24_32.pdf

[4] Vicor Power, "28V Wide Input Maxi Family DC-DC Converter Module Data Sheet" Vicor Maxi, Mini and Micro DC-DC Converters product page, 2010. [Online]. http://cdn.vicorpower.com/documents/datasheets/ds_28vin-maxi-family.pdf

[5] Underwriters Laboratory code 1741 (2007). Inverters, converters, controllers, and interconnection system equipment for use with distributed energy resources [Online]. Available: http://ulstandardsinfonet.ul.com/scopes/scopes.asp?fn=1741.html

[6] IEEE Standards Association (2010, Dec. 28). IEEE 1547 Standard for Interconnecting Distributed Resources with Electric Power Systems [Online]. Available: http://grouper.ieee.org/groups/scc21/1547/1547_index.html

[7] Pacific Gas and Electric Company (2004, Aug. 11). Summary of General Technical Requirements for the Interconnection of Distributed Generation (DG) to PG\&E's Distribution System [Online]. Available: http://www.pge.com/includes/docs/pdfs/shared/newgenerator/ebio/dg_technical_ white_paper_0419_attachment.pdf

[8] National Fire Protection Association (n.d.). NFPA 70® National Electric Code® 2011 Edition [Online]. Available: http://www.nfpa.org/onlinepreview/online_preview_document.asp?id=7011SB

[9] D. Braun. "Soft Switching DC-DC Conversion to Harvest Exercise Machine Electricty EISG Proposal," 2010.

[10] A.J. Hilario, "Energy Harvesting from Elliptical Machines Using Four-Switch Buck Boost Topology," Cal Poly Digital Commons. 2011. [Online]. http://digitalcommons.calpoly.edu/theses/511/ 
[11] N. Mohan, T. Undeland, et al., "Switching DC Power Supplies," in Power Electronics: Converters, Applications and Design, $2^{\text {nd }}$ Ed. New York: Wiley, 1995, pp. 310-319.

[12] A.J. Prabhakar; J.D. Bollinger; H.T. Ma; M. Ferdowsi; K. Corzine. "Efficiency analysis and comparative study of hard and soft switching DC-DC converters in a wind farm," Industrial Electronics, 2008. IECON 2008. 34th Annual Conference of IEEE, pp.2156-2160, 2008.

[13] NFN Taufik, Advanced Power Electronics Design, Winter 2010, San Luis Obispo, CA: California Polytechnic State University, 2010.

[14] N. Mohan, T. Undeland, et al., "DC-DC Switch-Mode Converters," in Power Electronics: Converters, Applications and Design, $2^{\text {nd }}$ Ed. New York: Wiley, 1995, pp. 179-188.

[15] M. Gaboriault; A. Notman. "A high efficiency, noninverting, buck-boost DC-DC converter," Applied Power Electronics Conference and Exposition, 2004. APEC '04. Nineteenth Annual IEEE, vol.3, pp. 1411- 1415, 2004.

[16] W. Huang; K. Yen; G. Roig; E. Lee. "Voltage divided noninverting Cuk converter with large conversion ratios," Southeastcon '91., IEEE Proceedings of, vol. 2, pp.1005-1007, 1991.

[17] M. Orellana; S. Petibon; B. Estibals; C. Alonso. "Four Switch Buck-Boost Converter for Photovoltaic DC-DC power applications," IECON 2010 - 36th Annual Conference on IEEE Industrial Electronics Society, pp.469-474, 2010.

[18] Wikipedia, "Single-ended primary-inductor converter", 2012. [Online]. http://en.wikipedia.org/SEPIC

[19] E. Niculescu; M.C. Niculescu; D.M. Purcaru. "Modelling the PWM Zeta converter in discontinuous conduction mode," Electrotechnical Conference, 2008. MELECON 2008. The 14th IEEE Mediterranean, pp.651-657.

[20] K.B. Park; H.W. Seong; H.S. Kim; G.W. Moon; M. J. Youn. "Integrated boost-sepic converter for high step-up applications," Power Electronics Specialists Conference, 2008. PESC 2008. IEEE, pp.944-950, 2008.

[21] Maxim IC, "SEPIC Equations and Component Ratings," Maxim Design Support, 2011. [Online]. http://www.maxim-ic.com/app-notes/index.mvp/id/1051 
[22] T.F. Wu; Y.K. Chen. "Modeling PWM DC/DC converters out of basic converter units," Power Electronics, IEEE Transactions on, vol.13, no.5, pp.870-881, 1998.

[23] J.P.M. Figueiredo; F.L. Tofoli; R.L. Alves. "Comparison of nonisolated dc-dc converters from the efficiency point of view," Power Electronics Conference (COBEP), 2011 Brazilian, pp.14-19, 2011.

[24] Texas Instruments, "Designing DC/DC converters based on ZETA topology." Texas Instruments. 2012. [Online]. http://www.ti.com/lit/an/slyt372/slyt372.pdf

[25] NFN Taufik, Introduction to Power Electronics, Fall 2009, San Luis Obispo, CA: California Polytechnic State University, 2009.

[26] A.C.-C. Hua; B.C.-y Tsai. "Design of a wide input range DC/DC converter based on SEPIC topology for fuel cell power conversion," Power Electronics Conference (IPEC), 2010 International, pp.311-316, 2010.

[27] Texas Instruments, "LM3478, LM3488 Application Note 1484 Designing A SEPIC Converter." DC/DC Controller (External Switch) - Step-Up Controller - LM3478 - TI.com. 2011. [Online]. http://www.ti.com/lit/an/slyt372/slyt372.pdf

[28] NFN Taufik, Switching Mode Power Supply: Components and Design, Spring 2010, San Luis Obispo, CA: California Polytechnic State University, 2010.

[29] J. Chen; R. Erickson; D. Maksimovic. "Averaged switch modeling of boundary conduction mode DC-to-DC converters," Industrial Electronics Society, 2001. IECON '01. The $27^{\text {th }}$ Annual Conference of the IEEE, vol.2, pp.844-849, 2001.

[30] C.H. Chia; P.S. Lei; R.C.-H. Chang. "A high-efficiency PWM DC-DC buck converter with a novel DCM control under light-load," Circuits and Systems (ISCAS), 2011 IEEE International Symposium on, pp.237-240, 2011.

[31] Y.T. Chen; C.H. Chen. "A DC-DC buck converter chip with integrated PWM/PFM hybrid mode control circuit," Power Electronics and Drive Systems, 2009. PEDS 2009. International Conference on, pp.181-186, 2009.

[32] G. Rizzoni, "Energy-Storage (Dynamic) Circuit Elements," in Principles and Applications of Electrical Engineering, $3^{\text {rd }}$ Ed. Boston: McGraw-Hill, 2000, p. 126.

[33] M. Sadiku and C.K. Alexander, "Inductors," in Fundamentals of Electric Circuits, $3^{\text {rd }}$ Ed. Boston: McGraw-Hill, 2005, pp. 212-213. 
[34] A. Pressman, K. Billings, et al., "MOSFET Basics," in Switching Power Supply Design, $3^{\text {rd }}$ Ed. New York: McGraw-Hill, 2009, pp. 459-464.

[35] Linear Technology, "Wide-Input Range, No Rsense Current Mode Boost, Flyback, and SEPIC Controller," LTC1871 product page, 2010. [Online].

http://cds.linear.com/docs/Datasheet/1871fe.pdf

[36] Vishay Siliconix, "Filter Inductors, High Current, Radial Leaded," Vishay IHV28BZ60 datasheet, 2012. [Online]. http://www.vishay.com/docs/34022/ihv.pdf

[37] S. Franco, "Stability," in Design with Operational Amplifiers and Analog Integrated Circuits, $3^{\text {rd }}$ Ed. Boston: McGraw-Hill, 2002, pp. 365-372.

[38] A.J. Yiin; R.D. Schrimpf; K.F. Galloway. "Gate-charge measurements for irradiated n channel DMOS power transistors," Nuclear Science, IEEE Transactions on, vol.38, no.6, pp.1352-1358, 1991.

[39] Infineon Technologies, "OptiMOSTM 3 Power-Transistor," IPP110N20N3 datasheet, 2011. [Online]. http://www.infineon.com/dgdl/IPP_I_110N20N3+G_IPB107N20N3+G+Rev2.3.p df?folderId=db3a3043163797a6011637d4bae7003b\&fileId=db3a3043243b5f170 $124968 \mathrm{e} 7 \mathrm{~d} 118 \mathrm{e} 7$

[40] Micro Commercial Components, "MBR2020CT Thru MBR20100CT," MBR20100CT product page, 2012. [Online]. http://61.222.192.61/mccsemi/up_pdf/MBR2020CT-MBR20100CT\%28TO$220 \mathrm{AB} \% 29$.pdf

[41] C.J. Tseng and C.L. Chen. "A passive lossless snubber cell for nonisolated PWM DC/DC converters," Industrial Electronics, IEEE Transactions on, vol.45, no.4, pp.593-601, 1998.

[42] A. Elasser and D. A. Torry, "Soft switching active snubbers for dc/dc converters," IEEE Trans. Power Electron., vol. 11, pp. 710-722, 1996.

[43] G. Hua and F. C. Lee, "Soft-switching techniques in PWM converters," IEEE Trans. Ind. Electron., vol. 42, pp. 595-603, 1995.

[44] R. L. Lin and F. C. Lee, "Novel zero-current-switching-zero-voltage switching converters," in Proc. PESC'96, pp. 438-442, 1996. 
[45] R. Streit and D. Tollik, "High efficiency telecom rectifier using a novel softswitched boost based input current shaper," in Proc. INTELEC'91, pp. 720-726, 1991.

[46] S. J. Finney, B. W. Williams, and T. C. Green, "The RCD snubber revisited," in Conf. Rec. IEEE-IAS Annu. Meeting, pp. 1267-1273, 1993.

[47] M. Ferranti, P. Ferraris, A. Fratta, and F. Profumo, "Solar energy supply system for induction motors and various loads," presented at INTELEC'89, Paper 15.7, 1989.

[48] N. Machin and T. Vescovi, "Very high efficiency techniques and their selective application to the design of a 70 A rectifier," in Proc. INTELEC'93, pp. 126-133, 1993.

[49] J. Lambert, J. Vieira, L. C. Freitas M. Vilela, and V. Farias, "A boost PWM softsingle switched converter without stresses of voltage and current," in Proc. APEC'96, pp. 469-474, 1996.

[50] X. He, B. W. Williams, S. J. Finney, Z. Qian, and T. C. Green, "New snubber circuit with passive energy recovery for power inverters," Proc. IEE-Elect. Power Applicat., vol. 143, no. 5, pp. 403-408, 1996.

[51] G. Carli, "Harmonic distortion reduction schemes for a new 100 A-48V power supply," in Proc. INTELEC'92, pp. 524-531, 1992.

[52] M. Sadiku and C.K. Alexander, "Capacitors," in Fundamentals of Electric Circuits, $3^{\text {rd }}$ Ed. Boston: McGraw-Hill, 2005, pp. 204.

[53] G. Hollister, "Elliptical Machine DC-DC Converter for the Energy Harvesting From Exercise Machines Project," Cal Poly Digital Commons. 2011. [Online]. http://www.digitalcommons.calpoly.edu/eesp/70/

[54] N.K. Lovgren, "Energy Harvesting From Exercise Machines: Forward Converters with a Central Inverter," Cal Poly Digital Commons. 2011. [Online]. http://www.digitalcommons.calpoly.edu/theses/520/

[55] Linear Technology, "LT4356-1/LT4356-2 Surge Stopper,” LT4356-1/LT4356-2 product page, 2010. [Online]. http://cds.linear.com/docs/Datasheet/4356fa.pdf

[56] Lumex, "SML-LX1206SRC-TR datasheet," Lumex: LED \& LCD Solutions: Product, 2012. [Online]. http://www.lumex.com/specs/SML-LX1206SRC-TR.pdf 
[57] IXYS Corporation, "GigaMOSTM Power MOSFET," IXFK230N20T product page, 2012. [Online]. http://ixdev.ixys.com/DataSheet/DS100133\%28IXFKFX230N20T\%29.pdf

[58] D. Gerke; W. Kimmel, "EMI and circuit components: Where the rubber meets the road." Electronic Design News. 2000. [Online].

http://www.edn.com/article/491125-

EMI_and_circuit_components_Where_the_rubber_meets_the_road.php

[59] Nippon Chemi-Con, "KHD251E156M99C0B00 datasheet," KHD251E156M99C0B00 product page, 2012. [Online]. http://www.chemicon.co.jp/e/catalog/pdf/ce-e/ce-sepa-e/ce-thd-e-110701.pdf

[60] CapSite 2009, "ESR," Introduction to Capacitors, 2012. [Online]. http://my.execpc.com/ endlr/esr.html

[61] Microsemi, "High Voltage Schottky Diode," APT30S20BG product page, 2012. [Online]. http://www2.microsemi.com/datasheets/30S20B_S.pdf

[62] Daycounter, Inc. Engineering Services, "Heatsink Temperature Calculator," Electronics Calculators, 2012. [Online]. http://www.daycounter.com/Calculators/Heat-Sink-Temperature-Calculator.phtml

[63] Ohmite, "M Series Heatsinks: Heatsinks with clips for TO-264 and TO-247," MV102-55E product page, 2012. [Online]. http://www.ohmite.com/cat/sink_m.pdf

[64] Aavid Thermalloy, “TO-220 \& TO-202 Heat Sinks,” 531202B02500G - Board level heatsinks, 2012. [Online]. http://www.aavid.com/sites/default/files/literature/Aavid-Board-Level-HeatsinksCatalog.pdf\#page $=59$

[65] Ohmite, "W Series Heatsinks For TO-220, TO-247, and TO-264 devices" WAT247-101E product page, 2012. [Online]. http://www.ohmite.com/cat/sink_w.pdf

[66] Aavid Thermalloy, “TO-220 \& TO-202 Heat Sinks," 574502B00000G - Board level heatsinks, 2012. [Online].

http://www.aavid.com/sites/default/files/literature/Aavid-Board-Level-HeatsinksCatalog.pdf\#page $=45$

[67] D. Rhodes; M. Davis-Marsh. "Proper PCB Layout Techniques Maximize Power Module Performance," 2010. [Online]. http://i.cmpnet.com/powermanagementdesignline/2010/08/C0610posted.pdf 
[68] S. Maniktala. "Proper Board Layout Makes Switchers Work Right the First Time." EE Times. 2002. [Online].

http://www.eetimes.com/electronicsnews/4164323/Proper-board-layout-makesswitchers-work-right-the-first-time

[69] Maxim Integrated Products. "Correct Board Layout Lowers EMI of Switchmode Converters." Maxim IC. 2005. [Online]. http://www.maxim-ic.com/appnotes/ index.mvp/id/3645

[70] ExpressPCB, "Tips for Designing PCBs." ExpressPCB - Free PCB Layout Software - Low Cost Circuit Boards - Top Quality PCB Manufacturing. 2010. [Online]. http://www.expresspcb.com/ExpressPCBHtm/Tips.htm

[71] Curious Inventor, "Tap \& Drill Sizes." Tools, Parts, Kits for DIY'ers - Curious Inventor. 2012. [Online]. http://store.curiousinventor.com/guides/tap_drill

[72] Axotron, "Crowbar circuit." Articles about electronics. 2012. [Online]. http://www.axotron.se/index_en.php?page=26

[73] Circuitmaniac.com, "Electronic crowbar protection." Power Plant. 2012. [Online]. http://www.circuitmaniac.com/2009/04/17/electronic-crowbar-protection/

[74] National Semiconductor (2007, May). Application Note AN-1628: Minimizing FET Losses for a High-Input Rail Buck Converter [Online]. Available:

http://web.archive.org/web/20070821192228/http://www.national.com/nationaled ge/may07/

[75] Linear Technology. Datasheet - LTC3862 - Multi-Phase Current Mode Step-Up DC/DC Controller [Online]. http://cds.linear.com/docs/Datasheet/3862fb.pdf

[76] ExpressPCB. Manufacturing Specs - Production Service [Online]. Available: http://www.expresspcb.com/ExpressPCBHtm/SpecsProduction.htm 